University of South Florida

DIGITAL COMMONS

Digital Commons @ University of

@ UNIVERSITY OF SOUTH FLORIDA

South Florida

3-23-2010

\title{
Theoretical and Experimental Simulation of Passive Vacuum Solar Flash Desalination
}

Mohammad Abutayeh

University of South Florida

Follow this and additional works at: https://digitalcommons.usf.edu/etd

Part of the American Studies Commons

\section{Scholar Commons Citation}

Abutayeh, Mohammad, "Theoretical and Experimental Simulation of Passive Vacuum Solar Flash Desalination" (2010). USF Tampa Graduate Theses and Dissertations.

https://digitalcommons.usf.edu/etd/1555

This Dissertation is brought to you for free and open access by the USF Graduate Theses and Dissertations at Digital Commons @ University of South Florida. It has been accepted for inclusion in USF Tampa Graduate Theses and Dissertations by an authorized administrator of Digital Commons @ University of South Florida. For more information, please contact digitalcommons@usf.edu. 
Theoretical and Experimental Simulation of Passive Vacuum Solar Flash Desalination

by

Mohammad Abutayeh

A dissertation submitted in partial fulfillment

of the requirements for the degree of

Doctor of Philosophy

Department of Chemical \& Biomedical Engineering

College of Engineering

University of South Florida

Major Professor: D. Yogi Goswami, Ph.D.

Elias K. Stefanakos, Ph.D.

Scott W. Campbell, Ph.D.

John T. Wolan, Ph.D.

Thomas L. Crisman, Ph.D.

Date of Approval:

March 23, 2010

Keywords: Solar Energy, Seawater Separation, Desalting, Distillation, Evaporation

(C) Copyright 2010, Mohammad Abutayeh 


\section{DEDICATION}

To the loving memory of my brother, Hussein 


\section{ACKNOWLEDGEMENTS}

I would like to first thank Dr. D. Yogi Goswami for his ideas and research assistance that made this exploration possible. I would also like to express my gratitude to Dr. Elias K. Stefanakos for his professional leadership and generous support.

Then, I would like to express my sincere appreciation to Dr. Scott W. Campbell for his tremendous knowledge that guided me throughout my career. I would also like to thank Dr. John T. Wolan for his valued suggestions and support all through my studies.

My thanks must also go to Dr. Thomas L. Crisman for his appreciated input and his well regarded encouragement. Finally, I would like to extend my deepest appreciation to my family and friends for their support and inspiration. 


\section{TABLE OF CONTENTS}

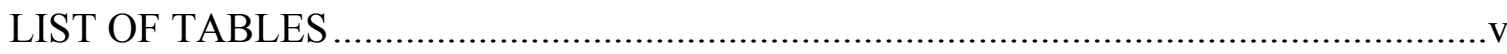

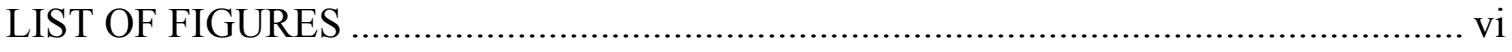

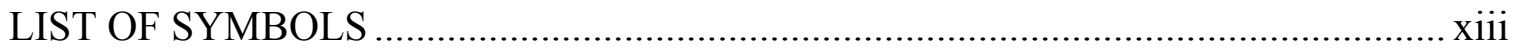

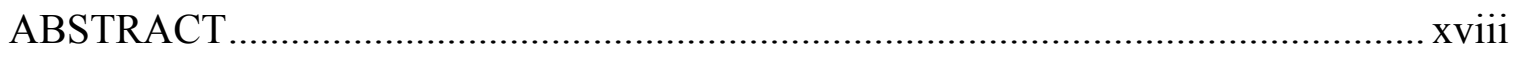

CHAPTER 1. INTRODUCTION ........................................................................

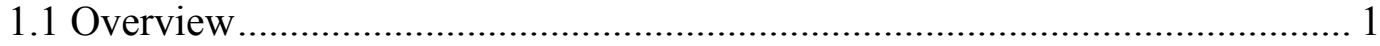

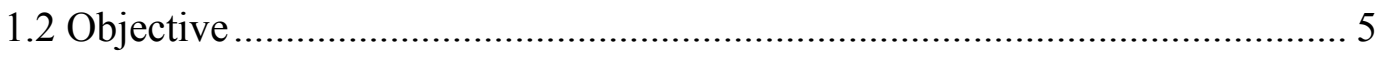

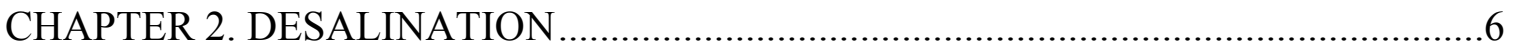

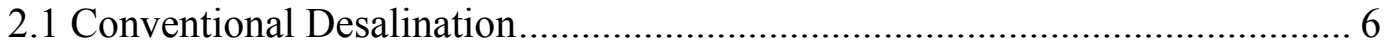

2.1.1 Multiple Effect Evaporation ..................................................... 9

2.1.2 Multi-Stage Flash ................................................................. 10

2.1.3 Vapor Compression .............................................................. 11

2.1.4 Indirect Contact Freezing ........................................................ 12

2.1.5 Reverse Osmosis .................................................................. 13

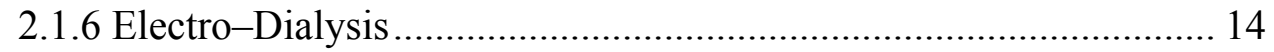

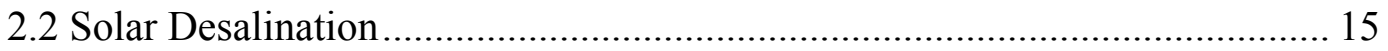

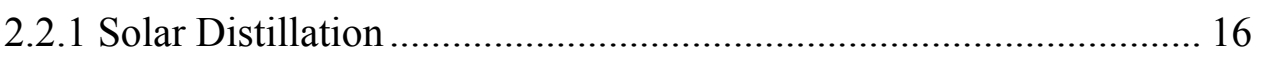

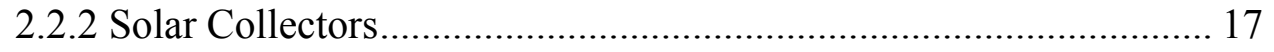

2.2.3 Thermal Energy Storage ......................................................... 18

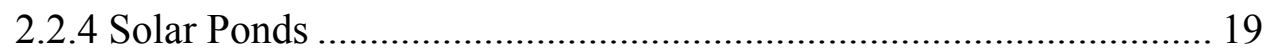

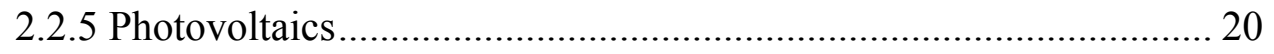

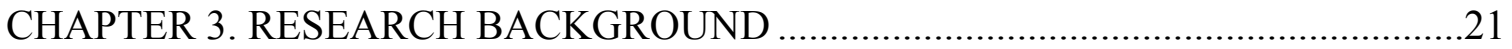

3.1 Renewable Energy Desalination Systems................................................ 21

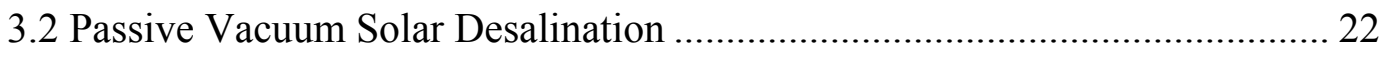

3.3 Passive Vacuum Solar Flash Desalination..................................................... 23

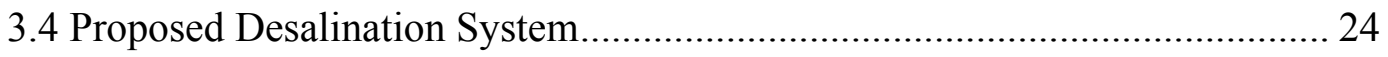




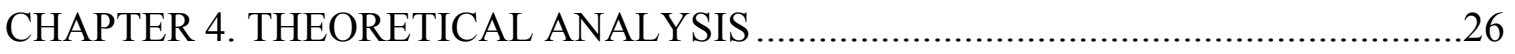

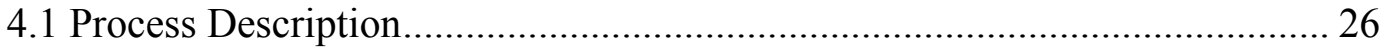

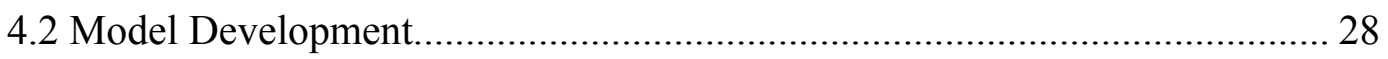

4.2.1 Mass and Energy Balance........................................................... 29

4.2.2 Equilibrium Distribution Coefficients ............................................. 35

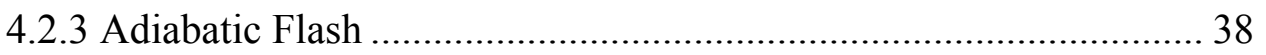

4.2.4 Heat Transfer ............................................................................ 40

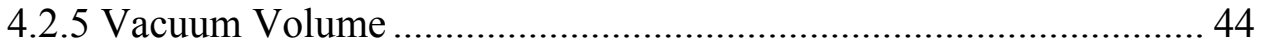

4.2.6 Vacuum Pressure ………………………………........................ 47

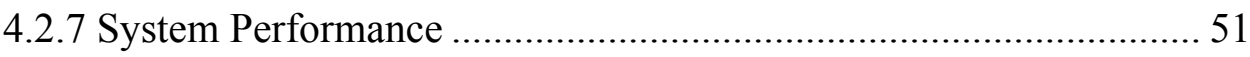

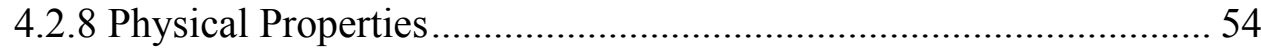

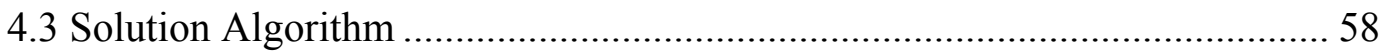

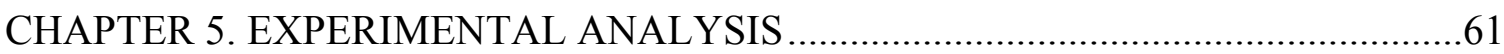

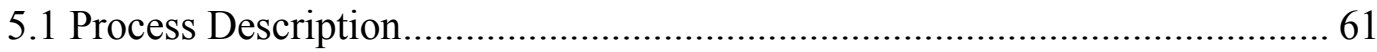

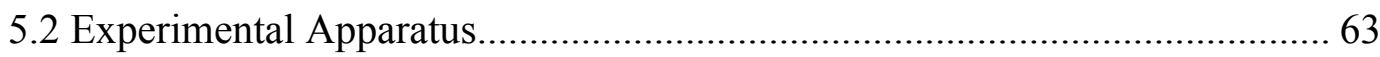

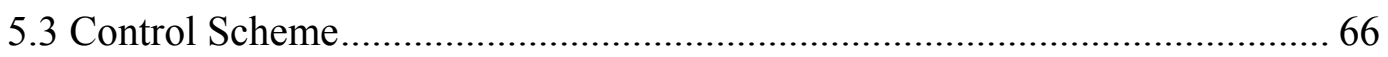

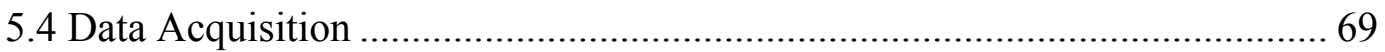

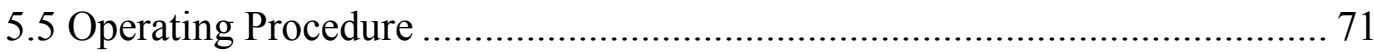

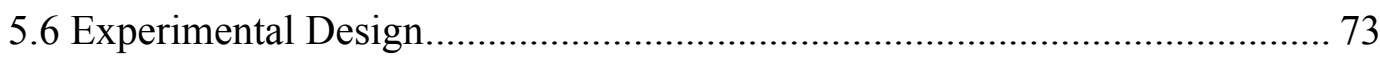

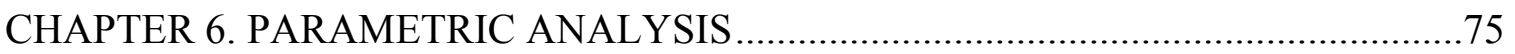

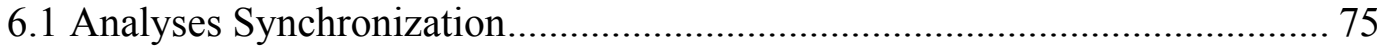

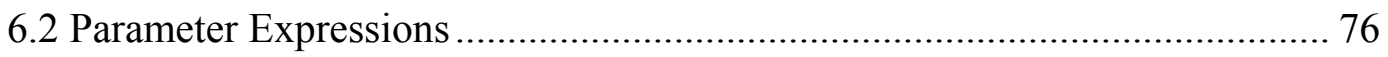

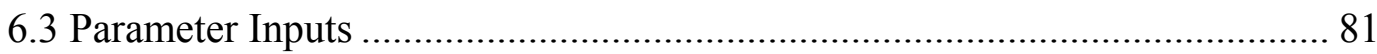

6.4 Equipment Specifications ……………………………........................... 85

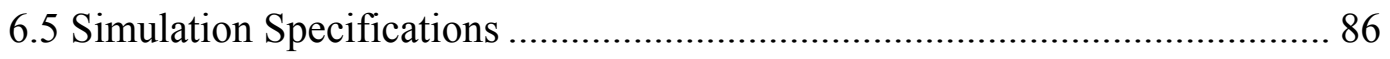

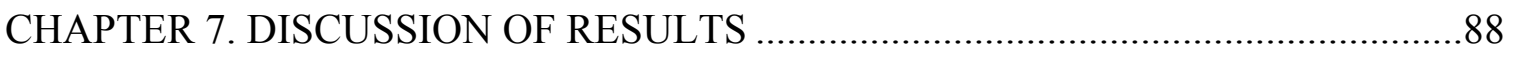

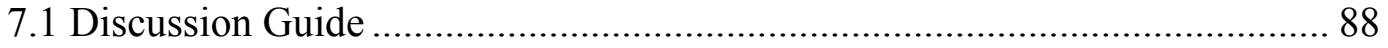

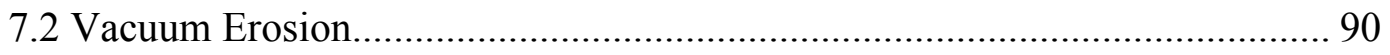

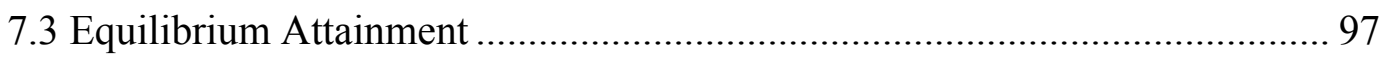

7.4 Equilibrium Departure ............................................................................ 104

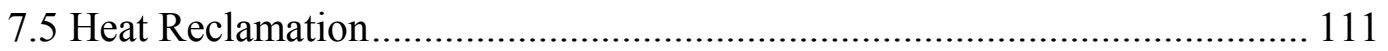




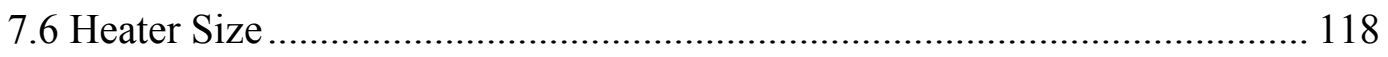

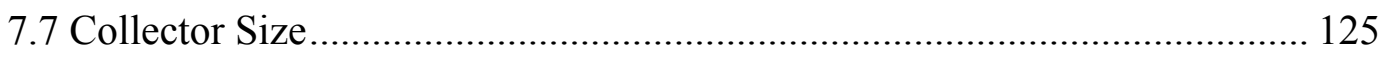

7.8 System Throughput .............................................................................. 132

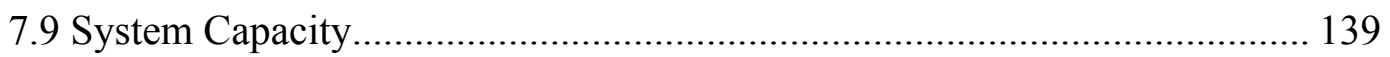

7.10 Process Feasibility ................................................................................ 146

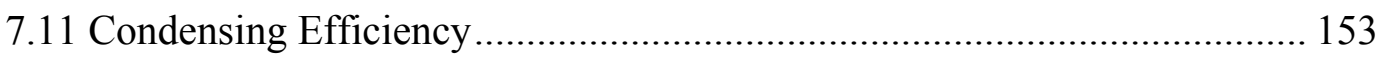

7.12 Recovery Efficiency............................................................................. 160

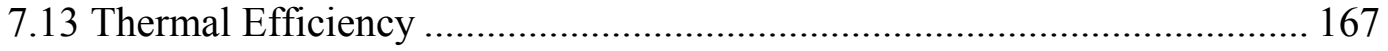

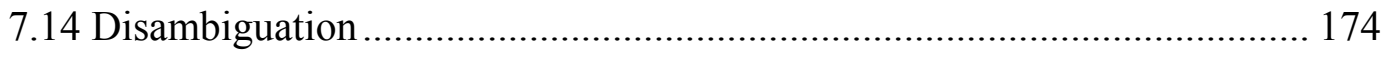

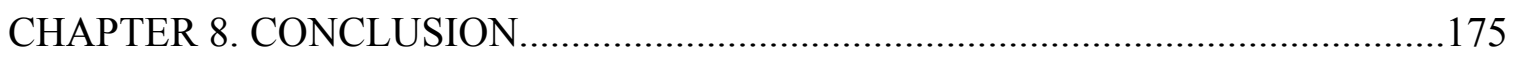

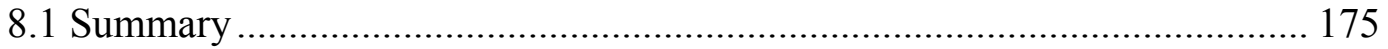

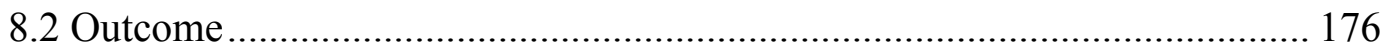

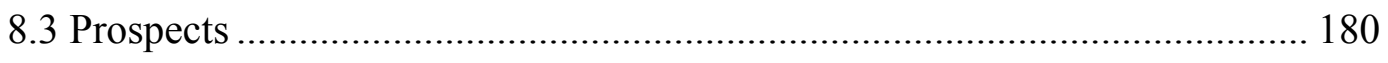

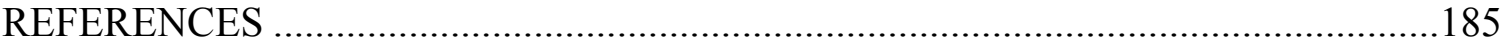

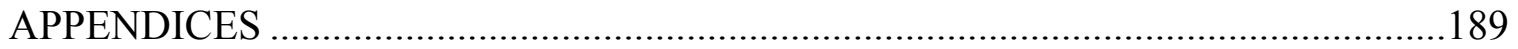

Appendix A. The operating procedure............................................................ 190

Appendix B. SUPERTRAPPTM code to generate K-values ................................ 195

Appendix C. Matlab code for $F_{C T}$ data regression............................................ 202

Appendix D. Matlab code for NEA data regression............................................ 203

Appendix E. Matlab code for $\gamma_{H 2 O}$ data regression............................................. 204

Appendix F. Matlab code for $\psi$ data regression ............................................... 205

Appendix G. Matlab code for $H C_{N 2}$ data regression........................................... 206

Appendix H. Matlab code for $\mathrm{HC}_{\mathrm{O} 2}$ data regression ........................................... 207

Appendix I. Matlab code for $H C_{A r}$ data regression........................................... 208

Appendix J. Matlab code for $\mathrm{HC}_{\mathrm{CO} 2}$ data regression.......................................... 209

Appendix K. Matlab code for $P_{\mathrm{H} 2 \mathrm{O}}{ }^{\text {sat }}$ data regression.......................................... 210

Appendix L. Sample TK Solver code for data mining ........................................ 211

Appendix M. Sample TK Solver code for model simulation .............................. 228

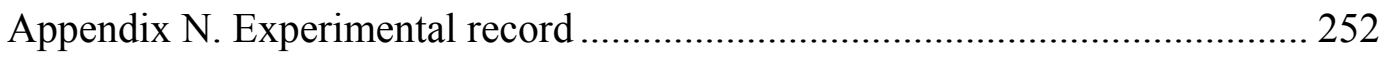

Appendix O. Experimental equipment specifications ....................................... 253

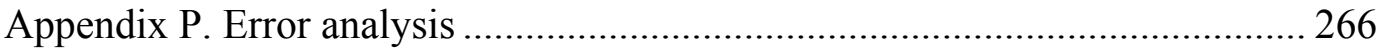


ABOUT THE AUTHOR End Page 


\section{LIST OF TABLES}

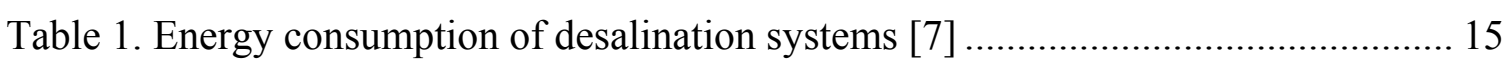

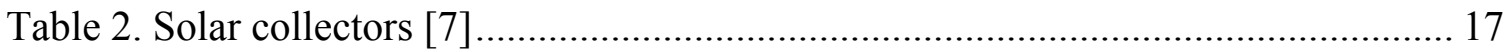

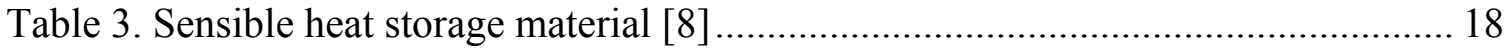

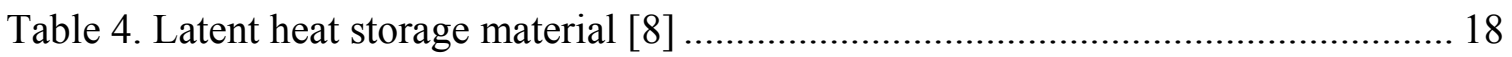

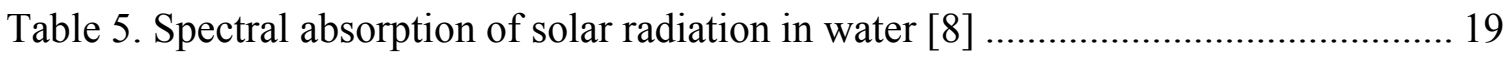

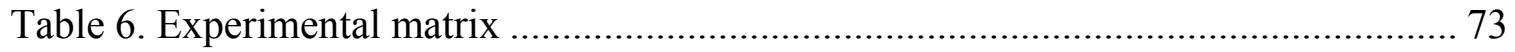

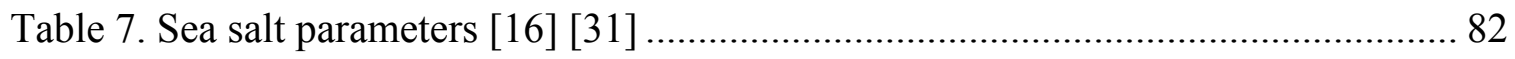

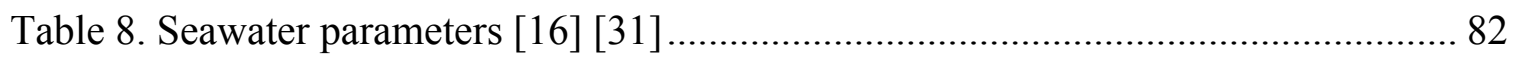

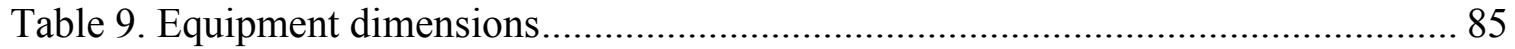

Table 10. Heat transfer equipment parameters .................................................... 85

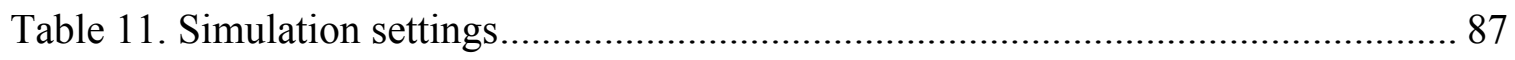

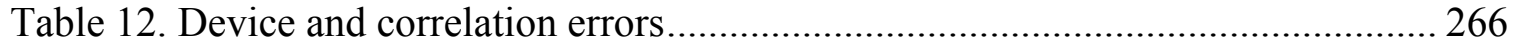

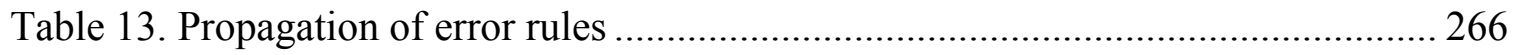




\section{LIST OF FIGURES}

Figure 1. Estimated water consumption of US counties for 2000 [1] ............................ 3

Figure 2. Estimated energy consumption per capita of US states for 2001 [1] .................. 3

Figure 3. Estimated energy consumption per capita of Florida and the US [1]................. 4

Figure 4. Monthly average daily solar insolation in the US [3] ..................................... 4

Figure 5. Global distribution of installed desalination capacity by technology [5]........... 8

Figure 6. Global distribution of installed desalination capacity by region [5] .................. 8

Figure 7. Multiple effect evaporation .................................................................. 9

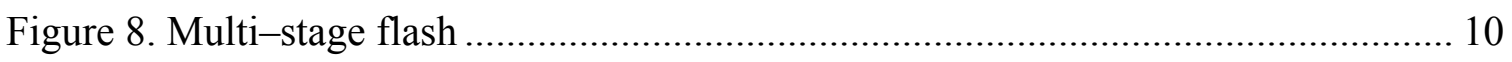

Figure 9. Mechanical vapor compression ................................................................. 11

Figure 10. Indirect contact freezing ......................................................................... 12

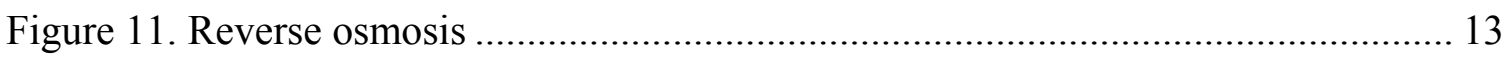

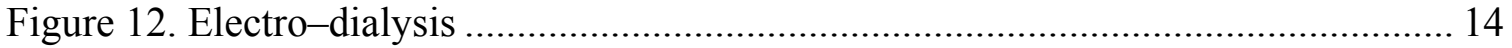

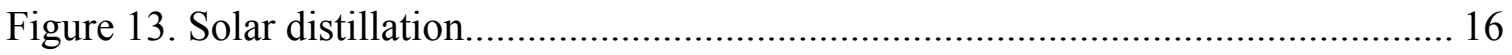

Figure 14. Vertical cross section of a solar pond........................................................ 19

Figure 15. Photovoltaic cell schematics.................................................................. 20

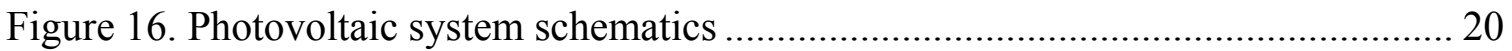

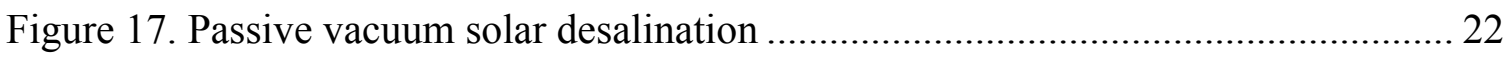

Figure 18. Passive vacuum solar flash desalination....................................................... 23

Figure 19. Single-stage solar flash desalination system................................................ 25

Figure 20. Multi-stage solar flash desalination system ................................................ 25

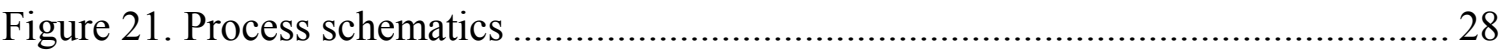

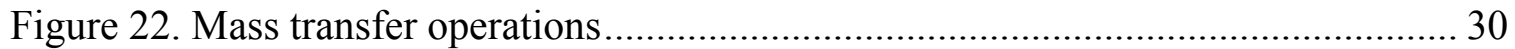

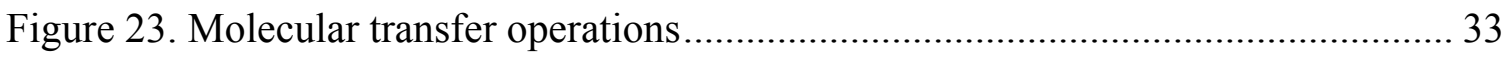

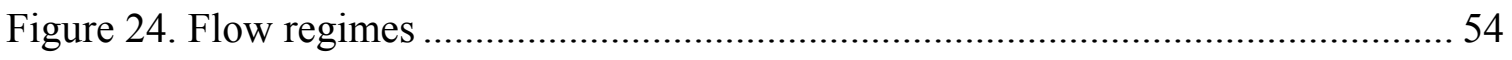

Figure 25. Developed model solution algorithm ........................................................ 59

Figure 26. Process and instrumentation diagram of the experimental unit....................... 62 


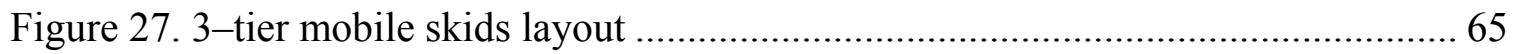

Figure 28. Feedback control loops of the experimental unit .......................................... 66

Figure 29. Block diagram of the flash temperature feedback control loop ...................... 68

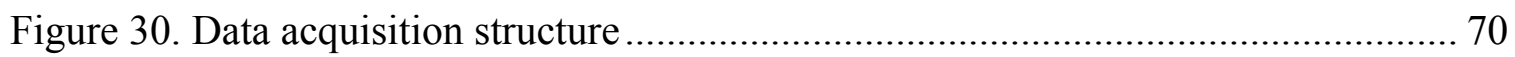

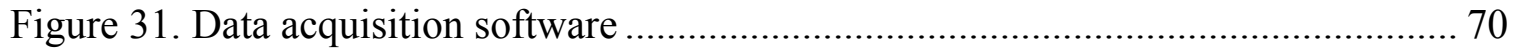

Figure 32. Overall view of the experimental unit .................................................... 74

Figure 33. Counter-current departure correction factor of condenser tube...................... 79

Figure 34. Non-equilibrium allowance representation.................................................. 79

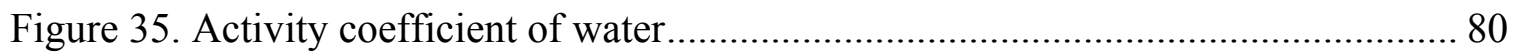

Figure 36. Gas phase molecular content correction factor .............................................. 80

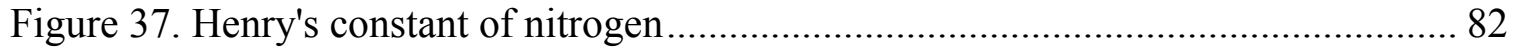

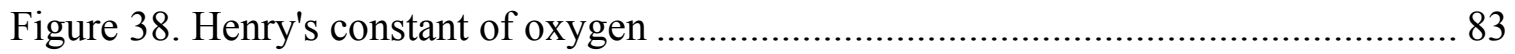

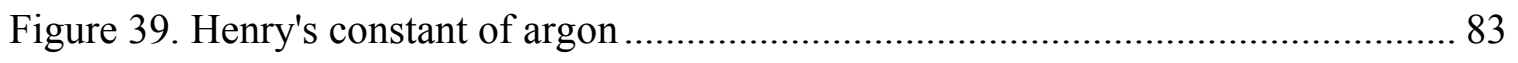

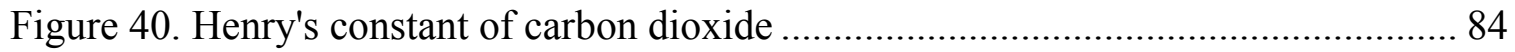

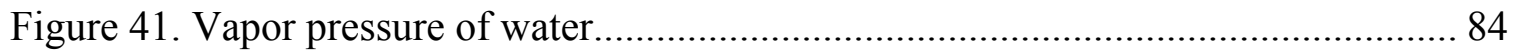

Figure 42. Modeled vacuum pressure profiles at lower flow .......................................... 91

Figure 43. Experimental vacuum pressure profiles at lower flow .................................... 91

Figure 44. Modeled vacuum pressure profiles at higher flow ……………………........... 92

Figure 45. Experimental vacuum pressure profiles at higher flow .................................. 92

Figure 46. Vacuum pressure at $50^{\circ} \mathrm{C}$ flash and lower flow .......................................... 93

Figure 47. Vacuum pressure at $50^{\circ} \mathrm{C}$ flash and higher flow .......................................... 93

Figure 48. Vacuum pressure at $60^{\circ} \mathrm{C}$ flash and lower flow ............................................. 94

Figure 49. Vacuum pressure at $60^{\circ} \mathrm{C}$ flash and higher flow ........................................ 94

Figure 50. Vacuum pressure at $70^{\circ} \mathrm{C}$ flash and lower flow ............................................ 95

Figure 51. Vacuum pressure at $70^{\circ} \mathrm{C}$ flash and higher flow ......................................... 95

Figure 52. Vacuum pressure at $80^{\circ} \mathrm{C}$ flash and lower flow ............................................ 96

Figure 53. Vacuum pressure at $80^{\circ} \mathrm{C}$ flash and higher flow ............................................ 96

Figure 54. Modeled equilibrium temperature profiles at lower flow.................................. 98

Figure 55. Experimental equilibrium temperature profiles at lower flow ........................ 98

Figure 56. Modeled equilibrium temperature profiles at higher flow ………………...... 99

Figure 57. Experimental equilibrium temperature profiles at higher flow ....................... 99 
Figure 58. Equilibrium temperature at $50^{\circ} \mathrm{C}$ flash and lower flow............................ 100

Figure 59. Equilibrium temperature at $50^{\circ} \mathrm{C}$ flash and higher flow ............................ 100

Figure 60. Equilibrium temperature at $60^{\circ} \mathrm{C}$ flash and lower flow............................. 101

Figure 61. Equilibrium temperature at $60^{\circ} \mathrm{C}$ flash and higher flow ............................ 101

Figure 62. Equilibrium temperature at $70^{\circ} \mathrm{C}$ flash and lower flow............................. 102

Figure 63. Equilibrium temperature at $70^{\circ} \mathrm{C}$ flash and higher flow ........................... 102

Figure 64. Equilibrium temperature at $80^{\circ} \mathrm{C}$ flash and lower flow.............................. 103

Figure 65. Equilibrium temperature at $80^{\circ} \mathrm{C}$ flash and higher flow ............................ 103

Figure 66. Modeled concentrated brine temperature profiles at lower flow.................. 105

Figure 67. Experimental concentrated brine temperature profiles at lower flow ........... 105

Figure 68. Modeled concentrated brine temperature profiles at higher flow ................ 106

Figure 69. Experimental concentrated brine temperature profiles at higher flow .......... 106

Figure 70. Concentrated brine temperature at $50^{\circ} \mathrm{C}$ flash and lower flow .................... 107

Figure 71. Concentrated brine temperature at $50^{\circ} \mathrm{C}$ flash and higher flow ................... 107

Figure 72. Concentrated brine temperature at $60^{\circ} \mathrm{C}$ flash and lower flow ................... 108

Figure 73. Concentrated brine temperature at $60^{\circ} \mathrm{C}$ flash and higher flow .................. 108

Figure 74. Concentrated brine temperature at $70^{\circ} \mathrm{C}$ flash and lower flow .................... 109

Figure 75. Concentrated brine temperature at $70^{\circ} \mathrm{C}$ flash and higher flow .................. 109

Figure 76. Concentrated brine temperature at $80^{\circ} \mathrm{C}$ flash and lower flow ................... 110

Figure 77. Concentrated brine temperature at $80^{\circ} \mathrm{C}$ flash and higher flow ................... 110

Figure 78. Modeled preheat temperature profiles at lower flow ................................ 112

Figure 79. Experimental preheat temperature profiles at lower flow .......................... 112

Figure 80. Modeled preheat temperature profiles at higher flow ............................... 113

Figure 81. Experimental preheat temperature profiles at higher flow .......................... 113

Figure 82. Preheat temperature at $50^{\circ} \mathrm{C}$ flash and lower flow .................................... 114

Figure 83. Preheat temperature at $50^{\circ} \mathrm{C}$ flash and higher flow .................................. 114

Figure 84. Preheat temperature at $60^{\circ} \mathrm{C}$ flash and lower flow .................................. 115

Figure 85. Preheat temperature at $60^{\circ} \mathrm{C}$ flash and higher flow ................................. 115

Figure 86. Preheat temperature at $70^{\circ} \mathrm{C}$ flash and lower flow .................................. 116

Figure 87. Preheat temperature at $70^{\circ} \mathrm{C}$ flash and higher flow ................................. 116

Figure 88. Preheat temperature at $80^{\circ} \mathrm{C}$ flash and lower flow .................................. 117 
Figure 89. Preheat temperature at $80^{\circ} \mathrm{C}$ flash and higher flow..... 117

Figure 90. Modeled heat load profiles at lower flow................................................. 119

Figure 91. Mined heat load profiles at lower flow..................................................... 119

Figure 92. Modeled heat load profiles at higher flow ................................................ 120

Figure 93. Mined heat load profiles at higher flow ................................................ 120

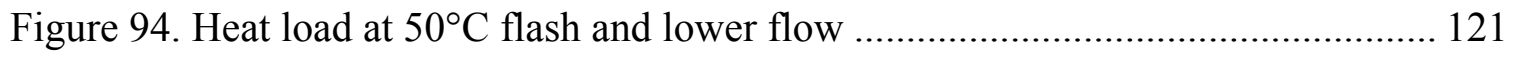

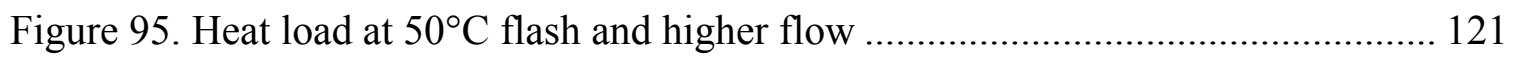

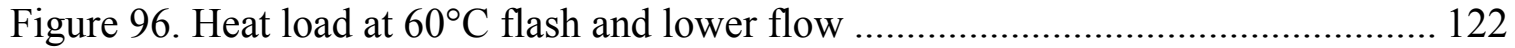

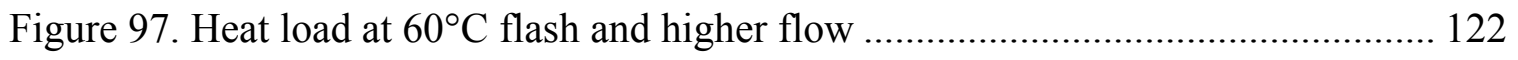

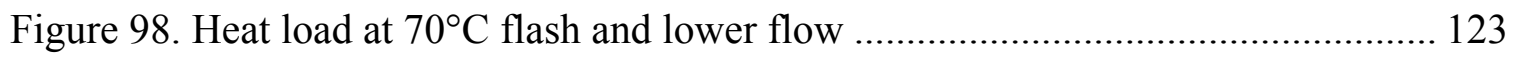

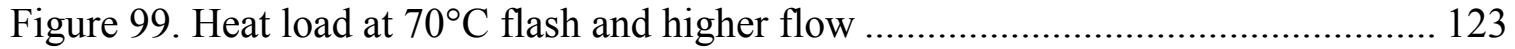

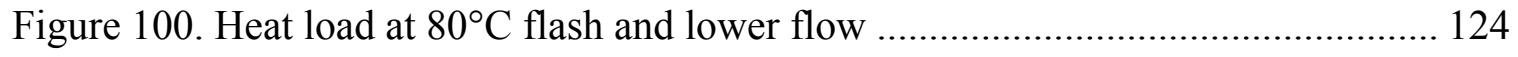

Figure 101. Heat load at $80^{\circ} \mathrm{C}$ flash and higher flow ............................................. 124

Figure 102. Modeled required solar collection area profiles at lower flow ................... 126

Figure 103. Mined required solar collection area profiles at lower flow...................... 126

Figure 104. Modeled required solar collection area profiles at higher flow .................. 127

Figure 105. Mined required solar collection area profiles at higher flow ..................... 127

Figure 106. Required solar collection area at $50^{\circ} \mathrm{C}$ flash and lower flow .................... 128

Figure 107. Required solar collection area at $50^{\circ} \mathrm{C}$ flash and higher flow ................... 128

Figure 108. Required solar collection area at $60^{\circ} \mathrm{C}$ flash and lower flow .................... 129

Figure 109. Required solar collection area at $60^{\circ} \mathrm{C}$ flash and higher flow ................... 129

Figure 110. Required solar collection area at $70^{\circ} \mathrm{C}$ flash and lower flow .................... 130

Figure 111. Required solar collection area at $70^{\circ} \mathrm{C}$ flash and higher flow ................... 130

Figure 112. Required solar collection area at $80^{\circ} \mathrm{C}$ flash and lower flow ..................... 131

Figure 113. Required solar collection area at $80^{\circ} \mathrm{C}$ flash and higher flow ................... 131

Figure 114. Modeled fresh water production rate profiles at lower flow ...................... 133

Figure 115. Mined fresh water production rate profiles at lower flow ......................... 133

Figure 116. Modeled fresh water production rate profiles at higher flow .................... 134

Figure 117. Mined fresh water production rate profiles at higher flow ........................ 134

Figure 118. Fresh water production rate at $50^{\circ} \mathrm{C}$ flash and lower flow ....................... 135

Figure 119. Fresh water production rate at $50^{\circ} \mathrm{C}$ flash and higher flow....................... 135 
Figure 120. Fresh water production rate at $60^{\circ} \mathrm{C}$ flash and lower flow ........................ 136

Figure 121. Fresh water production rate at $60^{\circ} \mathrm{C}$ flash and higher flow....................... 136

Figure 122. Fresh water production rate at $70^{\circ} \mathrm{C}$ flash and lower flow ......................... 137

Figure 123. Fresh water production rate at $70^{\circ} \mathrm{C}$ flash and higher flow....................... 137

Figure 124. Fresh water production rate at $80^{\circ} \mathrm{C}$ flash and lower flow ........................ 138

Figure 125. Fresh water production rate at $80^{\circ} \mathrm{C}$ flash and higher flow...................... 138

Figure 126. Modeled fresh water production amount profiles at lower flow ................ 140

Figure 127. Mined fresh water production amount profiles at lower flow ................... 140

Figure 128. Modeled fresh water production amount profiles at higher flow ............... 141

Figure 129. Mined fresh water production amount profiles at higher flow.................. 141

Figure 130. Fresh water production amount at $50^{\circ} \mathrm{C}$ flash and lower flow .................. 142

Figure 131. Fresh water production amount at $50^{\circ} \mathrm{C}$ flash and higher flow .................. 142

Figure 132. Fresh water production amount at $60^{\circ} \mathrm{C}$ flash and lower flow .................. 143

Figure 133. Fresh water production amount at $60^{\circ} \mathrm{C}$ flash and higher flow .................. 143

Figure 134. Fresh water production amount at $70^{\circ} \mathrm{C}$ flash and lower flow ................... 144

Figure 135. Fresh water production amount at $70^{\circ} \mathrm{C}$ flash and higher flow .................. 144

Figure 136. Fresh water production amount at $80^{\circ} \mathrm{C}$ flash and lower flow ................... 145

Figure 137. Fresh water production amount at $80^{\circ} \mathrm{C}$ flash and higher flow................. 145

Figure 138. Modeled prime energy consumption profiles at lower flow ...................... 147

Figure 139. Mined prime energy consumption profiles at lower flow ........................ 147

Figure 140. Modeled prime energy consumption profiles at higher flow .................... 148

Figure 141. Mined prime energy consumption profiles at higher flow ........................ 148

Figure 142. Prime energy consumption at $50^{\circ} \mathrm{C}$ flash and lower flow ........................ 149

Figure 143. Prime energy consumption at $50^{\circ} \mathrm{C}$ flash and higher flow ........................ 149

Figure 144. Prime energy consumption at $60^{\circ} \mathrm{C}$ flash and lower flow ......................... 150

Figure 145. Prime energy consumption at $60^{\circ} \mathrm{C}$ flash and higher flow ........................ 150

Figure 146. Prime energy consumption at $70^{\circ} \mathrm{C}$ flash and lower flow ......................... 151

Figure 147. Prime energy consumption at $70^{\circ} \mathrm{C}$ flash and higher flow ....................... 151

Figure 148. Prime energy consumption at $80^{\circ} \mathrm{C}$ flash and lower flow ......................... 152

Figure 149. Prime energy consumption at $80^{\circ} \mathrm{C}$ flash and higher flow ........................ 152

Figure 150. Modeled condenser efficiency profiles at lower flow .............................. 154 
Figure 151. Experimental condenser efficiency profiles at lower flow. 154

Figure 152. Modeled condenser efficiency profiles at higher flow ............................. 155

Figure 153. Experimental condenser efficiency profiles at higher flow....................... 155

Figure 154. Condenser efficiency at $50^{\circ} \mathrm{C}$ flash and lower flow ................................ 156

Figure 155. Condenser efficiency at $50^{\circ} \mathrm{C}$ flash and higher flow ................................ 156

Figure 156. Condenser efficiency at $60^{\circ} \mathrm{C}$ flash and lower flow ................................ 157

Figure 157. Condenser efficiency at $60^{\circ} \mathrm{C}$ flash and higher flow ............................... 157

Figure 158. Condenser efficiency at $70^{\circ} \mathrm{C}$ flash and lower flow ................................ 158

Figure 159. Condenser efficiency at $70^{\circ} \mathrm{C}$ flash and higher flow ............................... 158

Figure 160. Condenser efficiency at $80^{\circ} \mathrm{C}$ flash and lower flow ................................ 159

Figure 161. Condenser efficiency at $80^{\circ} \mathrm{C}$ flash and higher flow ............................... 159

Figure 162. Modeled recovery efficiency profiles at lower flow ................................ 161

Figure 163. Experimental recovery efficiency profiles at lower flow ......................... 161

Figure 164. Modeled recovery efficiency profiles at higher flow .............................. 162

Figure 165. Experimental recovery efficiency profiles at higher flow ......................... 162

Figure 166. Recovery efficiency at $50^{\circ} \mathrm{C}$ flash and lower flow.................................. 163

Figure 167. Recovery efficiency at $50^{\circ} \mathrm{C}$ flash and higher flow................................ 163

Figure 168. Recovery efficiency at $60^{\circ} \mathrm{C}$ flash and lower flow.................................. 164

Figure 169. Recovery efficiency at $60^{\circ} \mathrm{C}$ flash and higher flow................................ 164

Figure 170. Recovery efficiency at $70^{\circ} \mathrm{C}$ flash and lower flow................................. 165

Figure 171. Recovery efficiency at $70^{\circ} \mathrm{C}$ flash and higher flow............................... 165

Figure 172. Recovery efficiency at $80^{\circ} \mathrm{C}$ flash and lower flow................................. 166

Figure 173 . Recovery efficiency at $80^{\circ} \mathrm{C}$ flash and higher flow................................ 166

Figure 174. Modeled thermal efficiency profiles at lower flow ................................. 168

Figure 175. Mined thermal efficiency profiles at lower flow ................................... 168

Figure 176. Modeled thermal efficiency profiles at higher flow ................................ 169

Figure 177. Mined thermal efficiency profiles at higher flow ................................... 169

Figure 178. Thermal efficiency at $50^{\circ} \mathrm{C}$ flash and lower flow ................................ 170

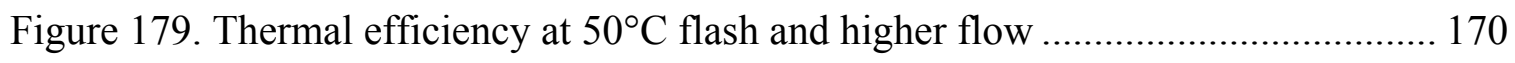

Figure 180. Thermal efficiency at $60^{\circ} \mathrm{C}$ flash and lower flow .................................. 171

Figure 181. Thermal efficiency at $60^{\circ} \mathrm{C}$ flash and higher flow ............................... 171 
Figure 182. Thermal efficiency at $70^{\circ} \mathrm{C}$ flash and lower flow ................................. 172

Figure 183. Thermal efficiency at $70^{\circ} \mathrm{C}$ flash and higher flow ................................. 172

Figure 184. Thermal efficiency at $80^{\circ} \mathrm{C}$ flash and lower flow ................................... 173

Figure 185. Thermal efficiency at $80^{\circ} \mathrm{C}$ flash and higher flow ................................. 173

Figure 186. Experimental and pseudo-experimental data acquisition ......................... 174

Figure 187. Seawater conversion dependence on flash temperature ........................... 179

Figure 188. Prime energy consumption dependence on flash temperature ................... 179

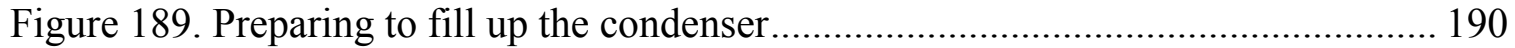

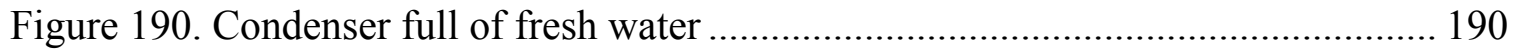

Figure 191. Preparing to fill up the evaporator........................................................ 191

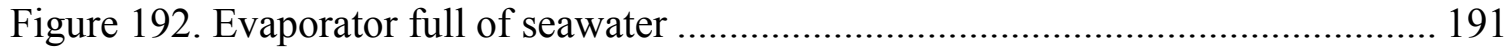

Figure 193. Switching the valve positions of the flash chamber ................................ 192

Figure 194. Flash chamber passively vacuumed ................................................... 192

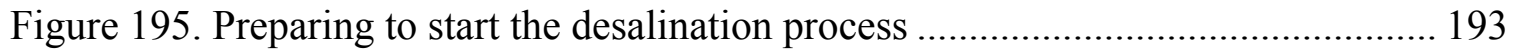

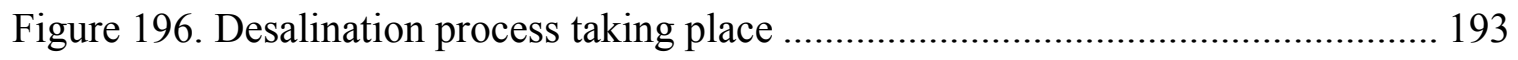

Figure 197. Flash chamber vented to terminate vacuum ......................................... 194

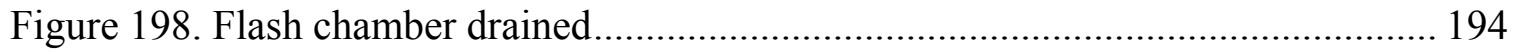




\section{LIST OF SYMBOLS}

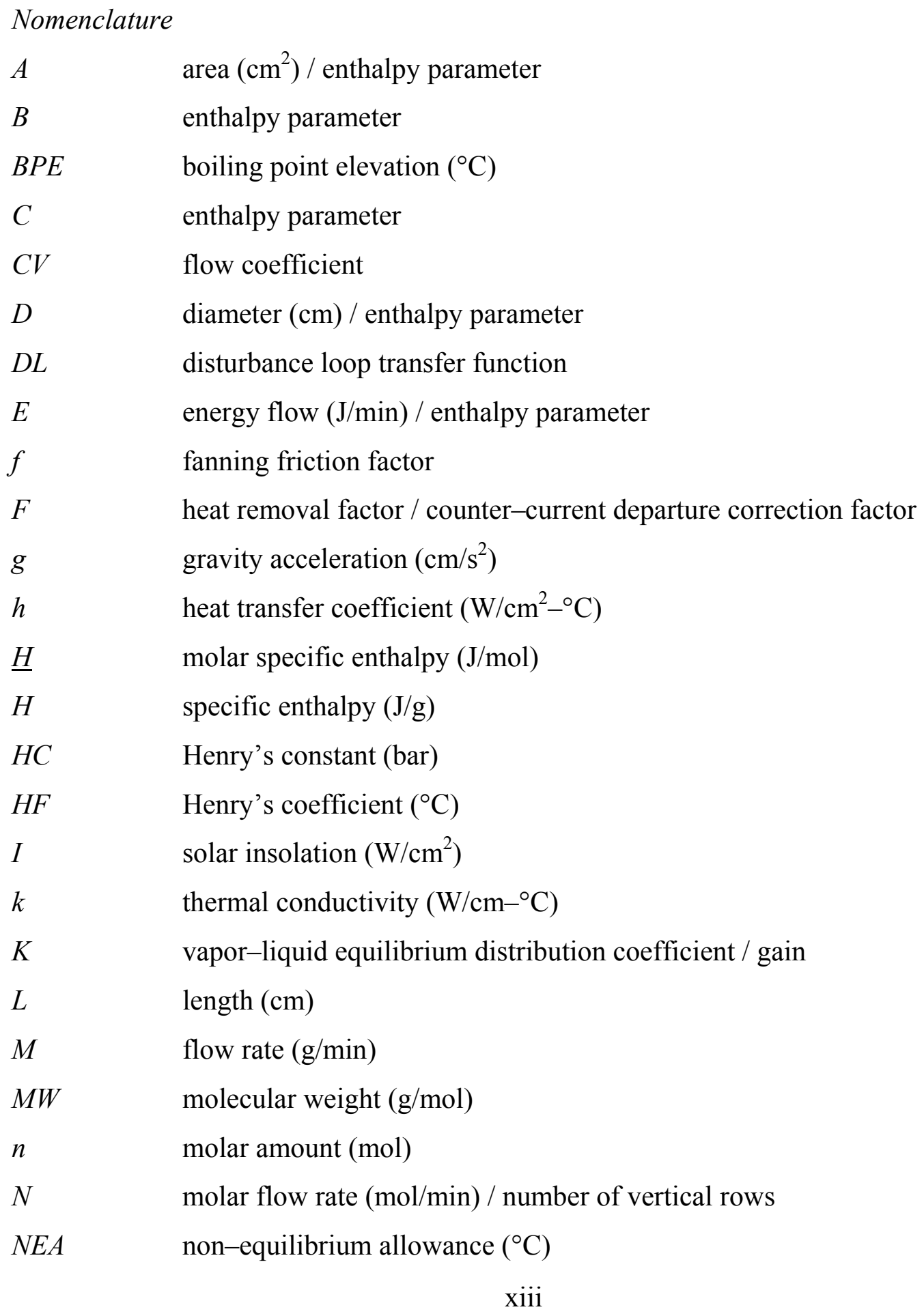




\begin{tabular}{|c|c|}
\hline$P$ & pressure (bar) \\
\hline$P A$ & saturated pressure coefficient \\
\hline$P B$ & saturated pressure coefficient \\
\hline$P C$ & saturated pressure coefficient \\
\hline$P D$ & nominal pipe diameter $(\mathrm{cm})$ \\
\hline$P E C$ & prime energy consumption \\
\hline$P L$ & equivalent pipe length $(\mathrm{cm}) /$ process loop transfer function \\
\hline$Q$ & heat input rate $(\mathrm{J} / \mathrm{min})$ \\
\hline$r$ & correlation coefficient \\
\hline$R$ & universal gas constant $\left(\mathrm{bar}-\mathrm{cm}^{3} / \mathrm{mol}-{ }^{\circ} \mathrm{C}\right)$ \\
\hline $\operatorname{Re}$ & Reynolds number \\
\hline$S$ & counter-current departure parameter \\
\hline$s$ & Laplace domain frequency $(1 / \mathrm{sec})$ \\
\hline$S G$ & specific gravity \\
\hline$T$ & temperature $\left({ }^{\circ} \mathrm{C}\right)$ \\
\hline$t$ & time (min) \\
\hline$T I C$ & temperature controller transfer function \\
\hline$U$ & overall heat transfer coefficient $\left(\mathrm{W} / \mathrm{cm}^{2}-^{\circ} \mathrm{C}\right)$ \\
\hline$V$ & volume $\left(\mathrm{cm}^{3}\right)$ \\
\hline$W$ & work output rate (J/min) \\
\hline$x$ & mole fraction in concentrated brine \\
\hline$X A$ & cross sectional area $\left(\mathrm{cm}^{2}\right)$ \\
\hline$y$ & mole fraction in flashed vapor \\
\hline$Z$ & level or elevation $(\mathrm{cm})$ \\
\hline$z$ & mole fraction before flash \\
\hline$\alpha$ & relativity factor / absorptance \\
\hline$\gamma$ & activity coefficient \\
\hline$\delta$ & thickness $(\mathrm{cm})$ \\
\hline$\Delta P$ & pressure drop (bar) \\
\hline$\Delta t$ & time increment (min) \\
\hline$\Delta T m$ & logarithmic mean temperature difference $\left({ }^{\circ} \mathrm{C}\right)$ \\
\hline
\end{tabular}




\begin{tabular}{ll}
$\varepsilon$ & Error \\
$\zeta$ & non-equilibrium allowance correlation parameter \\
$\eta$ & efficiency (\%) \\
$\mu$ & viscosity (Poise) \\
$\mu A$ & viscosity coefficient \\
$\mu B$ & viscosity coefficient \\
$\mu C$ & viscosity coefficient \\
$\mu D$ & viscosity coefficient \\
$\xi$ & non-equilibrium allowance correlation parameter \\
$\rho$ & density (g/cm ${ }^{3}$ ) \\
$\rho A$ & density coefficient \\
$\rho B$ & density coefficient \\
$\rho C$ & density coefficient \\
$\sigma$ & diffusion conductance parameter $\left(\mathrm{g}-{ }^{\circ} \mathrm{C}^{1 / 2} / \mathrm{bar}-\mathrm{min}-\mathrm{cm}^{2}\right)$ \\
$\tau$ & transmittance / time constant $($ sec $)$ \\
$\varphi$ & mass fraction in streams \\
$\psi$ & gas phase molecular content correction factor \\
$\omega$ & diffusion resistance parameter $(\mathrm{bar})$ \\
& mass fraction in sea salt \\
\hline &
\end{tabular}

\section{Subscripts}

0

dead time

Ar argon

$B \quad$ brine water tank

$\mathrm{BO}_{3} \quad$ borate

$B P \quad$ bubble point

$\mathrm{Br} \quad$ bromide

C condenser / controller

$\mathrm{Ca} \quad$ calcium

$\mathrm{Cl} \quad$ chlorine

$\mathrm{CO}_{2} \quad$ carbon dioxide 


\begin{tabular}{|c|c|}
\hline$C T$ & condenser tube \\
\hline$C V$ & condenser vacuum \\
\hline$D$ & derivative \\
\hline$D L$ & disturbance loop \\
\hline$D P$ & dew point \\
\hline$E$ & flashed vapor \\
\hline$E V$ & evaporator vacuum \\
\hline$F$ & fluoride / fresh water tank \\
\hline$H$ & heater \\
\hline $\mathrm{H}_{2} \mathrm{O}$ & water \\
\hline $\mathrm{HCO}_{3}$ & bicarbonate \\
\hline$H T$ & heater tube \\
\hline$I$ & integral \\
\hline$j$ & representative stream \\
\hline$K$ & potsium \\
\hline$M g$ & magnesium \\
\hline$N_{2}$ & nitrogen \\
\hline$N a$ & sodium \\
\hline$N C G$ & representative non-condensable gas \\
\hline$O$ & orifice \\
\hline $\mathrm{O}_{2}$ & oxygen \\
\hline$P$ & pump \\
\hline$P L$ & process loop \\
\hline$R$ & recovery \\
\hline$S$ & seawater tank \\
\hline Salt & sea salt \\
\hline$S C$ & solar collector \\
\hline $\mathrm{SO}_{4}$ & sulfate \\
\hline$S r$ & strontium \\
\hline$T$ & thermal \\
\hline$V$ & vacuum \\
\hline
\end{tabular}




$\begin{array}{ll}W & \text { evaporator } \\ X & \text { seawater preheat }\end{array}$

$\begin{array}{ll}\text { Superscripts } & \\ a & \text { accumulating } \\ C & \text { condensed vapor } \\ d & \text { diffusing } \\ i & \text { initial / inside } \\ \text { id } & \text { inside dirt } \\ \text { in } & \text { entering } \\ L & \text { latent } \\ o & \text { reference state / outside } \\ \text { od } & \text { outside dirt } \\ \text { out } & \text { existing } \\ \text { sat } & \text { saturated } \\ W & \text { concentrated brine } \\ w & \text { wall }\end{array}$




\title{
THEORETICAL AND EXPERIMENTAL SIMULATION OF PASSIVE VACUUM
}

\author{
SOLAR FLASH DESALINATION
}

Mohammad Abutayeh

\begin{abstract}
Experimental and theoretical simulations of a novel sustainable desalination process have been carried out. The simulated process consists of pumping seawater through a solar heater before flashing it under vacuum in an elevated chamber. The vacuum is passively created and then maintained by the hydrostatic balance between pressure inside the elevated flash chamber and outdoor atmospheric pressure.

The experimental simulations were carried out using a pilot unit built to depict the proposed desalination system. Theoretical simulations were performed using a detailed computer code employing fundamental physical and thermodynamic laws to describe the separation process, complimented by experimentally based correlations to estimate physical properties of the involved species and operational parameters of the proposed system setting it apart from previous empirical desalination models.

Experimental and theoretical simulation results matched well with one another, validating the developed model. Feasibility of the proposed system rapidly increased with flash temperature due to increased fresh water production and improved heat recovery. In addition, the proposed desalination system is naturally sustainable by solar radiation and gravity, making it very energy efficient.
\end{abstract}




\section{CHAPTER 1. INTRODUCTION}

\subsection{Overview}

Fresh water demand is persistently increasing both as populations around the world keep growing and as existing fresh water reserves keep declining due to consumption and pollution. Figure 1 shows the estimated water consumption of US counties for 2000 [1]. Marine waters represent an infinite water source since $98 \%$ of all global water is present in oceans; therefore, seawater desalination is the logical approach to meet rising fresh water demand.

Energy demand is also continually increasing due to relentless global industrialization. Oil and gas remain the primary sources of energy for most of the world; however, their reserves are dwindling, production is peaking, and consumption is harming the environment. Figure 2 illustrates the estimated energy consumption per capita of US states for 2001, while Figure 3 compares energy consumption per capita of Florida to the rest of the country for the past forty years [1]. Renewable energy sources are continually replenished by cosmic forces and can be used to produce sustainable and useful forms of energy with minimum environmental impact.

Serious economic and social disruptions are unfolding over the finite water and energy resources; hence, securing fresh water supply and employing renewable energy sources will help avoid catastrophic conflicts, continue modern lifestyles, and circumvent global warming and environmental pollution [2]. 
Desalination can be accomplished by separation techniques developed over the years to produce potable water. The most widespread desalination methods are given in CHAPTER 2. Momentous amounts of energy are required in all desalination processes; therefore, reducing energy demand, as well as employing renewable energy, is imperative to developing viable desalination processes. Various desalination systems driven by renewable energy have been developed over the last few years; nonetheless, most have not yet been commercially implemented due to high capital cost associated with utilizing renewable energy.

Solar radiation is a very appealing source of energy because it is available at no cost; furthermore, exploiting it has no notable adverse effect on the environment. Plenty of research and development have been undertaken to utilize this free form of energy to develop more efficient sustainable processes such as water desalination and power generation. Figure 4 illustrates the US share of solar radiation [3]. Solar energy is intermittent and requires storage; however, maximizing its use alongside developing energy efficient processes can greatly diversify energy resources, save the environment, and reduce imposed social cost [4].

Solar desalination is essentially a small-scale duplicate of the natural hydrologic cycle that produces rain, which is the primary source of fresh water worldwide. Solar insolation is preferred over other renewable energy sources to drive desalination systems because its thermal energy can be directly applied to thermal desalination schemes without adverse energy conversion requirements that usually entail certain energy losses. In addition, thermal desalination schemes are more suitable than mechanical desalination schemes for large-scale applications as will seen later. 


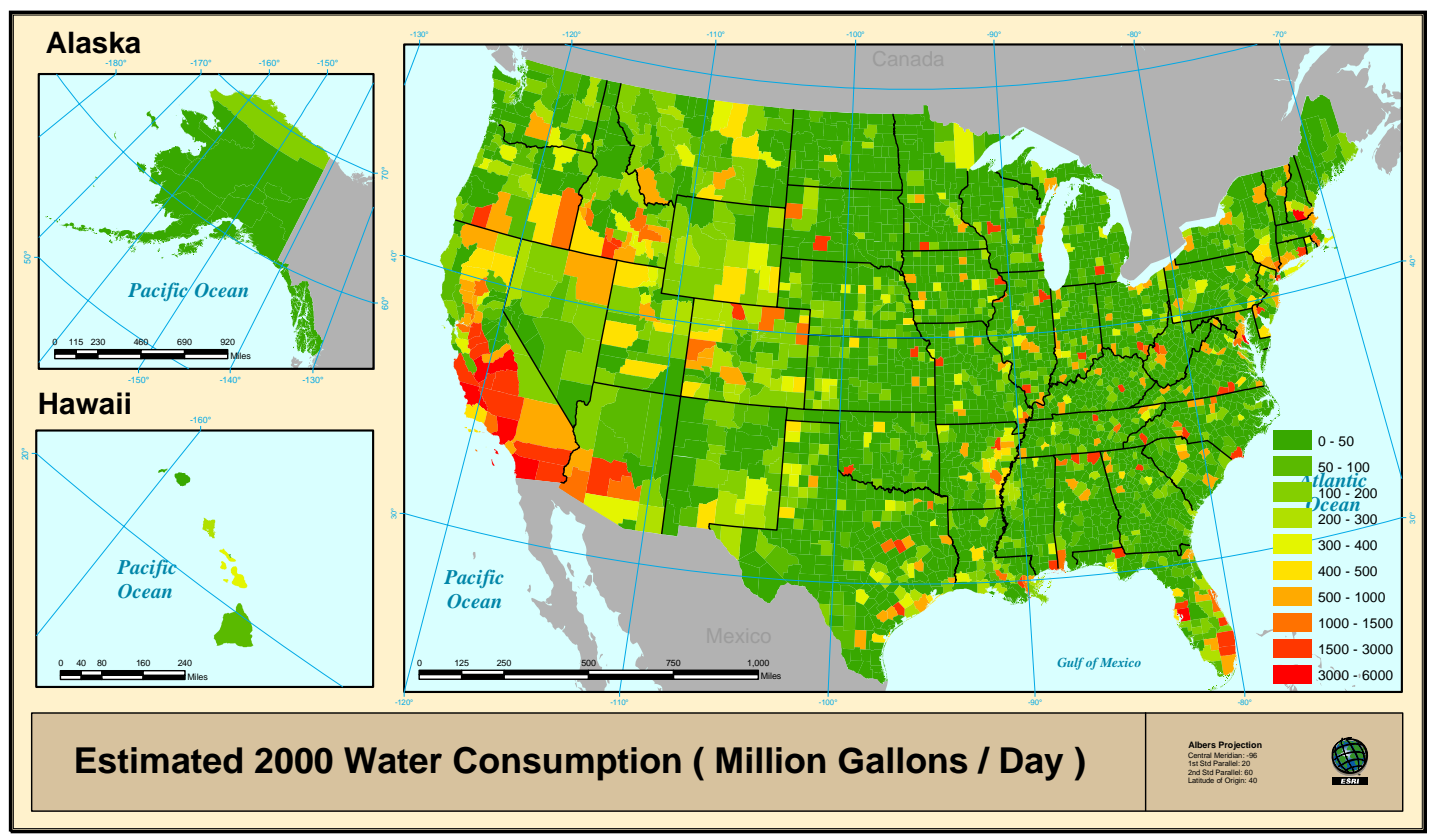

Figure 1. Estimated water consumption of US counties for 2000 [1]

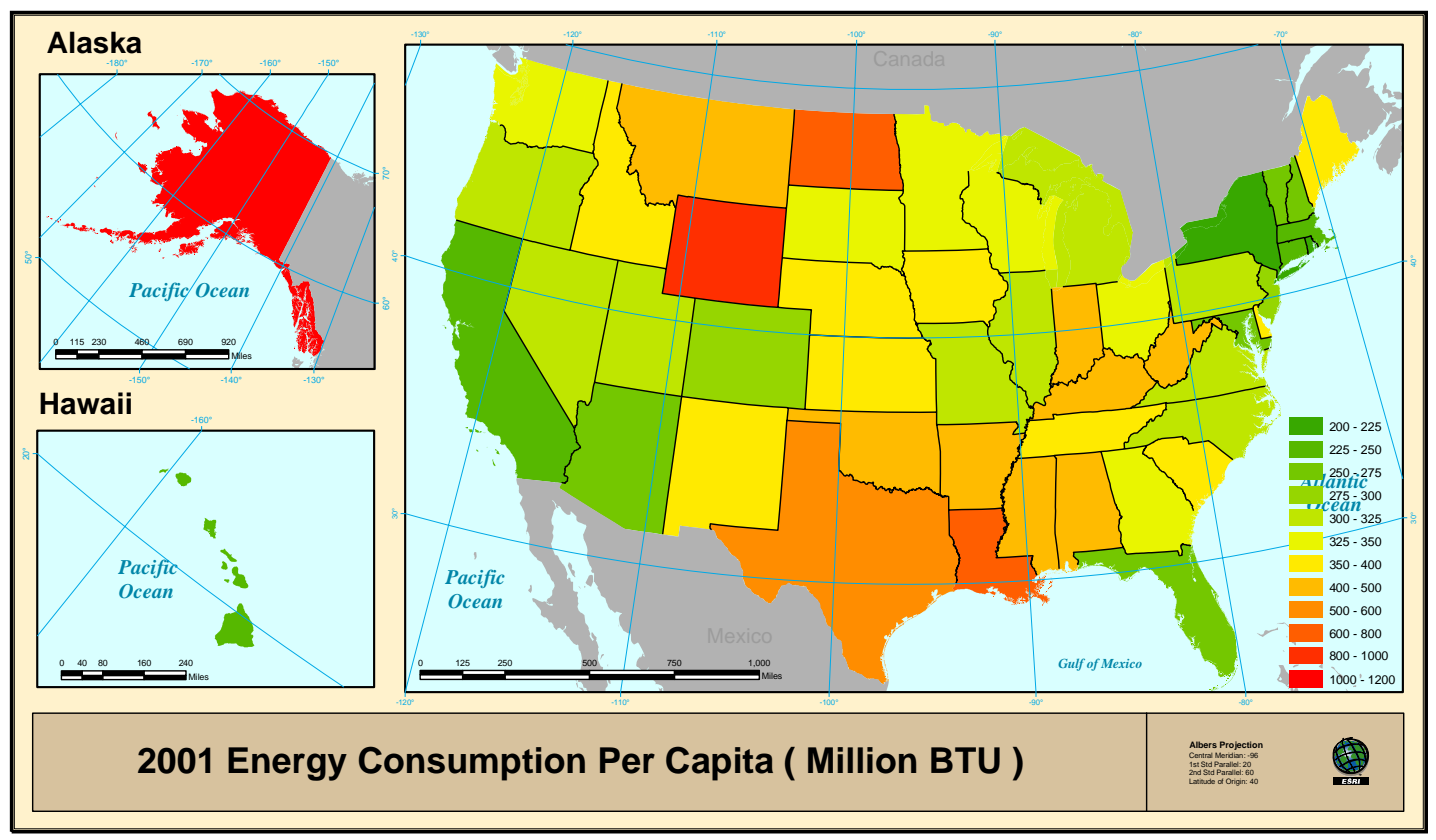

Figure 2. Estimated energy consumption per capita of US states for 2001 [1] 


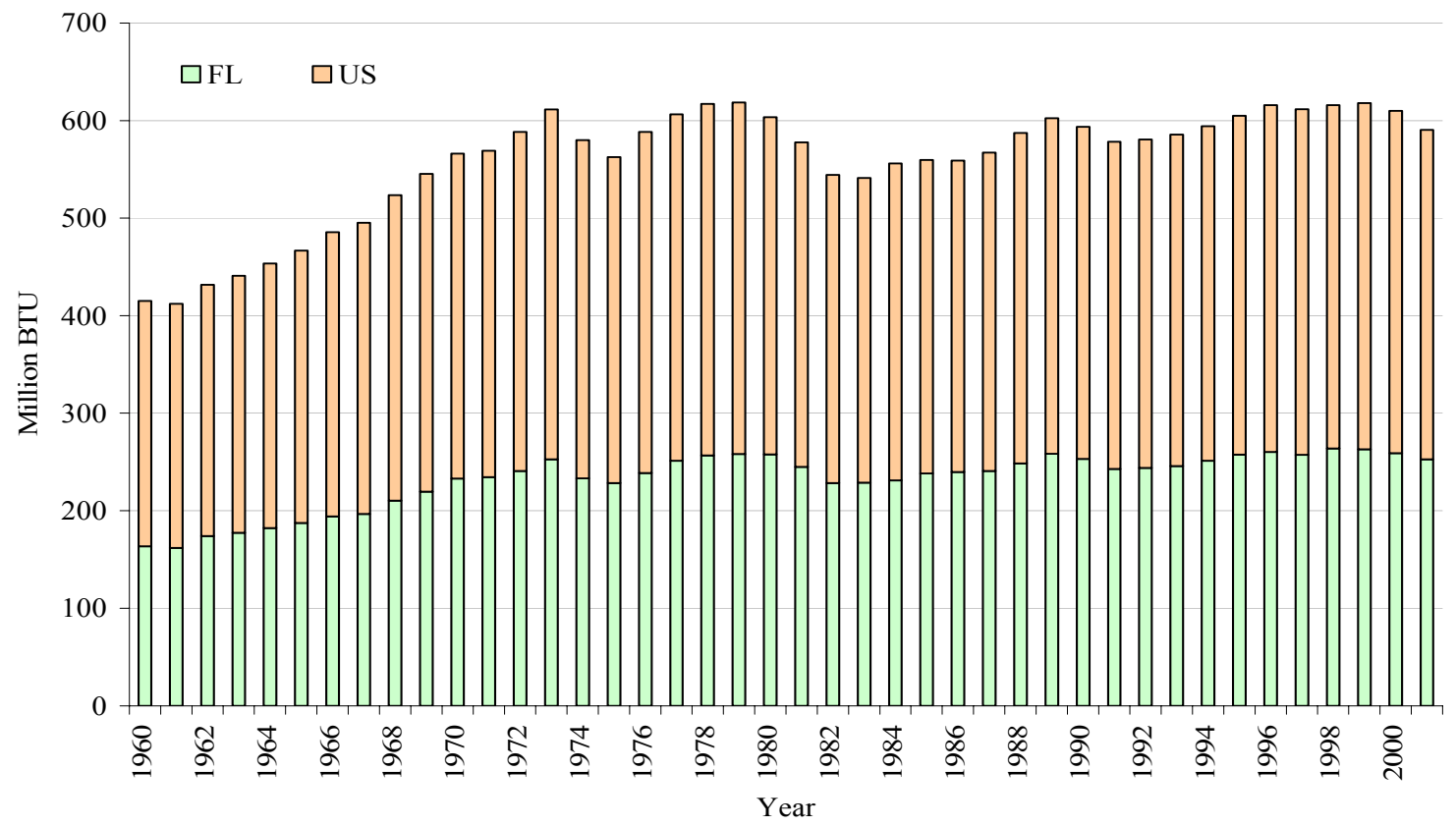

Figure 3. Estimated energy consumption per capita of Florida and the US [1]

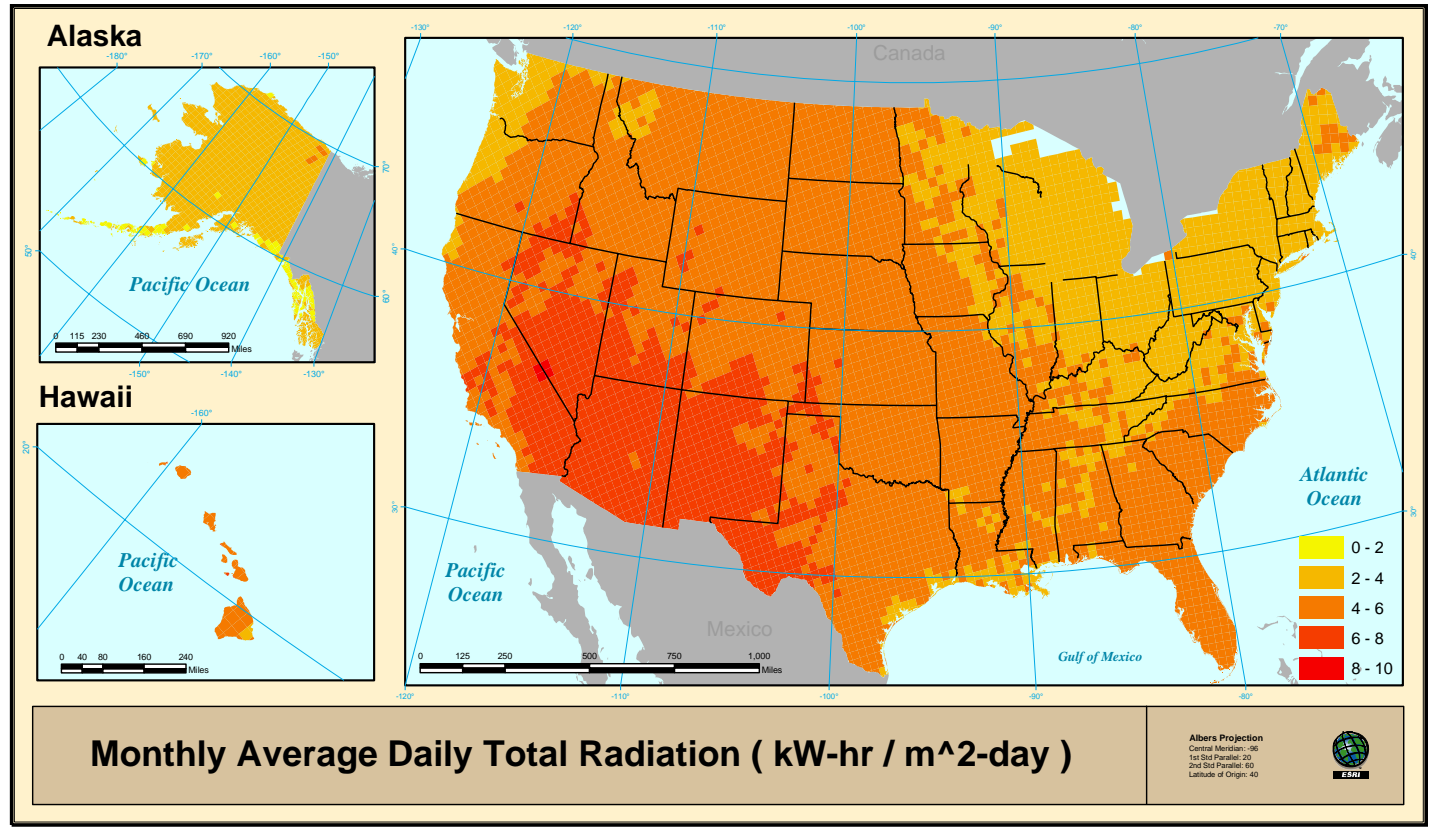

Figure 4. Monthly average daily solar insolation in the US [3] 


\subsection{Objective}

Developing an economically-viable and environmentally-friendly desalination system involves lowering its energy demand and employing renewable energy to drive its operation. In this study, the most common desalination technique, multi-stage flash, will be modified to have its system vacuum created passively and to have its thermal energy requirements drawn from solar insolation. The proposed modifications are expected to further the feasibility and broaden the applicability of the desalination process.

Creating vacuum conditions above liquids will increase their evaporation rates. This phenomenon can be integrated into a practical continuous desalination process by repeatedly flashing seawater in vacuumed chambers to produce water vapor that will be condensed to produce fresh water. Gravity can be used to balance hydrostatic pressure inside the flash chambers with the outdoor atmospheric pressure to maintain that vacuum, while low grade heat or solar radiation can be used to heat seawater before flashing it.

The objective of this study is to simulate theoretically and experimentally a solar flash desalination process under a hydrostatically sustained vacuum and analyze its controlling variables. Theoretical simulation is accomplished by a rigorous computer code employing fundamental physical and thermodynamic relationships to describe the process complimented by reliable empirical correlations to estimate physical properties of

the involved species and operational parameters of the proposed system. Experimental simulation is realized by constructing a pilot unit depicting the proposed desalination system. Theoretical and experimental simulations will be run under various analogous conditions, and their results will be compared and analyzed both to validate the developed model and to examine the feasibility of the proposed system. 


\section{CHAPTER 2. DESALINATION}

\subsection{Conventional Desalination}

Desalination is very energy intensive and requires costly infrastructure; therefore; several desalination processes have been developed over the years to produce fresh water from seawater economically. These can be classified according to the applied separation scheme into thermal, physical, and chemical processes.

Thermal desalination processes produce a fractional phase change of liquid seawater to either vapor or solid. The new phase is then separated from the bulk brine water producing fresh water, while the latent heat of phase change is reclaimed. Multiple

effect evaporation, multi-stage flash, vapor compression, and indirect contact freezing are examples of thermal desalination processes.

Physical desalination processes extract fresh water from seawater by applying pressure or electric potential across a membrane. Either fresh water or solute ions travel through the semi-permeable membrane due to the mechanically induced gradient yielding the desired separation. Reverse osmosis, electro-dialysis, and nano-filtration are examples of physical desalination processes.

Chemical desalination processes extract fresh water from seawater by precipitating its salts due to chemical reactions. These processes are less common because they are usually too expensive to produce fresh water. Ion exchange, gas hydrate, and liquid-liquid extraction are examples of chemical desalination processes. 
Selecting a suitable desalination process requires several design considerations such as: start-up time, seawater quality, seawater supply, maintenance requirements, energy efficiency, capital cost, operating cost, and other site specific factors [2]. Global distribution of these processes is illustrated categorically in Figure 5 and geographically in Figure 6 as percentages of total installed capacity [5].

Selecting a particular desalination process also requires knowledge of its specific design limitations. Discussing the design limitations of different desalination processes is beyond the scope of this study, but such discussions are widely available in academic and business literature [6]. A brief summary of these limitations is provided here.

The energy needed to recover fresh water from seawater increases with increased salinity; therefore, limiting recovery rates is one way to optimize the desalination process. Also, increasing process efficiency usually involves increasing equipment size, which entails capital cost increase. Optimum design of desalination plants generally includes analyzing the tradeoff between energy and capital costs to minimize production costs. In addition, scaling is a major issue in desalination because it fouls mass and heat transfer surface areas, reducing both capacity and efficiency. Scaling can be minimized by reducing the saturation limit of saline water via dropping the operating temperature and limiting the recovery rates in addition to chemical pretreatment and lime soda softening. Finally, desired water quality directly influences which desalination path to take [6].

Desalination is a continually evolving field with many of its processes under research and development. In addition, a wide variety of cost effective hybrid processes are proposed as alternatives to the rather expensive common commercial processes. A brief discussion of the major desalination processes is provided next. 


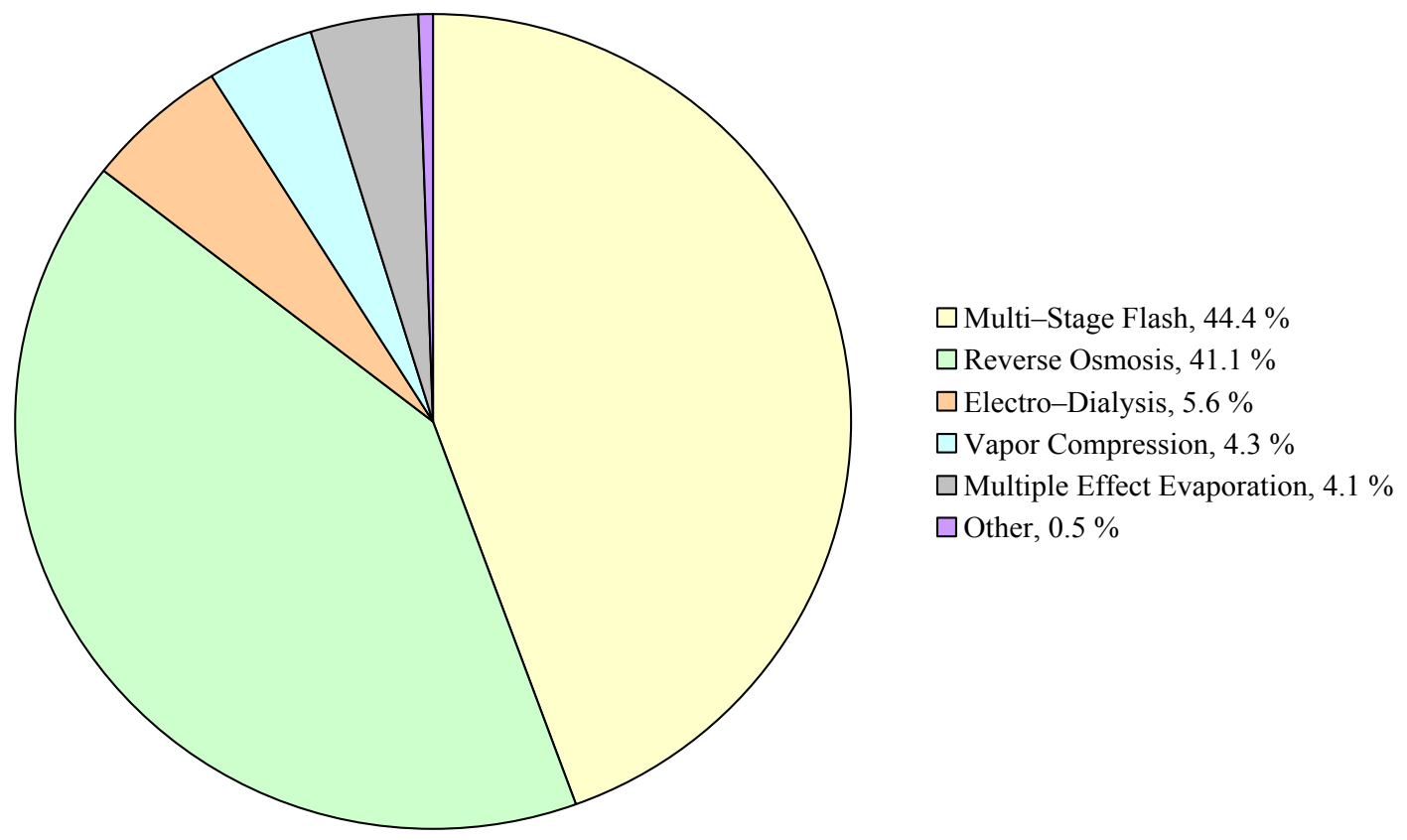

Figure 5. Global distribution of installed desalination capacity by technology [5]

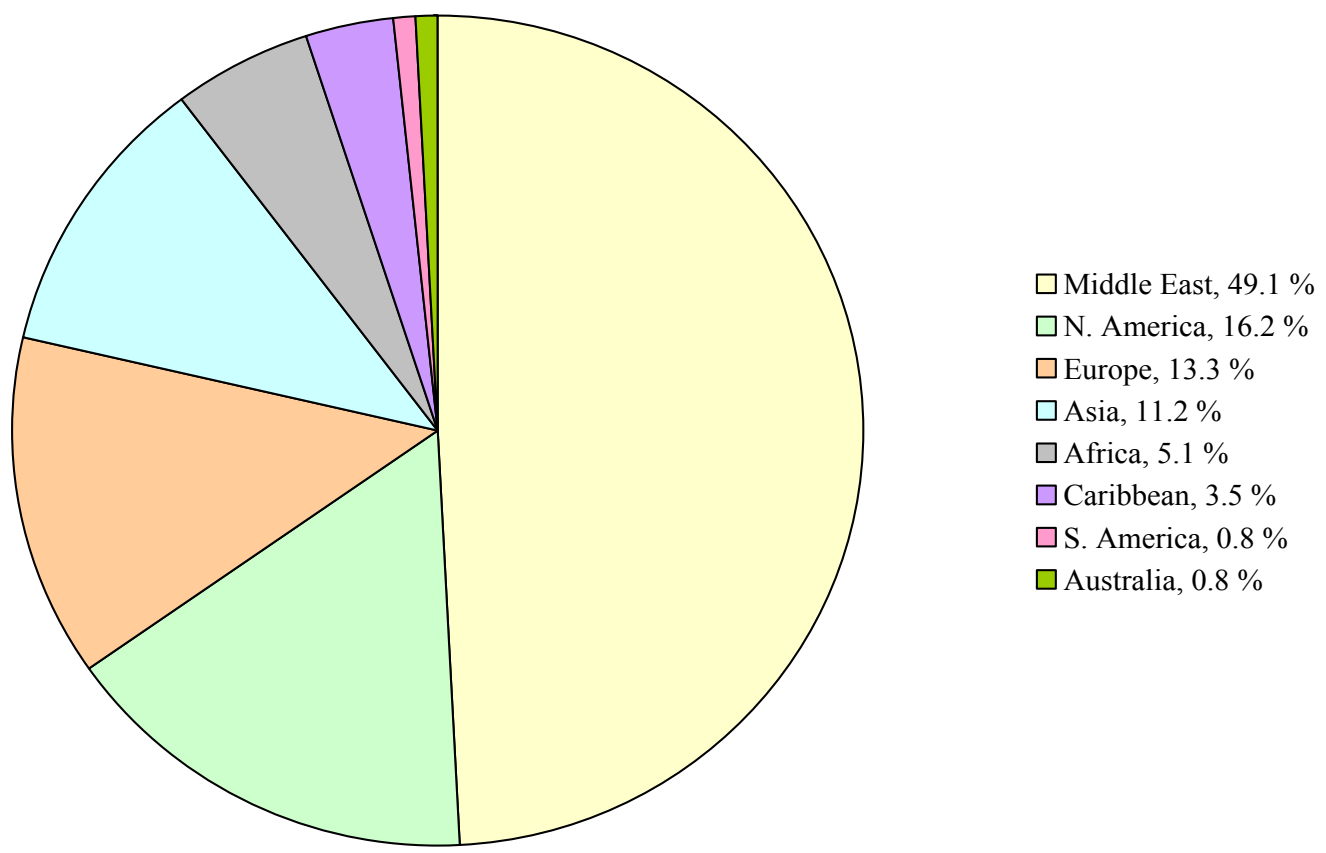

Figure 6. Global distribution of installed desalination capacity by region [5] 


\subsubsection{Multiple Effect Evaporation}

Originally developed in the 1820 s for concentrating sugar in sugar cane juice, multiple effect evaporation was used for desalination in the 1950s, making it the oldest desalination process still in operation. Multiple effect evaporation has been combined with other desalination methods, such as vapor compression, to increase its efficiency.

Seawater is distributed to a sequence of vacuumed vessels, known as effects, held at successively lower pressures. External heat is supplied to the first effect, and the generated vapor of each effect supplies its latent heat of condensation to the next. Condensed vapor of each effect is then collected as the fresh water product.

Multiple effect evaporation has a relatively good thermal performance since external heating is only required for the first effect, but its heat transfer tubes are very susceptible to scaling, making it a less attractive desalination option. Figure 7 provides a simple process flow diagram of the multiple effect evaporation desalination process [6].

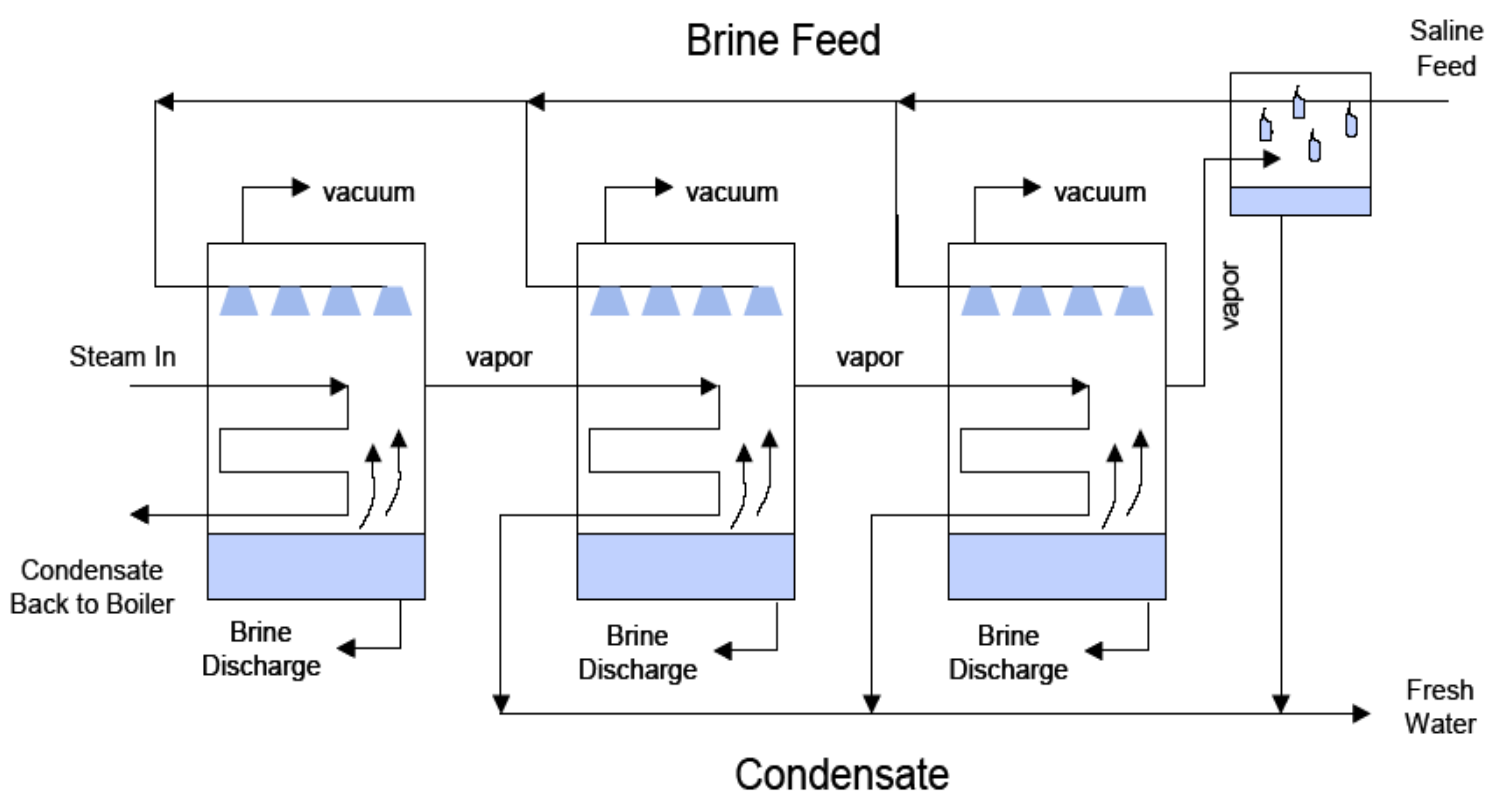

Figure 7. Multiple effect evaporation 


\subsubsection{Multi-Stage Flash}

Multi-stage flash is the most common desalination technique, accounting for over $40 \%$ of the global capacity [5]. It has been coupled with other processes, such as solar heating and steam turbine power generation, to increase its efficiency.

Seawater is moved through a sequence of vacuumed vessels, known as stages, held at successively lower pressures, where it is preheated. External heat is then supplied, heating the seawater to above its saturation point. Seawater is then successively passed from one stage to the next, where a small amount of water flashes to steam in each stage, and the remaining brine is forwarded to next stage for further flashing. The flashed steam of each stage condenses by losing its latent heat of condensation to the entering seawater. The condensed vapor of each stage is then collected as the fresh water product.

Multi-stage flash has a relatively low thermal performance due to bulk heating of seawater, but its heat transfer tubes are less susceptible to scaling because of that bulk heating, making it a more attractive desalination option. Figure 8 provides a simple process flow diagram of the multi-stage flash desalination process [6].

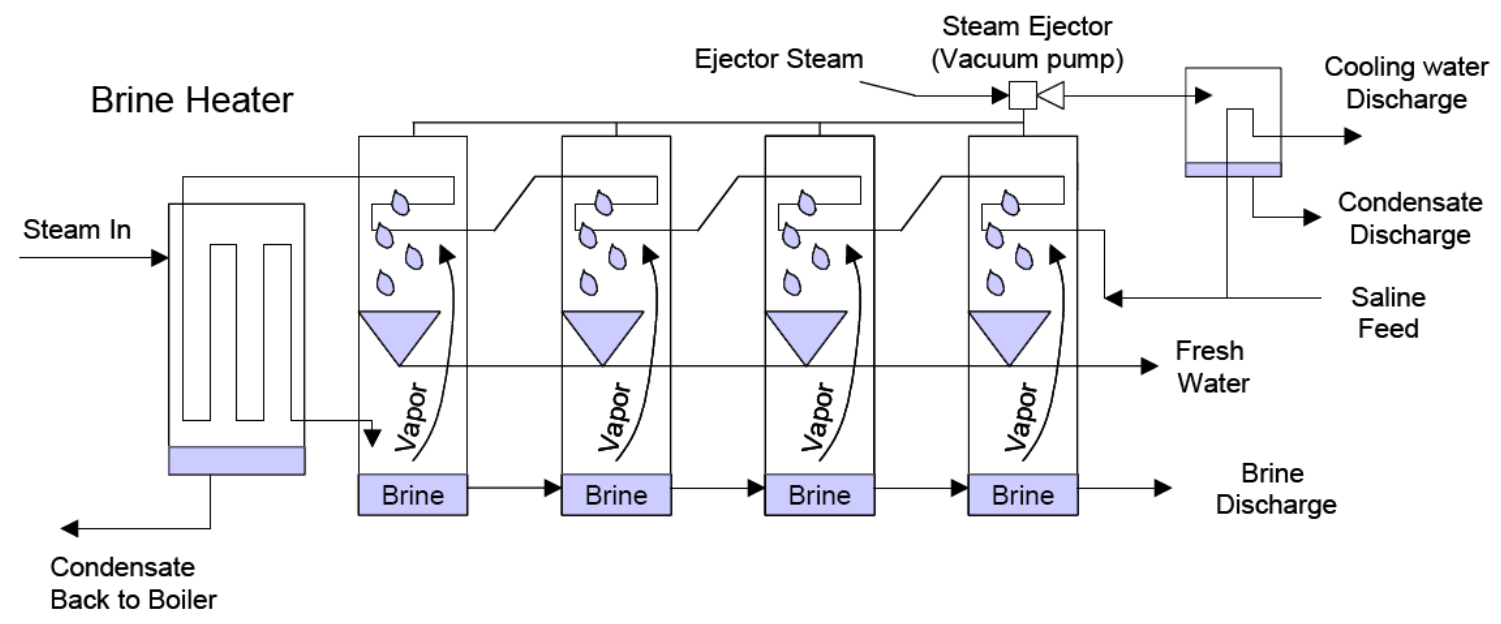

Figure 8. Multi-stage flash 


\subsubsection{Vapor Compression}

Seawater is preheated before entering a vacuumed vessel to be partially vaporized by the latent heat of a condensing steam obtained via compressing vaporized water. The process is dubbed mechanical vapor compression if steam compression is done by a compressor or thermal vapor compression if steam compression is done by an ejector.

Vapor compression has a relatively high thermal performance and can be applied in the desalination of extremely concentrated brines. Vapor compression is generally employed in small and medium capacity applications. Figure 9 provides a simple process flow diagram of the mechanical vapor compression desalination process [6].

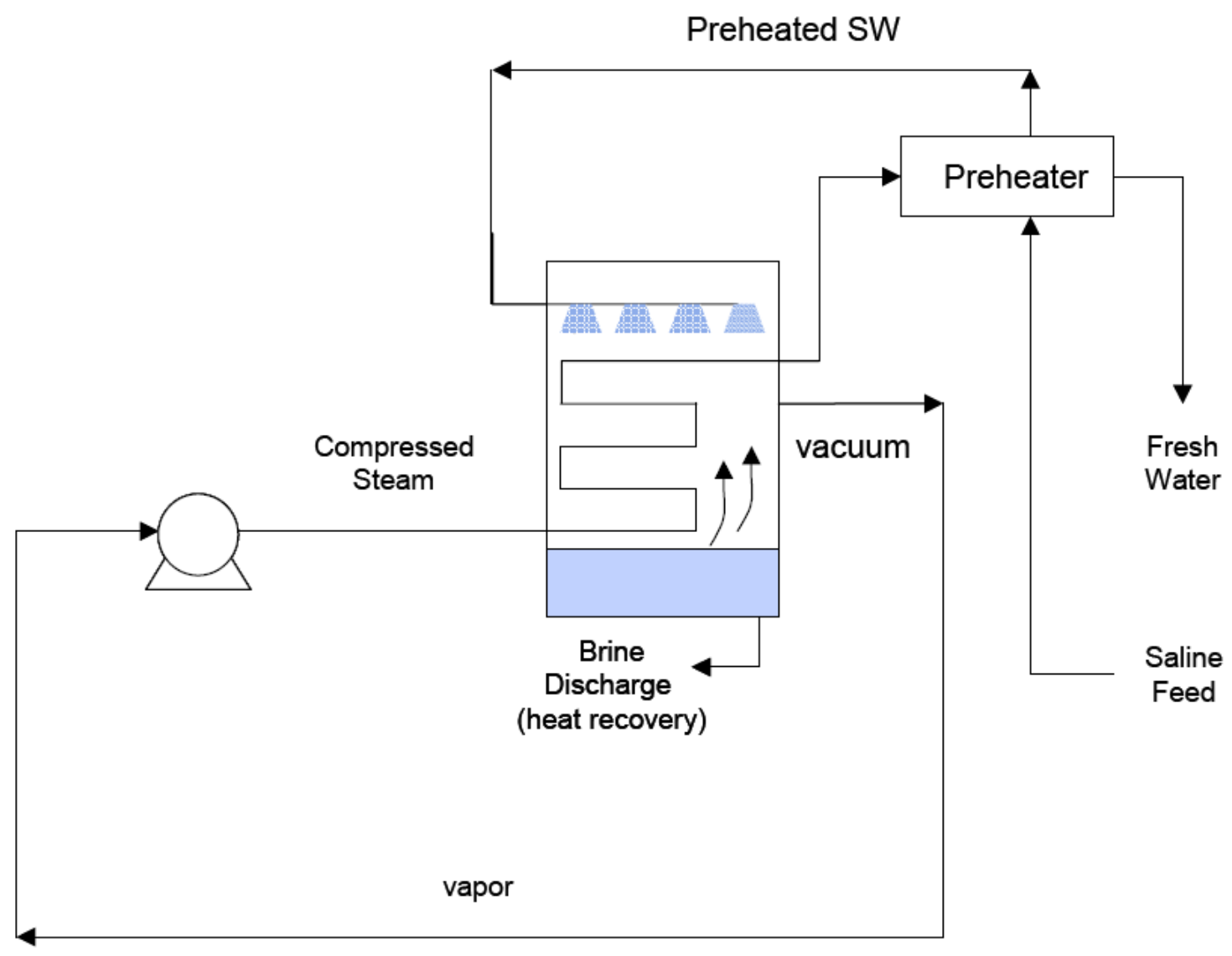

Figure 9. Mechanical vapor compression 


\subsubsection{Indirect Contact Freezing}

Seawater is cooled by cold outgoing fresh water and brine before it enters the evaporator of a separate refrigeration cycle, known as the freezer, where it is partially frozen by evaporating refrigerant. Crystallized ice is separated from the brine before it enters the condenser of the refrigeration cycle, known as the melter, where it melts by extracting its latent heat of fusion from condensing refrigerant. Cold outgoing fresh water and brine streams are used to cool the entering seawater in a heat exchanger.

Indirect contact freezing has a relatively high thermal performance and is less susceptible to scaling and corrosion due to its low temperature operation, but problems arise both from controlling solids handling operations and from the uncertain reliability of refrigerant compressors due to increased risk of oil slugging at low pressures. Figure 10 provides a simple schematic of the indirect contact freezing desalination process [6].

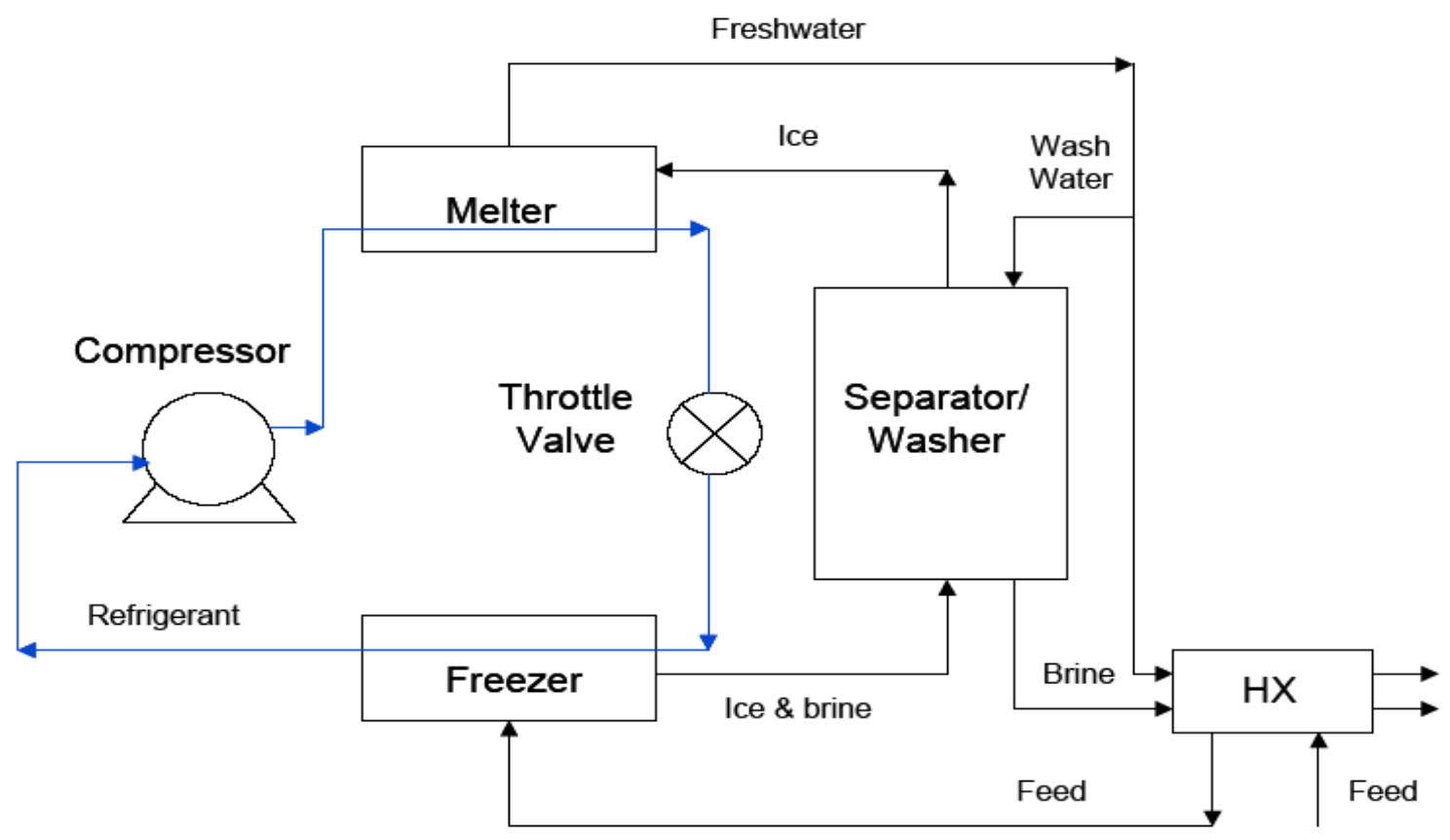

Figure 10. Indirect contact freezing 


\subsubsection{Reverse Osmosis}

Reverse osmosis is the most common desalination process nationally and the second most common internationally in terms of capacity. It is best used for brackish water and is usually combined with other filtration methods to increase its efficiency.

Seawater is initially treated to adjust its $\mathrm{pH}$ and to free it from particulates that negatively impact the membrane structure. Seawater is then pumped to a network of semi-permeable membranes, separating fresh water from concentrated brine. Seawater pressure is raised above its natural osmotic pressure, typically 25 bars, but is kept below the membrane tolerance pressure, typically between 60 and 80 bars, forcing pure water molecules through the membrane pores to the fresh water side. Separated water is then treated and collected as the fresh water product, while the concentrated brine is rejected.

Reverse osmosis is very efficient because the mechanical compression energy can be reclaimed from the rejected concentrated brine with a suitable turbine. Figure 11 provides a simple process flow diagram of the reverse osmosis desalination process [6].

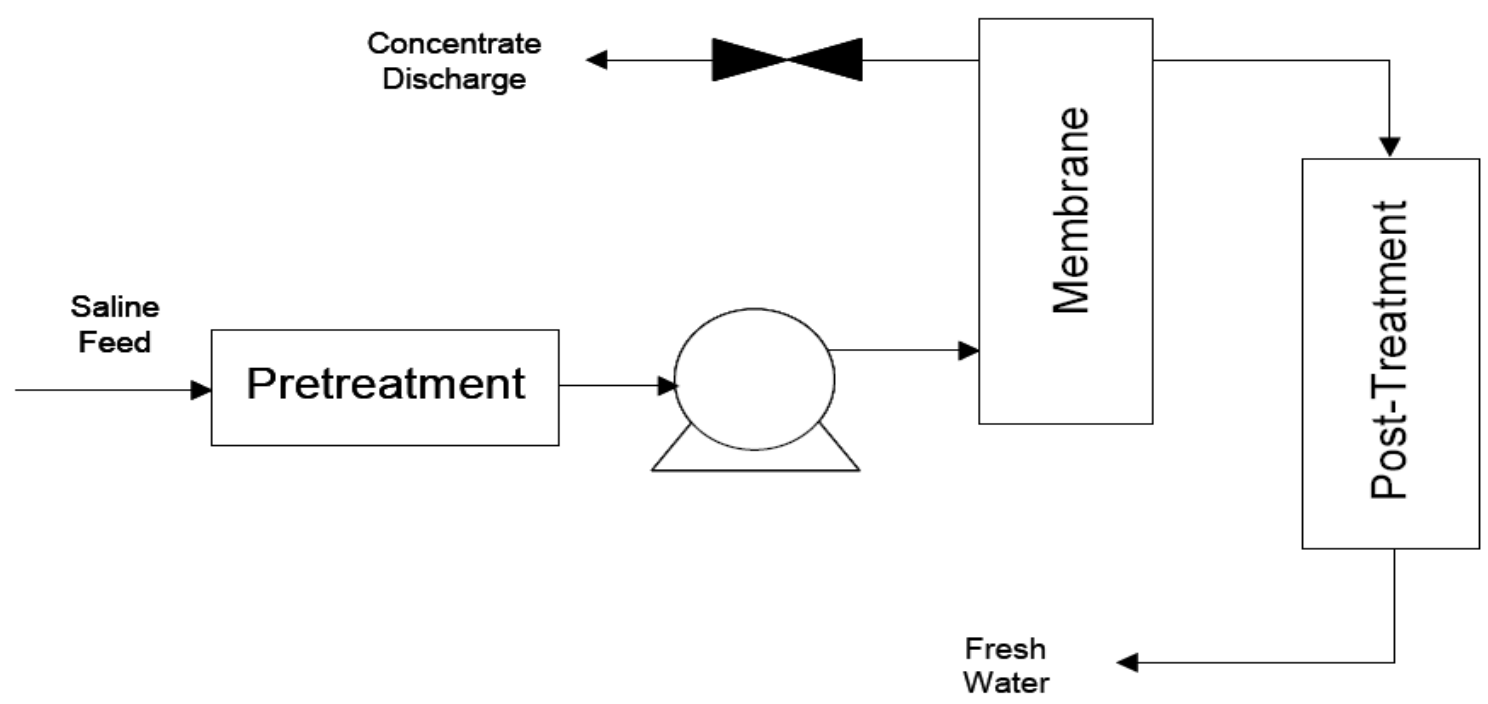

Figure 11. Reverse osmosis 


\subsubsection{Electro-Dialysis}

Seawater is passed through an electro-dialysis stack consisting of alternating layers of cationic and anionic ion exchange membranes in an electrical field. Cations and anions then migrate in opposite directions through ion selective membranes and away from the saline feed in response to applied voltage across the electro-dialysis stack, producing fresh water in the intermediary channels.

The electro-dialysis stack can be arranged in series to increase purification and in parallel to increase output. Electro-dialysis is best used in brackish water applications and is usually combined with other filtration methods to increase its efficiency. Figure 12 provides a simple process flow diagram of the electro-dialysis desalination process [6].

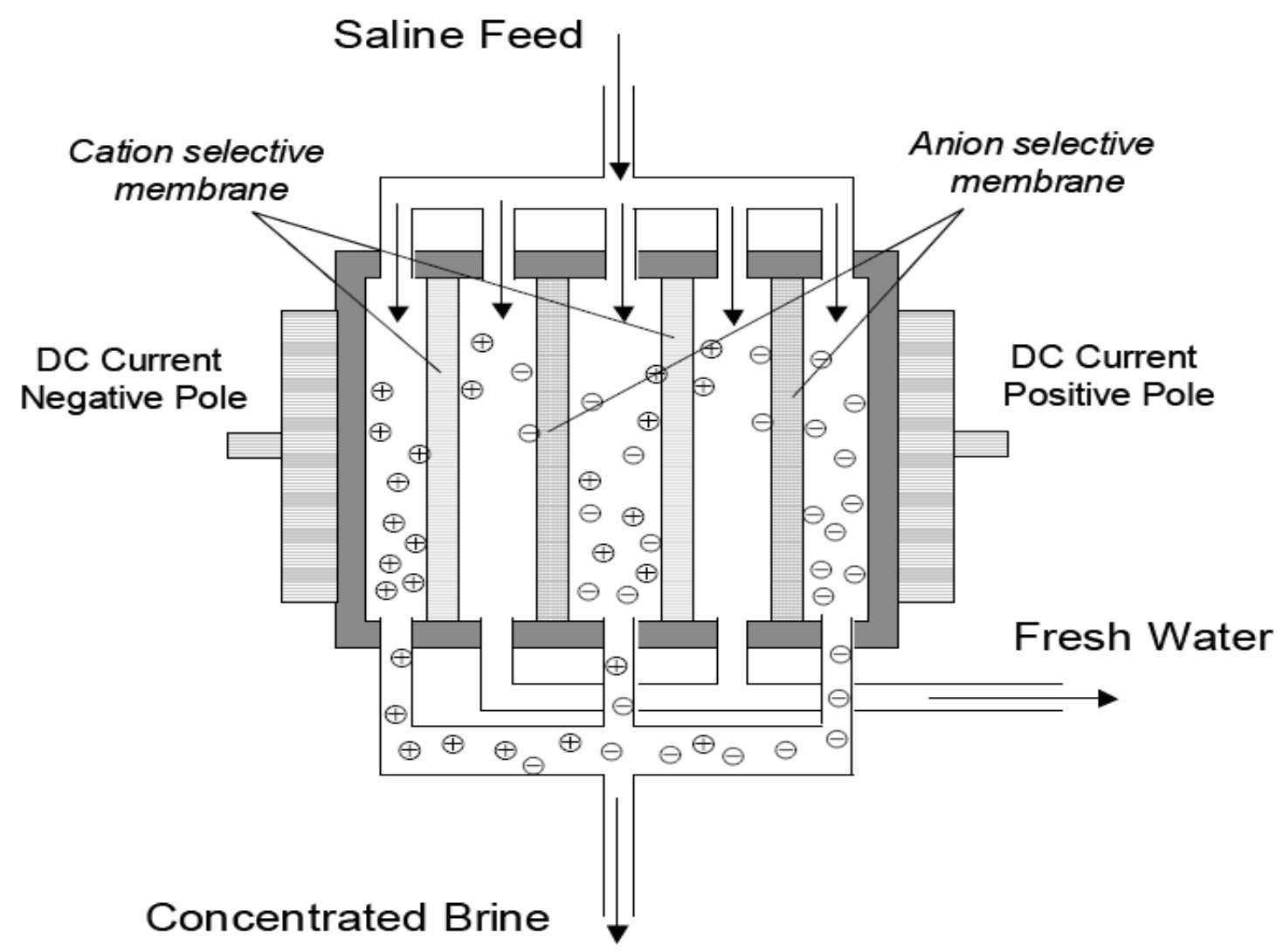

Figure 12. Electro-dialysis 


\subsection{Solar Desalination}

Extracting fresh water from seawater requires a great deal of energy, both thermal and mechanical, as detailed in Table 1 [7]. Renewable energy driven desalination is becoming more viable despite its expensive infrastructure because it employs free natural energy sources and releases no harmful effluents to the environment. Solar insolation is usually chosen over other renewable energy sources because its thermal energy can be directly applied to drive desalination systems without irreversible energy conversion that involves inevitable energy loss according to the second law of thermodynamics.

Solar desalination systems are classified into direct and indirect processes depending on the energy path to fresh water. Direct solar desalination systems combine solar energy collection and desalination in one process producing fresh water distillate by directly applying collected solar energy to seawater. Solar distillation using a solar still is an example of direct solar desalination. Indirect solar desalination systems comprise two sub-systems: a solar collection system and a desalination system. The solar collection sub-system is used either to collect heat using solar collectors and supply it via a heat exchanger to a thermal desalination process or convert heat to electricity using photovoltaic cells to power a physical desalination process. The desalination sub-system can be any of the previously mentioned conventional desalination systems.

Table 1. Energy consumption of desalination systems [7]

\begin{tabular}{lccc}
\hline Process & $\begin{array}{c}\text { Heat Input } \\
(\mathrm{kJ} / \mathrm{kg} \text { of product })\end{array}$ & $\begin{array}{c}\text { Power Input } \\
(\mathrm{kJ} / \mathrm{kg} \text { of product })\end{array}$ & $\begin{array}{c}\text { Prime Energy Consumption } \\
(\mathrm{kJ} / \mathrm{kg} \text { of product })\end{array}$ \\
\hline$M E E$ & 123 & 8 & 149 \\
\hline$M S F$ & 294 & $9-14$ & 338 \\
\hline$V C$ & - & $29-58$ & 192 \\
\hline$R O$ & - & $18-47$ & 120 \\
\hline$E D$ & - & 43 & 144 \\
\hline
\end{tabular}




\subsubsection{Solar Distillation}

Seawater is placed in a blackened basin inside an air tight transparent structure where it evaporates due to absorption of solar radiation then condenses on the sloping structure by losing its latent heat of condensation to the surroundings. Condensed droplets run down the cover to accumulating troughs to be collected as fresh water.

Solar distillation is a small scale hydrological cycle, and its efficiency is significantly dependent on meteorological limitations such as solar radiation, sky clearness, wind velocity, ambient temperature, and many others. Solar distillation requires large collection areas to maximize insolation and is usually combined with other desalination methods to increase its efficiency. Figure 13 provides a simple process flow diagram of the solar distillation desalination process [6].

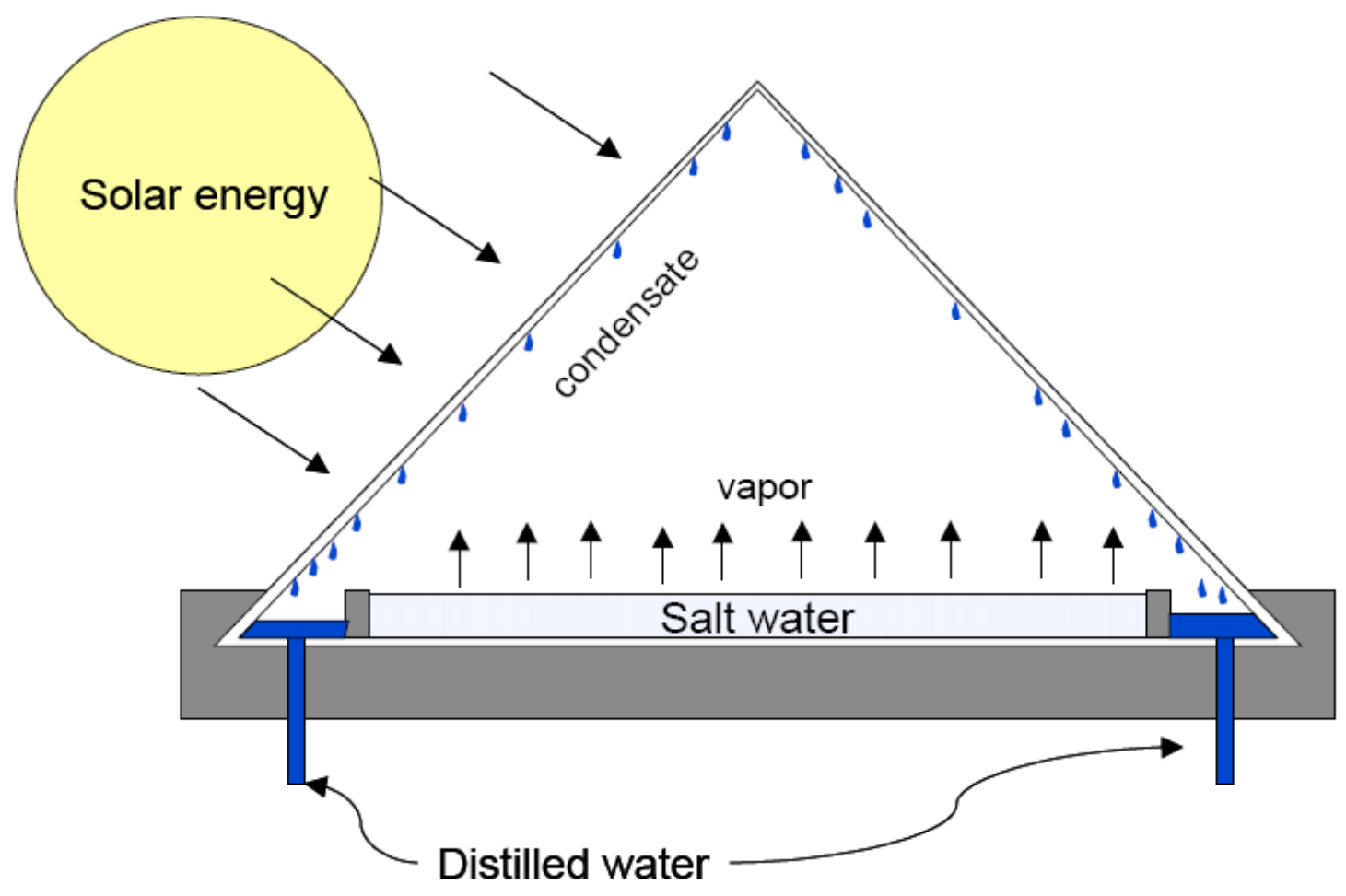

Figure 13. Solar distillation 


\subsubsection{Solar Collectors}

The solar collection sub-system of an indirect solar desalination system is essentially a solar collector that absorbs incident solar radiation and transfers heat to a fluid flowing through it. The working fluid of the collector can either be a medium to transfer heat to the process or to a thermal energy storage reservoir, or it can be the seawater itself before going through a thermal desalination system. Solar collectors can be either stationary or tracking. Tracking solar collectors can be designed to go after the rays of sunlight by moving around either a single axis or double axes.

Solar collectors can also be classified as concentrating and non-concentrating types. The concentration ratio of a solar collector is the relative amount of the solar flux on the receiver to flux on the aperture. Concentrating collectors have a highly reflective surface to reflect and concentrate solar radiation onto a receiver or an absorber, while non-concentrating collectors have a highly absorptive surface with low emittance to maximize heat transfer to the working fluid. Solar collectors are chosen according to the desired process temperature. Table 2 includes an extensive list of solar collectors and their operational temperature ranges [7].

Table 2. Solar collectors [7]

\begin{tabular}{lllll}
\hline Tracking & Collector Type & Absorber & Concentration Ratio & Operational Range \\
\hline \multirow{4}{*}{ Stationary } & Flat plate & Flat & 1 & $30-80{ }^{\circ} \mathrm{C}$ \\
\cline { 2 - 5 } & Evacuated tube & Flat & 1 & $50-200{ }^{\circ} \mathrm{C}$ \\
\cline { 2 - 5 } & Compound parabolic & Tubular & $1-5$ & $60-240{ }^{\circ} \mathrm{C}$ \\
\hline \multirow{5}{*}{ Single-axis } & Compound parabolic & Tubular & $5-15$ & $60-300{ }^{\circ} \mathrm{C}$ \\
\cline { 2 - 5 } & Linear Fresnel & Tubular & $10-40$ & $60-250{ }^{\circ} \mathrm{C}$ \\
\hline \multirow{2}{*}{ Double-axis } & Parabolic trough & Tubular & $15-45$ & $60-300{ }^{\circ} \mathrm{C}$ \\
\cline { 2 - 5 } & Cylindrical trough & Tubular & $10-50$ & $60-300^{\circ} \mathrm{C}$ \\
\cline { 2 - 5 } & Parabolic dish & Point & $100-1000$ & $100-500^{\circ} \mathrm{C}$ \\
\hline
\end{tabular}




\subsubsection{Thermal Energy Storage}

Thermal energy storage in various solid and liquid media is used to synchronize energy supply and demand due to the intermittent nature of solar energy. Energy can be stored as sensible heat, as shown in Table 3, or as latent heat, as shown in Table 4 [8]. Thermal storage design depends on the temperature range of the solar collection and desalination systems, charge and discharge rates, space, corrosivity, and many others.

Table 3. Sensible heat storage material [8]

\begin{tabular}{|c|c|c|c|c|}
\hline Medium & Range $\left({ }^{\circ} \mathrm{C}\right)$ & $\rho\left(\mathrm{kg} / \mathrm{m}^{3}\right)$ & $C p\left(\mathrm{~J} / \mathrm{kg}^{-}{ }^{\circ} \mathrm{C}\right)$ & $K\left(W / m-{ }^{\circ} C\right)$ \\
\hline Water & $0-100$ & 1000 & 4190 & 0.63 \\
\hline Water -10 bar & $0-180$ & 881 & 4190 & - \\
\hline $50 \%$ ethylene glycol & $0-100$ & 1075 & 3480 & - \\
\hline Dowtherm $A^{\circledR}$ & $12-260$ & 867 & 2200 & 0.12 \\
\hline Therminol $66^{\circledR}$ & $-9-343$ & 750 & 2100 & 0.11 \\
\hline Draw salt & $220-540$ & 1733 & 1550 & 0.57 \\
\hline Molten salt & $142-540$ & 1680 & 1560 & 0.61 \\
\hline Liquid sodium & $100-760$ & 750 & 1260 & 67.50 \\
\hline Cast iron & Up to 1150 & 7200 & 540 & 42 \\
\hline Taconite & - & 3200 & 800 & - \\
\hline Aluminum & Up to 660 & 2700 & 920 & 200 \\
\hline Fireclay & - & $2100-2600$ & 1000 & $1.0-1.5$ \\
\hline Rock & - & 1600 & 880 & - \\
\hline
\end{tabular}

Table 4. Latent heat storage material [8]

\begin{tabular}{|c|c|c|c|c|c|c|c|}
\hline \multirow[t]{2}{*}{ Medium } & \multirow{2}{*}{$\begin{array}{c}M P \\
\left({ }^{\circ} \mathrm{C}\right)\end{array}$} & \multirow{2}{*}{$\begin{array}{c}\Delta H^{L} \\
(\mathrm{~kJ} / \mathrm{kg})\end{array}$} & \multicolumn{2}{|c|}{$\rho\left(\mathrm{kg} / \mathrm{m}^{3}\right)$} & \multicolumn{2}{|c|}{$C p\left(\mathrm{~kJ} / \mathrm{kg}-{ }^{\circ} \mathrm{C}\right)$} & \multirow{2}{*}{$\begin{array}{c}k \\
\left(W / m-{ }^{\circ} C\right)\end{array}$} \\
\hline & & & Solid & Liquid & Solid & Liquid & \\
\hline $\mathrm{LiClO}_{3} \cdot 3 \mathrm{H}_{2} \mathrm{O}$ & 8.1 & 253 & 1720 & 1530 & - & - & - \\
\hline $\mathrm{Na}_{2} \mathrm{SO}_{4} \cdot 10 \mathrm{H}_{2} \mathrm{O}$ & 32.4 & 251 & 1460 & 1330 & 1.76 & 3.32 & 2.25 \\
\hline $\mathrm{Na}_{2} \mathrm{~S}_{2} \mathrm{O}_{3} \cdot 5 \mathrm{H}_{2} \mathrm{O}$ & 48 & 200 & 1730 & 1665 & 1.47 & 2.39 & 0.57 \\
\hline $\mathrm{NaCH}_{3} \mathrm{COO} \cdot 3 \mathrm{H}_{2} \mathrm{O}$ & 58 & 180 & 1450 & 1280 & 1.90 & 2.50 & 0.50 \\
\hline $\mathrm{Ba}(\mathrm{OH})_{2} \cdot 8 \mathrm{H}_{2} \mathrm{O}$ & 78 & 301 & 2070 & 1937 & 0.67 & 1.26 & $0.65(\ell)$ \\
\hline $\mathrm{MgNO}_{3} \cdot 6 \mathrm{H}_{2} \mathrm{O}$ & 90 & 163 & 1636 & 1550 & 1.56 & 3.68 & 0.61 \\
\hline $\mathrm{LiNO}_{3}$ & 252 & 530 & 2310 & 1776 & 2.02 & 2.04 & 1.35 \\
\hline $\mathrm{LiCO}_{3} / \mathrm{K}_{2} \mathrm{CO}_{3}$ & 505 & 345 & 2265 & 1960 & 1.34 & 1.76 & - \\
\hline $\mathrm{LiCO}_{3} / \mathrm{K}_{2} \mathrm{CO}_{3} / \mathrm{Na}_{2} \mathrm{CO}_{3}$ & 397 & 277 & 2300 & 2140 & 1.68 & 1.63 & - \\
\hline n-Tetradecane & 5.5 & 228 & 825 & 771 & - & - & 0.15 \\
\hline n-Octadecane & 28 & 244 & 814 & 774 & 2.16 & - & 0.15 \\
\hline$H D P E$ & 126 & 180 & 960 & 900 & 2.88 & 2.51 & 0.36 \\
\hline Steric Acid & 70 & 203 & 941 & 347 & - & 2.35 & $0.17(\ell)$ \\
\hline
\end{tabular}




\subsubsection{Solar Ponds}

Water absorbs solar radiation going through it causing its temperature to rise. The shorter the wave length of sunlight, the deeper it can penetrate the water column as shown in Table 5 [8]. Solar ponds are pools of water with a darkened bottom to maximize light absorption. They are designed to have increasing salinity with depth creating a density gradient that inhibits natural convection currents. The final outcome is a stratified pond with increasing temperature and salinity with depth, as shown in Figure 14 [7]. Solar ponds function as both solar collectors and thermal energy storage media.

Table 5. Spectral absorption of solar radiation in water [8]

\begin{tabular}{lccccc}
\hline \multirow{2}{*}{ Wavelength $(\mu \mathrm{m})$} & \multicolumn{5}{c}{ Layer Depth } \\
\cline { 2 - 6 } & 0 & $1 \mathrm{~cm}$ & $10 \mathrm{~cm}$ & $1 \mathrm{~m}$ & $10 \mathrm{~m}$ \\
\hline $0.2-0.6$ & 23.7 & 23.7 & 23.6 & 22.9 & 17.2 \\
\hline $0.6-0.9$ & 36.0 & 35.3 & 36.0 & 12.9 & 0.9 \\
\hline $0.9-1.2$ & 17.9 & 12.3 & 0.8 & 0.0 & 0.0 \\
\hline$>1.2$ & 22.4 & 1.7 & 0.0 & 0.0 & 0.0 \\
\hline Total & 100.0 & 73.0 & 54.9 & 35.8 & 18.1
\end{tabular}

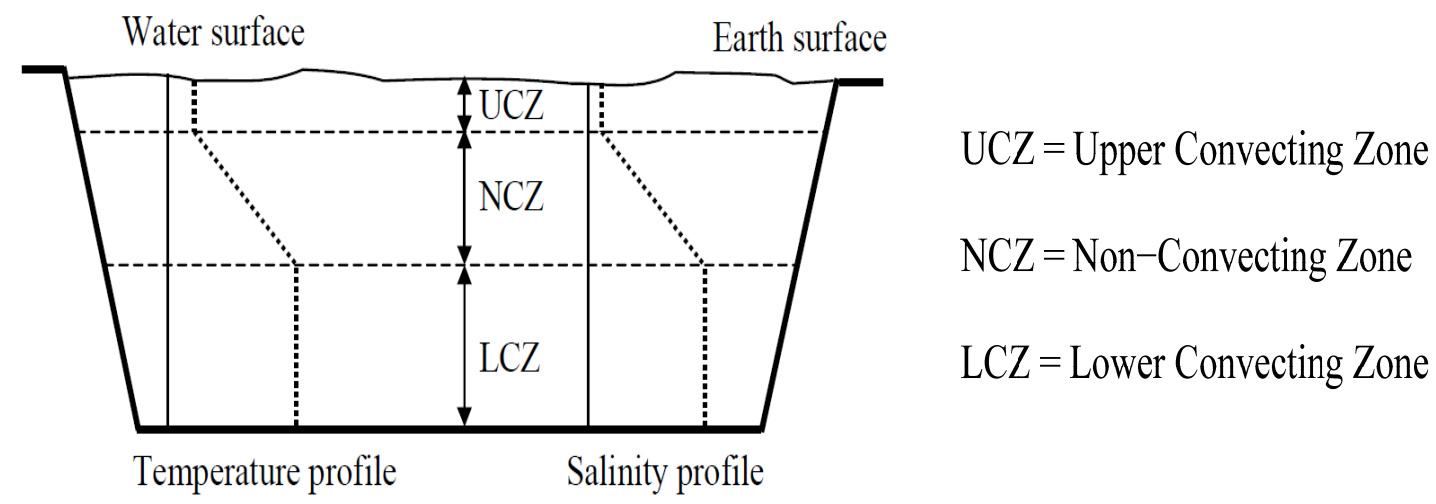

Figure 14. Vertical cross section of a solar pond 


\subsubsection{Photovoltaics}

Photovoltaic cells are made from common semiconductor compounds and can directly convert solar radiation into useful electricity, as shown in Figure 15 [8]. Cells are arranged to form modules that are combined to form panels. Photovoltaic systems include an array of joined panels to produce the required electrical output, as shown in Figure 16 [8]. Photovoltaics can be employed independently or jointly with other sources to generate electricity needed to power physical desalination systems.

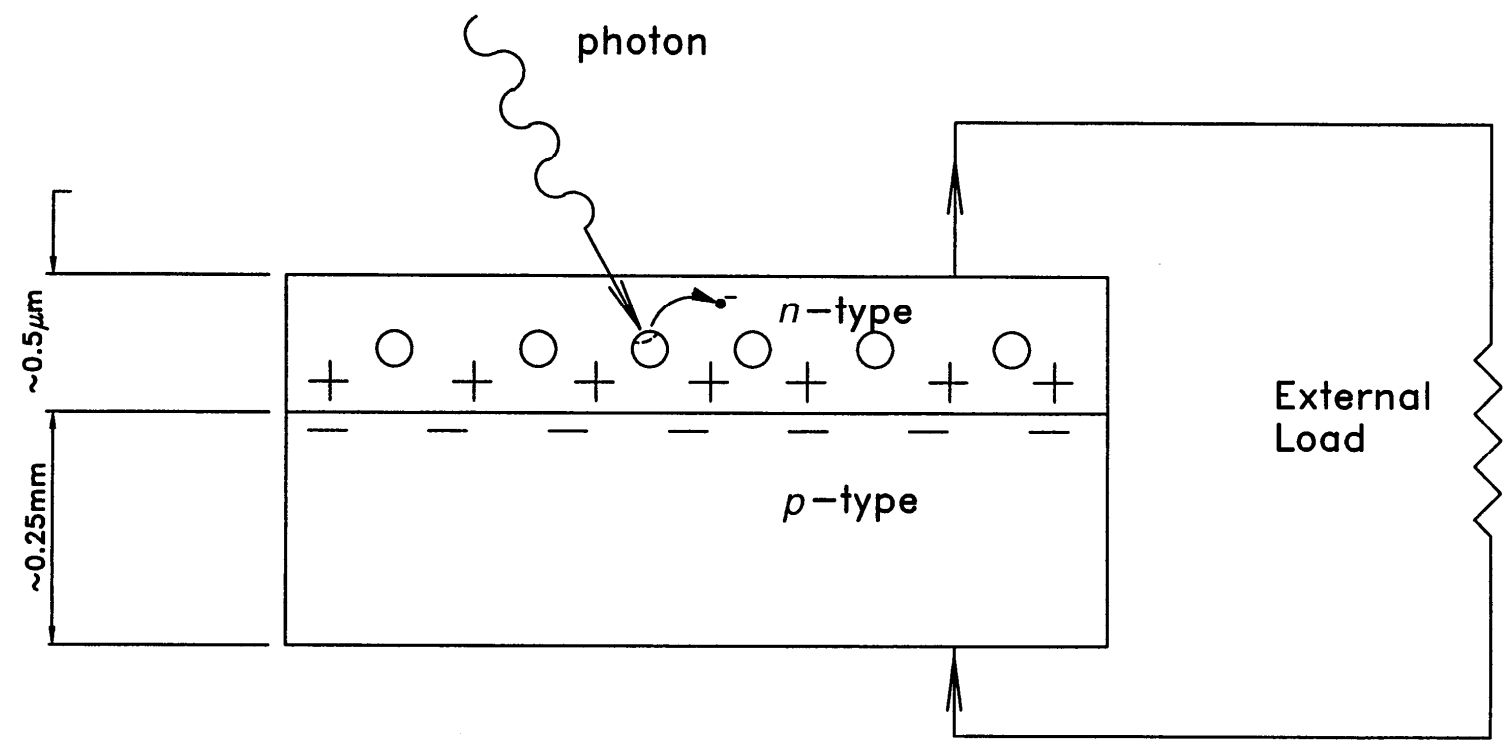

Figure 15. Photovoltaic cell schematics

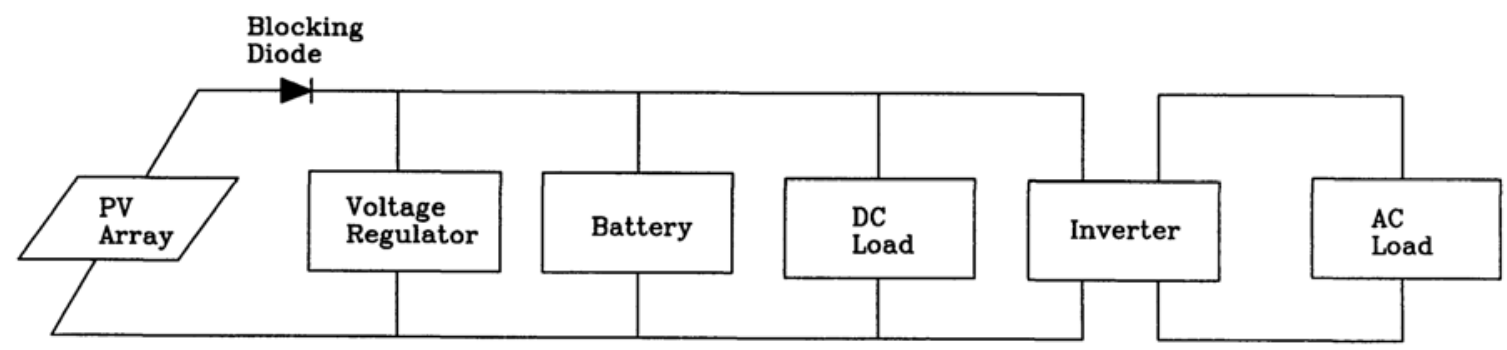

Figure 16. Photovoltaic system schematics 


\section{CHAPTER 3. RESEARCH BACKGROUND}

\subsection{Renewable Energy Desalination Systems}

Water and energy are the most essential ingredients of a flourishing civilization. Fresh water and energy reserves are increasingly exhausted as was mentioned earlier in CHAPTER 1; hence, seawater desalination using renewable energy sources is a very appealing research area. In addition, desalination is an enormously energy exhaustive process making fossil fuel based conventional desalination methods extremely unpopular especially in light of the growing impact of environmental pollution and global warming.

The worldwide capacity of desalination using renewable energy amounts to less than $1 \%$ of that of conventional desalination due to high capital and maintenance costs associated with using renewable energy sources [9]. Several renewable energy driven desalination plants were designed and constructed; however, most of them were geographically customized and built on pilot scale. A detailed record of renewable energy driven desalination plants was put together by Tzen and Morris [10].

Wind energy can be utilized to generate electricity via turbines to run physical and chemical desalination plants, while geothermal energy can be utilized to generate heat via underground heat exchangers to run thermal desalination plants. Solar energy is the most promising renewable energy source due to its ability to drive the more popular thermal desalination systems directly through solar collectors and to drive physical and chemical desalination systems indirectly through photovoltaic cells. 


\subsection{Passive Vacuum Solar Desalination}

The passive vacuum desalination concept was initially developed and examined by Goswami and Kharabsheh [11]. Atmospheric pressure forces seawater from a ground level tank into an elevated vacuumed chamber through an injection pipe where water starts to evaporate due to solar energy supplied to the chamber via a closed loop heat exchanger. The concentrated brine is then withdrawn through a withdrawal pipe annulus to the injection pipe to recover heat, while vapor moves towards a condenser due to a vapor pressure gradient through a finned pipe. Vapor then condenses by losing its latent heat of condensation to the ambient and flows down to a fresh water tank due to gravity. The vacuum is maintained by the hydrostatic balance amongst all of the joined vessels. Figure 17 provides a simple illustration of the passive vacuum solar desalination process.

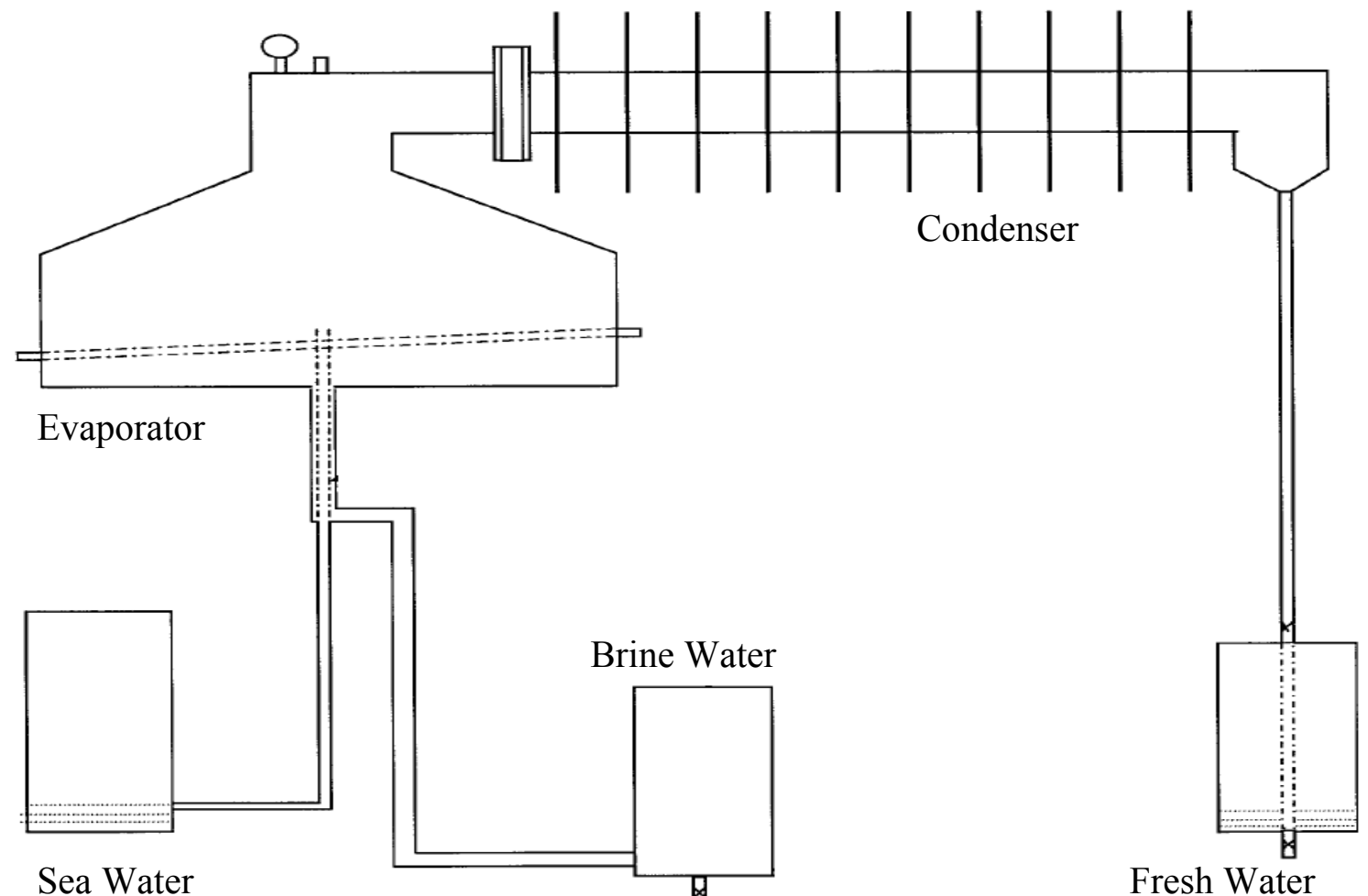

Figure 17. Passive vacuum solar desalination 


\subsection{Passive Vacuum Solar Flash Desalination}

The prior passive vacuum solar process was modified to overcome the big size of the evaporator and its large level fluctuations. Seawater is pumped through a condenser to preheat it before it enters a solar heater where it flashes into the vacuumed evaporator through an expansion orifice to produce water vapor and concentrated brine. The flashed vapor then condenses by losing its latent heat of condensation to the entering seawater in the condenser. The condensate and the concentrated brine flow down to ground tanks due to gravity, while the vacuum is naturally maintained by the hydrostatic balance between the ground and the elevated vessels. Figure 18 provides a simple process flow diagram of the passive vacuum solar flash desalination process that was developed and examined theoretically by Goswami and Maroo [12].

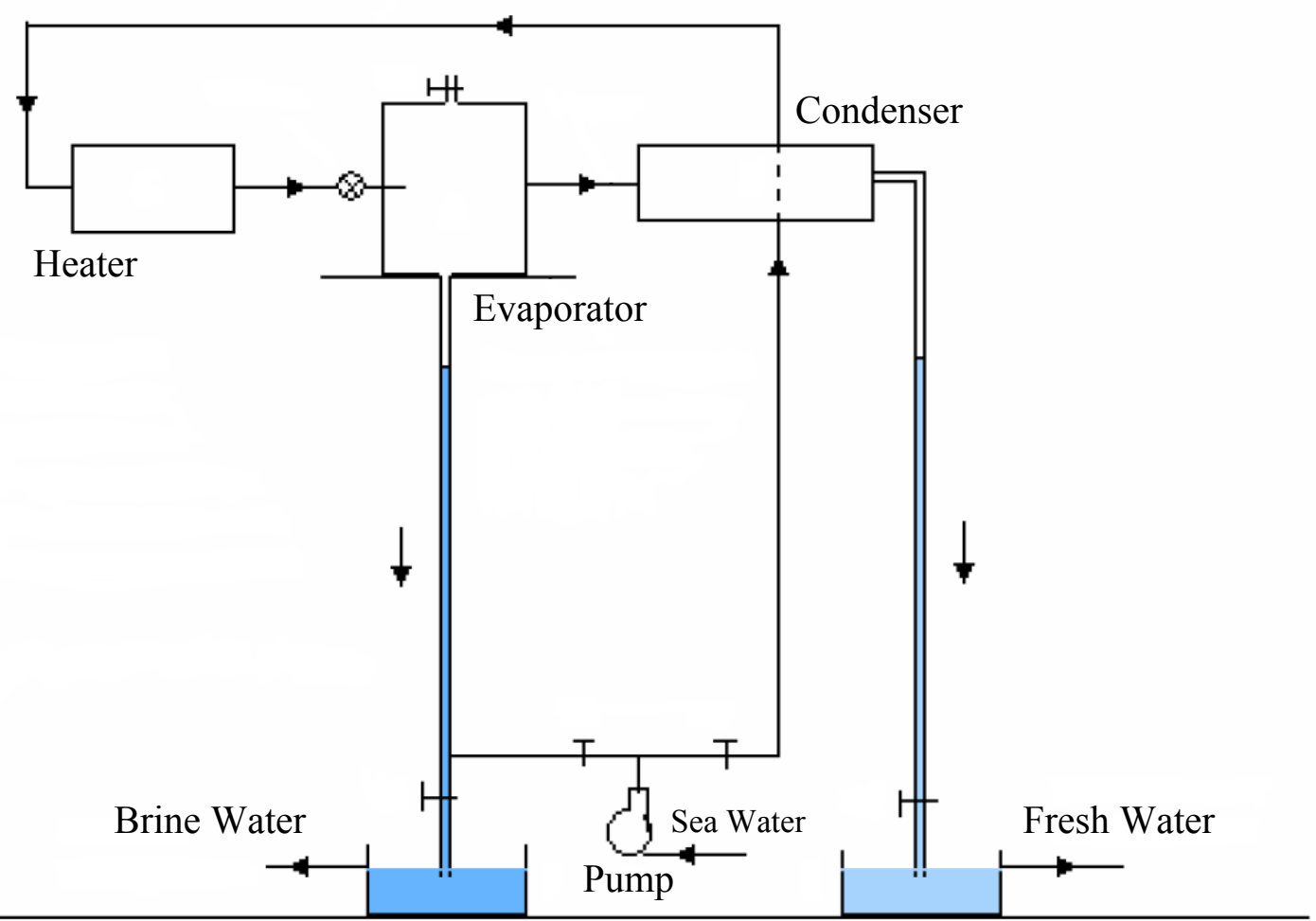

Figure 18. Passive vacuum solar flash desalination 


\subsection{Proposed Desalination System}

The proposed desalination system consists of a saline water tank, a concentrated brine tank, and a fresh water tank placed on ground level plus an evaporator and a condenser located at least ten meters above ground, as shown in Figure 19. The evaporator-condenser assembly, or flash chamber, is initially filled with saline water that later drops into the ground tanks by gravity, creating a vacuum above the water surface in the unit without a vacuum pump. The vacuum is maintained by the hydrostatic pressure balance among all of the connected vessels. The ground tanks are open to the atmosphere, while the flash chamber is insulated and sealed to retain both heat and vacuum.

In a continuous process, cool saline water is pumped through the condenser to preheat it before it enters a solar heater and flashes into a vacuumed evaporator through an expansion orifice or a pressure-reducing valve producing water vapor and concentrated brine. The water vapor then condenses by losing its heat of condensation to the entering saline water in the condenser. The fresh water condensate and concentrated brine flow down to the fresh water and brine water tanks, respectively, due to gravity through linking pipes. Each of the fresh water and the brine water tanks has a discharge pipe located a few centimeters above the level of the inlet water pipes, keeping their levels constant to maintain the vacuum in the flash chamber hydrostatically as well as to retrieve the fresh water product and reject the concentrating brine.

Multi-stage flash desalination scheme of the proposed system can be achieved by flashing seawater in sequentially lower pressure flash chambers, as shown in Figure 20. Employing the multi-stage flash desalination scheme will result in more evaporation and better recovery of heat of condensation, resulting in more fresh water output. 


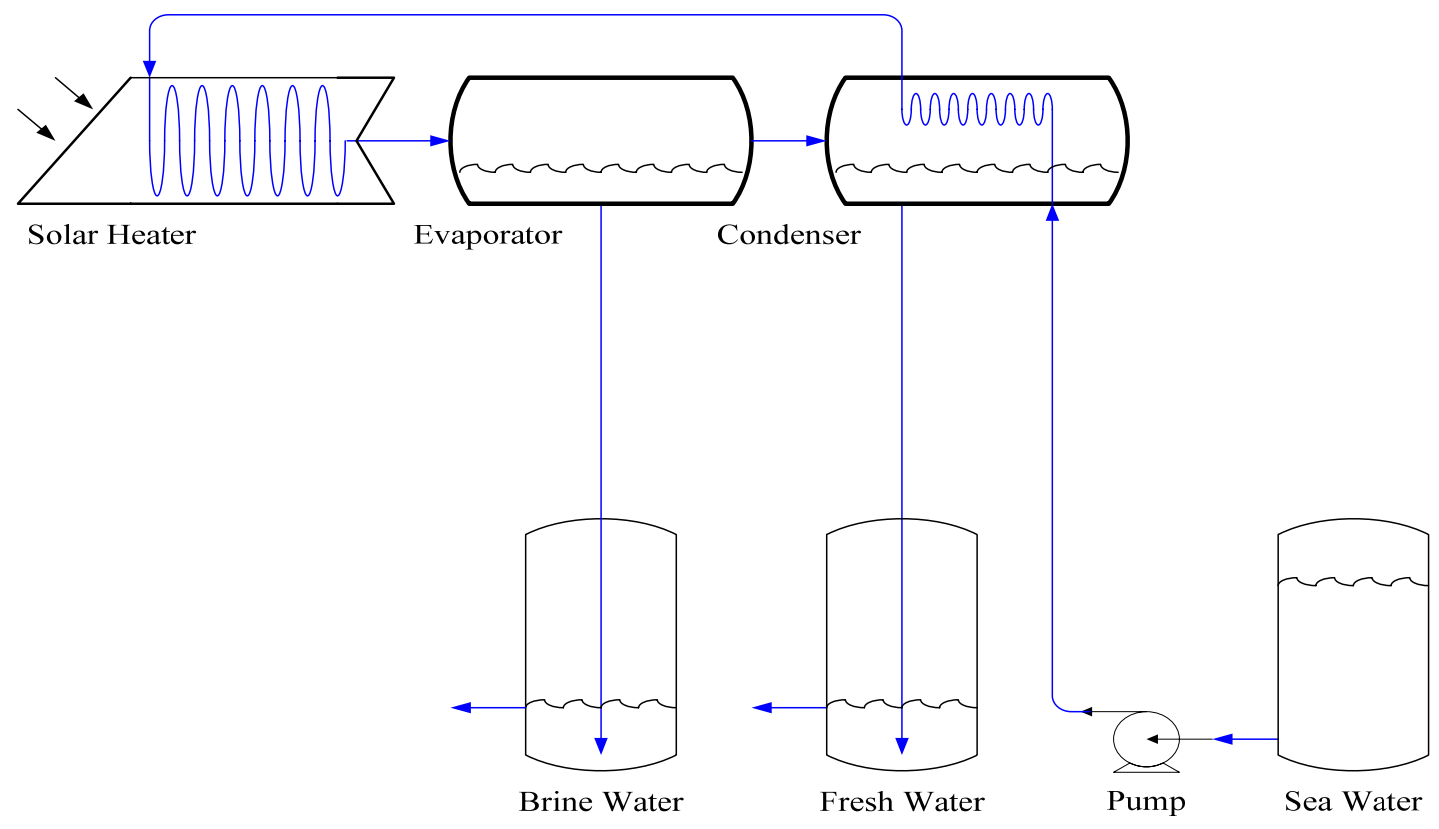

Figure 19. Single-stage solar flash desalination system

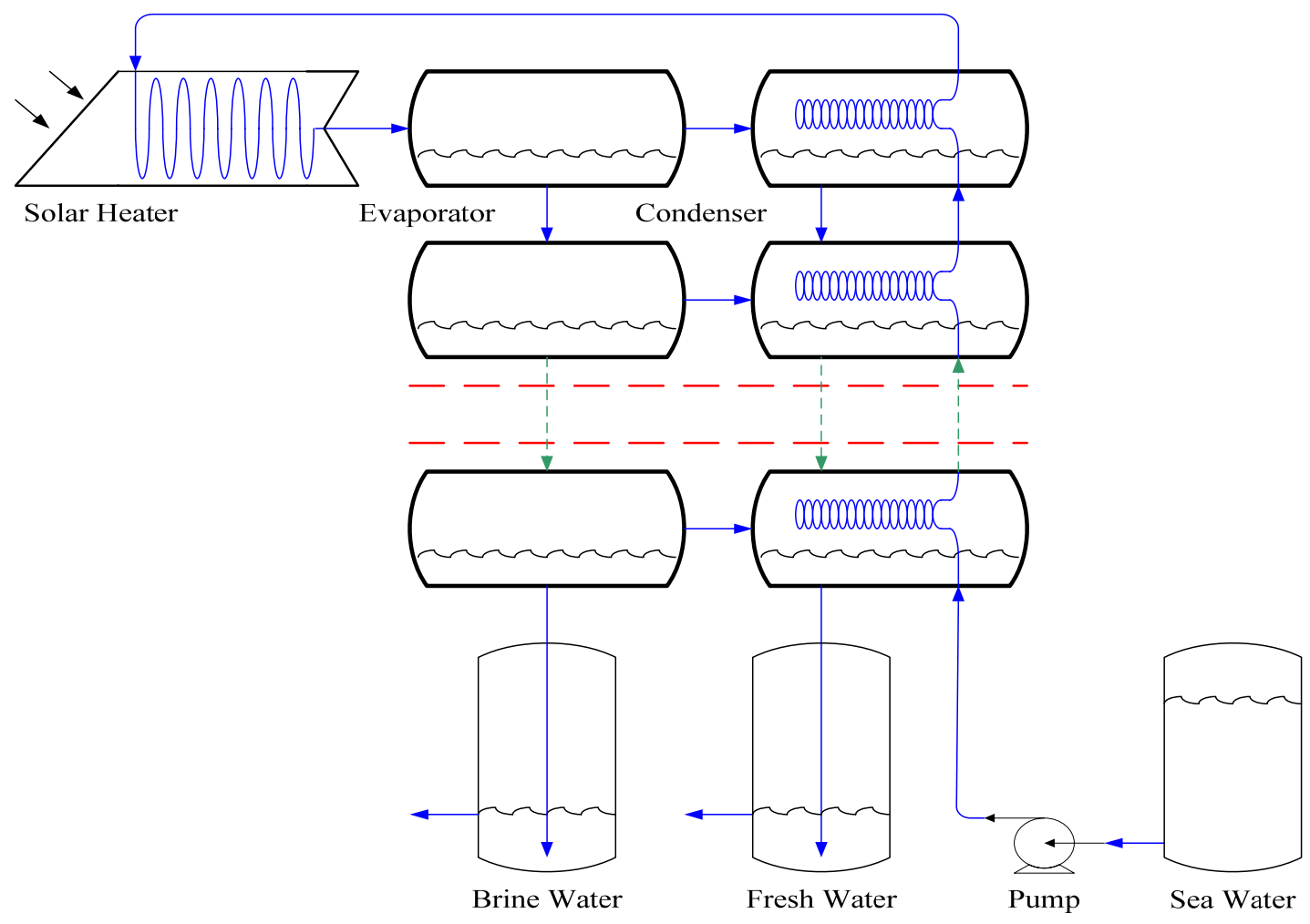

Figure 20. Multi-stage solar flash desalination system 


\section{CHAPTER 4. THEORETICAL ANALYSIS}

\subsection{Process Description}

The proposed desalination system with its designated stream labels is outlined in Figure 21. The desalination process includes two consecutive steps: a start-up procedure and a continuous operation. The start-up procedure is a simple process invoked prior to running the continuous operation and will not be included in the model. The continuous operation is the essential part of the desalination process, and a model will be built to simulate it. The valve positions shown depict the system in continuous operation mode.

The start-up procedure begins by separately pumping the condenser with fresh water and the evaporator with seawater, while their top valves are open and their bottom ones are closed until they are completely filled with water and free of air. Valve positions of both vessels of the flash chamber are then switched to let water drop under gravity, leaving behind a vacuum that is created without a vacuum pump.

The continuous operation begins right after the initial start-up procedure and it consists of pumping seawater through the condenser, preheating it before flowing it through the channels of a solar heater to reach a desired flash temperature. The desired flash temperature is controlled by manipulating the residence time of seawater in the solar heater by varying its flow rate in relation to available solar insolation. Hot seawater then flashes into an insulated vacuumed evaporator through an expansion orifice or a pressure-reducing valve, producing water vapor and concentrated brine. 
The produced water vapor flows to the condenser due to a vapor pressure gradient and condenses by losing its heat of condensation to seawater passing through the condenser while concentrated brine remains in the evaporator. The fresh water condensate and concentrated brine flow down to the fresh water and brine water ground tanks, respectively, due to gravity through linking pipes that stretch down till just above the bottom of the tanks. The fresh water and the brine water ground tanks have discharge pipes positioned a few centimeters higher than the lip of the linking pipes, keeping their levels constant to maintain the vacuum in the flash chamber by the hydrostatic balance with the levels in the flash chamber.

A comprehensive model will be developed to examine the dynamics of proposed continuous desalination operation. The model will employ fundamental laws to describe the process in addition to reliable empirical correlations to estimate physical properties of the involved species and operational parameters of the proposed system. The model will assume total steam condensation as well as quasi steady state operation, accounting for the build up of non-condensable gases in the flash chamber. The model will also account for the natural diffusion process of water vapor occurring because of a vapor pressure gradient present between the hot and cold sides of the flash chamber.

The model will include mass and energy balances around process equipment and geometrical formulas describing equipment layout and size. The Rachford-Rice method [13] will be employed to perform flash calculations, while Bernoulli's fluid equation will be used to perform hydrostatic balance relations. Thermodynamic equilibria and several physical property correlations will also be included in the model. In addition, an integrative equation of state will be used to express rising vacuum pressure. 


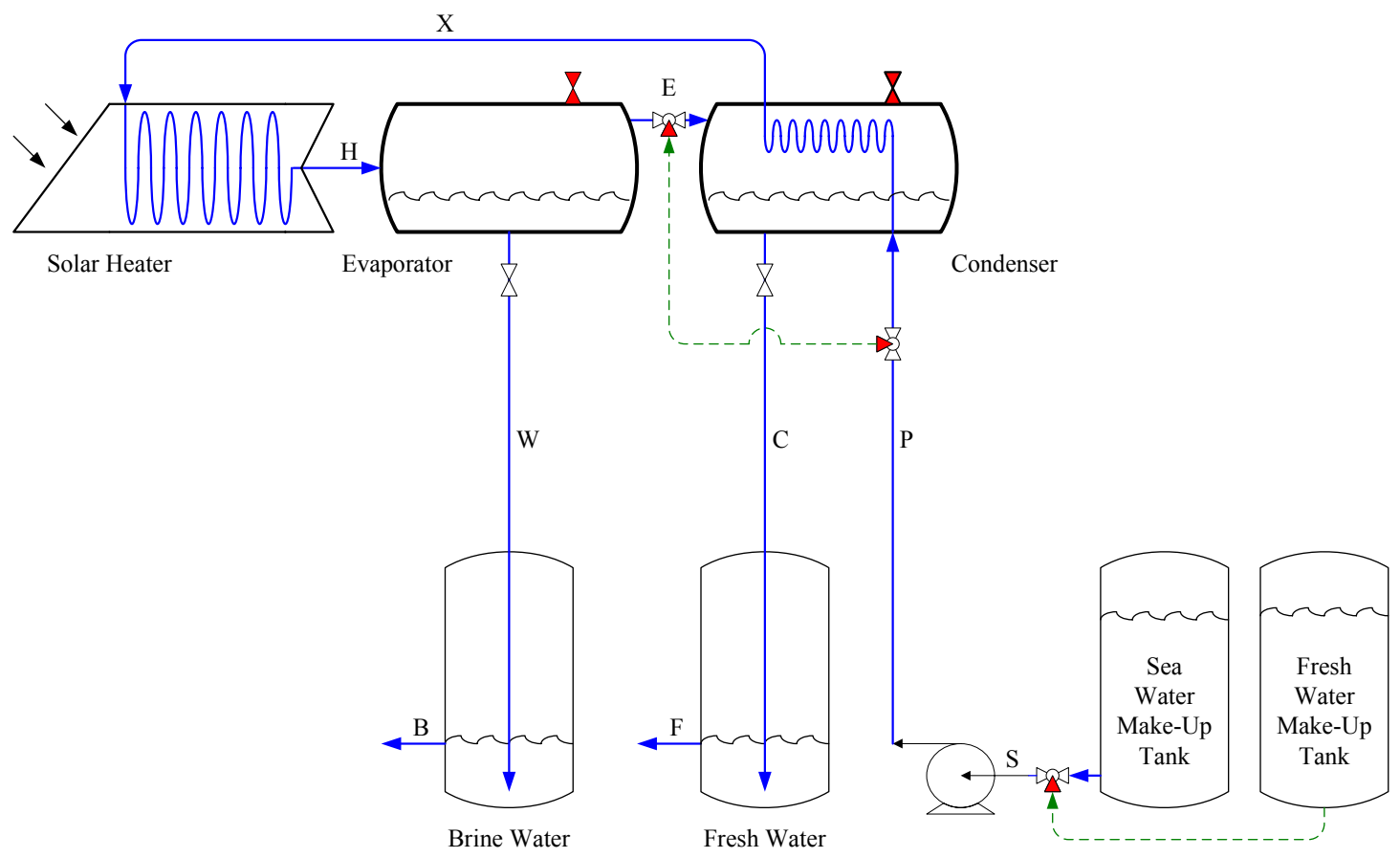

Figure 21. Process schematics

\subsection{Model Development}

Trace components in seawater may affect its kinetics but not its thermodynamic equilibrium; therefore, only major components will be considered in this theoretical analysis. The following sub-sections present all equations used in modeling the proposed system, while the next section sketches the solution algorithm.

The nomenclature and engineering units of all variables used in the model are detailed at the beginning of this dissertation in the LIST OF SYMBOLS section. In addition, stream symbols that appear on the process flow diagram of Figure 21 are used as subscripts for different stream property variables. Process equipment referred to in the model denote the pump, the condenser, the heater, and the evaporator. The complete code with its input and output values can be found in the APPENDICES section. 


\subsubsection{Mass and Energy Balance}

Salt balances around process equipment are given by

$\varphi_{S} \cdot M_{S}=\varphi_{P} \cdot M_{P}$

$\varphi_{P} \cdot M_{P}=\varphi_{X} \cdot M_{X}$

$\varphi_{X} \cdot M_{X}=\varphi_{H} \cdot M_{H}$

$\varphi_{H} \cdot M_{H}=\varphi_{W} \cdot M_{W}$

Overall energy balances around process equipment are given by

$$
\begin{aligned}
& Q_{P}-W_{P}+E_{P}^{\text {in }}-E_{P}^{\text {out }}=0 \\
& Q_{C}-W_{C}+E_{C}^{\text {in }}-E_{C}^{\text {out }}=E_{C}^{a} \\
& Q_{H}-W_{H}+E_{H}^{\text {in }}-E_{H}^{\text {out }}=0 \\
& Q_{E}-W_{E}+E_{E}^{\text {in }}-E_{E}^{\text {out }}=E_{E}^{d}
\end{aligned}
$$

Energy flow inputs to process equipment are given by

$E_{P}^{i n}=M_{S} \cdot H_{S}$

$E_{C}^{i n}=M_{P} \cdot H_{P}+M_{E} \cdot\left(H_{E}+H_{E}^{L}\right)$

$E_{H}^{i n}=M_{X} \cdot H_{X}$

$E_{E}^{i n}=M_{H} \cdot H_{H}$

Energy flow outputs from process equipment are given by

$$
\begin{aligned}
& E_{P}^{\text {out }}=M_{P} \cdot H_{P} \\
& E_{C}^{\text {out }}=M_{X} \cdot H_{X}+M_{C} \cdot H_{C} \\
& E_{H}^{\text {out }}=M_{H} \cdot H_{H} \\
& E_{E}^{\text {out }}=M_{W} \cdot H_{W}+M_{E} \cdot\left(H_{E}+H_{E}^{L}\right)
\end{aligned}
$$


Energy accumulation in the condenser due to non-condensable gases is given by

$E_{C}^{a}=M_{C}^{a} \cdot H_{C}^{a}$

Energy transmitted by the diffusing water molecules from the concentrated brine phase to the fresh water vapor phase is accounted for in the above energy input and output expressions; therefore, an offset term is included in the energy balance of the evaporator to neutralize the effect of that transmitted energy on the flashing process. In other words, offsetting transmitted energy of diffusing water molecules effectively altered the boundary of the above energy balance from the evaporator to expansion orifice. Figure 22 illustrates the mass transfer operations of the proposed system, where flash and diffusion operations occur in the evaporator. The transmitted energy of the diffusing water molecules offset term in the evaporator is given by

$E_{E}^{d}=M_{E}^{d} \cdot\left(H_{W}-H_{E}-H_{E}^{L}\right)$

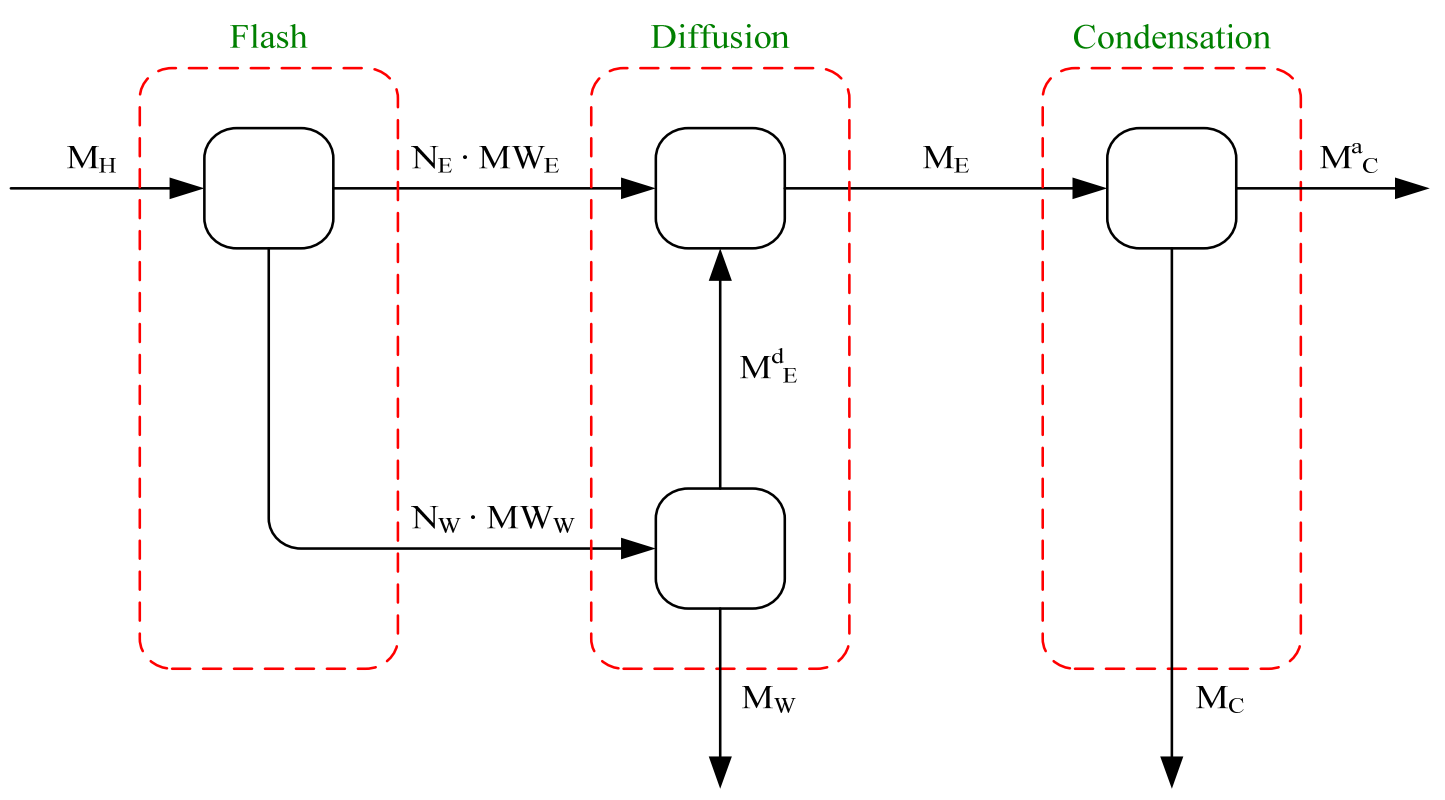

Figure 22. Mass transfer operations 
Demisting is a standard unit operation in industry accomplished by devices called demisters that are fitted to process vessels to ensure a full removal of liquid droplets from vapor streams. No demister was attached to the experimental unit and no demisting is considered in the model; however, flashed vapor can be safely assumed free from entrained brine droplets yielding zero salinity expressed by

$\varphi_{E}=\varphi_{C}=0$

The experimental simulation will be thoroughly discussed in CHAPTER 5 and its output will be comprehensively disclosed in CHAPTER 7; nonetheless, a significant observation regarding the produced amount of fresh water vapor was made and needs to be mentioned here since it will be included in the model.

The maximum amount of fresh water that can be produced by flashing seawater can be approximated by the expression $\int\left[M_{H} \cdot\left(H_{H}-H_{W}\right) /\left(H_{E}+H_{E}^{L}-H_{W}\right)\right] d t$, which is obtained by conducting an energy balance around the expansion orifice assuming seawater to be a single component fluid and ignoring heat losses. Maximum amounts that can be produced were computed using experimental flow and temperature values, then they were compared to actual collected amounts.

Actual amounts of fresh water produced at lower flash temperatures were considerably less than predicted amounts by the single component flash calculation, indicating that a sizeable quantity of the flashed vapor condenses prematurely in the evaporator before making it to the condenser. In contrast, the actual amounts of fresh water produced at higher flash temperatures were much more than predicted amounts by the single component flash calculation, suggesting the presence of a diffusion process of vaporized water molecules from the evaporator to the condenser. 
Bemporad [14] developed a correlation that estimates the diffusion rate of water vapor between two joined chambers under vacuum, where one chamber contains saline water and the other contains fresh water. The correlation was experimentally based with one empirical coefficient, and it identified the gradient $\Delta P_{H 2 O} / \sqrt{ } T$ as the driving force for diffusion. The correlation was slightly modified to properly correspond to the current experimental results yielding the following expression

$$
M_{E}^{d}=\sigma \cdot X A_{E} \cdot\left[\frac{\left(1-0.54 \cdot \varphi_{W}\right) \cdot P_{H_{2} O}^{W}}{\sqrt{T_{W}+273.15}}-\frac{P_{H_{2} O}^{C}+\Omega}{\sqrt{T_{C}+273.15}}\right]
$$

Parameter $\sigma$ serves as a diffusion coefficient, while parameter $\Omega$ serves as a diffusion barrier and both can be adjusted using experimental results. The two parameters can be thought of as conductance and resistance terms, and it is imperative to reiterate that their obtained values pertain to the geometry of the experimental set-up and should be readjusted whenever applied to different geometries using experimental records.

The vapor pressures corresponding to the brine and fresh water temperatures are needed to evaluate the above expression and can be calculated by [15]

$$
\begin{aligned}
& P_{\mathrm{H}_{2} \mathrm{O}}^{W}=\exp \left[P A-\left(\frac{P B}{T_{W}+P C}\right)\right] \\
& P_{\mathrm{H}_{2} \mathrm{O}}^{C}=\exp \left[P A-\left(\frac{P B}{T_{C}+P C}\right)\right]
\end{aligned}
$$

Flash and accumulation computations will be carried out on molar basis; therefore, a mole balance is included in the model to represent both operations by

$$
\begin{aligned}
& N_{H}=N_{W}+N_{E} \\
& N_{E}=N_{C}+N_{C}^{a}
\end{aligned}
$$


The flash operation is the heart of the desalination process and will be thoroughly discussed later. The condensation operation is considered a quasi-steady state operation where the formed non-condensable gases accumulate in the flash chamber, and all of the flashed water vapor condenses forming the fresh water product. This quasi-steady state operation is expressed by

$N_{C}^{a}=\left(1-y_{H_{2} \mathrm{O}}\right) \cdot N_{E}$

Note that the last equation can be replaced by $N_{C}=y_{H 2 O} \cdot N_{E}$ because total accumulation of non-condensable gases and total condensation of water vapor are interchangeable statements. Figure 23 represents a transformation of Figure 22 from mass to a molecular basis to correspond to the above mole balance and is accomplished by dividing the mass flow rates by the stream molecular weights presented next.

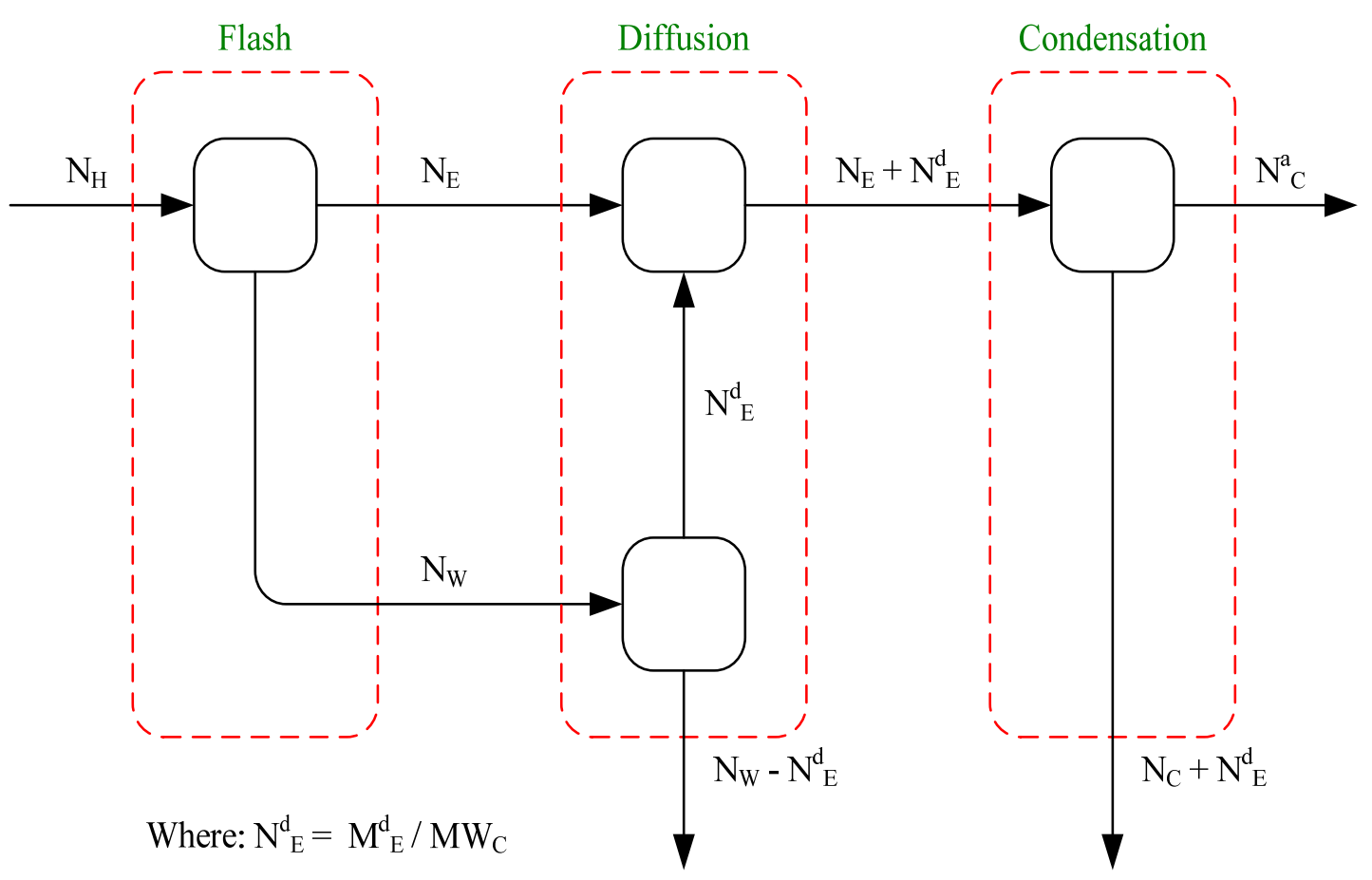

Figure 23. Molecular transfer operations 
Mass flow rate and composition of process streams prior to flashing are considered constant, and their values will be input to the model

$M_{S}=M_{P}=M_{X}=M_{H}$

Both molar and mass flow rates are interchangeably used in this model to allow for flash and accumulation computations on a molar basis and for diffusion and production computations on a mass basis. They can be related using the average molecular weight of process streams that will be introduced later as follows

$$
\begin{aligned}
& M_{H}=N_{H} \cdot M W_{H} \\
& M_{W}=N_{W} \cdot M W_{W}-M_{E}^{d} \\
& M_{E}=N_{E} \cdot M W_{E}+M_{E}^{d} \\
& M_{C}=N_{C} \cdot M W_{C}+M_{E}^{d} \\
& M_{C}^{a}=N_{C}^{a} \cdot M W_{C}^{a}
\end{aligned}
$$

Seawater is a solution of many salts and contains a small amount of dissolved gases. To simplify calculations, seawater salt will be treated as one substance with nitrogen, oxygen, argon, and carbon dioxide making up the dissolved gases. The average molecular weights of seawater salt and process streams are used in relating molar and mass flow rates and can be estimated by considering their major components as [16]

$$
\begin{gathered}
\frac{1}{M W_{\text {Salt }}}=\left(\begin{array}{l}
\frac{\omega_{\mathrm{Cl}}}{M W_{\mathrm{Cl}}}+\frac{\omega_{\mathrm{Na}}}{M W_{\mathrm{Na}}}+\frac{\omega_{\mathrm{SO}_{4}}}{M W_{\mathrm{SO}_{4}}}+\frac{\omega_{\mathrm{Mg}}}{M W_{\mathrm{Mg}}}+\frac{\omega_{\mathrm{Ca}}}{M W_{\mathrm{Ca}}}+\frac{\omega_{K}}{M W_{K}} \\
+\frac{\omega_{\mathrm{HCO}_{3}}}{M W_{\mathrm{HCO}_{3}}}+\frac{\omega_{\mathrm{Br}}}{M W_{\mathrm{Br}}}+\frac{\omega_{B O_{3}}}{M W_{\mathrm{BO}_{3}}}+\frac{\omega_{\mathrm{Sr}}}{M W_{\mathrm{Sr}}}+\frac{\omega_{F}}{M W_{F}}
\end{array}\right) \\
M W_{H}=\left(\begin{array}{l}
z_{\mathrm{N}_{2}} \cdot M W_{\mathrm{N}_{2}}+z_{\mathrm{O}_{2}} \cdot M W_{\mathrm{O}_{2}}+z_{\mathrm{Ar}} \cdot M W_{A r} \\
+z_{\mathrm{CO}_{2}} \cdot M W_{\mathrm{CO}_{2}}+z_{\text {Salt }} \cdot M W_{\text {Salt }}+z_{\mathrm{H}_{2} \mathrm{O}} \cdot M W_{\mathrm{H}_{2} \mathrm{O}}
\end{array}\right)
\end{gathered}
$$




$$
\begin{aligned}
& M W_{W}=\left(\begin{array}{l}
x_{\mathrm{N}_{2}} \cdot M W_{\mathrm{N}_{2}}+x_{\mathrm{O}_{2}} \cdot M W_{\mathrm{O}_{2}}+x_{A r} \cdot M W_{A r} \\
+x_{\mathrm{CO}_{2}} \cdot M W_{\mathrm{CO}_{2}}+x_{\text {Salt }} \cdot M W_{\text {Salt }}+x_{\mathrm{H}_{2} \mathrm{O}} \cdot M W_{\mathrm{H}_{2} \mathrm{O}}
\end{array}\right) \\
& M W_{E}=\left(\begin{array}{l}
y_{\mathrm{N}_{2}} \cdot M W_{\mathrm{N}_{2}}+y_{\mathrm{O}_{2}} \cdot M W_{\mathrm{O}_{2}}+y_{\mathrm{Ar}} \cdot M W_{A r} \\
+y_{\mathrm{CO}_{2}} \cdot M W_{\mathrm{CO}_{2}}+y_{\mathrm{H}_{2} \mathrm{O}} \cdot M W_{\mathrm{H}_{2} \mathrm{O}}
\end{array}\right) \\
& M W_{C}=M W_{H_{2} O} \\
& M W_{C}^{a}=\frac{\left(y_{N_{2}} \cdot M W_{N_{2}}+y_{O_{2}} \cdot M W_{O_{2}}+y_{A r} \cdot M W_{A r}+y_{C_{2}} \cdot M W_{C_{2}}\right)}{\left(1-y_{H_{2} O}\right)}
\end{aligned}
$$

\subsubsection{Equilibrium Distribution Coefficients}

The distribution of non-condensable gases between the flashed vapor and concentrated brine in the flash chamber can be estimated by assuming equilibrium between the two phases. Salt is considered non-volatile and therefore is not present in the flashed vapor. Henry's constants for non-condensable gases and saturation pressure of water are needed to describe this assumed equilibrium.

Henry's constants for the non-condensable gases are given by [17]

$$
\begin{aligned}
& H C_{N_{2}}=H C_{N_{2}}^{o} \cdot \exp \left[-H F_{N_{2}} \cdot\left(\frac{1}{T_{E}+273.15}-\frac{1}{298.15}\right)\right] \\
& H C_{O_{2}}=H C_{O_{2}}^{o} \cdot \exp \left[-H F_{O_{2}} \cdot\left(\frac{1}{T_{E}+273.15}-\frac{1}{298.15}\right)\right] \\
& H C_{A r}=H C_{A r}^{o} \cdot \exp \left[-H F_{A r} \cdot\left(\frac{1}{T_{E}+273.15}-\frac{1}{298.15}\right)\right] \\
& H C_{C_{2}}=H C_{C_{2}}^{o} \cdot \exp \left[-H F_{C O_{2}} \cdot\left(\frac{1}{T_{E}+273.15}-\frac{1}{298.15}\right)\right]
\end{aligned}
$$


The saturated pressure of water is given by [15]

$$
P_{\mathrm{H}_{2} \mathrm{O}}=\exp \left[P A-\left(\frac{P B}{T_{E}+P C}\right)\right]
$$

The equilibrium distribution coefficients are used in mass transfer computations to determine the distribution of chemicals between phases in equilibrium [18]. These are also known as the partition coefficients in the literature or more commonly as K-values. The mentioned vapor-liquid equilibrium distribution coefficient of species $i$ is defined as $K_{i}=y_{i} / x_{i}=\gamma_{i} \cdot P_{i}^{\text {sat }} / P$

The $\mathrm{K}$-value of seawater salt is zero due to its non-volatility, while those of the non-condensable gases as well as water can be approximated using the above temperature-based correlations as follows

$$
\begin{aligned}
& K_{N_{2}}=\frac{y_{N_{2}}}{x_{N_{2}}}=\frac{\frac{P_{N_{2}}}{P_{V}}}{x_{N_{2}}}=\frac{\frac{H C_{N_{2}} \cdot x_{N_{2}}}{P_{V}}}{x_{N_{2}}}=\frac{H C_{N_{2}}}{P_{V}} \\
& K_{O_{2}}=\frac{y_{O_{2}}}{x_{O_{2}}}=\frac{\frac{P_{O_{2}}}{P_{V}}}{x_{O_{2}}}=\frac{\frac{H C_{O_{2}} \cdot x_{O_{2}}}{P_{V}}}{x_{O_{2}}}=\frac{H C_{O_{2}}}{P_{V}} \\
& K_{A r}=\frac{y_{A r}}{x_{A r}}=\frac{\frac{P_{A r}}{P_{V}}}{x_{A r}}=\frac{\frac{H C_{A r} \cdot x_{A r}}{P_{V}}}{x_{A r}}=\frac{H C_{A r}}{P_{V}} \\
& K_{\mathrm{CO}_{2}}=\frac{y_{\mathrm{CO}_{2}}}{x_{\mathrm{CO}_{2}}}=\frac{\frac{P_{\mathrm{CO}_{2}}}{P_{V}}}{x_{\mathrm{CO}_{2}}}=\frac{\frac{\mathrm{HC}_{\mathrm{CO}_{2}} \cdot x_{\mathrm{CO}_{2}}}{P_{V}}}{x_{\mathrm{CO}_{2}}}=\frac{H C_{\mathrm{CO}_{2}}}{P_{V}} \\
& K_{\mathrm{H}_{2} \mathrm{O}}=\frac{\gamma_{\mathrm{H}_{2} \mathrm{O}} \cdot P_{\mathrm{H}_{2} \mathrm{O}}}{P_{V}}
\end{aligned}
$$


Vapor-liquid equilibrium distribution coefficients were obtained using the SUPERTRAPPTM program, an interactive computer code distributed by the National Institute of Standards and Technology that calculates thermodynamic properties of mixtures based on the Peng-Robinson equation of state. SUPERTRAPPTM was employed to perform isobaric phase equilibria flash calculations for water with an average content of non-condensable gas as reported in literature [16] at various temperatures to produce a dataset of $\mathrm{K}$-values. Least squares regression was then used to fit the data to the above equilibrium equations by adjusting values of $H C^{\circ}{ }_{i}, H F_{i}, P A, P B$, and $P C$ producing correlation coefficients very close to unity as will be seen later in CHAPTER 6.

The SUPERTRAPPTM code used in generating the vapor-liquid equilibrium distribution coefficient data and the Matlab ${ }^{\mathrm{TM}}$ codes used in regressing that data to adjust the vapor-liquid equilibrium parameters are in the APPENDICES section.

SUPERTRAPPTM simulations are fresh water based, and no salts were included in their flash calculations. To adjust phase equilibria computations of the current model for saline water, $\mathrm{K}$-values are multiplied by a relativity parameter that can be defined as $\alpha_{i}=$ solubility in fresh water / solubility in seawater for solutes and $\alpha_{H 2 O}=$ seawater saturated pressure / fresh water saturated pressure for water. The relativity factor is a single constant obtained by averaging literature data given over the operating temperature range to simplify calculations [19].

Activity is a way for expressing the effective concentrations of species to account for their deviation from ideal behavior. Activity can be applied to any concentration scales such as molality, molarity, or fractional scales; however, molar fraction is the most common concentration scale used in flash calculation. 
Activity accounts for deviations from ideal behavior by multiplying the concentration by an activity coefficient that can be experimentally determined or empirically computed using several available models. The value of an activity coefficient approaches unity as molecular interactions behave more ideally.

The activity coefficient of water is needed to calculate its $\mathrm{K}$-value. Experimental data can be used to perform adiabatic flash calculations, generating activity coefficient data that are then used to find an activity coefficient correlation resembling

$$
\gamma_{\mathrm{H}_{2} \mathrm{O}}=f\left(\frac{P_{V}}{P_{\mathrm{H}_{2} \mathrm{O}}}\right)
$$

\subsubsection{Adiabatic Flash}

The flash operation of the proposed desalination process is an adiabatic expansion operation where the temperature of seawater drops upon entering the flash chamber due to the drawn enthalpy of vaporization by the flashing water vapor, attaining a saturation temperature used in the above equilibrium calculations. Flash computations are carried out on a molar basis, and the molar composition of the stream entering the flash chamber can be calculated from the average composition of seawater reported on mass basis [16]

$$
\begin{aligned}
& z_{N_{2}}=\frac{\frac{\varphi_{N_{2}}}{M W_{N_{2}}}}{\frac{\varphi_{N_{2}}}{M W_{N_{2}}}+\frac{\varphi_{O_{2}}}{M W_{O_{2}}}+\frac{\varphi_{A r}}{M W_{A r}}+\frac{\varphi_{C O_{2}}}{M W_{C O_{2}}}+\frac{\varphi_{H}}{M W_{\text {Salt }}}+\frac{\varphi_{\mathrm{H}_{2} \mathrm{O}}}{M W_{\mathrm{H}_{2} \mathrm{O}}}} \\
& z_{\mathrm{O}_{2}}=\frac{\frac{\varphi_{\mathrm{O}_{2}}}{M W_{\mathrm{O}_{2}}}}{\frac{\varphi_{\mathrm{N}_{2}}}{M W_{\mathrm{N}_{2}}}+\frac{\varphi_{\mathrm{O}_{2}}}{M W_{\mathrm{O}_{2}}}+\frac{\varphi_{\mathrm{Ar}}}{M W_{A r}}+\frac{\varphi_{\mathrm{CO}_{2}}}{M W_{\mathrm{CO}_{2}}}+\frac{\varphi_{\mathrm{H}}}{M W_{\text {Salt }}}+\frac{\varphi_{\mathrm{H}_{2} \mathrm{O}}}{M W_{\mathrm{H}_{2} \mathrm{O}}}}
\end{aligned}
$$




$$
\begin{aligned}
& z_{A r}=\frac{\frac{\varphi_{A r}}{M W_{A r}}}{\frac{\varphi_{N_{2}}}{M W_{N_{2}}}+\frac{\varphi_{O_{2}}}{M W_{O_{2}}}+\frac{\varphi_{A r}}{M W_{A r}}+\frac{\varphi_{C O_{2}}}{M W_{C O_{2}}}+\frac{\varphi_{H}}{M W_{\text {Salt }}}+\frac{\varphi_{H_{2} O}}{M W_{H_{2} O}}} \\
& z_{\mathrm{CO}_{2}}=\frac{\frac{\varphi_{\mathrm{CO}_{2}}}{M W_{\mathrm{CO}_{2}}}}{\frac{\varphi_{\mathrm{N}_{2}}}{M W_{\mathrm{N}_{2}}}+\frac{\varphi_{\mathrm{O}_{2}}}{M W_{\mathrm{O}_{2}}}+\frac{\varphi_{\mathrm{Ar}}}{M W_{\mathrm{Ar}}}+\frac{\varphi_{\mathrm{CO}_{2}}}{M W_{\mathrm{CO}_{2}}}+\frac{\varphi_{\mathrm{H}}}{M W_{\text {Salt }}}+\frac{\varphi_{\mathrm{H}_{2} \mathrm{O}}}{M W_{\mathrm{H}_{2} \mathrm{O}}}} \\
& z_{\text {Salt }}=\frac{\frac{\varphi_{\mathrm{H}}}{M W_{\text {Salt }}}}{\frac{\varphi_{\mathrm{N}_{2}}}{M W_{\mathrm{N}_{2}}}+\frac{\varphi_{\mathrm{O}_{2}}}{M W_{\mathrm{O}_{2}}}+\frac{\varphi_{\mathrm{Ar}}}{M W_{\mathrm{Ar}}}+\frac{\varphi_{\mathrm{CO}_{2}}}{M W_{\mathrm{CO}_{2}}}+\frac{\varphi_{\mathrm{H}}}{M W_{\text {Salt }}}+\frac{\varphi_{\mathrm{H}_{2} \mathrm{O}}}{M W_{\mathrm{H}_{2} \mathrm{O}}}}
\end{aligned}
$$

The molar composition of the concentrated brine is given by

$$
\begin{aligned}
& x_{N_{2}}=\frac{z_{N_{2}} \cdot N_{H}}{N_{W}+N_{E} \cdot \alpha_{N_{2}} \cdot K_{N_{2}}} \\
& x_{O_{2}}=\frac{z_{O_{2}} \cdot N_{H}}{N_{W}+N_{E} \cdot \alpha_{O_{2}} \cdot K_{O_{2}}} \\
& x_{A r}=\frac{z_{A r} \cdot N_{H}}{N_{W}+N_{E} \cdot \alpha_{A r} \cdot K_{A r}} \\
& x_{C O_{2}}=\frac{z_{C O_{2}} \cdot N_{H}}{N_{W}+N_{E} \cdot \alpha_{C O_{2}} \cdot K_{C O_{2}}} \\
& x_{S a l t}=\frac{z_{S a l t} \cdot N_{H}}{N_{W}} \\
& x_{H_{2} O}=\frac{z_{H_{2} O} \cdot N_{H}}{N_{W}+N_{E} \cdot \alpha_{H_{2} O} \cdot K_{H_{2} O}}
\end{aligned}
$$

Similarly, the molar composition of the flashed vapor is given by

$$
y_{N_{2}}=x_{N_{2}} \cdot \alpha_{N_{2}} \cdot K_{N_{2}}
$$




$$
\begin{aligned}
& y_{\mathrm{O}_{2}}=x_{\mathrm{O}_{2}} \cdot \alpha_{\mathrm{O}_{2}} \cdot K_{\mathrm{O}_{2}} \\
& y_{\mathrm{Ar}}=x_{\mathrm{Ar}} \cdot \alpha_{\mathrm{Ar}} \cdot K_{\mathrm{Ar}} \\
& y_{\mathrm{CO}_{2}}=x_{\mathrm{CO}_{2}} \cdot \alpha_{\mathrm{CO}_{2}} \cdot K_{\mathrm{CO}_{2}} \\
& y_{\mathrm{H}_{2} \mathrm{O}}=x_{\mathrm{H}_{2} \mathrm{O}} \cdot \alpha_{\mathrm{H}_{2} \mathrm{O}} \cdot K_{\mathrm{H}_{2} \mathrm{O}}
\end{aligned}
$$

Fraction summations are given by

$$
\begin{aligned}
& \varphi_{\mathrm{N}_{2}}+\varphi_{\mathrm{O}_{2}}+\varphi_{\mathrm{Ar}}+\varphi_{\mathrm{CO}_{2}}+\varphi_{\mathrm{H}}+\varphi_{\mathrm{H}_{2} \mathrm{O}}=1 \\
& z_{\mathrm{N}_{2}}+z_{\mathrm{O}_{2}}+z_{\mathrm{Ar}}+z_{\mathrm{CO}_{2}}+z_{\mathrm{Salt}}+z_{\mathrm{H}_{2} \mathrm{O}}=1 \\
& x_{\mathrm{N}_{2}}+x_{\mathrm{O}_{2}}+x_{\mathrm{Ar}}+x_{\mathrm{CO}_{2}}+x_{\mathrm{Salt}}+x_{\mathrm{H}_{2} \mathrm{O}}=1 \\
& y_{\mathrm{N}_{2}}+y_{\mathrm{O}_{2}}+y_{\mathrm{Ar}}+y_{\mathrm{CO}_{2}}+y_{\mathrm{H}_{2} \mathrm{O}}=1
\end{aligned}
$$

\subsubsection{Heat Transfer}

Computing temperatures of streams exiting the flash chamber properly is essential in accurately evaluating performance of the proposed desalination system; therefore, heat transfer calculations are included in the model, complimenting the above energy balance to solve for those temperatures. Heat transfer calculations are included to estimate the amount of heat transferred from the condensing vapor to the entering seawater feed through the condenser tube as well as the heat loss from both compartments of the flash chamber through the walls of the condenser and evaporator.

The condenser is exposed to maximize heat loss, while the evaporator is insulated to minimize heat loss, and the entire flash chamber is vacuum sealed. The vapor pressure gradient between the two compartments of the flash chamber is the driving force of vapor transfer from the hot evaporator to the cold condenser to produce fresh water. 
The condenser will be modeled as a shell and tube heat exchanger, both where the cold seawater is flowing inside a coiled tube placed in an exposed shell and where the flashed vapor is condensing on the outer surface of that coiled tube by losing its latent heat of condensation to the entering cold seawater. The evaporator will be modeled as an insulated vessel, where heated seawater is flashing producing fresh water vapor that moves to the condenser due to lower vapor pressure through a connecting duct.

Heat transfer is a complex process, particularly when phase change is involved. Heat transfer can come about in different modes; however, the current model will use the overall heat transfer approach to simplify computations.

The inside and outside fluid film coefficients can be estimated by the following correlations that were developed specifically for water and stagnant air [20] as well as evaporating and condensing steam [15]

$$
\begin{aligned}
& h_{C T}^{i}=3.0525 \cdot 10^{-4} \cdot\left(\frac{M_{X}}{D_{C T} \cdot \rho_{X}}\right)^{0.8} \cdot\left(\frac{1.35+0.02 \cdot T_{X}}{D_{C T}}\right) \\
& h_{C T}^{o}=0.725 \cdot\left(\frac{g \cdot H_{E}^{L} \cdot \rho_{X}{ }^{2} \cdot k_{X}{ }^{3}}{N_{C T} \cdot \mu_{X} \cdot\left(D_{C T}+\delta_{C T}\right) \cdot\left(T_{E}-T_{X}\right)}\right)^{0.25} \\
& h_{C}^{i}=1.13 \cdot\left(\frac{g \cdot H_{E}^{L} \cdot \rho_{C}{ }^{2} \cdot k_{C}{ }^{3}}{\mu_{C} \cdot L_{C} \cdot\left(T_{E}-T\right)}\right)^{0.25} \\
& h_{C}^{o}=0.0448 \cdot\left(\frac{T_{E}-T}{L_{C}}\right)^{0.25} \\
& h_{E}^{i}=1.13 \cdot\left(\frac{g \cdot H_{E}^{L} \cdot \rho_{W}{ }^{2} \cdot k_{W}{ }^{3}}{\mu_{W} \cdot L_{E} \cdot\left(T_{E}-T\right)}\right)^{0.25} \\
& h_{E}^{o}=0.0448 \cdot\left(\frac{T_{E}-T}{L_{E}}\right)^{0.25}
\end{aligned}
$$


The overall heat transfer coefficient is a simplified parameter used in gauging overall convective and conductive resistance to heat transfer. Overall heat transfer coefficients are computed by the following correlations [20]

$$
\begin{aligned}
& \frac{1}{U_{C T}}=\frac{1}{h_{C T}^{o}}+\frac{1}{h_{C T}^{o d}}+\frac{D_{C T}+\delta_{C T}}{D_{C T} \cdot h_{C T}^{i}}+\frac{D_{C T}+\delta_{C T}}{D_{C T} \cdot h_{C T}^{i d}}+\frac{D_{C T}+\delta_{C T}}{2 \cdot k_{C T}^{w}} \cdot \operatorname{Ln}\left[\frac{D_{C T}+\delta_{C T}}{D_{C T}}\right] \\
& \frac{1}{U_{C}}=\frac{1}{h_{C}^{o}}+\frac{1}{h_{C}^{o d}}+\frac{D_{C}+\delta_{C}}{D_{C} \cdot h_{C}^{i}}+\frac{D_{C}+\delta_{C}}{D_{C} \cdot h_{C}^{i d}}+\frac{D_{C}+\delta_{C}}{2 \cdot k_{C}^{w}} \cdot \operatorname{Ln}\left[\frac{D_{C}+\delta_{C}}{D_{C}}\right] \\
& \frac{1}{U_{E}}=\frac{1}{h_{E}^{o}}+\frac{1}{h_{E}^{\text {od }}}+\frac{D_{E}+\delta_{E}}{D_{E} \cdot h_{E}^{i}}+\frac{D_{E}+\delta_{E}}{D_{E} \cdot h_{E}^{i d}}+\frac{D_{E}+\delta_{E}}{2 \cdot k_{E}^{w}} \cdot \operatorname{Ln}\left[\frac{D_{E}+\delta_{E}}{D_{E}}\right]
\end{aligned}
$$

Heat transfer area is assumed to be equal to that of the inner surface of the heat transfer medium, with the end sections ignored; therefore, heat exchange surface areas are given by the following geometrical relationships

$$
\begin{aligned}
& A_{C T}=\pi \cdot D_{C T} \cdot L_{C T} \\
& A_{C}=\pi \cdot D_{C} \cdot L_{C} \\
& A_{E}=\pi \cdot D_{E} \cdot L_{E}
\end{aligned}
$$

The log mean temperature difference is a logarithmic average of the temperature difference between the hot and cold streams of a heat exchanger. It represents the driving force for heat transfer in heat exchangers, since that heat transfer is directly proportional to its value. The log mean temperature difference expressions are given by

$$
\begin{gathered}
\Delta \operatorname{Tm}_{C T}=\frac{T_{X}-T_{P}}{\operatorname{Ln}\left[\frac{T_{E}-T_{P}}{T_{E}-T_{X}}\right]} \\
\Delta \operatorname{Tm}_{C}=\frac{T_{C}-T}{\operatorname{Ln}\left[\frac{T_{E}-T}{T_{E}-T_{C}}\right]}
\end{gathered}
$$




$$
\Delta T m_{E}=\frac{T_{W}-T}{\operatorname{Ln}\left[\frac{T_{E}-T}{T_{E}-T_{W}}\right]}
$$

The counter-current departure parameters are dimensionless ratios used in correcting the log mean temperature difference expressions and are given by

$S_{C T}=\frac{T_{X}-T_{P}}{T_{E}-T_{P}}$

$S_{C}=\frac{T_{C}-T}{T_{E}-T}$

$S_{E}=\frac{T_{W}-T}{T_{E}-T}$

The heat transferred from the condensing vapor to the entering seawater, as well as the heat loss from the condenser and from the evaporator, are given by

$$
\begin{aligned}
& M_{X} \cdot H_{X}-M_{P} \cdot H_{P}=60 \cdot U_{C T} \cdot A_{C T} \cdot F_{C T} \cdot \Delta T m_{C T} \\
& -Q_{C}=60 \cdot U_{C} \cdot A_{C} \cdot F_{C} \cdot \Delta T m_{C} \\
& -Q_{E}=60 \cdot U_{E} \cdot A_{E} \cdot F_{E} \cdot \Delta T m_{E}
\end{aligned}
$$

The counter-current departure correction factors are dimensionless variables used in correcting log mean temperature difference expressions. The counter-current departure correction factors are widely available in literature as look-up charts for many types of heat exchangers and can be computed empirically as functions of counter-current departure parameters

$$
\begin{aligned}
& F_{C T}=f\left(S_{C T}\right) \\
& F_{C}=f\left(S_{C}\right) \\
& F_{E}=f\left(S_{E}\right)
\end{aligned}
$$


The vapor pressure of seawater is $1.84 \%$ lower than that of pure water at the same temperature due to non-volatile salts, and therefore, the boiling point of seawater is slightly higher than that of fresh water. This phenomenon is known as the boiling point elevation or vapor pressure depression. Boiling point elevation is a function of salinity and does not depend on properties of solute or solvent [14].

The temperature of seawater drops upon entering the flash chamber to attain equilibrium; however, equilibrium is not always fully achieved. This phenomenon is known as the non-equilibrium allowance, and it depends on several factors such as flash temperature, flow rates, concentrated brine depth, and chamber geometry.

Correlations for boiling point elevation [21] and non-equilibrium allowance [22] can be incorporated into the model to account for flash efficiency as follows $T_{W}=T_{E}+B P E+N E A$

\subsubsection{Vacuum Volume}

All vessels will be modeled as right circular cylinders with specified dimensions. The fresh water and the brine water tanks are equipped with discharge pipes located slightly above the level of the inlet water pipes, keeping their levels constant. Conversely, levels of the seawater tank, as well as condenser and evaporator, are constantly changing during operation; therefore, they need to be computed and included in the model. The level of the feed seawater tank is used in calculating the vertical discharge pressure head that will be used in determining the pumping requirements, while levels of the condenser and evaporator are used in calculating the vacuum volume that will be used in calculating the vacuum pressure. 
The circular cross sectional areas of these vessels are needed to calculate their levels and are determined by

$$
\begin{aligned}
& X A_{S}=\frac{\pi}{4} D_{S}^{2} \\
& X A_{E}=\frac{\pi}{4} D_{E}^{2} \\
& X A_{C}=\frac{\pi}{4} D_{C}^{2}
\end{aligned}
$$

The level of the seawater tank decreases with time because of the continuous pumping of seawater to the process, and it can be geometrically computed by

$$
Z_{S}=Z_{S}^{i}-\frac{\int M_{S} d t}{\rho_{S} \cdot X A_{S}}
$$

If the seawater flow rate remains constant during operation, the numerator of the second term of the above equation simplifies to $\int M_{S} d t=M_{S} \cdot t$.

The level in the condenser is hydrostatically balanced with the level in the fresh water tank. Since the level in the fresh water tank is kept constant, the vacuum pressure inside the flash chamber is the only variable controlling the level in the condenser. The level in the condenser decreases as vacuum pressure increases due to non-condensable gases building up in the flash chamber.

The initial and the dynamic levels in the condenser can be estimated using Bernoulli's fluid equation given by

$$
\begin{aligned}
& Z_{C}^{i}=-\left(\frac{P-P_{V}^{i}-\Delta P_{C}}{\rho_{C} \cdot g}\right) \cdot 10^{6}-P L_{C}+Z_{F} \\
& Z_{C}=-\left(\frac{P-P_{V}-\Delta P_{C}}{\rho_{C} \cdot g}\right) \cdot 10^{6}-P L_{C}+Z_{F}
\end{aligned}
$$


Similarly, the level in the evaporator is hydrostatically balanced with the level in the brine water tank. Since the level in the brine water tank is kept constant, the vacuum pressure inside the flash chamber is the only variable controlling the level in the evaporator. The level in the evaporator decreases as vacuum pressure increases due to non-condensable gases building up in the flash chamber.

The initial and the dynamic levels in the evaporator can be estimated using Bernoulli's fluid equation given by

$$
\begin{aligned}
& Z_{E}^{i}=-\left(\frac{P-P_{V}^{i}-\Delta P_{W}}{\rho_{W} \cdot g}\right) \cdot 10^{6}-P L_{W}+Z_{B} \\
& Z_{E}=-\left(\frac{P-P_{V}-\Delta P_{W}}{\rho_{W} \cdot g}\right) \cdot 10^{6}-P L_{W}+Z_{B}
\end{aligned}
$$

The initial and the dynamic volumes of the vacuum in the condenser depend on corresponding initial and dynamic levels of the condenser. They are geometrically computed by subtracting corresponding fresh water volume from total volume of the right circular horizontal cylinder condenser as follows

$$
\begin{aligned}
& V_{C V}^{i}=L_{C} \cdot\left(\frac{\pi \cdot D_{C}{ }^{2}}{8}+\frac{D_{C}{ }^{2}}{4} \cdot \operatorname{Arcsin}\left(1-\frac{2 \cdot Z_{C}^{i}}{D_{C}}\right)+\left(\frac{D_{C}}{2}-Z_{C}^{i}\right) \cdot\left(Z_{C}^{i} \cdot\left(D_{C}-Z_{C}^{i}\right)\right)^{\frac{1}{2}}\right) \\
& V_{C V}=L_{C} \cdot\left(\frac{\pi \cdot D_{C}{ }^{2}}{8}+\frac{D_{C}{ }^{2}}{4} \cdot \operatorname{Arcsin}\left(1-\frac{2 \cdot Z_{C}}{D_{C}}\right)+\left(\frac{D_{C}}{2}-Z_{C}\right) \cdot\left(Z_{C} \cdot\left(D_{C}-Z_{C}\right)\right)^{\frac{1}{2}}\right)
\end{aligned}
$$

Initial and the dynamic volumes of the vacuum in the evaporator depend on corresponding initial and dynamic levels of the evaporator. They are geometrically computed by subtracting the corresponding brine water volume from total volume of the right circular horizontal cylinder evaporator as follows 


$$
\begin{aligned}
& V_{E V}^{i}=L_{E} \cdot\left(\frac{\pi \cdot D_{E}^{2}}{8}+\frac{D_{E}^{2}}{4} \cdot \operatorname{Arcsin}\left(1-\frac{2 \cdot Z_{E}^{i}}{D_{E}}\right)+\left(\frac{D_{E}}{2}-Z_{E}^{i}\right) \cdot\left(Z_{E}^{i} \cdot\left(D_{E}-Z_{E}^{i}\right)\right)^{\frac{1}{2}}\right) \\
& V_{E V}=L_{E} \cdot\left(\frac{\pi \cdot D_{E}^{2}}{8}+\frac{D_{E}^{2}}{4} \cdot \operatorname{Arcsin}\left(1-\frac{2 \cdot Z_{E}}{D_{E}}\right)+\left(\frac{D_{E}}{2}-Z_{E}\right) \cdot\left(Z_{E} \cdot\left(D_{E}-Z_{E}\right)\right)^{\frac{1}{2}}\right)(
\end{aligned}
$$

Vacuum volume is the total space occupied by vapor in the flash chamber and can be computed by adding the vapor space of both condenser and evaporator to the volume of the connecting duct and subtracting the volume of the coiled tube of the condenser. Therefore, the initial and dynamic vacuum volumes can be calculated by

$$
\begin{aligned}
& V_{V}^{i}=V_{E V}^{i}+V_{C V}^{i}+\frac{\pi}{4} \cdot\left(P L_{E} \cdot P D_{E}^{2}-L_{C T} \cdot D_{C T}^{2}\right) \\
& V_{V}=V_{E V}+V_{C V}+\frac{\pi}{4} \cdot\left(P L_{E} \cdot P D_{E}^{2}-L_{C T} \cdot D_{C T}{ }^{2}\right)
\end{aligned}
$$

\subsubsection{Vacuum Pressure}

Seawater flow rate and the flash temperature are the only controlled variables of the proposed continuous desalination operation, and their effects on the system will be analyzed later. Seawater flow rate determines the amount of non-condensable gases accumulated, while the flash temperature determines the equilibrium temperature reached inside the flash chamber in line with the above mass and energy balance.

The accumulated amount of non-condensable gases and the reached equilibrium temperature, as well as the calculated vacuum volume, determine vacuum pressure according to any equation of state. It is imperative to express the vacuum pressure properly to simulate the proposed continuous desalination operation accurately because of the profound impact of vacuum pressure on the outcome of the flash operation. 
The ideal gas model describes fluid properties without considering molecular size or intermolecular attractions; therefore, its accuracy diminishes at higher pressures and lower temperatures. Low vacuum pressure marginalizes the effect of molecular size, while the fairly high flash temperature, manifested in higher thermal kinetic energy, weakens the relative importance of intermolecular attractions. Consequently, the ideal gas law becomes a suitable equation of state to express rising vacuum pressure inside the flash chamber due to build up of non-condensable gases in the flash chamber.

Initial vacuum pressure is an input value and should be very close to or equal to the saturated pressure of water at ambient conditions, while the initial vacuum volume can be determined by the above mentioned relations, knowing initial levels in the flash chamber. The gas phase primarily consists of water molecules at first, and their amount can then be estimated by

$n_{V}^{i}=\frac{P_{V}^{i} \cdot V_{V}^{i}}{R \cdot(T+273.15)}$

Non-condensable gas molecules progressively accumulate in the flash chamber, and their amount must be added to the initial amount computed above to express the dynamic amount of molecules in the gas phase as

$$
n_{V}=n_{V}^{i}+\int N_{C}^{a} d t
$$

Initial and the dynamic saturated pressures of water are needed to express vacuum pressure in a little while. The dynamic saturated pressure of water is given above as a function of dynamic equilibrium temperature, while initial saturated pressure of water is a function of ambient temperature and can be calculated by [15]

$$
P_{\mathrm{H}_{2} \mathrm{O}}^{i}=\exp \left[P A-\left(\frac{P B}{T+P C}\right)\right]
$$


The vacuum pressure needs to be specified to proceed with the flash calculations ultimately leading to convergence of the whole model; therefore, the simulation is executed incrementally, where the computed vacuum pressure of one time increment becomes the input vacuum pressure to the next time increment. The known initial vacuum pressure value is input to the first time increment to initialize this progression. This scheme is known as the Iterative and Incremental Development in the art of software development. Consequently, the incremented vacuum pressure is expressed by

$$
\begin{aligned}
& \left.P_{V}\right|_{t}=\frac{\psi \cdot n_{V} \cdot R \cdot\left(T_{E}+273.15\right)}{V_{V}}+\left[P_{\mathrm{H}_{2} \mathrm{O}}-P_{\mathrm{H}_{2} \mathrm{O}}^{i}\right] \\
& P_{V}=\left.P_{V}\right|_{t-\Delta t}
\end{aligned}
$$

To illustrate the Iterative and Incremental Development concept as it pertains to the current model, consider the ensuing paradigm. The known initial vacuum pressure is fed to the computer code as dynamic vacuum pressure of the first increment resulting in a solution for the dynamic vacuum pressure of the second increment that is then fed to the computer code resulting in a solution for the vacuum pressure of the third increment, and so forth until the last increment is reached.

The dynamic quantity of molecules in the gas phase incorporates accumulated non-condensable gas molecules plus water molecules present at the initial ambient point; however, there are more water molecules in the gas phase not account for due to the temperature increase from ambient to equilibrium. Consequently, the second term on right hand side of the vacuum pressure expression, $P_{\mathrm{H} 2 \mathrm{O}}-P_{\mathrm{H} 2 \mathrm{O}}^{i}$, is added to correct the dynamic amount of water molecules in the gas phase by accounting for the increase in vapor pressure due to temperature rise from ambient to equilibrium. 
The model assumes total accumulation of non-condensable gases in the flash chamber; however, water vapor dissolves a small quantity of non-condensable gases as it condenses. In addition, average values for seawater content of dissolved gases are input to the model, as the real seawater content of dissolved gases is indefinite and could be somewhat different from the average values. Moreover, the true vapor-liquid equilibrium for carbon dioxide involves more than just the afore mentioned $\mathrm{K}$-values due to presence of several carbonates in seawater that are also in equilibrium with carbon dioxide.

Consequently, a correction factor for the dynamic amount of molecules in the gas phase is included in the vacuum pressure expression. Experimental data can be used to perform adiabatic flash calculations, generating correction factor data that are then used to find a correction factor correlation resembling

$\psi=f\left(\frac{P_{V}}{P_{\mathrm{H}_{2} \mathrm{O}}}\right)$

Other expressions of vacuum pressure can be worked out, but it is very important for the expressed vacuum pressure to match experimental values closely due to its strong impact on the outcome of the simulation as mentioned earlier.

Operating pressure inside the flash chamber has to be between the dew point and the bubble point to carry out a successful flash separation. Dew point and bubble point pressures are estimated by

$$
\begin{aligned}
& \frac{P_{B P}}{P_{V}}=z_{N_{2}} \alpha_{N_{2}} K_{N_{2}}+z_{O_{2}} \alpha_{\mathrm{O}_{2}} K_{\mathrm{O}_{2}}+z_{A r} \alpha_{A r} K_{A r}+z_{\mathrm{CO}_{2}} \alpha_{\mathrm{CO}_{2}} K_{\mathrm{CO}_{2}}+z_{\mathrm{H}_{2} \mathrm{O}} \alpha_{\mathrm{H}_{2} \mathrm{O}} K_{\mathrm{H}_{2} \mathrm{O}} \\
& \frac{P_{V}}{P_{D P}}=\frac{z_{\mathrm{N}_{2}}}{\alpha_{\mathrm{N}_{2}} \cdot K_{\mathrm{N}_{2}}}+\frac{z_{\mathrm{O}_{2}}}{\alpha_{\mathrm{O}_{2}} \cdot K_{\mathrm{O}_{2}}}+\frac{z_{A r}}{\alpha_{A r} \cdot K_{A r}}+\frac{z_{\mathrm{CO}_{2}}}{\alpha_{\mathrm{CO}_{2}} \cdot K_{\mathrm{CO}_{2}}}+\frac{z_{\mathrm{H}_{2} \mathrm{O}}}{\alpha_{\mathrm{H}_{2} \mathrm{O}} \cdot K_{\mathrm{H}_{2} \mathrm{O}}}
\end{aligned}
$$




\subsubsection{System Performance}

Pressure drop is a design parameter used in accounting for pressure reduction due to friction. Total pressure drop of seawater flow from the seawater tank to flash chamber can be determined by summing up the pressure drops of each upstream pipe

$$
\Delta P=\Delta P_{S}+\Delta P_{P}+\Delta P_{X}+\Delta P_{H}+\Delta P_{H T}+\Delta P_{C T}
$$

If a throttling valve is used to control flow rate of seawater, the pump will run at full capacity, and work exerted on seawater by the pump is a direct function of the power of the pump, that is $W_{P}=-44742 \cdot H P_{P}$. If a variable-frequency drive is used to control flow rate of seawater, the pump will run at modulated speeds, and work exerted on seawater by the pump is a function of the head pressure.

The proposed desalination system will consider a variable-frequency drive to control flow rate of seawater due to its superior energy efficiency over a throttling valve; therefore, work exerted on seawater by the pump can be estimated using Bernoulli's fluid equation as

$W_{P}=-M_{P} \cdot\left[g \cdot\left(Z_{S}-Z_{O}\right) \cdot 10^{-7}+\frac{P_{V}-P-\Delta P}{10 \cdot \rho_{P}}\right]$

An appropriate circulation pump can be selected from the catalog of any process equipment manufacture based on required flow rate and total head. Selecting the pump will set many parameters including its power and suction force. The procedure of selecting a pump or any required piece of equipment for the process is beyond the scope of this analysis; however, equipment sizing is a common straightforward practice. Formulae for sizing pumps, valves, vessels, pipes, expansion orifices, and many other process equipment are abundantly available in literature [23]. 
A solar heater is employed in the proposed desalination system to heat the preheated seawater coming out of the condenser further before flashing it in the evaporator. Solar heating can be accomplished in a variety of ways; however, the present model assumes the heater to be a single-glazed flat-plate solar collector directly heating seawater flowing through its absorbing tubes.

Solar insolation is geographically referenced and continually varying due to dynamic solar angles. In addition, solar insolation incident on the collector varies with plate geometry, sky clearness, ground reflectivity, and many other factors. Average values for a generic single-glazed flat-plate solar collector will be used to simplify comparison among the different simulation scenarios.

The solar insolation area of the collector needed to meet the required heating load can be found using the Hottel-Whillier-Bliss correlation [8]

$$
A_{S C}=\frac{Q_{H}}{60 \cdot F_{S C} \cdot\left[\tau_{S C} \cdot \alpha_{S C} \cdot I-U_{S C} \cdot\left(T_{X}-T\right)\right]}
$$

Solar heating is usually accomplished indirectly by an intermediary heat exchanger that transfers heat from a solar collector loop to a process loop. The proposed desalination system drops this intermediary heat exchanger by flowing seawater directly through the absorbing tubes of the solar collector; therefore, increasing the efficiency and reducing the cost of the solar heater. On the other hand, this direct heating scheme has its drawbacks by increasing the risk of corrosion and scale formation causing equipment damage and inhibiting heat transfer. Hermann-Koschikowski-Rommel [24] developed corrosion-free solar collectors for thermal desalination systems use composed of a series of coated glass tubes mounted inside a conventional flat-plate solar collector enclosure; therefore, flowing seawater directly through the collector is a viable alternative. 
Condenser efficiency is defined as the percent of the ratio of the temperature gradient on the cold tube side to the temperature gradient on the hot shell side as $\eta_{C}=\frac{T_{X}-T_{P}}{T_{E}-T_{C}} \times 100 \%$

Heat recovery efficiency is defined as the percent of total enthalpy change that was essentially accomplished by reclaiming heat from the condensing vapor as

$\eta_{R}=\frac{H_{X}-H_{S}}{H_{H}-H_{S}} \times 100 \%$

Thermal efficiency of the proposed desalination system is defined as the percent of the total thermal energy supplied that was actually used to vaporize water as

$\eta_{T}=\frac{M_{E} \cdot\left(H_{E}+H_{E}^{L}\right)}{M_{H} \cdot H_{H}} \times 100 \%$

Prime energy consumption is a very important parameter in evaluating feasibility of any desalination system and is defined as the ratio of the amount of energy exhausted to the amount of fresh water produced. The total amount of energy exhausted is the heat supplied by the heater plus power supplied by the pump.

Prime energy consumption can be expressed as a constantly shifting parameter by $P E C=\left(Q_{H}+W_{P}\right) / M_{C}$ on instantaneous basis; however, it is typically desired to express prime energy consumption as a single value attained on a totalized basis by integrating the implicated dynamic process variables over the entire operating period. Total prime energy consumption of the proposed unit is given by

$P E C=\frac{\int Q_{H} d t+\int W_{P} d t}{\int M_{C} d t}$ 


\subsubsection{Physical Properties}

Laminar flow is a smooth flow pattern, where fluid layers are flowing in parallel concentric cylindrical layers without any inter-layer mixing in a manner determined by the viscosity of the fluid [25]. Turbulent flow is a rough flow pattern, where fluid particles are randomly fluctuating in transverse to the general flow direction in a manner determined by inertial forces of the fluid [25]. Figure 24 illustrates the streaming profile of both flow regimes.

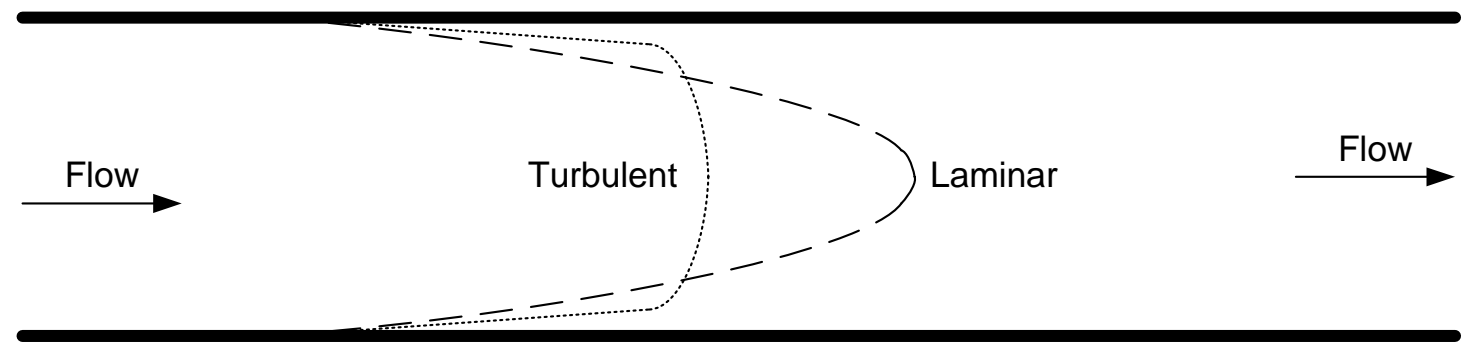

Figure 24. Flow regimes

Reynolds number is a dimensionless quantity that represents the ratio of inertial forces to viscous forces and is used to classify different flow regimes as either laminar or turbulent. Laminar flow behavior occurs at low Reynolds numbers, while turbulent flow behavior occurs at high Reynolds numbers. The critical Reynolds number of 2300 is generally accepted as the midpoint of the transition range between laminar and turbulent flows in cylindrical pipes.

Reynolds number of process streams is given by

$$
R e_{j}=\frac{M_{j}}{15 \cdot \pi \cdot P D_{j} \cdot \mu_{j}}
$$


The pressure of a flowing fluid inside a pipe inevitably drops due to gravity and wall drag. This pressure drop and loss can be approximated depending on the flow pattern by the Hagen-Poiseuille equation: $\Delta P_{j}=6.79 \cdot 10^{-7} \cdot\left(\mu_{j} \cdot P L_{j} \cdot M_{j}\right) /\left(\rho_{j} \cdot P D_{j}^{4}\right)$ for laminar or by the Moody equation: $\Delta P_{j}=9.01 \cdot 10^{-10} \cdot\left(f_{j} \cdot P L_{j} \cdot M_{j}^{2}\right) /\left(\rho_{j} \cdot P D_{j}^{5}\right)$ for turbulent flows [15], where $f$ is the dimensionless Fanning Friction Factor available in literature as a function of both Reynolds number and pipe roughness. The current desalination process will be designed to include streams exhibiting laminar flow conditions to simplify experimental simulations later; hence, the model will employ the Hagen-Poiseuille equation to estimate average pressure drops of process streams as

$$
\Delta P_{j}=\frac{6.79 \cdot 10^{-7} \cdot \mu_{j} \cdot P L_{j} \cdot M_{j}}{\rho_{j} \cdot P D_{j}^{4}}
$$

Thermal conductivity is a property that gauges heat conduction ability of a given substance. Dependence of thermal conductivity on temperature relates to the freedom of movement molecules enjoy; therefore, thermal conductivity varies with temperature in fluids but remains fairly constant in solids. Thermal conductivities of process streams are calculated empirically by the Caldwell Relation [26]

$$
k_{j}=\left(5711.16+17.1335 \cdot T_{j}-0.0585 \cdot T_{j}^{2}-1656.2364 \cdot \varphi_{j}\right) \cdot 10^{-6}
$$

Furthermore, thermal conductivities of flashing water vapor and accumulating non-condensable gases are not required but can be calculated empirically by [27]

$$
\begin{aligned}
& k_{E}=\left[\begin{array}{l}
2.2744 e-12 \cdot\left(1.8 \cdot T_{j}+32\right)^{3}-5.8518 e-10 \cdot\left(1.8 \cdot T_{j}+32\right)^{2} \\
+3.8912 e-7 \cdot\left(1.8 \cdot T_{j}+32\right)+1.6943 e-4
\end{array}\right] \\
& k_{C}^{a}=\left[\begin{array}{l}
1.5207 e-13 \cdot\left(T_{j}+273.15\right)^{3}-4.8574 e-10 \cdot\left(T_{j}+273.15\right)^{2} \\
+1.0184 e-6 \cdot\left(T_{j}+273.15\right)-3.9333 e-6
\end{array}\right]
\end{aligned}
$$


The stream densities used in several correlations above will be calculated by an empirical relationship experimentally developed by the Rosenstiel School of Marine and Atmospheric Science at the University of Miami to calculate density of seawater as a function of temperature and salinity [28]. Densities of process streams are given by

$$
\begin{aligned}
& \rho A_{j}=0.82+T_{j} \cdot\left(-4.09 \cdot 10^{-3}+T_{j} \cdot\left(7.64 \cdot 10^{-5}+T_{j} \cdot\left(-8.25 \cdot 10^{-7}+T_{j} \cdot 5.39 \cdot 10^{-9}\right)\right)\right) \\
& \rho B_{j}=-5.72466 \cdot 10^{-3}+T_{j} \cdot\left(1.0227 \cdot 10^{-4}-1.6546 \cdot 10^{-6} \cdot T_{j}\right) \\
& \rho C_{j}=\left(6.79 \cdot 10^{-2}-\left(9.10 \cdot 10^{-3}-\left(10^{-4}-\left(1.12 \cdot 10^{-6}-6.54 \cdot 10^{-9} T_{j}\right) \cdot T_{j}\right) \cdot T_{j}\right) \cdot T_{j}\right) \cdot T_{j} \\
& \rho_{j}=\frac{999.84+\rho C_{j}}{1000}+\varphi_{j} \cdot\left(\rho A_{j}+\rho B_{j} \cdot\left(1000 \cdot \varphi_{j}\right)^{\frac{1}{2}}+0.48314 \cdot \varphi_{j}\right)
\end{aligned}
$$

Stream viscosities used in several correlations above will be calculated by an empirical relationship that was experimentally developed to calculate the viscosity of seawater as a function of temperature and salinity [29]. Viscosities of process streams are given by

$$
\begin{aligned}
& \mu A_{j}=1.0675 \cdot 10^{-4}+5.185 \cdot 10^{-5} \cdot T_{j} \\
& \mu B_{j}=2.591 \cdot 10^{-3}+3.3 \cdot 10^{-5} \cdot T_{j} \\
& \mu C_{j}=553.5413 \cdot \rho_{j} \cdot \varphi_{j} \\
& \mu D_{j}=0.01002 \cdot 10^{\left[\frac{1.1709 \cdot\left(20-T_{j}\right)-1.827 \cdot 10^{-3} \cdot\left(T_{j}-20\right)^{2}}{T_{j}+89.93}\right]} \\
& \mu_{j}=\left(1+\mu A_{j} \cdot \mu C_{j}^{0.5}+\mu B_{j} \cdot \mu C_{j}\right) \cdot \mu D_{j}
\end{aligned}
$$

stream enthalpies used in the energy balances above will be calculated by an empirical relationship that was experimentally developed to calculate heat capacity of seawater as a function of temperature and salinity [30] as follows 


$$
H_{j}=\left[\begin{array}{l}
+\left(0.0530-1.6853 \cdot \varphi_{j}+8.5367 \cdot \varphi_{j}^{2}\right) \cdot 10^{-4} \cdot T_{j}^{3} \\
-\left(0.0007-0.0310 \cdot \varphi_{j}+0.1624 \cdot \varphi_{j}^{2}\right) \cdot T_{j}^{2} \\
+\left(4.2045-6.7823 \cdot \varphi_{j}+14.7532 \cdot \varphi_{j}^{2}\right) \cdot T_{j}-0.04881
\end{array}\right]
$$

Seawater flashes in the evaporator producing water vapor that acquires its latent enthalpy of vaporization from the concentrated brine. Then, the produced water vapor condenses in the condenser by losing its latent enthalpy of condensation to the entering saline water. Latent enthalpy of vaporization and the latent enthalpy of condensation are numerically equal but have opposite signs and can be estimated by [27]

$H_{E}^{L}=2496.2101-1.9535 \cdot T_{E}-0.0042 \cdot T_{E}^{2}$

Nitrogen, oxygen, argon, and carbon dioxide are the only non-condensable gases considered in the model, since they make up more than $99.9 \%$ of the total dissolved gases in seawater [19]. The US National Institute of Standards and Technology provides the following correlation to calculate molar enthalpy of non-condensable gases [31]

$$
\underline{H}_{N C G}=\left(\begin{array}{l}
A_{N C G} \cdot\left(T_{E}+273.15\right)+\frac{B_{N C G}}{2 e 3} \cdot\left(T_{E}+273.15\right)^{2}+\frac{C_{N C G}}{3 e 6} \cdot\left(T_{E}+273.15\right)^{3} \\
+\frac{D_{N C G}}{4 e 9} \cdot\left(T_{E}+273.15\right)^{4}-\frac{1 e 6 \cdot E_{N C G}}{\left(T_{E}+273.15\right)}+1 e 3 \cdot F_{N C G}
\end{array}\right)
$$

Overall enthalpy of the accumulating non-condensable gases can be computed by adding molar enthalpies of each composing species weighted on a water-free basis, in relation to the assumption of total condensation of flashed water vapor. In addition, the average molecular weight of the accumulating gases referenced before was employed to convert its enthalpy units from molar to mass based. Thus, overall enthalpy of accumulating non-condensable gases is given by

$$
H_{C}^{a}=\frac{y_{N_{2}} \cdot \underline{H}_{N_{2}}+y_{\mathrm{O}_{2}} \cdot \underline{H}_{\mathrm{O}_{2}}+y_{A r} \cdot \underline{H}_{A r}+y_{C_{2}} \cdot \underline{H}_{C O_{2}}}{\left(1-y_{\mathrm{H}_{2} \mathrm{O}}\right) \cdot M W_{C}^{a}}
$$




\subsection{Solution Algorithm}

A computer code featuring the above equations, plus other correlations and parameters given later in CHAPTER 6 can be found in the APPENDICES section along with sample input and output values of process variables. Computer code execution is incremental due to time-based numerical integration used above to account for accumulation of non-condensable gases in the flash chamber, while convergence process is iterative due to interdependence of equations of the model. The increment size should be carefully selected to simplify convergence and reduce processing without jeopardizing the integrity of the simulation. A concise block diagram outlining the general scheme to solve the above model is shown in Figure 25.

Time is embedded in the model by flow rates of different streams; moreover, integration operations of the model are based on small time increments that evenly divide the entire run. Ambient temperature and pressure, as well as physical properties and geometrical dimensions of process pipes and vessels, are input to the model. Universal values such as gas constant and gravity acceleration, plus seawater composition and the molecular weights of the involved species, are also input to the model. Parameters for enthalpy and vapor-liquid equilibrium relations, as well as average values for a generic single-glazed flat-plate solar collector, are also supplied to the code.

Flash temperature is a controlled variable and will be supplied to code as a single set value. Initial vacuum pressure is a known quantity and will also be supplied to code to launch the simulation process. Initial vacuum pressure will be fed to the first increment, producing vacuum pressure for the second increment producing the vacuum pressure for the third increment and so forth. 


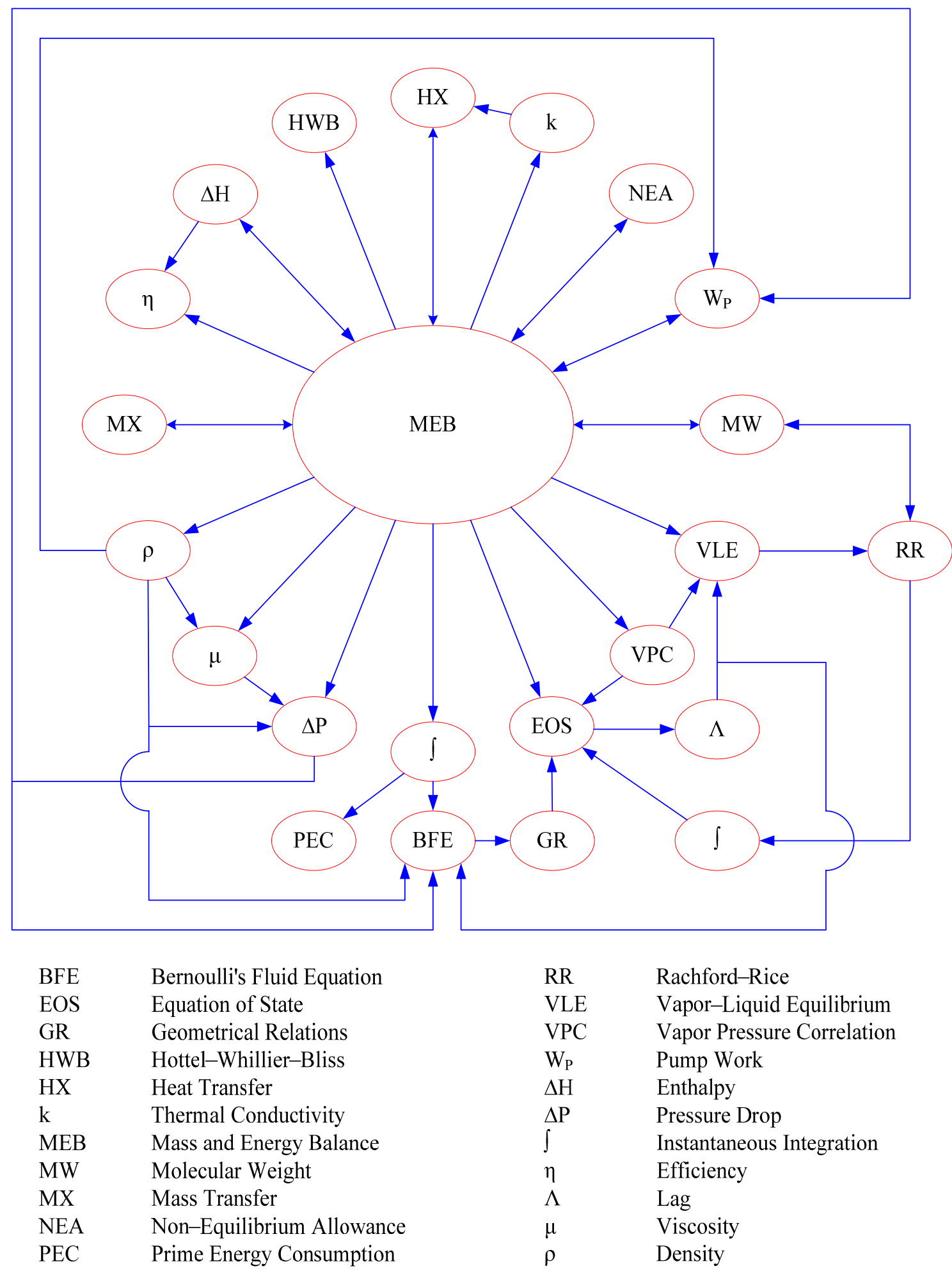

Figure 25. Developed model solution algorithm 
The mass and energy balance simultaneously solves with mass and heat transfer relations, as well as enthalpy and non-equilibrium allowance correlations. Mass and energy balance indirectly solves with the pump work relation via density and pressure drop correlations and with molecular weight relations via Rachford-Rice calculations.

Mass and energy balance provides inputs for density, viscosity, and pressure drop correlations, producing outputs that are fed to the pump work relation and Bernoulli's fluid equation. In addition, mass and energy balance provides inputs for thermal conductivity correlations used in heat transfer calculations and for efficiency relations that use those inputs along with other inputs from the enthalpy correlations to evaluate system performance. The mass and energy balance also provides input values for the Hottel-Whillier-Bliss correlation to estimate solar collection area needed and for an integrator that totalizes system variables before forwarding them both to the prime energy consumption function and to Bernoulli's fluid equation.

Mass and energy balance and the Rachford-Rice calculations are linked via molecular weight relations and concurrently solve for equilibrium temperature that is fed to vapor-liquid equilibrium relations, a vapor pressure correlation, and an equation of state. The equation of state estimates system vacuum pressure before it is lagged and forwarded to vapor-liquid equilibrium relations, where $\mathrm{K}$-values are generated and fed to Rachford-Rice calculations to calculate the rate of accumulation of non-condensable gases that is integrated and fed back to the equation of state to calculate the pressure of the next time increment. The lagged system pressure is also fed to Bernoulli's fluid equation, where tank levels are calculated and forwarded to geometrical relations to compute volume of the vacuum before forwarding it to the equation of state. 


\section{CHAPTER 5. EXPERIMENTAL ANALYSIS}

\subsection{Process Description}

A small pilot unit has been built to simulate the proposed continuous desalination system described previously experimentally. Figure 26 outlines a general process and instrumentation diagram of the small pilot unit. Experimental simulations were performed inside a laboratory to simplify operation and maintenance. Due to this indoor process, solar heating was hard to implement due to lack of solar insolation, and passive vacuum was difficult to produce due to limited elevation.

Solar heating is widely used in several applications, including desalination systems as was mentioned in CHAPTER 2; therefore, replicating it with an electric heater is considered acceptable, since the concept of solar heating does not require further proof. In addition, vacuum was passively generated by Goswami and Kharabsheh [11] for their desalination unit as was mentioned in CHAPTER 3; therefore, producing it with a vacuum pump is considered acceptable, since the phenomenon of passive vacuum generation using gravity has been experimentally established.

Placing the flash chamber at a low elevation in the experimental unit removed hydraulic head of the proposed unit; thus, a circulation pump is no longer required to pass seawater through the unit. The pressure difference between the vacuumed flash chamber and open seawater feed tank becomes the driving force of seawater flow, which was manually controlled by manipulating valve positions. 


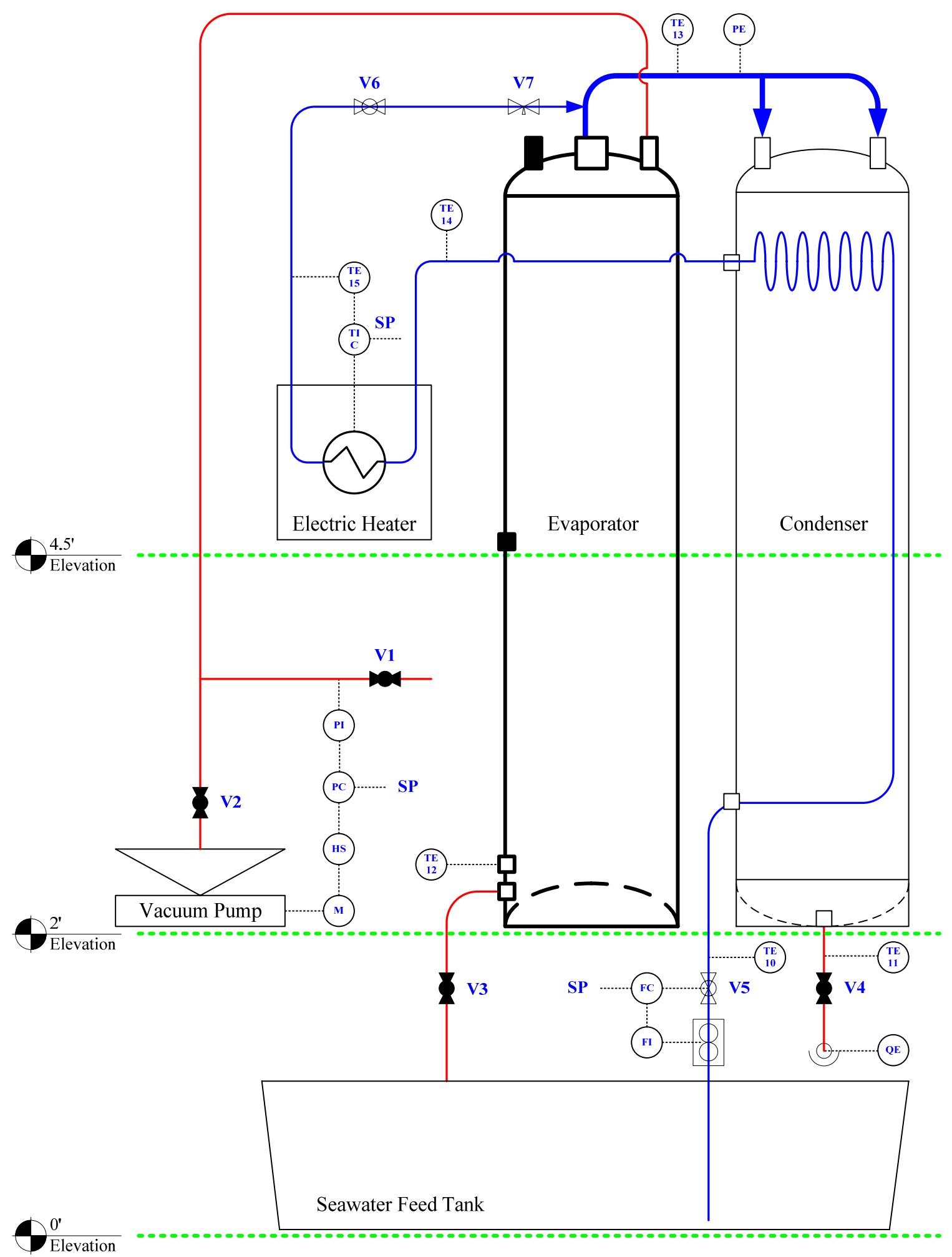

Figure 26. Process and instrumentation diagram of the experimental unit 


\subsection{Experimental Apparatus}

The entire experimental unit is mounted on three-tier mobile skids built from slotted and unslotted struts with linking joints and brackets as shown in Figure 27. The three tiers are connected by four upright bars, the bottom two tiers include plywood for weight distribution, and the bottom tier is outfitted with four wheels for mobility.

The seawater feed tank is a 50-gallon open-top, horizontal polyethylene trough placed on the bottom tier of skids. The condenser is a 40-gallon painted-steel, upright cylinder, while the evaporator is a 40-gallon galvanized-steel, upright cylinder, and they are both placed on the middle tier of the skids directly above the seawater feed tank. Condenser, evaporator, and 2" Y-shaped CPVC pipe connecting them from the top make up the flash chamber. The condenser was cut open around its upper section to install a $43 / 4$ $\mathrm{m}$ long $1 / 2 "$ " copper pipe coiled to provide the necessary condensing surface, then welded back to its original shape. In addition, a small hole was drilled at the bottom of the condenser to retrieve the condensed water. The evaporator and the 2" Y-shaped CPVC pipe are wrapped with sheets of insolating material to minimize heat loss. The condenser and evaporator have discharge pipes that drain into the seawater feed tank.

The heater is a $4.5 \mathrm{~kW}$ zinc-plated, copper electric heating element placed inside a copper shell, where seawater coming out of the condenser passes through it on its way to be flashed in the evaporator. A $1 / 2 \mathrm{HP}$ vacuum pump is piped to the evaporator to create the initial vacuum, and a $1 / 2$ " polypropylene needle valve, V7 in Figure 26, is placed right before the evaporator to function as an expansion orifice for the incoming heated seawater to be flashed. The $1 / 2$ " CPVC pipe is used in connecting all of the above equipment as well as several $1 / 2$ " CPVC and copper pipe fittings. 
Several instruments have been integrated into the experimental unit to manage system variables as shown in Figure 26. These are used in monitoring and controlling system vacuum, seawater flow, and flash temperature, as well as monitoring temperature of each process stream. Nomenclature used in the P\&ID of Figure 26 is consistent with the International Society of Automation symbol standards.

Pressure indicator PI is a liquid-filled analog vacuum gauge, while pressure element PE is a high-precision vacuum transmitter. The PI was used to help establish the initial system vacuum and to monitor its gradual erosion. The PE was used to continually supply the value of the system vacuum to a data acquisition system.

Flow indicator FI is an acrylic in-line flowmeter, while quantitative element QE is a glass $500 \mathrm{ml}$ graduated cylinder. The FI was used to help establish and monitor the seawater flow through the system. The quantitative element QE was used to collect and measure the amount of fresh water produced at the end of each experiment.

Temperature elements TE10, TE11, TE12, TE13, and TE14 are single-output, while temperature element TE15 is dual-output 1/8" diameter T-Type thermocouples. All used to supply the value of the temperature of each process stream continually to a data acquisition system. In addition, TE15 is used to supply the temperature controller with the value of its controlled variable.

Pressure controller PC and flow controller FC are imaginary pressure and flow manual controllers, while temperature indicating controller TIC is an LED-equipped digital PID automatic controller. Regulators V1 and V4 are 1/4", while regulators V2, V3, V5, and V6 are $1 / 2$ " full port ball valves. Detailed descriptions of all of the above apparatus taken from their vendors are in the APPENDICES section. 

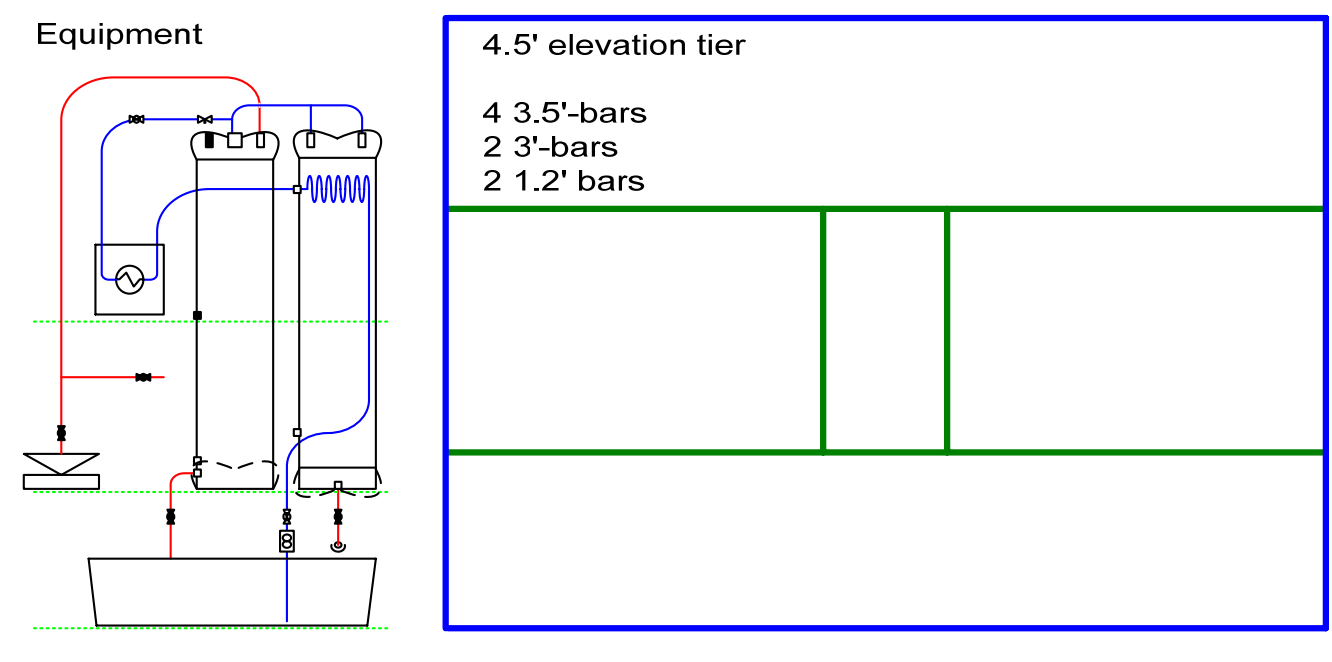

Legend
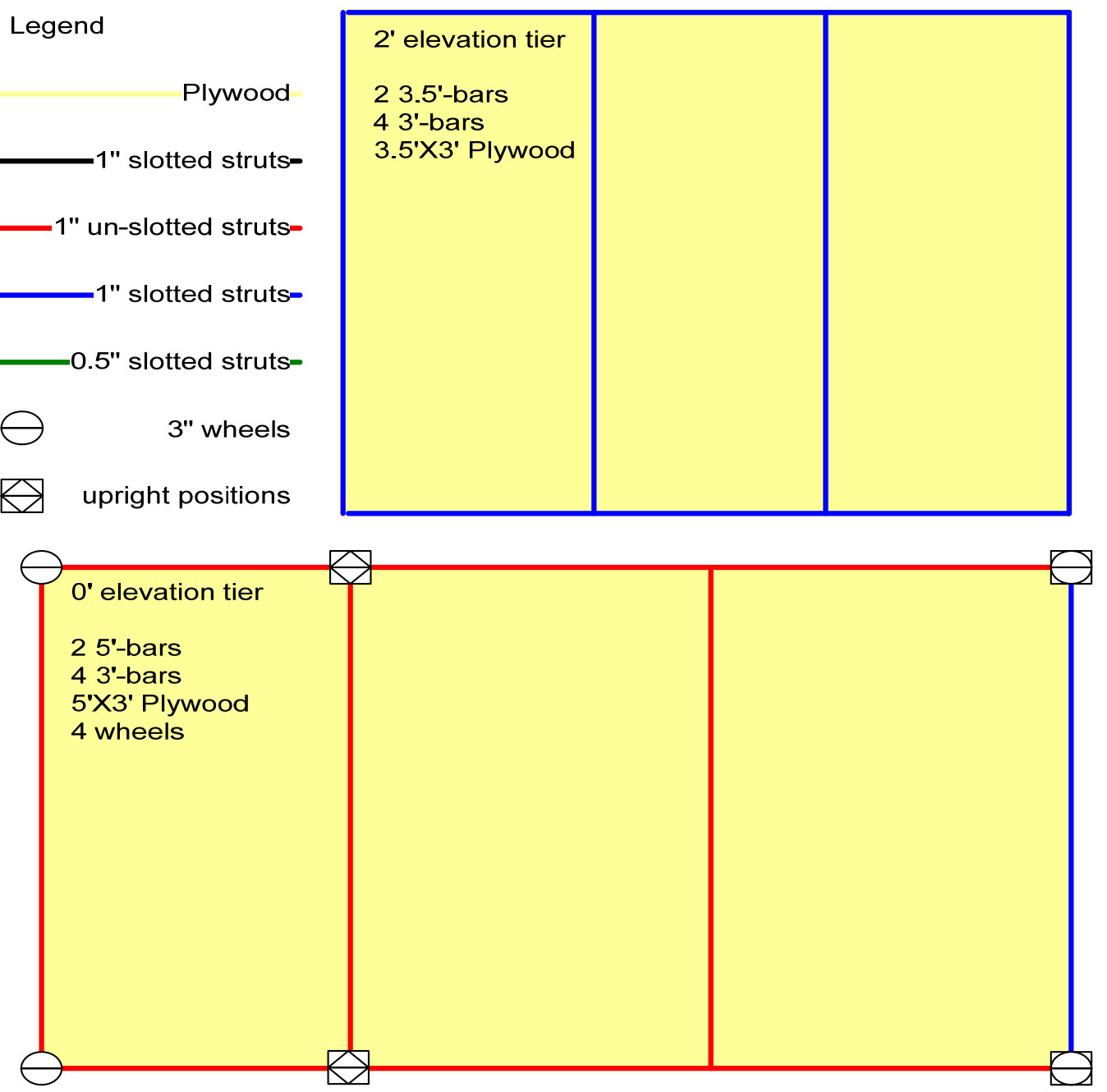

Figure 27. 3-tier mobile skids layout 


\subsection{Control Scheme}

The three feedback control loops pertaining to system vacuum, seawater flow, and flash temperature shown in Figure 26 are replicated in Figure 28 in isolation to clarify their control techniques. System vacuum and seawater flow are manually adjusted, while flash temperature is automatically controlled.

The system vacuum feedback control loop is invoked prior to running the experiment to attain the desired initial vacuum. It consists of measuring the vacuum with pressure indicator PI while the vacuum pump is running. Once the desired vacuum set point SP is reached, hand switch HS is manually switched off to shut down motor M of the vacuum pump, which remains shut during the entire experiment.

The seawater flow feedback control loop is invoked at the beginning of the experiment to attain the desired seawater flow rate, which remains constant throughout the experiment. It consists of measuring flow with flow indicator FI, while manually manipulating the valve position of V5 until the desired flow rate set point SP is realized. The valve position is kept constant throughout the experiment.
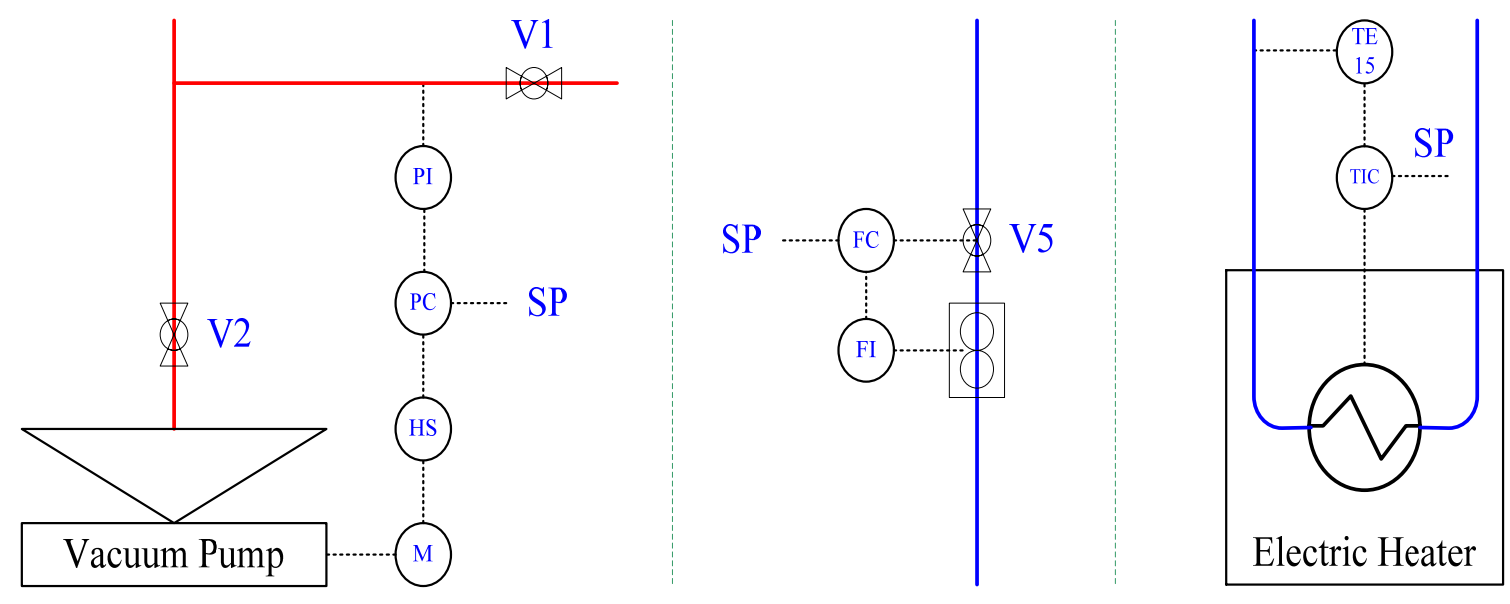

Figure 28. Feedback control loops of the experimental unit 
The flash temperature feedback control loop is constantly active to stabilize flash temperature during the experiment. It consists of measuring temperature of seawater coming out of the heater with temperature element TE15, then supplying that measurement to temperature indicating controller TIC that automatically manipulates the current input into the heat element of the heater, effectively varying its heat output to the incoming seawater until the desired flash temperature set point SP is achieved.

A simplified block diagram of the flash temperature feedback control loop is given in Figure 29. The assigned arrows SP, E, CO, TO, and $\mathrm{U}$ are the frequency-domain Laplace transform functions of the set point, error, controller output, transmitter output, and disturbance signals, respectively.

Block TIC represents the transfer function of the digital PID automatic temperature controller given generically as

$$
T I C(s)=K_{C} \cdot\left(1+\frac{1}{\tau_{I} \cdot s}+\tau_{D} \cdot s\right)
$$

Automatic temperature controller TIC is equipped with an automatic tuning ability that was used to tune its parameters during a dry run, yielding the following values $\mathrm{PB}=20 \% \mathrm{TO} / \% \mathrm{CO}$ $\mathrm{K}_{\mathrm{C}}=100 / \mathrm{PB}=5 \% \mathrm{CO} / \% \mathrm{TO}$ $\tau_{\mathrm{I}}=60$ seconds $\tau_{\mathrm{D}}=2$ seconds

The cycle time or total period that controller output cycles on and off when the controlled variable is within the PB was set to 1 second. In addition, a derivative approach control of $2.5 \times \mathrm{PB}$ was used to remove derivative action at system start-up. The fail-safe mode of the controller was set to turn off SP upon input signal loss. 
Block PL represents the transfer function of the process loop between the controlled and manipulated variables, which is usually represented by a first order model with dead time compensation as follows

$$
P L(s)=\frac{K_{P L} \cdot \operatorname{EXP}\left(-t_{0} \cdot s\right)}{\tau_{P L} \cdot s+1}
$$

Block DL represents the transfer function of the disturbance loop between the controlled variable and disturbance, which is usually represented by a first order model with dead time compensation as follows

$$
D L(s)=\frac{K_{D L} \cdot \operatorname{EXP}\left(-t_{0} \cdot s\right)}{\tau_{D L} \cdot s+1}
$$

Block PL and block DL are actually combinations of several transfer functions that were lumped into a single first order model to simplify representing the dynamic response of the process. Block PL merges a sequence of transfer functions characterizing thermocouple TE15, the heating process, and electric heater. Block DL merges a sequence of transfer functions characterizing thermocouple TE15 and flowing process. The automatic tuning ability of controller TIC is based on obtaining the parameters of the first order models representing block PL and block DL.

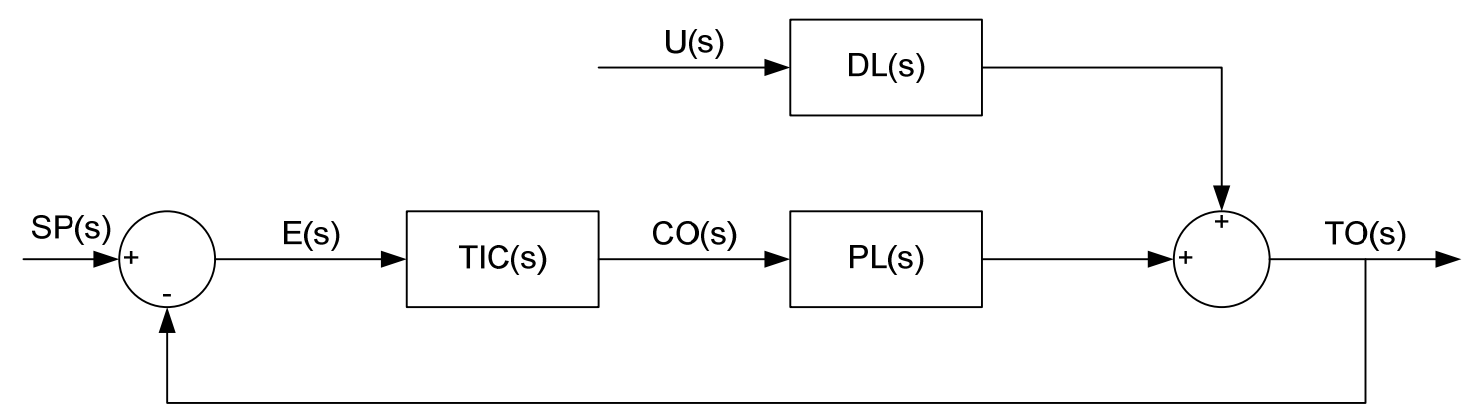

Figure 29. Block diagram of the flash temperature feedback control loop 


\subsection{Data Acquisition}

A data acquisition system designed to sample and record process variables was assembled and attached to the experimental apparatus. Configuration of the data acquisition system is illustrated in Figure 30.

Pressure element PE outputs an analog current signal corresponding to system vacuum with a range from 4-20 mA to fieldbus module FBM1 through a 2-wire cable. Temperature elements TE10, TE11, TE12, TE13, TE14, and TE15 output analog voltage signals corresponding to system temperatures, each with a range from $-0.001-0.01 \mathrm{mV}$ to fieldbus module FBM2, through thermocouple extension wires.

Fieldbus module FBM1 is a multiplexing signal conditioner, where the analog current signal of pressure element PE is converted to a corresponding analog voltage signal with a range from $1-5 \mathrm{~V}$. Fieldbus module FBM2 is a multiplexing signal conditioner, where the analog voltage signals of temperature elements TE10, TE11, TE12, TE13, TE14, and TE15 are converted to corresponding analog voltage signals with a range of $1-5 \mathrm{~V}$. The conditioned analog output signals of fieldbus modules FBM1 and FBM2 are multiplexed via multiplexer MUX, which is a DC-37 ribbon connecting cable.

Analog to digital converter ADC is a 16-bit data acquisition system that converts continuous analog signals supplied by multiplexer MUX to discrete digital signals and forwards them to human-machine interface HMI through an enhanced parallel port LPT cable. Human-machine interface HMI is a notebook PC running a data acquisition software that converts acquired data from its conditioned voltage units to its corresponding physical attributes. The data acquisition software also displays and stores the acquired data for later analysis as shown in Figure 31. 


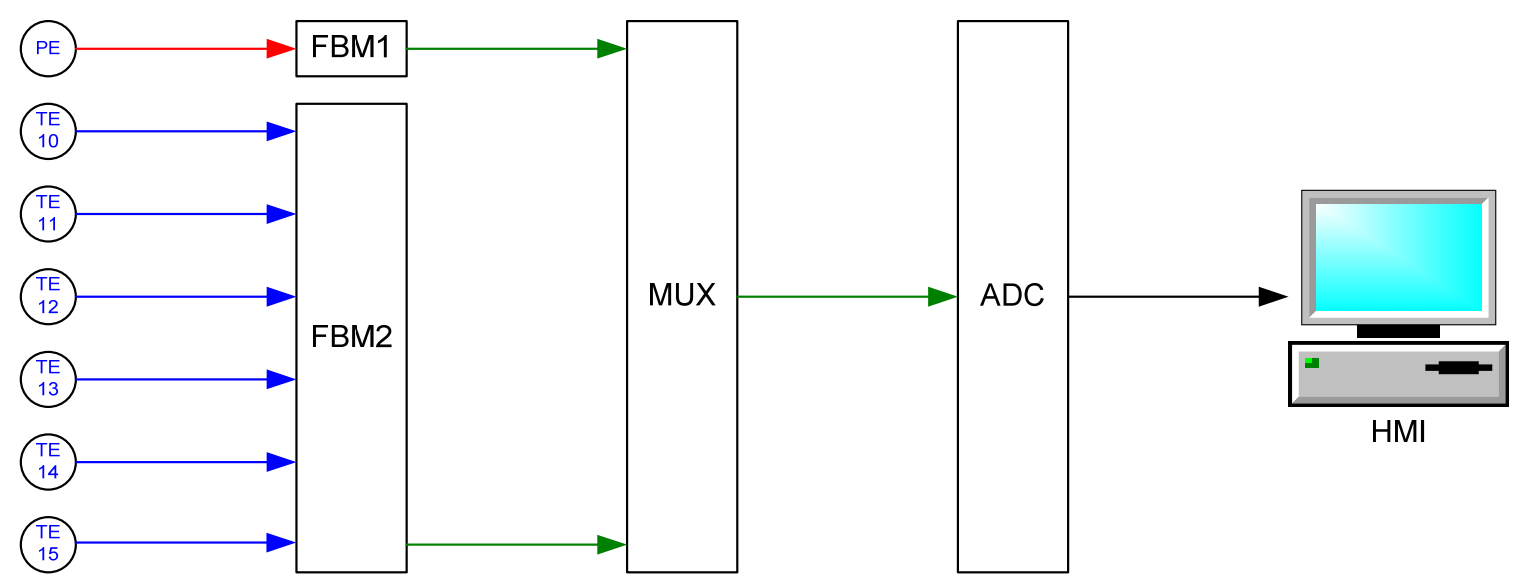

Figure 30. Data acquisition structure

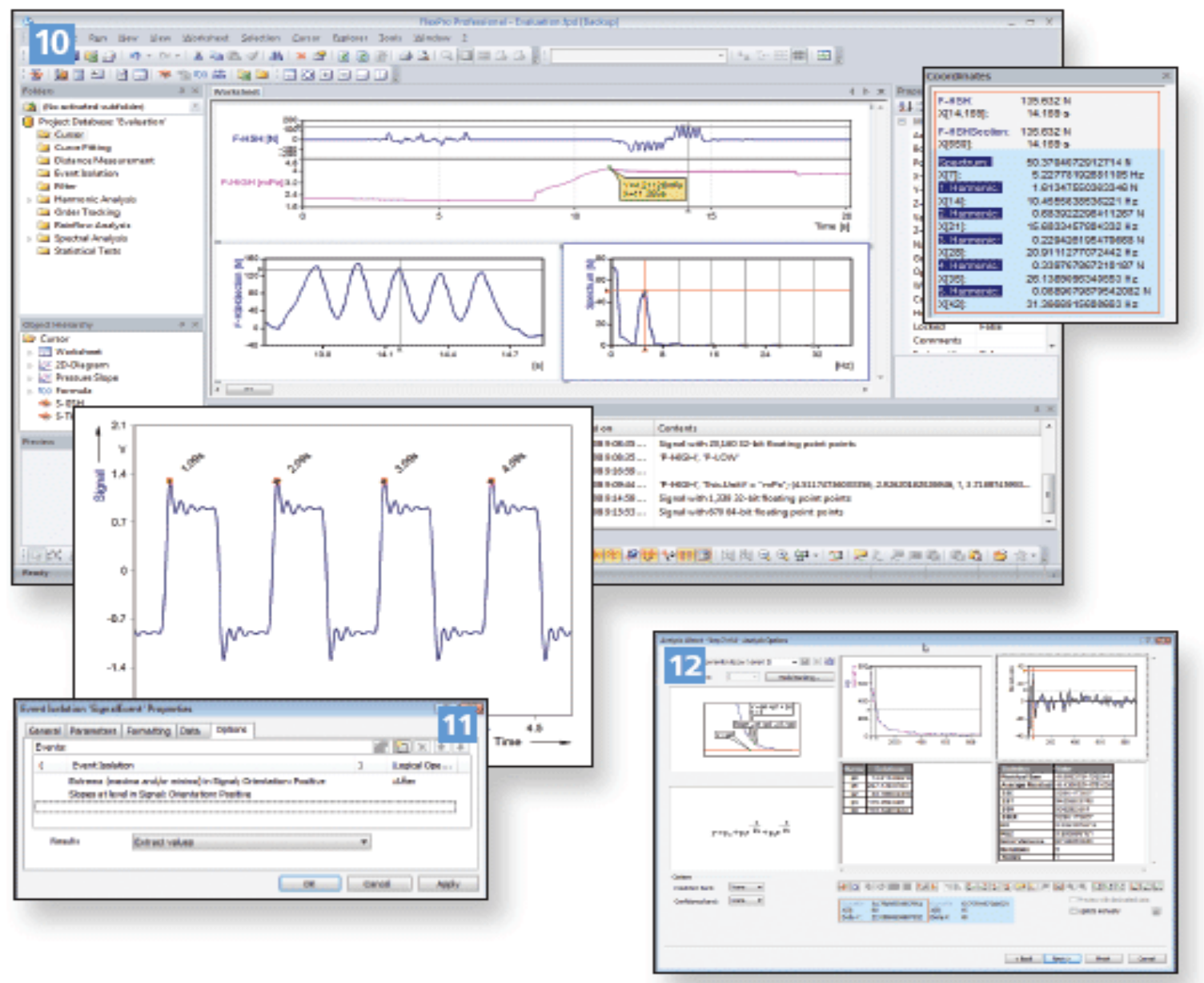

Figure 31. Data acquisition software 


\subsection{Operating Procedure}

Operation of the experimental unit closely resembled that of the proposed desalination system described in CHAPTER 4. Initial vacuum was created by running the vacuum pump until a reasonable vacuum was reached. Running the vacuum pump further will trap moisture in its oil reservoir significantly inhibiting its performance.

Synthetic seawater was prepared by mixing 13 pounds of commercial-grade sea salt with 40 gallons of tap water. The seawater mix was stirred well before each experiment to ensure full solution of sea salt. The seawater trough has a large open surface that enhances evaporation; therefore, small amounts of fresh water were often added before running experiments to reach a 40 -gallon level mark in the trough.

Temperature indicating controller TIC is not interlocked with seawater flow; thus, ensuring seawater flow through the electric heater is a very critical safety measure. Regulators V5 and V6, plus needle valve V7, are instruments that control seawater flow. The valve position of needle valve V7 was kept constant at about $90 \%$ open for all runs, because narrowing valve position caused flow oscillations regardless of the valve positions of regulators $\mathrm{V} 5$ and $\mathrm{V} 6$. The valve position of regulator $\mathrm{V} 6$ was used to start and stop the experiment; therefore, it was toggled between fully open and fully close. Valve position of regulator V5 was used to manipulate the flow as was mentioned above.

Pressure element PE is calibrated by the manufacture, while Fieldbus module FBM2 contains a built-in cold junction compensation that automatically calibrates thermocouple outputs. In addition, data acquisition software wais set to execute one scan per second and to average every ten scans to reduce signal noise. The acquired data were saved to an assigned ASCII formatted file on the Human-machine interface HMI. 
The experimental unit must be kept motionless during operation due to its high center of gravity, while its normal operating procedure is as follows

1. Start data acquisition system

2. Open regulator V2 fully

3. Start vacuum pump until desired vacuum is reached

4. Close regulator V2 fully

5. Stop vacuum pump

6. Slowly open regulator V6 fully

7. Set seawater flow rate through regulator V5 and flow indicator FI

8. Activate temperature indicating controller TIC

9. Trigger data recording function of data acquisition software

10. Run unit until the specified period of the experiment is reached

11. Stop data recording function of data acquisition software

12. Disable temperature indicating controller TIC

13. Quickly close regulator V6 fully

14. Stop data acquisition system

15. Open regulator V1 to terminate the vacuum

16. Open regulator $\mathrm{V} 3$ to drain brine into the seawater trough

17. Open regulator $\mathrm{V} 4$ to drain fresh water into quantitative element $\mathrm{QE}$

18. Record amount produced then drain into the seawater trough

19. Fully close regulator V1

20. Fully close regulator V3

21. Fully close regulator V4 


\subsection{Experimental Design}

Experiments were conducted at analogous conditions to simplify design evaluation but with different values of the controlling variables to enhance process analysis and modeling. All experiments were run for a period of three hours starting with an initial system vacuum of 0.14 bars. Experiments were carried out at two different seawater flow rate ranges and at four different flash temperatures of $50,60,70$, and 80 degrees centigrade. In addition, each experiment was duplicated three times to estimate its variation. Table 6 illustrates the experimental matrix, while Figure 32 gives an overall view of the experimental unit.

Table 6. Experimental matrix

\begin{tabular}{|c|c|c|c|c|c|c|}
\hline Number & Start Time & Stop Time & Initial PI (bar) & $F I(L P M)$ & $\operatorname{TIC~SP}\left({ }^{\circ} \mathrm{C}\right)$ & $Q E(m l)$ \\
\hline 1 & & & \multirow{24}{*}{0.14} & \multirow{12}{*}{$\begin{array}{l}\text { lower } \\
\text { flow } \\
\text { around } \\
\sim 0.50\end{array}$} & \multirow{3}{*}{50} & \\
\hline 2 & & & & & & \\
\hline 3 & & & & & & \\
\hline 4 & & & & & \multirow{3}{*}{60} & \\
\hline 5 & & & & & & \\
\hline 6 & & & & & & \\
\hline 7 & & & & & \multirow{3}{*}{70} & \\
\hline 8 & & & & & & \\
\hline 9 & & & & & & \\
\hline 10 & & & & & \multirow{3}{*}{80} & \\
\hline 11 & & & & & & \\
\hline 12 & & & & & & \\
\hline 13 & & & & \multirow{12}{*}{$\begin{array}{l}\text { higher } \\
\text { flow }\end{array}$} & \multirow{3}{*}{50} & \\
\hline 14 & & & & & & \\
\hline 15 & & & & & & \\
\hline 16 & & & & & \multirow{3}{*}{60} & \\
\hline 17 & & & & & & \\
\hline 18 & & & & & & \\
\hline 19 & & & & & \multirow{3}{*}{70} & \\
\hline 20 & & & & & & \\
\hline 21 & & & & & & \\
\hline 22 & & & & & \multirow{3}{*}{80} & \\
\hline 23 & & & & & & \\
\hline 24 & & & & & & \\
\hline
\end{tabular}




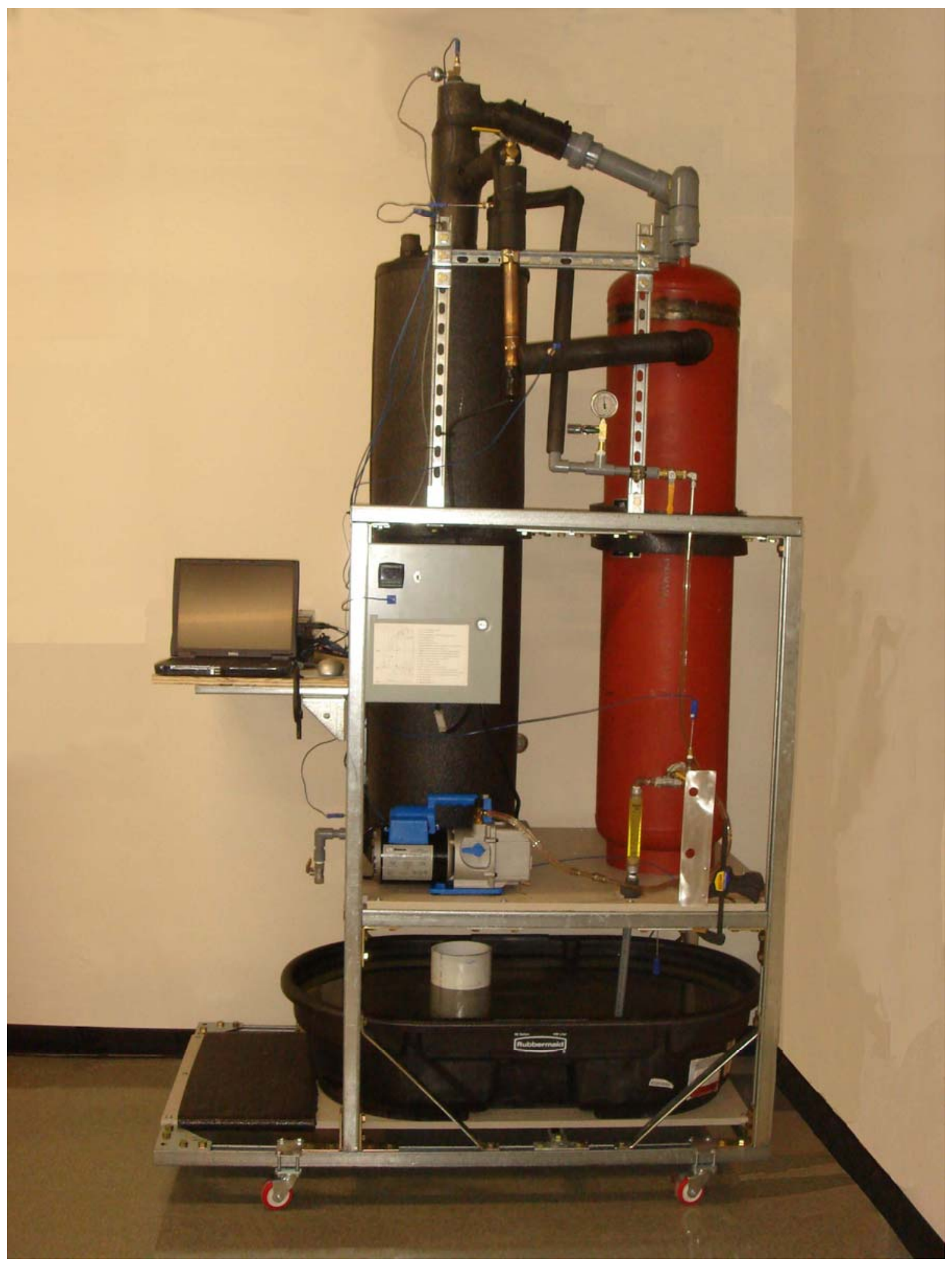

Figure 32. Overall view of the experimental unit 


\section{CHAPTER 6. PARAMETRIC ANALYSIS}

\subsection{Analyses Synchronization}

Theoretical and experimental analyses must be synchronized to compare their outputs properly. The model developed in CHAPTER 4 holds for the proposed desalination system outlined in Figure 21; however, it needs to be modified to represent the experimental unit outlined in Figure 26 to validate its predictions.

The flash chamber of the experimental unit is not elevated for passive vacuum generation, and levels of the flash chamber are not hydrostatically balanced as was

mentioned in CHAPTER 5. Vacuum is created before running the unit by a vacuum pump; furthermore, the flash chamber is closed during operation to maintain that vacuum, since it can not be maintained hydrostatically. Consequently, Bernoulli's fluid equation can not be used to estimate initial and the dynamic levels in the evaporator and condenser. Initial levels are equal to zero as vessels are drained before operation while dynamic levels are functions of totalized, or integrated, inlet flows. Therefore, Equation 98 through Equation 101 are substituted with

$Z_{C}^{i}=0$

(Alternate 98)

$Z_{C}=Z_{C}^{i}-\frac{\int M_{C} d t}{\rho_{C} \cdot X A_{C}}$

(Alternate 99)

$Z_{E}^{i}=0$

(Alternate 100)

$Z_{E}=Z_{E}^{i}-\frac{\int M_{W} d t}{\rho_{W} \cdot X A_{E}}$

(Alternate 101) 
The condenser and evaporator are modeled as horizontally-mounted right circular cylinders as can be seen in Figure 21; however, the condenser and evaporator of the experimental unit are vertically-mounted right circular cylinders as can be seen in Figure 26. Consequently, the geometry of the vacuum volume needs to adapt; therefore, Equation 102 through Equation 105 are substituted with

$$
\begin{aligned}
& V_{C V}^{i}=X A_{C} \cdot\left(L_{C}-Z_{C}^{i}\right) \\
& V_{C V}=X A_{C} \cdot\left(L_{C}-Z_{C}\right) \\
& V_{E V}^{i}=X A_{E} \cdot\left(L_{E}-Z_{E}^{i}\right) \\
& V_{E V}=X A_{E} \cdot\left(L_{E}-Z_{E}\right)
\end{aligned}
$$

The experimental unit does not include a feed pump as was mentioned in CHAPTER 5; therefore, Equation 117 is substituted with

$W_{P}=0$

(Alternate 117)

\subsection{Parameter Expressions}

The model developed in CHAPTER 4 along with the above alternate equations were coded and executed using experimental temperature, pressure, and flow rate values as inputs generating pseudo-experimental data of model parameters. This data mining process is used to uncover patterns in model parameters so they can be properly expressed in the model via correlations obtained using non-linear regression.

The counter-current departure correction factor for the condenser tube is used to correct its log mean temperature difference to solve accurately for the temperature of preheated seawater before it enters the heater, which is essential for estimating prime energy consumption and efficiency of the condenser and heat recovery. 
A correlation for the counter-current departure correction factor for the condenser tube is obtained by regressing the mined data as shown in Figure 33 yielding $F_{C T}=0.0293+0.1655 \cdot S_{C T}+2.9102 \cdot S_{C T}^{2}-6.1629 \cdot S_{C T}^{3}+4.2518 \cdot S_{C T}^{4}$ (Alternate 90)

The counter-current departure correction factor for the condenser is used to correct its log mean temperature difference to solve for the temperature of the condensed fresh water accurately; however, experimental data show that the temperature of the condensed fresh water remained rather constant with a value about two degrees above ambient regardless of how high the equilibrium temperature was. This outcome is most likely due to a good heat rejection by the condenser, in addition to the fact that the amount of cool seawater flowing through the condenser tube vastly exceeds that of the condensing water vapor outside the condenser tube.

Consequently, a correlation for the counter-current departure correction factor for the condenser will be replaced by

$T_{C}=T+2$

(Alternate 91)

The counter-current departure correction factor for the evaporator is used in correcting its log mean temperature difference to estimate its heat loss accurately. Temperatures of the flashed vapor and concentrated brine diverge due to boiling point elevation and non-equilibrium allowance as mentioned in CHAPTER 4 as well as a small amount of heat loss from the insulated evaporator.

To simplify calculations, heat loss from the evaporator is ignored, and a correlation for the non-equilibrium allowance is obtained by regressing the experimental data as shown in Figure 34 yielding

$Q_{E}=0$

(Alternate 92) 


$$
\begin{aligned}
& \xi=1.6836 \cdot \zeta^{2}-3.3898 \cdot \zeta+2.7785-0.1399 \cdot \exp \left(-5.9154 \cdot \zeta^{-29.3208}\right) \\
& \text { Where } \xi=\frac{T_{W}+273.15}{T_{E}+273.15} \quad \zeta=\frac{T_{H}+273.15}{25+273.15}
\end{aligned}
$$

The activity coefficient of water is used to correct its molar fractions to solve for its $\mathrm{K}$-value accurately. Theoretical expressions for activity coefficients of species in electrolyte solutions, such as seawater, are available in literature but very challenging to implement due to the large number of interactions present among all ions and molecules. Those interactions are characterized by parameters that in most cases must be experimentally determined.

To simplify calculations, a correlation for the activity coefficient of water is obtained by regressing the mined data as shown in Figure 35 yielding

$$
\gamma_{\mathrm{H}_{2} \mathrm{O}}=0.0020+1.0385 \cdot \frac{P_{V}}{P_{\mathrm{H}_{2} \mathrm{O}}}
$$

The gas phase molecular content correction factor is used in correcting the equation of state to solve for the vacuum pressure accurately as was mentioned earlier in CHAPTER 4. It accounts for both gases dissolving in the condensing water vapor and any possible discrepancy in the input seawater content of dissolved gases or the calculated vapor-liquid equilibrium for carbon dioxide.

To simplify calculations, a correlation for gas phase molecular content correction factor is obtained by regressing the mined data as shown in Figure 36 yielding

$$
\psi=1-2.2861 \cdot \exp \left(-\frac{P_{V}}{P_{\mathrm{H}_{2} \mathrm{O}}}\right)
$$

The Matlab ${ }^{\mathrm{TM}}$ codes used for regressing all of the above mined data are found in the APPENDICES section. 


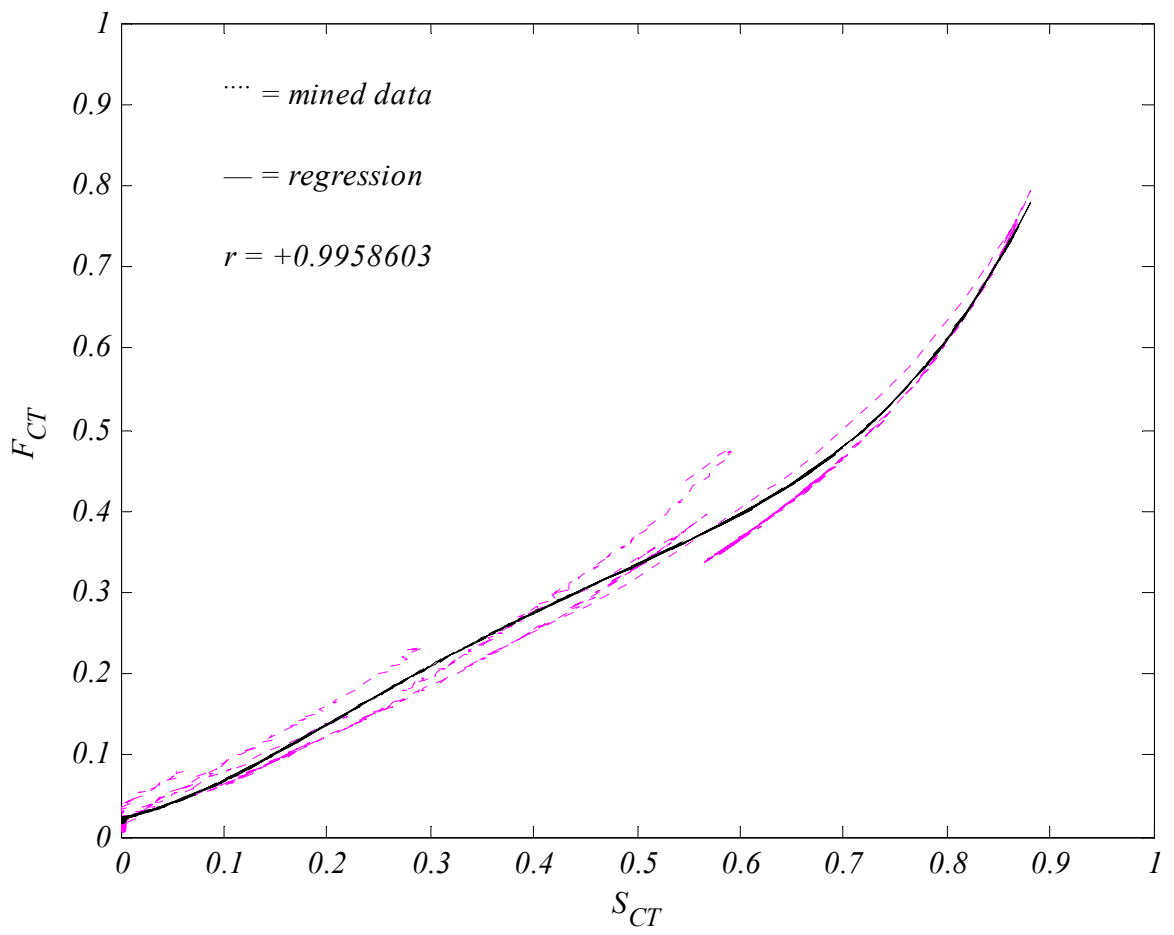

Figure 33. Counter-current departure correction factor of condenser tube

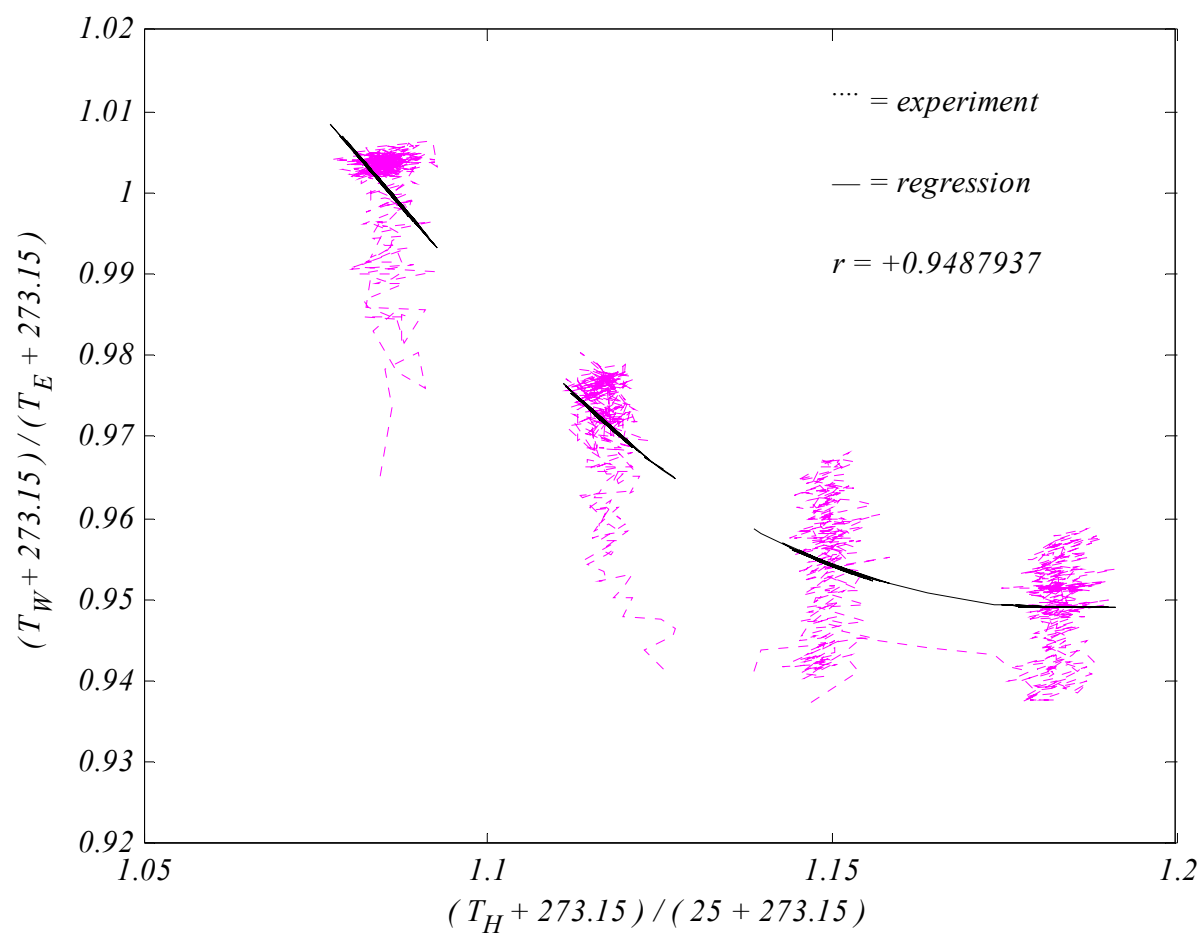

Figure 34. Non-equilibrium allowance representation 


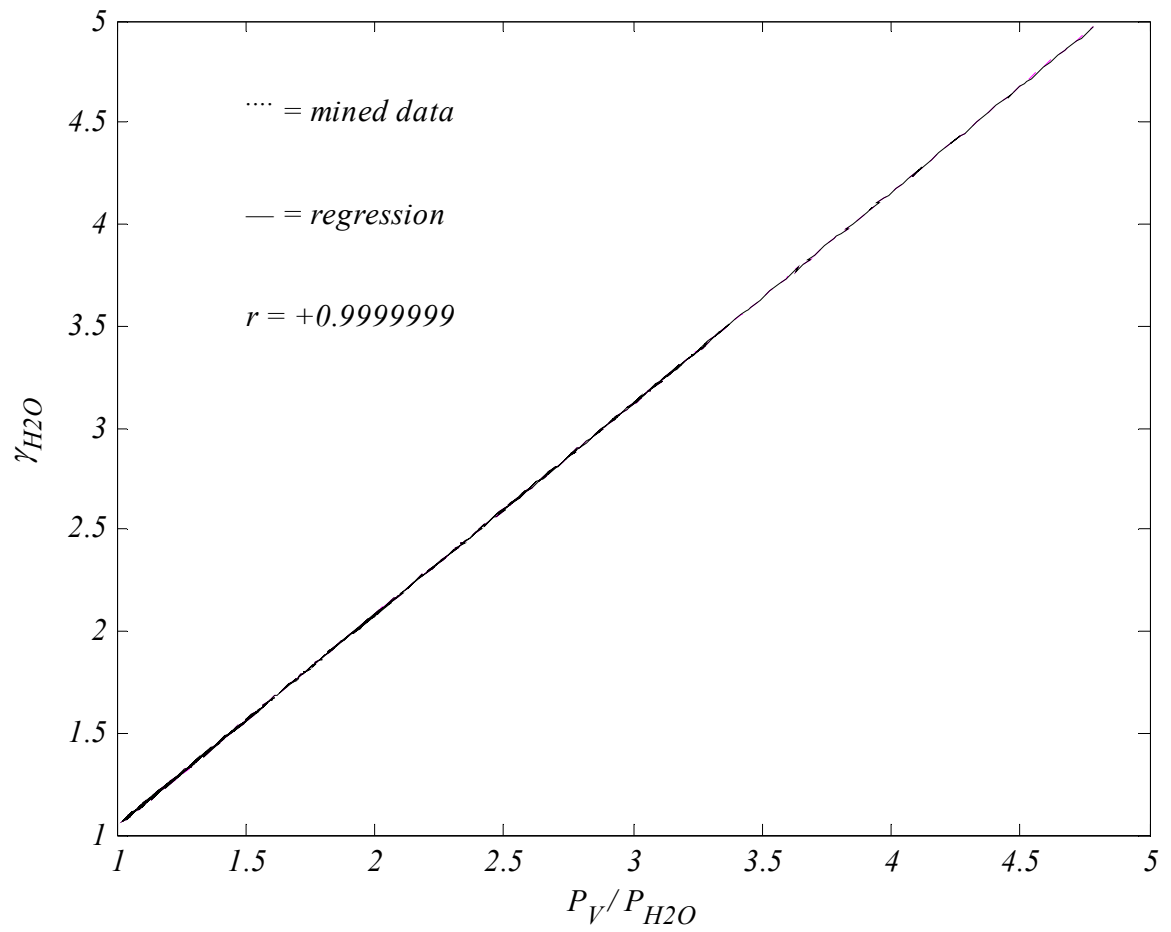

Figure 35. Activity coefficient of water

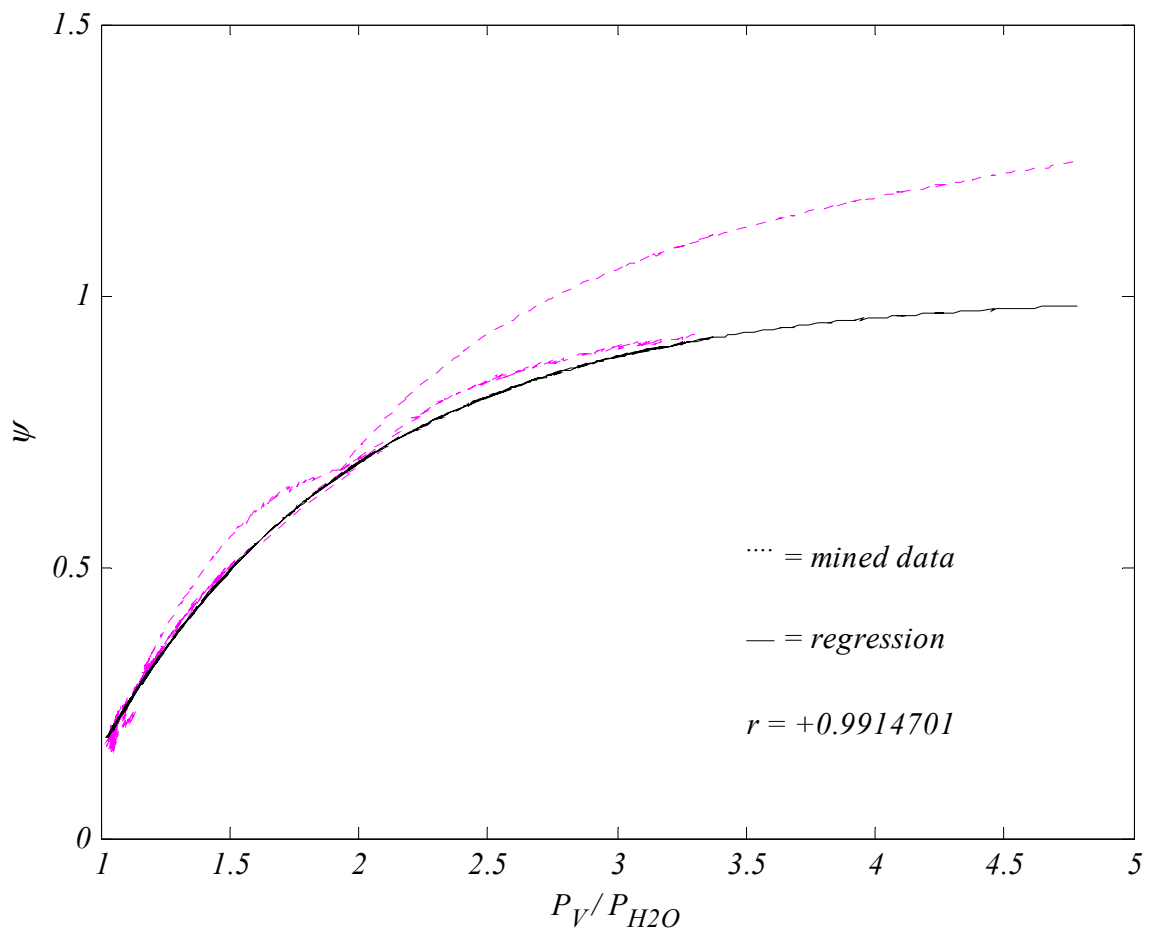

Figure 36. Gas phase molecular content correction factor 


\subsection{Parameter Inputs}

Seawater is a solution of many salts and contains a small amount of dissolved gases as mentioned earlier in CHAPTER 4. Input parameters used for sea salt are given in Table 7, while input parameters used for seawater are given in Table 8. The molecular weights given in both tables are obtained from NIST [31], while mass fractions given in both tables are obtained from an oceanography manual [16]. In addition, the relativity factors given in Table 8 are found by averaging temperature-stamped data [19], while enthalpy parameters given in Table 8 are obtained from NIST [31].

As mentioned in CHAPTER 4, the SUPERTRAPPTM code was employed to perform isobaric phase equilibria flash calculations for water with an average content of non-condensable gas as reported in literature [16] at various temperatures to produce a dataset of K-values. SUPERTRAPPTM flash calculations were executed at a constant pressure of 1 bar; therefore, the produced K-values are equivalent to Henry's constant for non-condensable gases and vapor pressure for water. The reported values of $H C^{\circ}{ }_{i}$ and $H F_{i}$ [17] plus $P A, P B$, and $P C$ [15] were used as initial guesses in Matlab ${ }^{\mathrm{TM}}$ least squares regression codes to adjust their values to best fit the produced $\mathrm{K}$-value dataset to Henry's constant and vapor pressure correlations.

The SUPERTRAPPTM code used for generating the $\mathrm{K}$-value dataset and Matlab $^{\text {TM }}$ codes used for regressing them are in the APPENDICES section. The performed regressions yielded excellent results with correlation coefficients very close to unity as can be seen in Figure 37 through Figure 41. Better estimates of K-values denote better representation of the vapor-liquid equilibrium, ultimately resulting in more reliable flash calculations. 
Table 7. Sea salt parameters [16] [31]

\begin{tabular}{l|cc}
\hline & $M W$ & $\omega$ \\
\hline $\mathrm{Cl}$ & 35.45 & 0.55030 \\
\hline $\mathrm{Na}$ & 22.99 & 0.30590 \\
\hline $\mathrm{SO}_{4}$ & 96.06 & 0.07680 \\
\hline $\mathrm{Mg}$ & 24.31 & 0.03680 \\
\hline
\end{tabular}

\begin{tabular}{l|cc}
\hline & $M W$ & $\omega$ \\
\hline $\mathrm{Ca}$ & 40.08 & 0.01180 \\
\hline $\mathrm{K}$ & 39.10 & 0.01110 \\
\hline $\mathrm{HCO}_{3}$ & 61.02 & 0.00410 \\
\hline $\mathrm{Br}$ & 79.90 & 0.00190 \\
\hline
\end{tabular}

\begin{tabular}{l|cc}
\hline & $M W$ & $\omega$ \\
\hline $\mathrm{BO}_{3}$ & 58.81 & 0.00080 \\
\hline $\mathrm{Sr}$ & 87.62 & 0.00040 \\
\hline$F$ & 19.00 & 0.00003 \\
\hline & & \\
\hline
\end{tabular}

Table 8. Seawater parameters [16] [31]

\begin{tabular}{|c|c|c|c|c|c|c|c|c|c|c|c|c|c|c|}
\hline & $M W$ & $\varphi$ & $\alpha$ & $A$ & $B$ & $C$ & $D$ & $E$ & $F$ & $H C^{\circ}$ & $H F$ & $P A$ & $P B$ & $P C$ \\
\hline$N_{2}$ & 28.01 & $1.26 \mathrm{E}-05$ & 1.21 & 26.0920 & 8.2188 & -1.9761 & 0.1593 & 0.0444 & -7.9892 & 8067573 & -3546 & & & \\
\hline $\mathrm{O}_{2}$ & 32.00 & $7.70 \mathrm{E}-06$ & 1.22 & 29.6590 & 6.1373 & -1.1865 & 0.0958 & -0.2197 & -9.8614 & 358815 & -2209 & & & \\
\hline$A r$ & 39.94 & $4.00 \mathrm{E}-07$ & 1.23 & 20.7860 & $2.83 \mathrm{E}-07$ & $-1.46 \mathrm{E}-07$ & $1.09 \mathrm{E}-08$ & $-3.66 \mathrm{E}-08$ & -6.1974 & 384073 & -2308 & & & \\
\hline $\mathrm{CO}_{2}$ & 44.01 & $2.20 \mathrm{E}-07$ & 1.17 & 24.9974 & 55.1870 & -33.6914 & 7.9484 & -0.1366 & -10.0851 & 10915 & -445 & & & \\
\hline Salt & & $3.50 \mathrm{E}-02$ & & & & & & & & & & & & \\
\hline $\mathrm{H}_{2} \mathrm{O}$ & 18.01 & & 0.9816 & 30.0920 & 6.8325 & 6.7934 & -2.5345 & 0.0821 & -9.0546 & & & 13 & 4391 & 245 \\
\hline
\end{tabular}

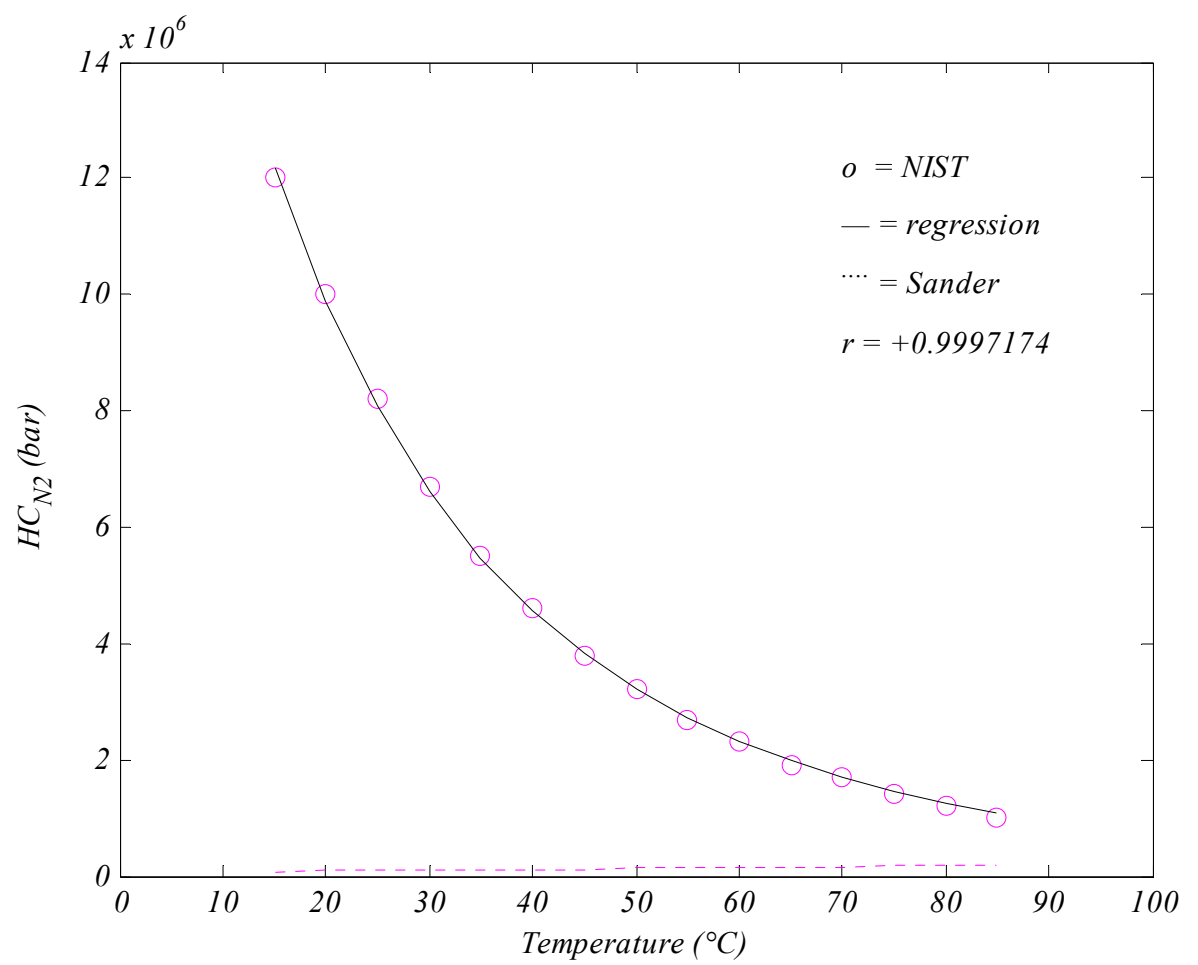

Figure 37. Henry's constant of nitrogen 


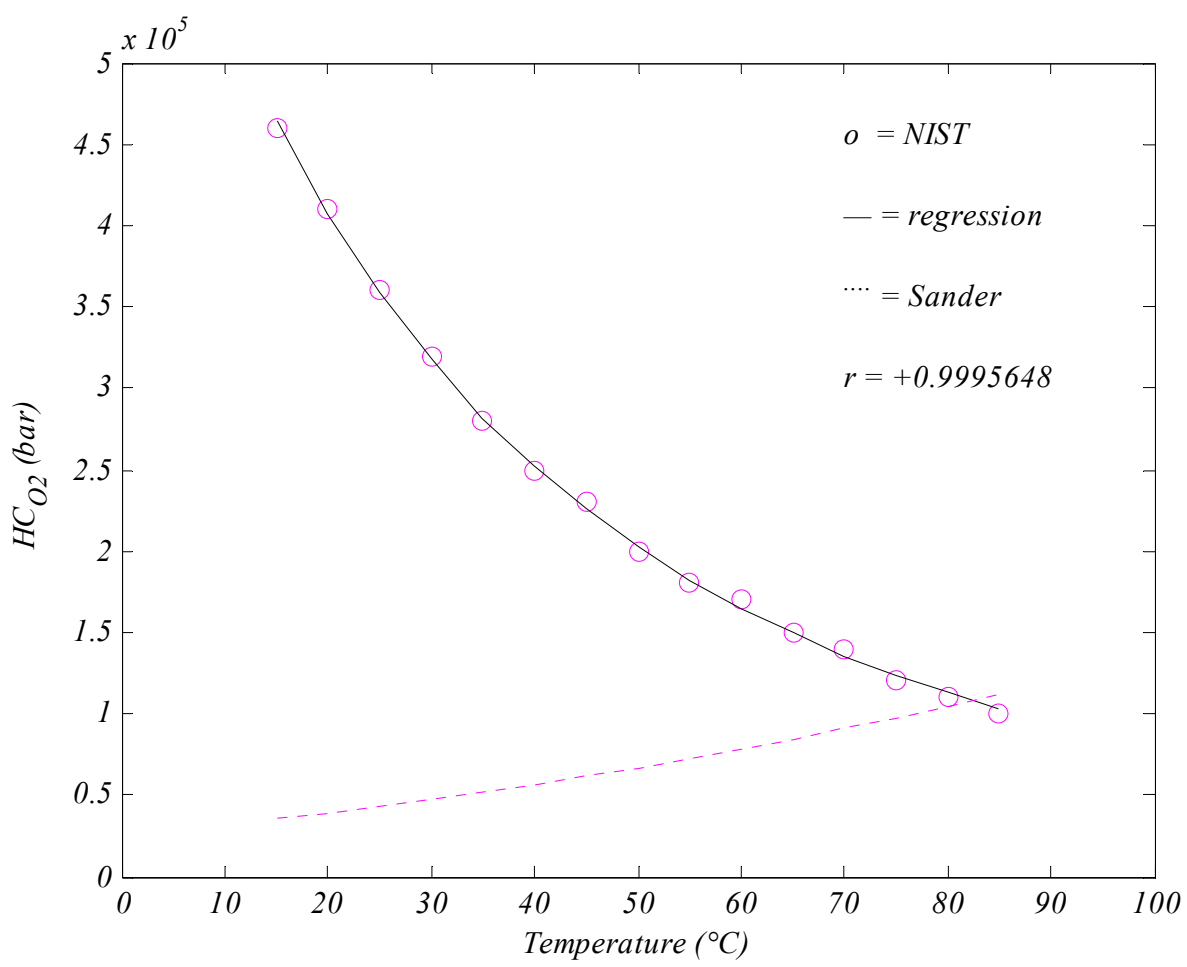

Figure 38. Henry's constant of oxygen

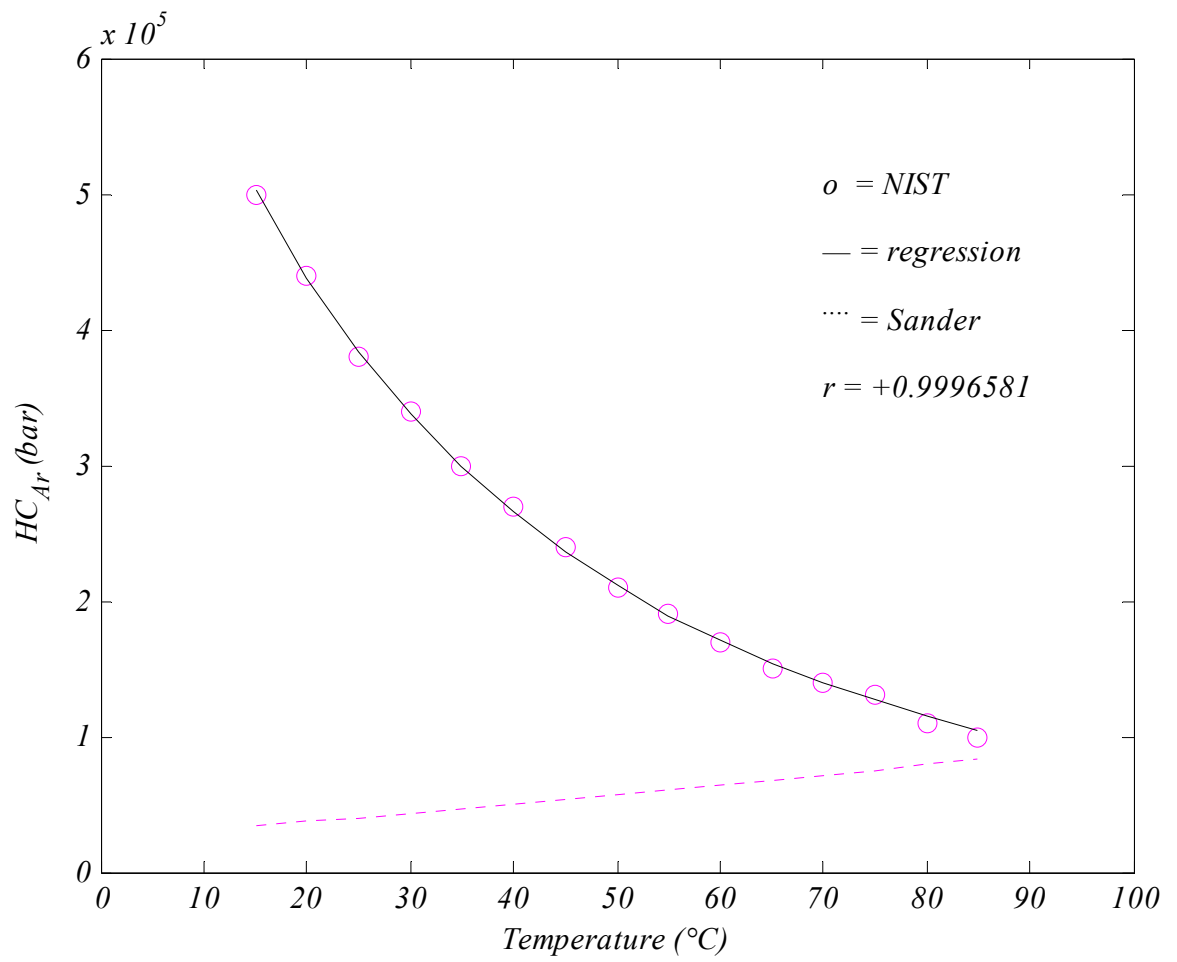

Figure 39. Henry's constant of argon 


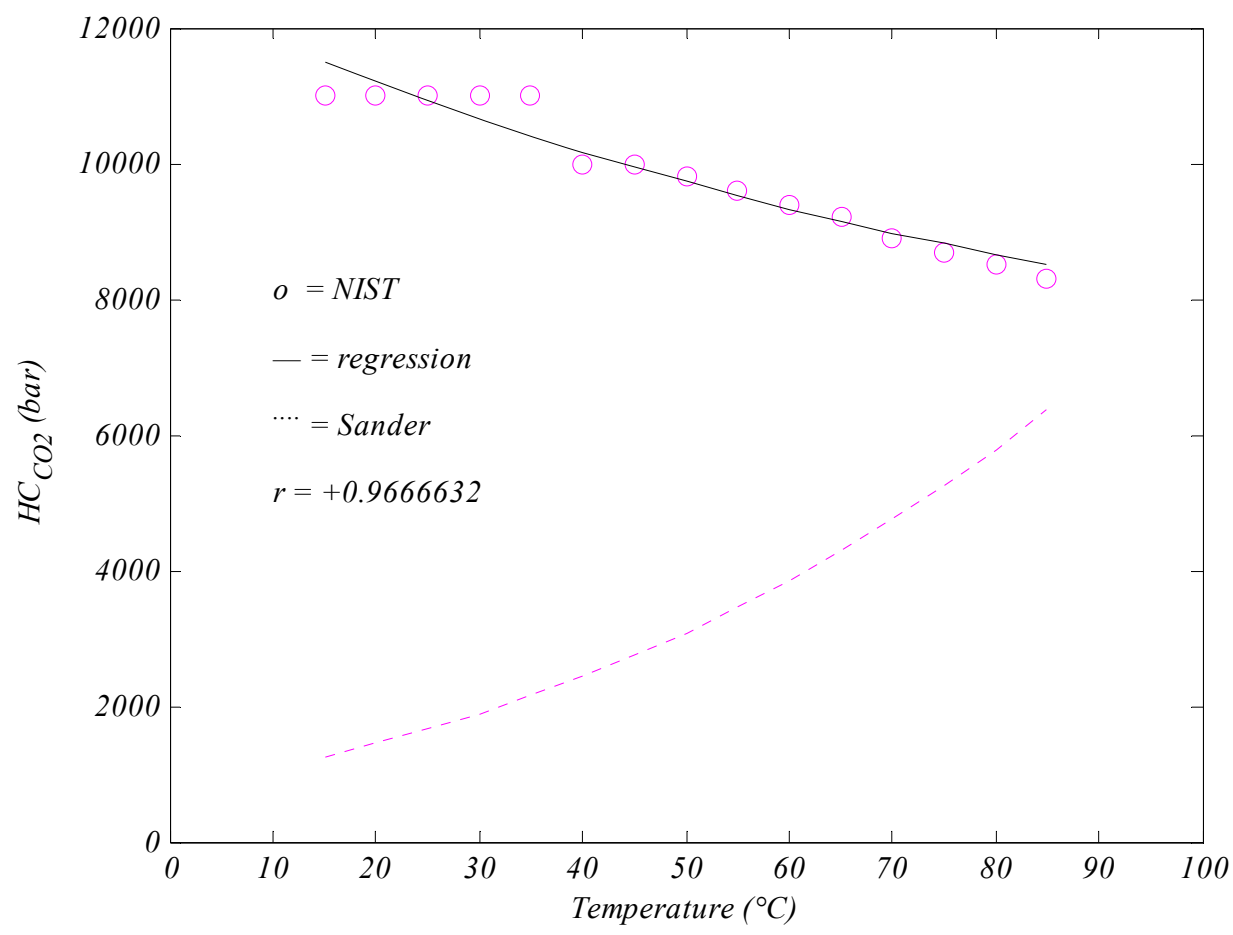

Figure 40. Henry's constant of carbon dioxide

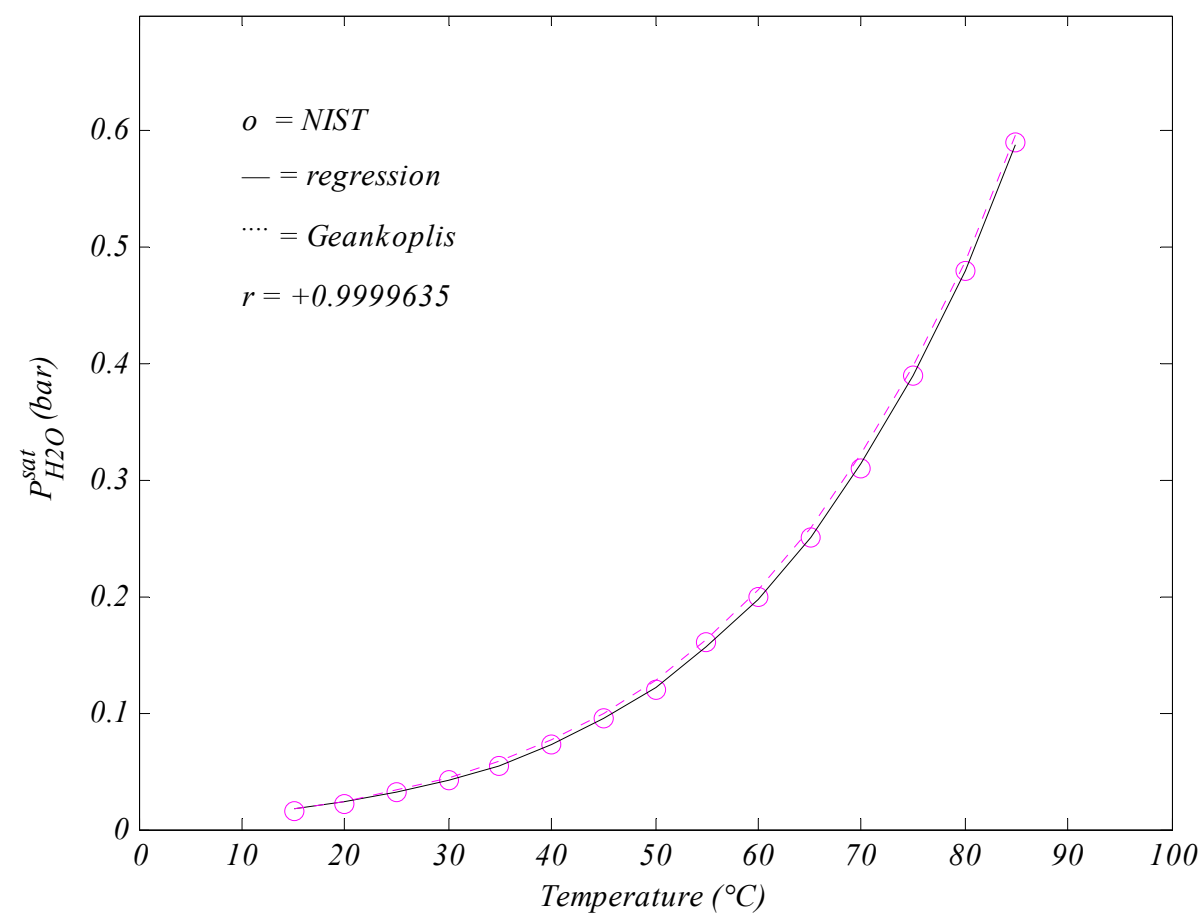

Figure 41. Vapor pressure of water 


\subsection{Equipment Specifications}

The seawater feed as well as brine and fresh water tanks are modeled as horizontal polyethylene troughs; moreover, the condenser and evaporator are modeled as painted-steel and galvanized-steel upright cylinders, respectively. The connecting pipes are modeled as CPVC with copper tubing used inside the condenser and heater. The condenser tube is a protracted copper tube vertically coiled inside the condenser in four loops, that is $N_{C T}=4$. Copper is not suited for seawater due to its corrosivity and should not be used in desalination systems; however, for theoretical and short-term experimental simulations, it is considered acceptable. Dimensions and the heat transfer parameters of the experimental unit are given in Table 9 and Table 10, and these values will be input to the model as well. In addition, the model neglects any heat input by any pump as well as any work output by the heater, condenser, or evaporator, that is $Q_{P}=W_{C}=W_{H}=W_{E}=0$.

Table 9. Equipment dimensions

\begin{tabular}{l|cc}
\hline Vessel & $D$ & $L$ \\
\hline Seawater & 90 & 30 \\
\hline Brine Water & 90 & 30 \\
\hline Fresh Water & 90 & 30 \\
\hline Evaporator & 35 & 160 \\
\hline Condenser & 35 & 160 \\
\hline
\end{tabular}

\begin{tabular}{l|cc}
\hline Pipe & $D$ & $L$ \\
\hline$S$ & 1.27 & 95 \\
\hline$P$ & 1.27 & 25 \\
\hline$X$ & 1.27 & 60 \\
\hline$H$ & 1.27 & 75 \\
\hline$W$ & 1.27 & 30 \\
\hline
\end{tabular}

\begin{tabular}{l|cc}
\hline Pipe & $D$ & $L$ \\
\hline$E$ & 5.08 & 180 \\
\hline$C$ & 0.32 & 35 \\
\hline$C T$ & 1.27 & 475 \\
\hline$H T$ & 1.27 & 13 \\
\hline & & \\
\hline
\end{tabular}

Table 10. Heat transfer equipment parameters

\begin{tabular}{lcccc}
\hline & $h^{\text {id }}$ & $h^{\text {od }}$ & $\delta$ & $k^{w}$ \\
\hline Condenser Tube & 0.20 & 0.50 & 0.125 & 3.810 \\
\hline Condenser & 0.50 & 0.75 & 0.250 & 0.450 \\
\hline Evaporator & 0.50 & 0.75 & 0.250 & 0.001 \\
\hline
\end{tabular}


The model also uses typical absorptance and transmittance values plus average heat transfer and removal factors pertaining to a single-glazed flat-plate solar collector, in addition to a standard value for the intensity of solar insolation, to estimate the solar collection area needed to meet the computed heater load adequately. Explicitly declaring: $I=600 \mathrm{~W} / \mathrm{m}^{2}, F_{S C}=0.82, U_{S C}=0.92 \mathrm{~W} / \mathrm{m}^{2}-^{\circ} \mathrm{C}, \alpha_{S C}=0.92, \tau_{S C}=0.90$.

\subsection{Simulation Specifications}

Model simulations will be carried out at conditions corresponding to those of the experimental simulations so they can be compared. Simulated operations will run for a period of three hours and will be modeled using one minute increments with the same initial seawater tank level and vacuum pressure. Simulations were carried out at two different seawater flow rate ranges and at four different flash temperatures.

Mass flow rate of a fluid across an orifice is given by $M=\rho \cdot C V \cdot \sqrt{ }(\Delta P / S G)$. The flow rate was manually adjusted during experiments using a ball valve. The valve position was kept constant for each flow rate range; however, flow rate slightly varied within each range due to varying fluid densities caused by different fluid temperatures and varying differential pressures across the valve caused by the different vacuum pressures. In addition, flow rates were progressively decreasing during each experiment due to declining differential pressure caused by the increasing vacuum pressure. An average flow rate value was computed for each experiment and input to the corresponding model simulation as a constant value to simplify calculations. The average flow rate value was obtained by dividing the estimated amount of seawater transferred from the seawater feed tank by the duration of the experiment. 
The diffusion rate correlation given in CHAPTER 4 includes two adjustable parameters. Parameter $\sigma$ serves as a diffusion coefficient and can be thought of as a conductance term, while parameter $\Omega$ serves as a diffusion barrier and can be thought of as a resistance term. Both parameters were adjusted using the same code mentioned earlier for generating pseudo-experimental data of model parameters by arbitrarily assigning a value for parameter $\sigma$ while tuning parameter $\Omega$ to match the output amount of fresh water produced by the code to the actual amount of fresh water produced. It is important to point out once more that those obtained diffusion rate correlation parameter values pertain to the geometry of the current experimental set-up and should be readjusted whenever applied to different geometries using new experimental records.

The above mentioned experimental and adjusted simulation settings are given in Table 11 and will be input to the model. The experimental simulations were conducted in a laboratory; consequently, ambient conditions are considered accordingly, $T=20^{\circ} \mathrm{C}$ and $P=1.01325$ bar. Finally, temperature in the seawater feed tank is assumed equal to ambient, $T_{S}=T$, while common literature values were used for the universal gas constant and the gravity acceleration, $R=83.14472 \mathrm{bar}-\mathrm{cm}^{3} / \mathrm{mol}-{ }^{\circ} \mathrm{C}$ and $\mathrm{g}=980.0665 \mathrm{~cm} / \mathrm{s}^{2}$.

Table 11. Simulation settings

\begin{tabular}{llllllc}
\hline Simulation & $Z_{S}^{i}$ & $P_{V}^{i}$ & $M_{S}$ & $T_{H}$ & $\sigma$ & $\Omega$ \\
\hline 1 & 24 & 0.14 & 496 & 50 & 2 & 0.118 \\
\hline 2 & 24 & 0.14 & 474 & 60 & 2 & 0.150 \\
\hline 3 & 24 & 0.14 & 453 & 70 & 2 & 0.120 \\
\hline 4 & 24 & 0.14 & 388 & 80 & 2 & 0.019 \\
\hline \hline 5 & 24 & 0.14 & 711 & 50 & 2 & 0.135 \\
\hline 6 & 24 & 0.14 & 690 & 60 & 2 & 0.197 \\
\hline 7 & 24 & 0.14 & 668 & 70 & 2 & 0.229 \\
\hline 8 & 24 & 0.14 & 582 & 80 & 2 & 0.103 \\
\hline
\end{tabular}




\section{CHAPTER 7. DISCUSSION OF RESULTS}

\subsection{Discussion Guide}

Model and experimental results will be compared and discussed throughout this chapter. Model results are obtained by executing a code comprised of the equations given in CHAPTER 4 as well as the alternate equations and the parameter values given in CHAPTER 6. Experimental results correspond to two sets of experiments, where one set was conducted at lower seawater flow rates, and the other set was conducted at higher seawater flow rates. In addition, each set of experiments includes four variations of flash temperature, where each variation was duplicated three times to validate its outcome. So, each table value and figure curve given in this dissertation designated as an experimental result is in fact the averaged outcome of three matching experiments. The experimental matrix and conditions were provided earlier in Table 6 and Table 11. Experimental results will sometimes correspond to pseudo-experimental data generated by a code comprised of the equations and the alternate equations mentioned above, but with the mass and energy balance relations replaced by experimental temperature and pressure as well as flow rate values. Each figure will use a solid line to represent model data and a dotted line fitted with a translucent error band to represent experimental data. A detailed error analysis can be found in the APPENDICES section. The figures will also indicate if the experimental data correspond to real experimental data or to pseudo-experimental data by dubbing the data as either "experiment" or "mined", respectively, in their legend. 
The controlled variables of the current desalination system are seawater flow rate and flash temperature. Their effects on the desalination process will be analyzed through graphical representations of several system variables that illustrate their dynamics during each simulation. Twelve time-varying system variables will be examined by a set of twelve figures provided for each one. Every set includes four figures, each corresponding to a single flash temperature at lower seawater flow rates, as well as four figures, each corresponding to a single flash temperature at higher seawater flow rates. Furthermore, each figure will sketch two profiles, where one corresponds to model simulation, and the other corresponds to experimental simulation, both at analogous conditions. Each set also includes four figures, where the four different flash temperature profiles of each seawater flow rate range are joined on one figure for both model and experimental simulations.

The legend of each figure includes the correlation coefficient that measures the linear dependence between the modeled and experimental datasets. The correlation coefficient is also known as the Pearson product moment correlation coefficient and is computed by dividing the covariance of two variables by the product of their standard deviations, yielding a value between -1 and +1 . The computation process of the correlation coefficient is rather cumbersome; however, it is available as a built-in function in many software packages. A correlation coefficient of 0 indicates the total lack of correlation, while a correlation coefficient of -1 indicates a perfect negative linear correlation and a correlation coefficient of +1 indicates a perfect positive linear correlation. A correlation coefficient less than -0.8 or greater than 0.8 typically indicates a strong correlation, while a correlation coefficient between -0.5 and +0.5 typically indicates a weak correlation. 


\subsection{Vacuum Erosion}

System vacuum pressure accounts for the water vapor in addition to the accumulating non-condensable gases as was mentioned in CHAPTER 4. System vacuum gradually eroded for both seawater flow rates; moreover, it eroded a little faster at higher seawater flow rates. Vacuum pressure increased with flash temperatures for both seawater flow rates, since vapor pressure is directly proportional to flashing temperature.

The initial rapid increase of vacuum pressure was caused by the early rapid increase of water vapor pressure caused by the early rapid increase of temperature inside the flash chamber. Vacuum pressure continued to increase afterwards but at a much lower rate due to slow accumulation of non-condensable gases. The rate of increase of vacuum pressure, $\partial P_{V} / \partial t$, was decelerating for higher flash temperatures but was accelerating for lower flash temperatures for both seawater flow rates. This is especially obvious for higher flow experiments flashing at $50{ }^{\circ} \mathrm{C}$ indicating that higher flow rates entail a higher rate of accumulation of non-condensable gases. Decreasing pressure at a given temperature increases vapor-liquid equilibrium coefficient value resulting in more overall evaporation. Consequently, flashing seawater at lower flow rates slowed the rate accumulation of non-condensable gases, which decelerated vacuum erosion rate, resulting in more evaporation and more fresh water production.

Model prediction of vacuum pressure resembled the experimental results but was generally lower, and the discrepancy increased with temperature. This is probably due to the constant seawater flow rate assumed by the model, while it was progressively decreasing during experiments due to the declining differential pressure caused by the eroding vacuum. Vacuum pressure profiles are shown in Figure 42 through Figure 53. 


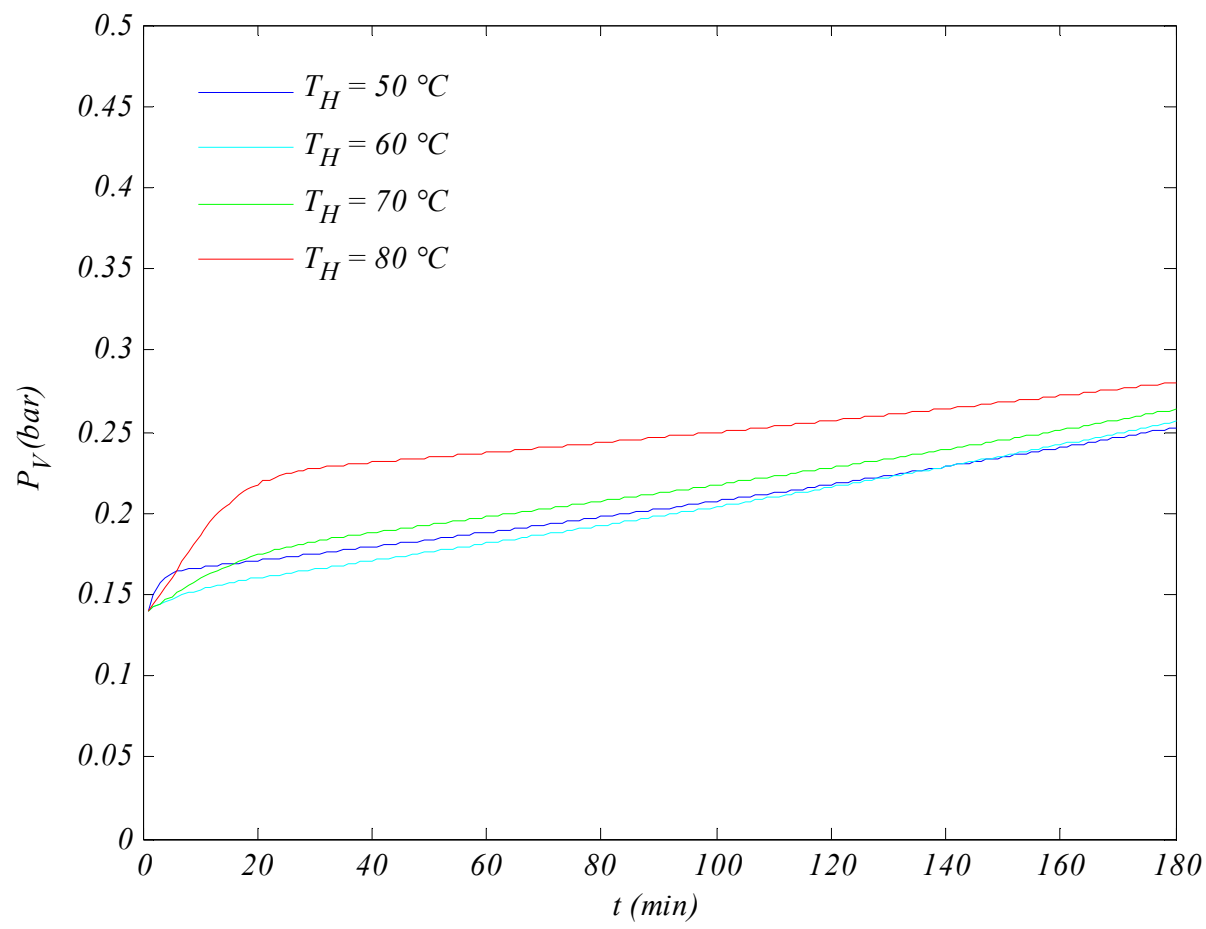

Figure 42. Modeled vacuum pressure profiles at lower flow

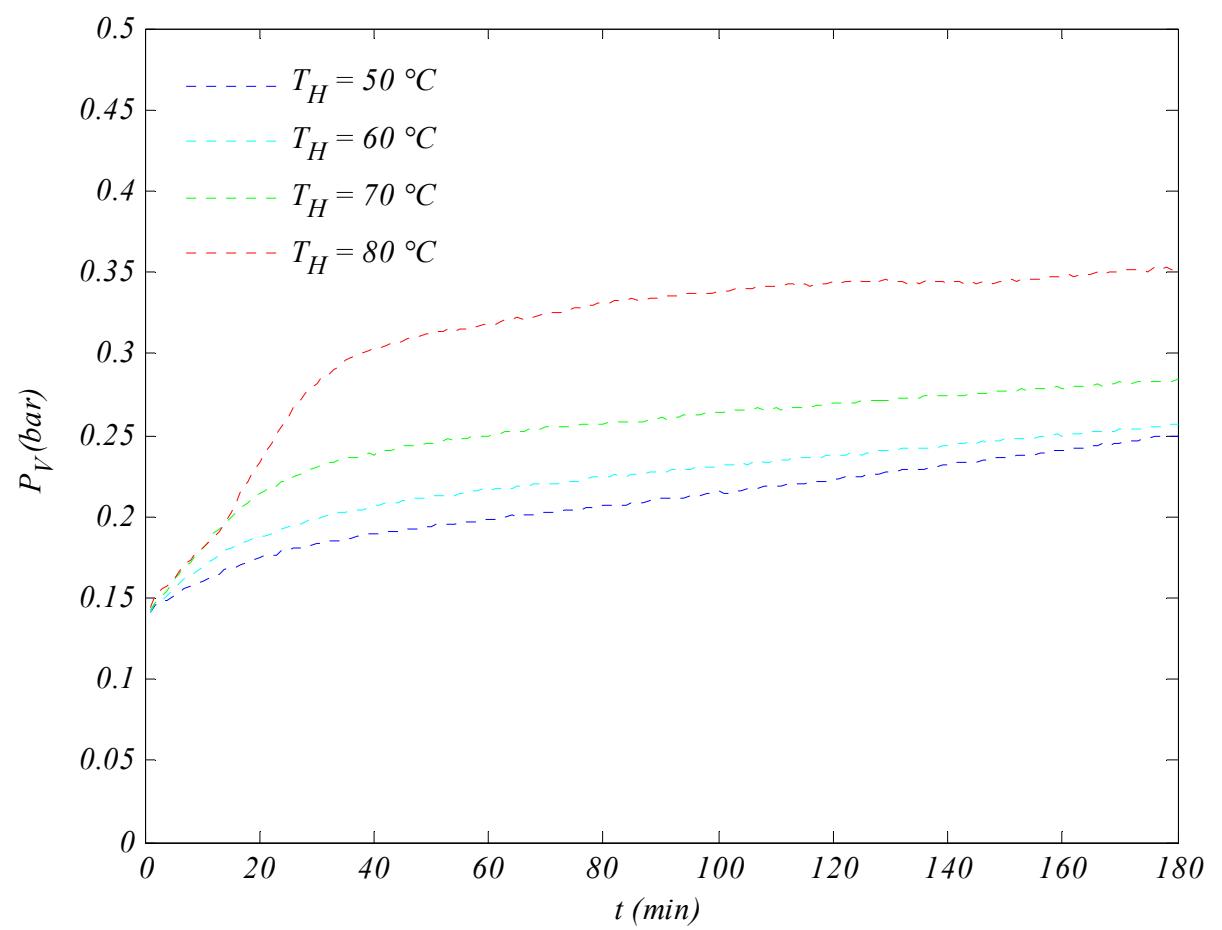

Figure 43. Experimental vacuum pressure profiles at lower flow 


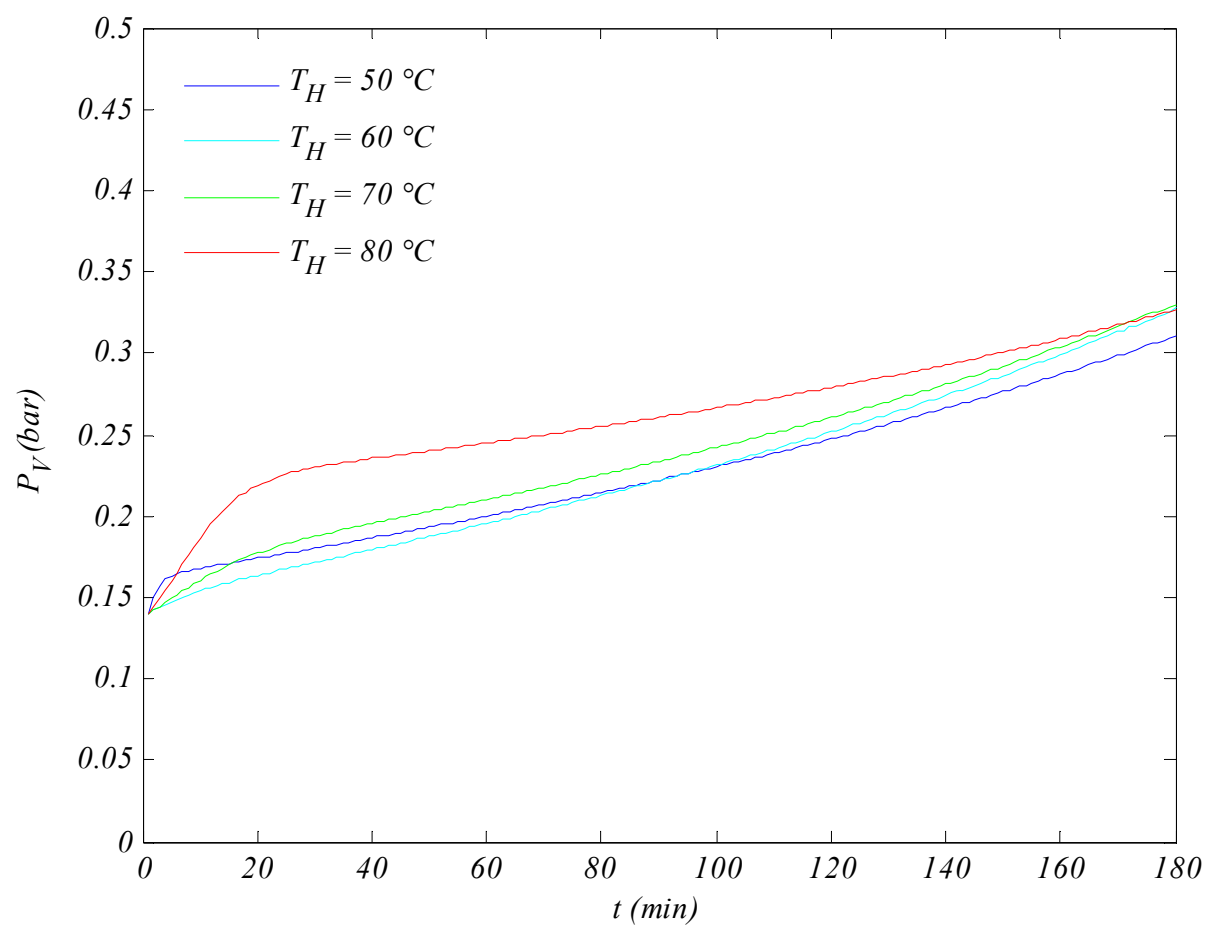

Figure 44. Modeled vacuum pressure profiles at higher flow

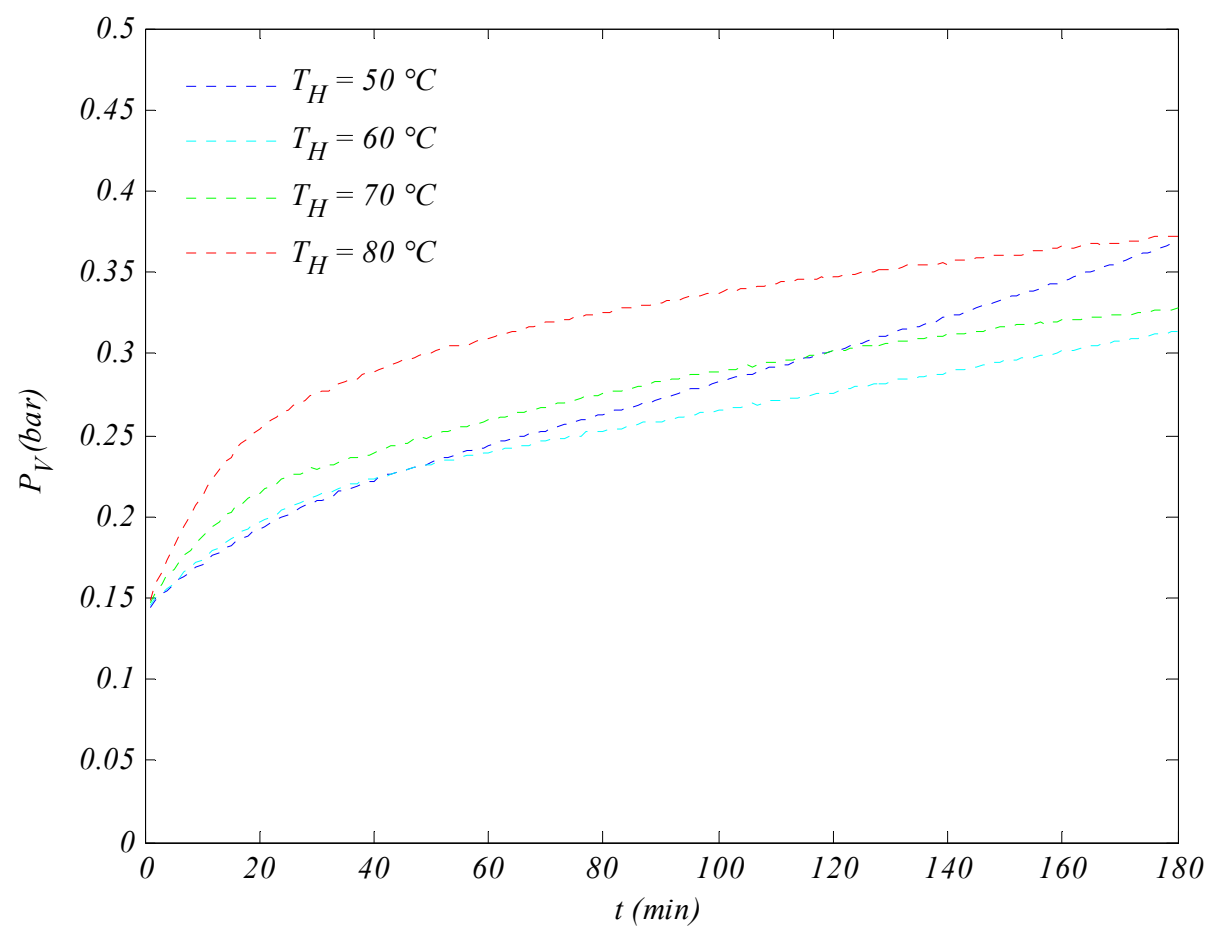

Figure 45. Experimental vacuum pressure profiles at higher flow 


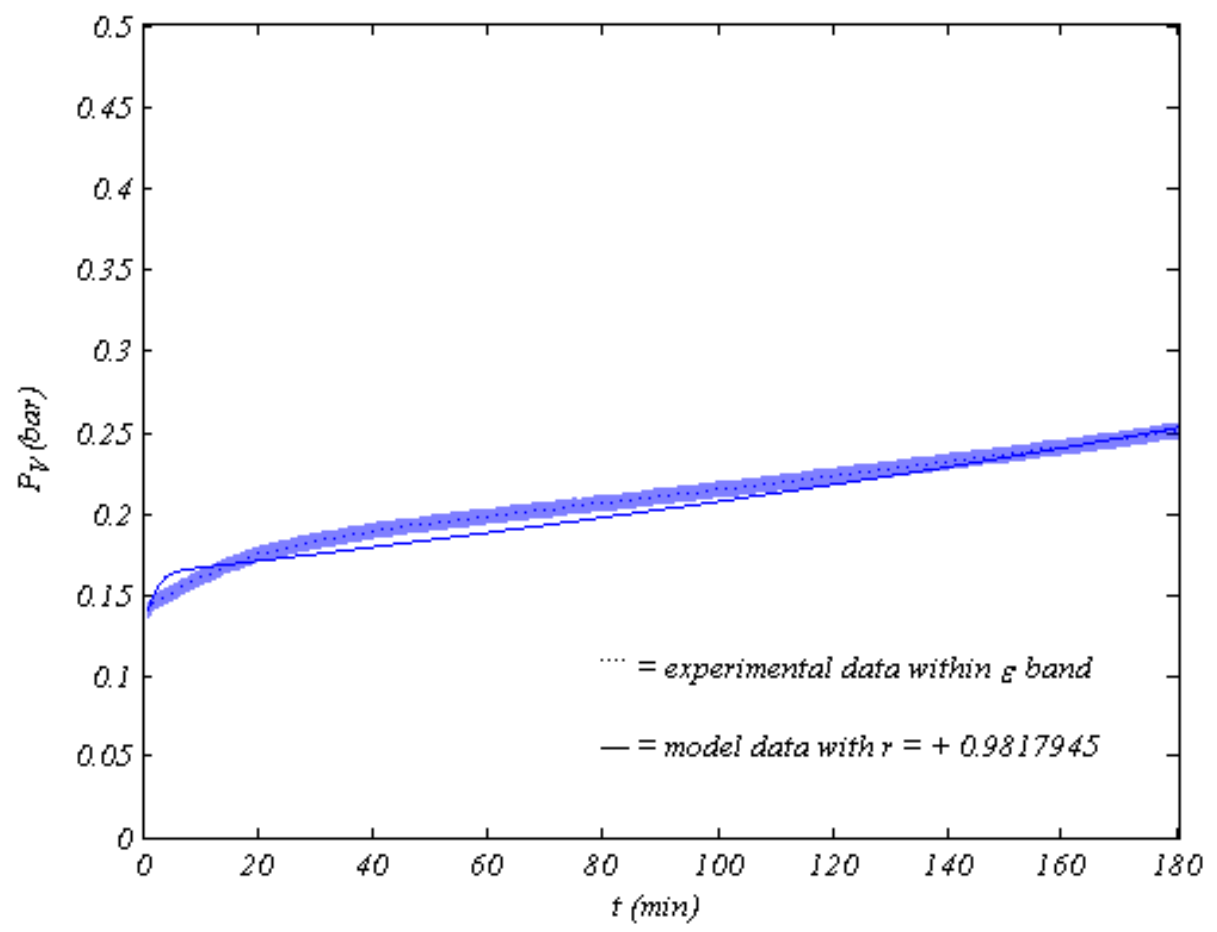

Figure 46 . Vacuum pressure at $50^{\circ} \mathrm{C}$ flash and lower flow

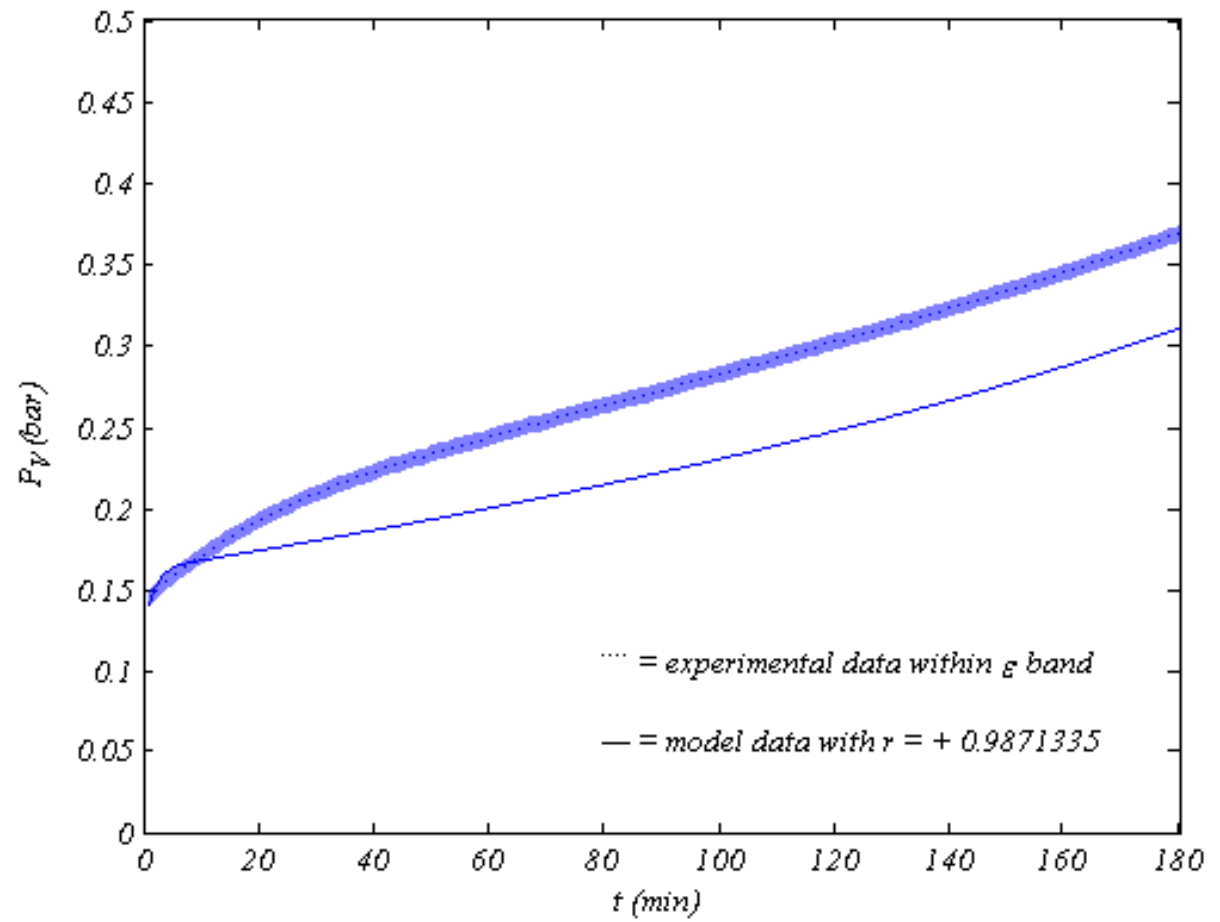

Figure 47. Vacuum pressure at $50^{\circ} \mathrm{C}$ flash and higher flow 


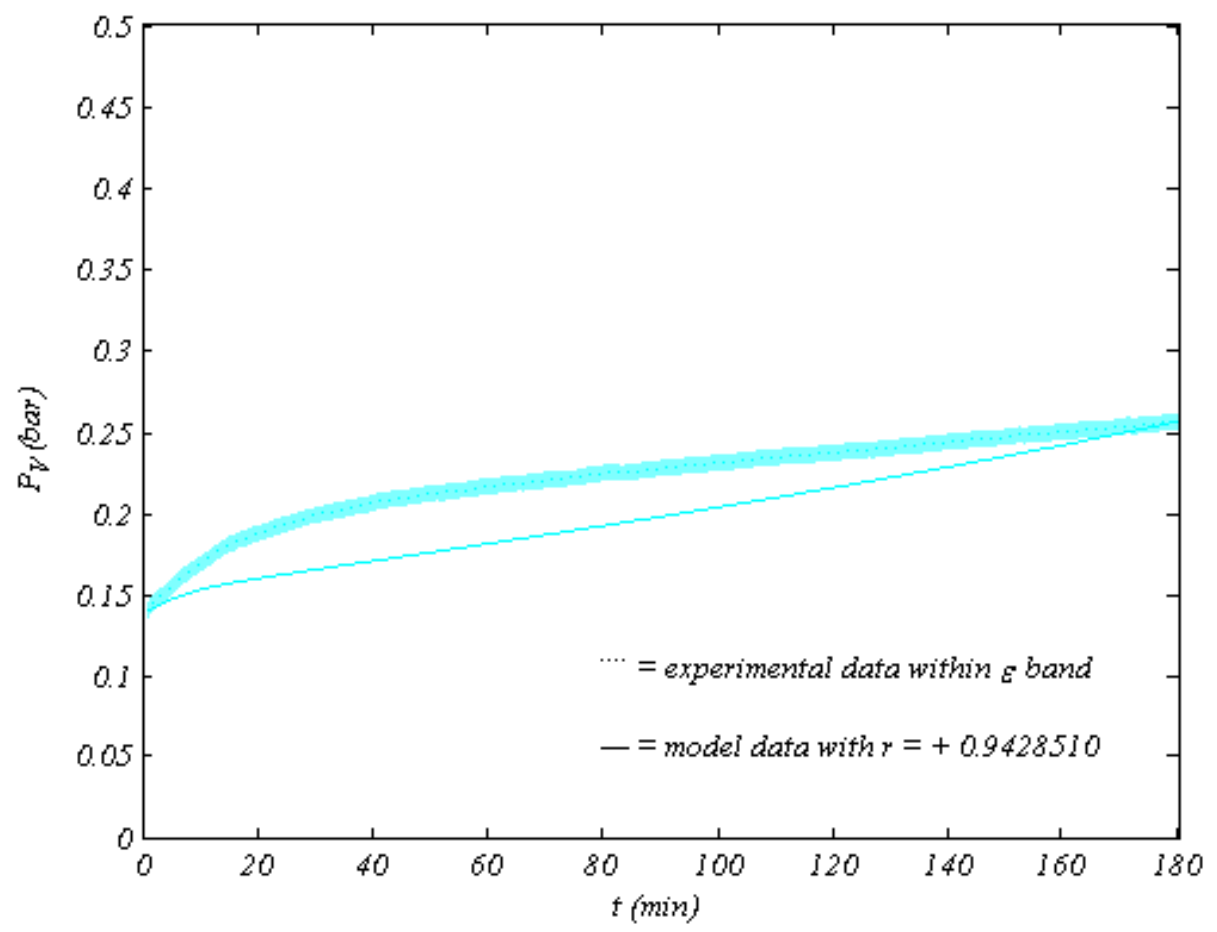

Figure 48 . Vacuum pressure at $60^{\circ} \mathrm{C}$ flash and lower flow

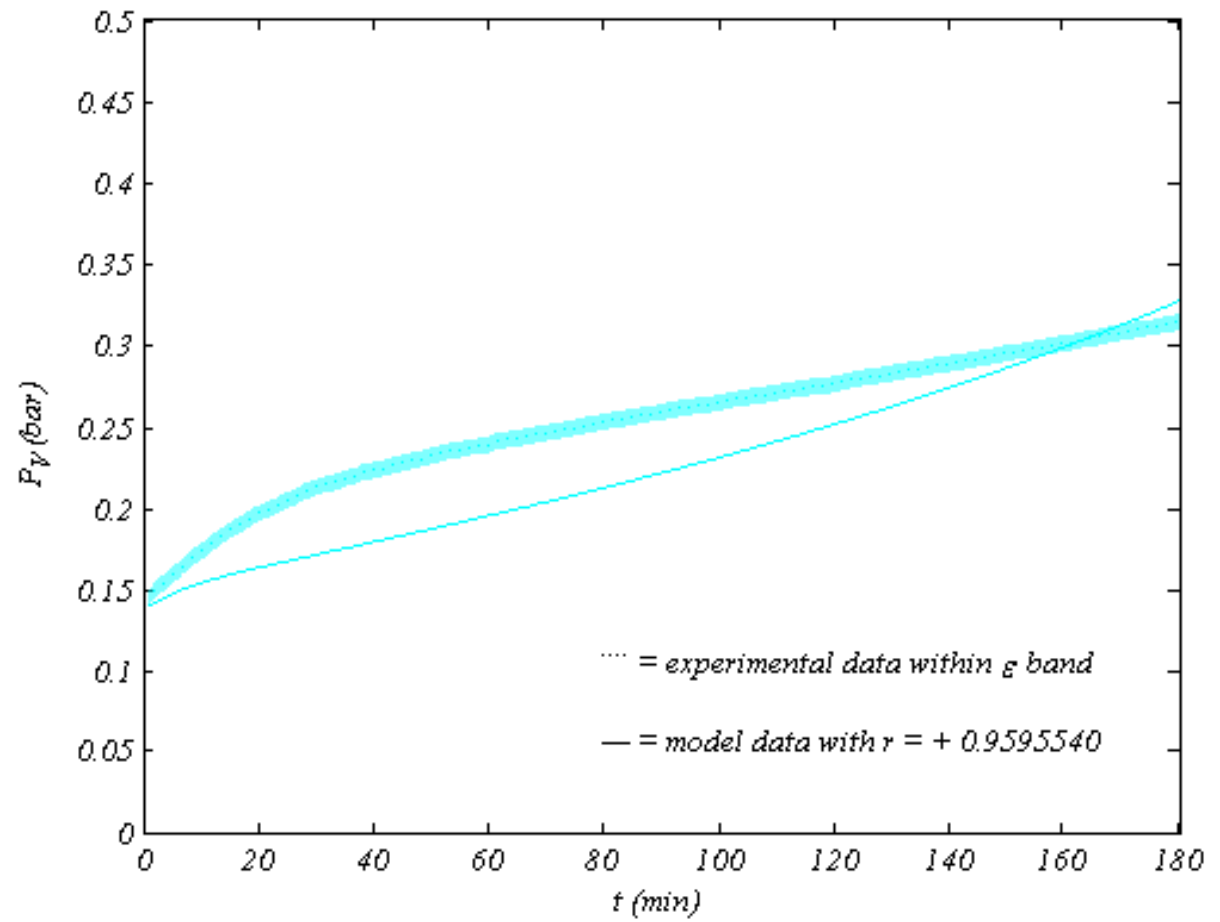

Figure 49. Vacuum pressure at $60^{\circ} \mathrm{C}$ flash and higher flow 


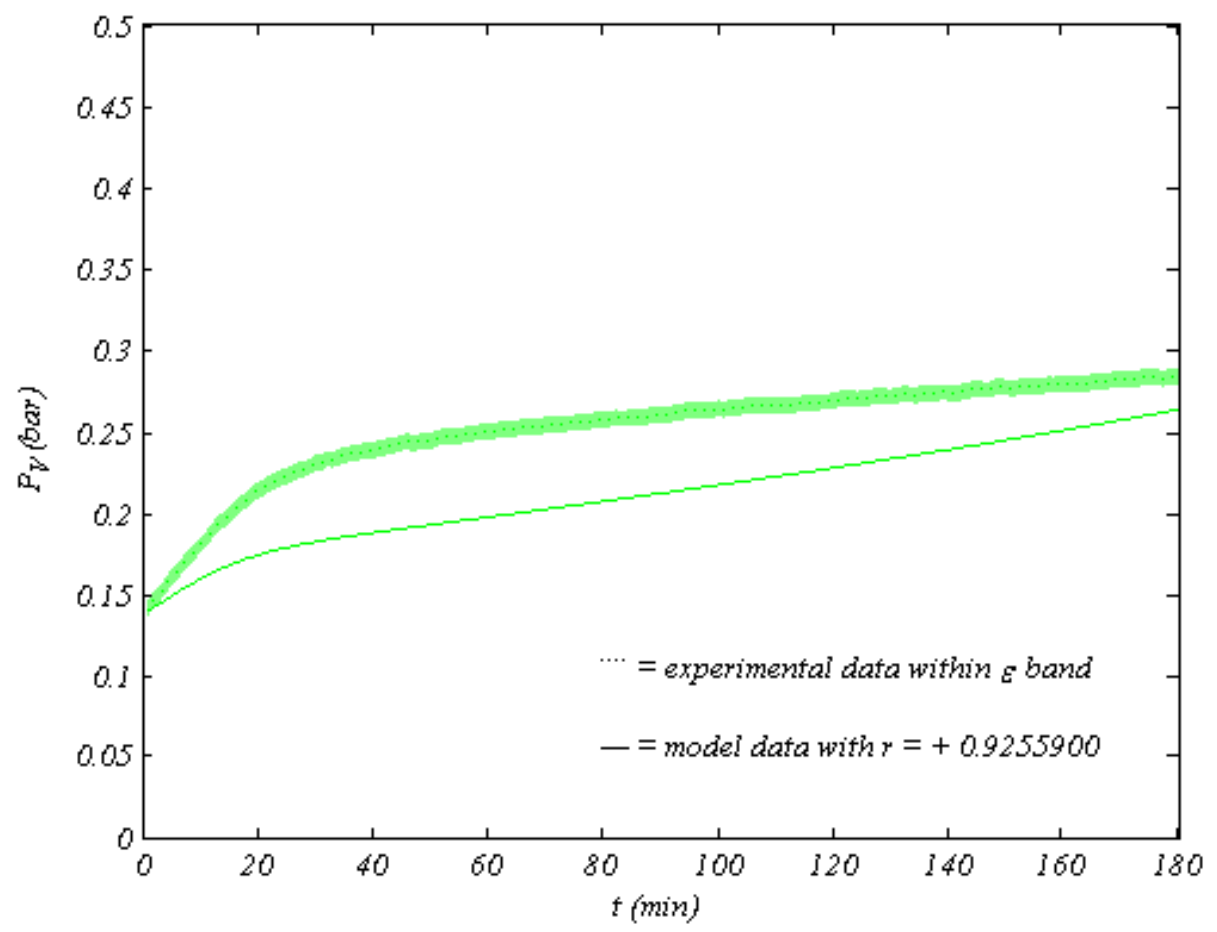

Figure 50 . Vacuum pressure at $70^{\circ} \mathrm{C}$ flash and lower flow

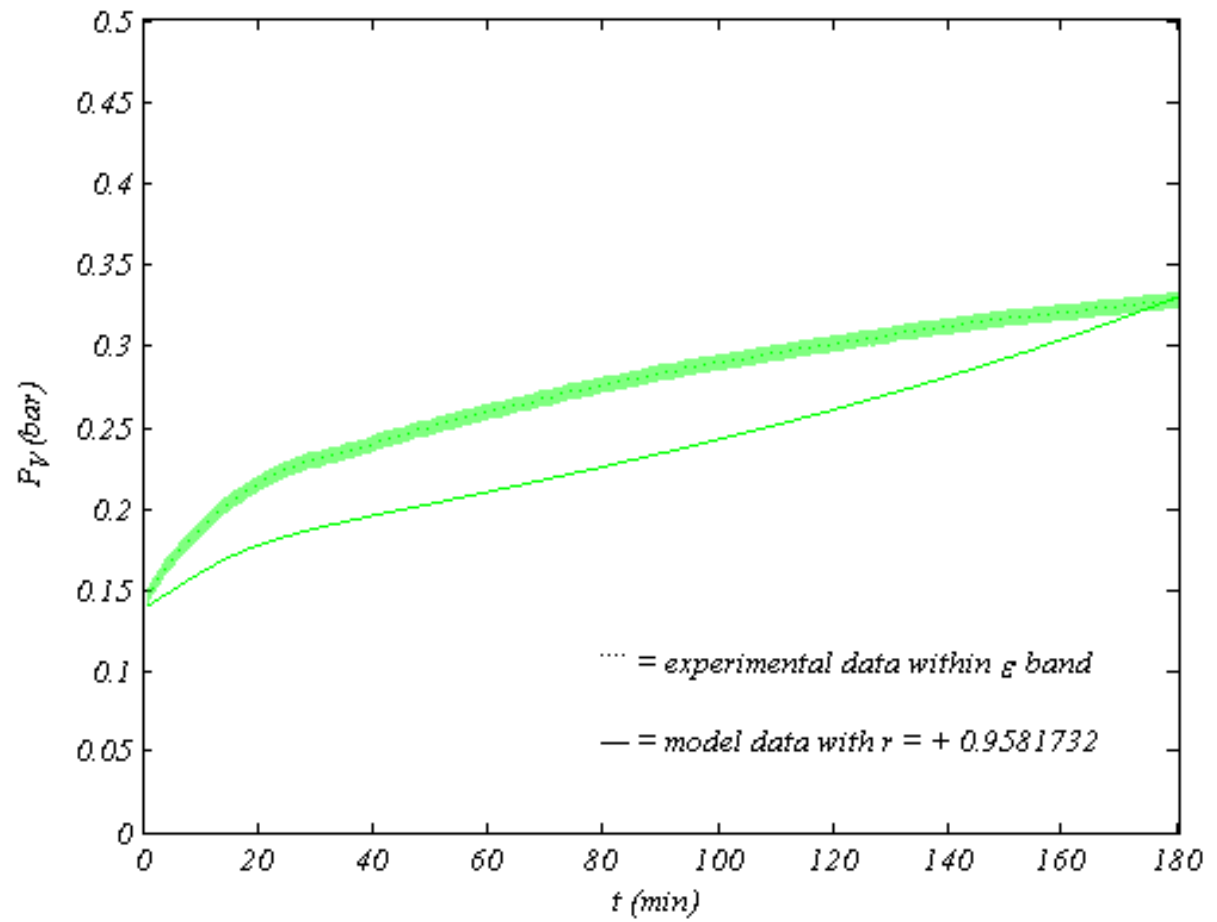

Figure 51 . Vacuum pressure at $70^{\circ} \mathrm{C}$ flash and higher flow 


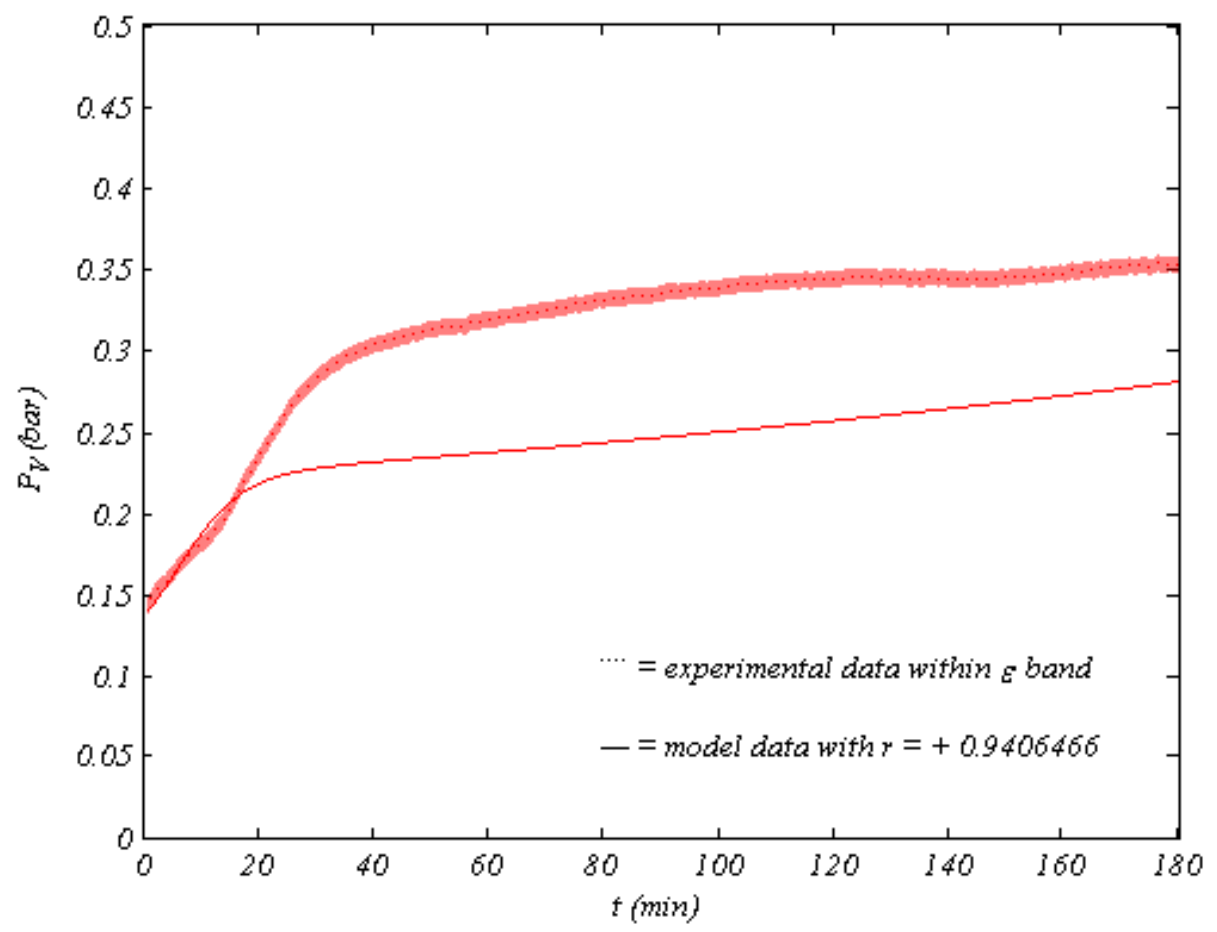

Figure 52. Vacuum pressure at $80^{\circ} \mathrm{C}$ flash and lower flow

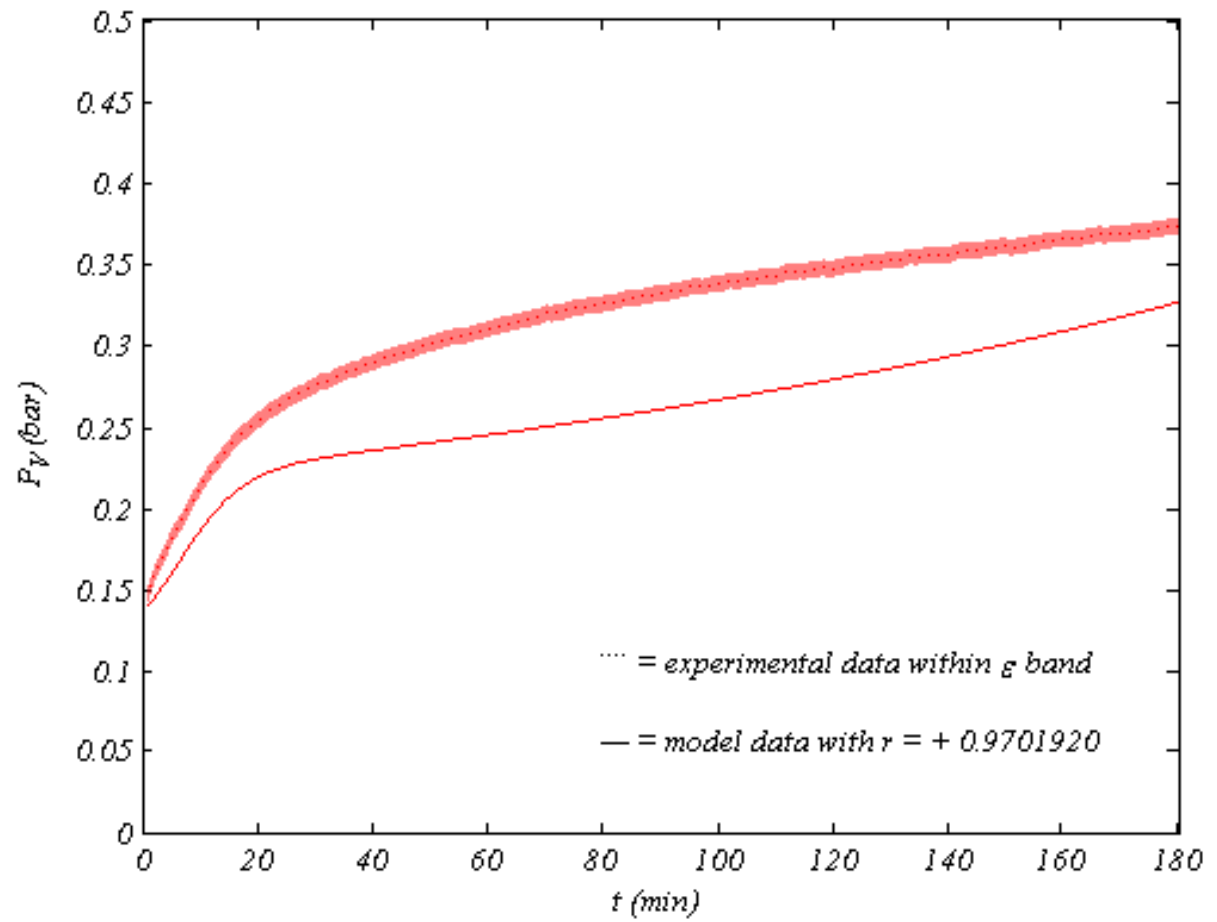

Figure 53. Vacuum pressure at $80^{\circ} \mathrm{C}$ flash and higher flow 


\subsection{Equilibrium Attainment}

The temperature of seawater drops from a set flash temperature to an equilibrium temperature corresponding to the system vacuum pressure as it enters the evaporator. Equilibrium temperature quickly increased, then mostly leveled for both seawater flow rates; moreover, it was higher to some extent at higher seawater flow rates. In addition, equilibrium temperatures increased with elevated flash temperatures for both seawater flow rates in compliance with the energy balance around the evaporator.

The equilibrium temperature was close to ambient at first; however, it rapidly increased as hot seawater was introduced to the evaporator, reaching a plateau comparable to the flash temperature for both seawater flow rates. Flash operation of the proposed desalination process is an adiabatic expansion, where temperature of seawater drops upon entering the flash chamber due to the drawn enthalpy of vaporization, forming fresh water vapor at an equilibrium status corresponding to the vacuum pressure.

The model utilizes the Iterative and Incremental Development scheme as mentioned in CHAPTER 4, where the computed vacuum pressure of a previous time increment becomes the input vacuum pressure to the executing time increment, solving for the equilibrium temperature and vacuum pressure of the next time increment. The entire model execution progression is initiated by the known initial vacuum pressure.

The model prediction of equilibrium temperature resembled the experimental results but was slightly lower, and the discrepancy increased with temperature. This observation understandably matches that of vacuum pressure seen earlier, since the attained equilibrium temperature depends on the system vacuum pressure. Equilibrium temperature profiles are shown in Figure 54 through Figure 65. 


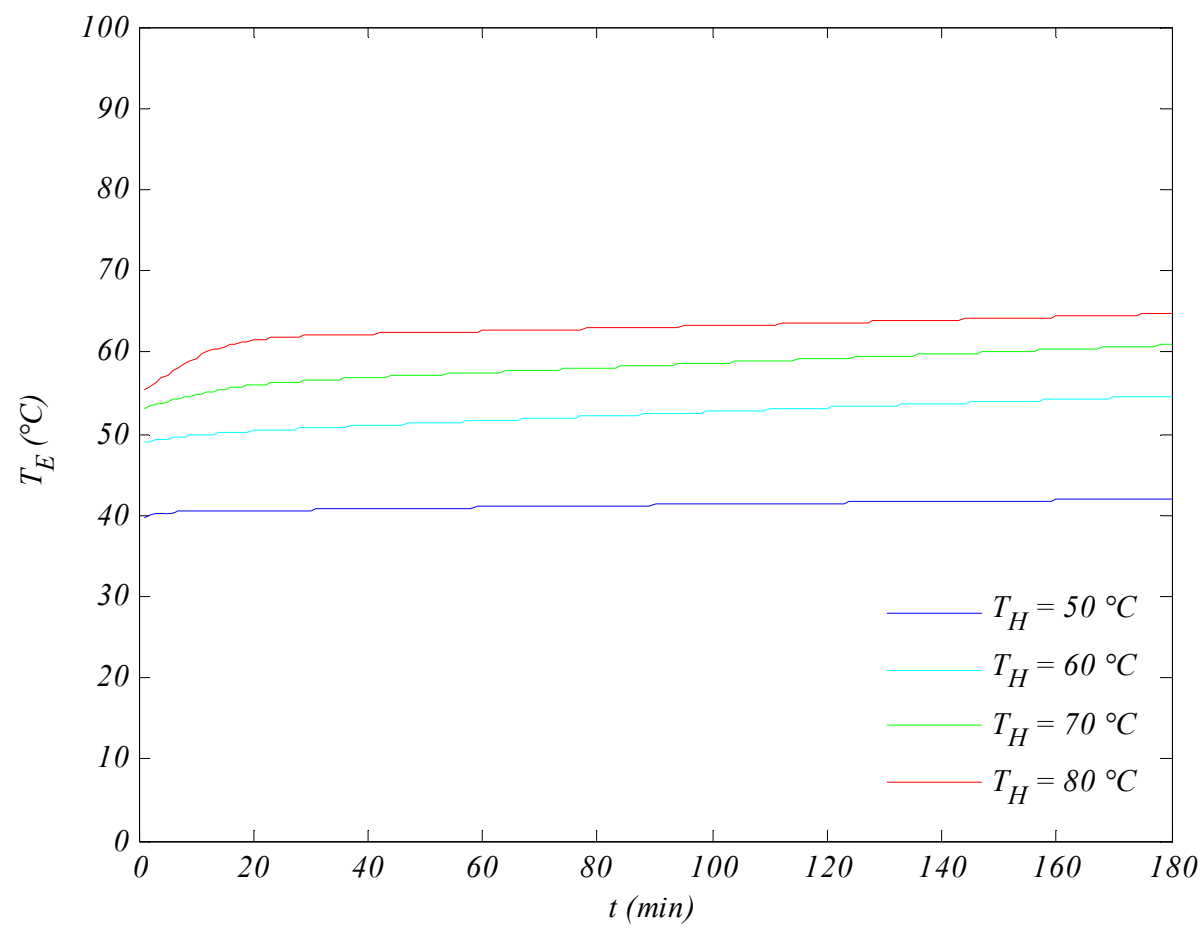

Figure 54. Modeled equilibrium temperature profiles at lower flow

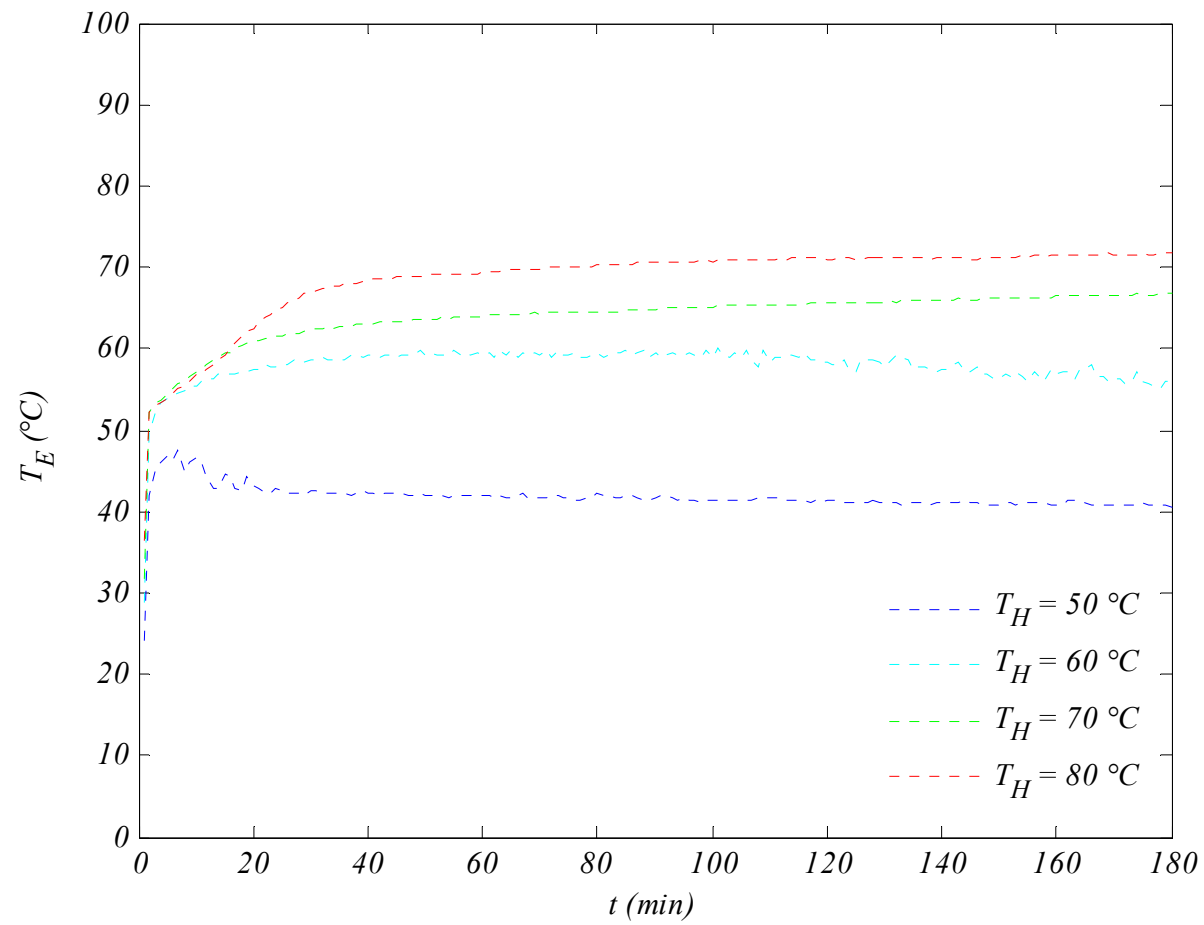

Figure 55. Experimental equilibrium temperature profiles at lower flow 


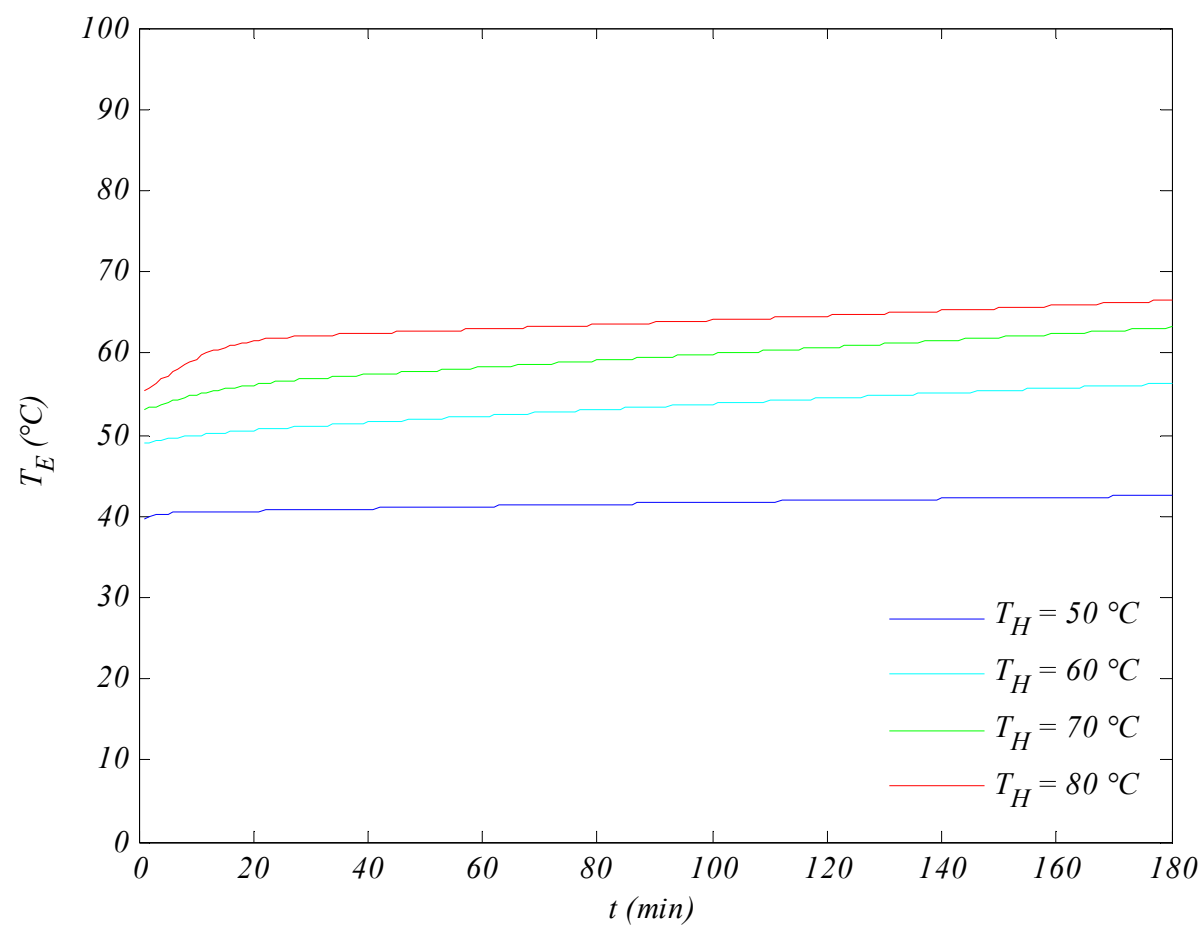

Figure 56. Modeled equilibrium temperature profiles at higher flow

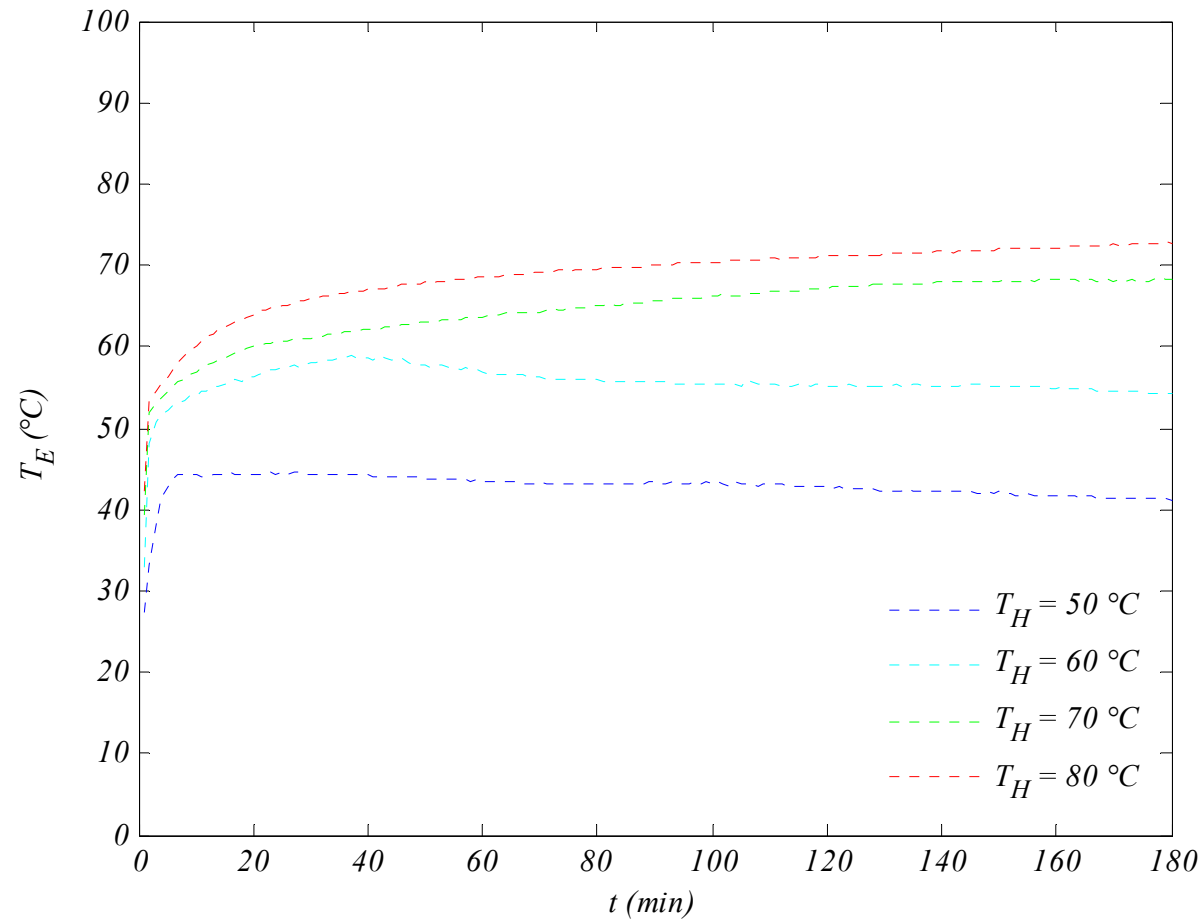

Figure 57. Experimental equilibrium temperature profiles at higher flow 


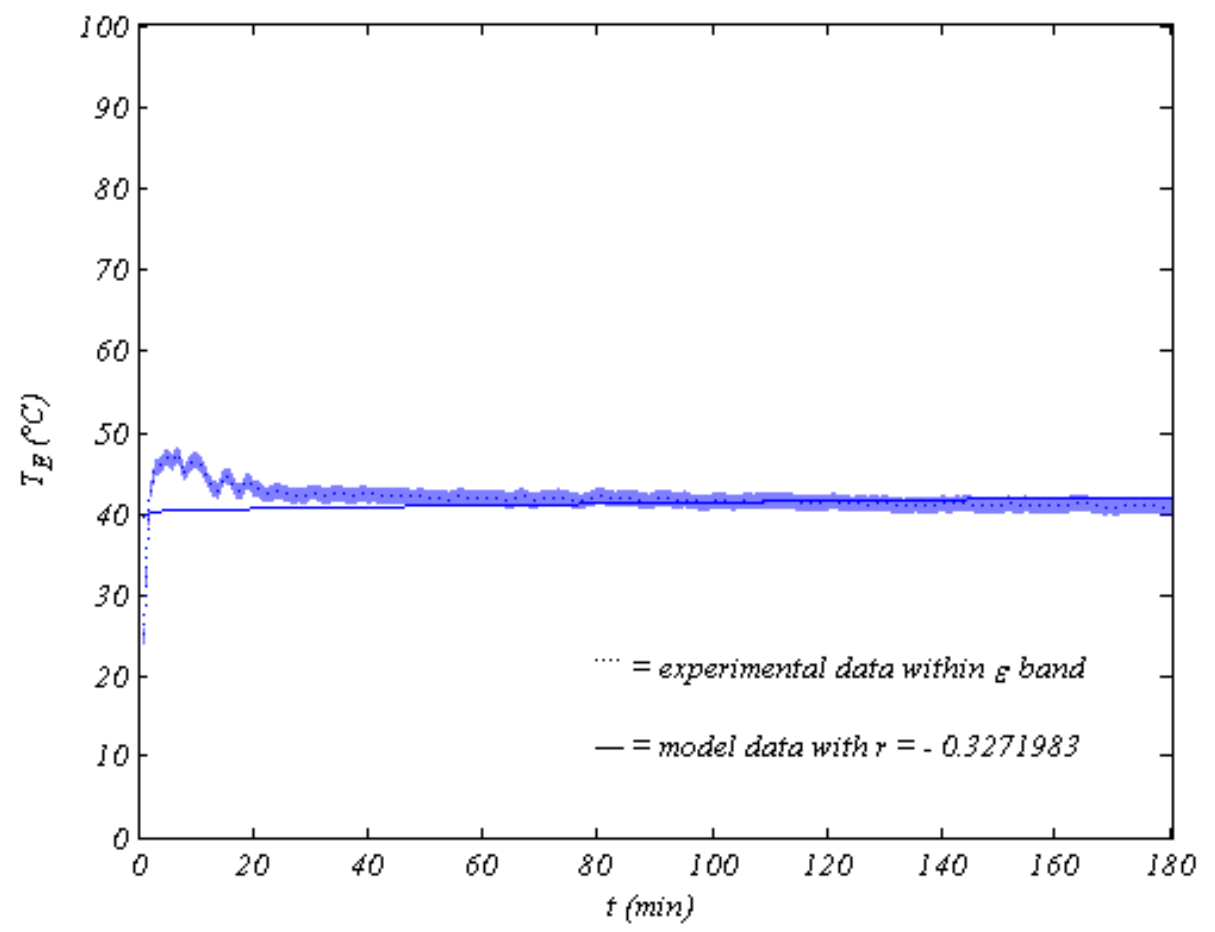

Figure 58. Equilibrium temperature at $50^{\circ} \mathrm{C}$ flash and lower flow

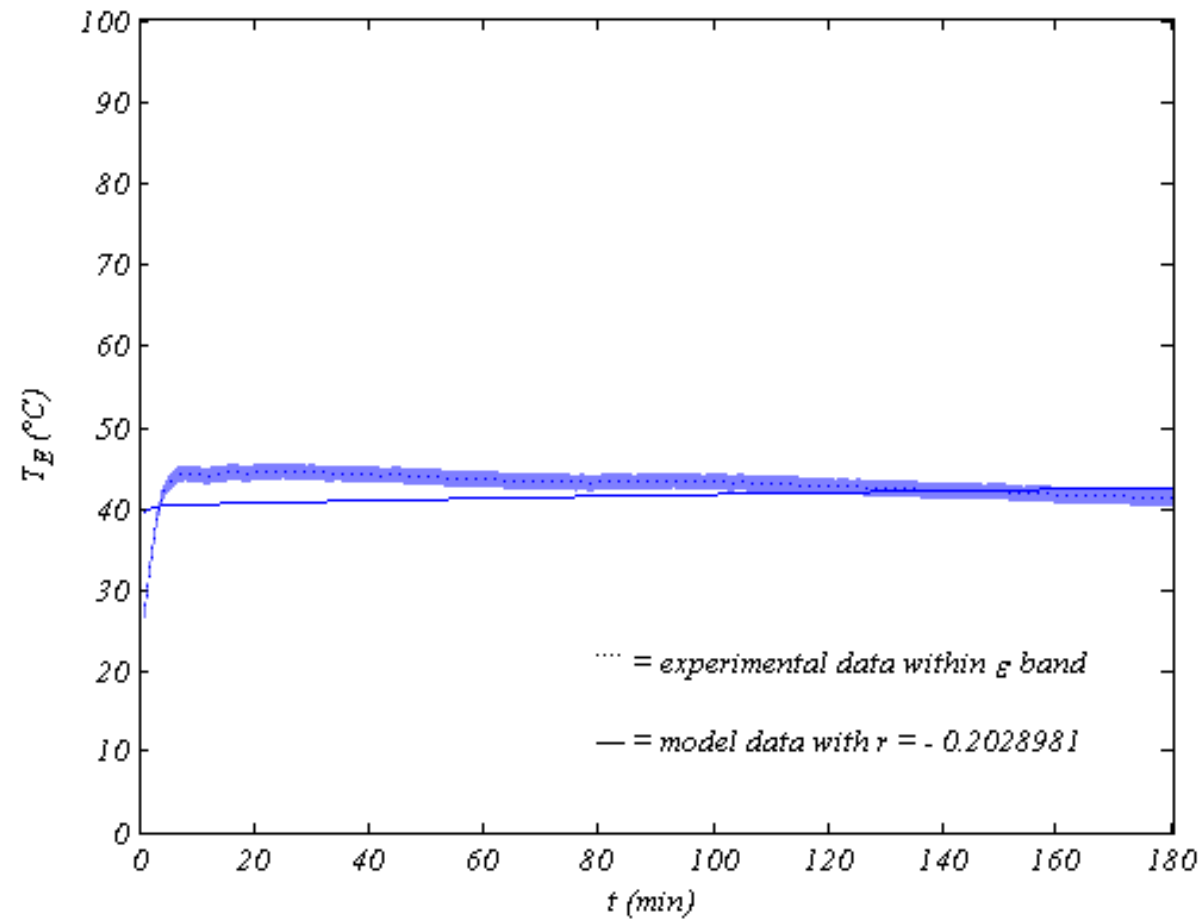

Figure 59. Equilibrium temperature at $50^{\circ} \mathrm{C}$ flash and higher flow 


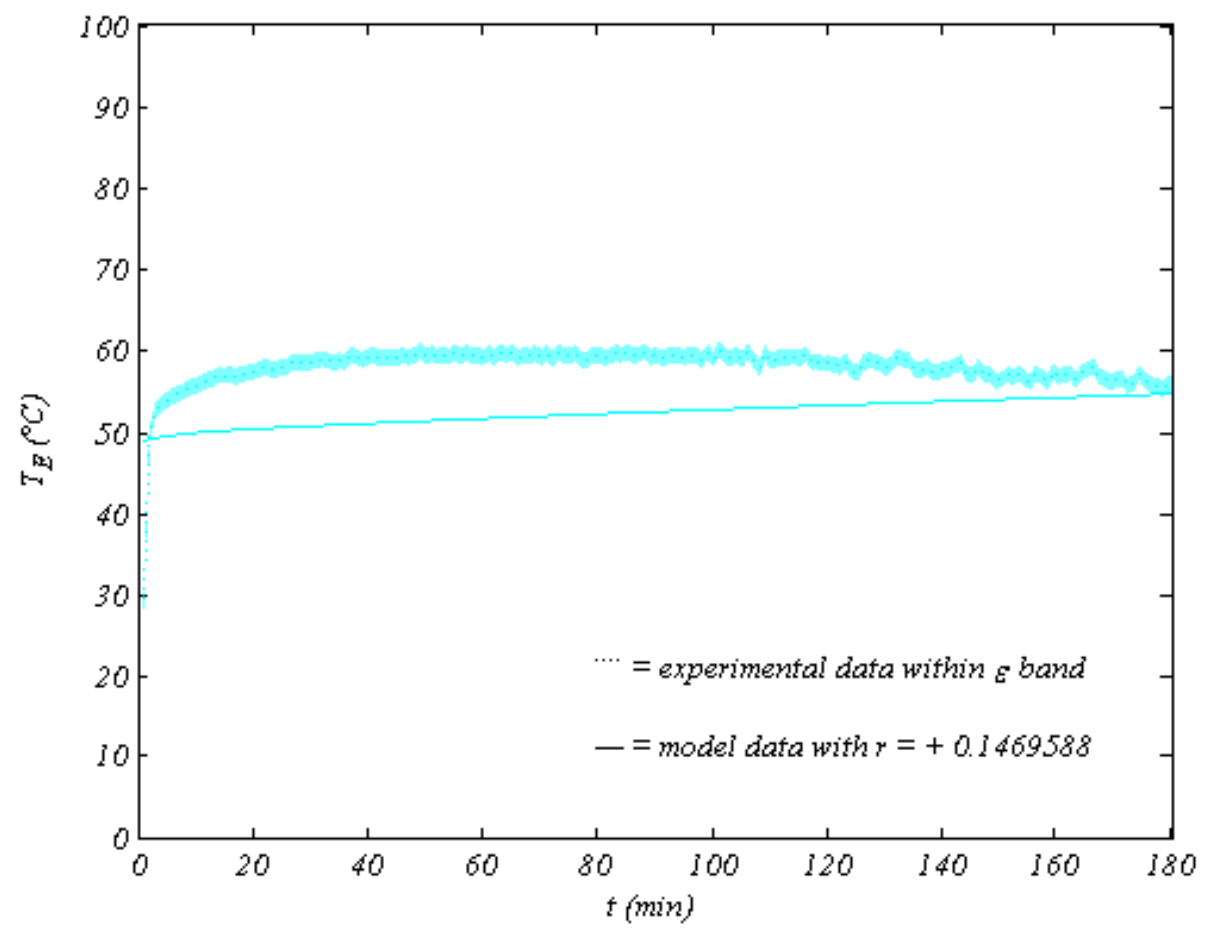

Figure 60. Equilibrium temperature at $60^{\circ} \mathrm{C}$ flash and lower flow

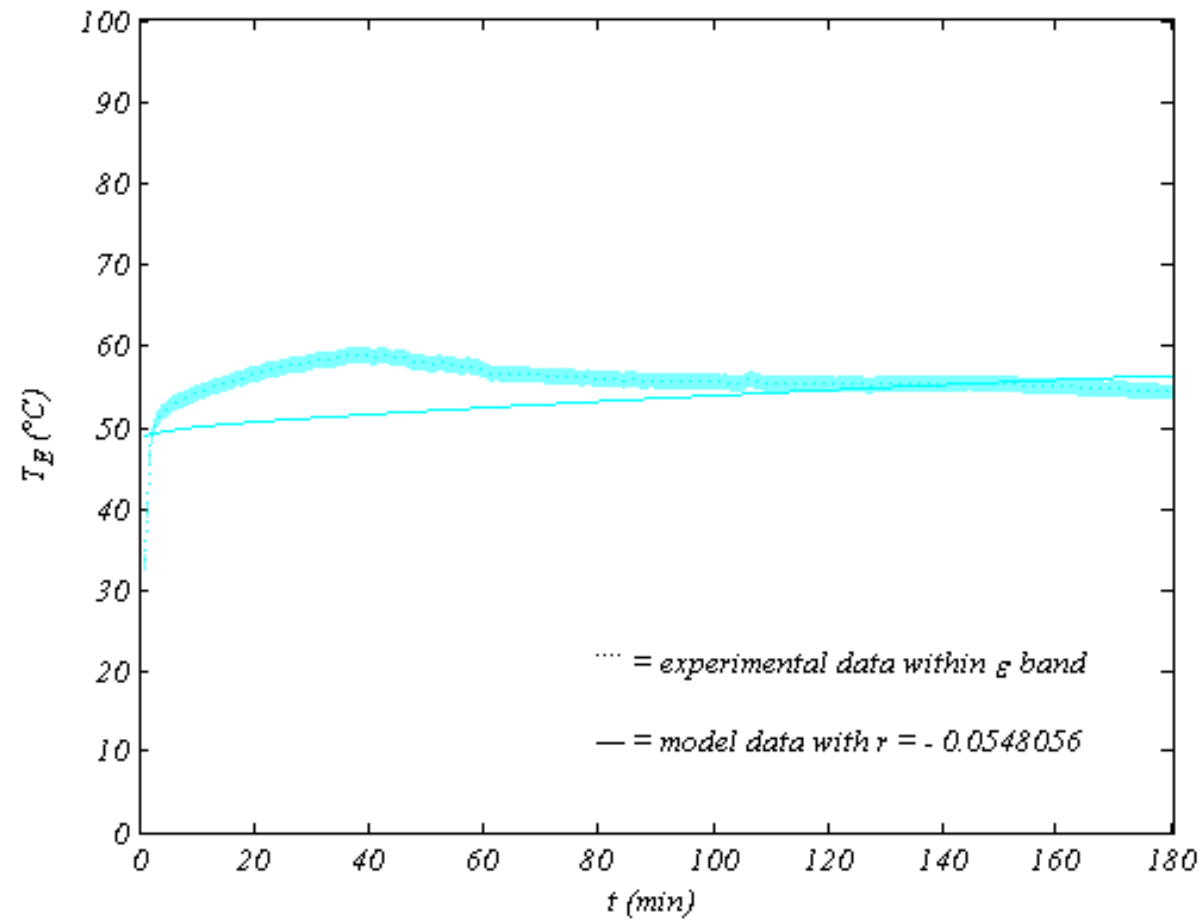

Figure 61 . Equilibrium temperature at $60^{\circ} \mathrm{C}$ flash and higher flow 


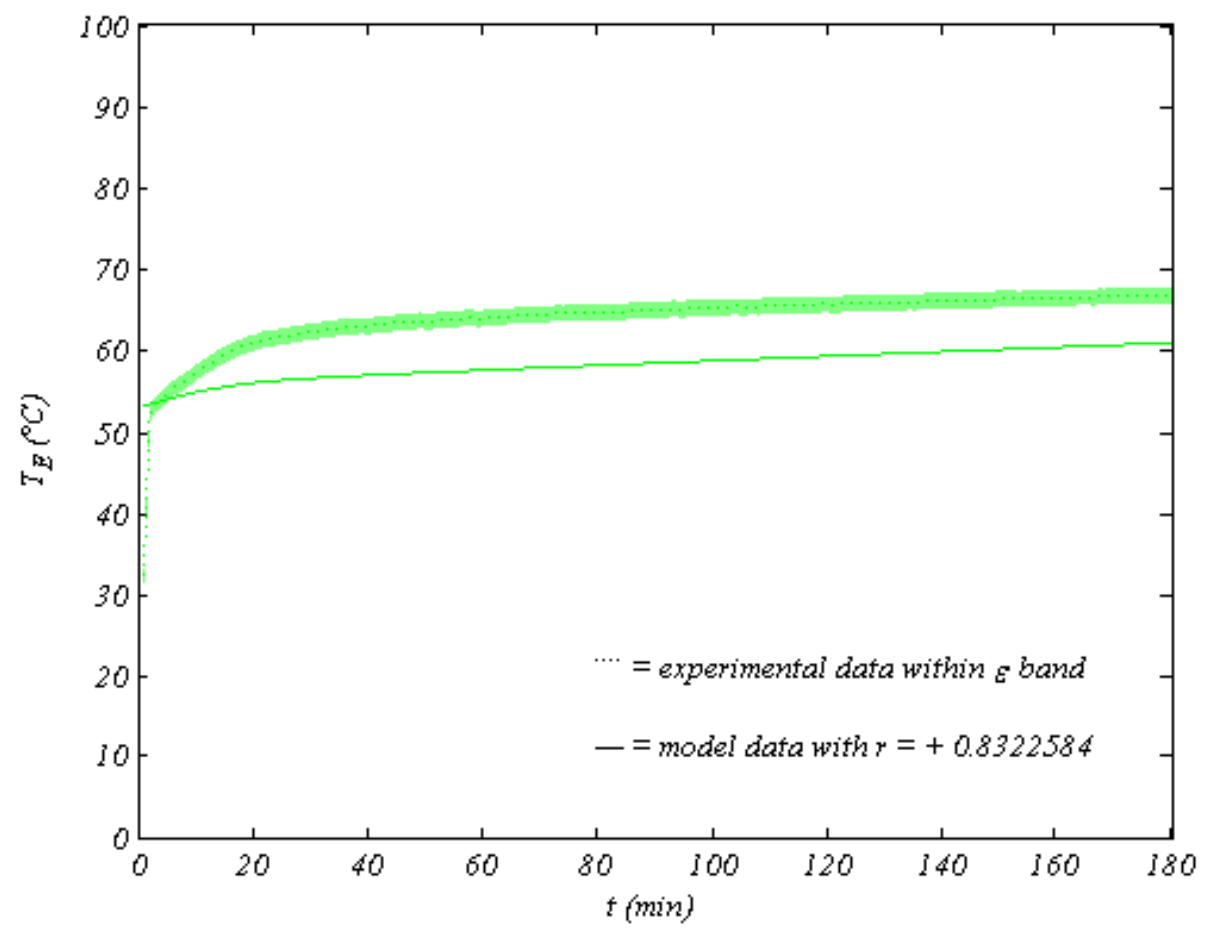

Figure 62. Equilibrium temperature at $70^{\circ} \mathrm{C}$ flash and lower flow

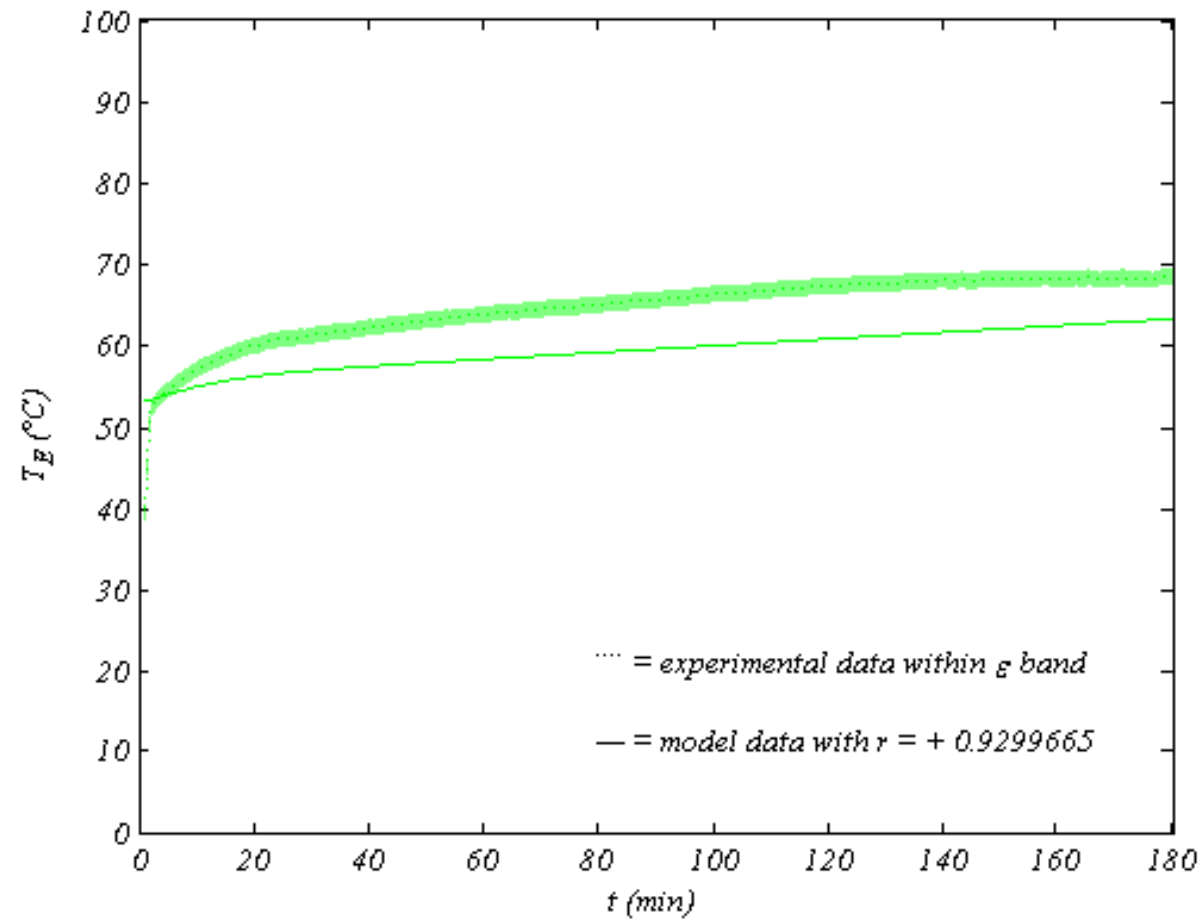

Figure 63. Equilibrium temperature at $70^{\circ} \mathrm{C}$ flash and higher flow 


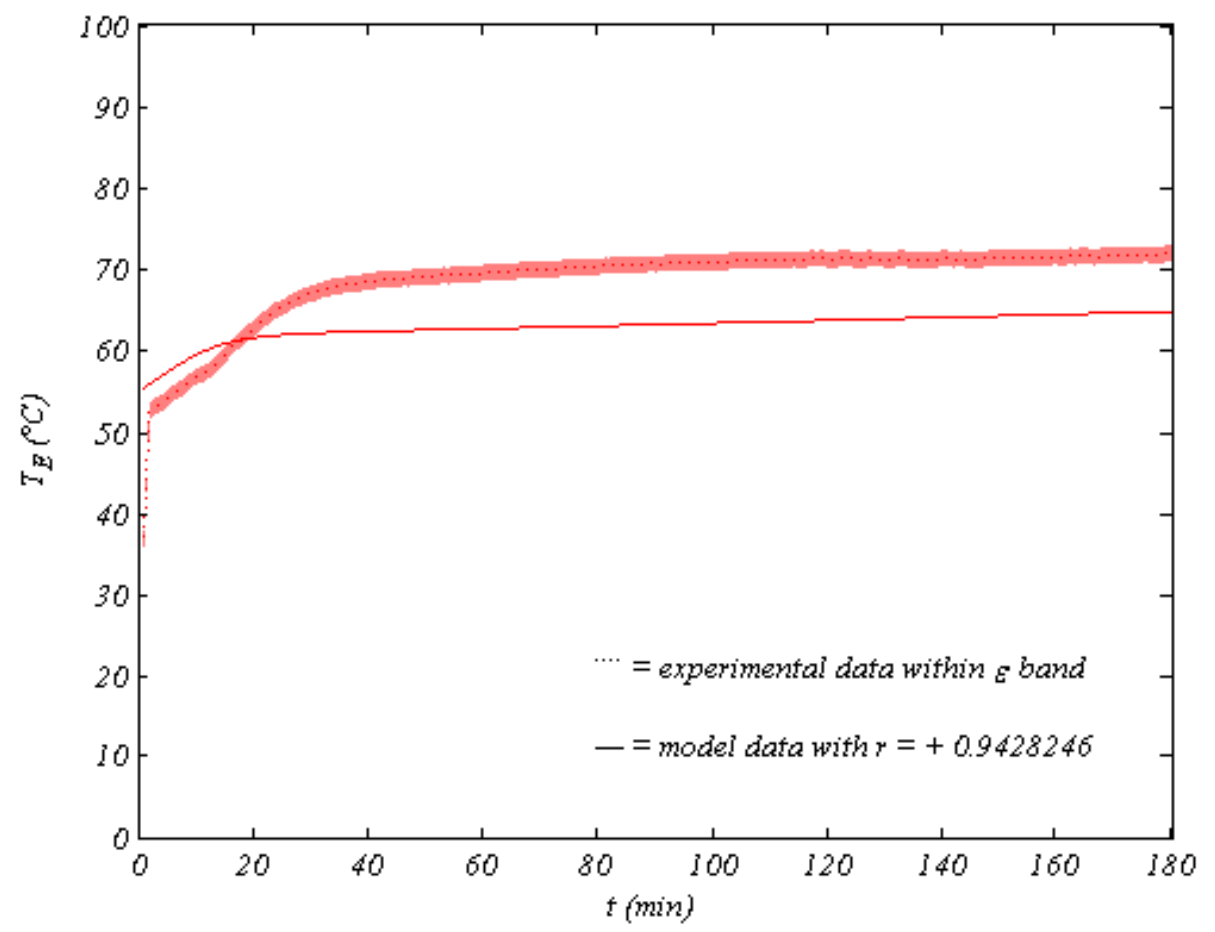

Figure 64. Equilibrium temperature at $80^{\circ} \mathrm{C}$ flash and lower flow

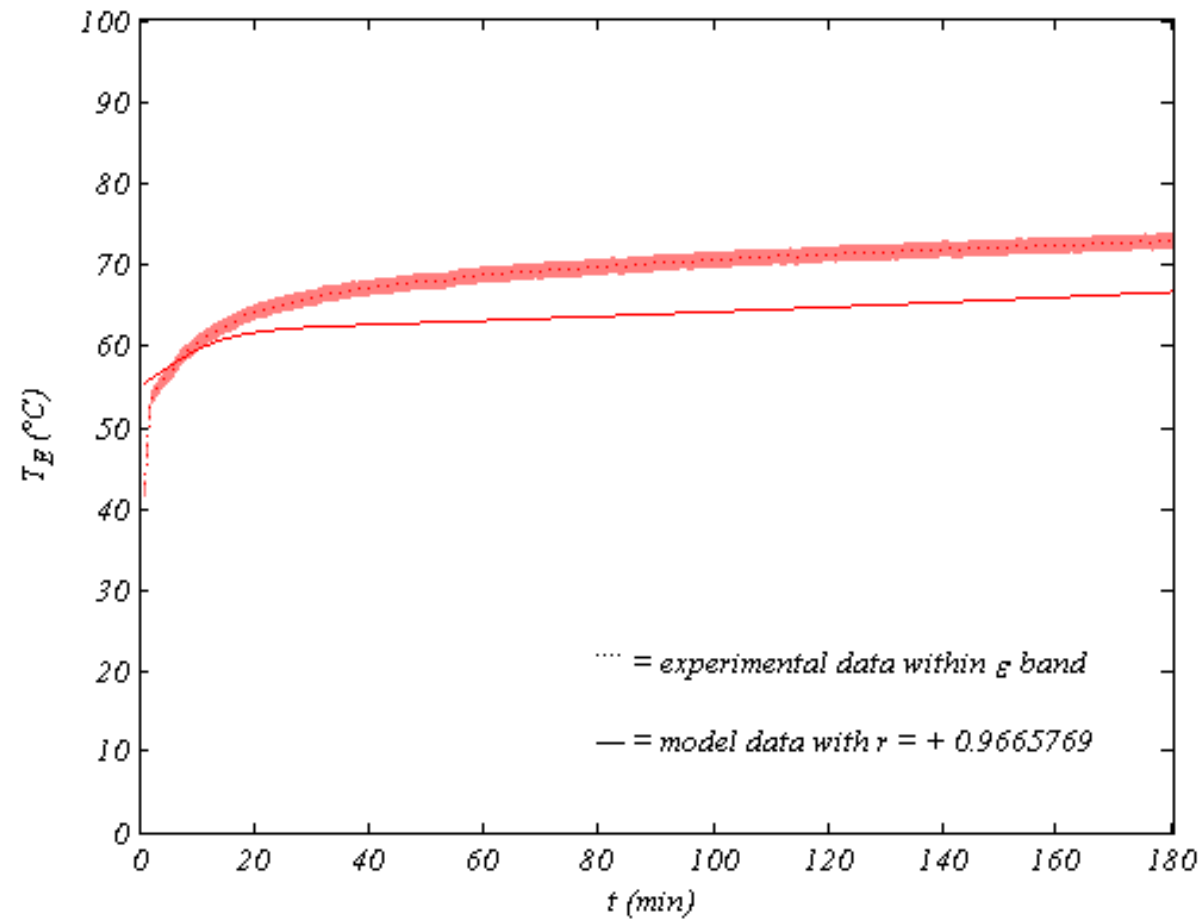

Figure 65. Equilibrium temperature at $80^{\circ} \mathrm{C}$ flash and higher flow 


\subsection{Equilibrium Departure}

Concentrated brine temperature is usually a bit lower than equilibrium temperature due to boiling point elevation and non-equilibrium allowance plus a small amount of heat loss from the insulated evaporator as mentioned in CHAPTER 4. Concentrated brine temperature quickly increased then leveled for both seawater flow rates; moreover, it was slightly higher at lower seawater flow rates. In addition, concentrated brine temperatures increased with elevated flash temperatures for both seawater flow rates in compliance with the energy balance around the evaporator.

The concentrated brine temperature was close to ambient at first; however, it rapidly increased as hot seawater was introduced to the evaporator, reaching a plateau parallel to equilibrium temperature for both seawater flow rates. The concentrated brine temperature remained lower than the equilibrium temperature except for the lowest flash temperature for both seawater flow rates, where concentrated brine temperature started lower but ended higher than equilibrium temperature. This can be attributed to some heat loss from the flashed water vapor along with diminishing vaporization rates as vacuum pressure rises at a relative low equilibrium temperature since the flashed vapor obtains its heat of vaporization from the concentrated brine.

The model prediction of concentrated brine temperature resembled the experimental results but was slightly higher, with the discrepancy rising with increasing flash temperatures. This can be attributed to the neglected small amount of heat loss from the insulated hot evaporator to the cool ambiance combined with the imprecision of the non-equilibrium allowance correlation used in the model. Concentrated brine temperature profiles are shown in Figure 66 through Figure 77. 


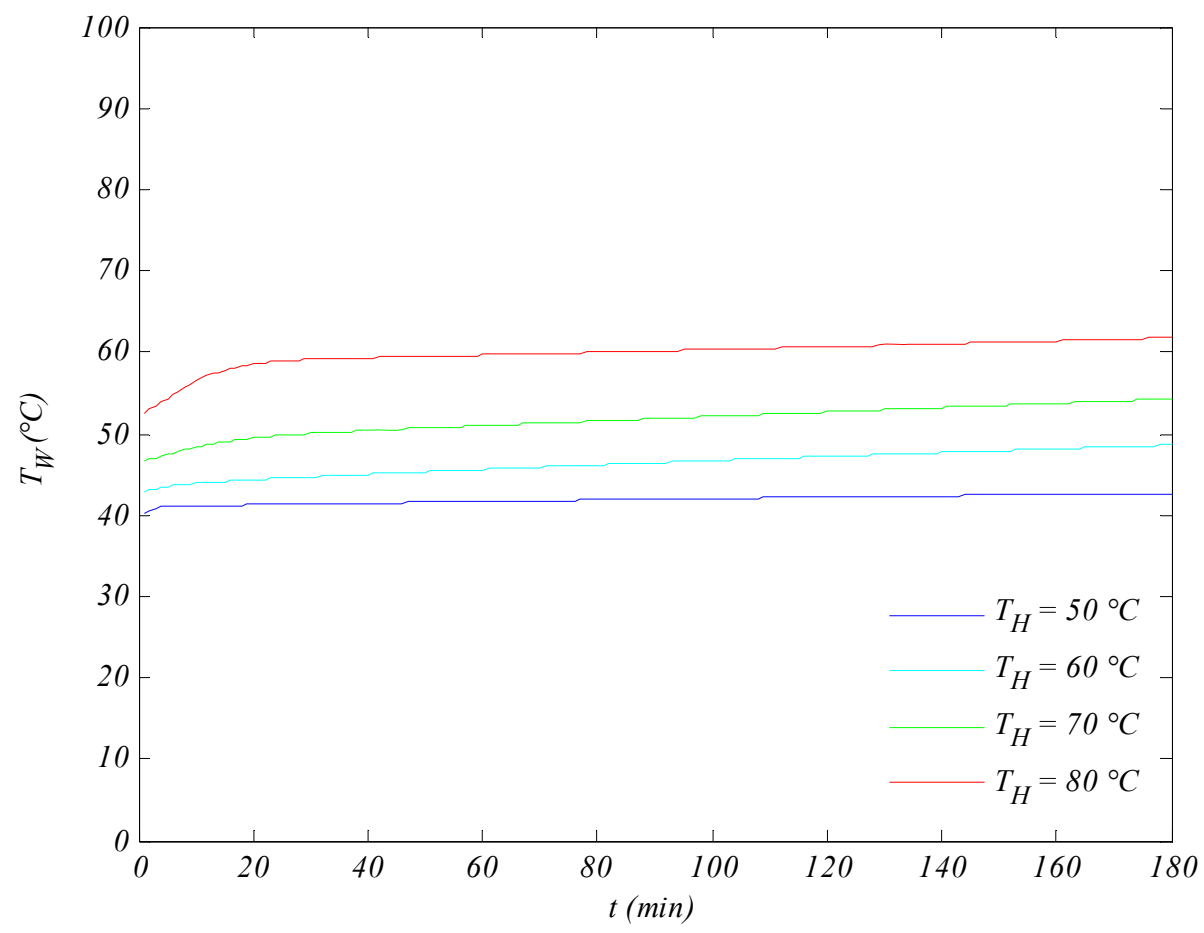

Figure 66. Modeled concentrated brine temperature profiles at lower flow

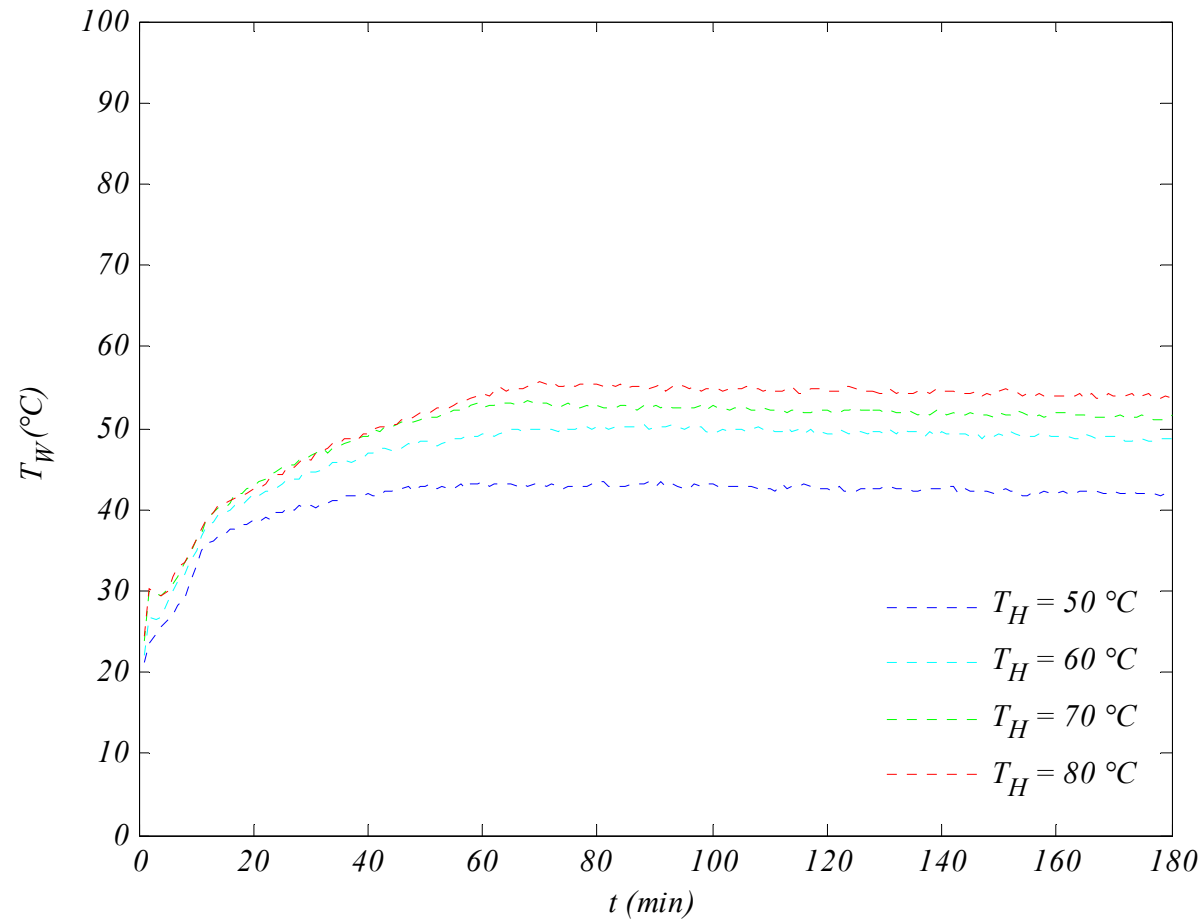

Figure 67. Experimental concentrated brine temperature profiles at lower flow 


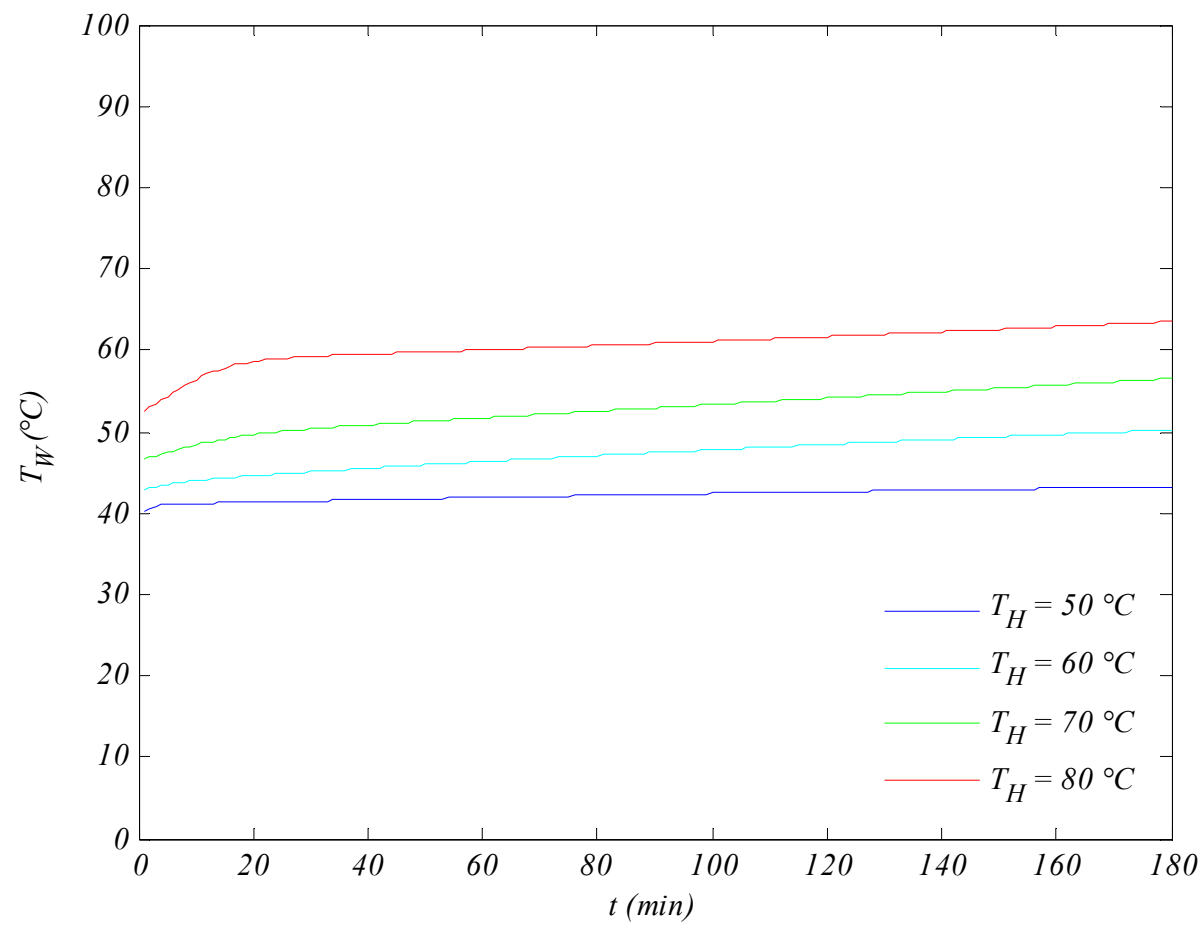

Figure 68. Modeled concentrated brine temperature profiles at higher flow

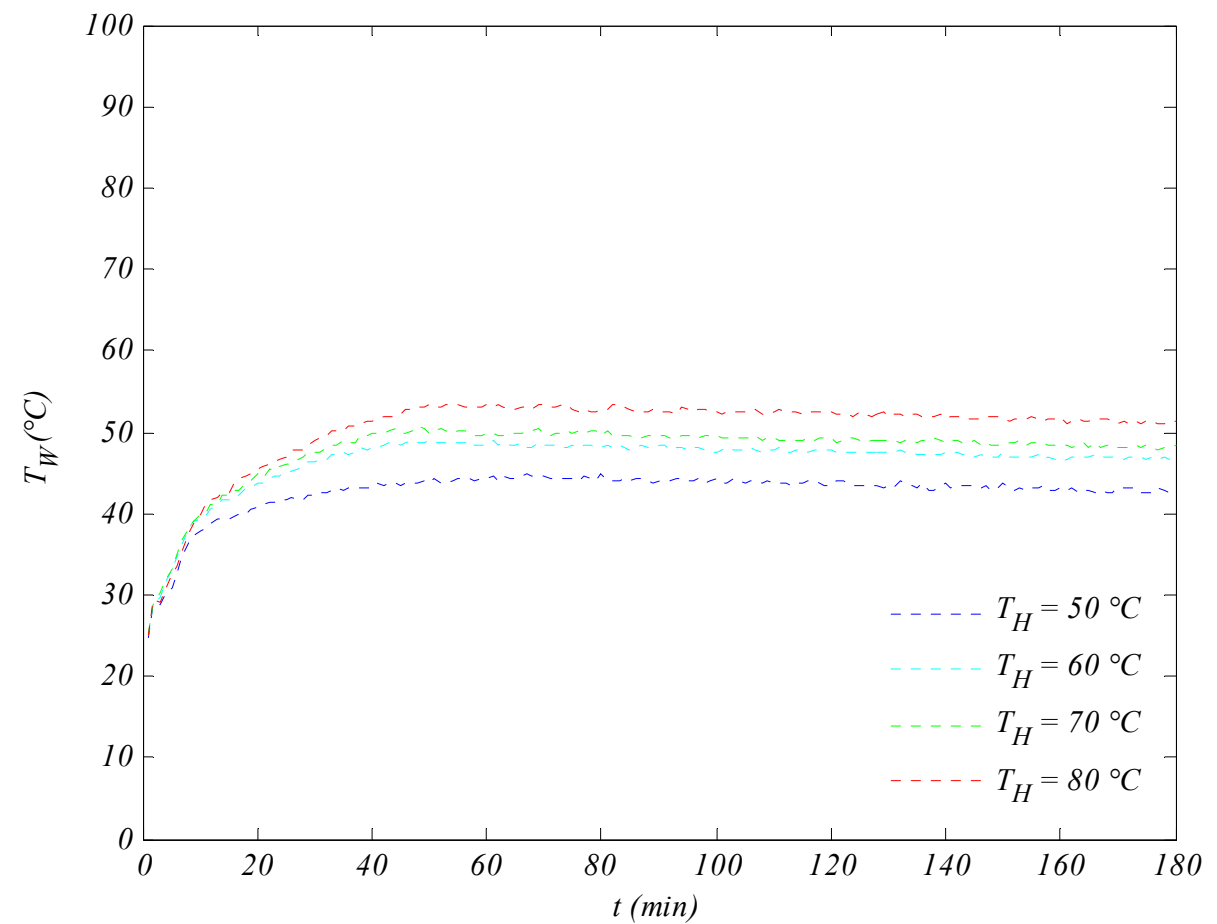

Figure 69. Experimental concentrated brine temperature profiles at higher flow 


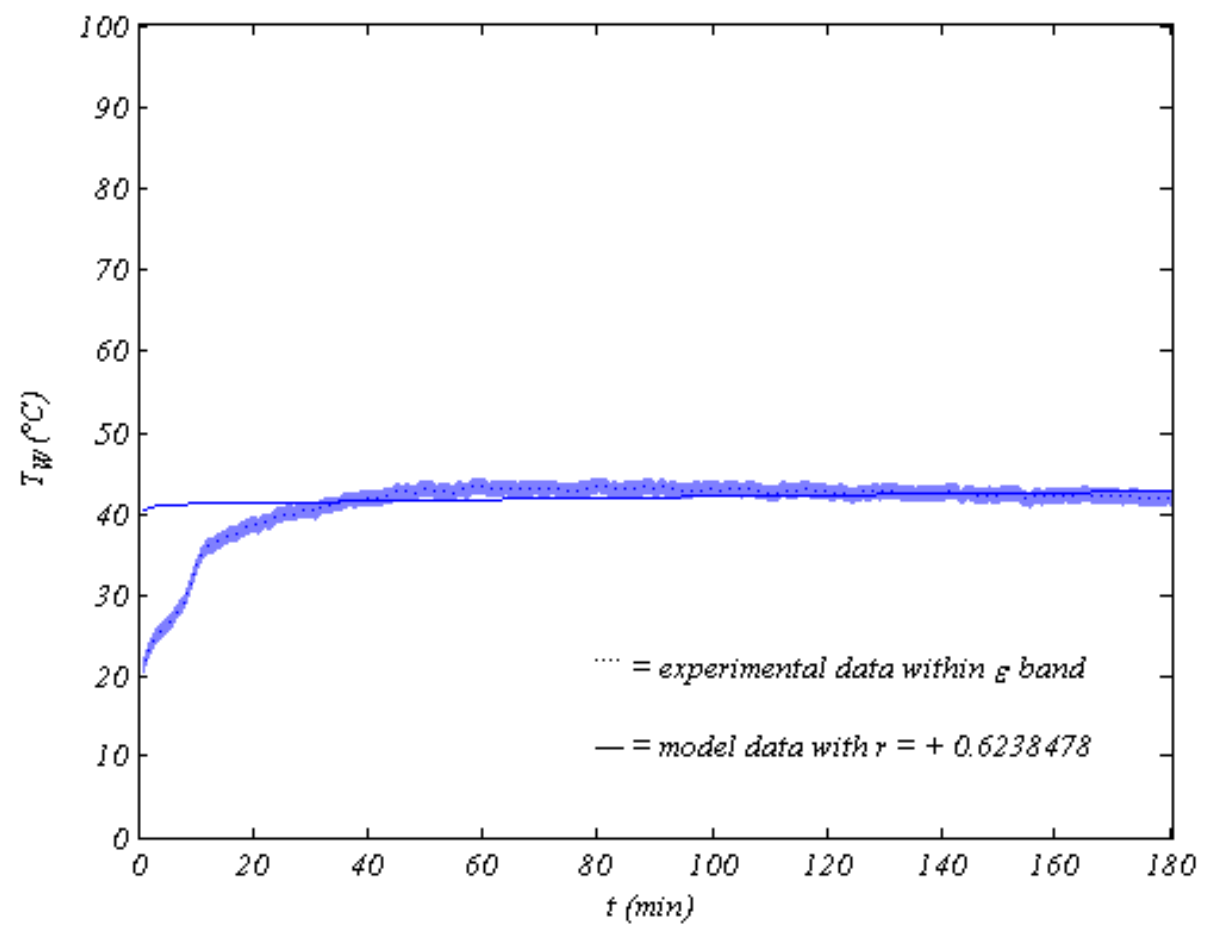

Figure 70. Concentrated brine temperature at $50^{\circ} \mathrm{C}$ flash and lower flow

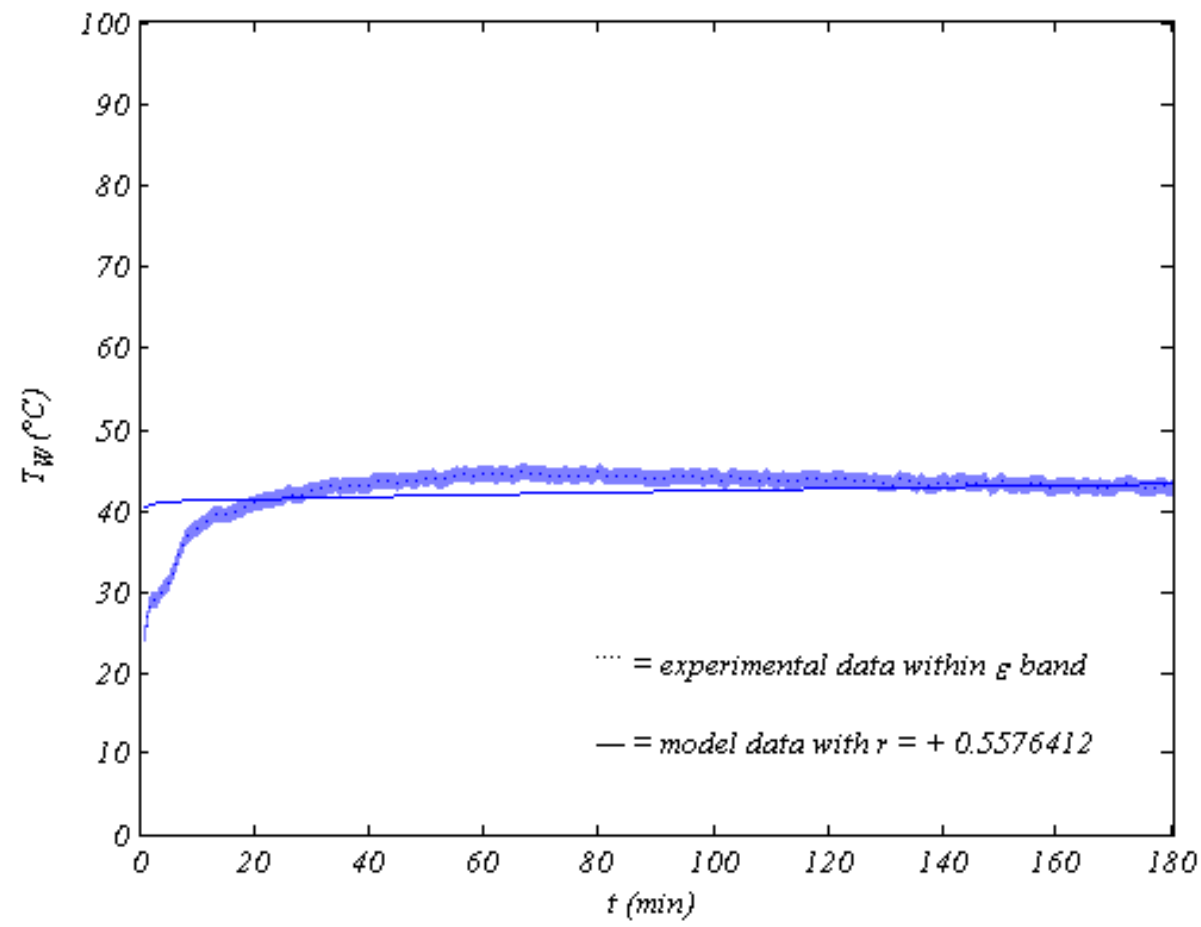

Figure 71. Concentrated brine temperature at $50^{\circ} \mathrm{C}$ flash and higher flow 


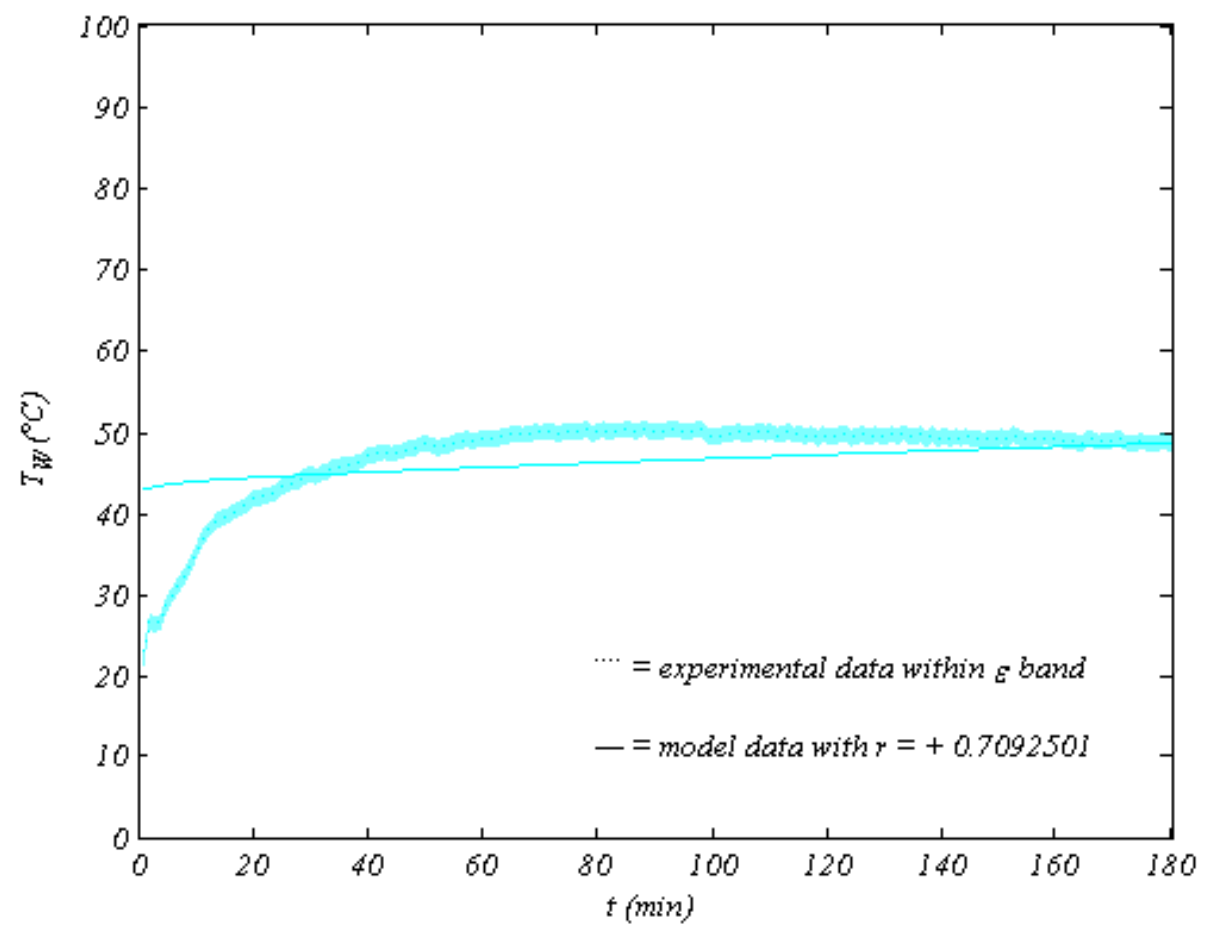

Figure 72. Concentrated brine temperature at $60^{\circ} \mathrm{C}$ flash and lower flow

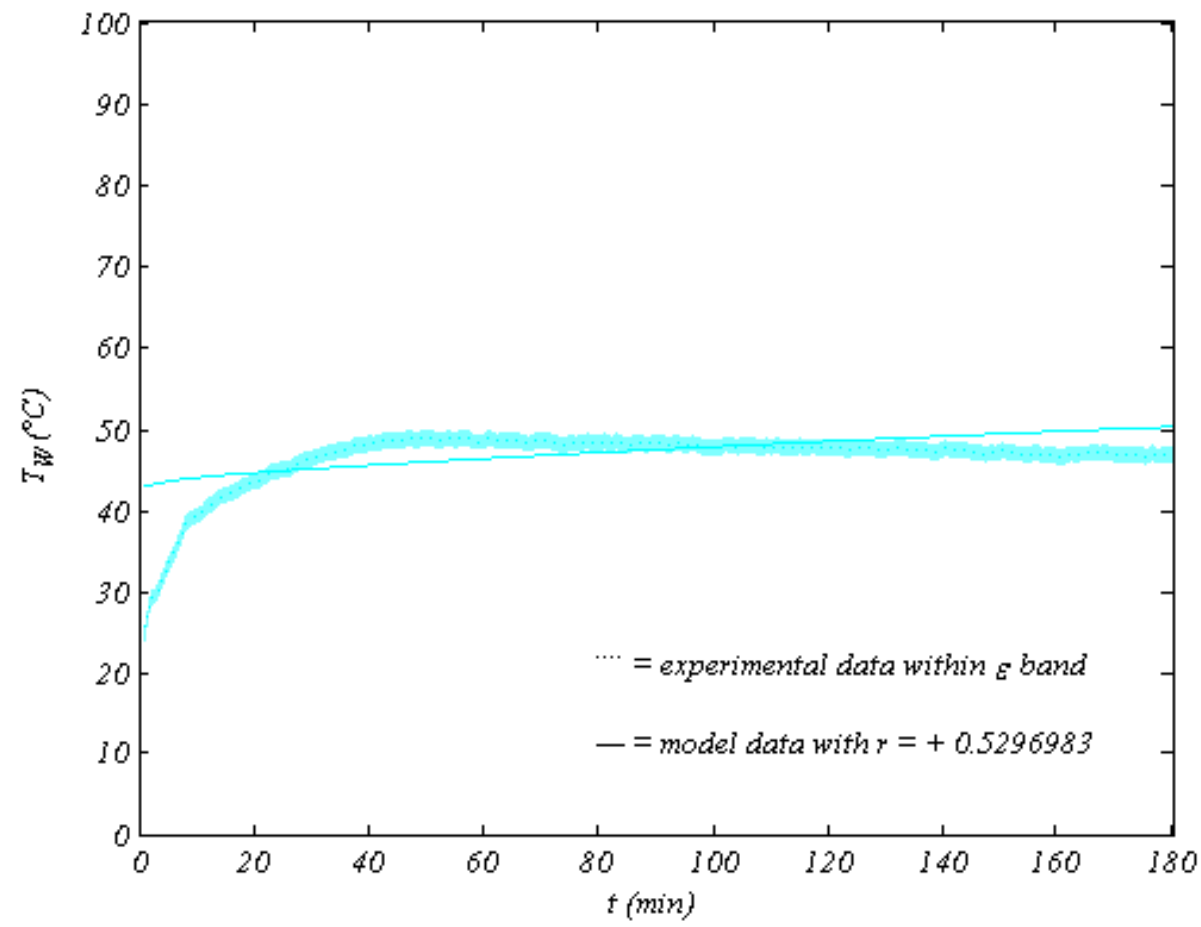

Figure 73. Concentrated brine temperature at $60^{\circ} \mathrm{C}$ flash and higher flow 


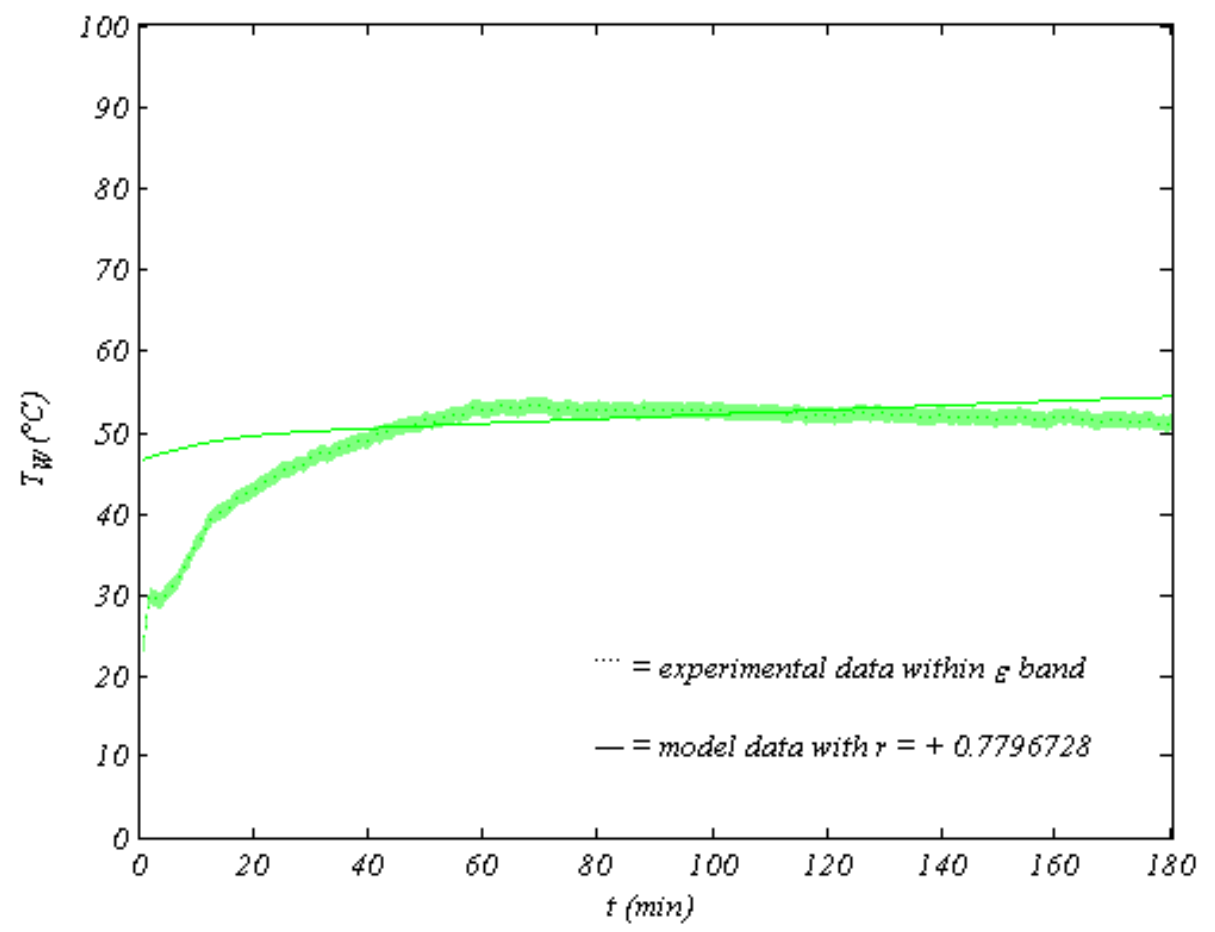

Figure 74. Concentrated brine temperature at $70^{\circ} \mathrm{C}$ flash and lower flow

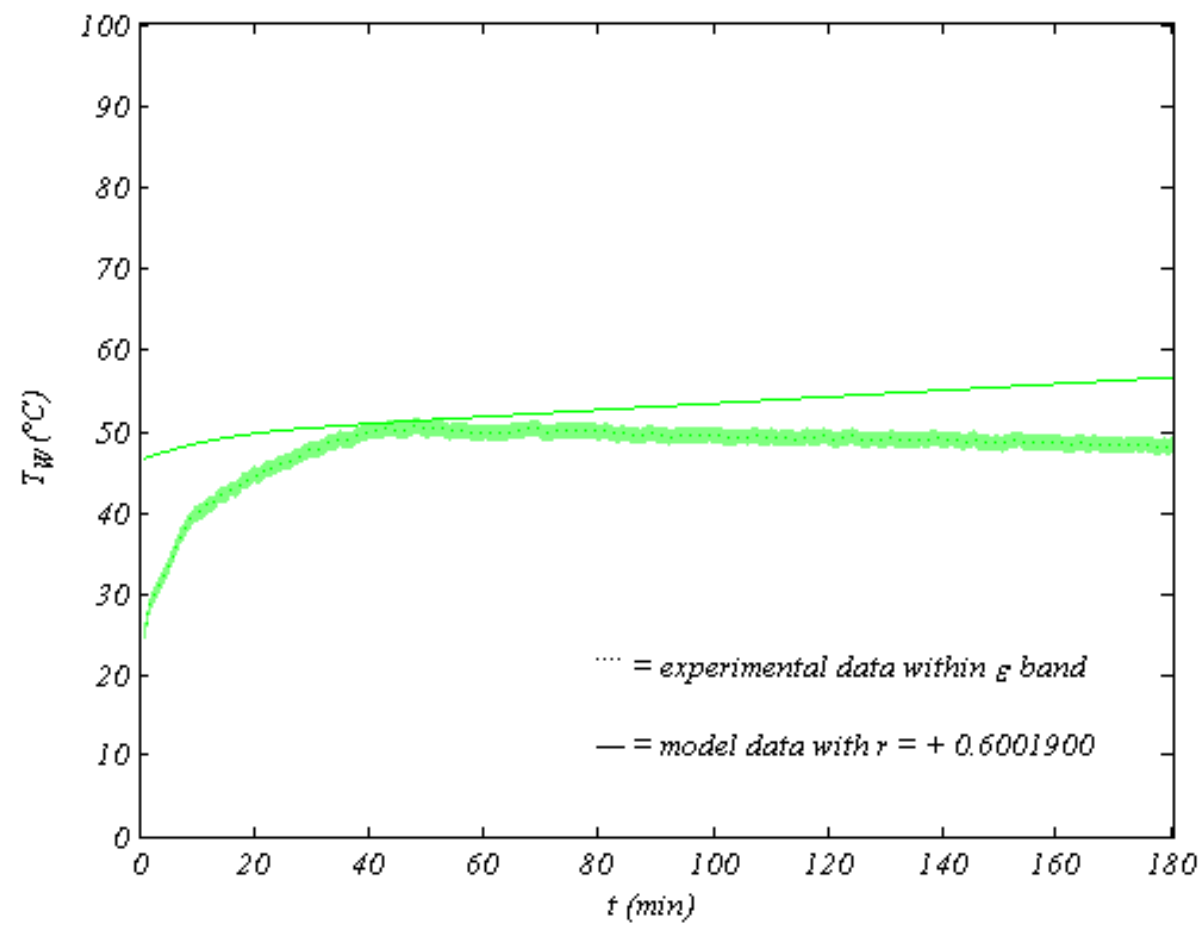

Figure 75. Concentrated brine temperature at $70^{\circ} \mathrm{C}$ flash and higher flow 


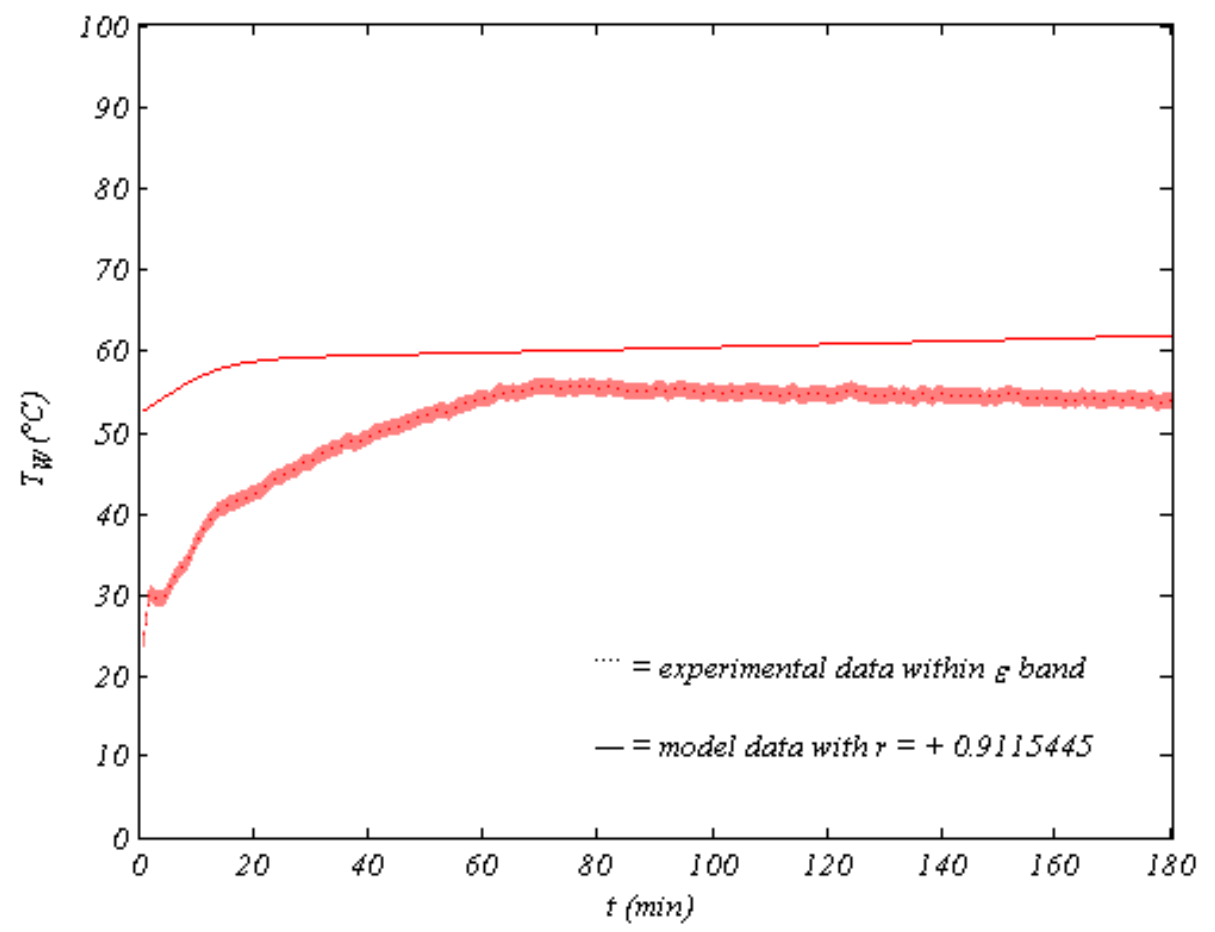

Figure 76. Concentrated brine temperature at $80^{\circ} \mathrm{C}$ flash and lower flow

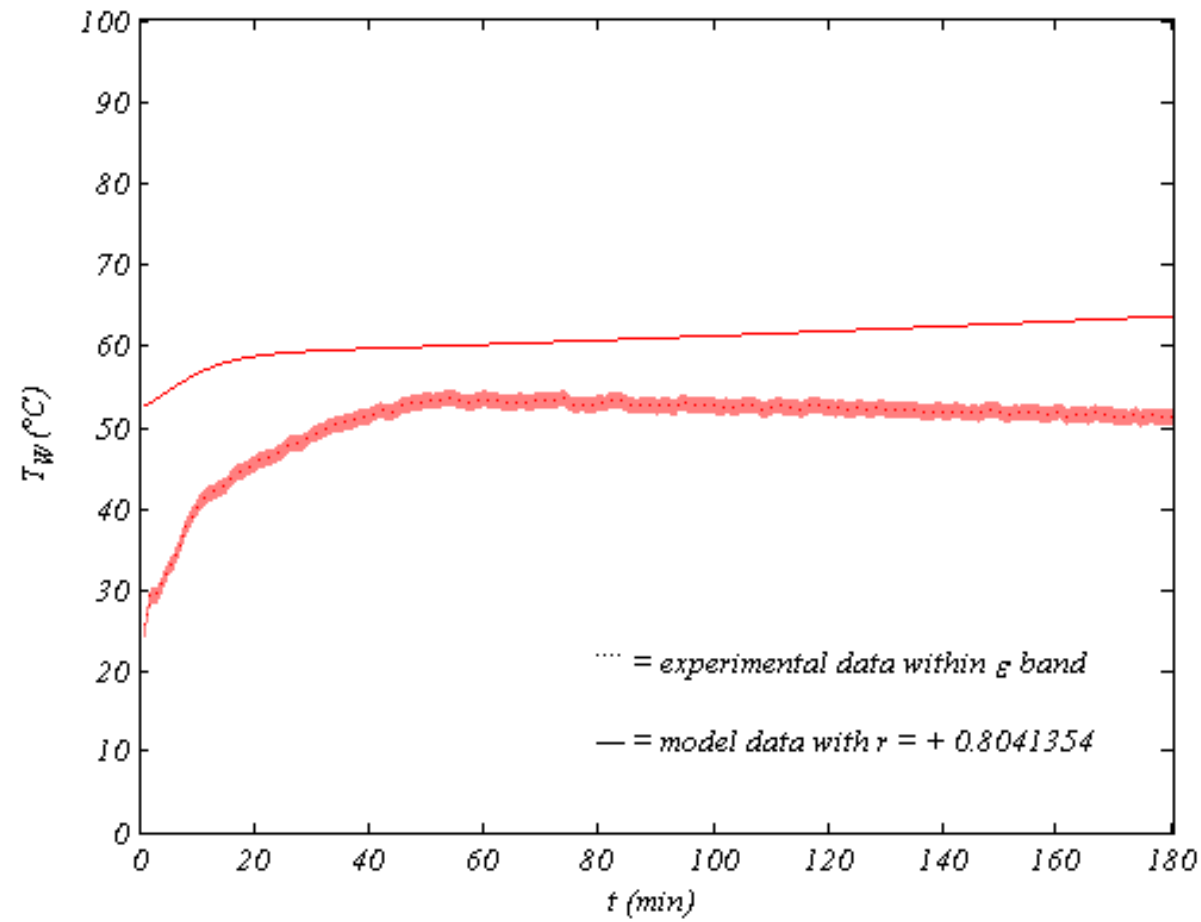

Figure 77. Concentrated brine temperature at $80^{\circ} \mathrm{C}$ flash and higher flow 


\subsection{Heat Reclamation}

Cold incoming seawater is preheated in the condenser by hot water vapor condensing on the surface of the condenser tube before it enters the heater as mentioned in CHAPTER 4. The preheat temperature rapidly increased to reach a maximum early, before it slowly declined for both seawater flow rates; moreover, the preheat temperature attained at lower seawater flow rates, was higher. It increased with flash temperatures for both seawater flow rates since the amount and temperature of the condensing water vapor are directly proportional to flashing temperature.

Hot water vapor condenses by losing its latent heat of condensation to the entering seawater in the condenser; hence, preheat temperature indirectly denotes the rate of water vaporization and condensation. The preheat temperature rapidly increased due to high initial rate of vaporization caused by rapidly increasing equilibrium temperature at lower vacuum pressures, then it slowly declined due to the decreasing rate of vaporization caused by the stabilizing equilibrium temperature at rising vacuum pressures for both seawater flow rates as was shown previously. The preheat temperature profiles for both seawater flow rates are similar; however, they were higher for lower flow rates due to more condensation caused by more vaporization as will be seen later.

Model prediction of preheat temperature loosely resembled the experimental results due to the inability of the condenser tube heat transfer module to capture the rate of condensation. Modeling a heat transfer operation with a phase change is extremely complex, especially in the presence of non-condensable gases. The precision of the model in predicting the preheat temperature affects the quality of its evaluation of system performance. Preheat temperature profiles are shown in Figure 78 through Figure 89. 


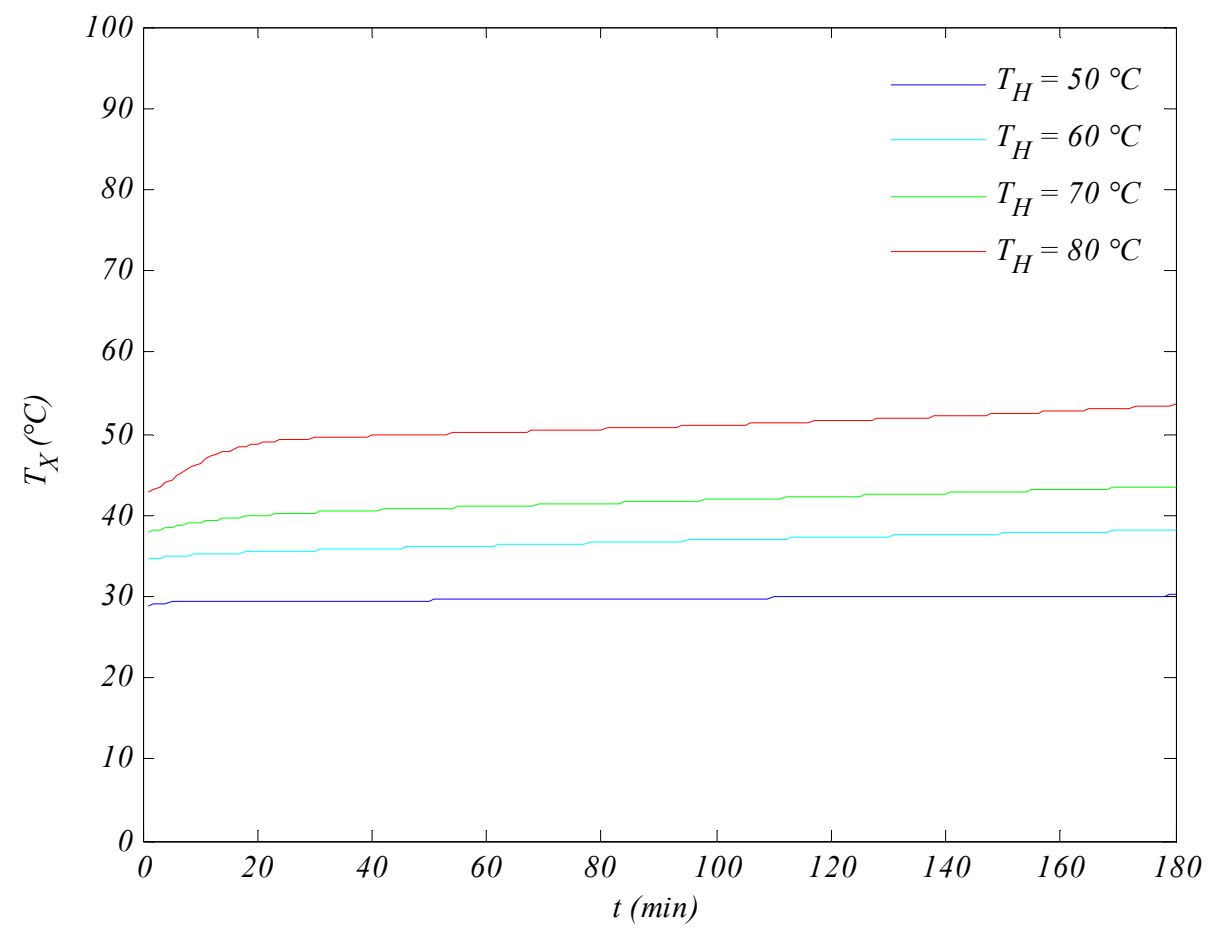

Figure 78. Modeled preheat temperature profiles at lower flow

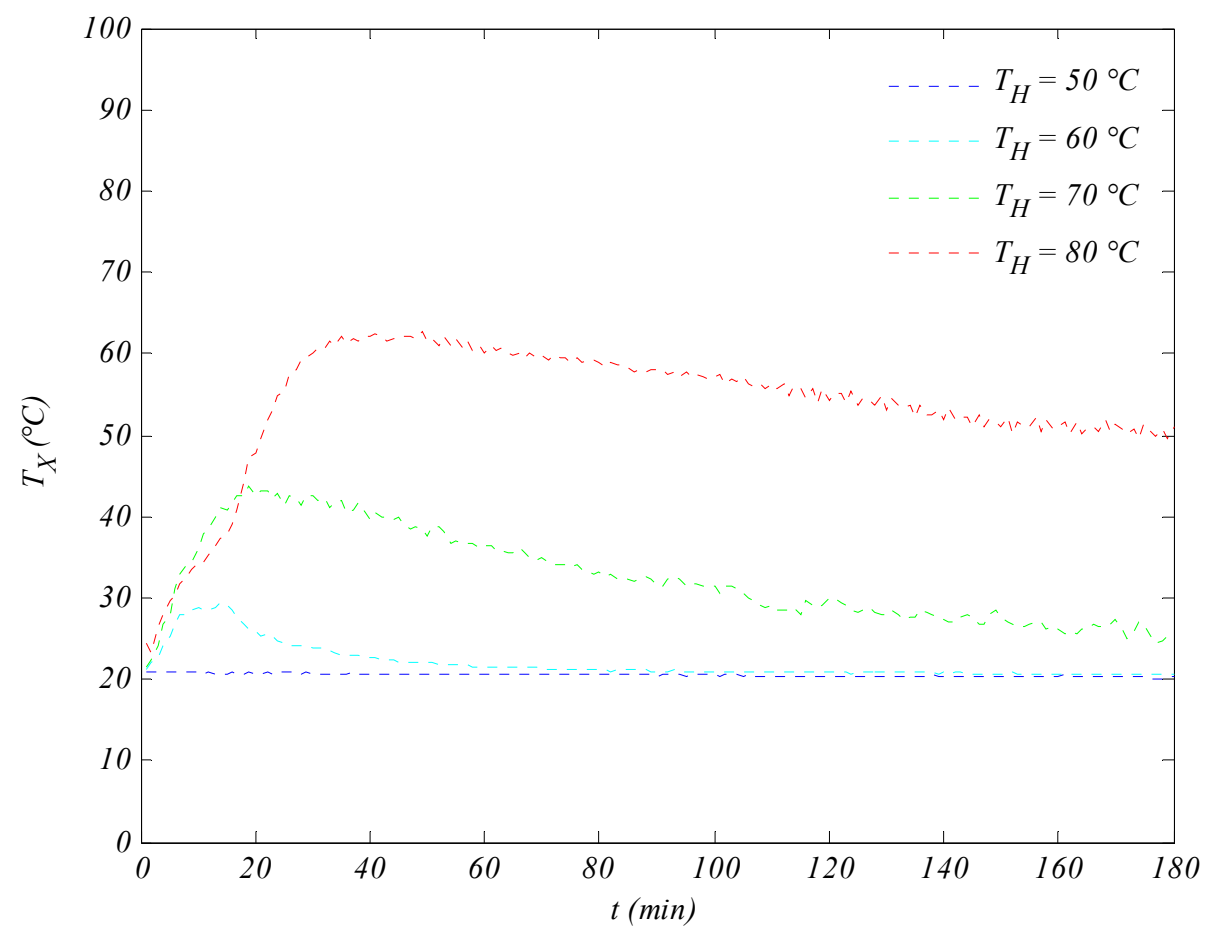

Figure 79. Experimental preheat temperature profiles at lower flow 


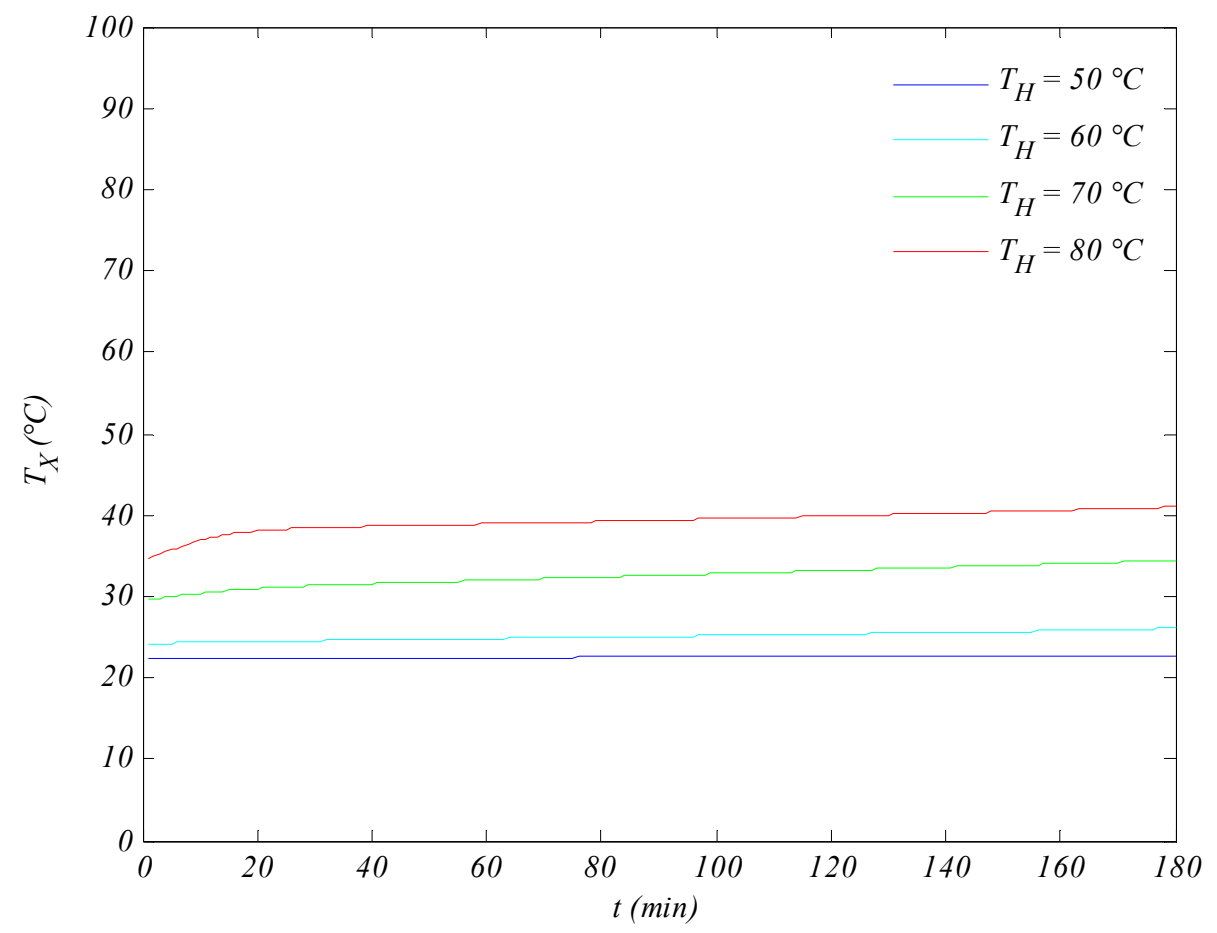

Figure 80 . Modeled preheat temperature profiles at higher flow

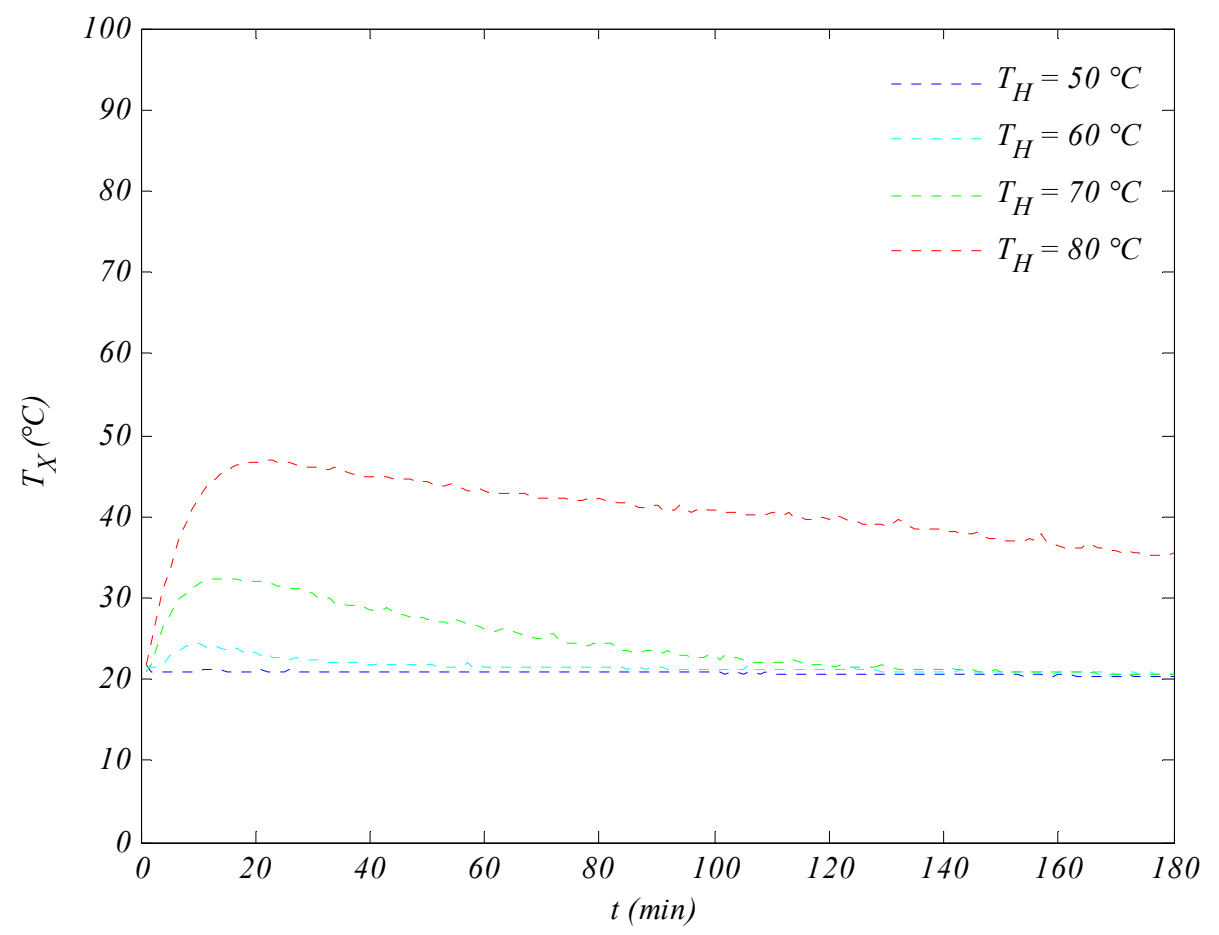

Figure 81. Experimental preheat temperature profiles at higher flow 


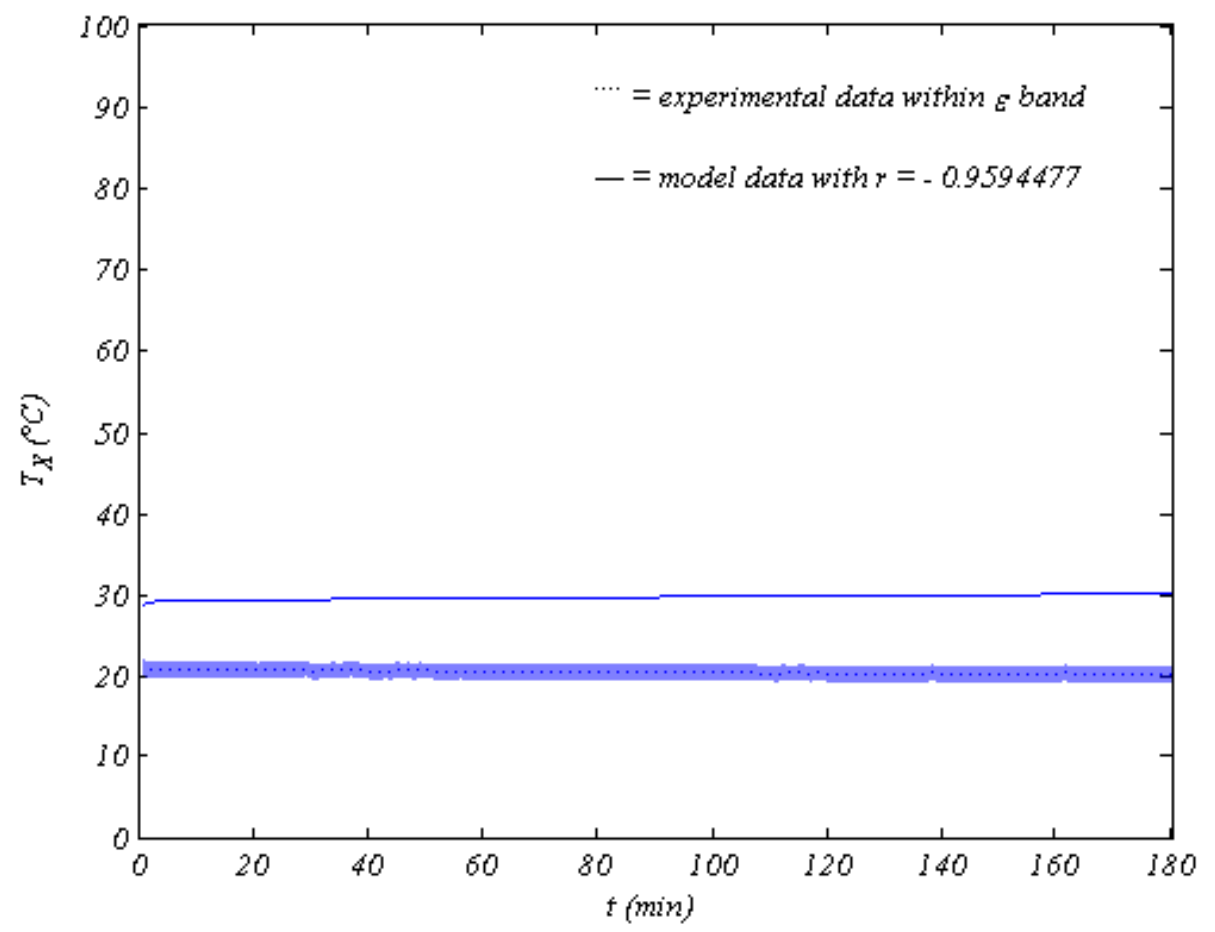

Figure 82 . Preheat temperature at $50^{\circ} \mathrm{C}$ flash and lower flow

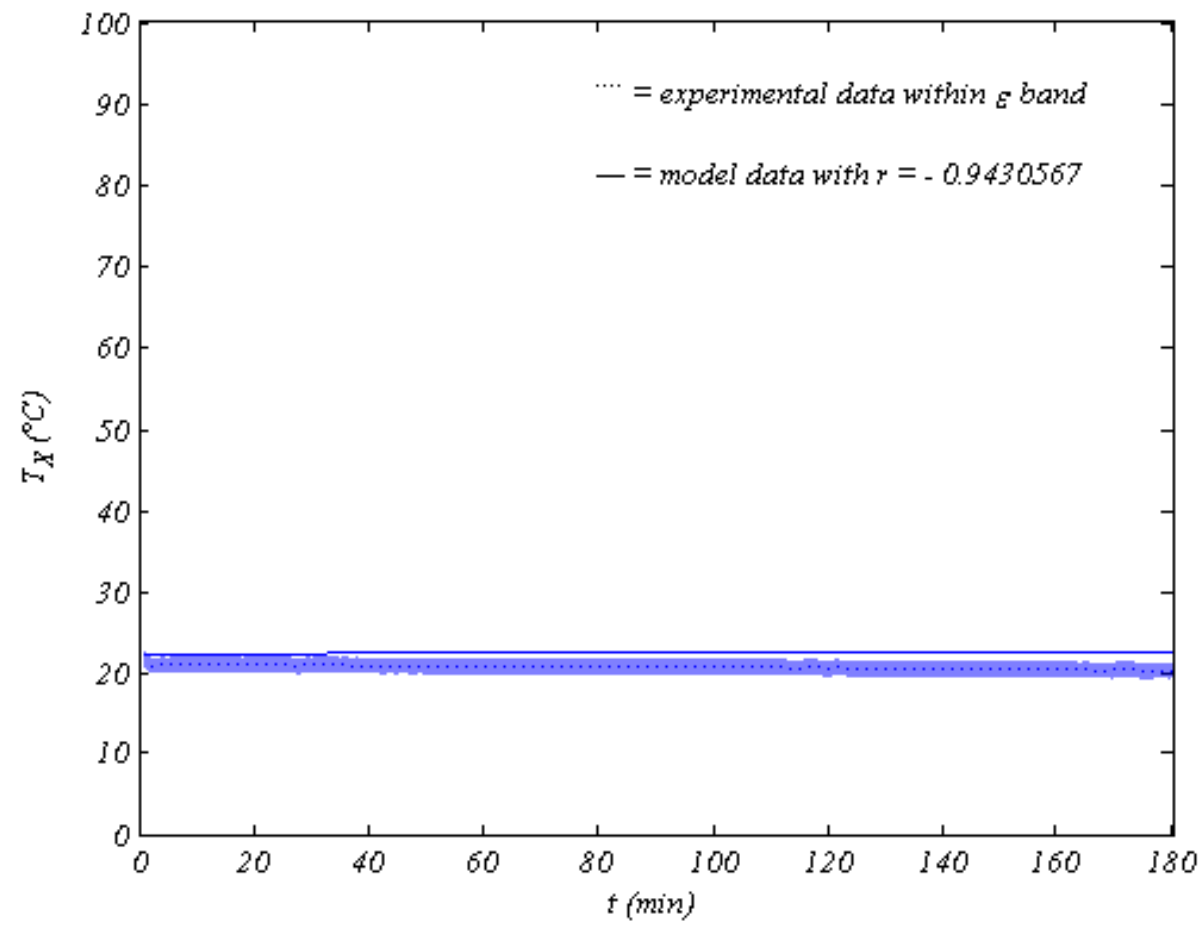

Figure 83. Preheat temperature at $50^{\circ} \mathrm{C}$ flash and higher flow 


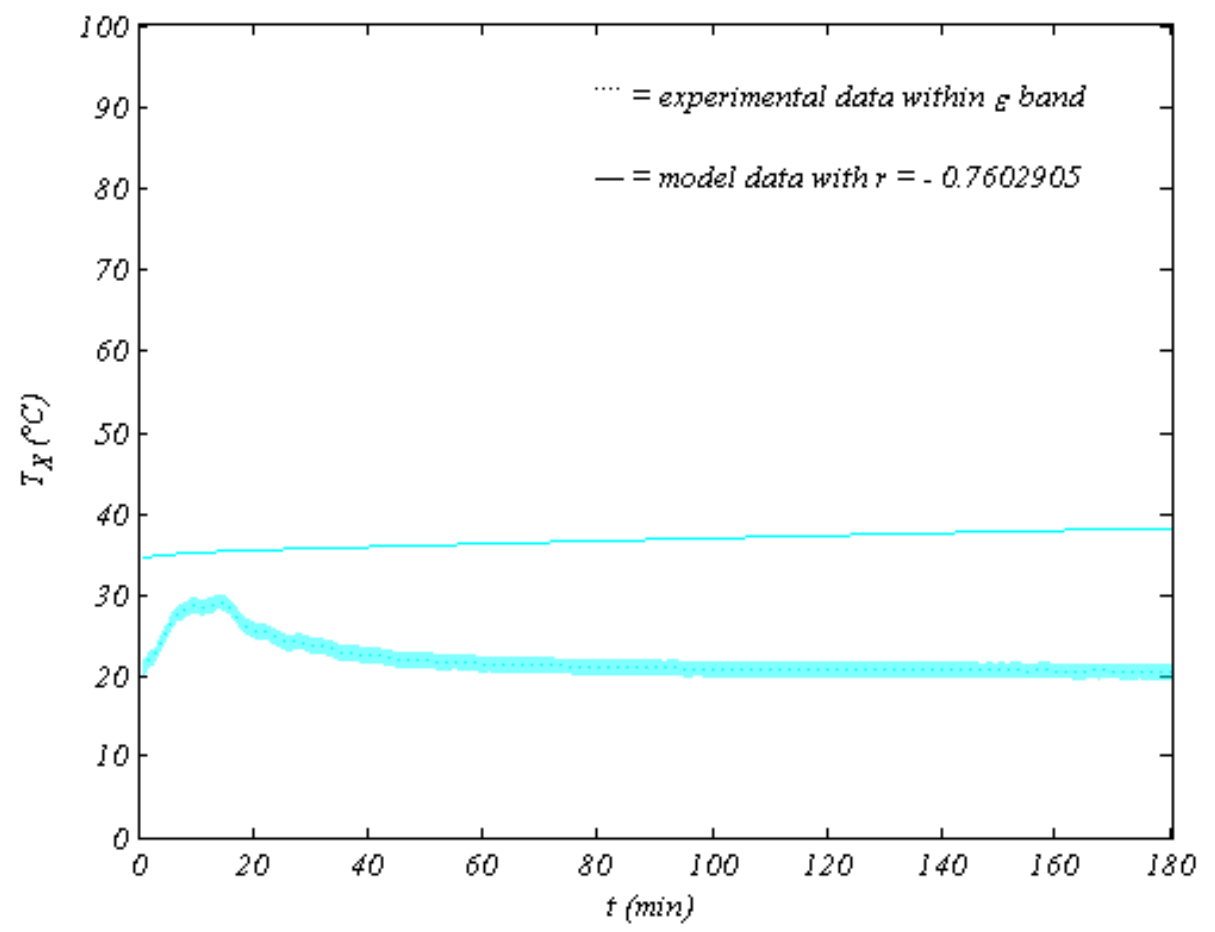

Figure 84. Preheat temperature at $60^{\circ} \mathrm{C}$ flash and lower flow

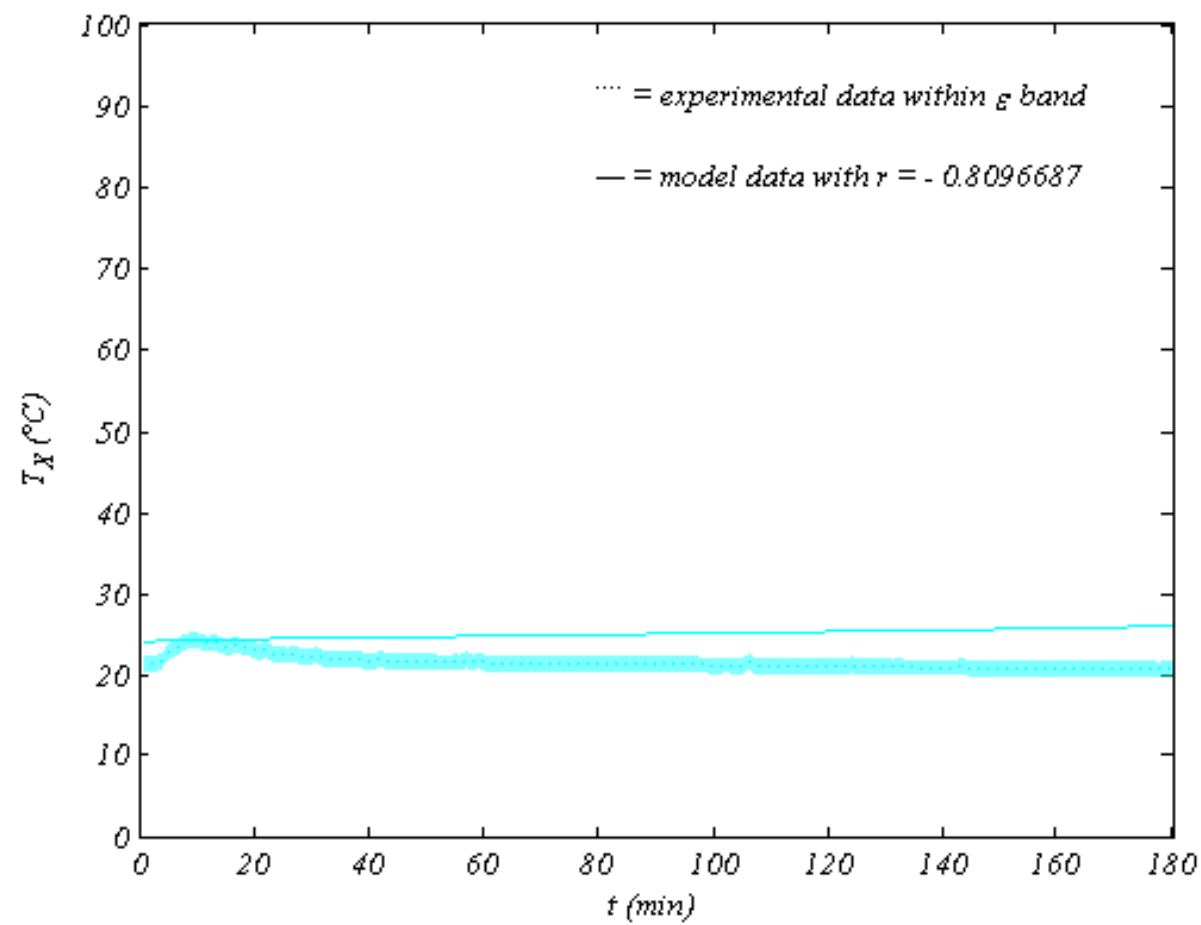

Figure 85 . Preheat temperature at $60^{\circ} \mathrm{C}$ flash and higher flow 


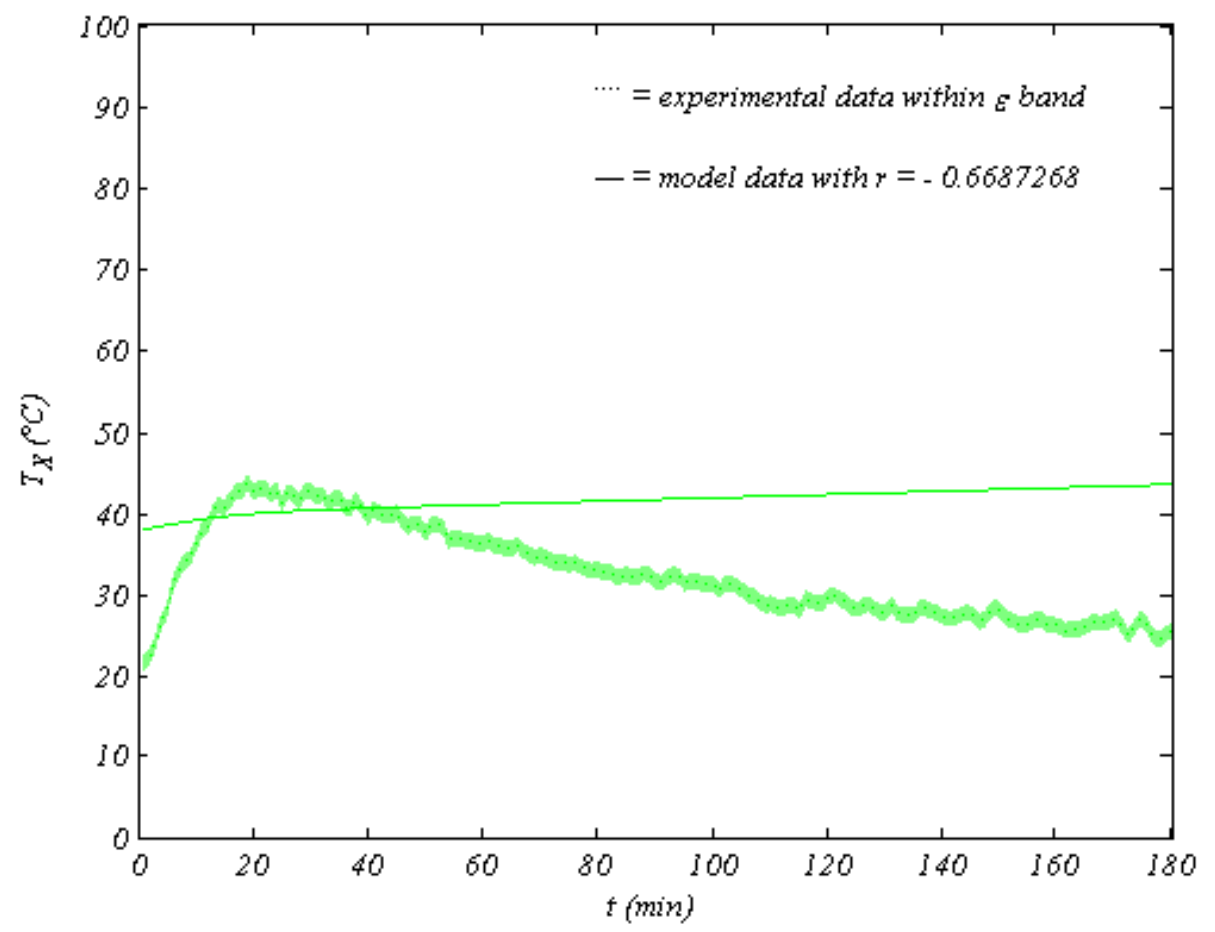

Figure 86. Preheat temperature at $70^{\circ} \mathrm{C}$ flash and lower flow

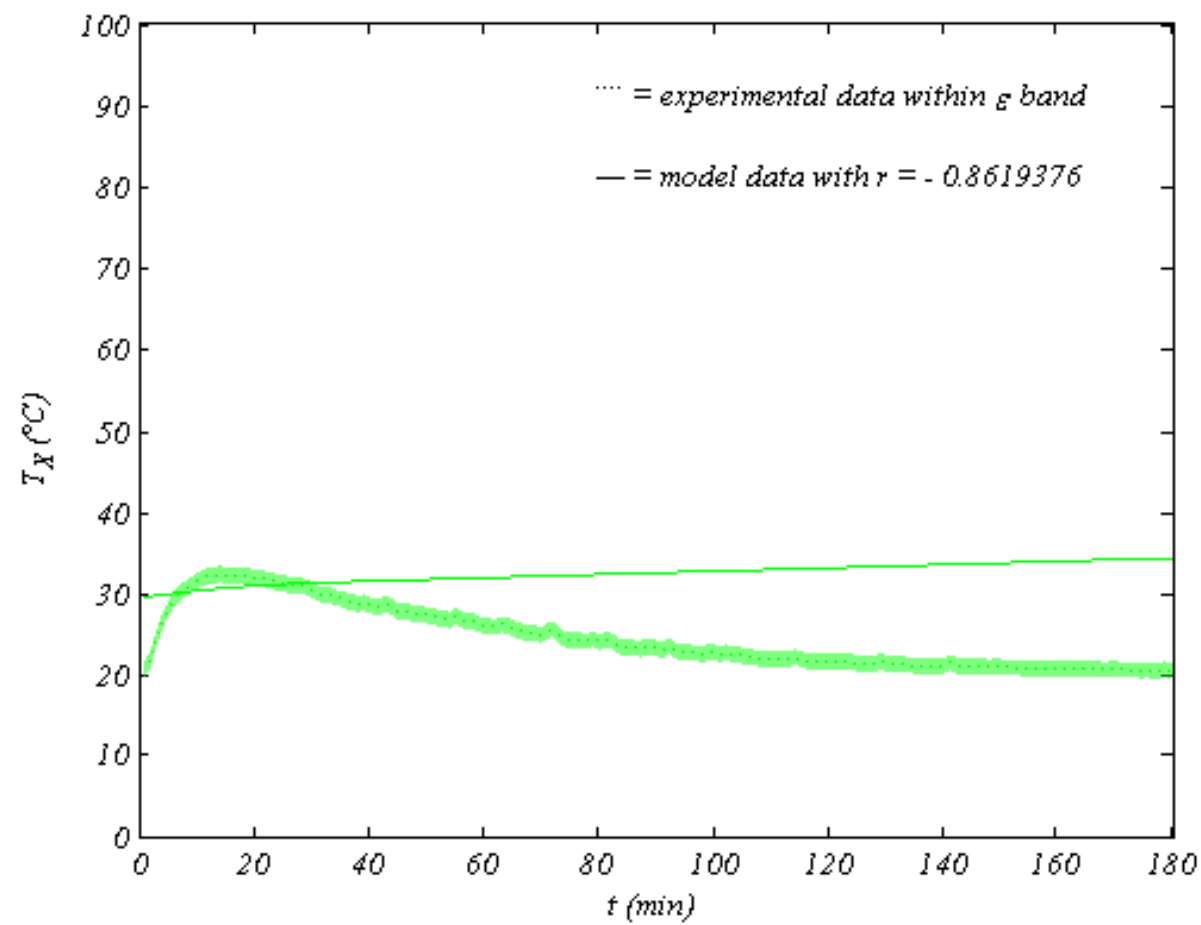

Figure 87. Preheat temperature at $70^{\circ} \mathrm{C}$ flash and higher flow 


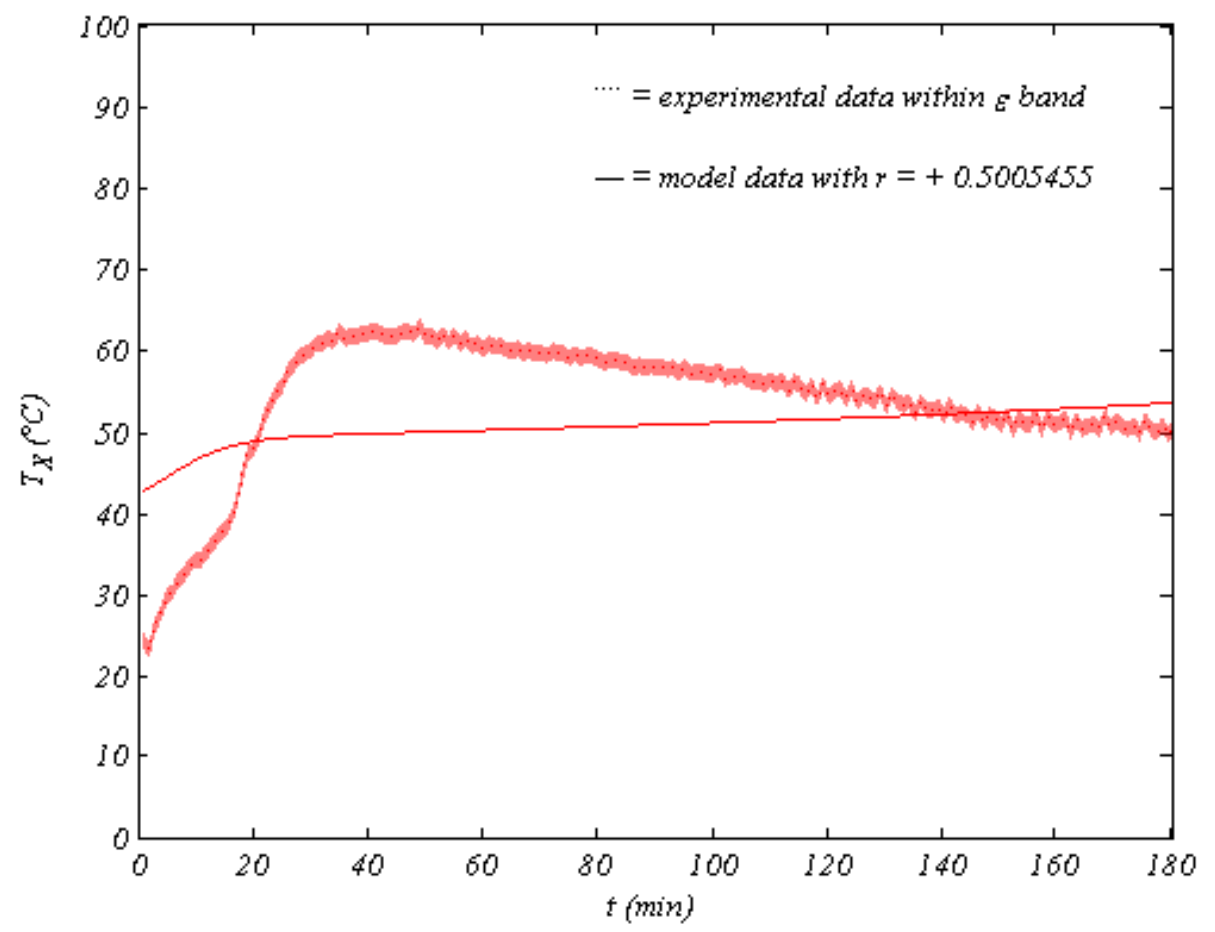

Figure 88 . Preheat temperature at $80^{\circ} \mathrm{C}$ flash and lower flow

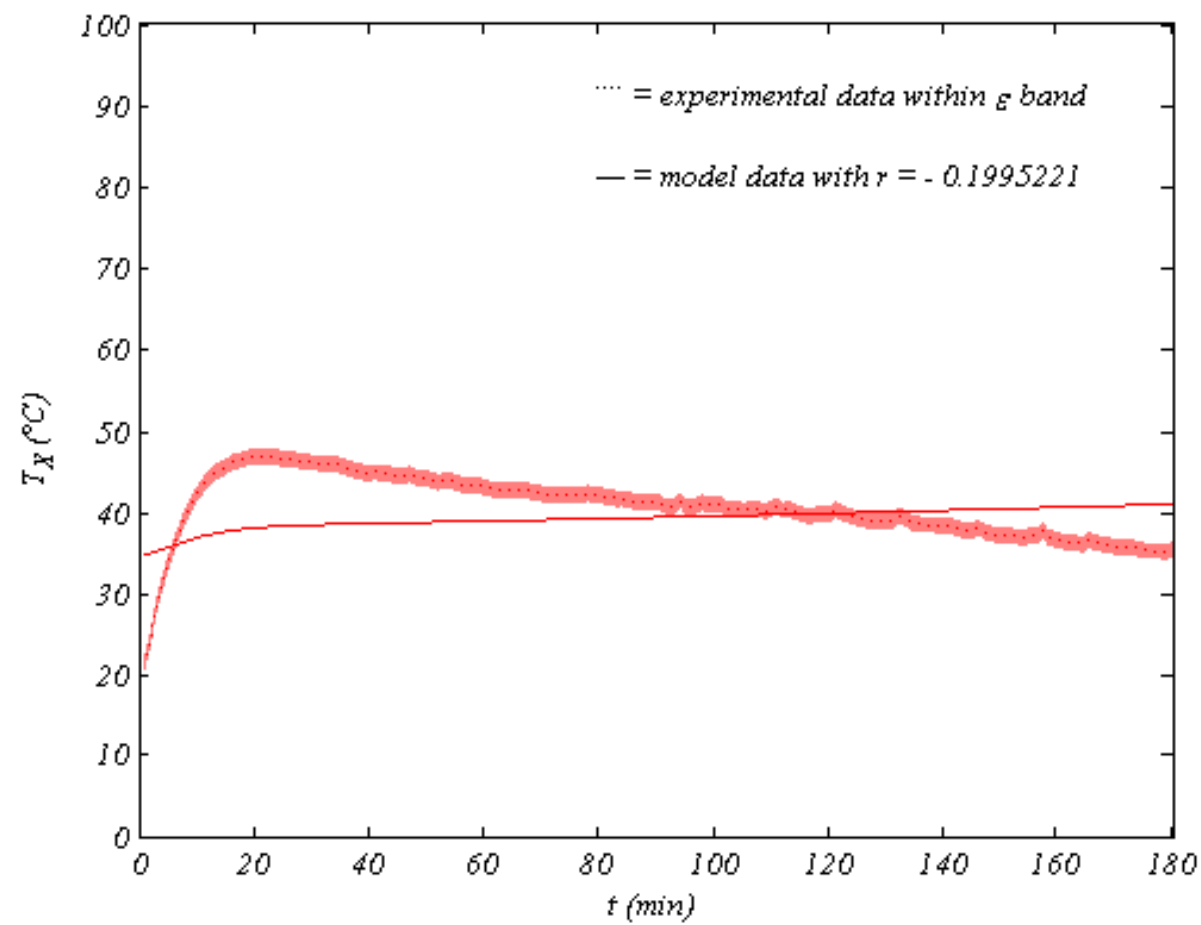

Figure 89 . Preheat temperature at $80^{\circ} \mathrm{C}$ flash and higher flow 


\subsection{Heater Size}

Preheated seawater coming out of the condenser is further heated by the solar heater to a set flash temperature as mentioned in CHAPTER 4. The heater load was fairly constant at lower flash temperatures but spiked, then rapidly decreased, reaching a minimum before it steadily increased at higher flash temperatures for both seawater flow rates. Moreover, heater loads at lower seawater flow rates were lower. The heater load increased with flash temperatures, reaching a maximum between 60 and $70{ }^{\circ} \mathrm{C}$, after which it decreased for both seawater flow rates due to improved heat recovery caused by increased condensation experienced at higher flashing temperature as was mentioned.

The heater load makes up nearly all energy input to the desalination system due to the relatively small pumping work; hence, curtailing it enhances the feasibility of the process. The heater load logically increased with flash temperature at first but started to decrease later at higher flash temperature due to improved heat recovery caused by increased condensation for both seawater flow rates. Increased vaporization and the subsequent condensation improve heat recovery manifested in higher preheat temperatures that reduce the temperature gradient around the heater, ultimately reducing the heater load in line with the energy balance. The heater load profiles for both seawater flow rates are similar; however, they were lower for lower flow rates, since there was less volume to heat as well as the superior heat recovery as was seen earlier.

Model prediction of heater load loosely resembled the pseudo-experimental results due to the inexact preheat temperature calculation seen earlier. The precision of the model in predicting the heater load affects the quality of its evaluation of system performance. Heater load profiles are shown in Figure 90 through Figure 101. 


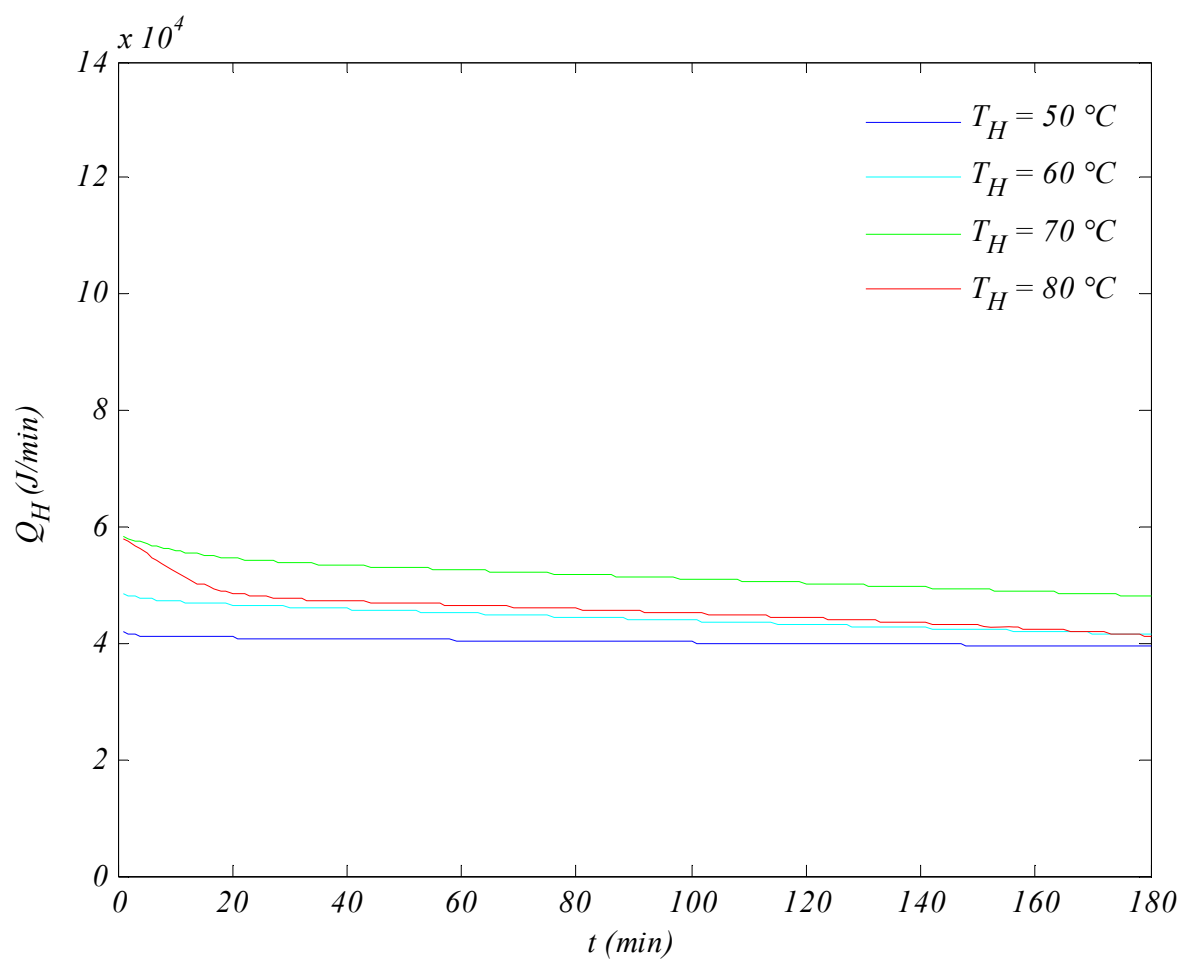

Figure 90. Modeled heat load profiles at lower flow

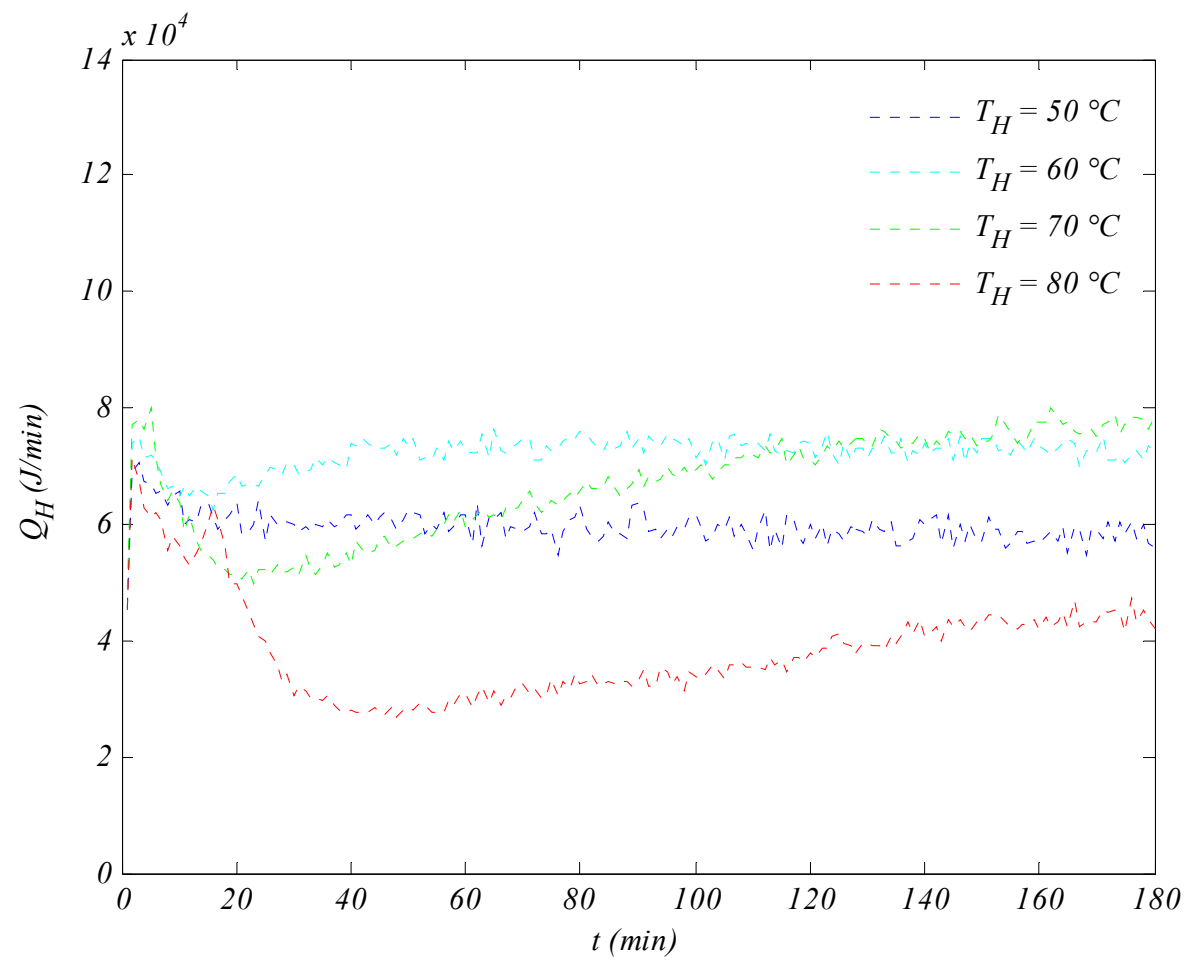

Figure 91. Mined heat load profiles at lower flow 


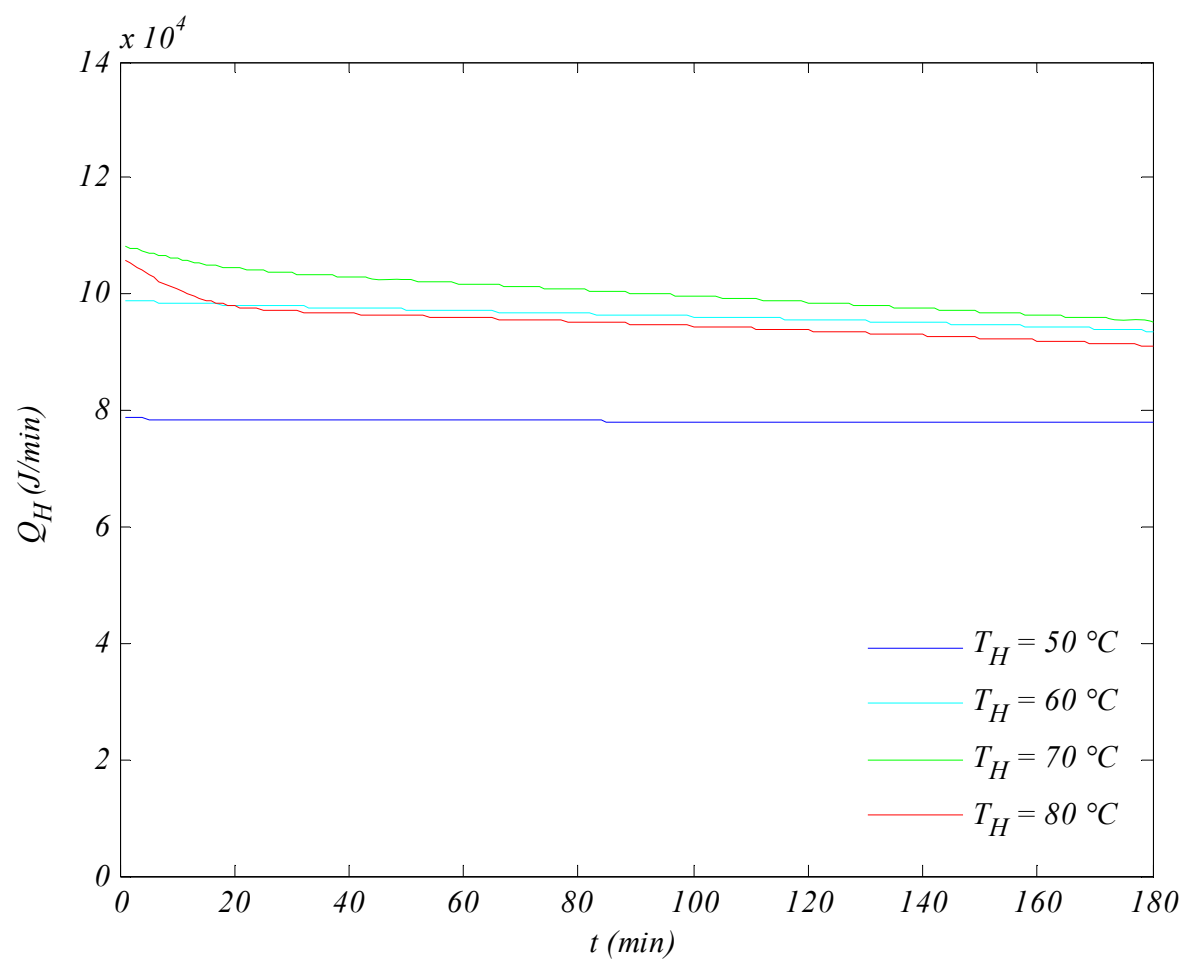

Figure 92. Modeled heat load profiles at higher flow

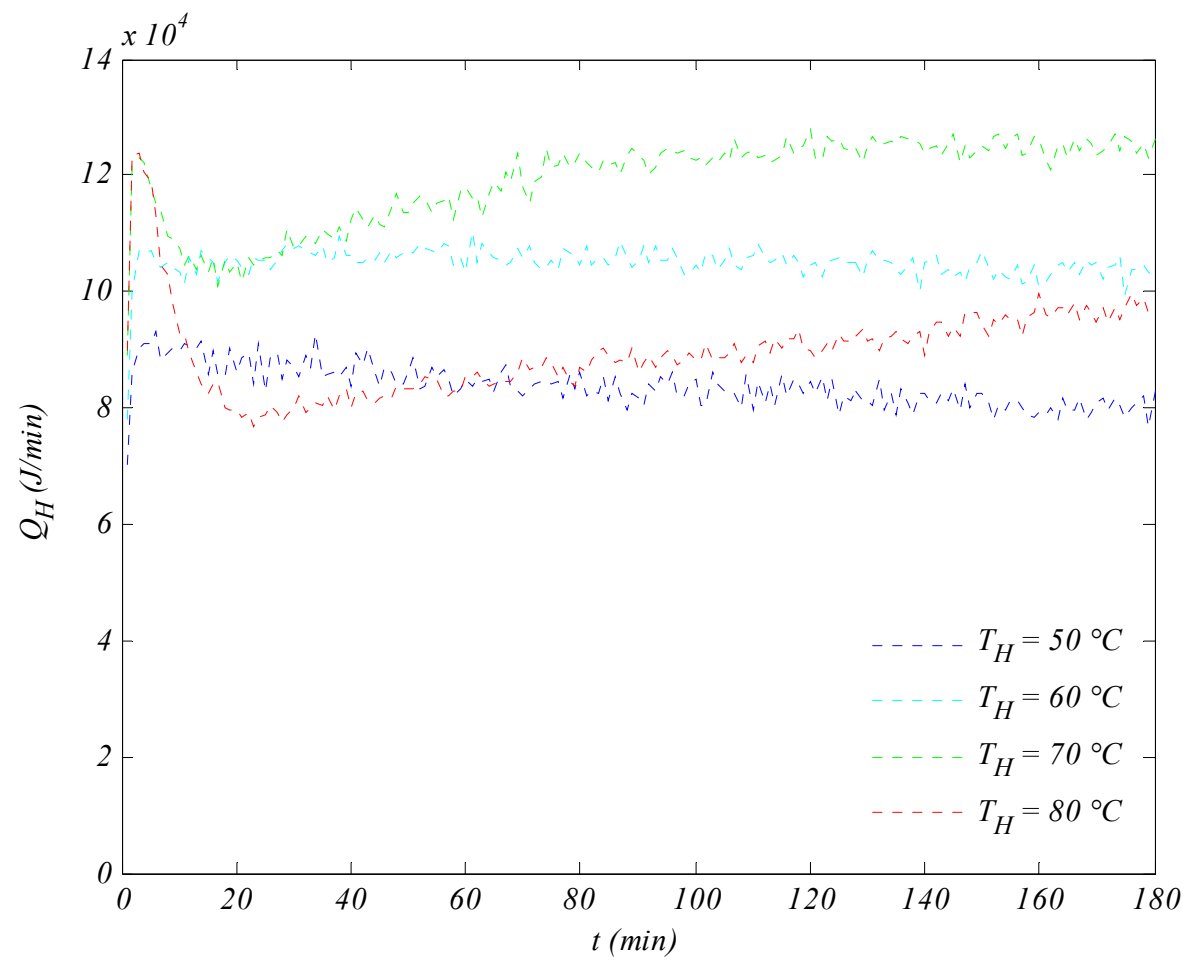

Figure 93. Mined heat load profiles at higher flow 


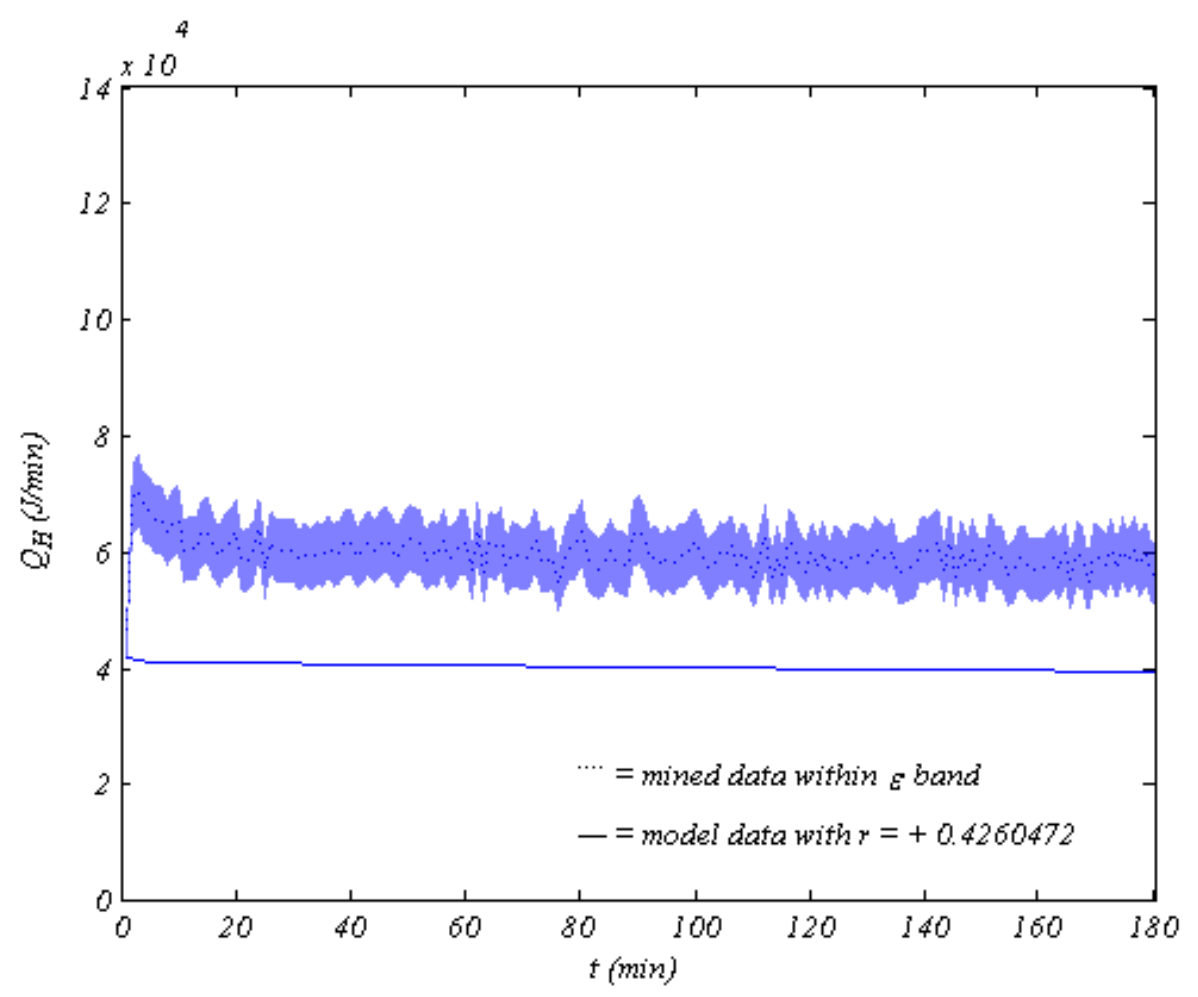

Figure 94. Heat load at $50^{\circ} \mathrm{C}$ flash and lower flow

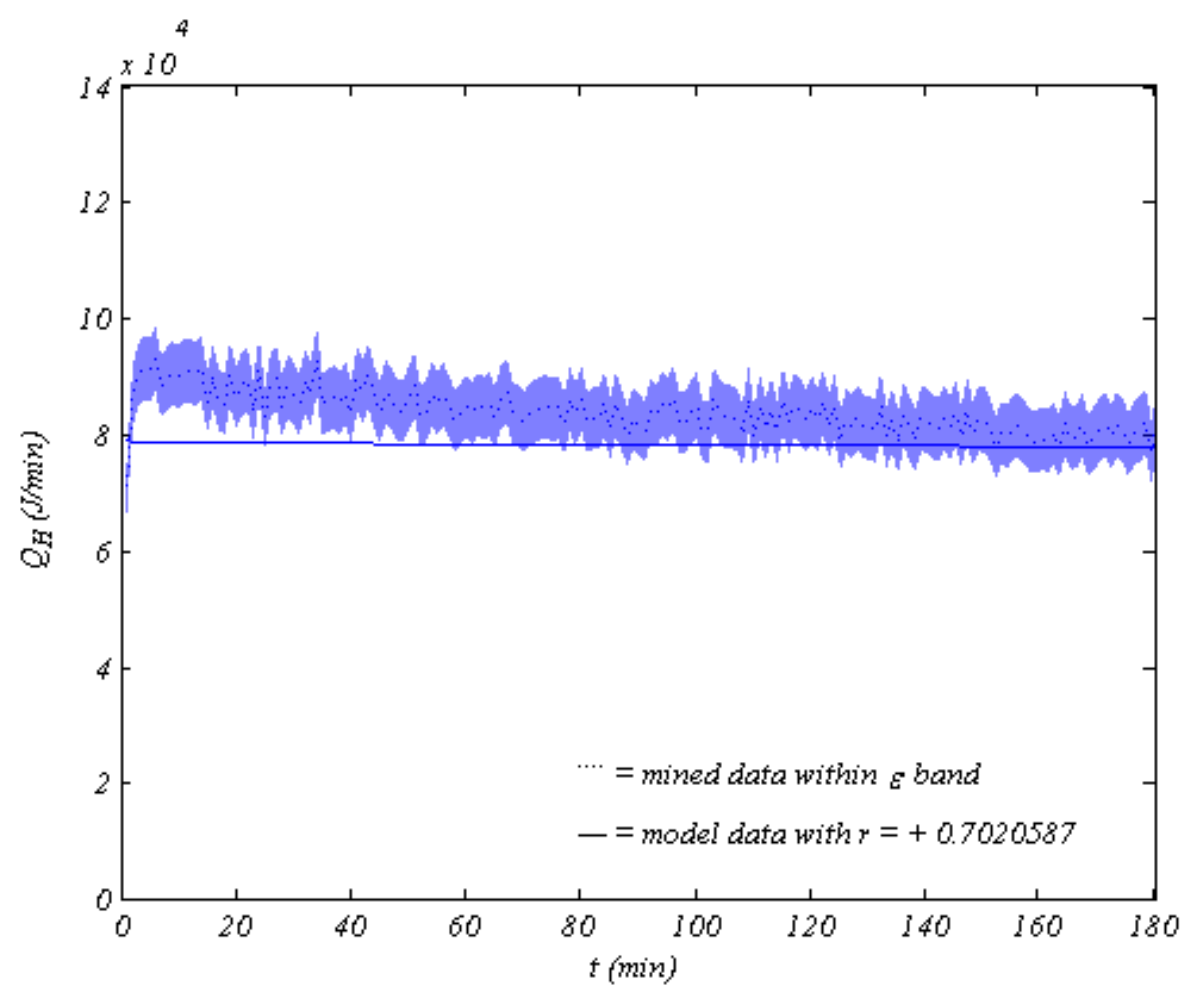

Figure 95 . Heat load at $50^{\circ} \mathrm{C}$ flash and higher flow 


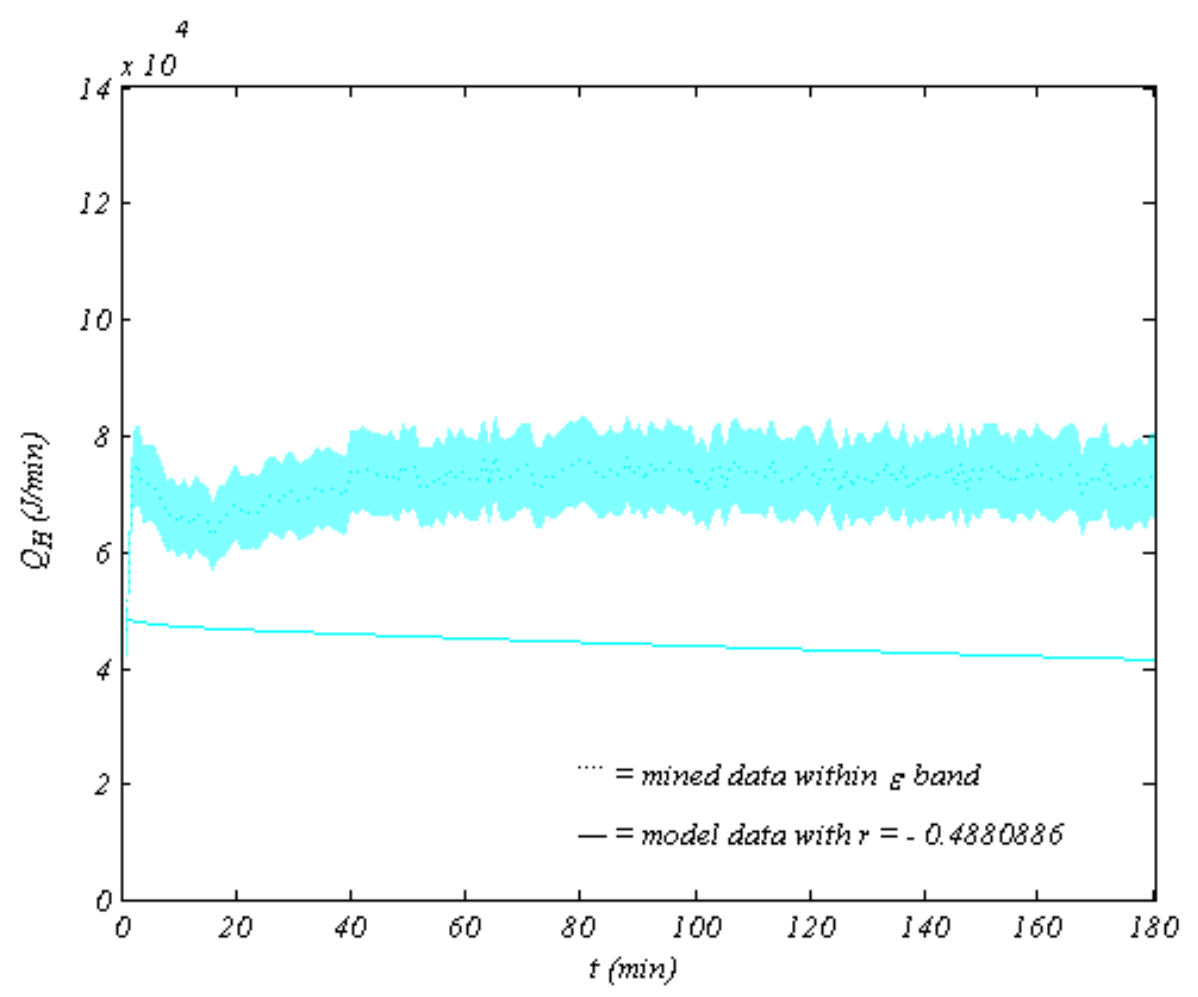

Figure 96. Heat load at $60^{\circ} \mathrm{C}$ flash and lower flow

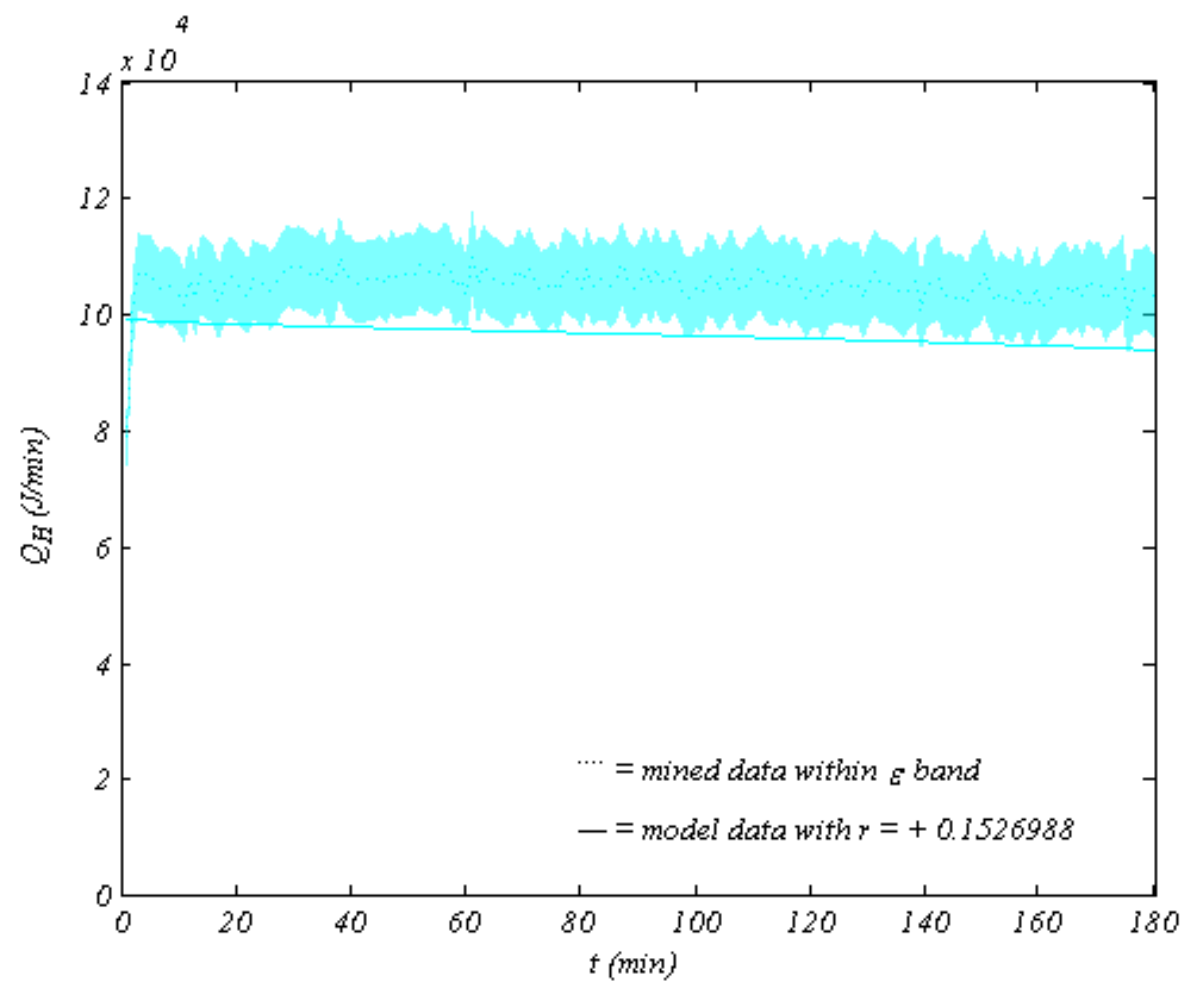

Figure 97 . Heat load at $60^{\circ} \mathrm{C}$ flash and higher flow 


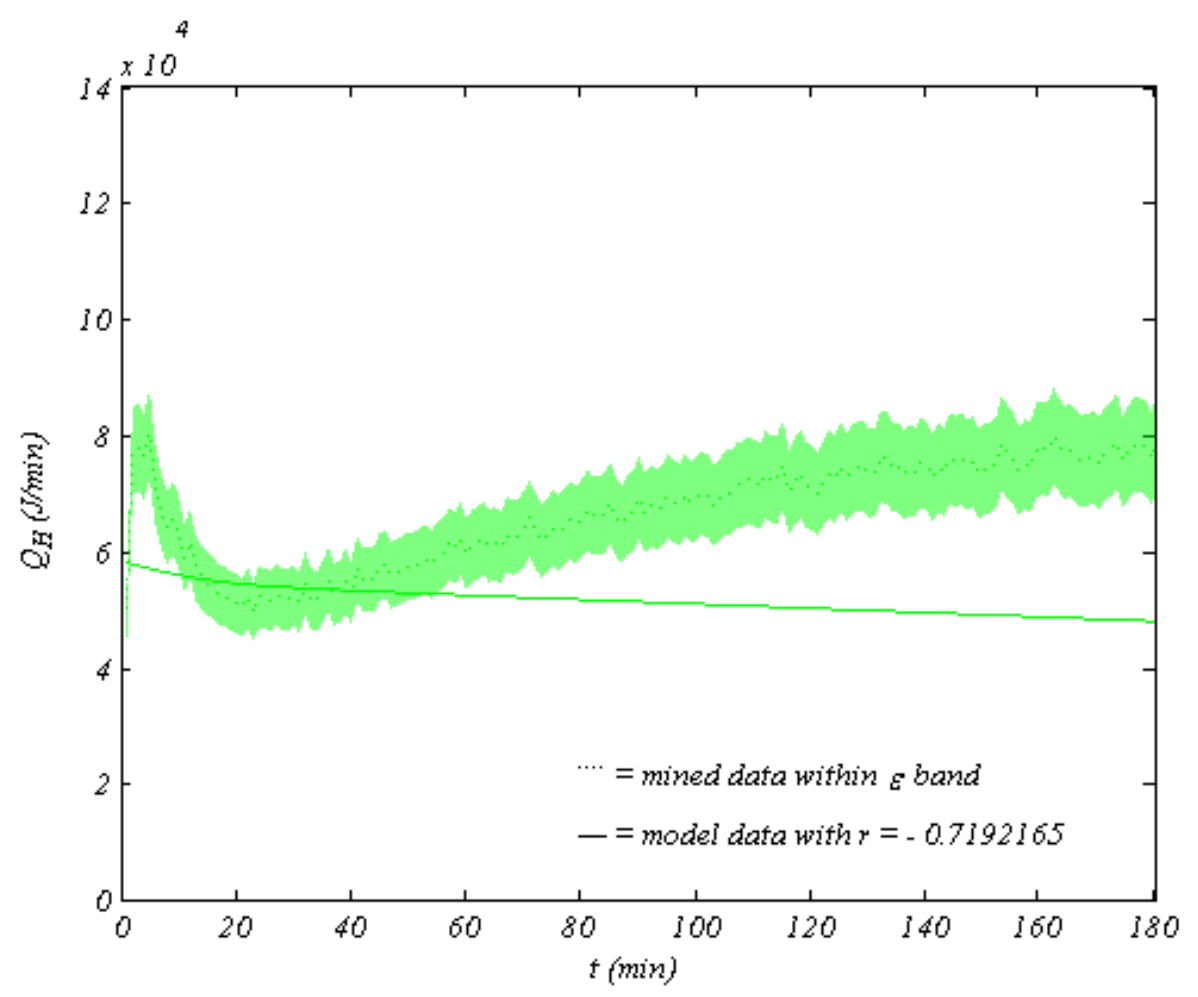

Figure 98 . Heat load at $70^{\circ} \mathrm{C}$ flash and lower flow

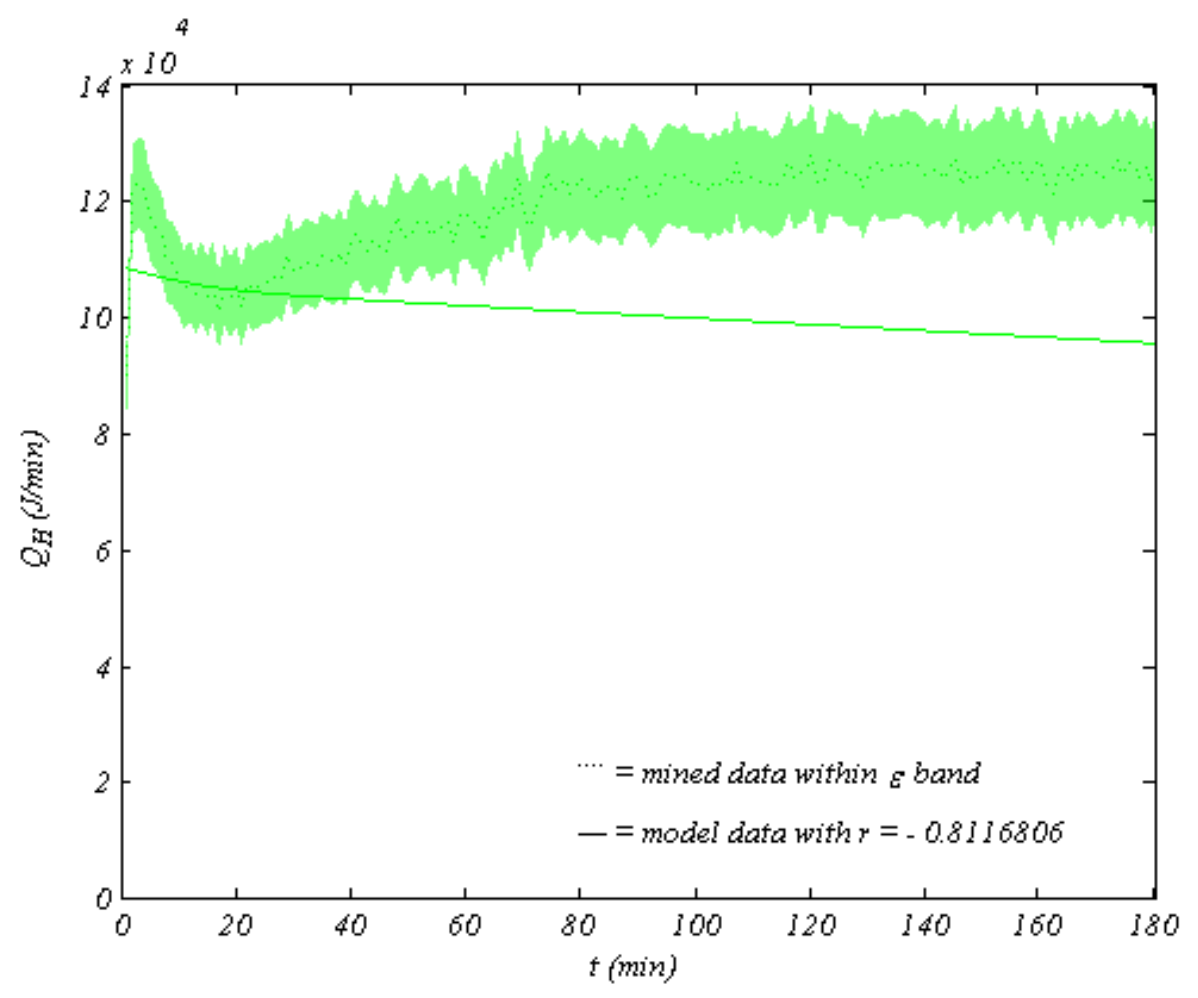

Figure 99 . Heat load at $70^{\circ} \mathrm{C}$ flash and higher flow 


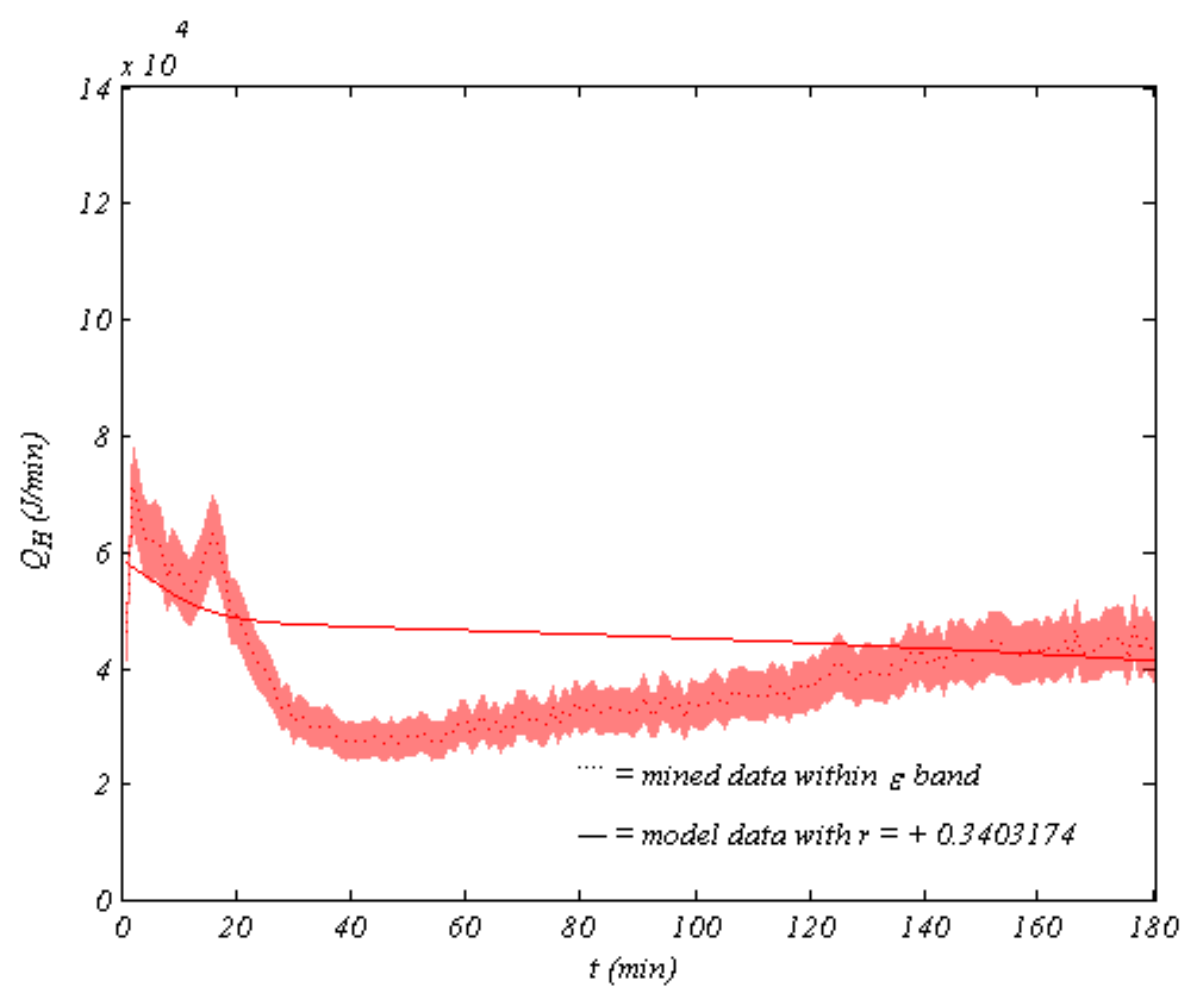

Figure 100. Heat load at $80^{\circ} \mathrm{C}$ flash and lower flow

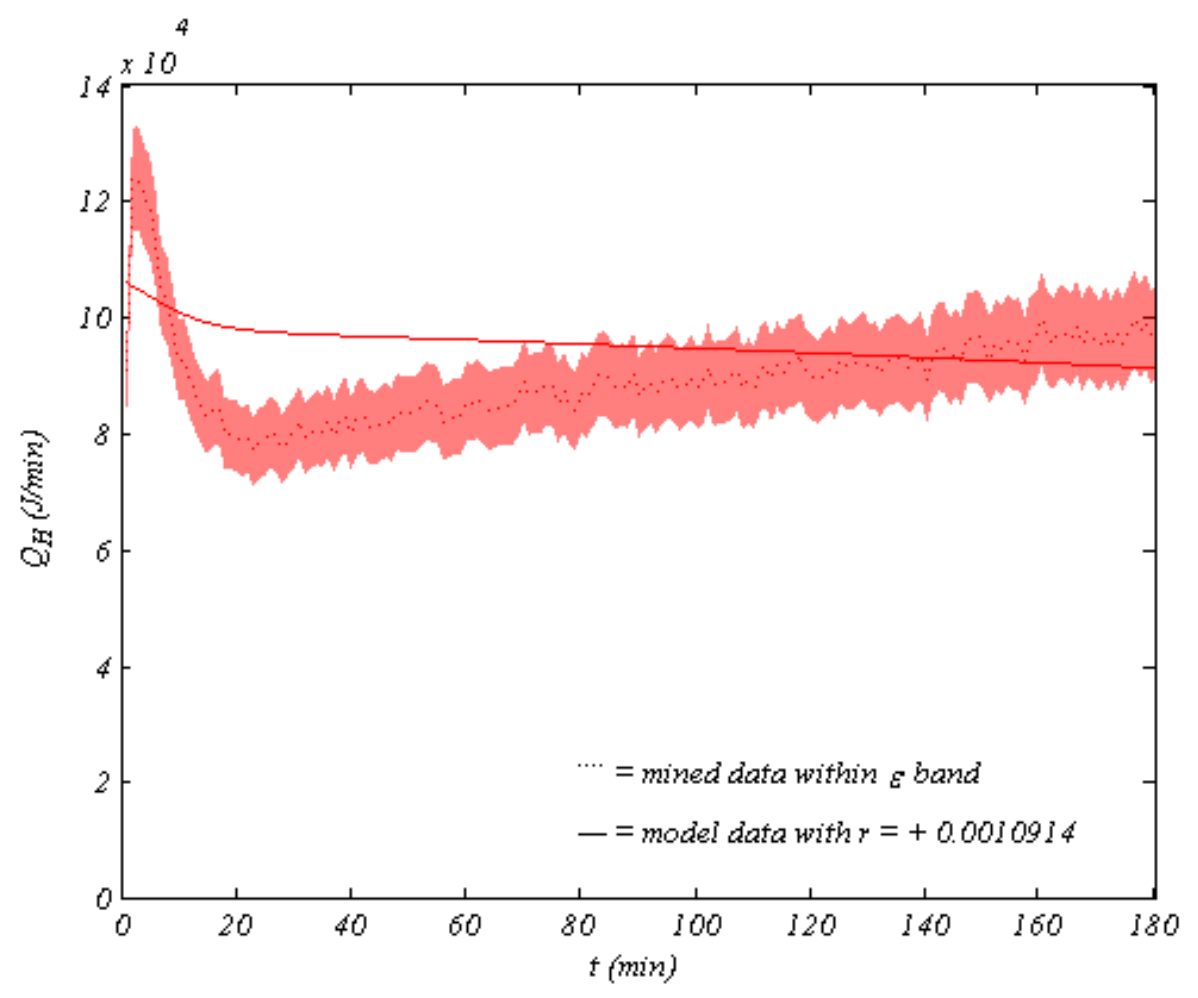

Figure 101. Heat load at $80^{\circ} \mathrm{C}$ flash and higher flow 


\subsection{Collector Size}

The heater is modeled as a single-glazed flat-plate solar collector directly heating seawater flowing through its absorbing tubes; moreover, it is sized by the solar collection area needed to meet the heater load computed by a correlation given in CHAPTER 4 [8]. The required solar collection area is directly proportional to the heater load, resulting in matching profiles of both variables. The required solar collection area was fairly constant at lower flash temperatures, but spiked, then rapidly decreased, reaching a minimum before it steadily increased at higher flash temperatures for both seawater flow rates; moreover, the required solar collection areas at lower seawater flow rates were lower. Required solar collection area increased with flash temperatures, reaching a maximum between 60 and $70{ }^{\circ} \mathrm{C}$, after which it decreased for both seawater flow rates due to improved heat recovery caused by increased condensation at higher flashing temperature.

The required solar collection area increased with flash temperature at first but started to decrease later at higher flash temperature, matching the above detailed profile of heater load for both seawater flow rates. The required solar collection area profiles for both seawater flow rates were similar; however, they were lower for lower flow rates due to reduced heater load, since there was less volume to heat as was seen earlier.

Model prediction of required solar collection area did not closely resemble the pseudo-experimental results because of poorly estimated heater load values caused by inexact preheat temperature calculation as was mentioned earlier. Reliability of model estimates of the required solar collection area depends on the accuracy of heater load computations, which relies on precision of preheat temperature computations. Required solar collection area profiles are shown in Figure 102 through Figure 113. 


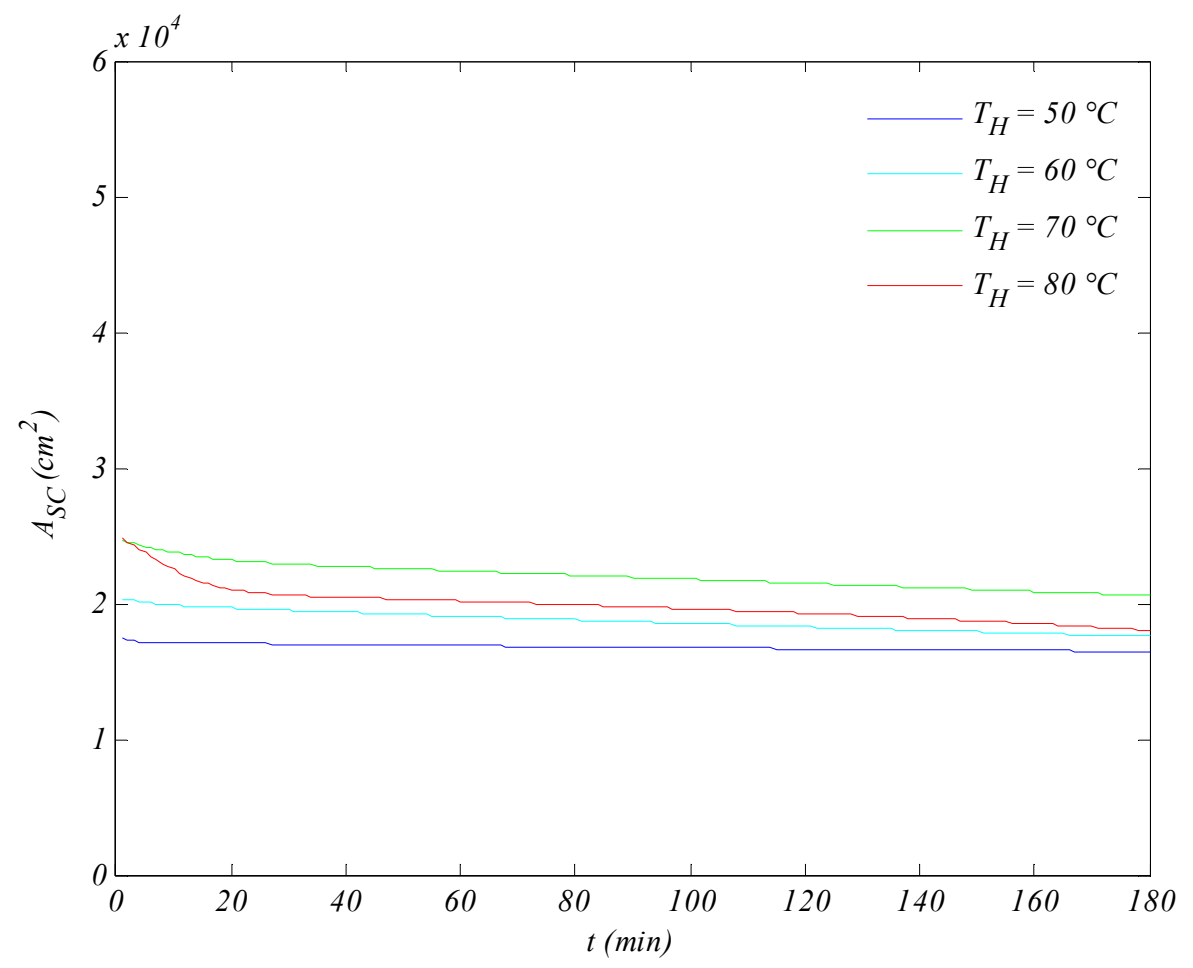

Figure 102. Modeled required solar collection area profiles at lower flow

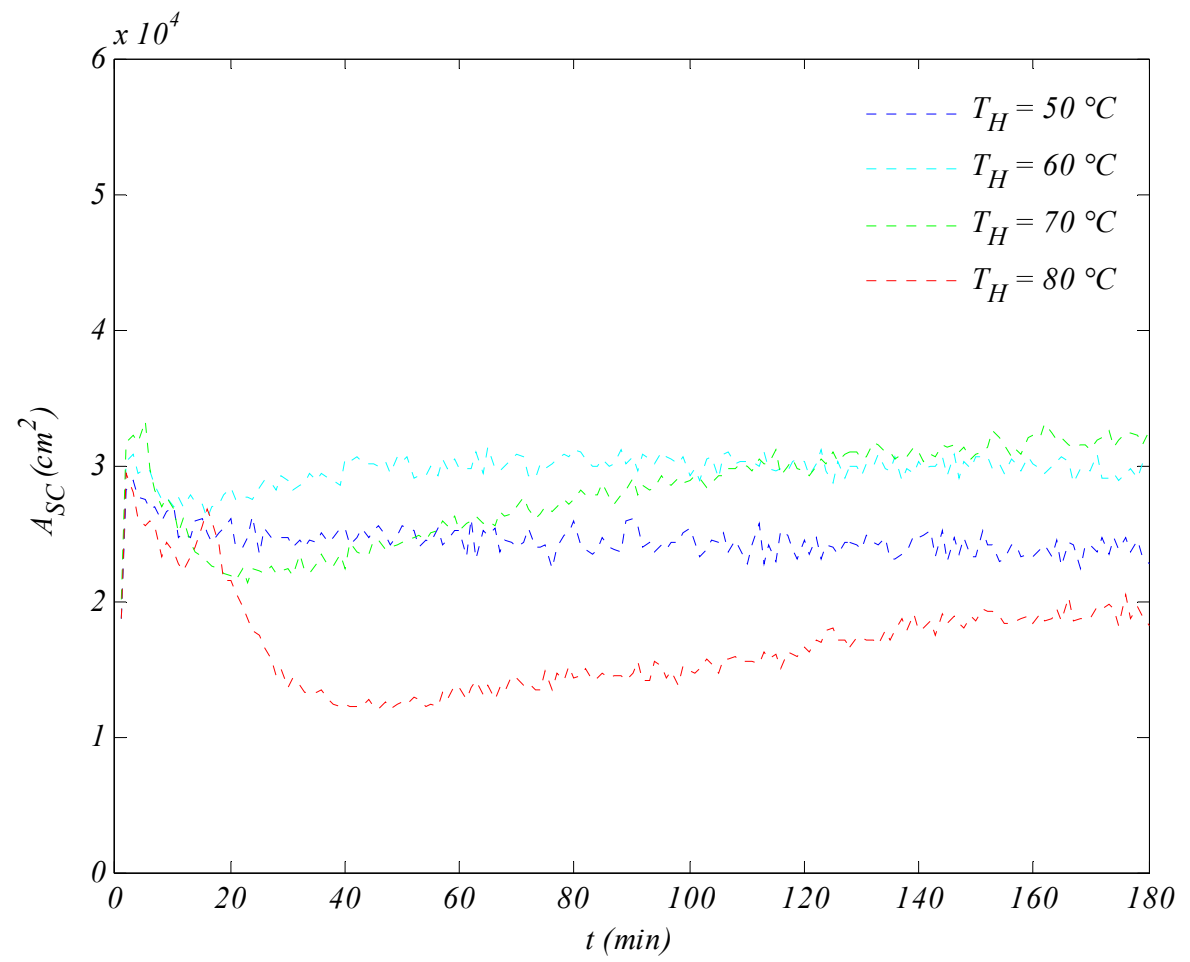

Figure 103. Mined required solar collection area profiles at lower flow 


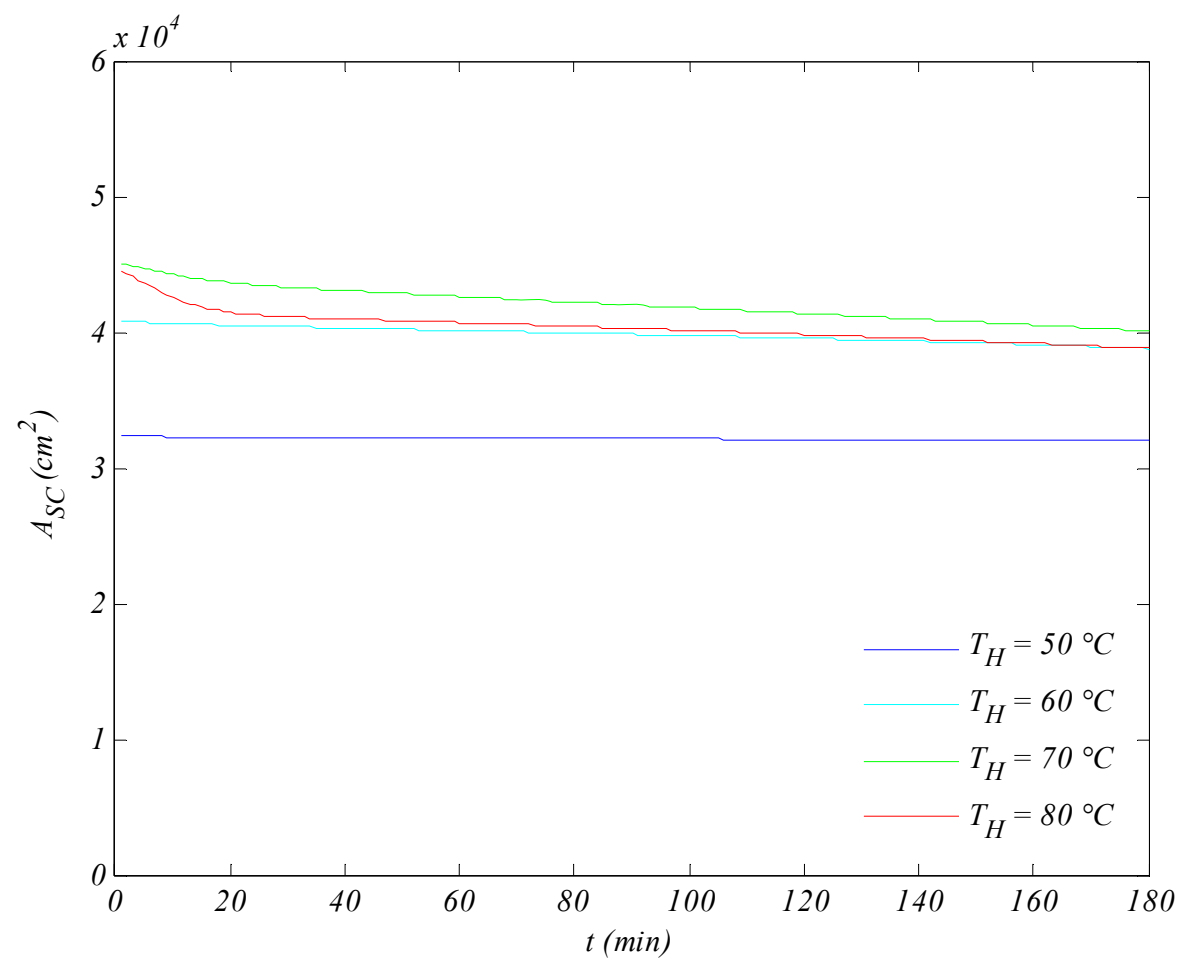

Figure 104. Modeled required solar collection area profiles at higher flow

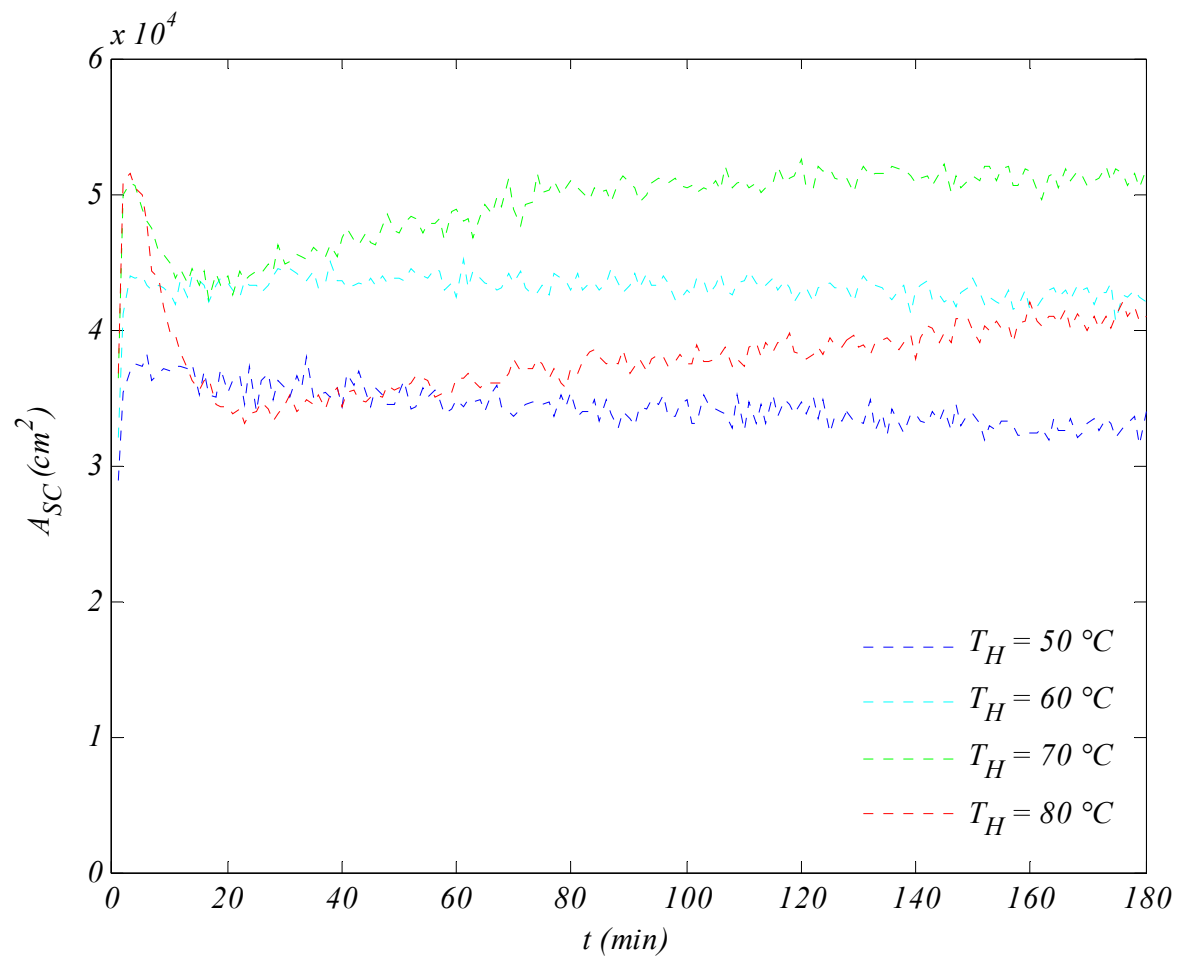

Figure 105. Mined required solar collection area profiles at higher flow 


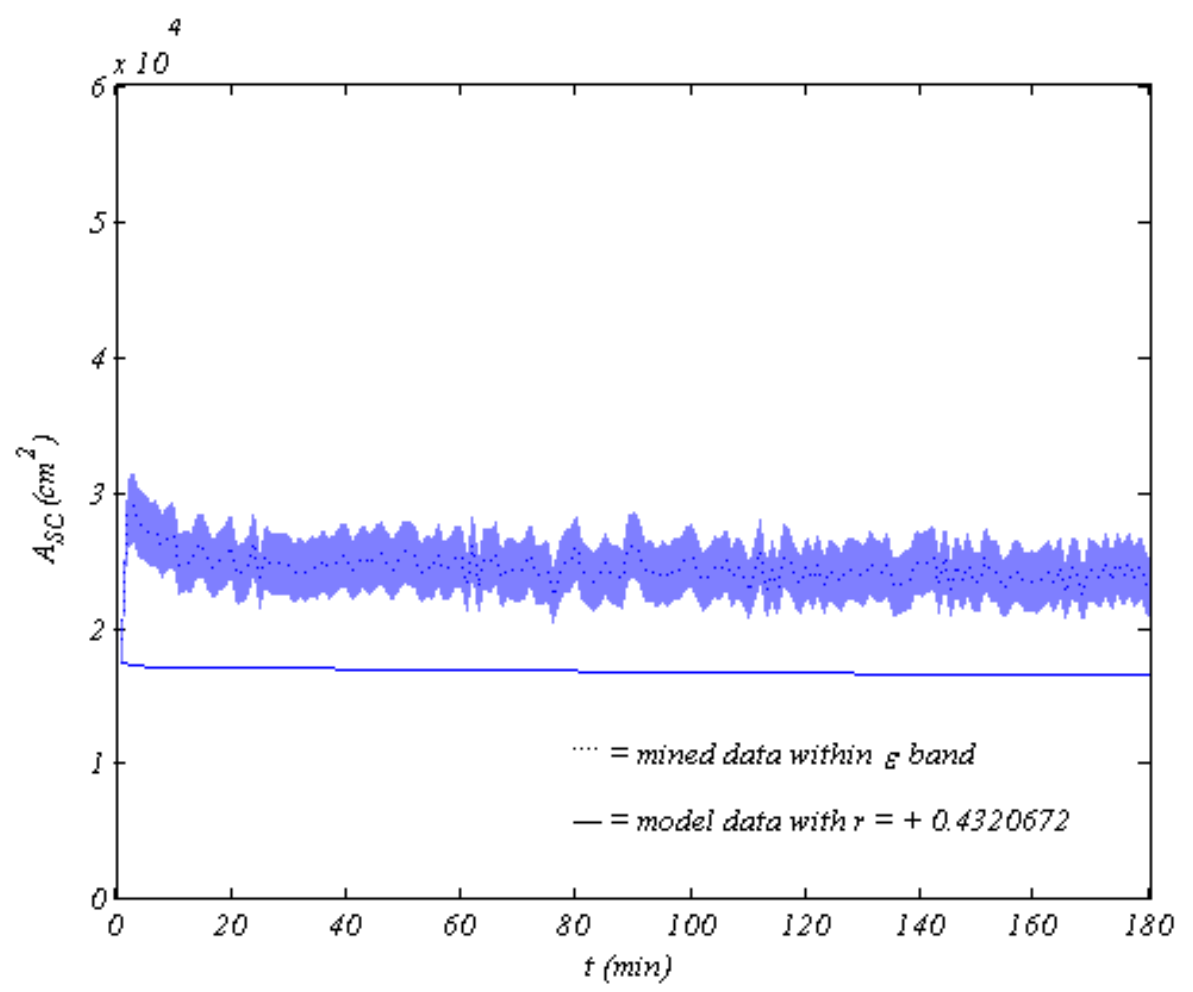

Figure 106. Required solar collection area at $50^{\circ} \mathrm{C}$ flash and lower flow

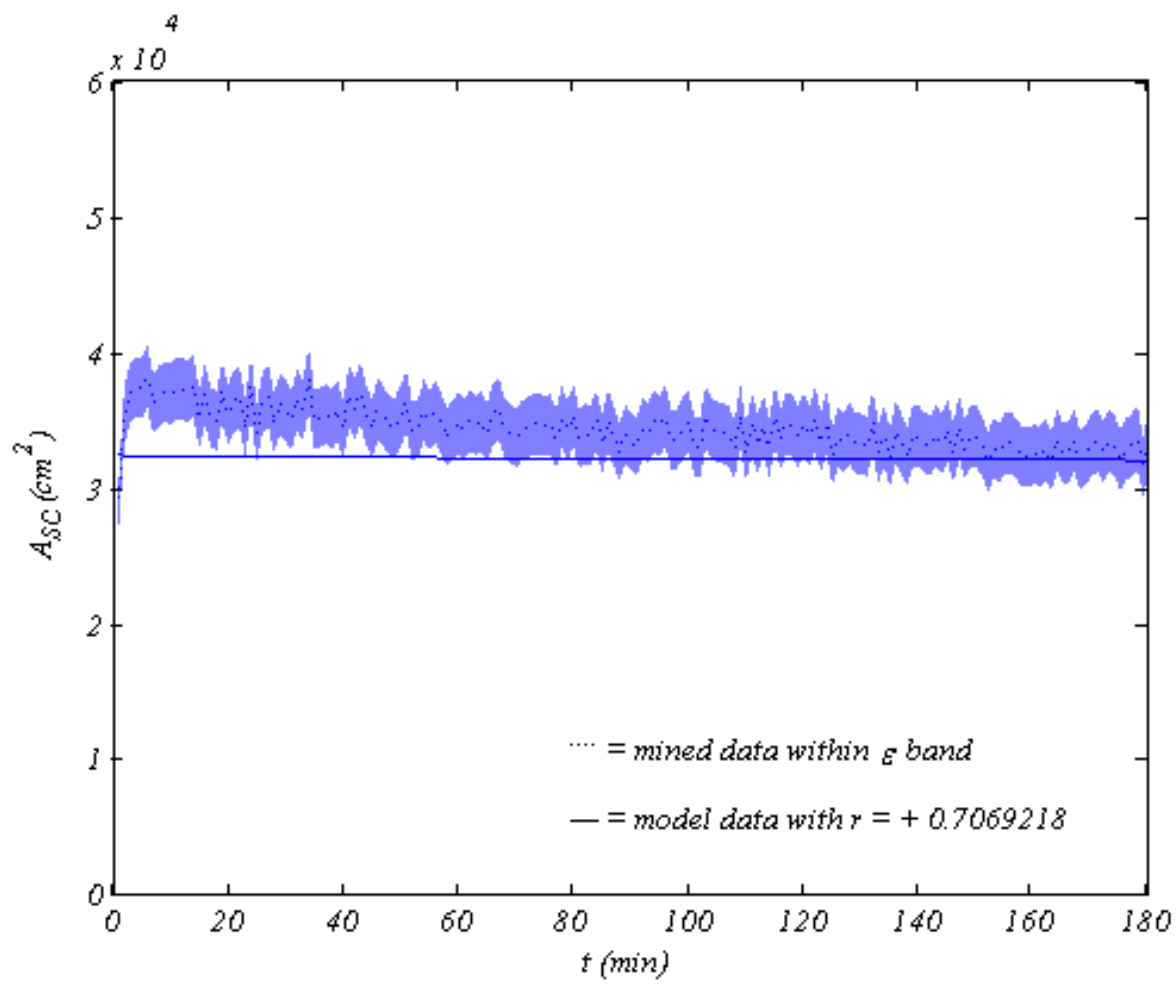

Figure 107. Required solar collection area at $50^{\circ} \mathrm{C}$ flash and higher flow 


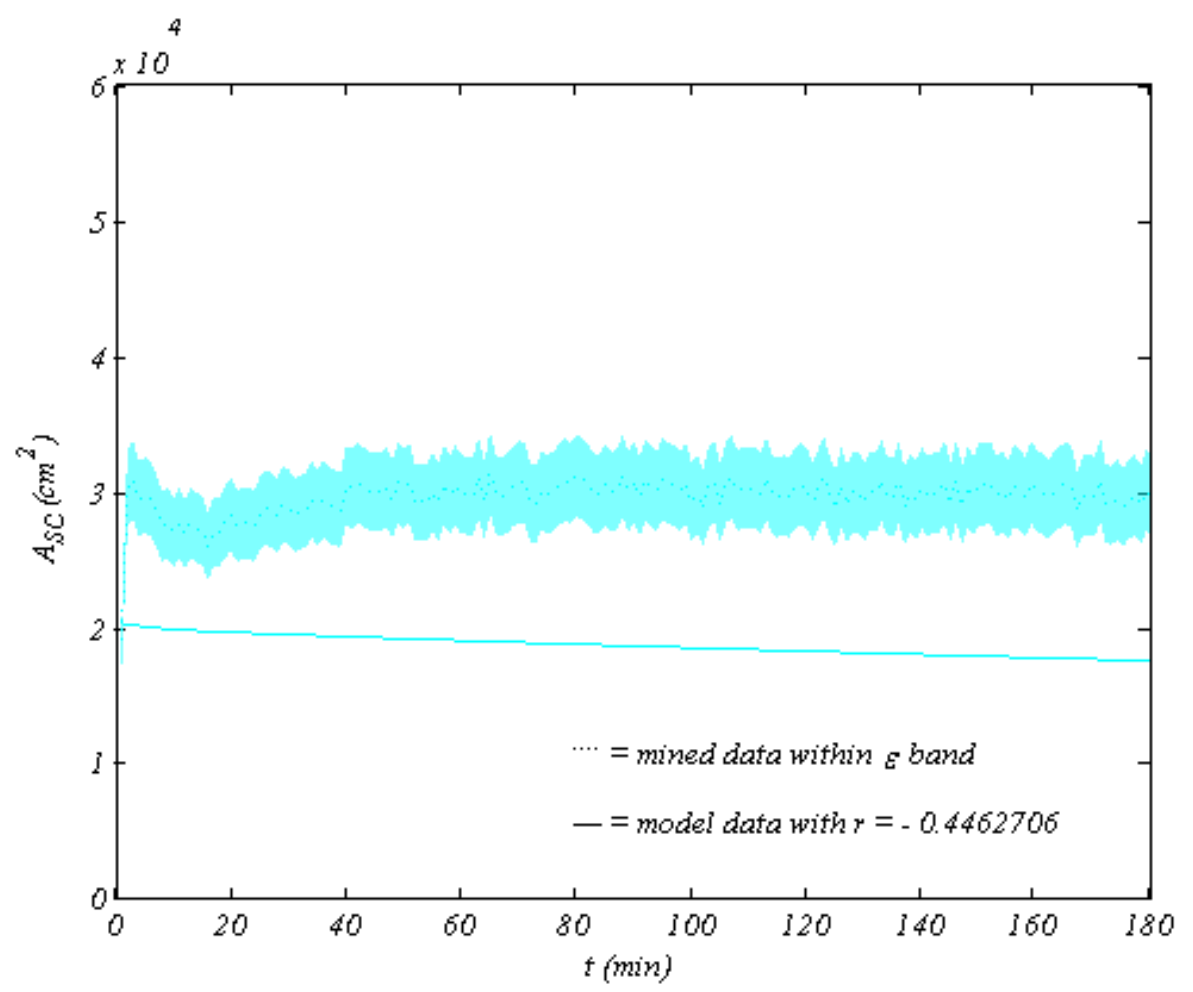

Figure 108. Required solar collection area at $60^{\circ} \mathrm{C}$ flash and lower flow

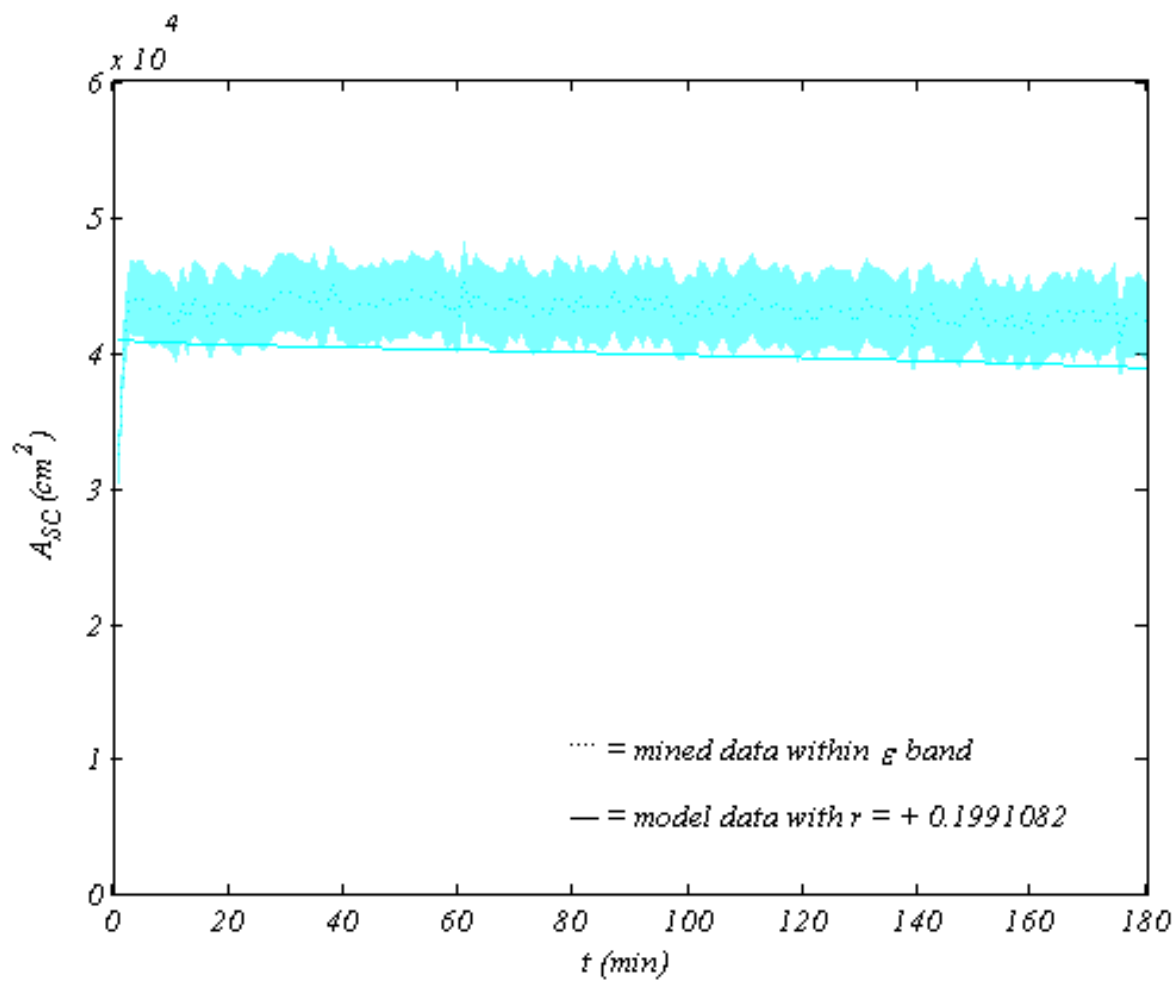

Figure 109. Required solar collection area at $60^{\circ} \mathrm{C}$ flash and higher flow 


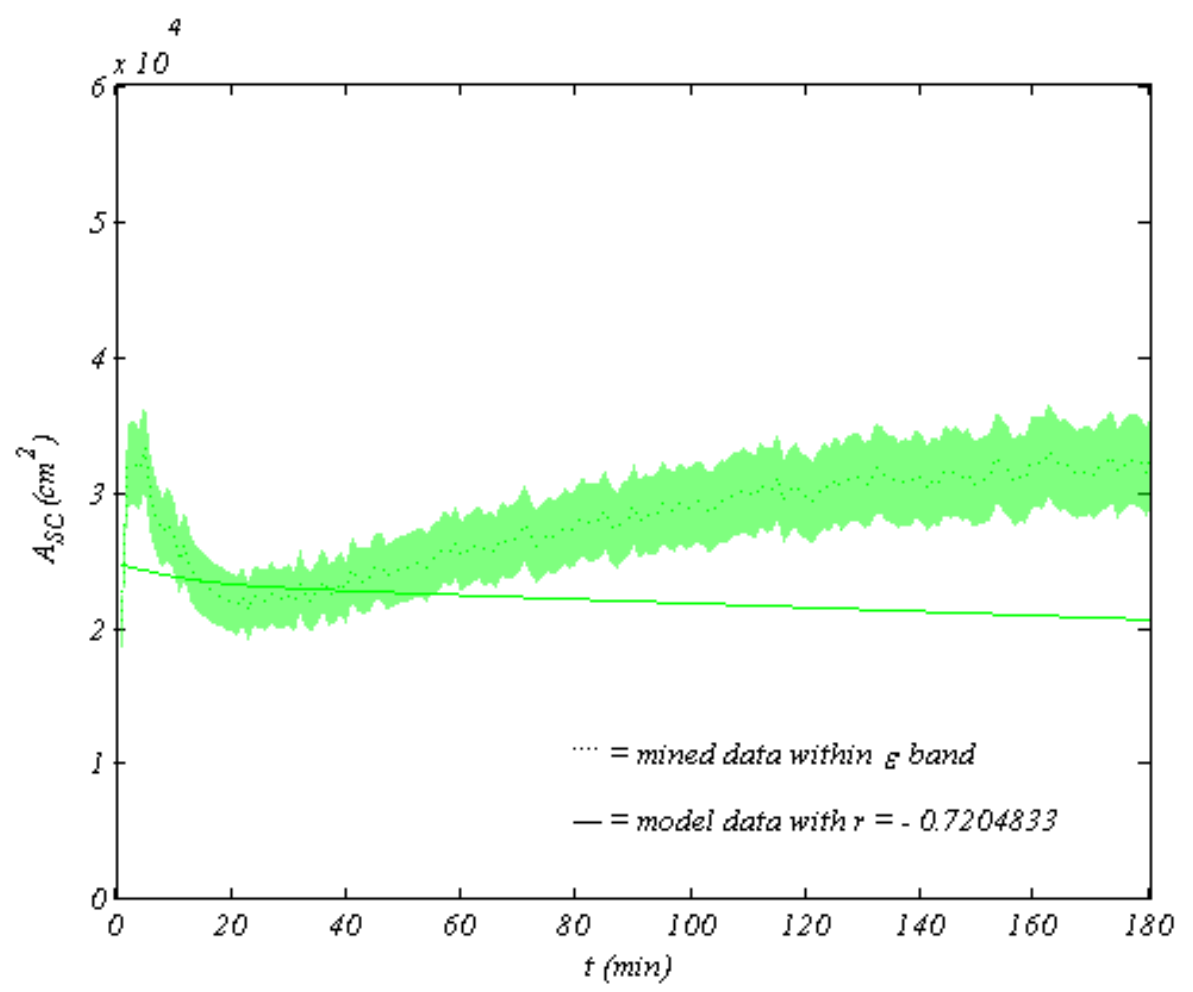

Figure 110. Required solar collection area at $70^{\circ} \mathrm{C}$ flash and lower flow

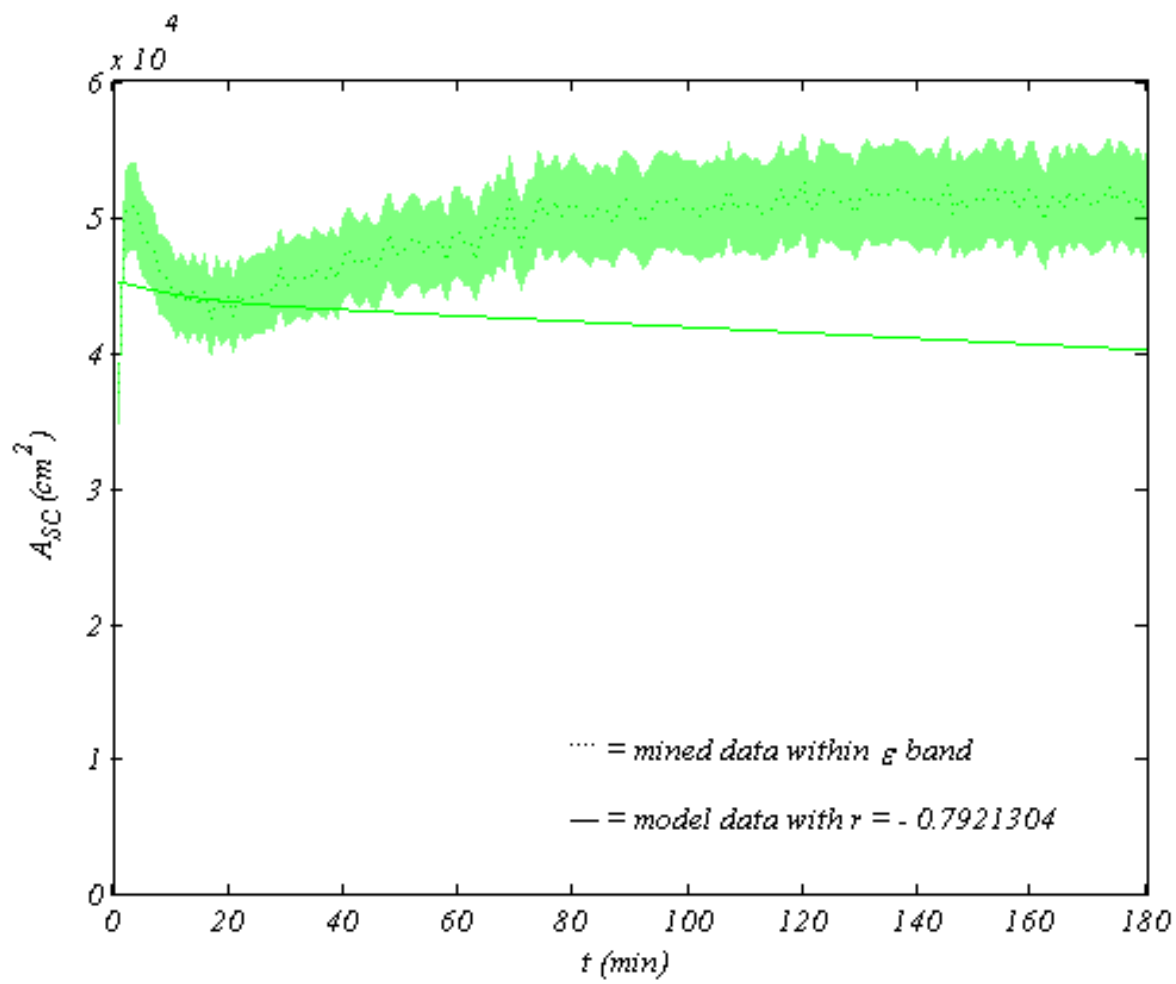

Figure 111. Required solar collection area at $70^{\circ} \mathrm{C}$ flash and higher flow 


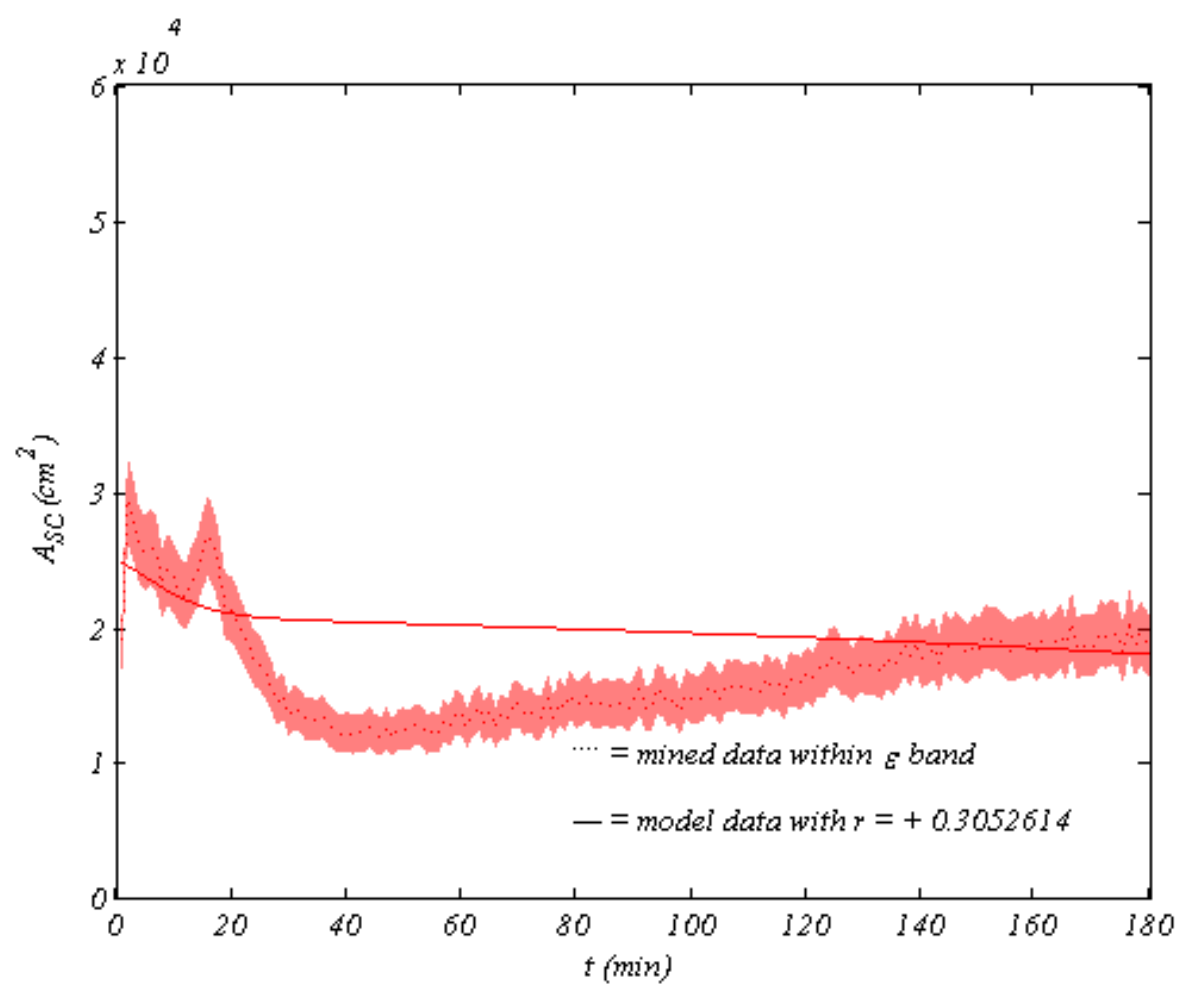

Figure 112. Required solar collection area at $80^{\circ} \mathrm{C}$ flash and lower flow

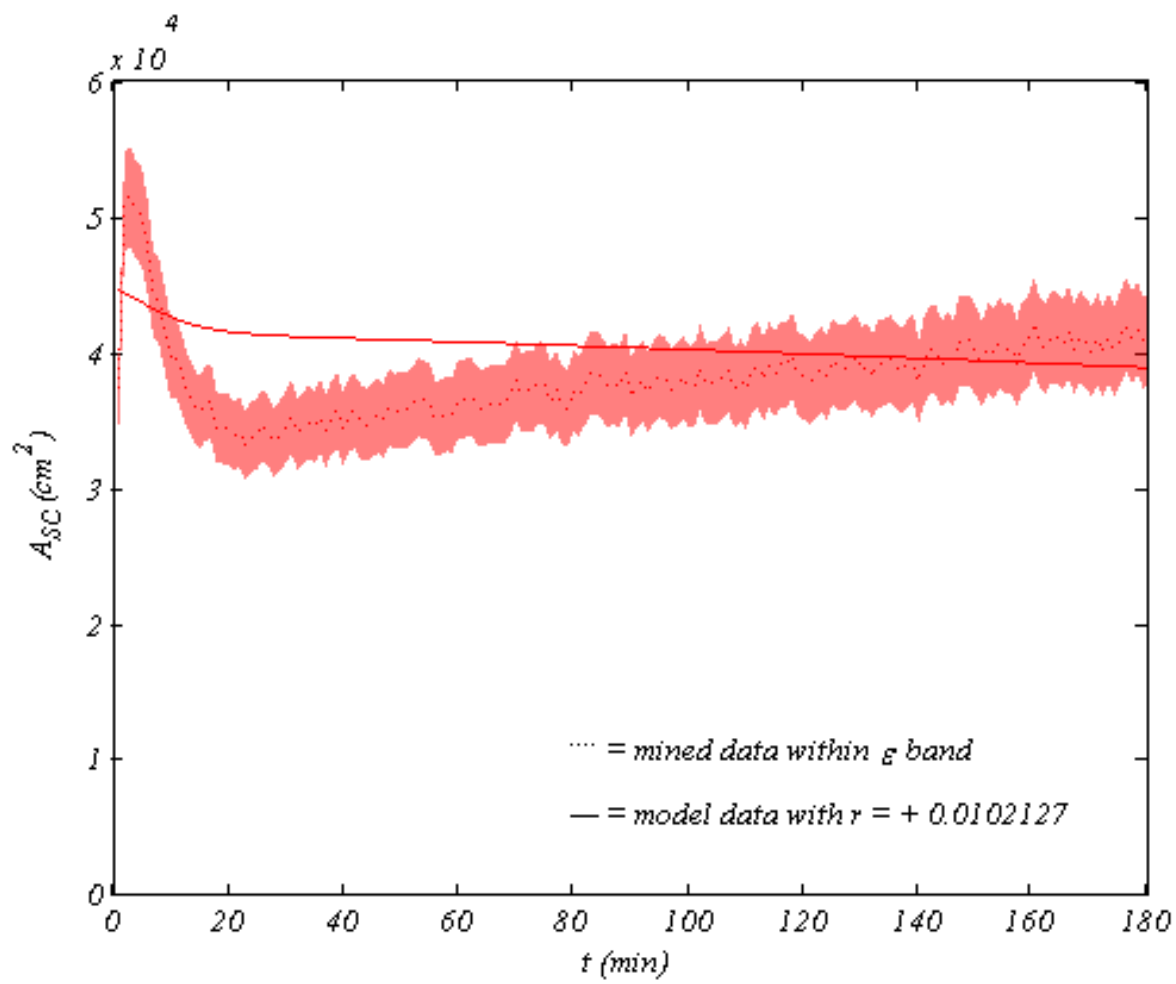

Figure 113. Required solar collection area at $80^{\circ} \mathrm{C}$ flash and higher flow 


\subsection{System Throughput}

The fresh water produced is due to condensation of flashed and diffused water vapor moving from the evaporator to condenser as was mentioned in CHAPTER 4. Fresh water production rate was highest at first, then slowly declined for both seawater flow rates; moreover, it was generally higher for lower seawater flow rates. The difference between fresh water production rates of both seawater flow rates is more significant at medium flash temperatures and seems to diminish at both low and high flash temperatures. Fresh water production rate increased with flash temperatures for both seawater flow rates, since the amounts of flashing and diffusing water vapor are directly proportional to flashing temperature and the temperature-induced vapor pressure gradient between the evaporator and condenser, respectively.

Fresh water production rate was high at first due to the high initial rate of vaporization caused by the rapidly increasing equilibrium temperature at lower vacuum pressures, then it slowly declined due to the decreasing rate of vaporization caused by the stabilizing equilibrium temperature at rising vacuum pressures, almost reaching a plateau comparable to the flash temperature for both seawater flow rates. Fresh water production rate profiles for both seawater flow rates are similar; however, they were higher for lower flow rates due to more condensation caused by more vaporization.

Model prediction of fresh water production rate appropriately resembled the pseudo-experimental results due to adequate prediction of system vacuum and equilibrium temperature. Furthermore, adjusted parameters played a significant role in shifting the profile of fresh water production rate to match pseudo-experimental results. Fresh water production rate profiles are shown in Figure 114 through Figure 125. 


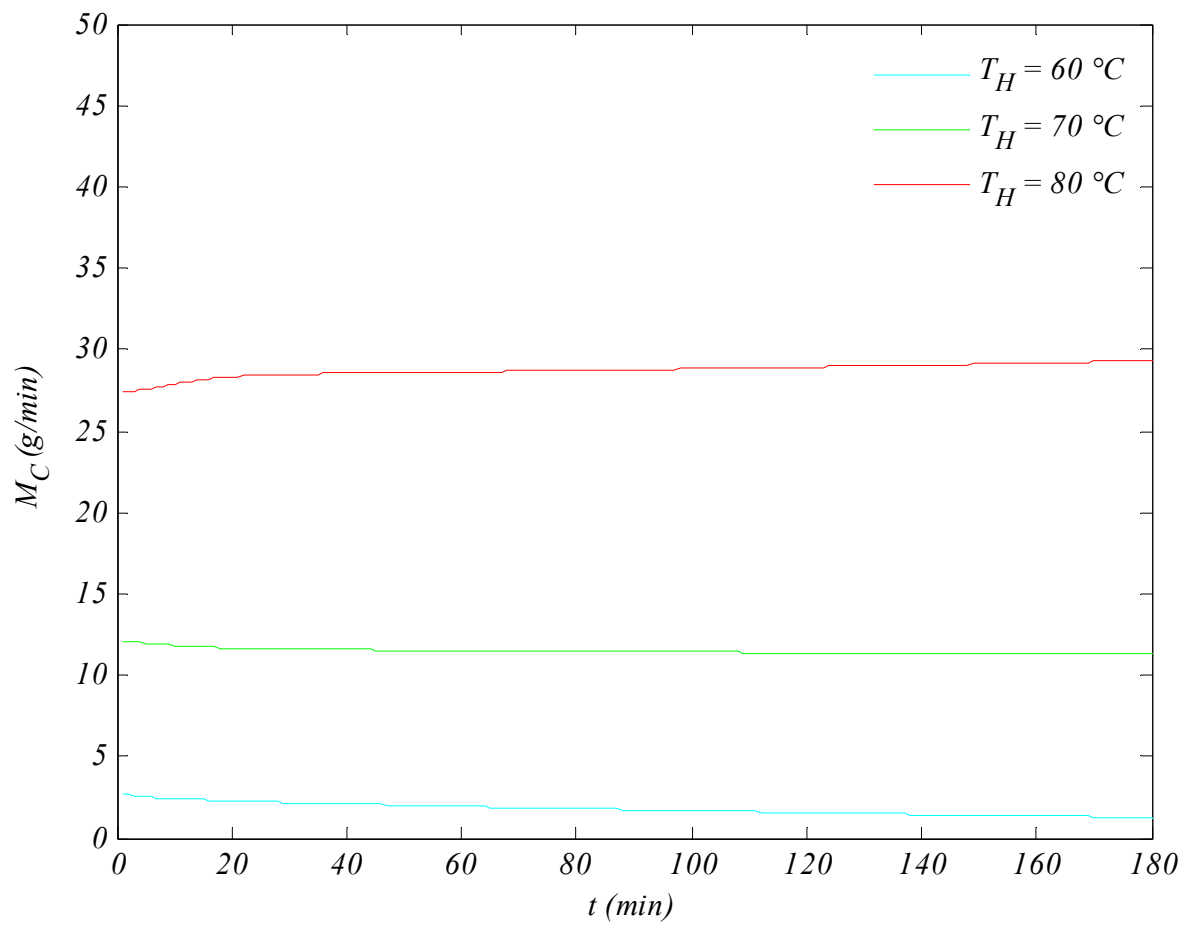

Figure 114. Modeled fresh water production rate profiles at lower flow

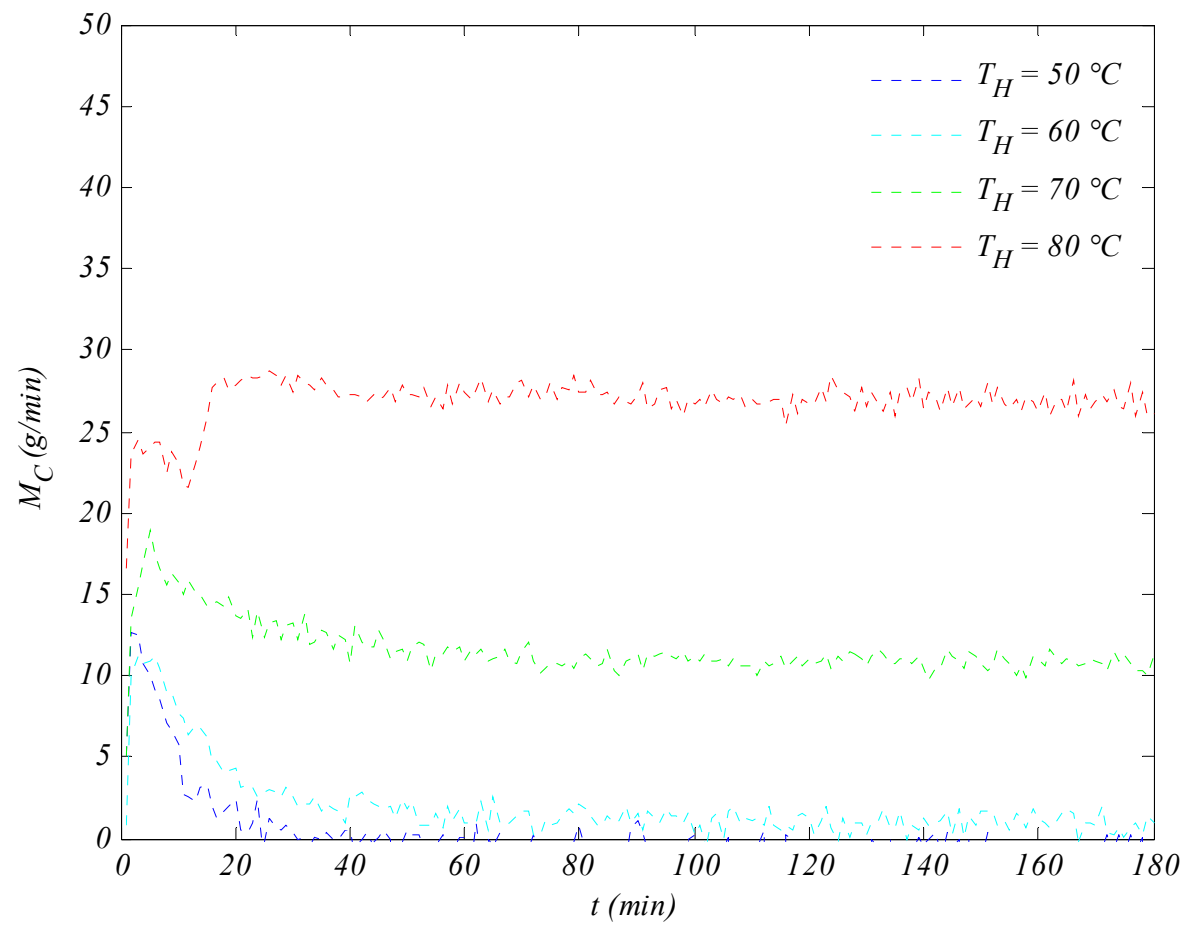

Figure 115. Mined fresh water production rate profiles at lower flow 


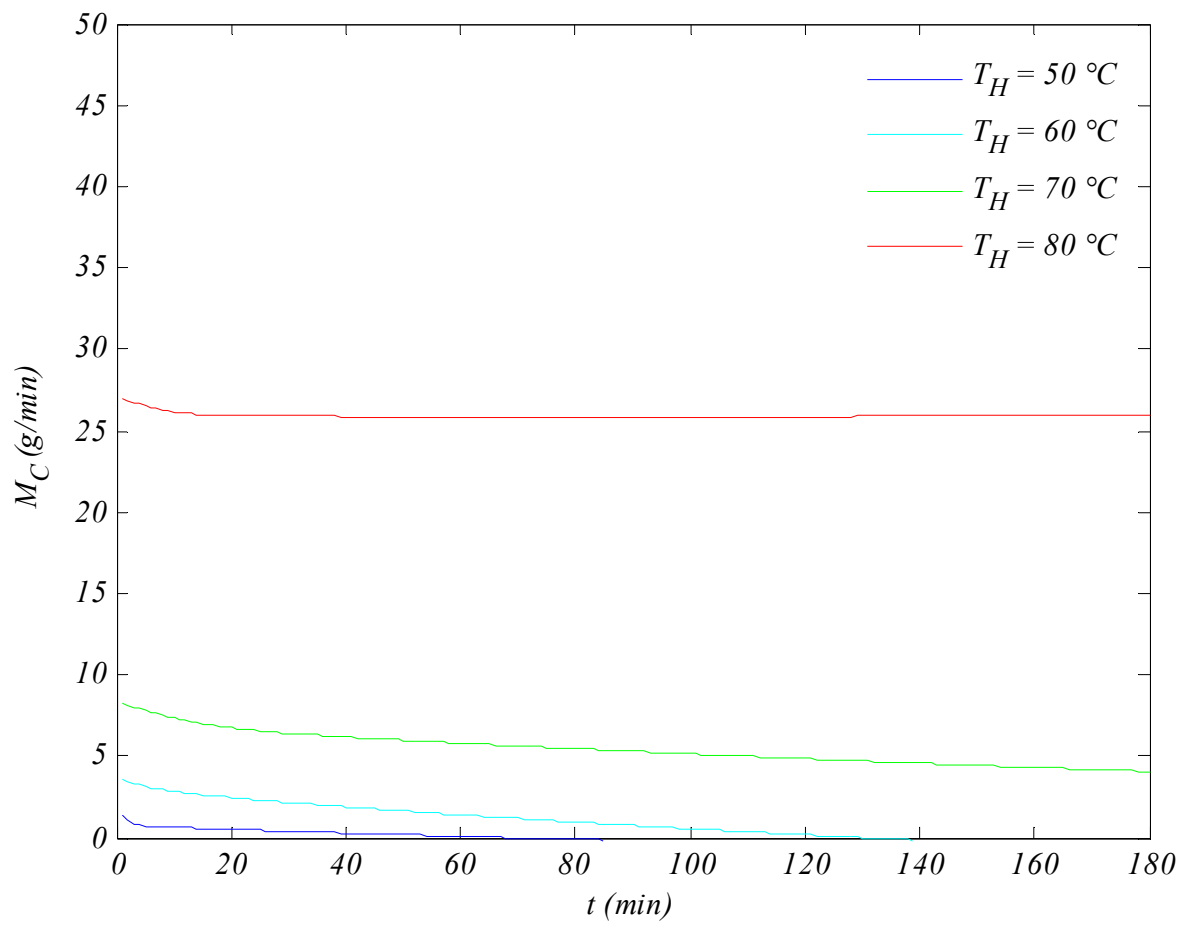

Figure 116. Modeled fresh water production rate profiles at higher flow

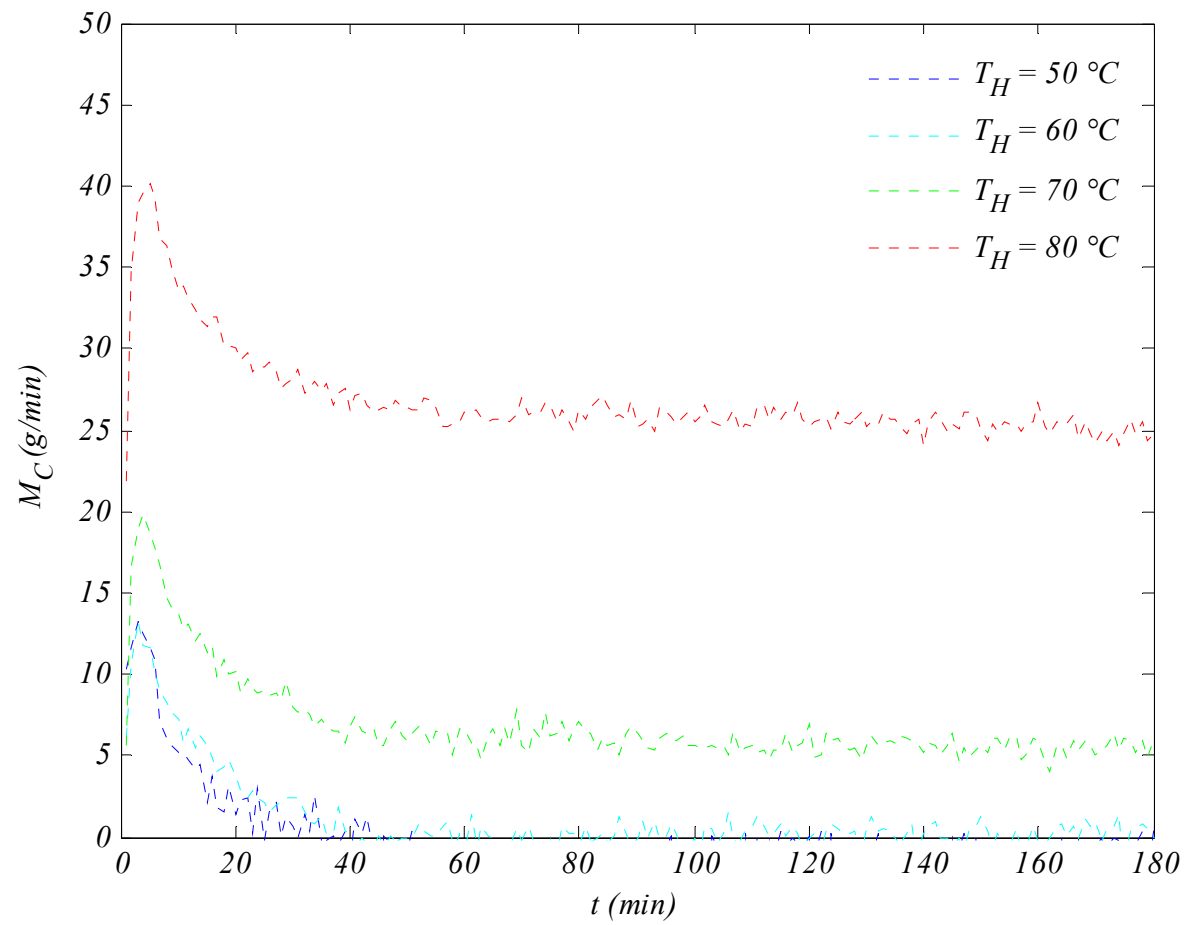

Figure 117. Mined fresh water production rate profiles at higher flow 


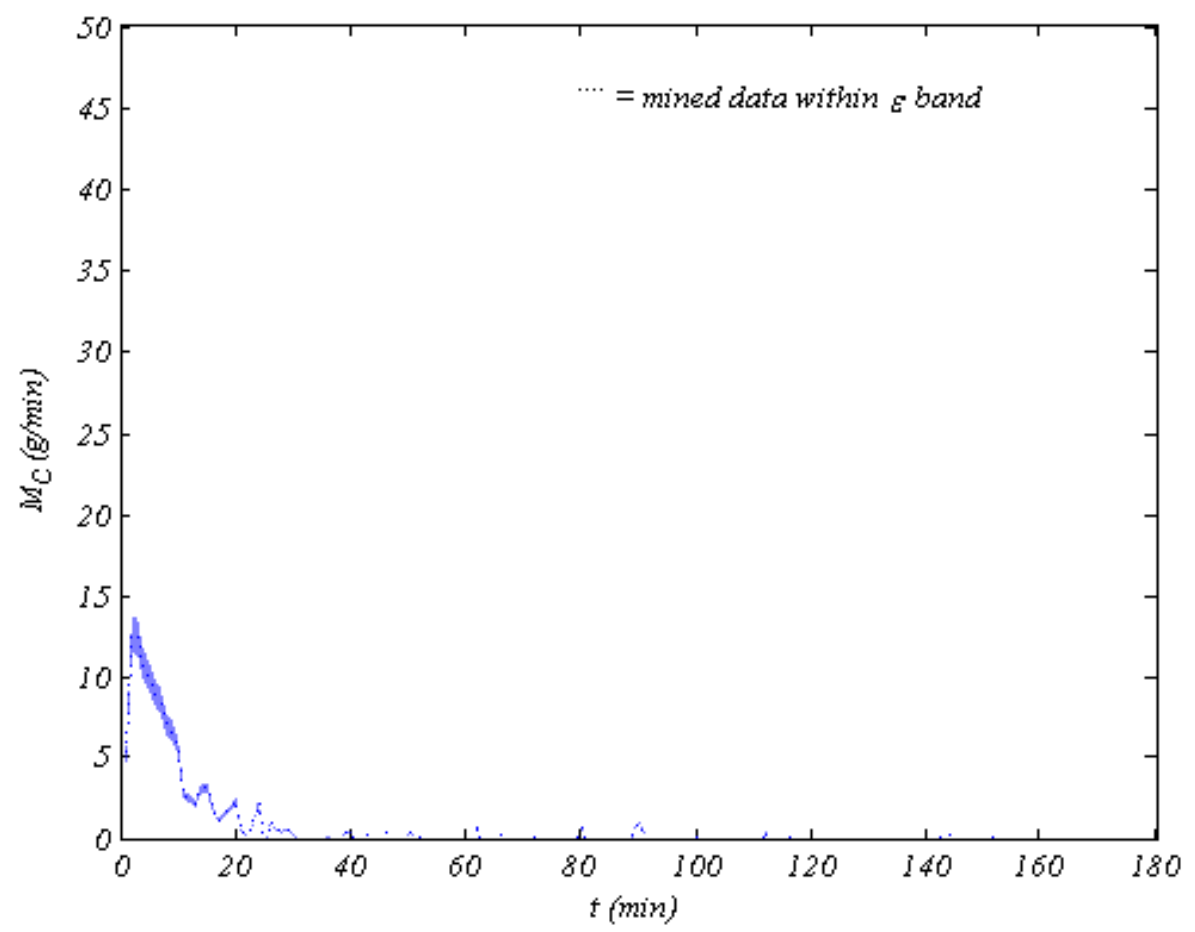

Figure 118. Fresh water production rate at $50^{\circ} \mathrm{C}$ flash and lower flow

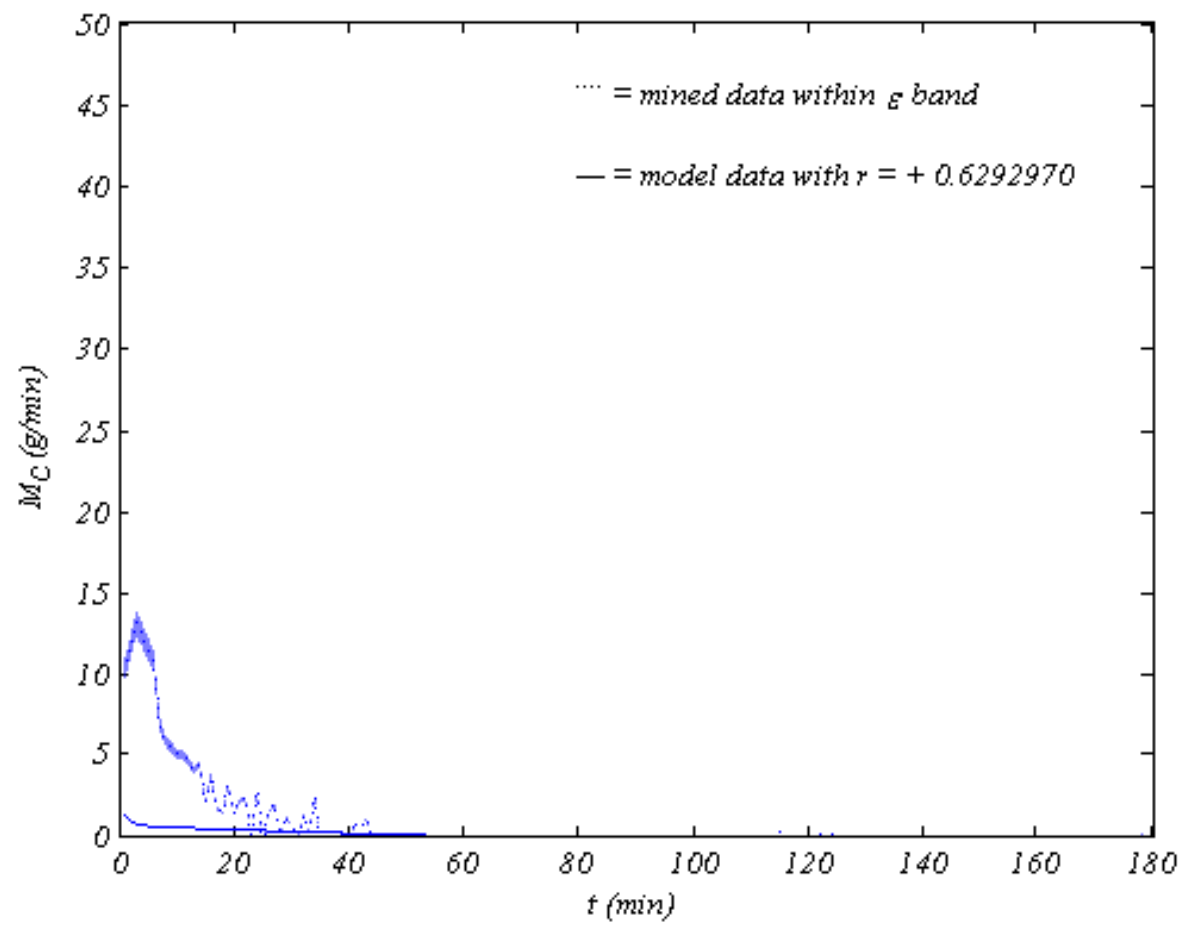

Figure 119. Fresh water production rate at $50^{\circ} \mathrm{C}$ flash and higher flow 


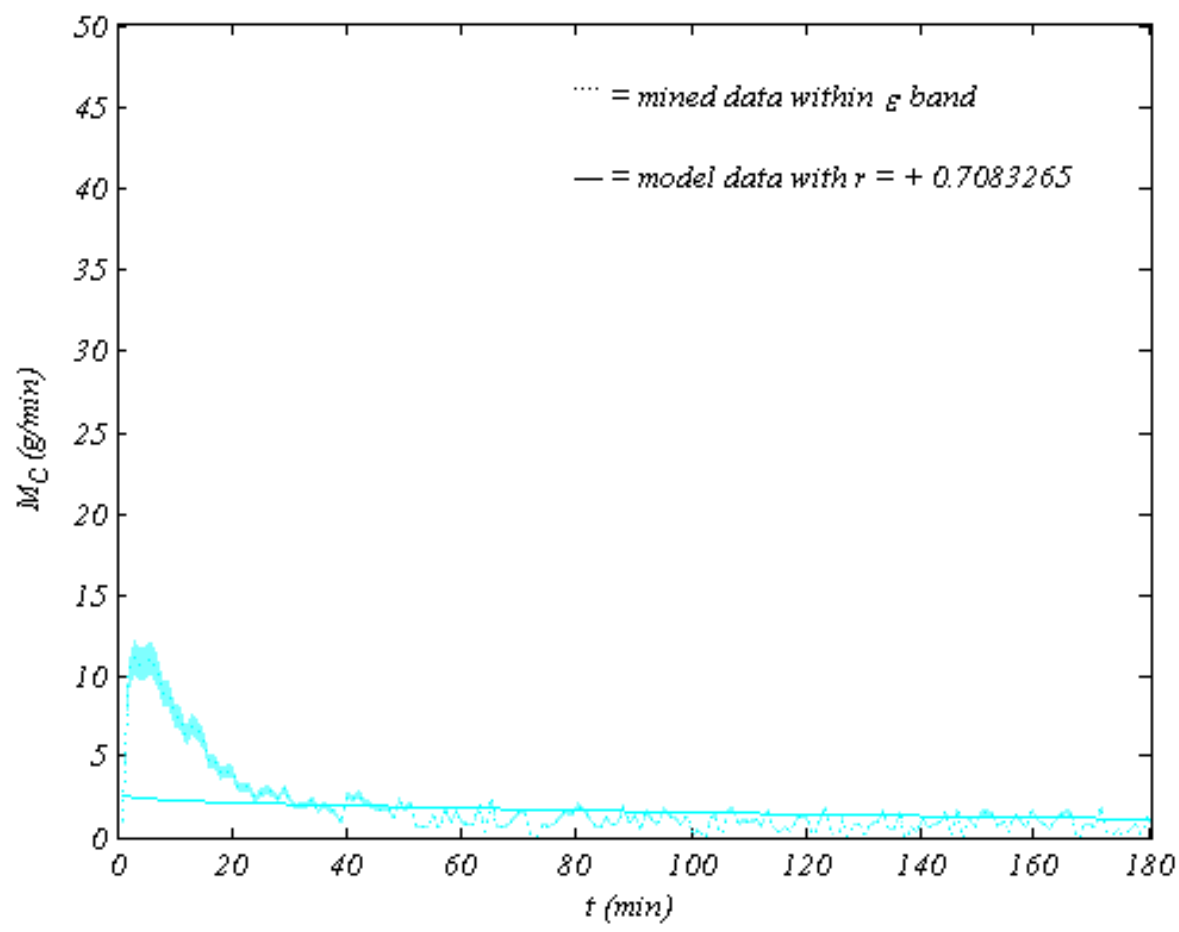

Figure 120. Fresh water production rate at $60^{\circ} \mathrm{C}$ flash and lower flow

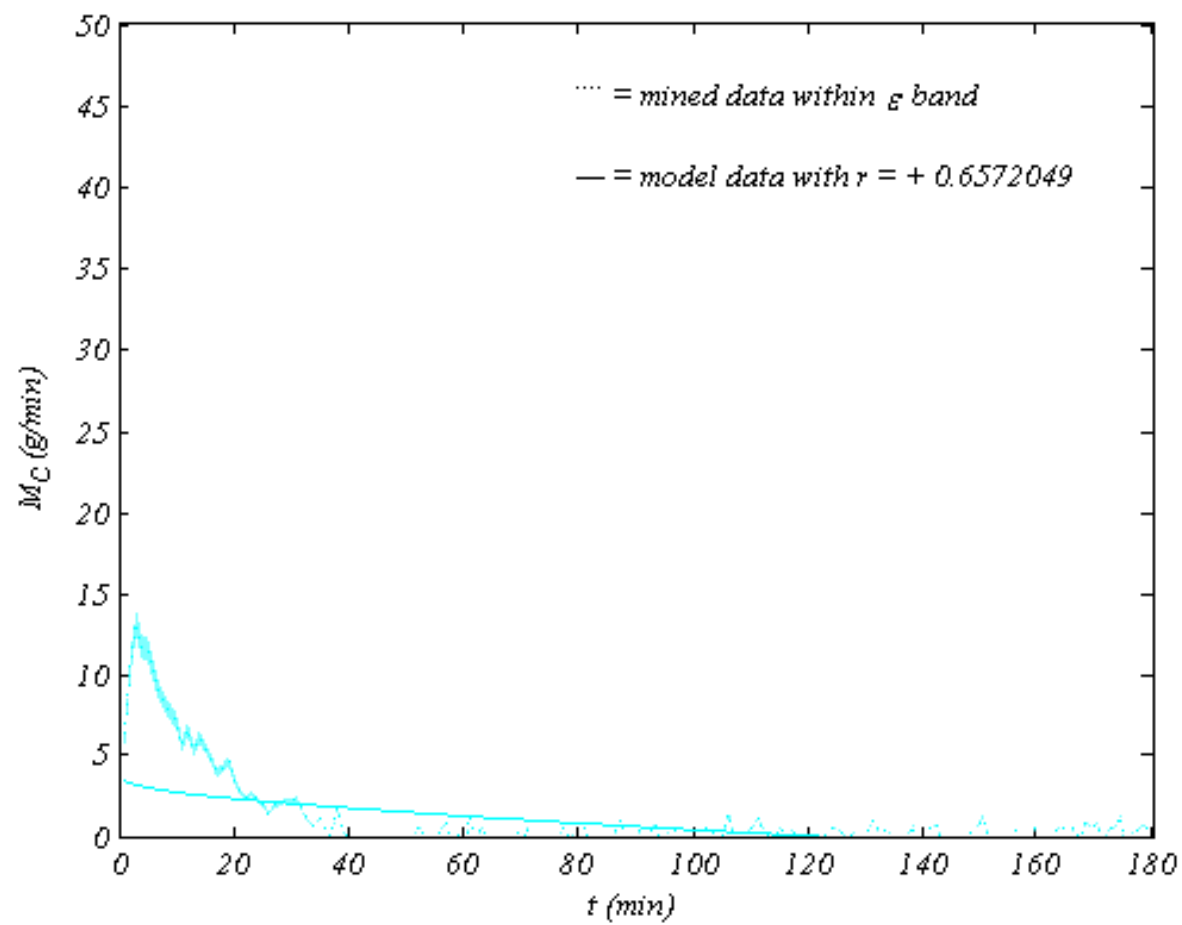

Figure 121. Fresh water production rate at $60^{\circ} \mathrm{C}$ flash and higher flow 


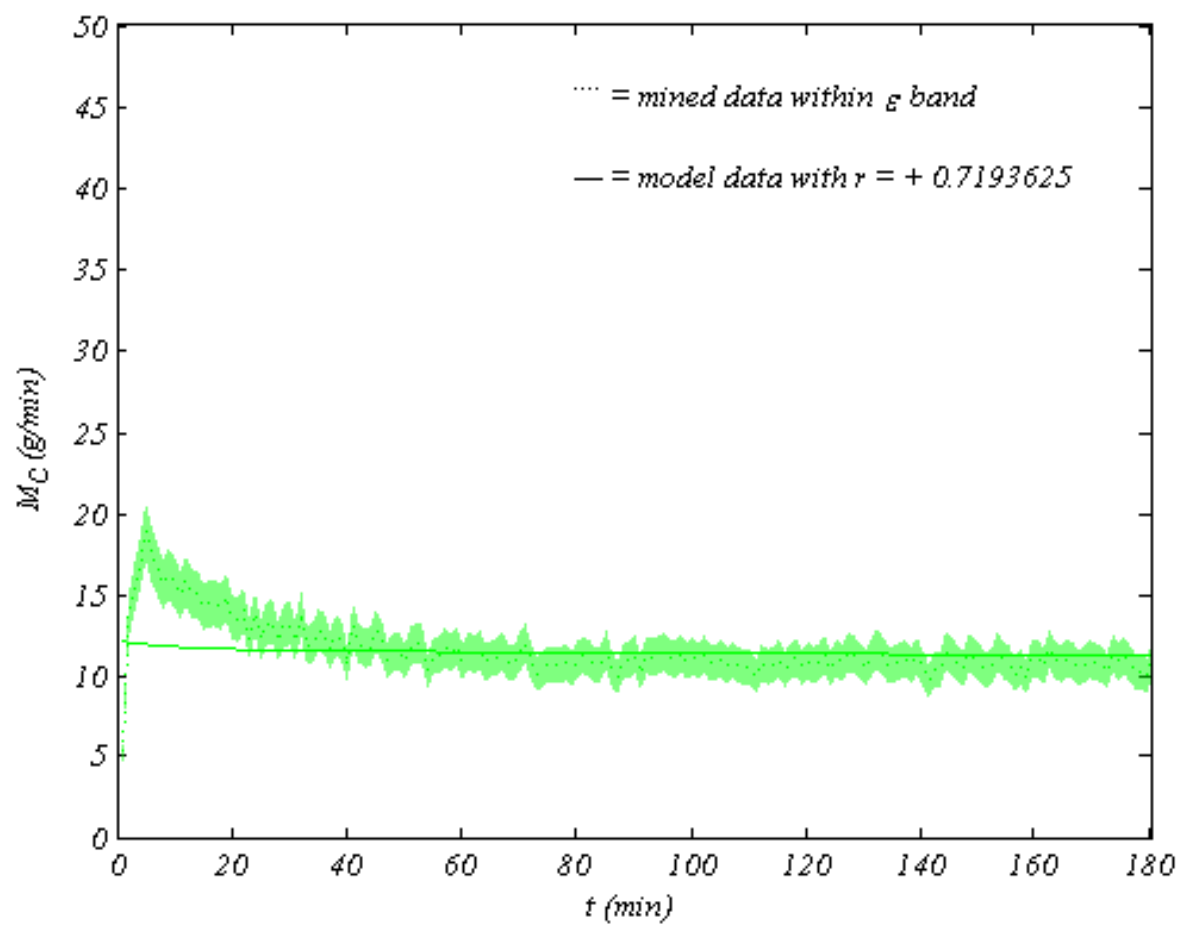

Figure 122. Fresh water production rate at $70^{\circ} \mathrm{C}$ flash and lower flow

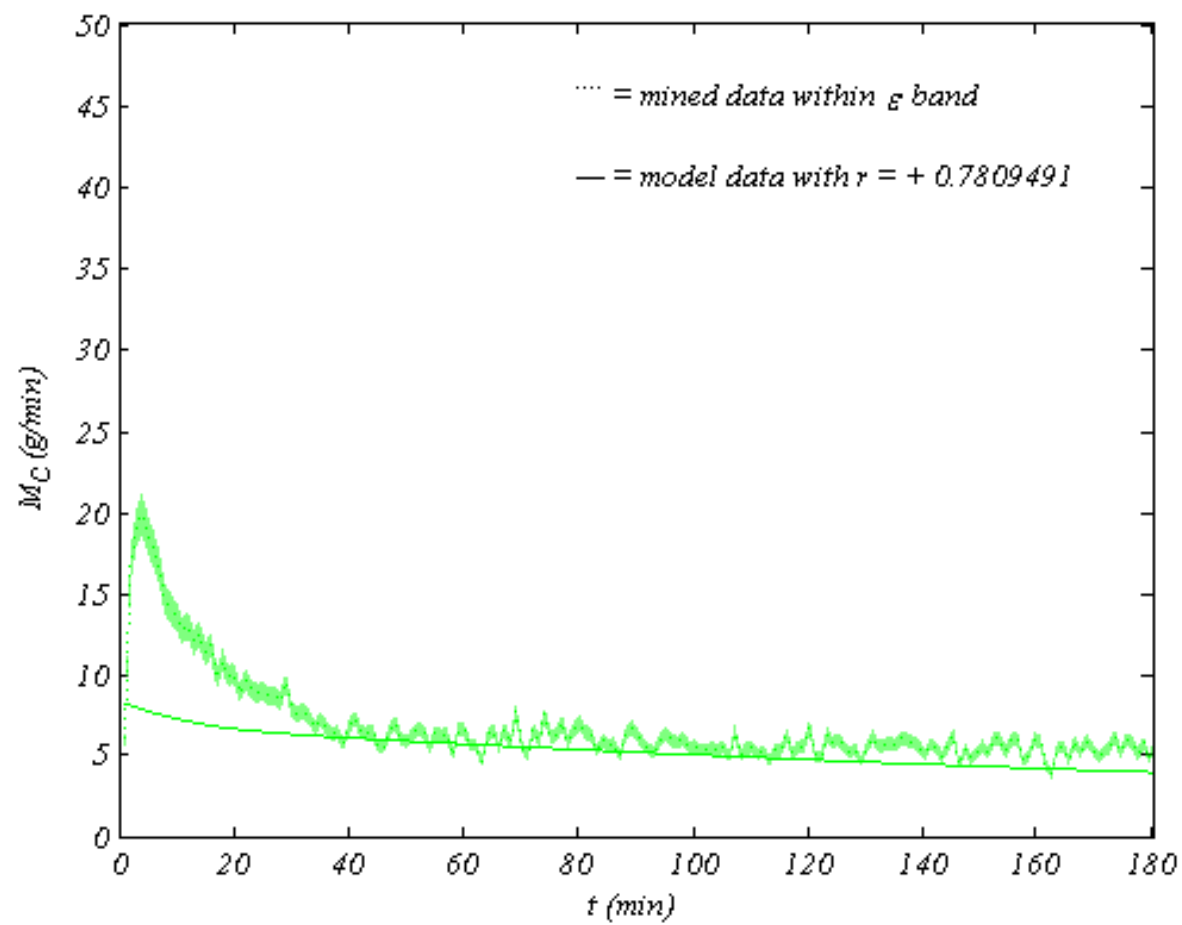

Figure 123. Fresh water production rate at $70^{\circ} \mathrm{C}$ flash and higher flow 


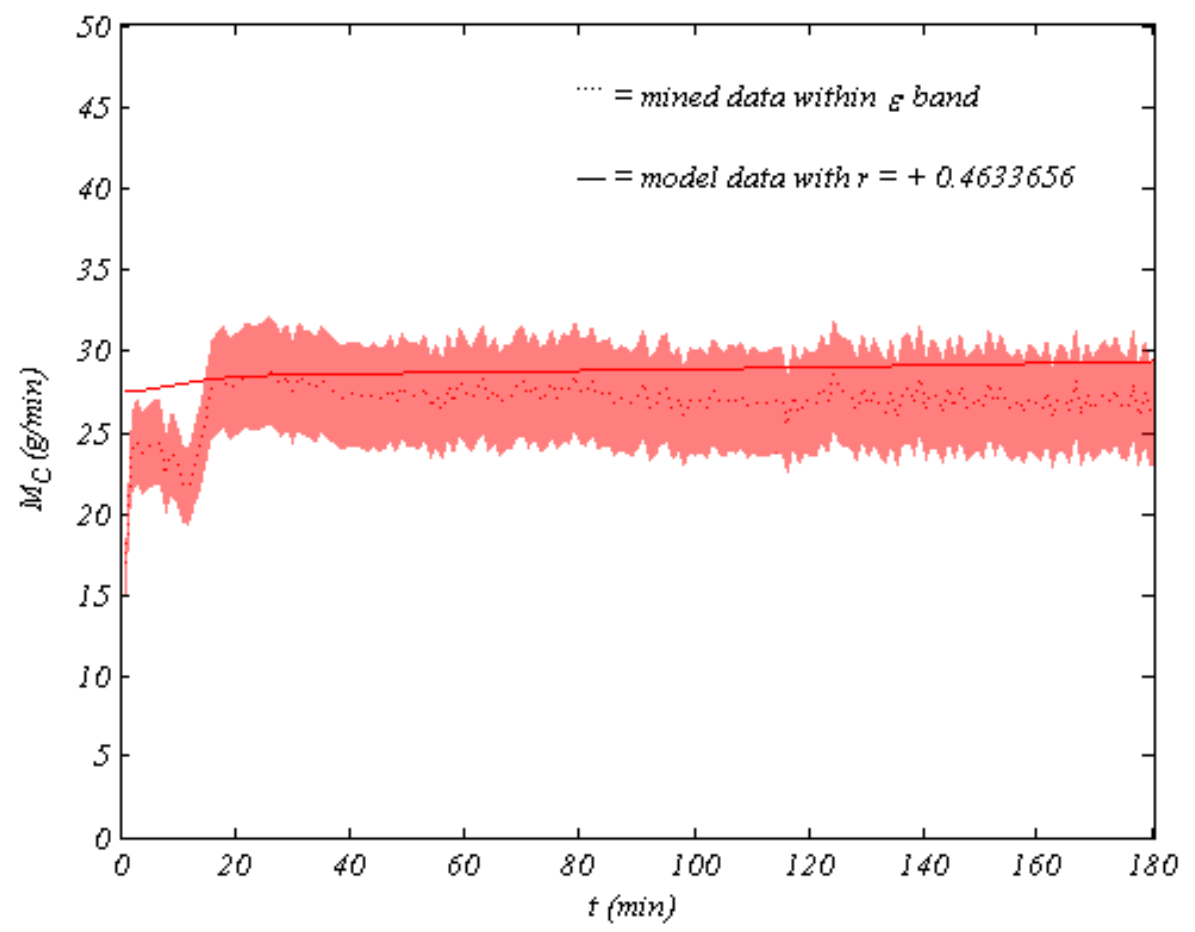

Figure 124. Fresh water production rate at $80^{\circ} \mathrm{C}$ flash and lower flow

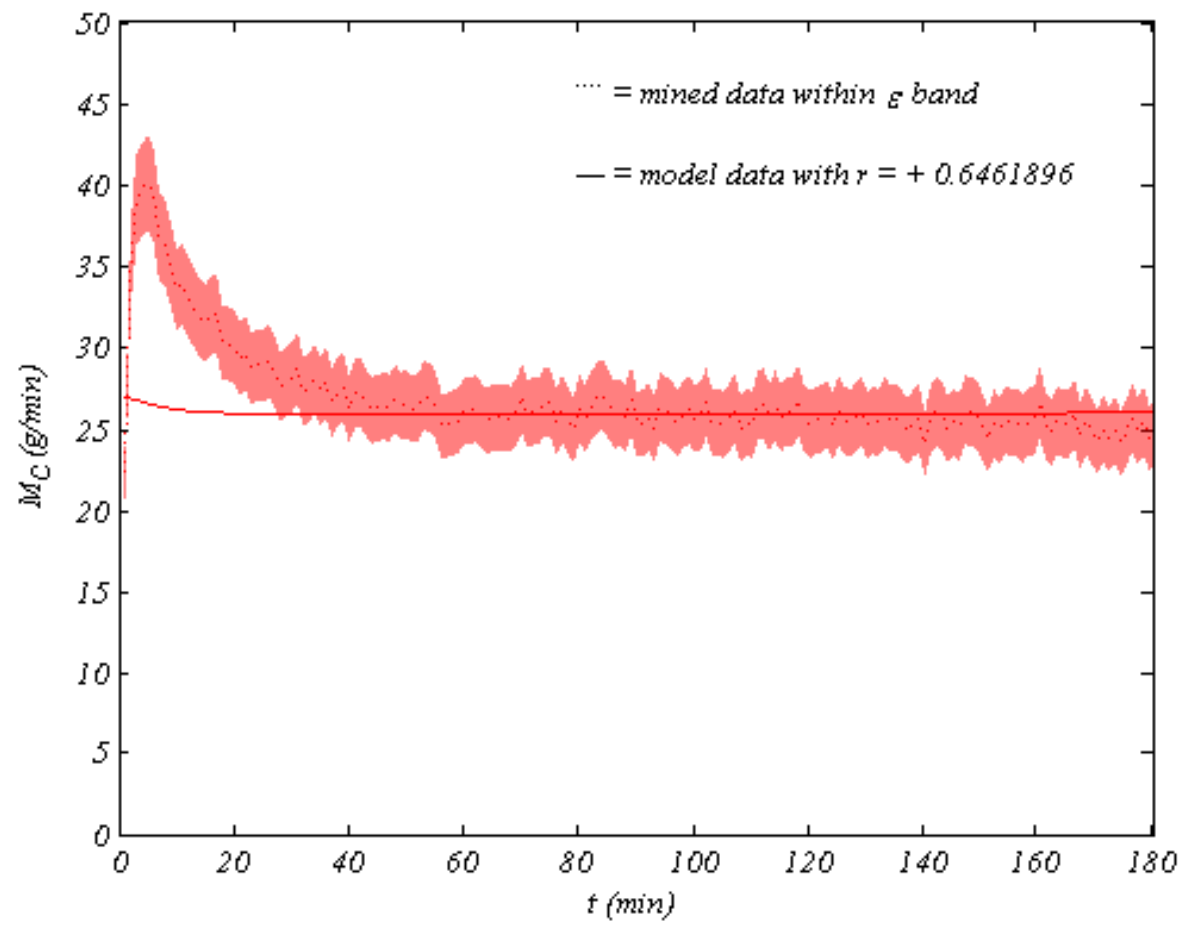

Figure 125. Fresh water production rate at $80^{\circ} \mathrm{C}$ flash and higher flow 


\subsection{System Capacity}

The total amount of fresh water produced is calculated by numerically integrating the dynamic fresh water production rate over the entire operating period as was revealed in CHAPTER 4. Fresh water production amount was rising for both seawater flow rates; moreover, it was generally higher for lower seawater flow rates. The difference between fresh water production amounts of both seawater flow rates is more obvious at medium flash temperatures and diminishes at both low and high flash temperatures corresponding to the fresh water production rate results. The fresh water production amount increased with flash temperatures for both seawater flow rates in line with the fresh water production rate results due to enhanced evaporation rates at higher flash temperatures according to thermodynamic phase equilibria resulting in more fresh water production.

The profile of fresh water production amount is not exactly linear, as it was rising at a higher rate at first due to the high initial fresh water production rate, as seen earlier for both seawater flow rates. Fresh water production amount profiles for both seawater flow rates are similar; however, they were higher for lower seawater flow rates due to higher fresh water production rates, as seen earlier.

Model prediction of fresh water production amount properly resembled the pseudo-experimental results due to accurate portrayal of fresh water production rate as a result of adequate prediction of system vacuum and equilibrium temperature mentioned earlier; furthermore, adjusted parameters played a significant role in shifting the fresh water production rate profile to match pseudo-experimental results, resulting in good estimates of fresh water production amount. Fresh water production amount profiles are shown in Figure 126 through Figure 137. 


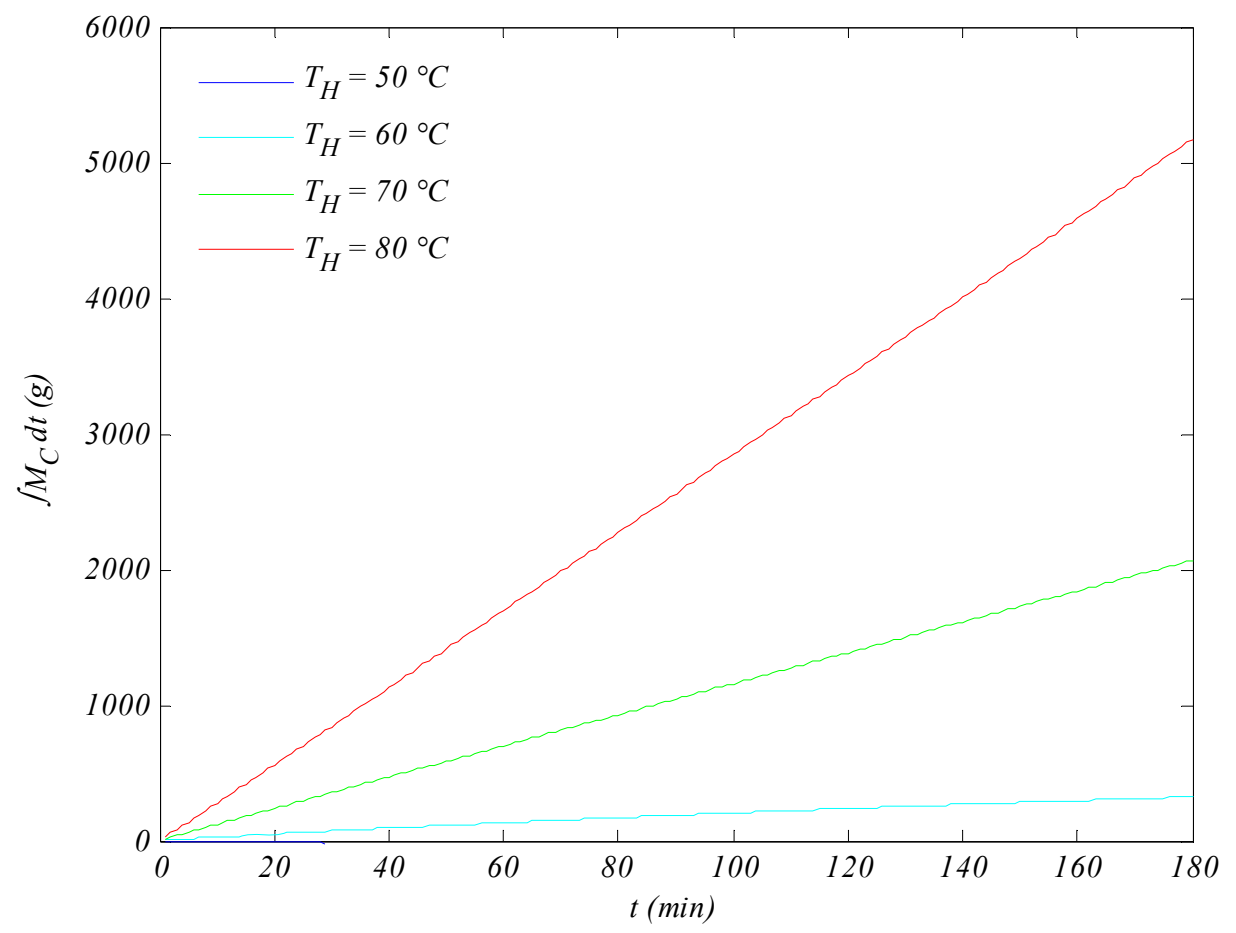

Figure 126. Modeled fresh water production amount profiles at lower flow

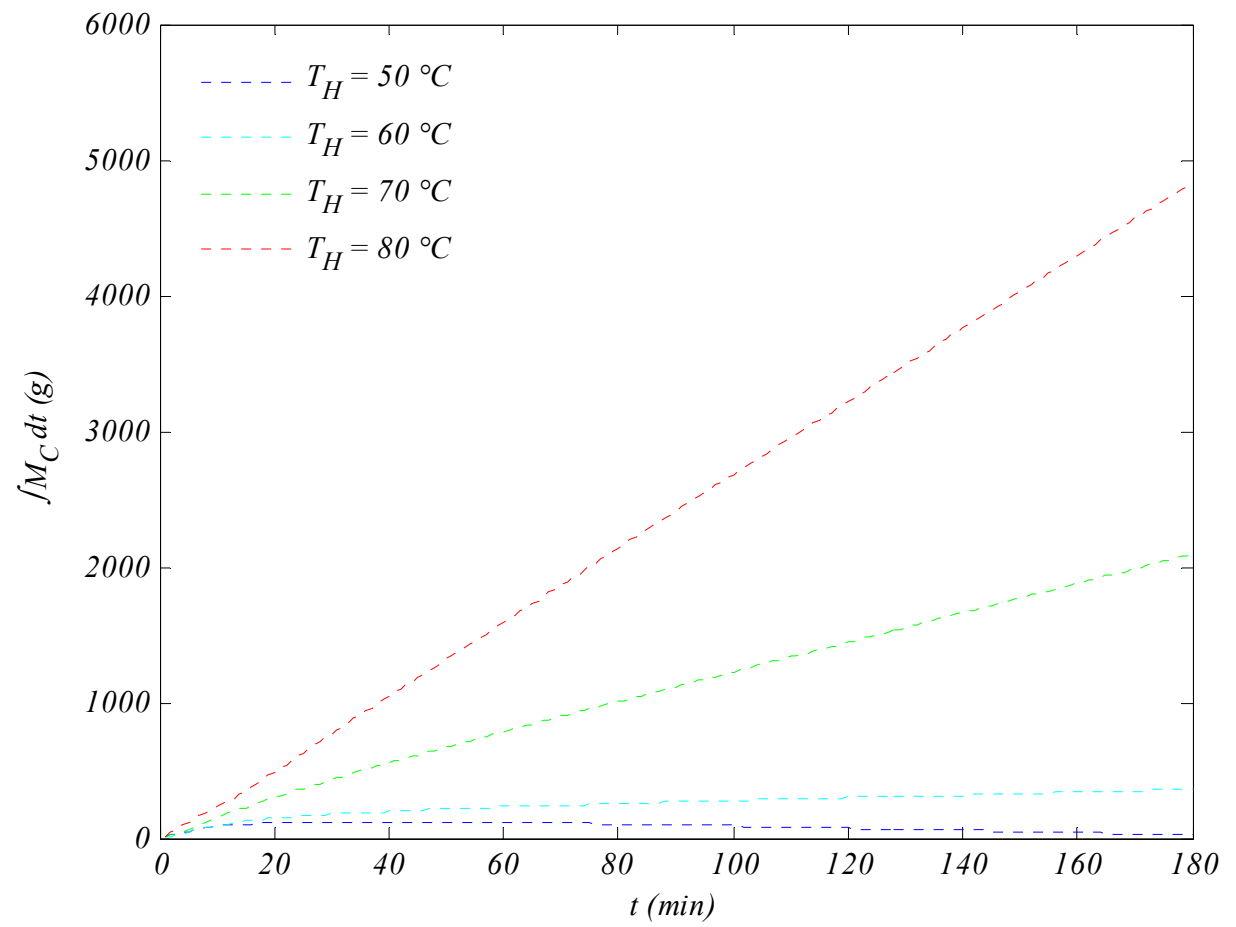

Figure 127. Mined fresh water production amount profiles at lower flow 


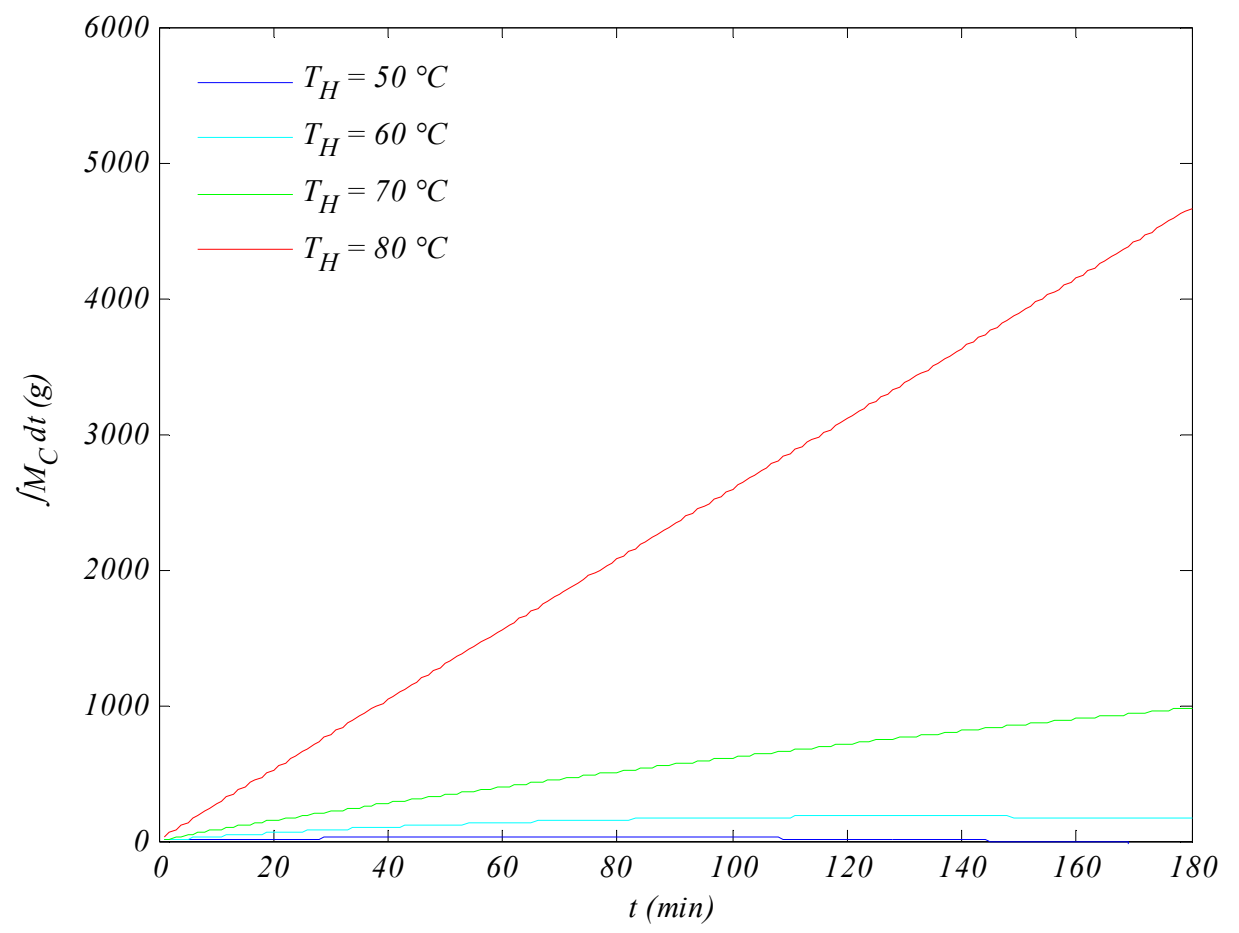

Figure 128. Modeled fresh water production amount profiles at higher flow

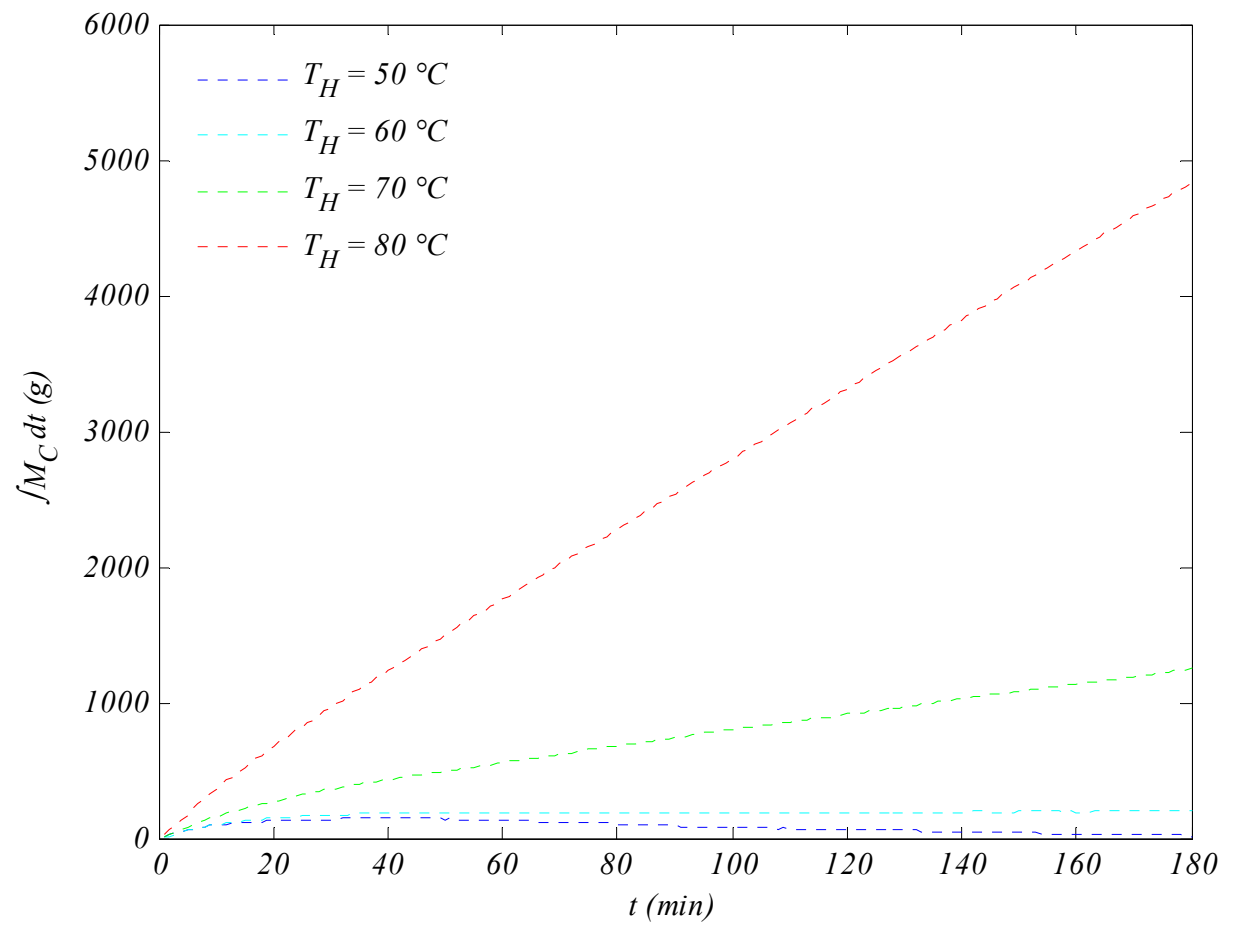

Figure 129. Mined fresh water production amount profiles at higher flow 


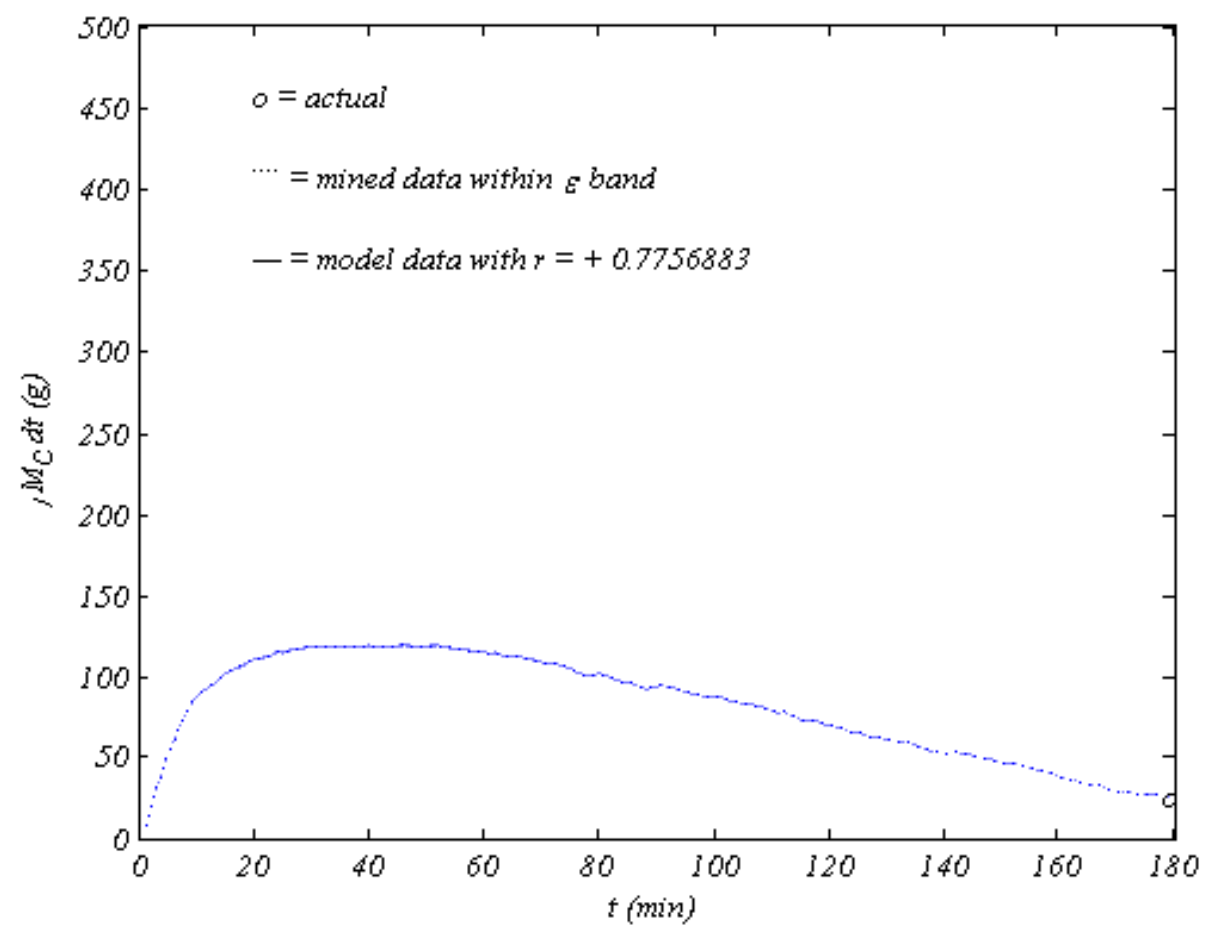

Figure 130. Fresh water production amount at $50^{\circ} \mathrm{C}$ flash and lower flow

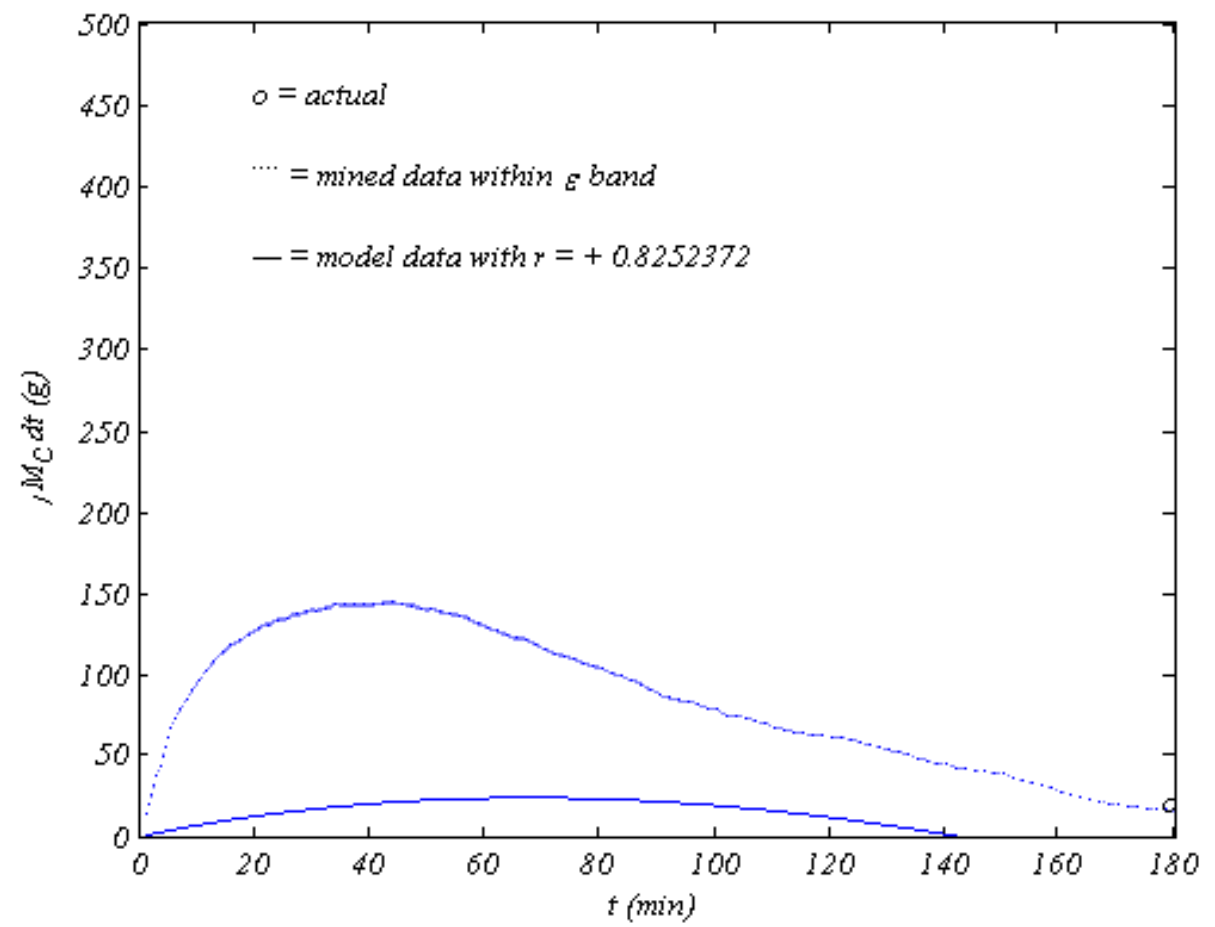

Figure 131. Fresh water production amount at $50^{\circ} \mathrm{C}$ flash and higher flow 


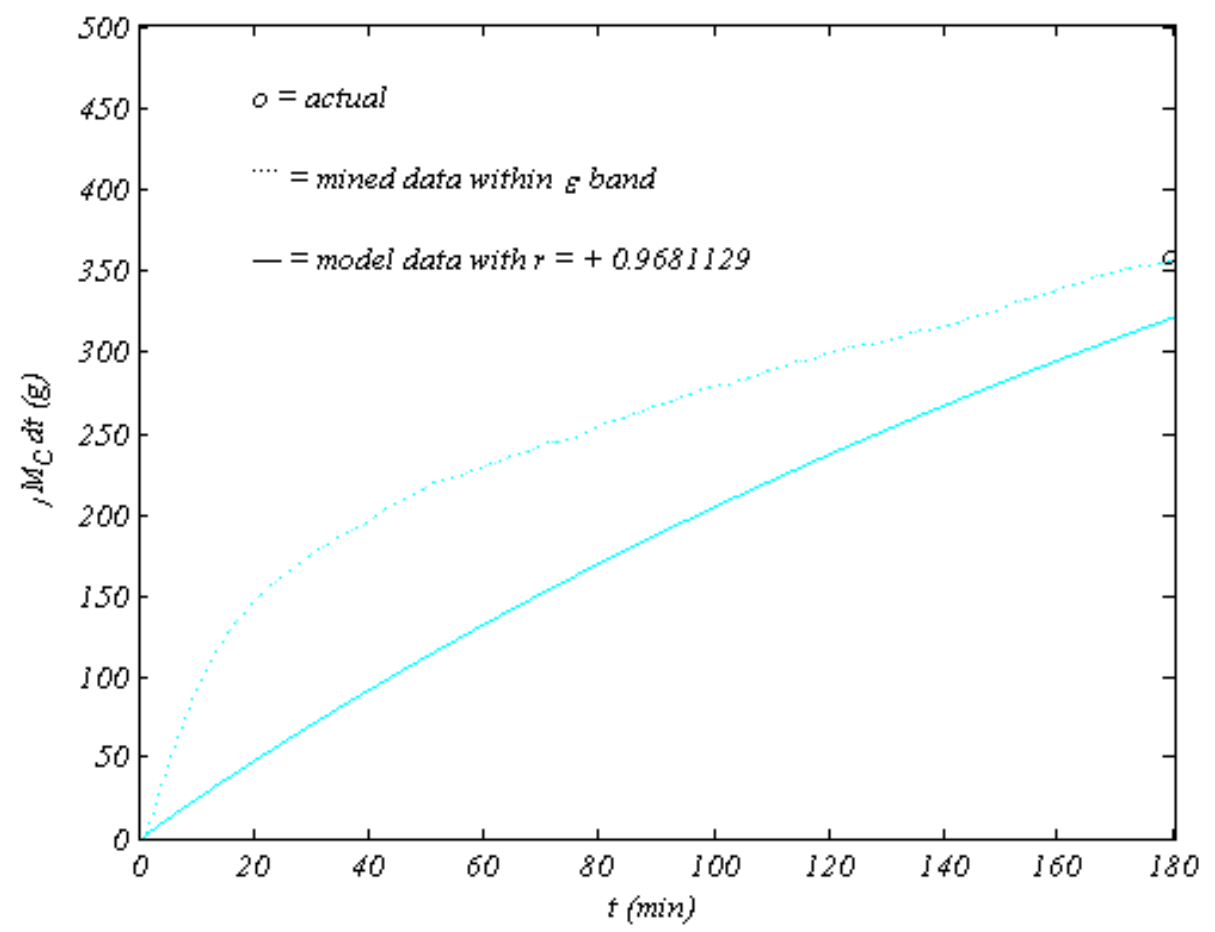

Figure 132. Fresh water production amount at $60^{\circ} \mathrm{C}$ flash and lower flow

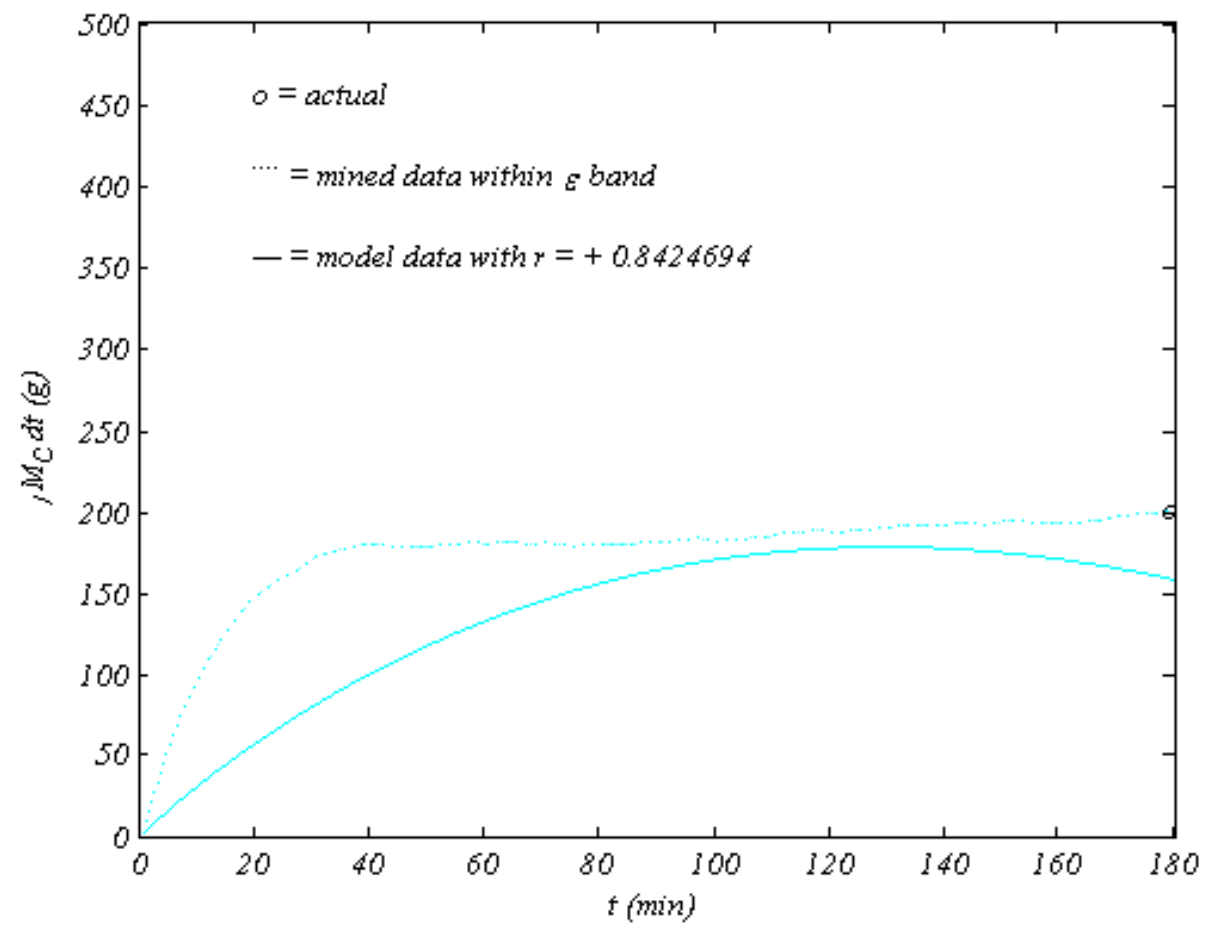

Figure 133. Fresh water production amount at $60^{\circ} \mathrm{C}$ flash and higher flow 


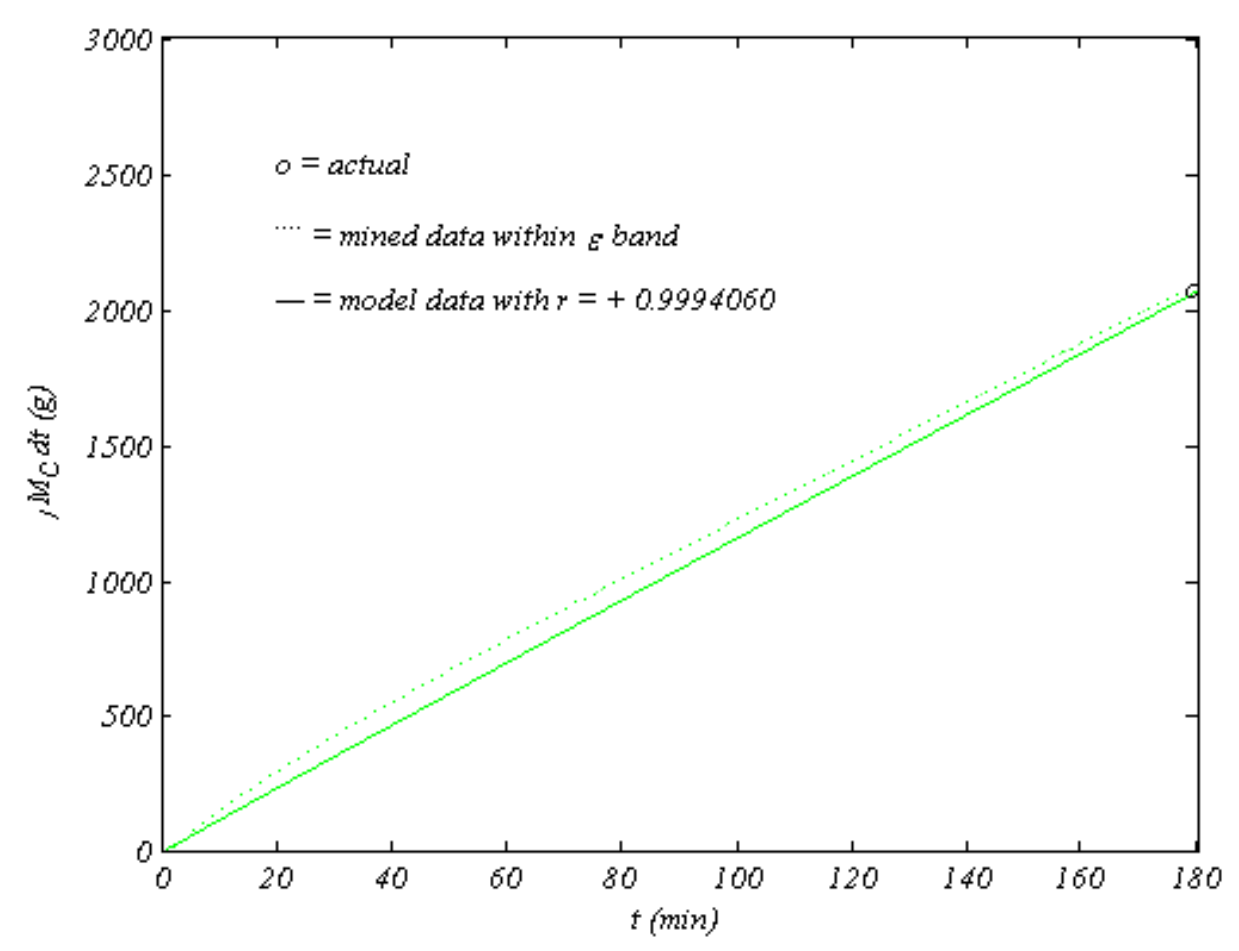

Figure 134. Fresh water production amount at $70^{\circ} \mathrm{C}$ flash and lower flow

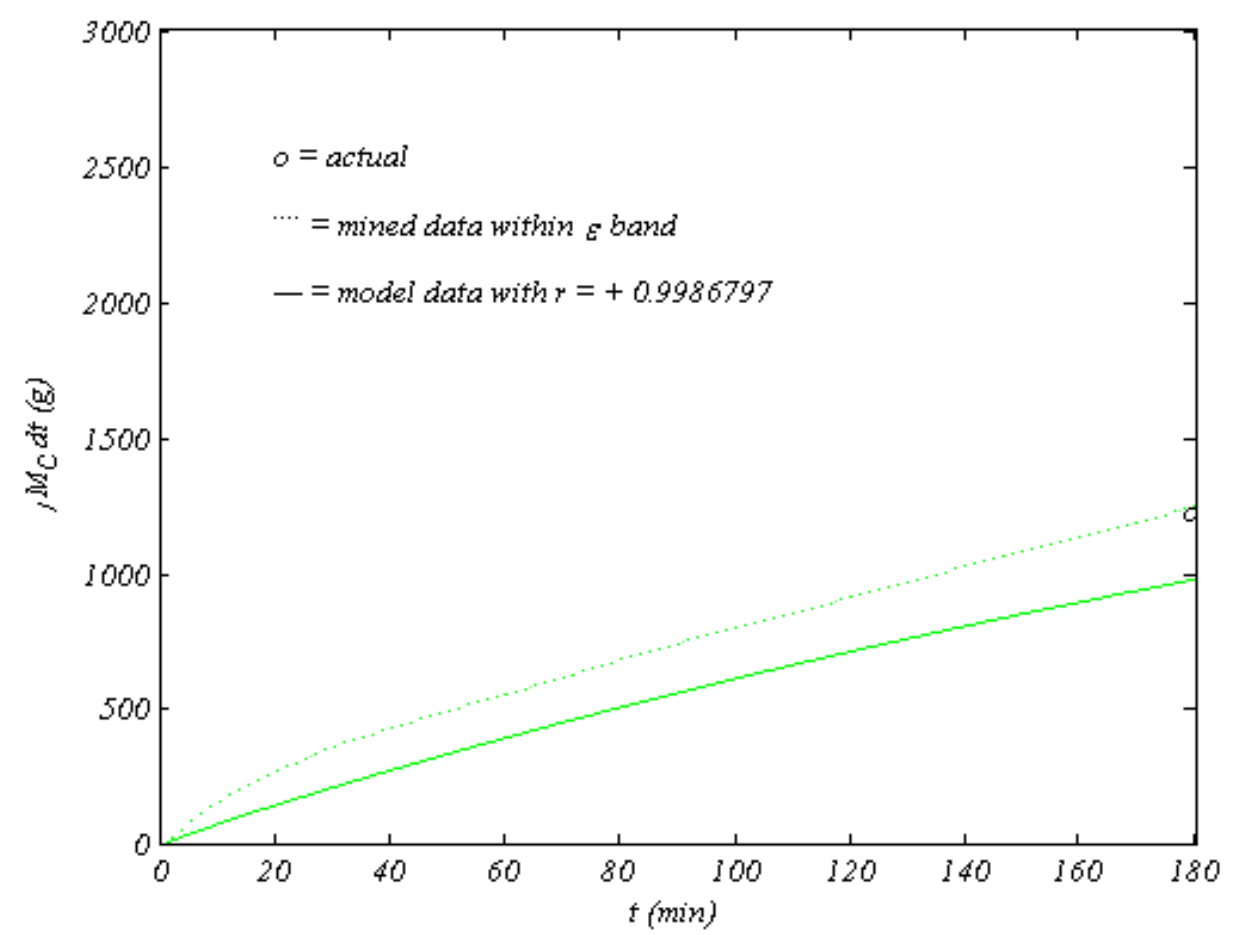

Figure 135. Fresh water production amount at $70^{\circ} \mathrm{C}$ flash and higher flow 


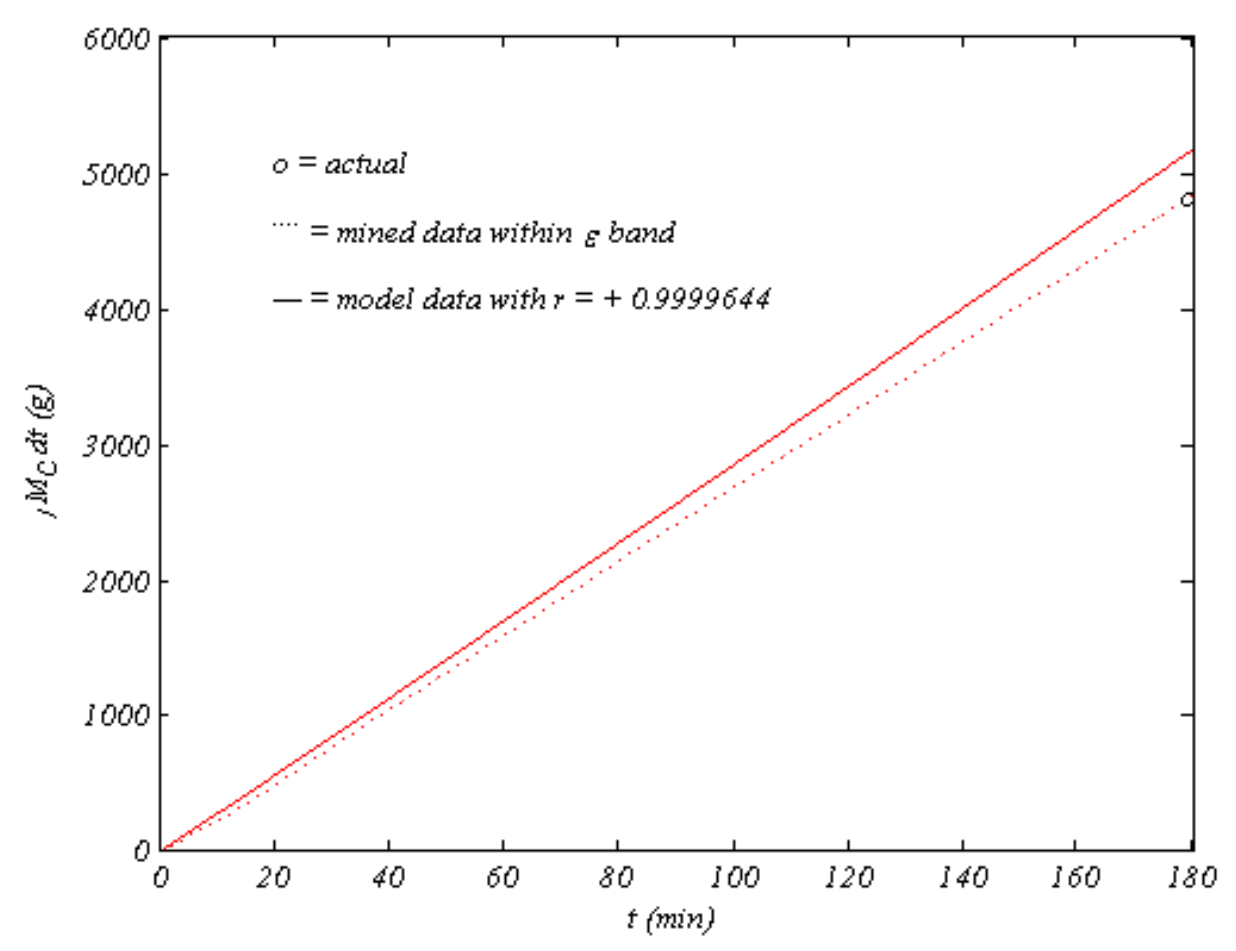

Figure 136. Fresh water production amount at $80^{\circ} \mathrm{C}$ flash and lower flow

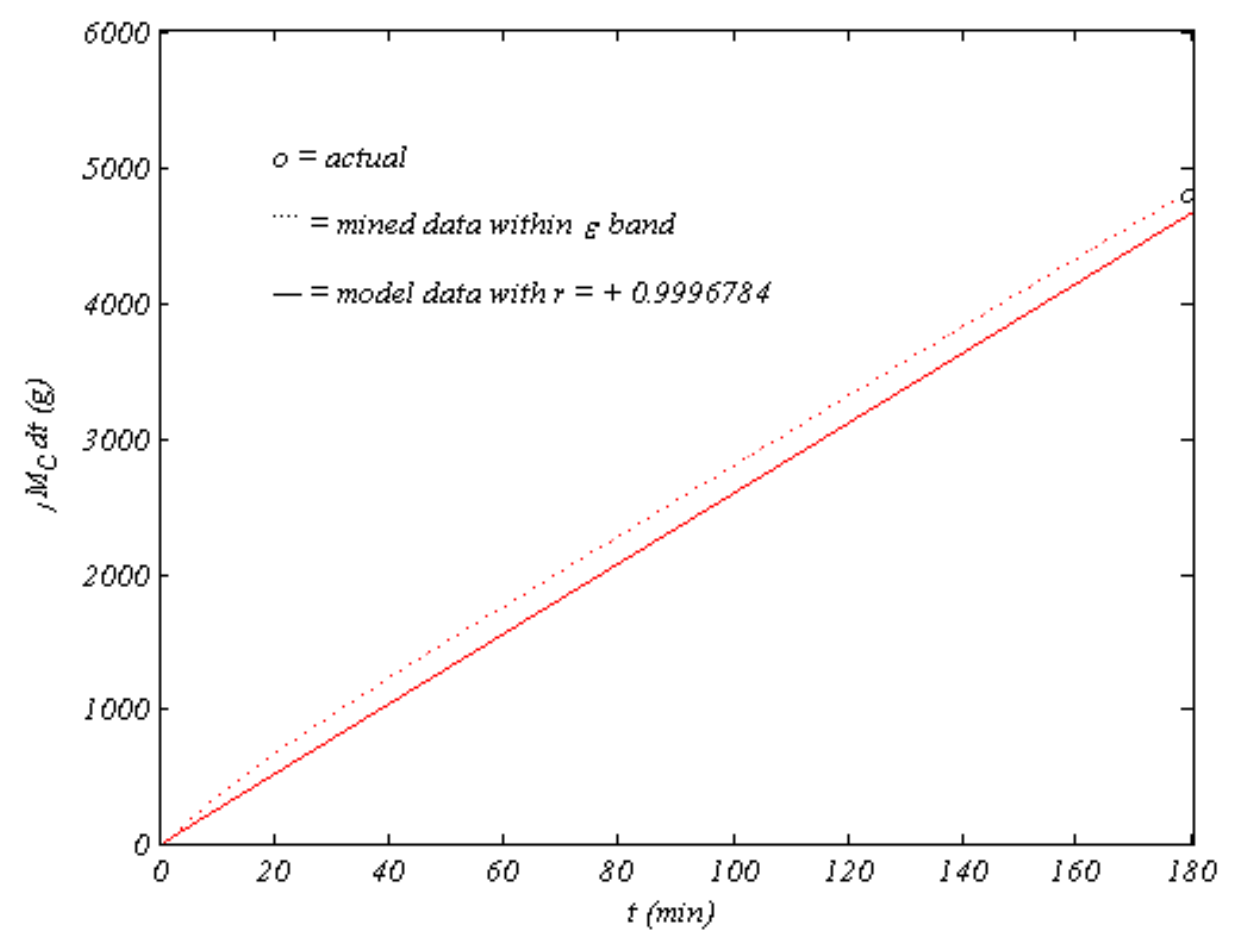

Figure 137. Fresh water production amount at $80^{\circ} \mathrm{C}$ flash and higher flow 


\subsection{Process Feasibility}

Feasibility of the proposed desalination system can be evaluated using its resulting prime energy consumption, defined as the ratio of the total amount of energy exhausted to total amount of fresh water produced, as mentioned in CHAPTER 4. Energy consumed is attributed to heat provided by the heater plus work supplied by the pump. The pumping work of the proposed desalination system was insignificant relative to the heater load whether the flow rate of seawater was controlled with a throttling valve or a variable-frequency drive; hence, the presented prime energy consumption computations ignore the pumping work, that is $P E C \approx \int Q_{H} d t / \int M_{C} d t$. No economic analysis was performed in this venture, but the optimization process of the proposed desalination system lies within minimizing the prime energy consumption via maximizing production and minimizing heater load.

Prime energy consumption steadily increased for both seawater flow rates; however, it was higher at higher seawater flow rates due to higher heater loads. It declined rapidly with flash temperature due to the increasing fresh water production and decreasing heater load due to the improved heat recovery caused by the increased condensation associated with higher flash temperatures. In addition; the difference between prime energy consumption experienced at both seawater flow rates was more significant at low flash temperatures and diminished at higher flash temperatures.

Model prediction of prime energy consumption deteriorated with decreasing flash temperatures but improved with increasing flash temperatures due to contrasting effects of poor heater load prediction and good production amount prediction. Prime energy consumption profiles are shown in Figure 138 through Figure 149. 


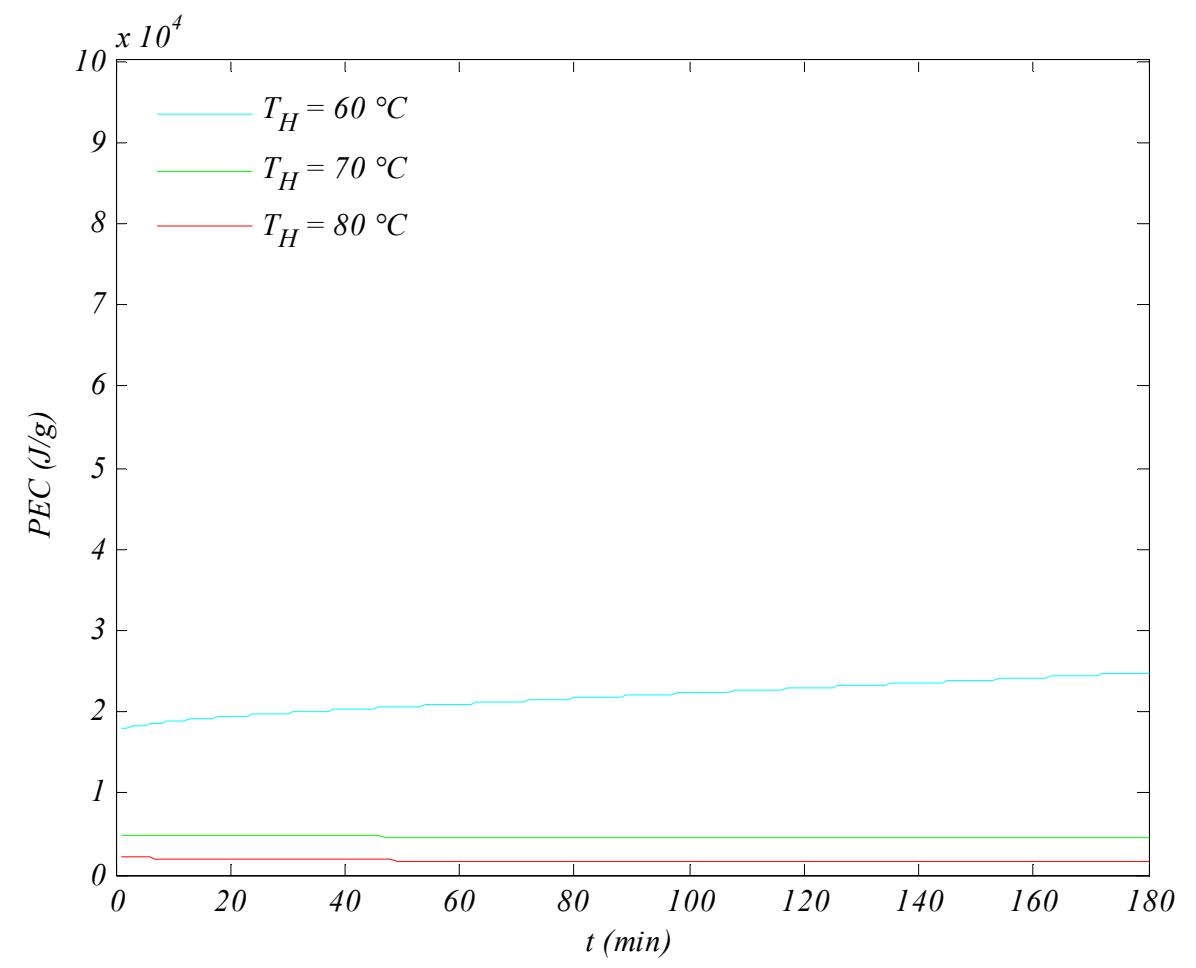

Figure 138. Modeled prime energy consumption profiles at lower flow

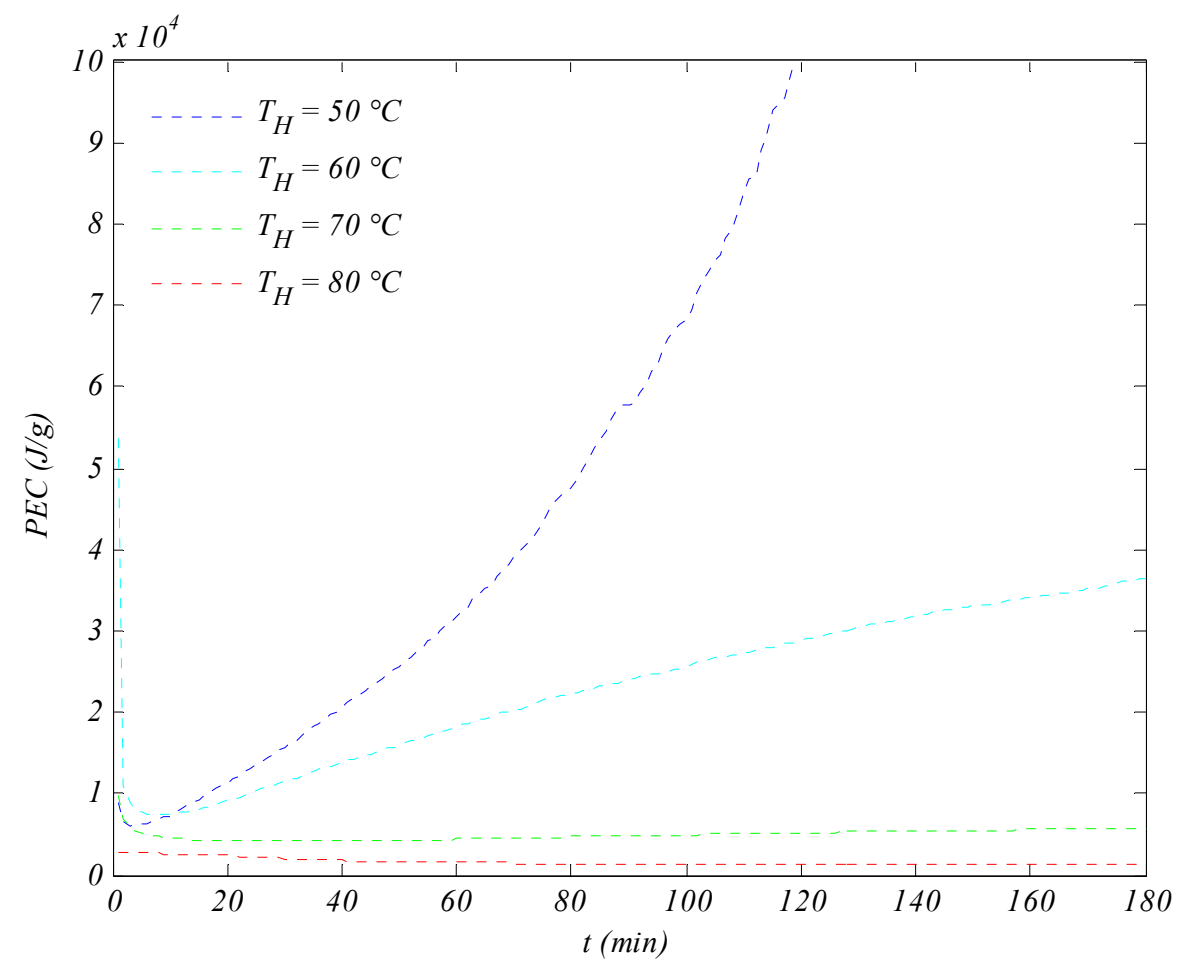

Figure 139. Mined prime energy consumption profiles at lower flow 


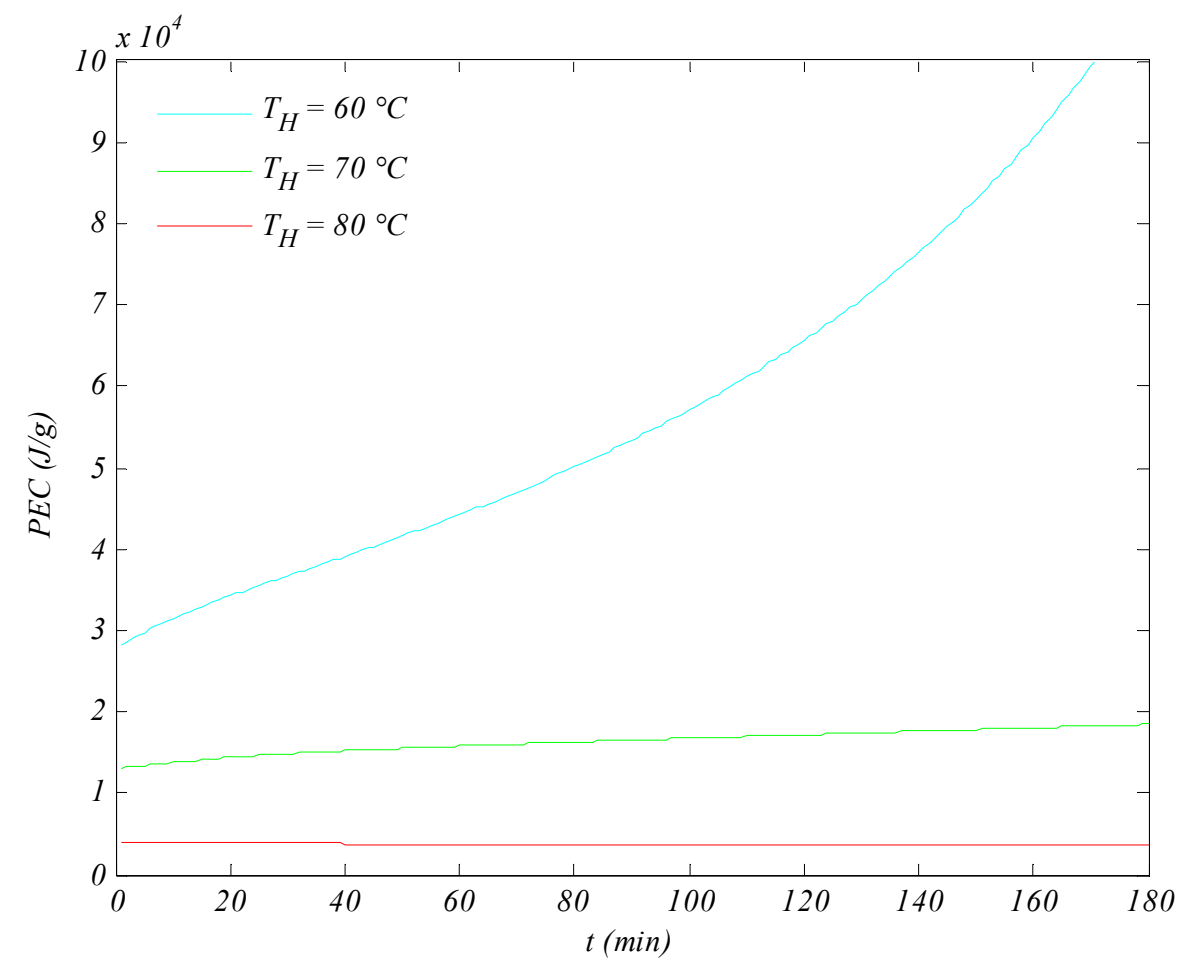

Figure 140. Modeled prime energy consumption profiles at higher flow

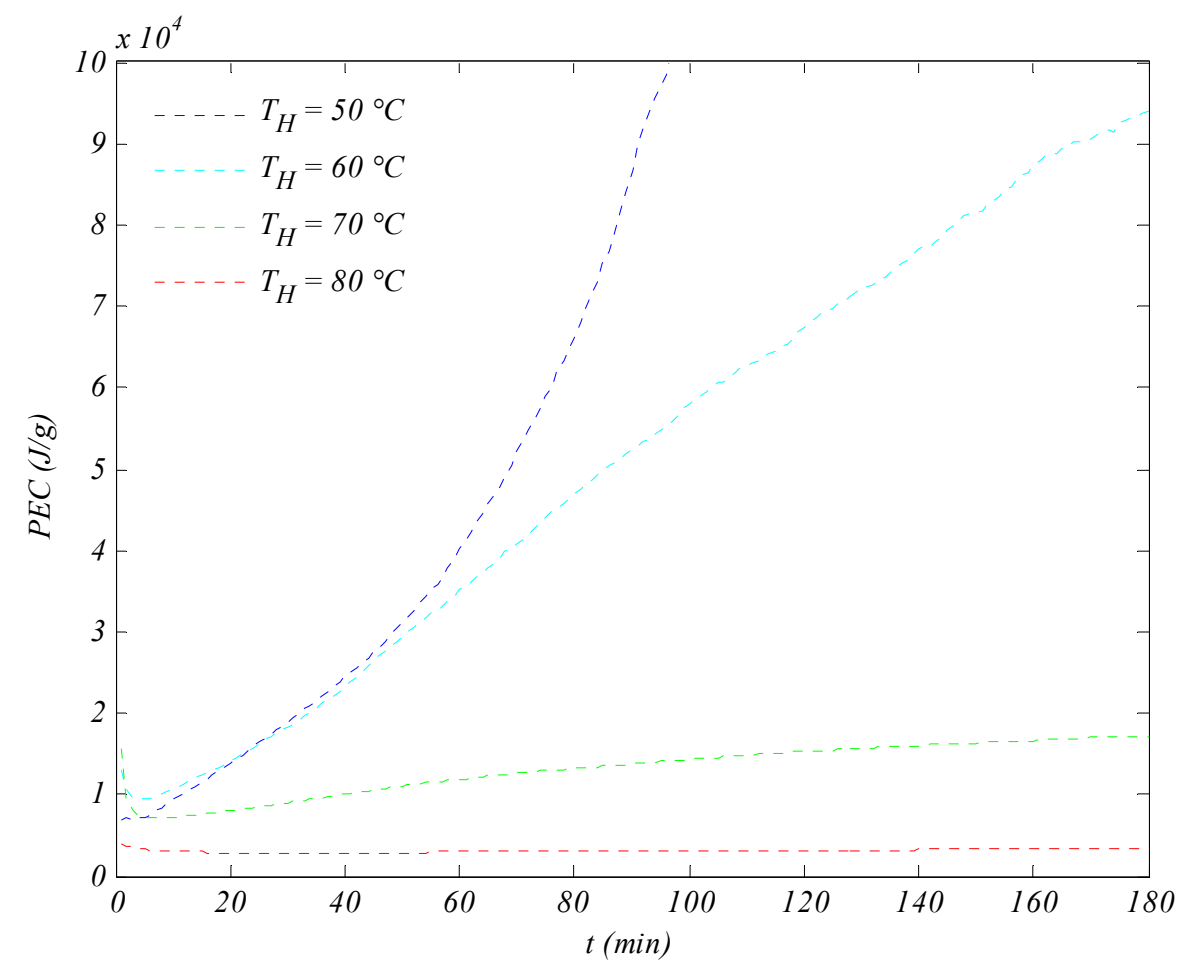

Figure 141. Mined prime energy consumption profiles at higher flow 


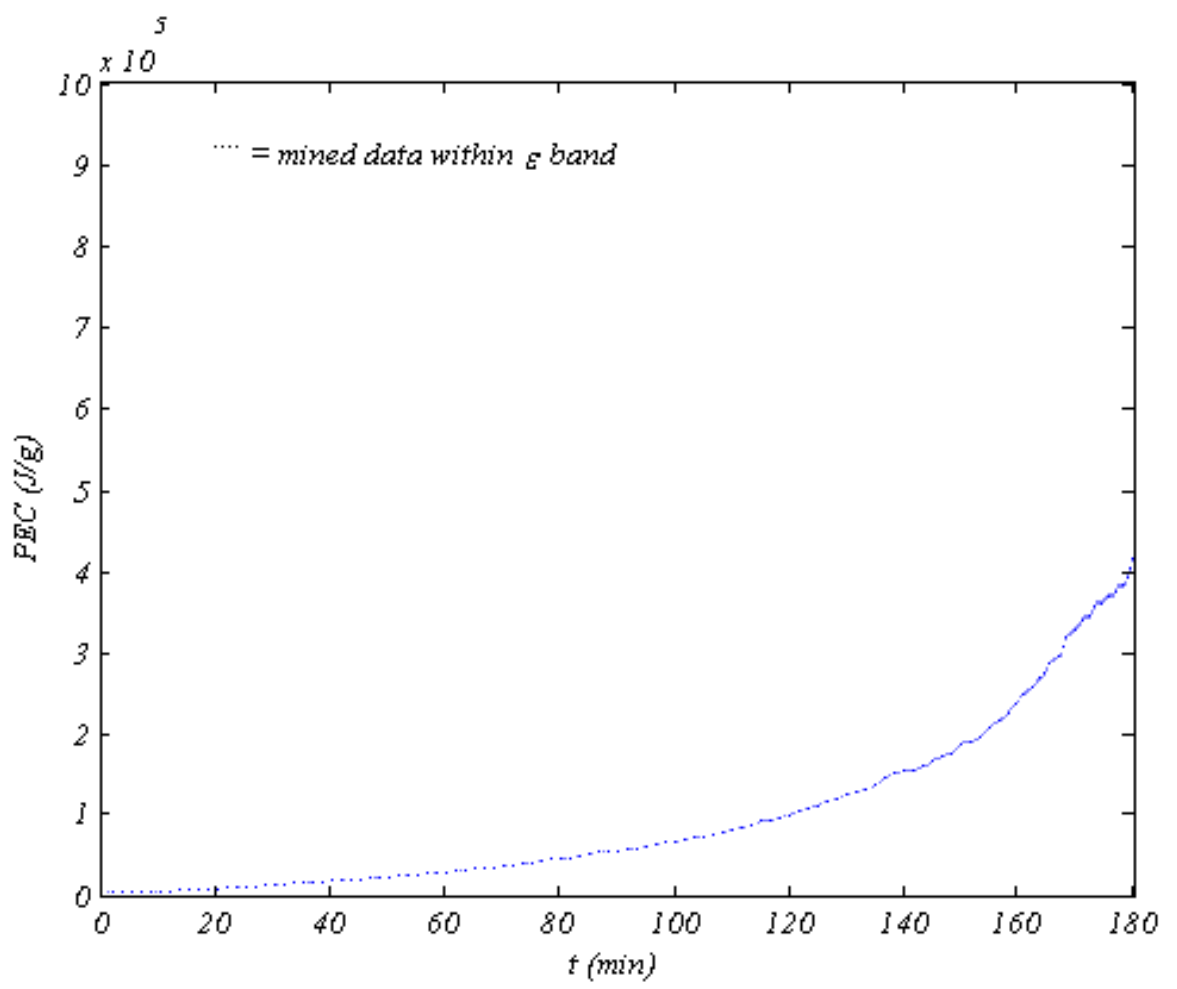

Figure 142. Prime energy consumption at $50^{\circ} \mathrm{C}$ flash and lower flow

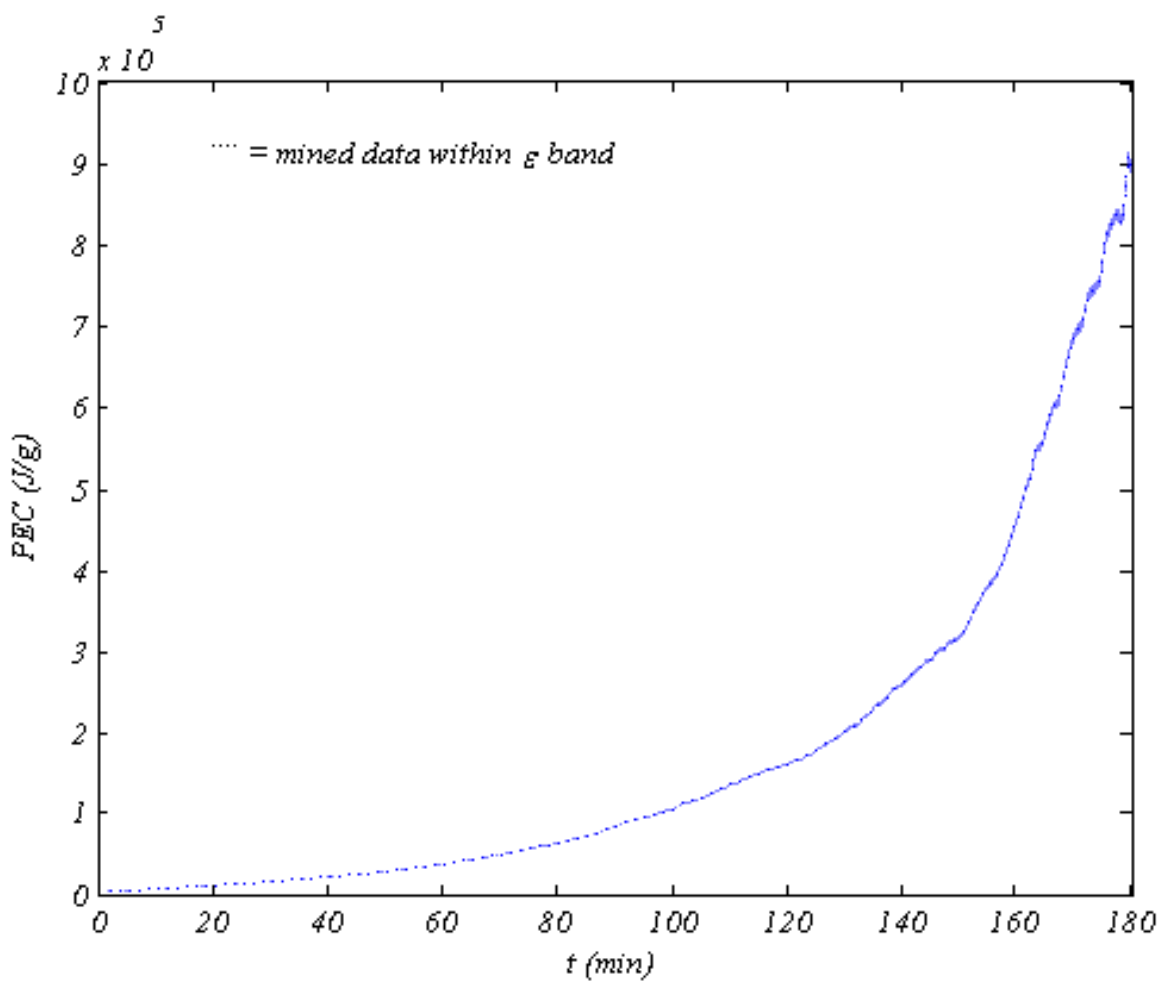

Figure 143. Prime energy consumption at $50^{\circ} \mathrm{C}$ flash and higher flow 


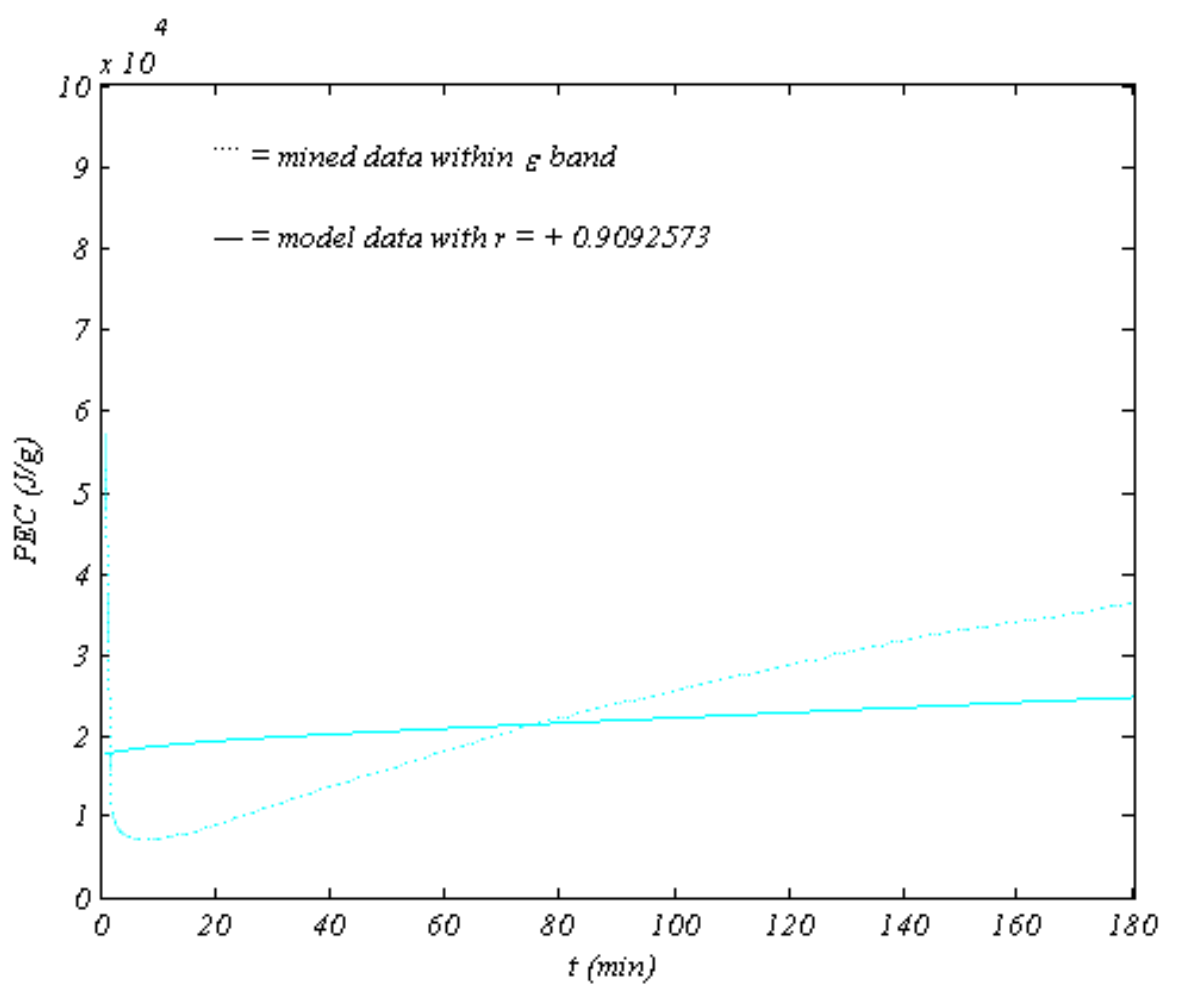

Figure 144. Prime energy consumption at $60^{\circ} \mathrm{C}$ flash and lower flow

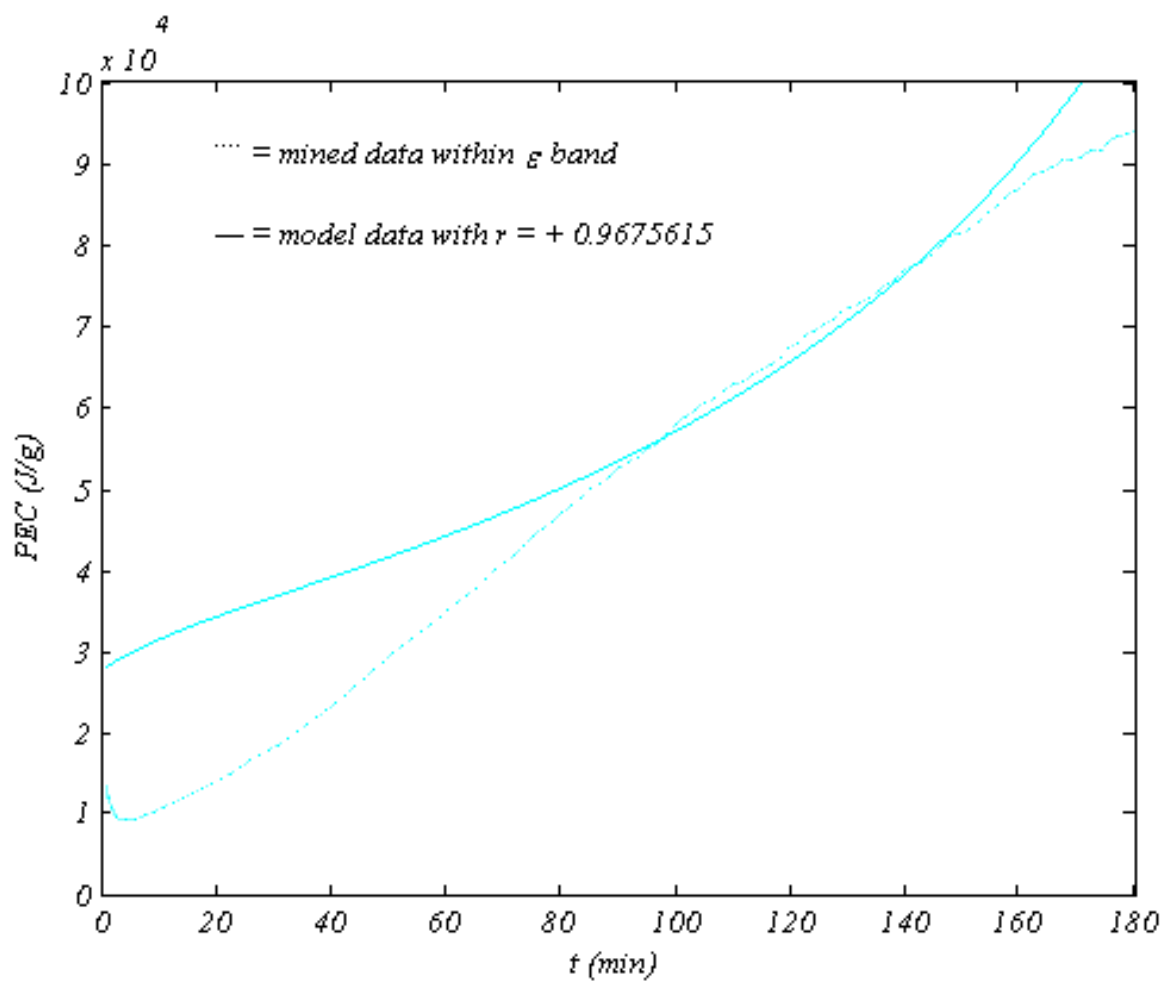

Figure 145. Prime energy consumption at $60^{\circ} \mathrm{C}$ flash and higher flow 


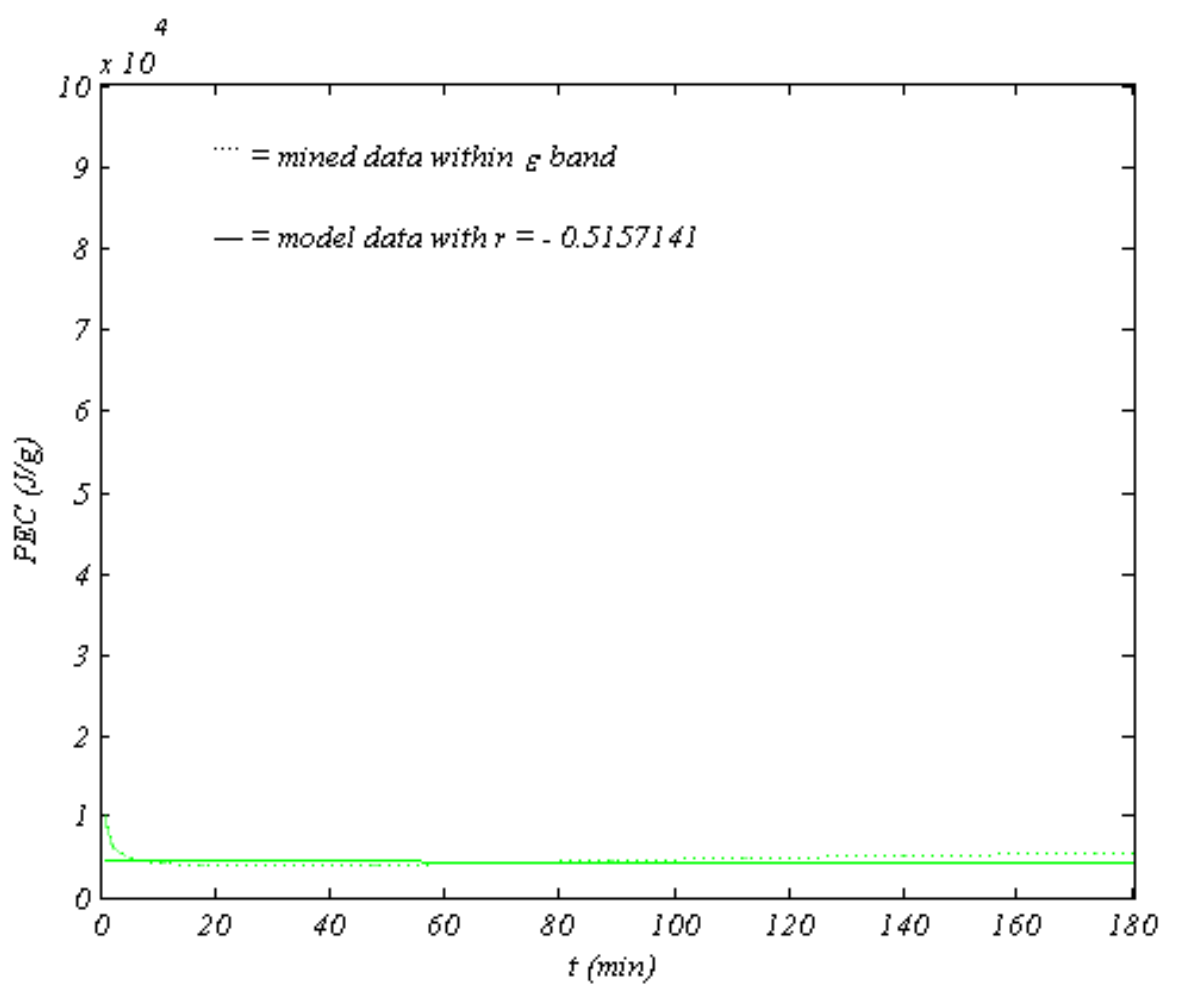

Figure 146. Prime energy consumption at $70^{\circ} \mathrm{C}$ flash and lower flow

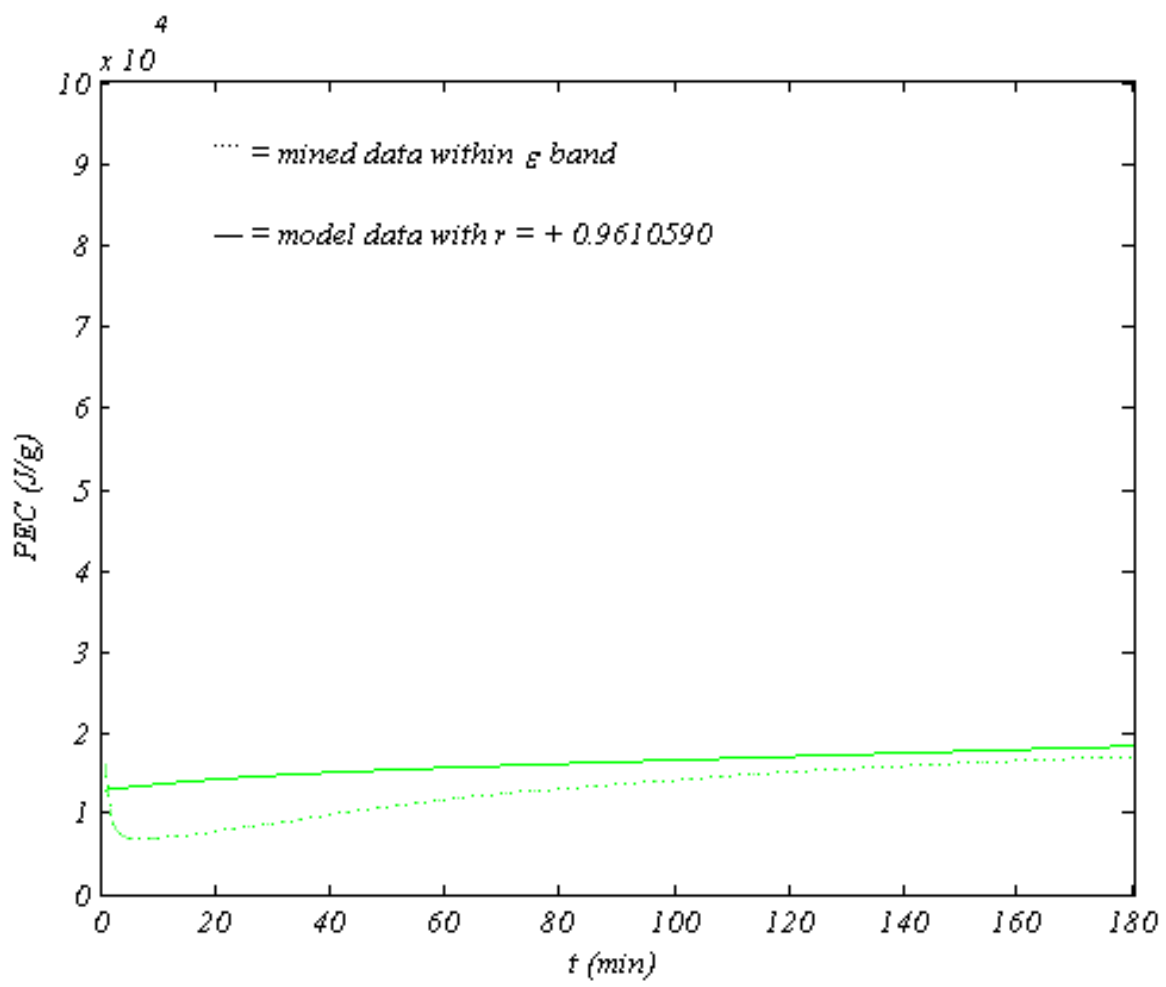

Figure 147. Prime energy consumption at $70^{\circ} \mathrm{C}$ flash and higher flow 


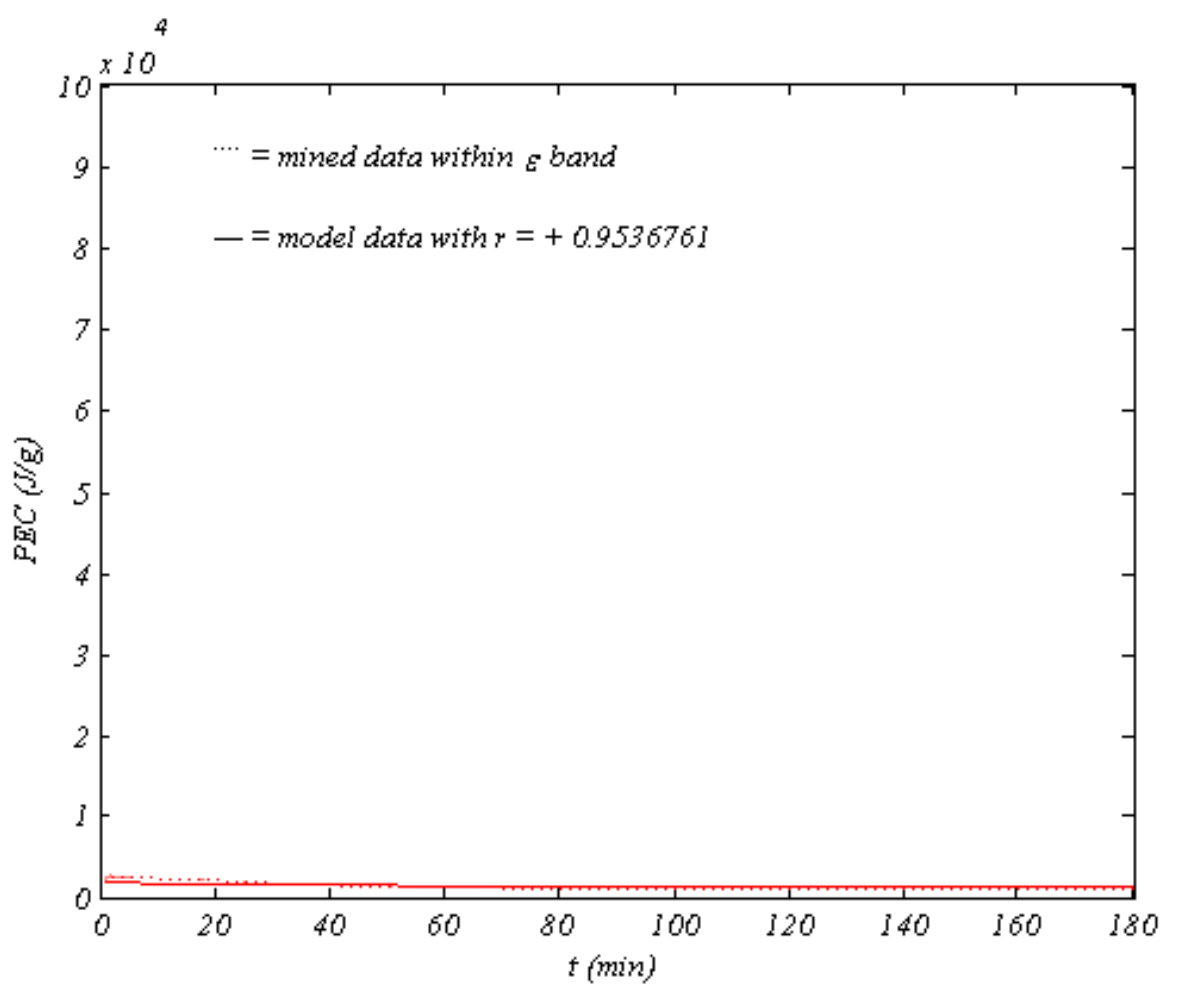

Figure 148. Prime energy consumption at $80^{\circ} \mathrm{C}$ flash and lower flow

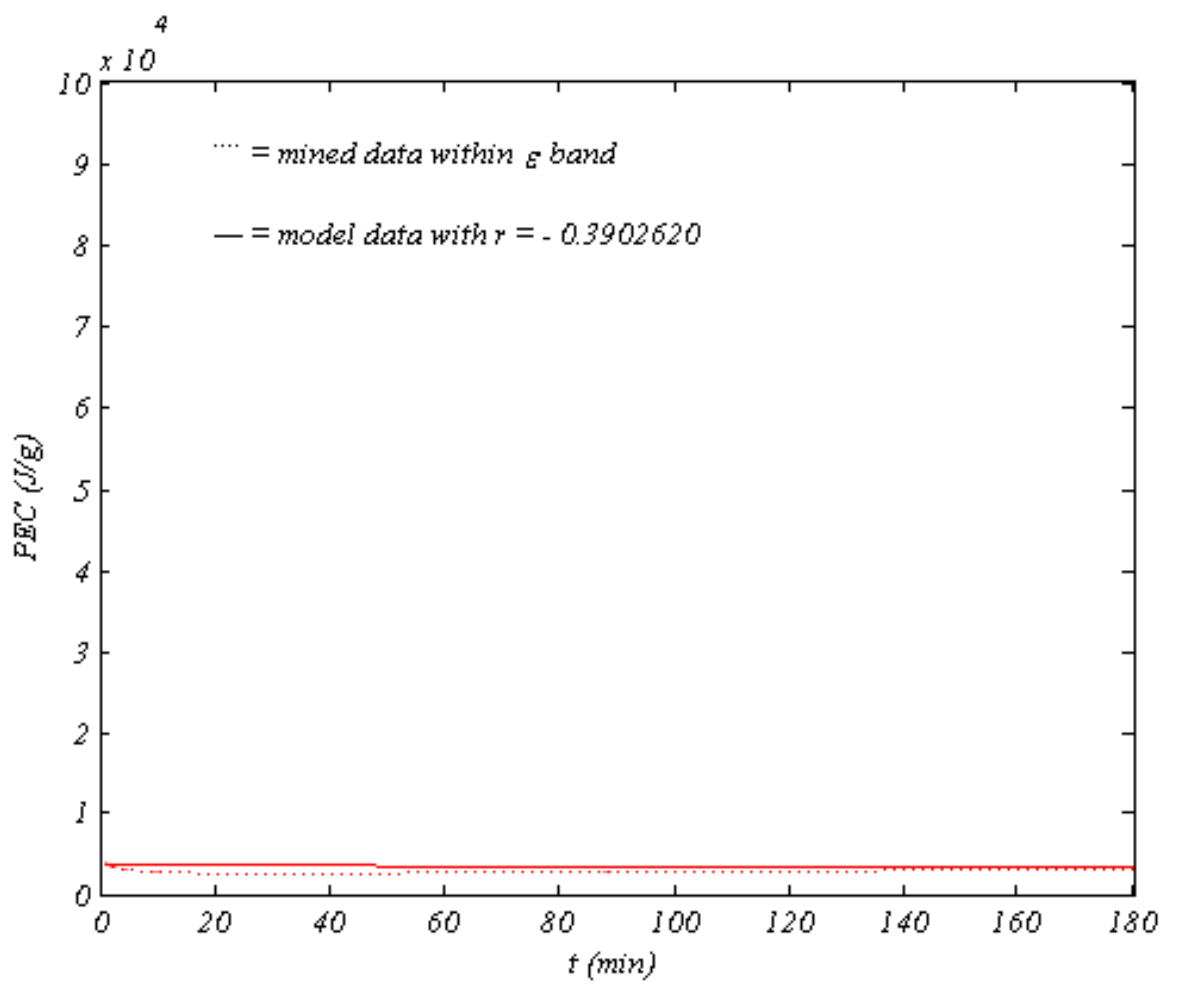

Figure 149. Prime energy consumption at $80^{\circ} \mathrm{C}$ flash and higher flow 


\subsection{Condensing Efficiency}

Condenser efficiency describes its heat transfer effectiveness and is defined as the ratio of the temperature gradient on the cold tube side to the temperature gradient on the hot shell side written as a percentage, that is $\eta_{C}=\left[\left(T_{X}-T_{P}\right) /\left(T_{E}-T_{C}\right)\right] \cdot 100 \%$, as was mentioned in CHAPTER 4. Condenser efficiency rapidly increased to reach a maximum early before it slowly declined for both seawater flow rates; however, condenser efficiency attained at lower seawater flow rates was higher. The condenser efficiency increased with flash temperatures for both seawater flow rates, since the preheat temperature is directly proportional to the flashing temperature.

The condenser efficiency indirectly conveys the percent of available heat that was utilized for preheating seawater. It rapidly increased because of the rapidly rising preheat temperature due to the high initial rate of vaporization caused by rapidly increasing equilibrium temperature at lower vacuum pressures, then it slowly declined because of the decreasing preheat temperature due to the decreasing rate of vaporization caused by stabilizing equilibrium temperature at rising vacuum pressures for both seawater flow rates. The condenser efficiency profiles for both seawater flow rates are similar; however, they are higher for lower flow rates because of the higher preheat temperature experienced at lower seawater flow rates due to more condensation caused by more vaporization as seen before.

Model prediction of condenser efficiency loosely resembled experimental results because of the loose depiction of the preheat temperature due to the inability of the condenser tube heat transfer module of the model to capture the rate of condensation. Condenser efficiency profiles are shown in Figure 150 through Figure 161. 


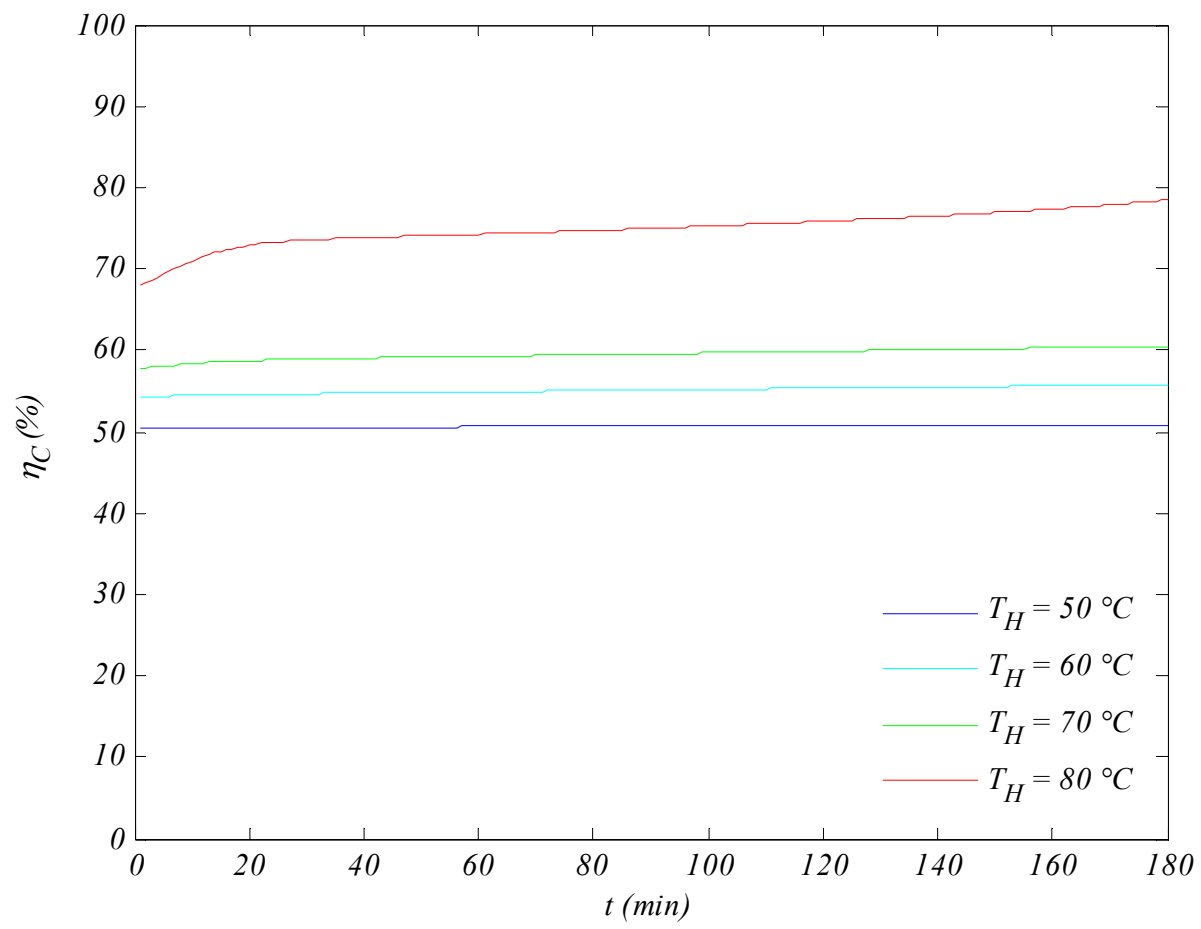

Figure 150. Modeled condenser efficiency profiles at lower flow

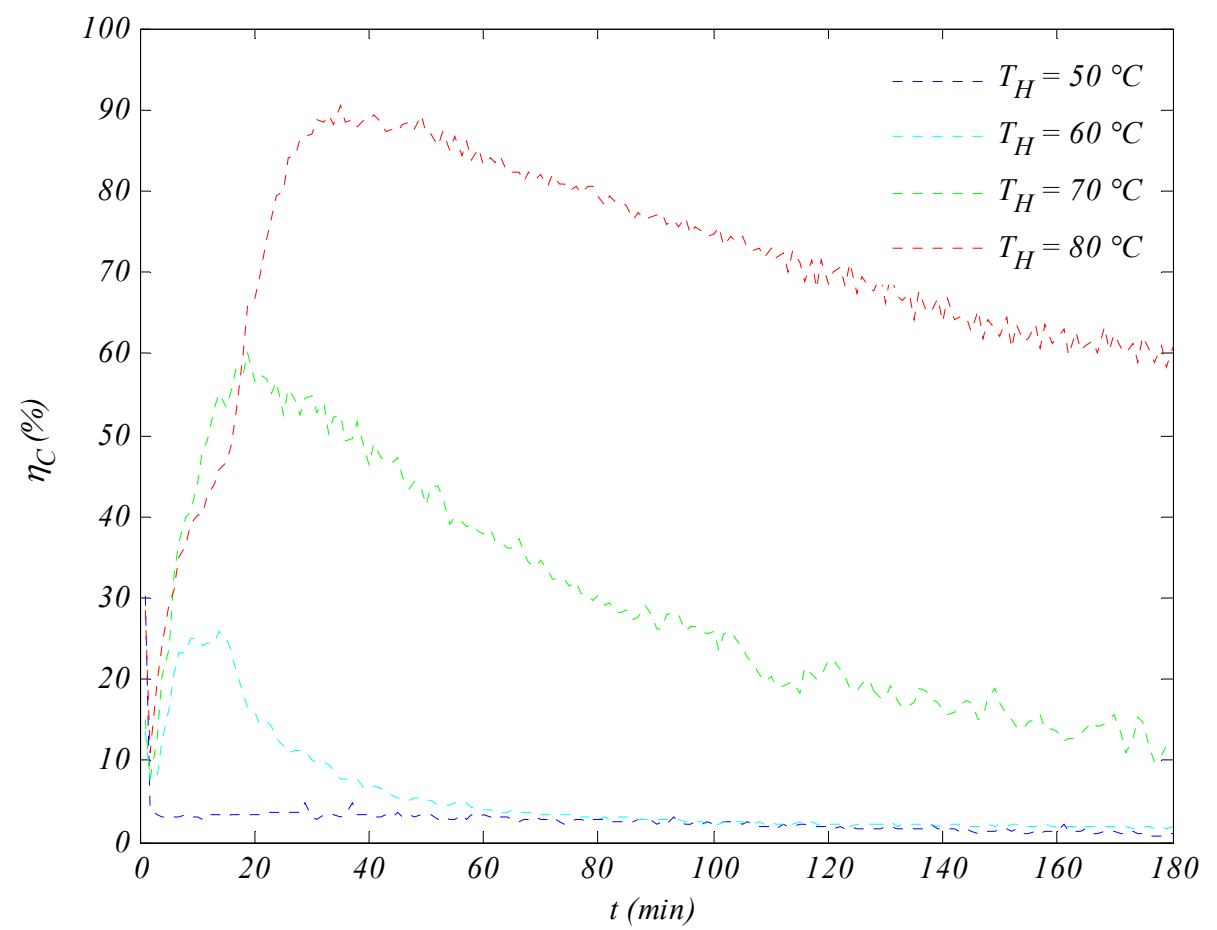

Figure 151. Experimental condenser efficiency profiles at lower flow 


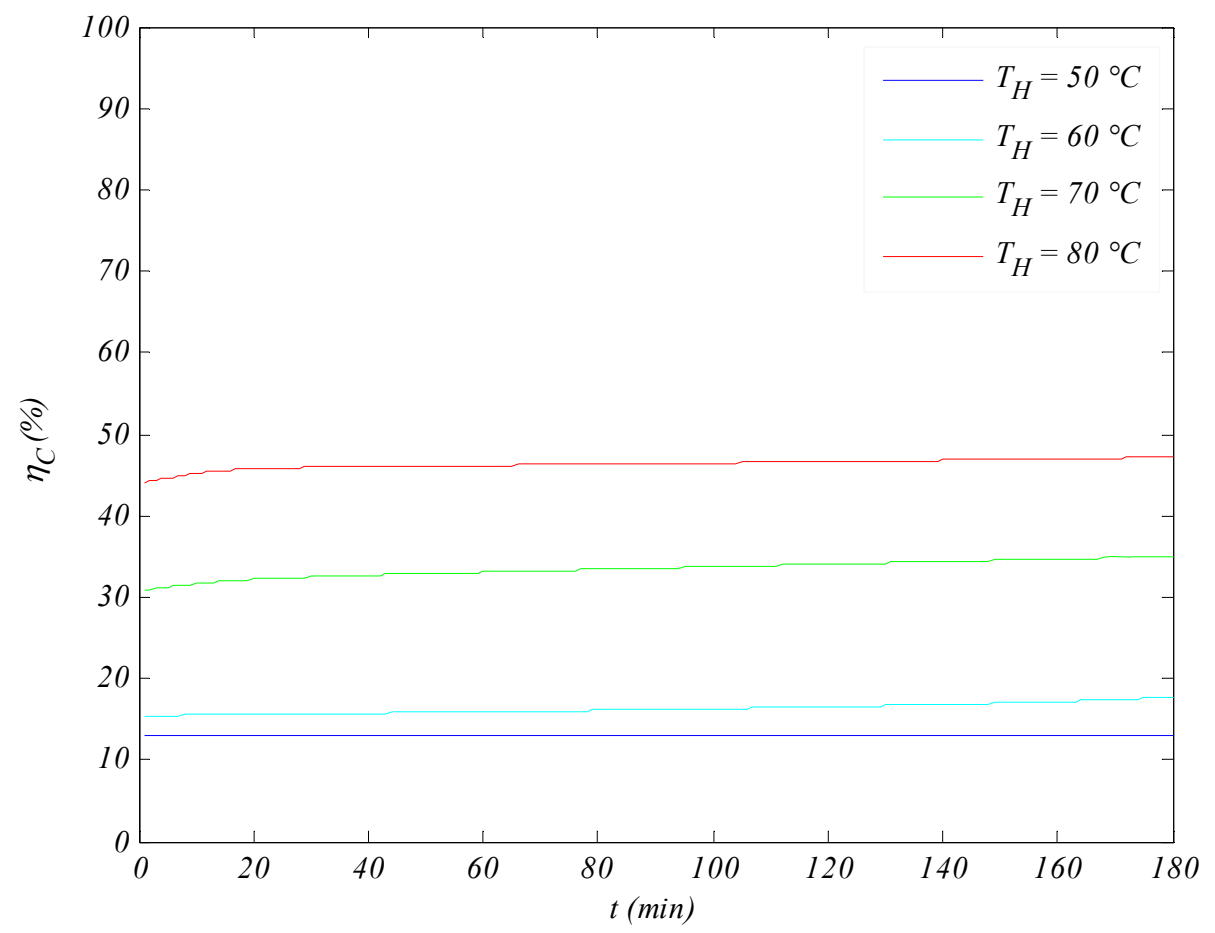

Figure 152. Modeled condenser efficiency profiles at higher flow

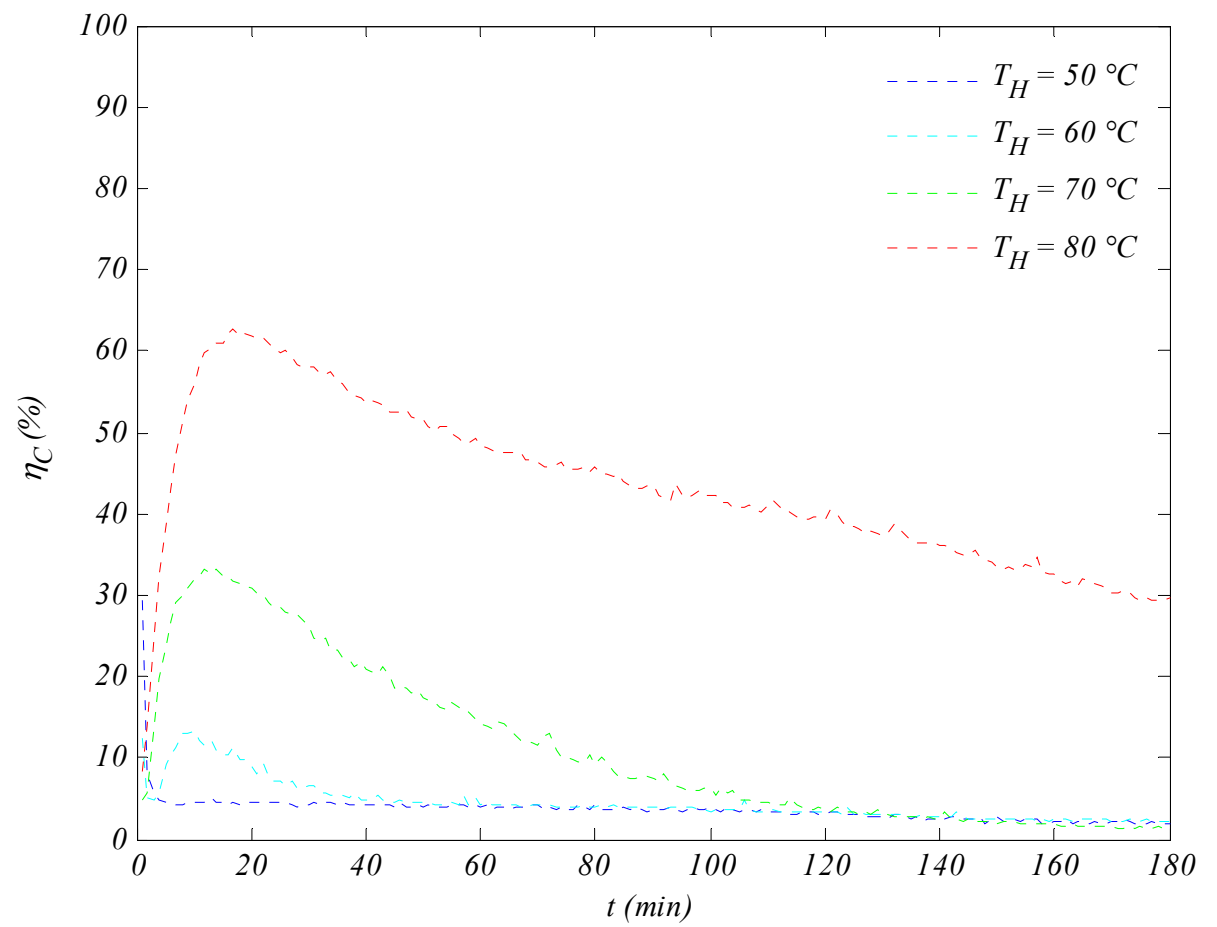

Figure 153. Experimental condenser efficiency profiles at higher flow 


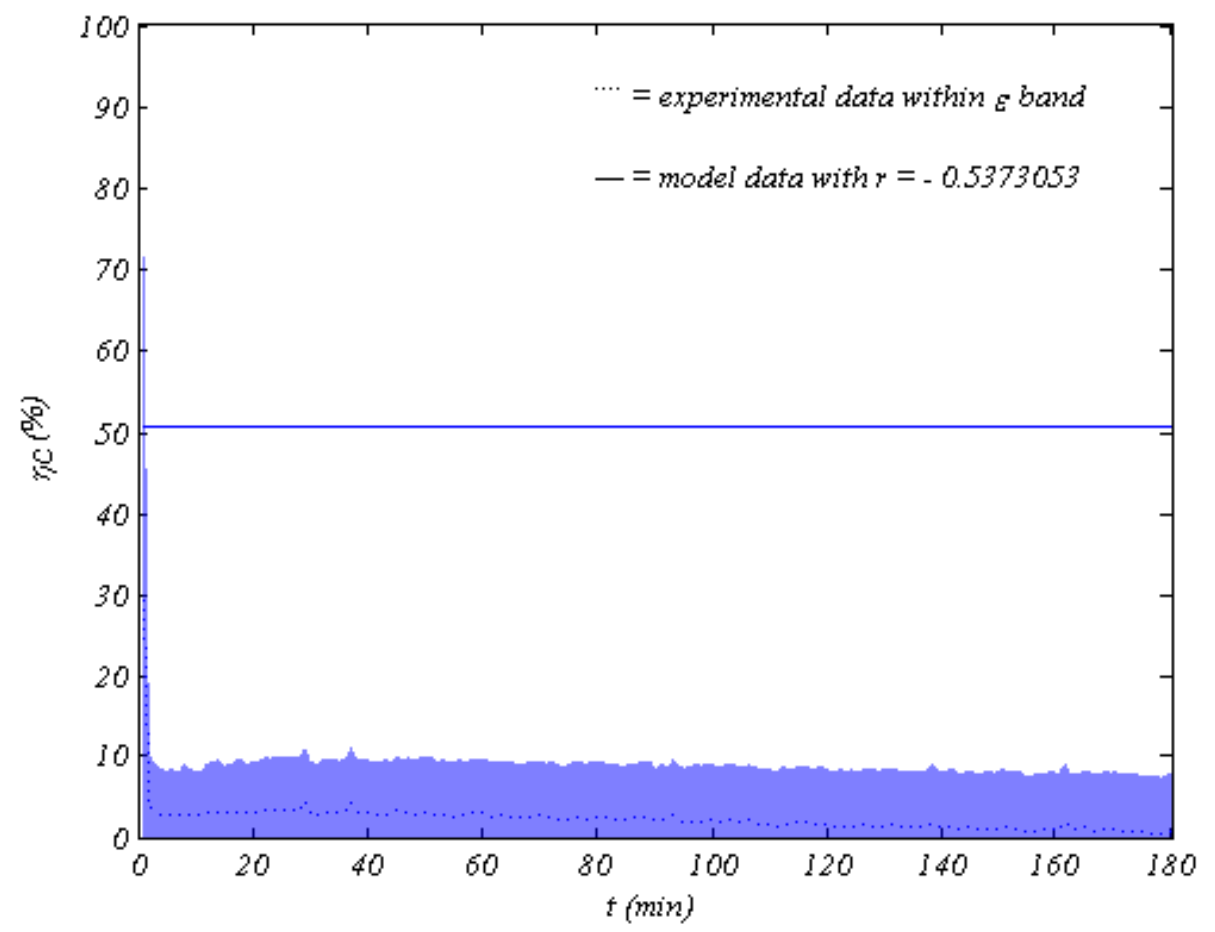

Figure 154. Condenser efficiency at $50^{\circ} \mathrm{C}$ flash and lower flow

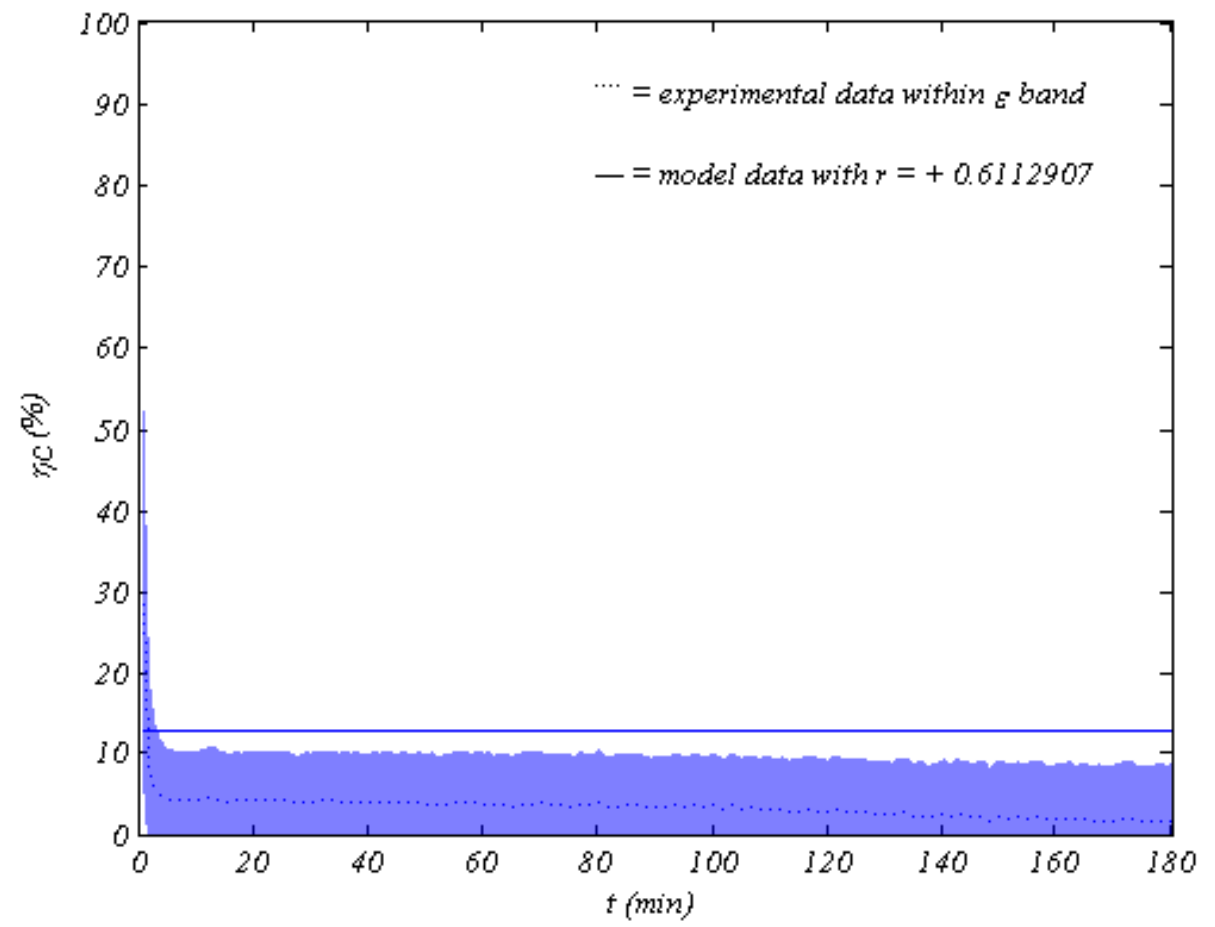

Figure 155. Condenser efficiency at $50^{\circ} \mathrm{C}$ flash and higher flow 


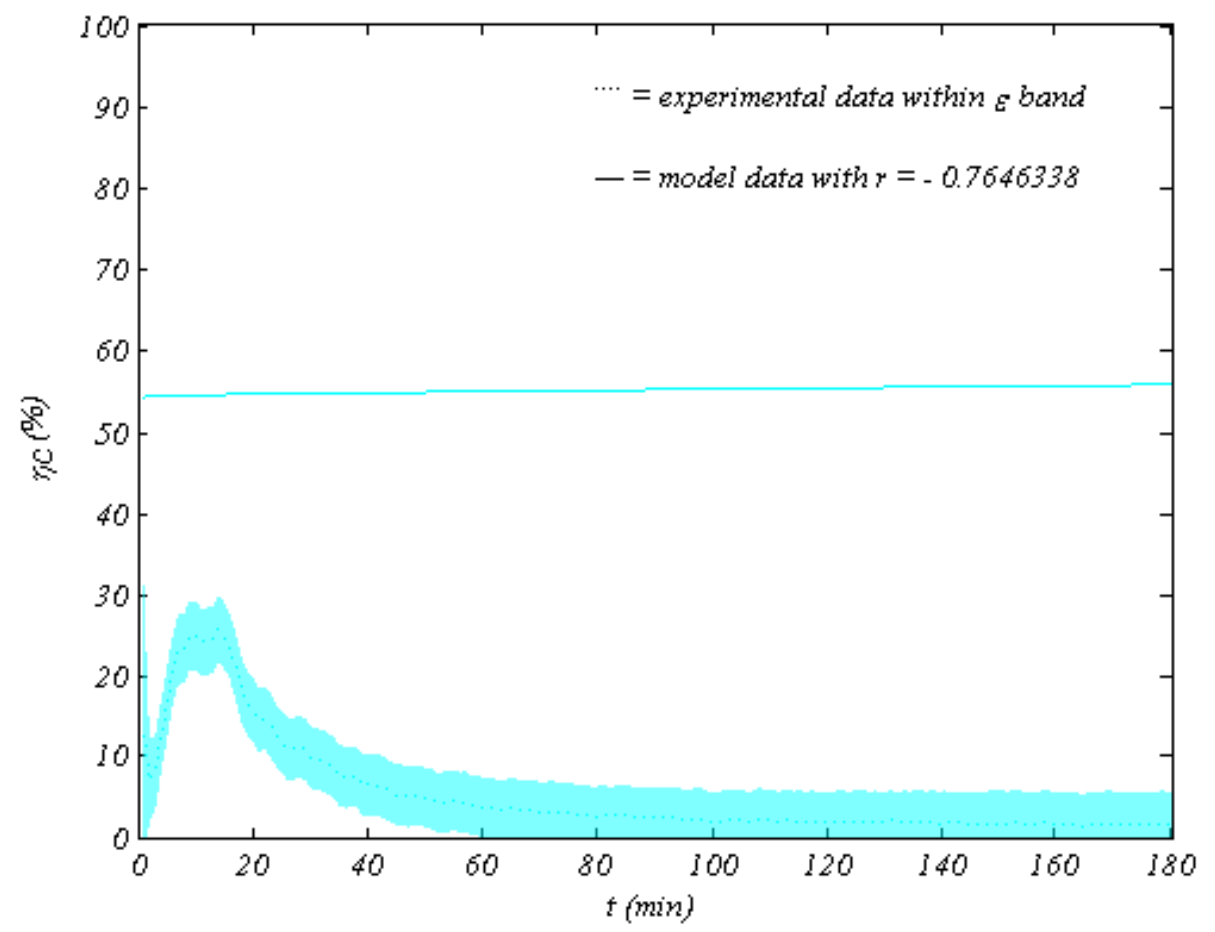

Figure 156. Condenser efficiency at $60^{\circ} \mathrm{C}$ flash and lower flow

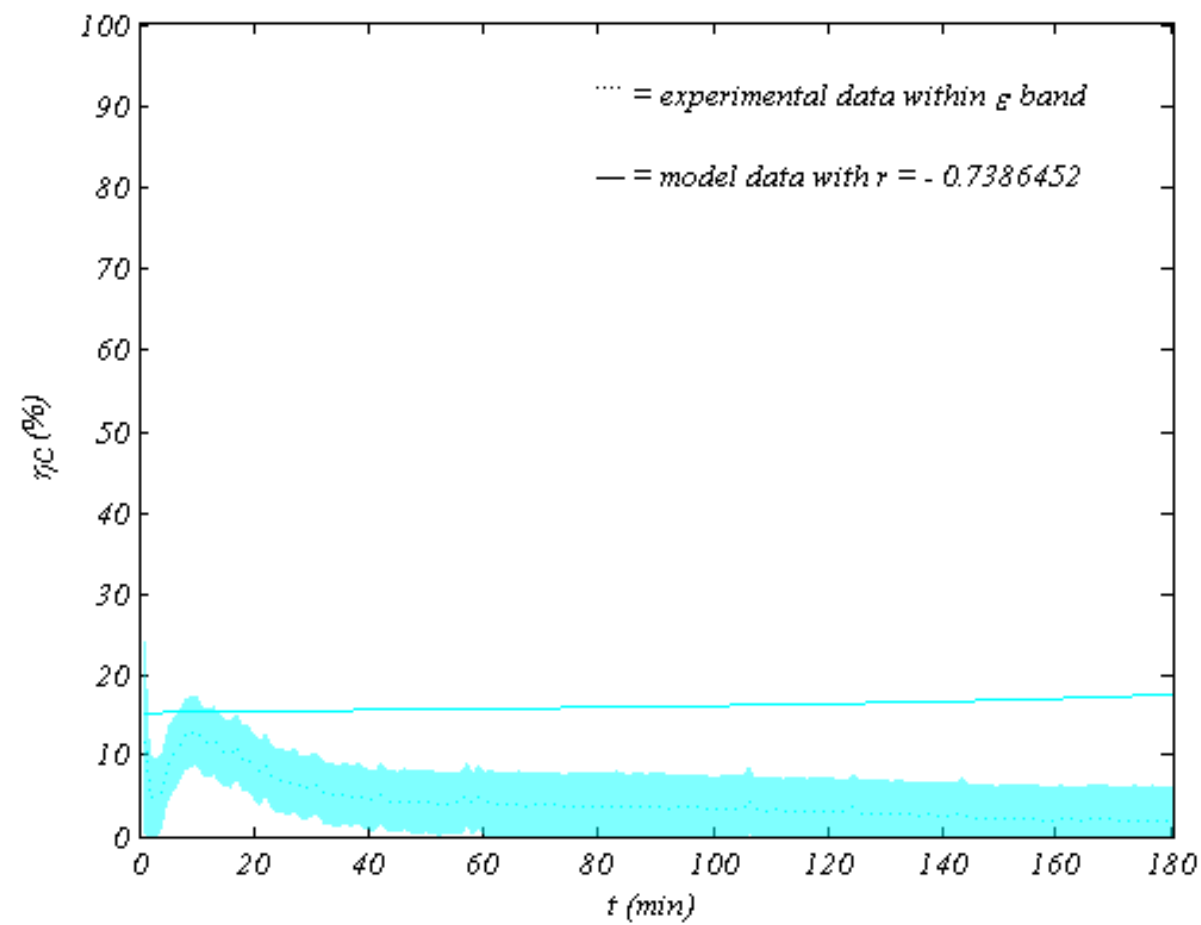

Figure 157. Condenser efficiency at $60^{\circ} \mathrm{C}$ flash and higher flow 


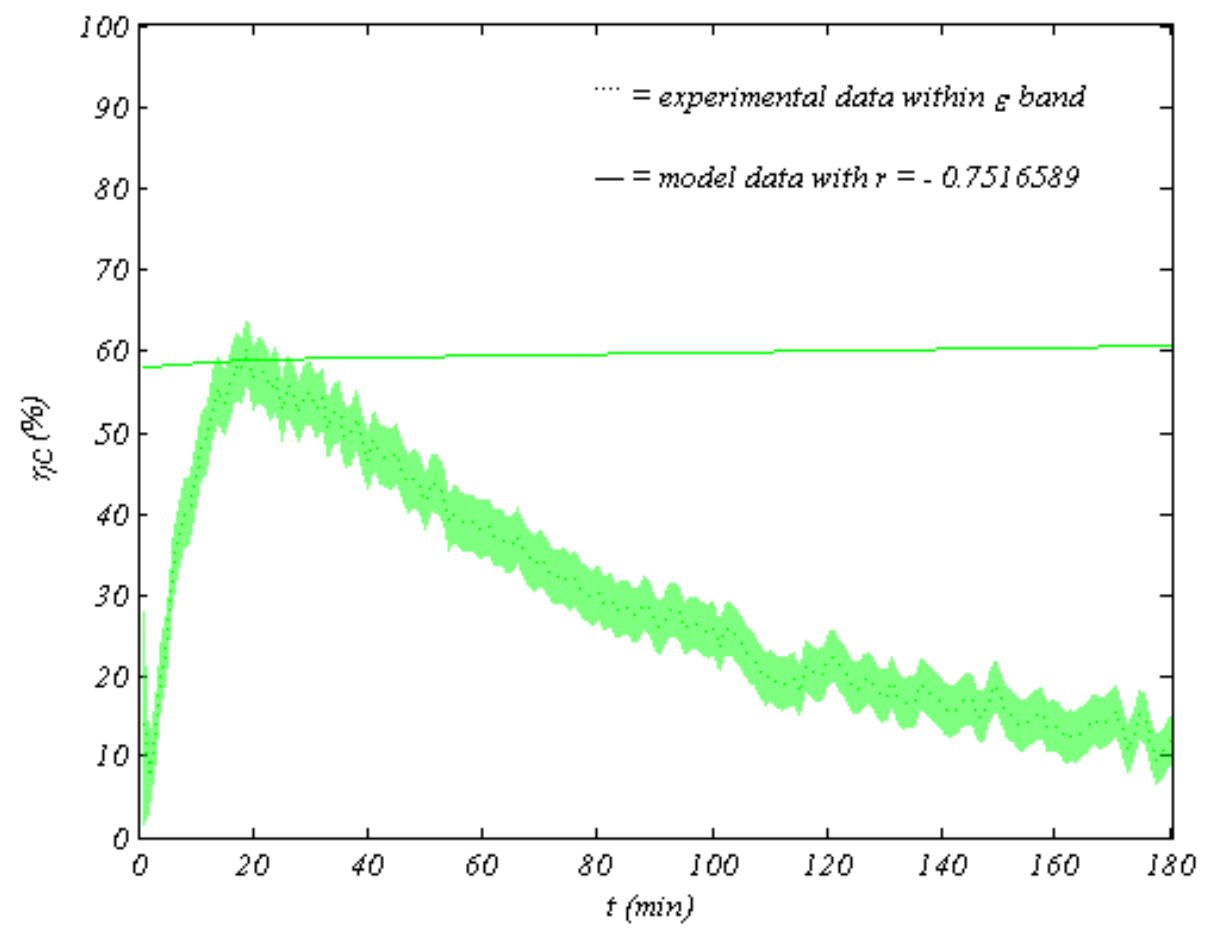

Figure 158. Condenser efficiency at $70^{\circ} \mathrm{C}$ flash and lower flow

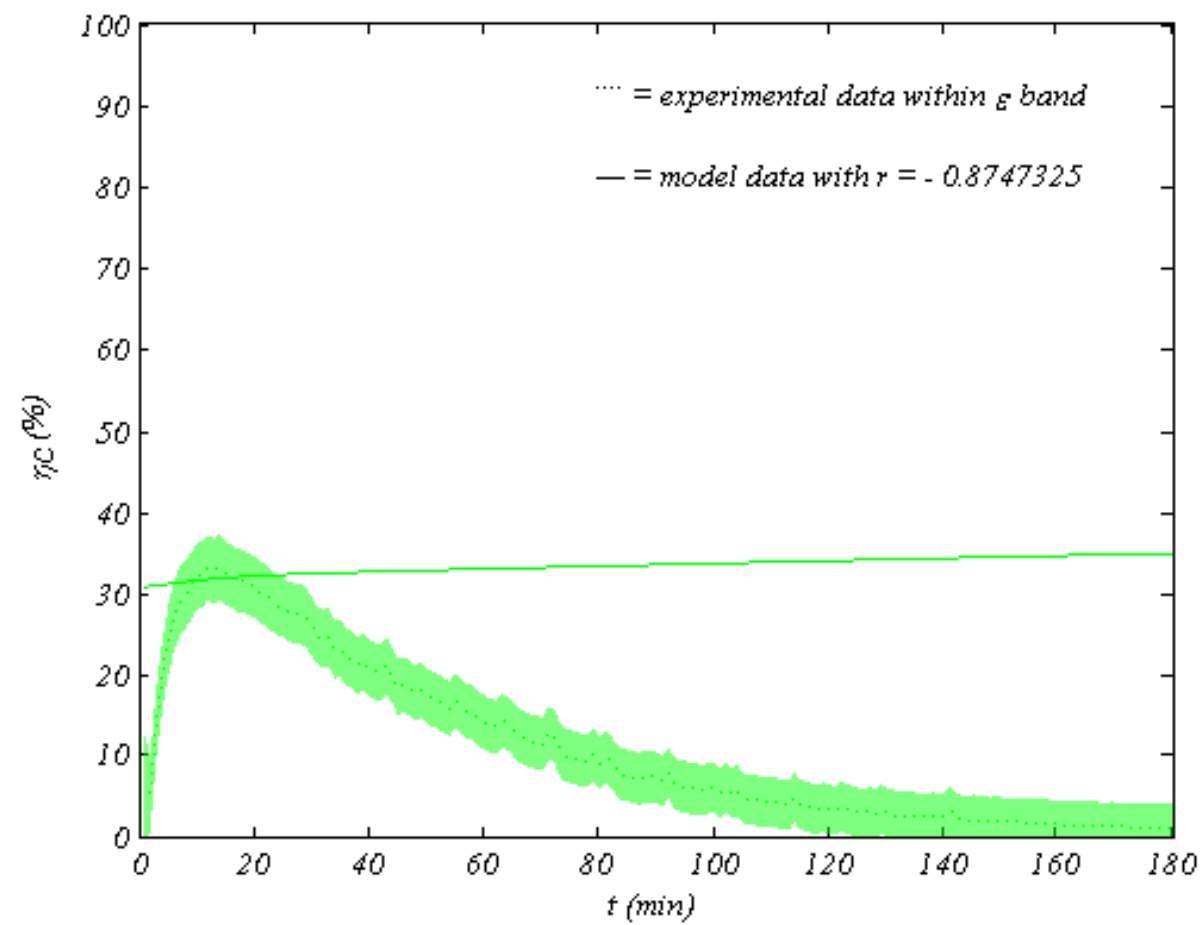

Figure 159. Condenser efficiency at $70^{\circ} \mathrm{C}$ flash and higher flow 


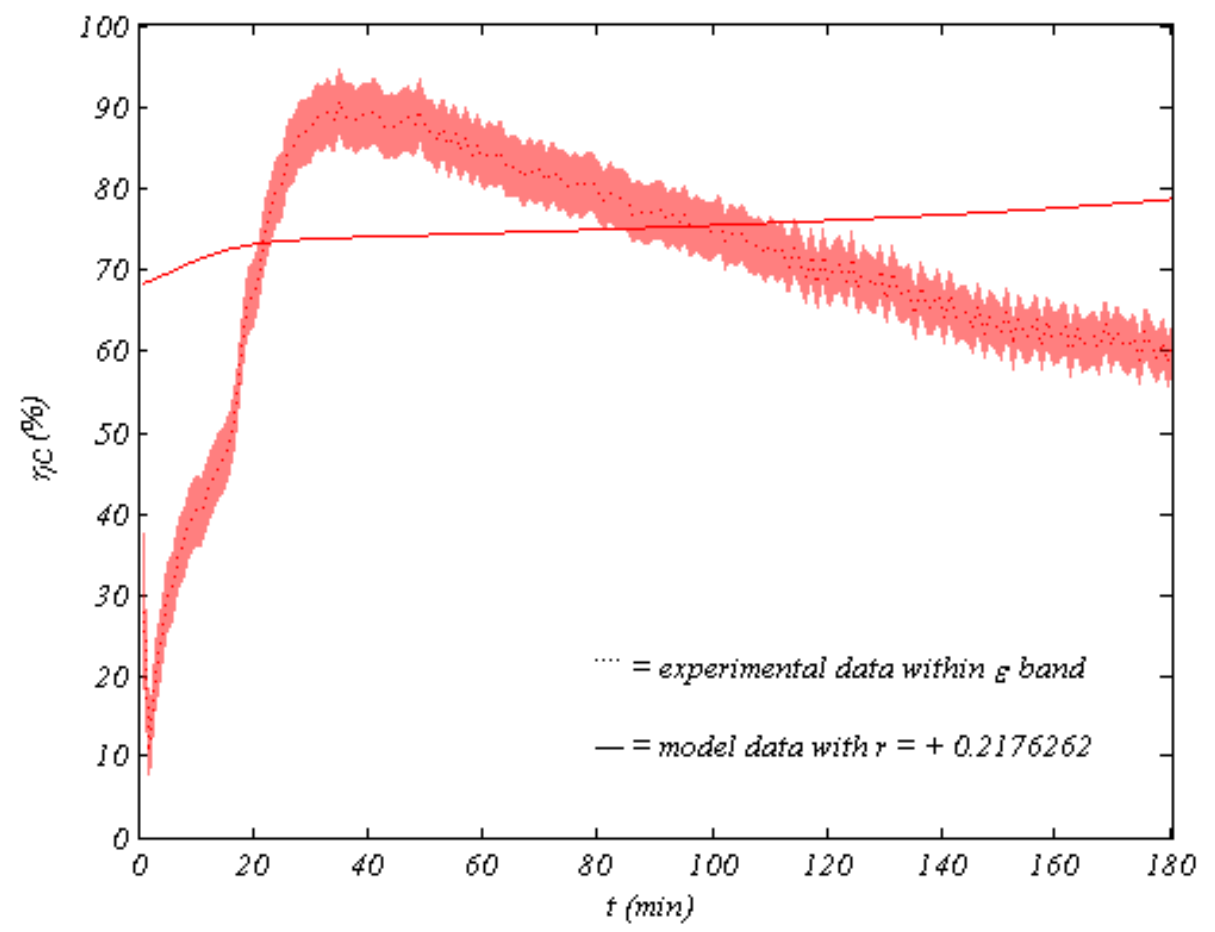

Figure 160. Condenser efficiency at $80^{\circ} \mathrm{C}$ flash and lower flow

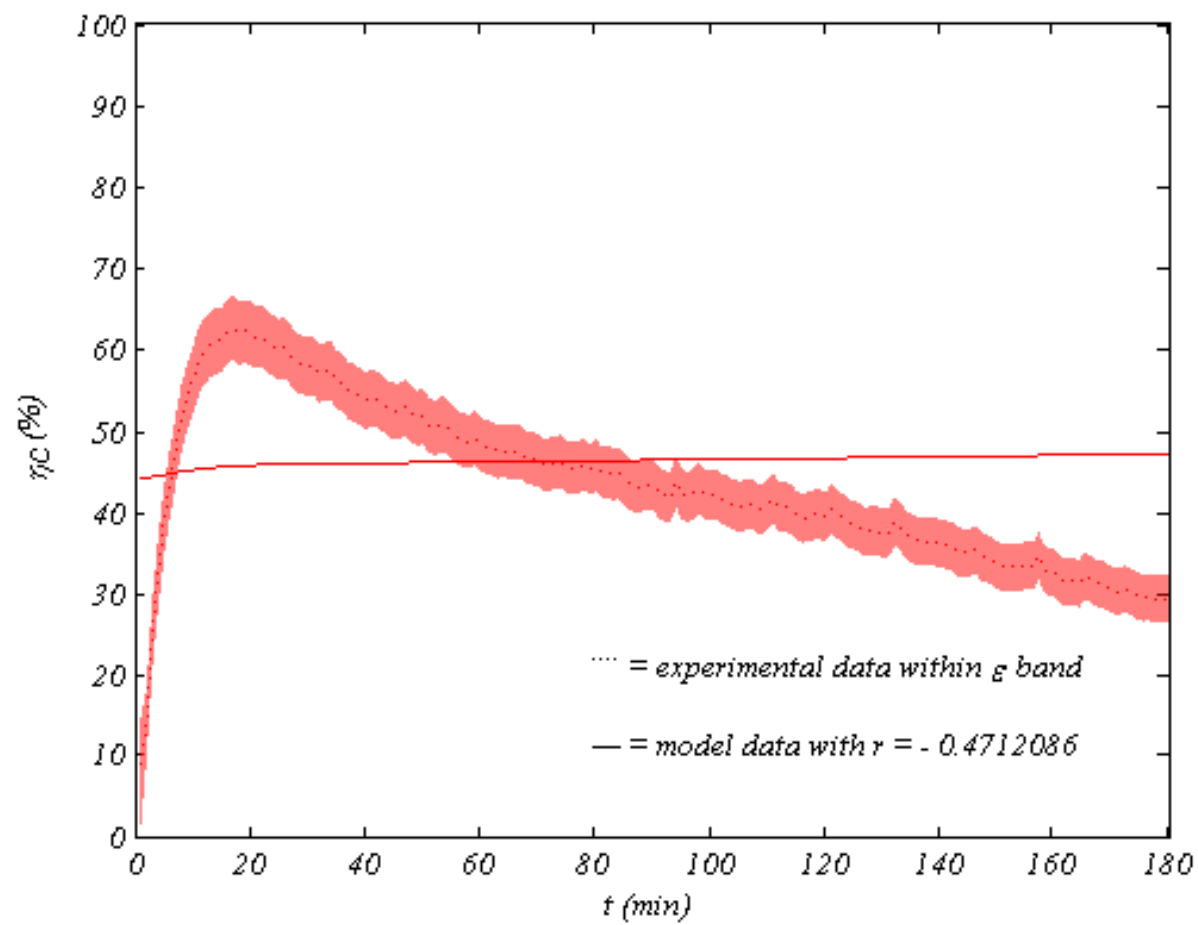

Figure 161. Condenser efficiency at $80^{\circ} \mathrm{C}$ flash and higher flow 


\subsection{Recovery Efficiency}

Recovery efficiency is defined as the ratio of seawater enthalpy change due to condensing water vapor to the overall enthalpy change required to attain the set flash temperature written as a percentage, that is $\eta_{R}=\left[\left(H_{X}-H_{S}\right) /\left(H_{H}-H_{S}\right)\right] \cdot 100 \%$, as was mentioned in CHAPTER 4. In other words, it is the percent of total enthalpy change that was essentially accomplished by reclaiming heat from condensing vapor. Recovery efficiency rapidly increased to reach a maximum early before it slowly declined for both seawater flow rates; however, recovery efficiency was higher at lower seawater flow rates. It increased with flash temperatures for both seawater flow rates, since preheat enthalpy is directly proportional to preheat temperature that is directly proportional to flashing temperature as seen earlier.

Recovery efficiency directly expresses the percent of required heat that is reclaimed from condensing vapor. Recovery efficiency rapidly increased because of rapidly rising preheat enthalpy due to rapidly rising preheat temperature, then it slowly declined because of decreasing preheat enthalpy due to decreasing preheat temperature. Recovery efficiency profiles for both seawater flow rates are similar; however, they were higher for lower flow rates because of higher preheat enthalpy due to higher preheat temperature experienced at lower seawater flow rates due to more condensation caused by more vaporization.

Model prediction of recovery efficiency loosely resembled experimental results because of loose depiction of preheat temperature due to inability of the condenser tube heat transfer module of the model to capture the rate of condensation. Recovery efficiency profiles are shown in Figure 162 through Figure 173. 


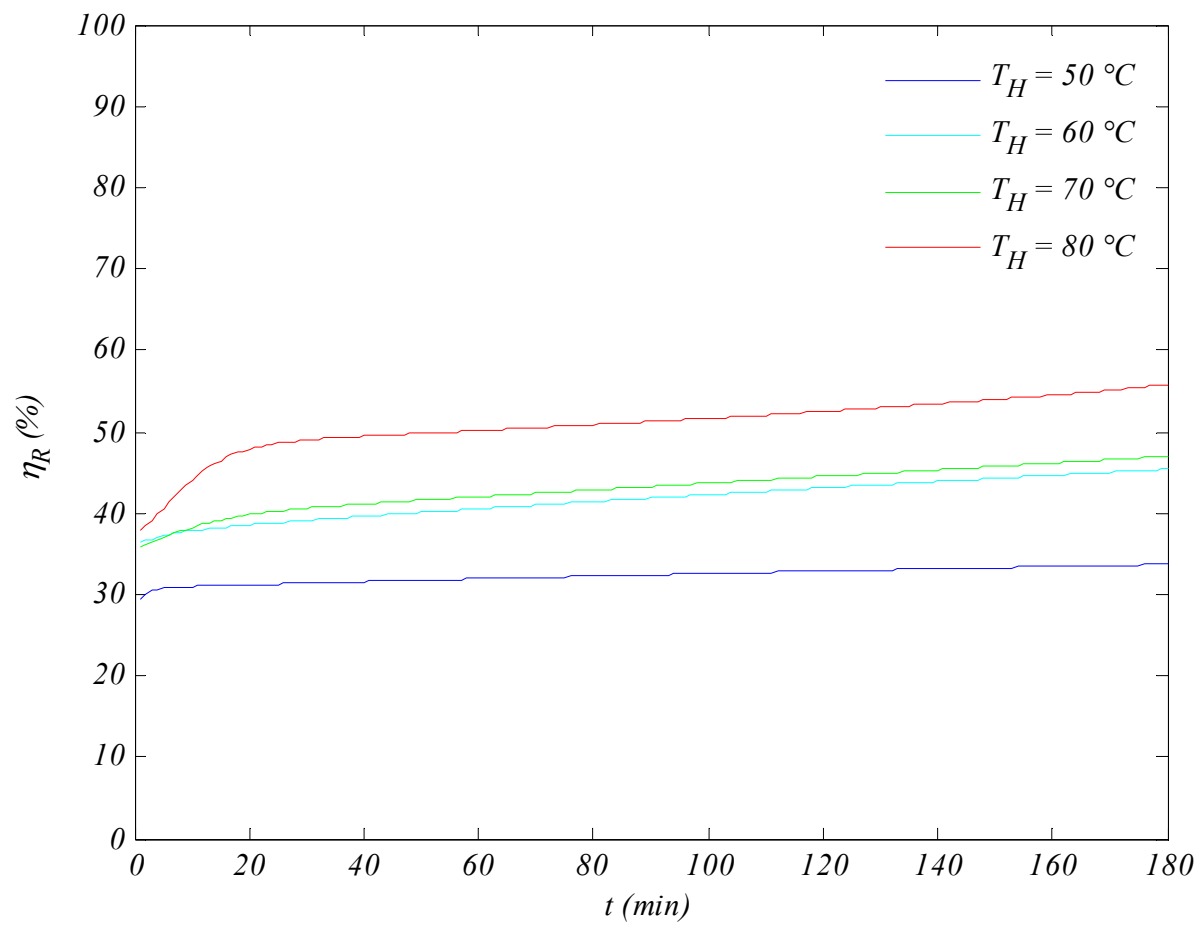

Figure 162. Modeled recovery efficiency profiles at lower flow

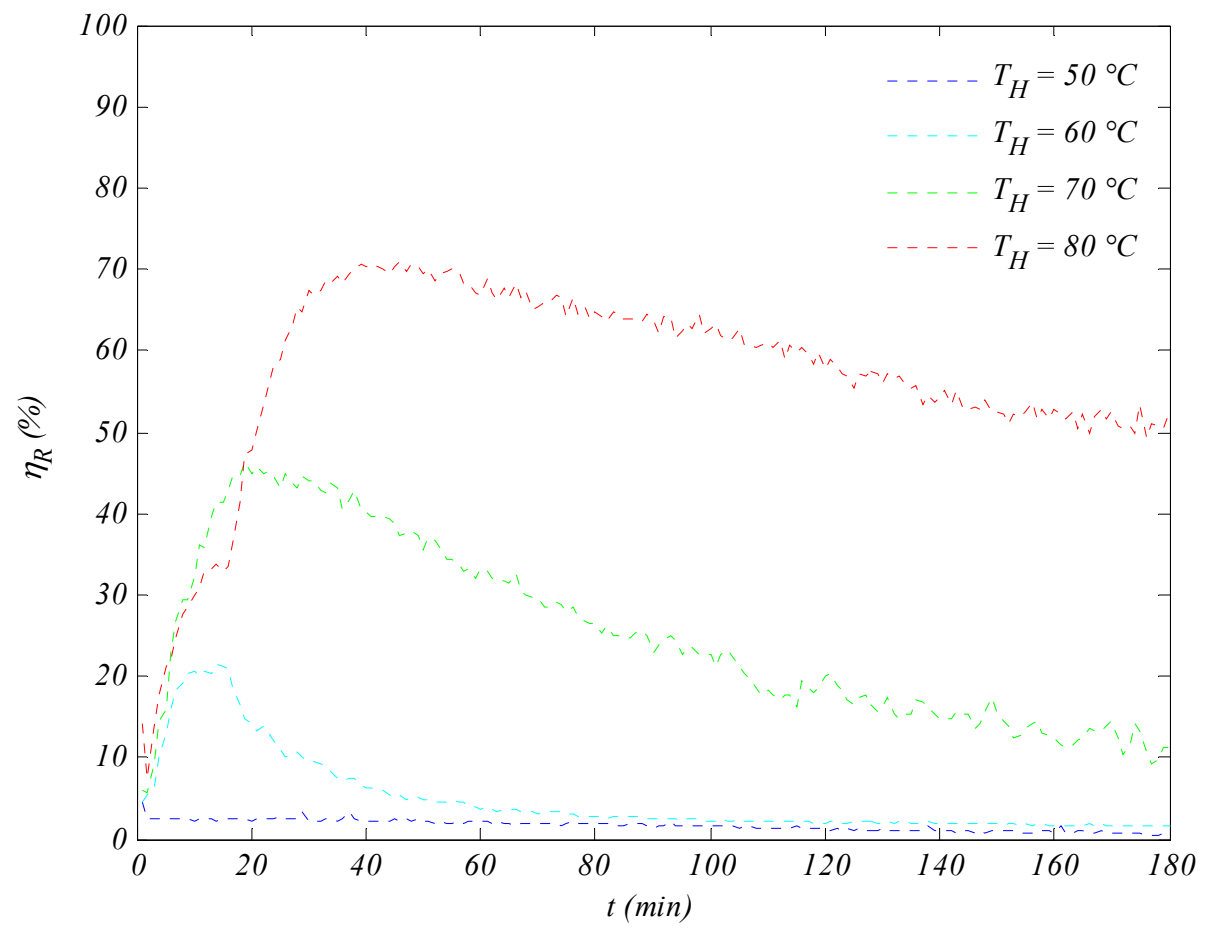

Figure 163. Experimental recovery efficiency profiles at lower flow 


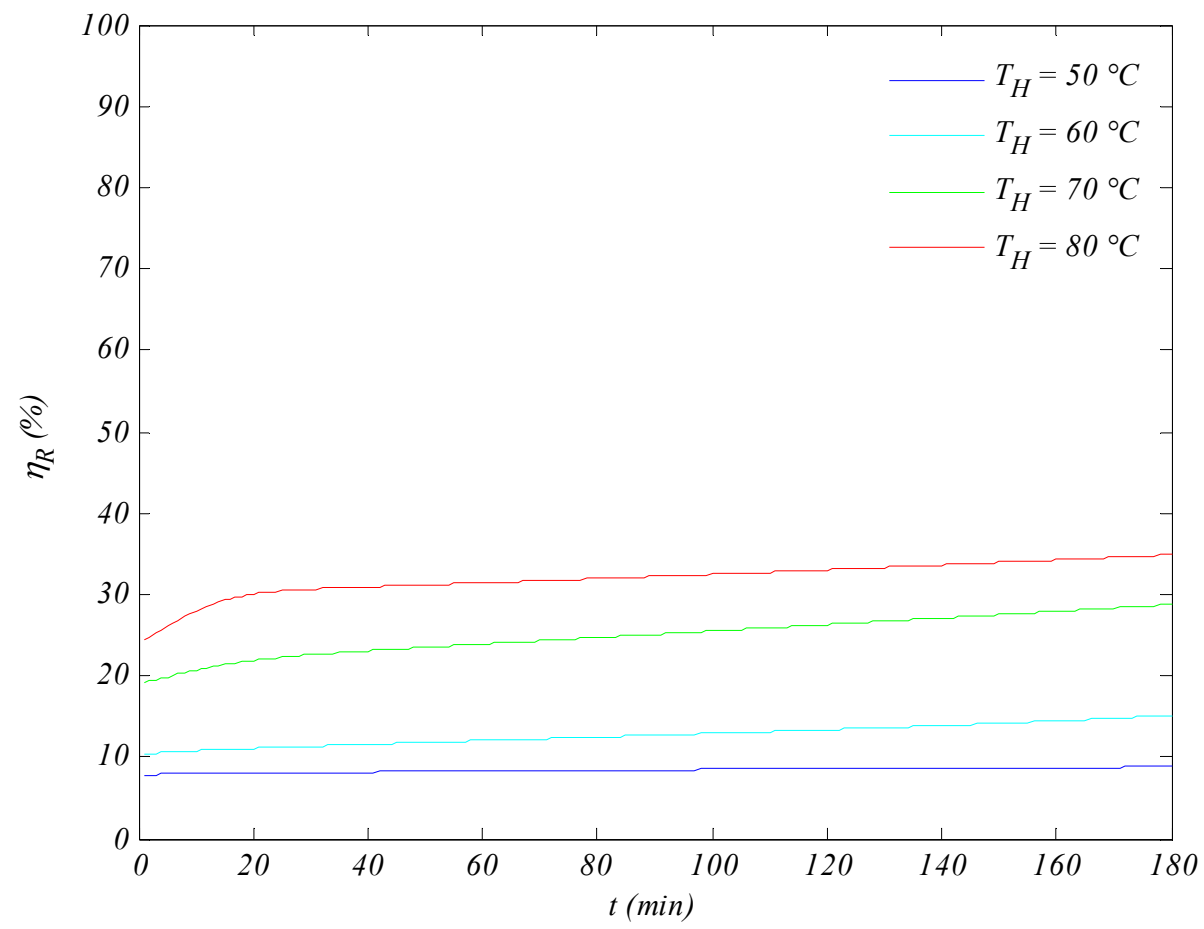

Figure 164. Modeled recovery efficiency profiles at higher flow

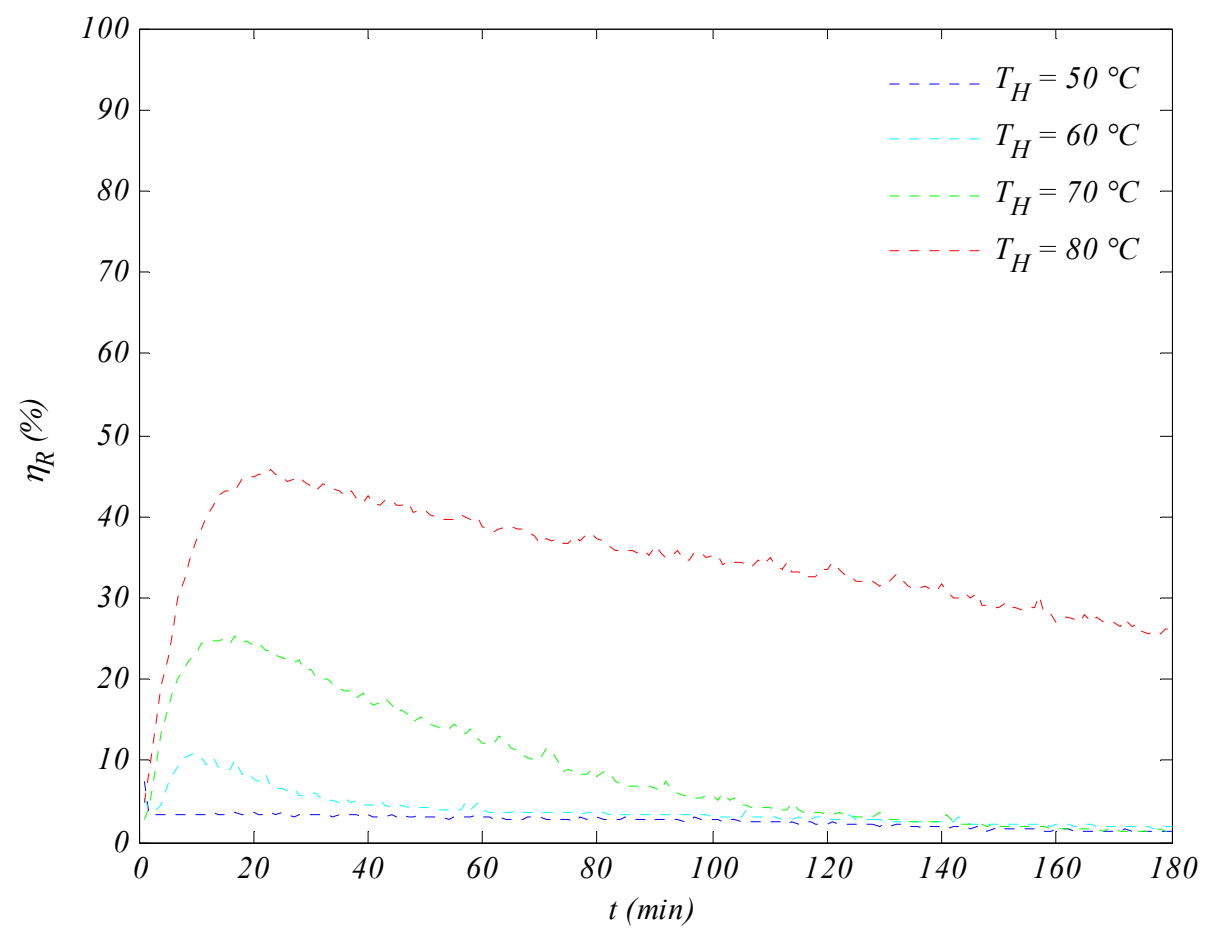

Figure 165. Experimental recovery efficiency profiles at higher flow 


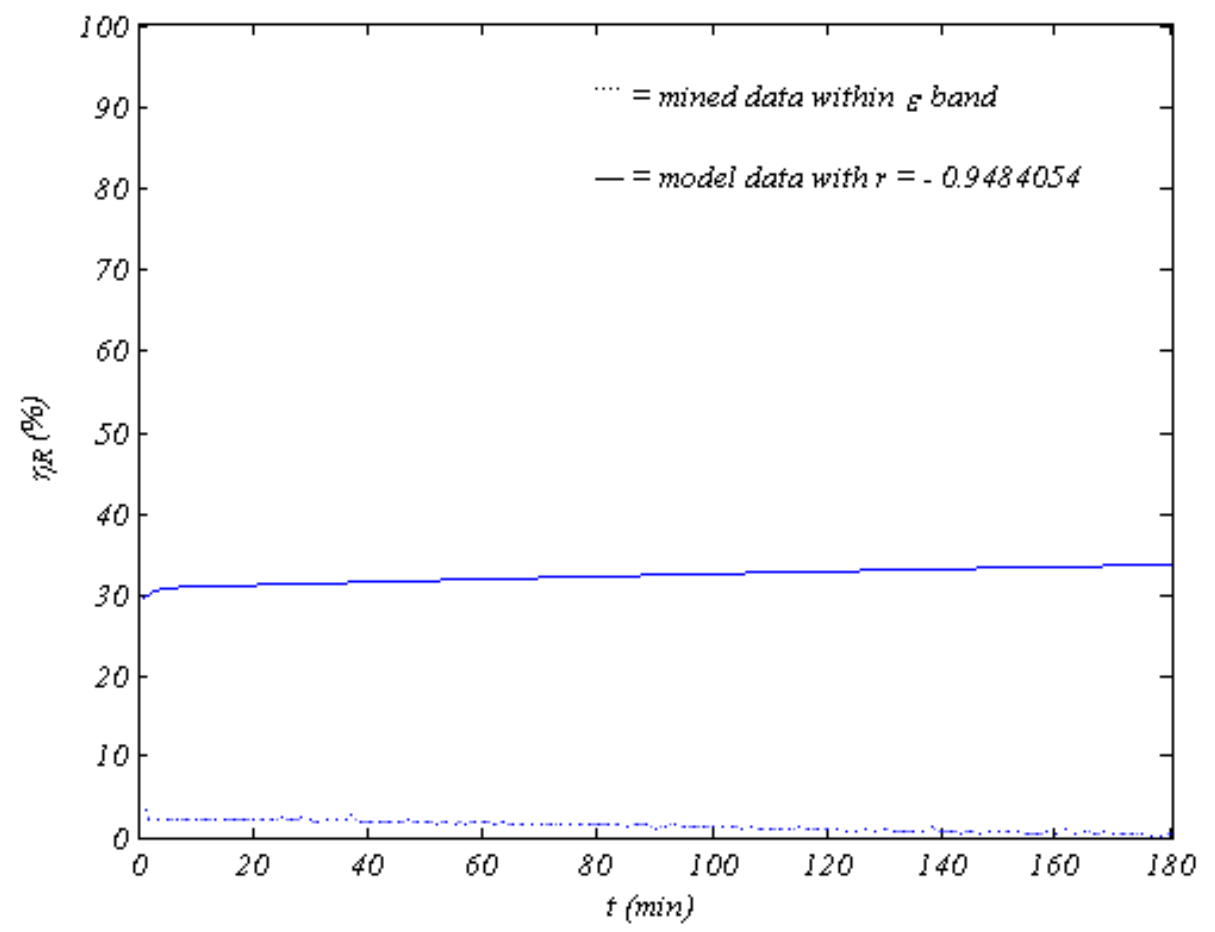

Figure 166 . Recovery efficiency at $50^{\circ} \mathrm{C}$ flash and lower flow

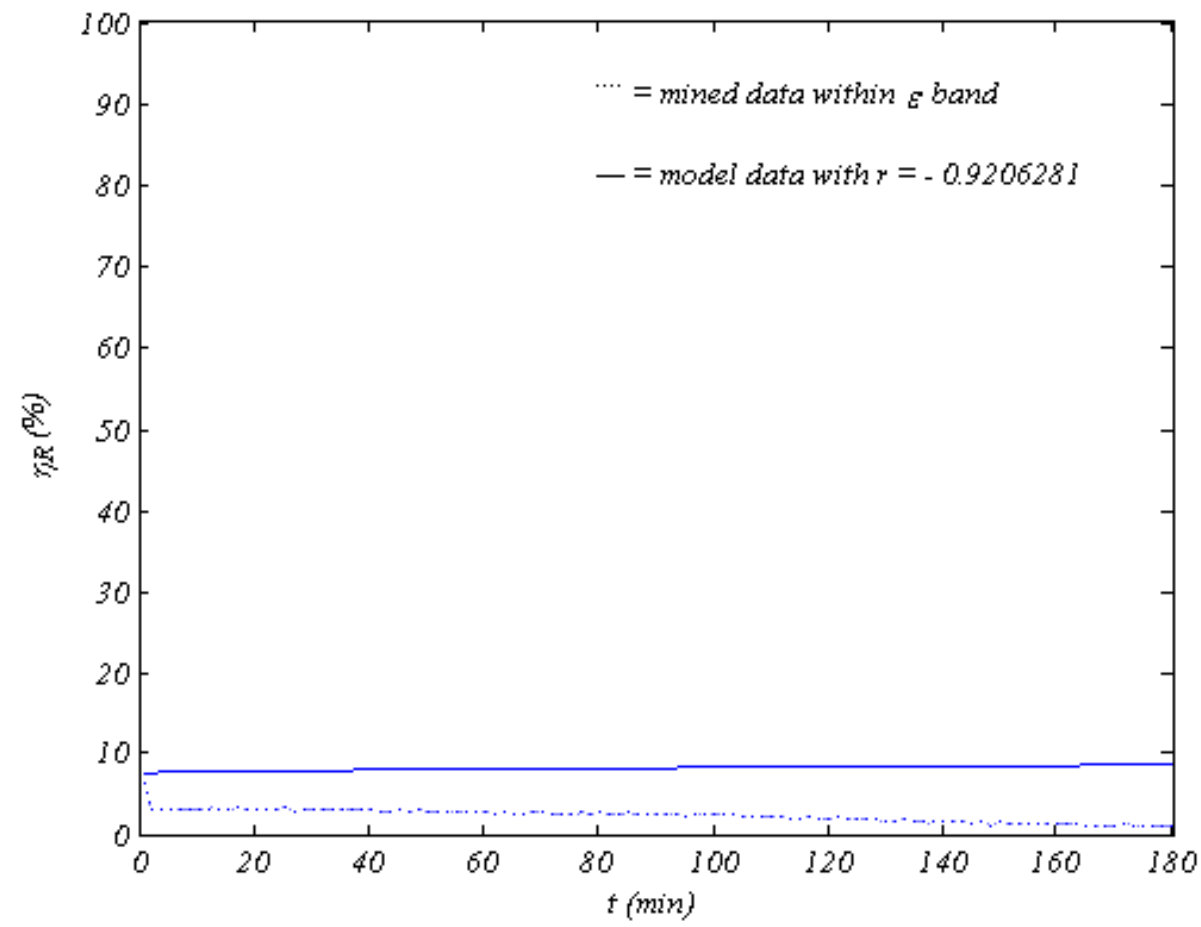

Figure 167. Recovery efficiency at $50^{\circ} \mathrm{C}$ flash and higher flow 


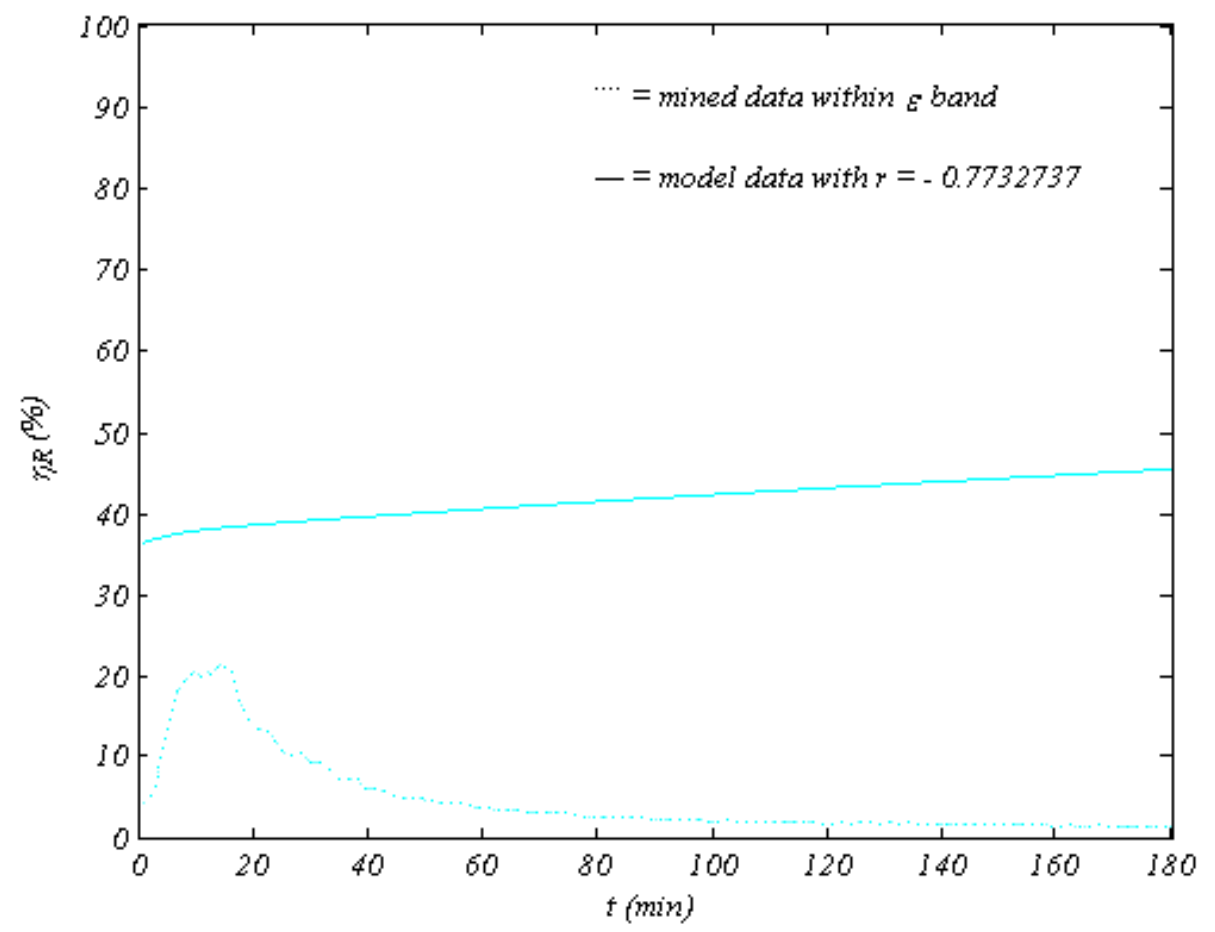

Figure 168 . Recovery efficiency at $60^{\circ} \mathrm{C}$ flash and lower flow

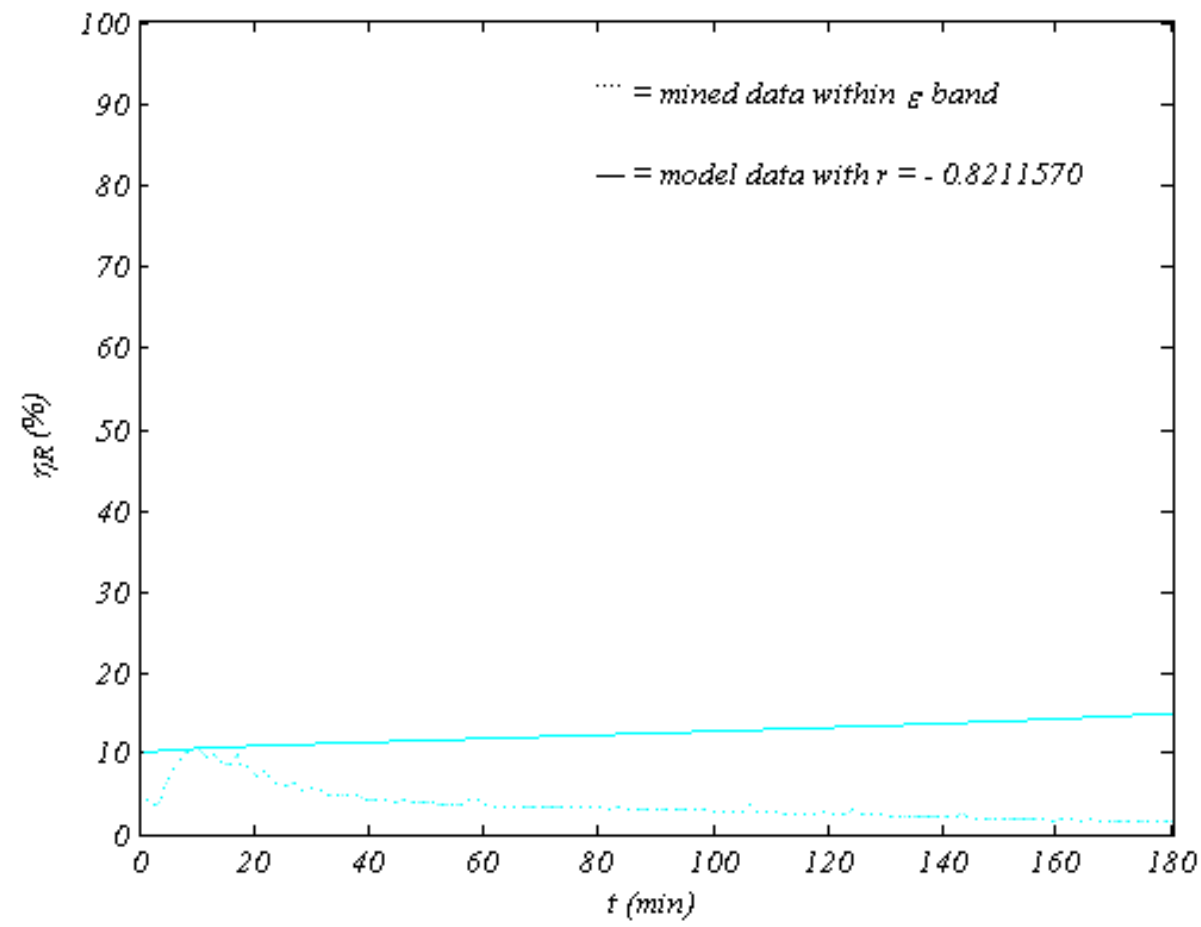

Figure 169. Recovery efficiency at $60^{\circ} \mathrm{C}$ flash and higher flow 


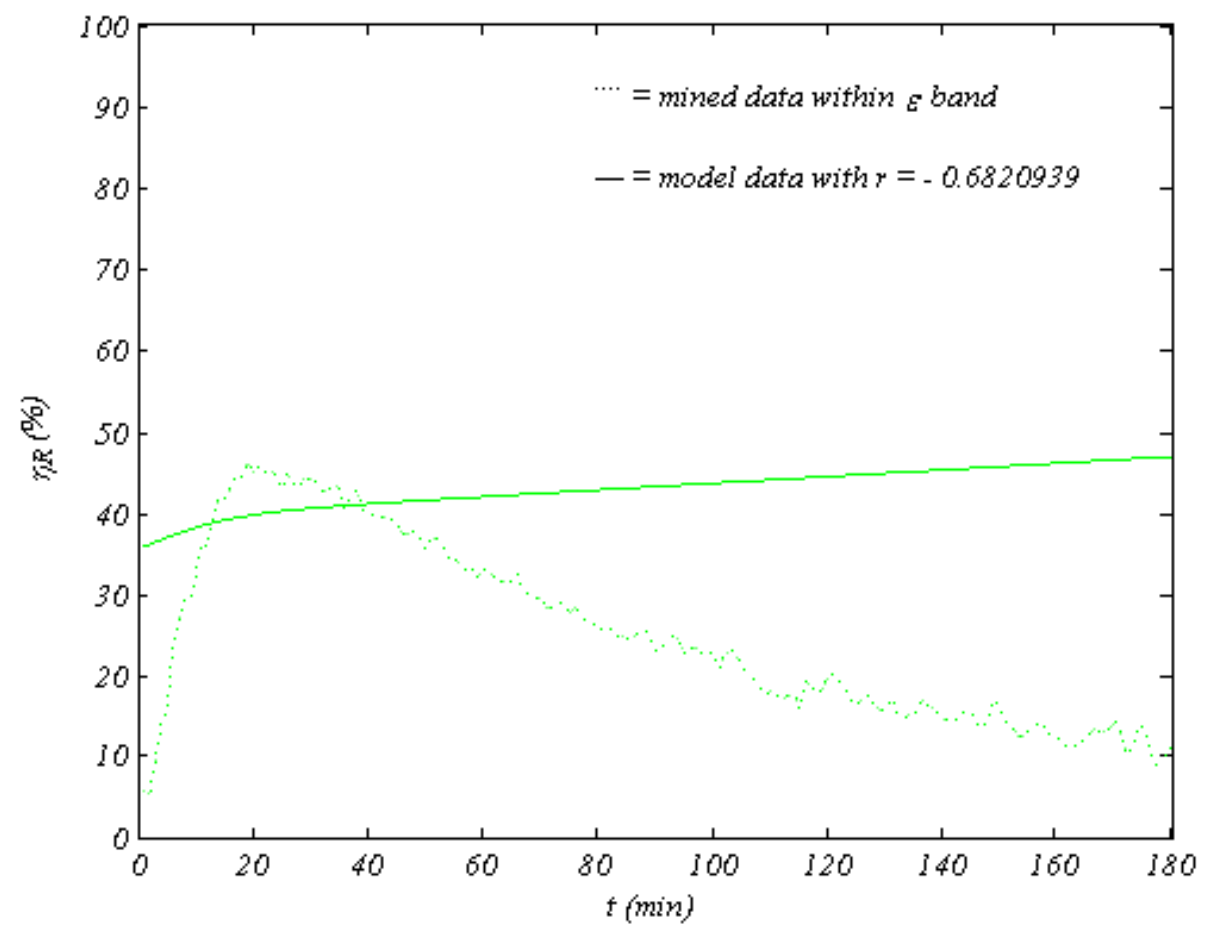

Figure 170 . Recovery efficiency at $70^{\circ} \mathrm{C}$ flash and lower flow

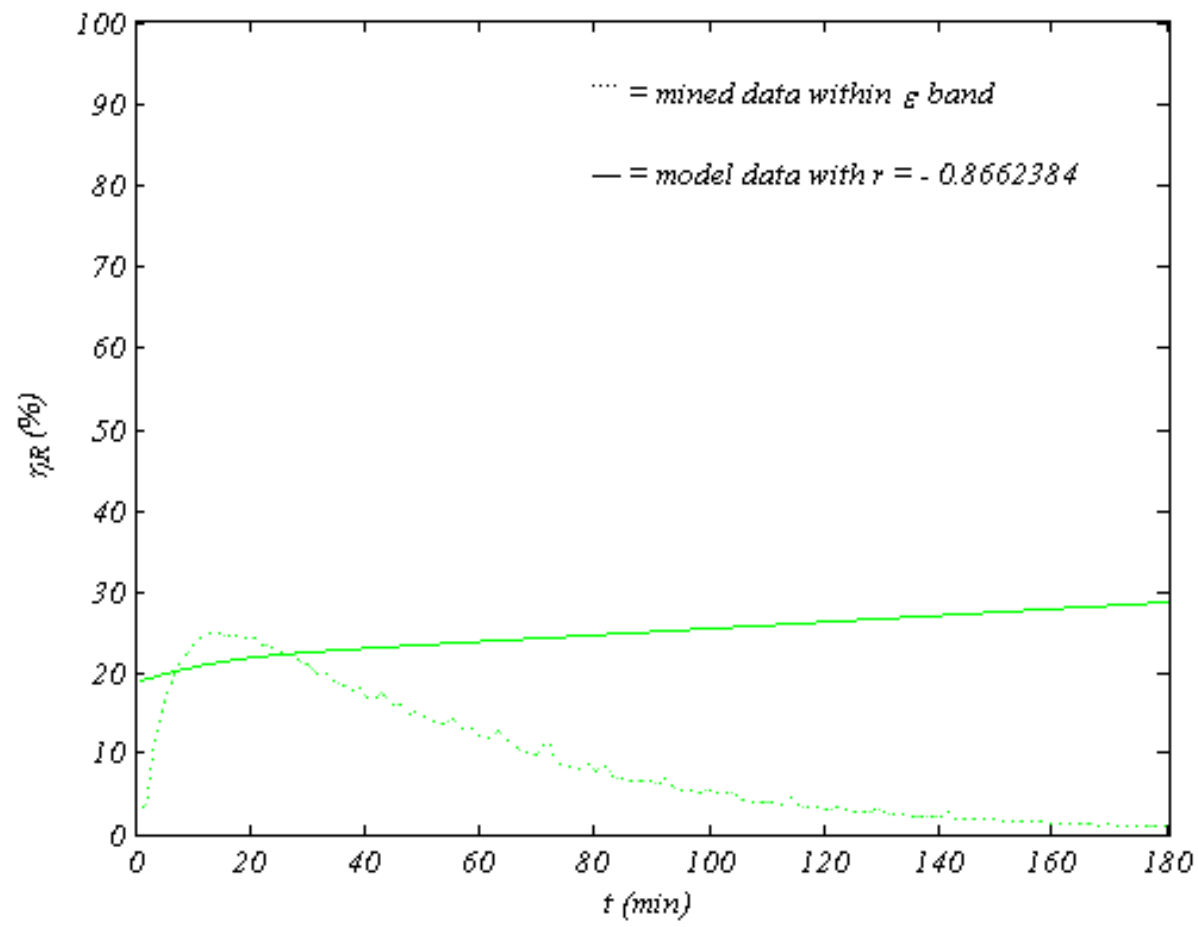

Figure 171 . Recovery efficiency at $70^{\circ} \mathrm{C}$ flash and higher flow 


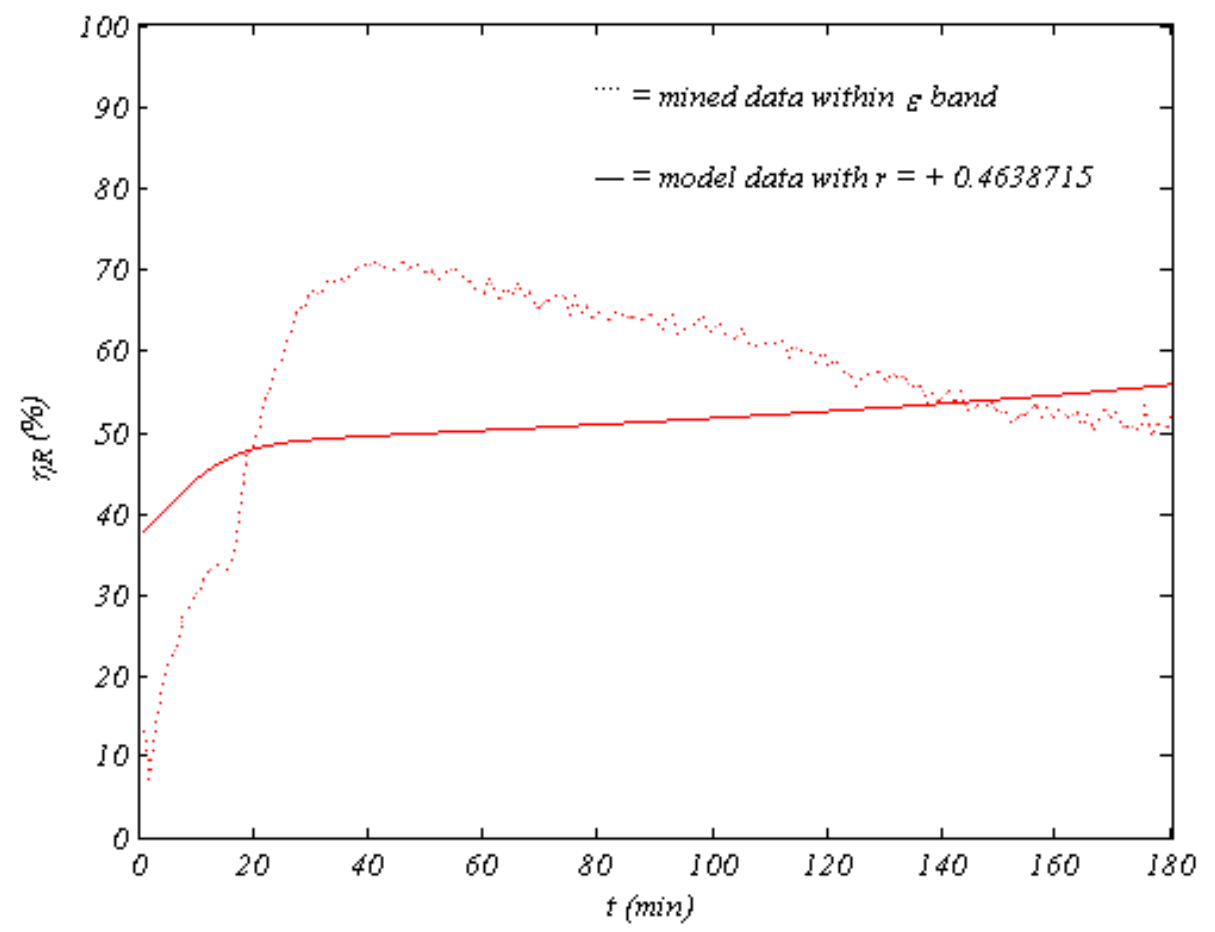

Figure 172 . Recovery efficiency at $80^{\circ} \mathrm{C}$ flash and lower flow

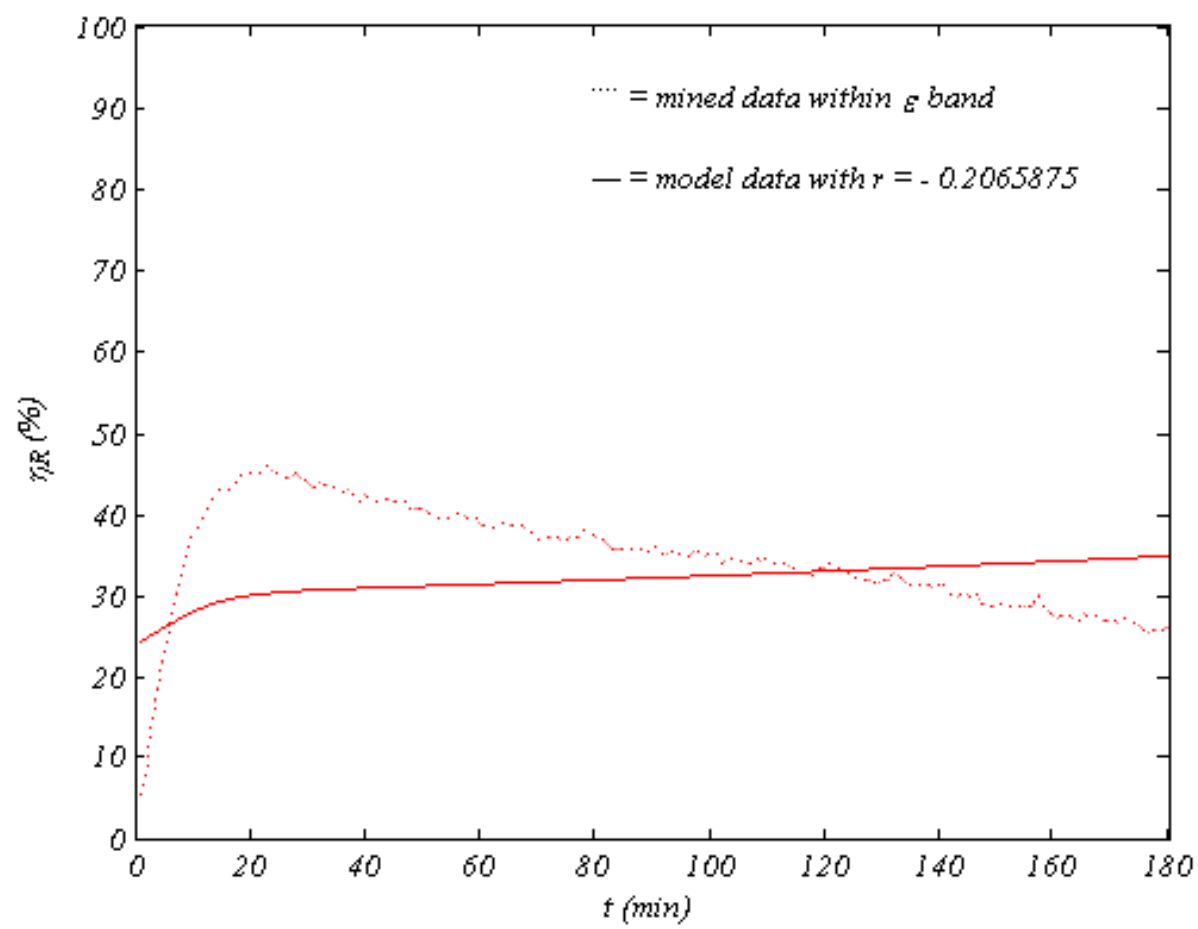

Figure 173. Recovery efficiency at $80^{\circ} \mathrm{C}$ flash and higher flow 


\subsection{Thermal Efficiency}

Thermal efficiency of the desalination process is a performance measure defined as the ratio of heat used in vaporizing water to overall heat added to bulk seawater written as a percentage, that is $\eta_{T}=\left[M_{E} \cdot\left(H_{E}+H_{E}^{L}\right) /\left(M_{H} \cdot H_{H}\right)\right] \cdot 100 \%$, as was mentioned in CHAPTER 4. In other words, it is the percent of total thermal energy supplied that was actually used to vaporize water. Thermal efficiency rapidly increased to reach a maximum early before it slowly declined, then stabilized for both seawater flow rates; however, thermal efficiency was higher at lower seawater flow rates. The thermal efficiency increased with flash temperatures for both seawater flow rates, since the amounts and the temperatures of the water vapor are directly proportional to the flashing temperature as seen earlier.

Thermal efficiency rapidly increased because of rapidly increasing water vapor enthalpy due to its rising amount and temperature, and then it slowly declined, reaching a plateau because of slowly stabilizing water vapor enthalpy due to gradually declining vaporization rates, but gradually rising temperatures, of the water vapor for both seawater flow rates. Thermal efficiency profiles for both seawater flow rates are similar; however, they were higher for lower flow rates due to higher vaporization rates.

Model prediction of thermal efficiency of the proposed desalination process properly resembled pseudo-experimental results due to accurate portrayal of water vaporization rates; furthermore, adjusted parameters and correlations played a significant role in shifting vaporization rate profiles to match pseudo-experimental results, resulting in excellent thermal efficiency estimates for the proposed desalination process. Thermal efficiency profiles are shown in Figure 174 through Figure 185. 


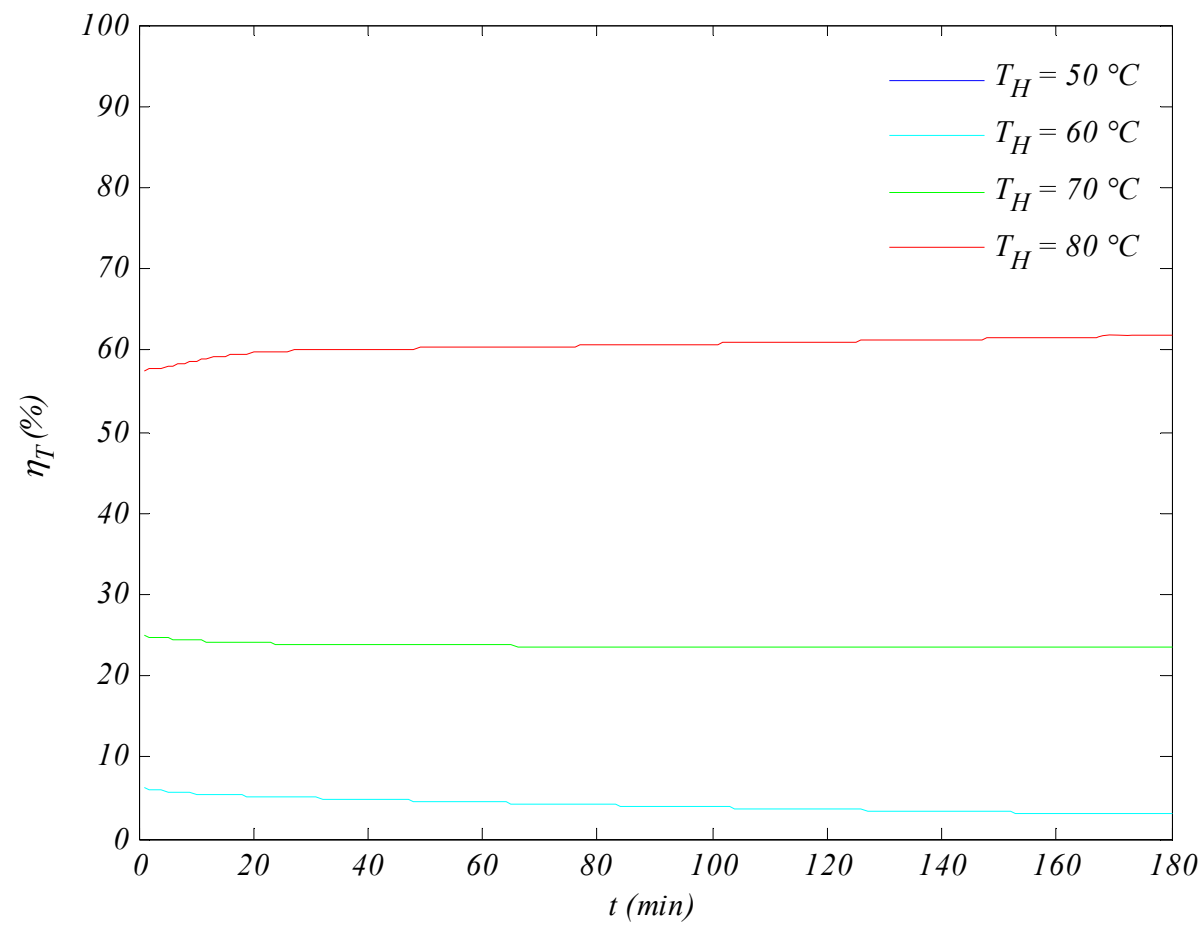

Figure 174. Modeled thermal efficiency profiles at lower flow

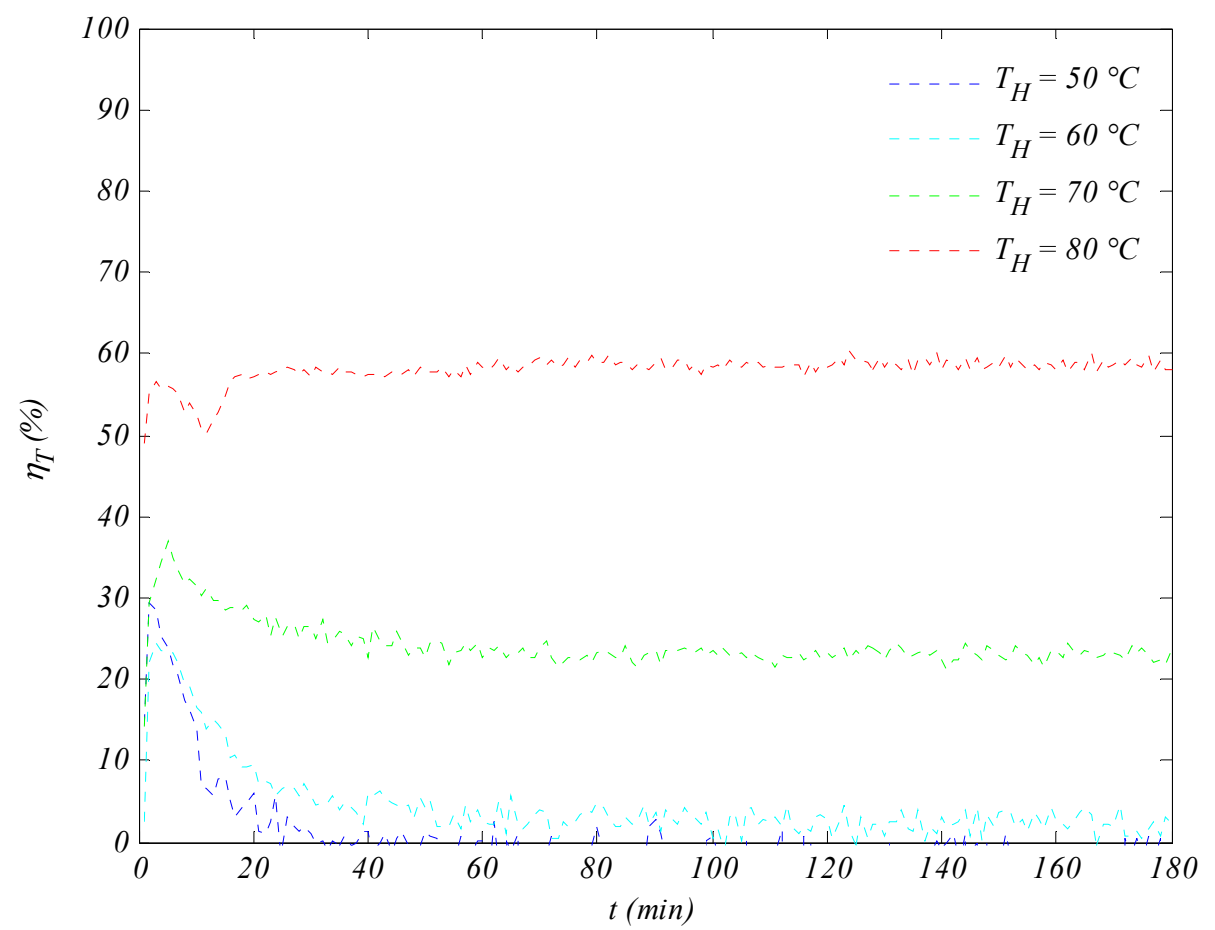

Figure 175. Mined thermal efficiency profiles at lower flow 


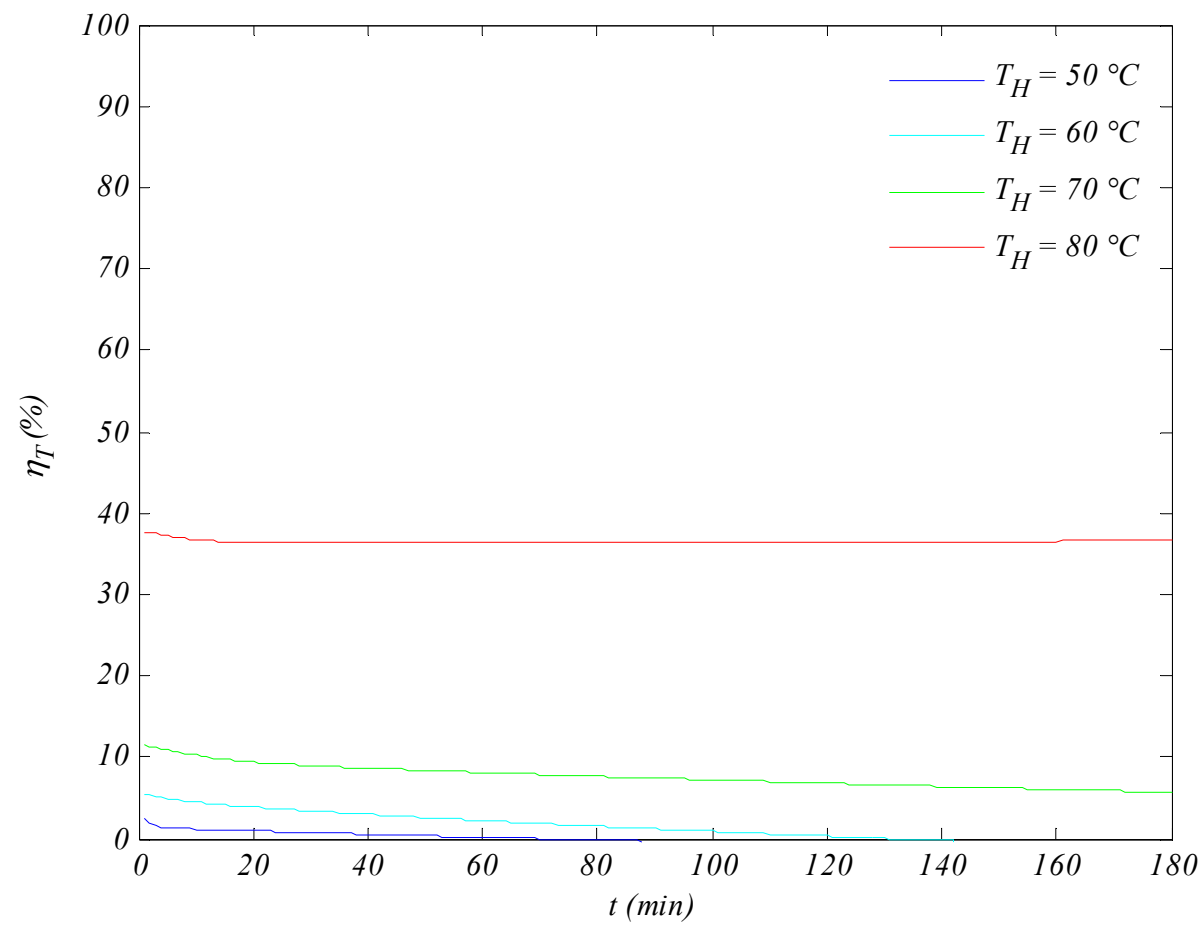

Figure 176. Modeled thermal efficiency profiles at higher flow

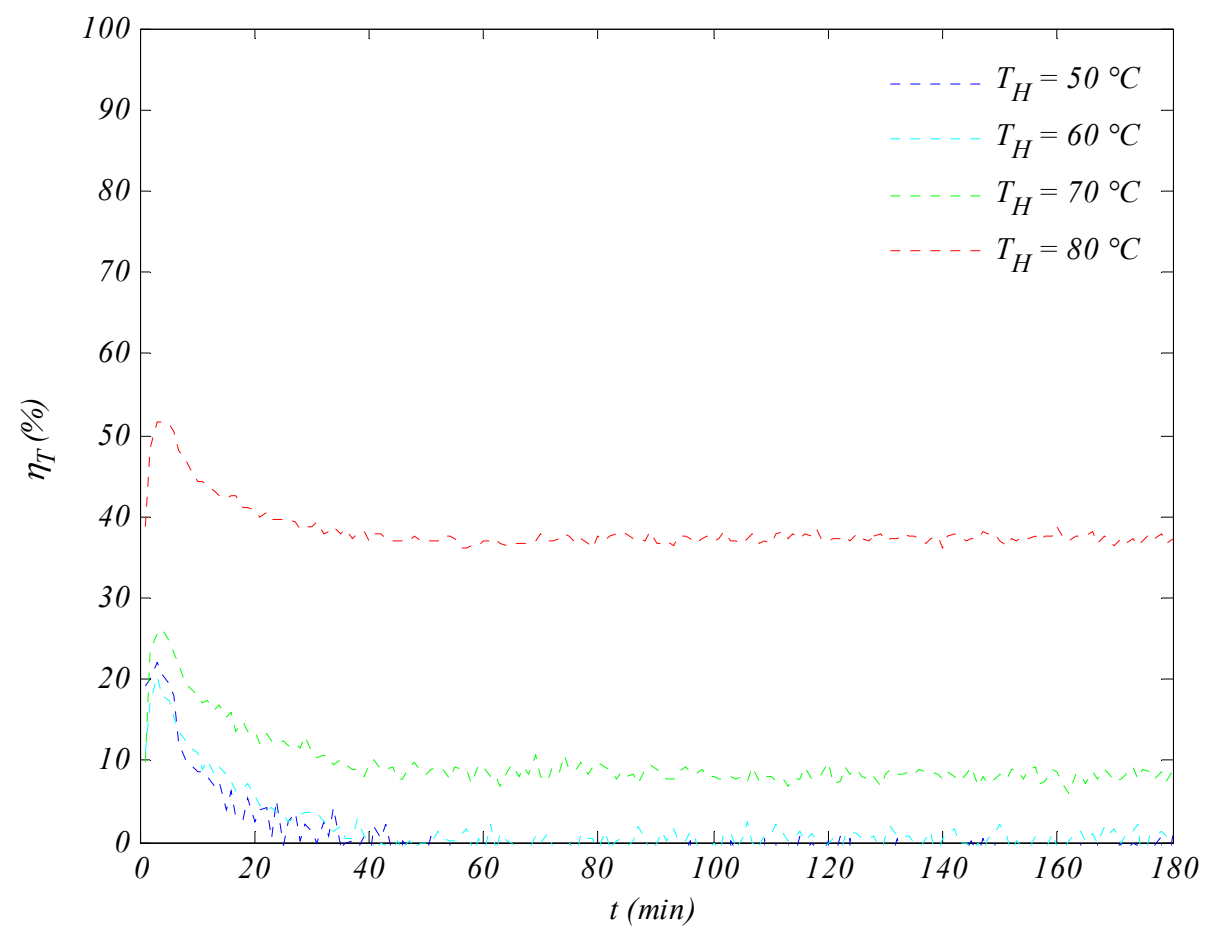

Figure 177. Mined thermal efficiency profiles at higher flow 


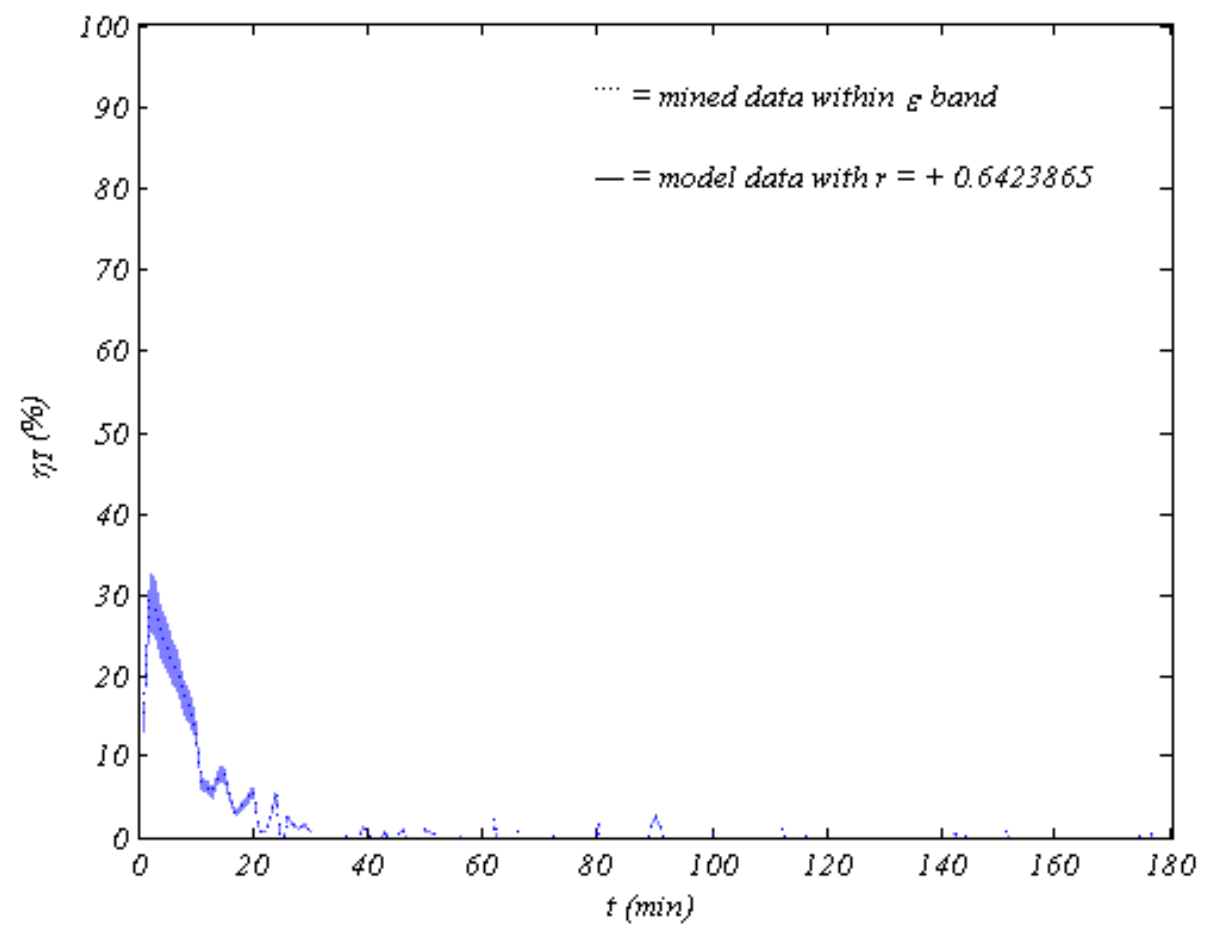

Figure 178. Thermal efficiency at $50^{\circ} \mathrm{C}$ flash and lower flow

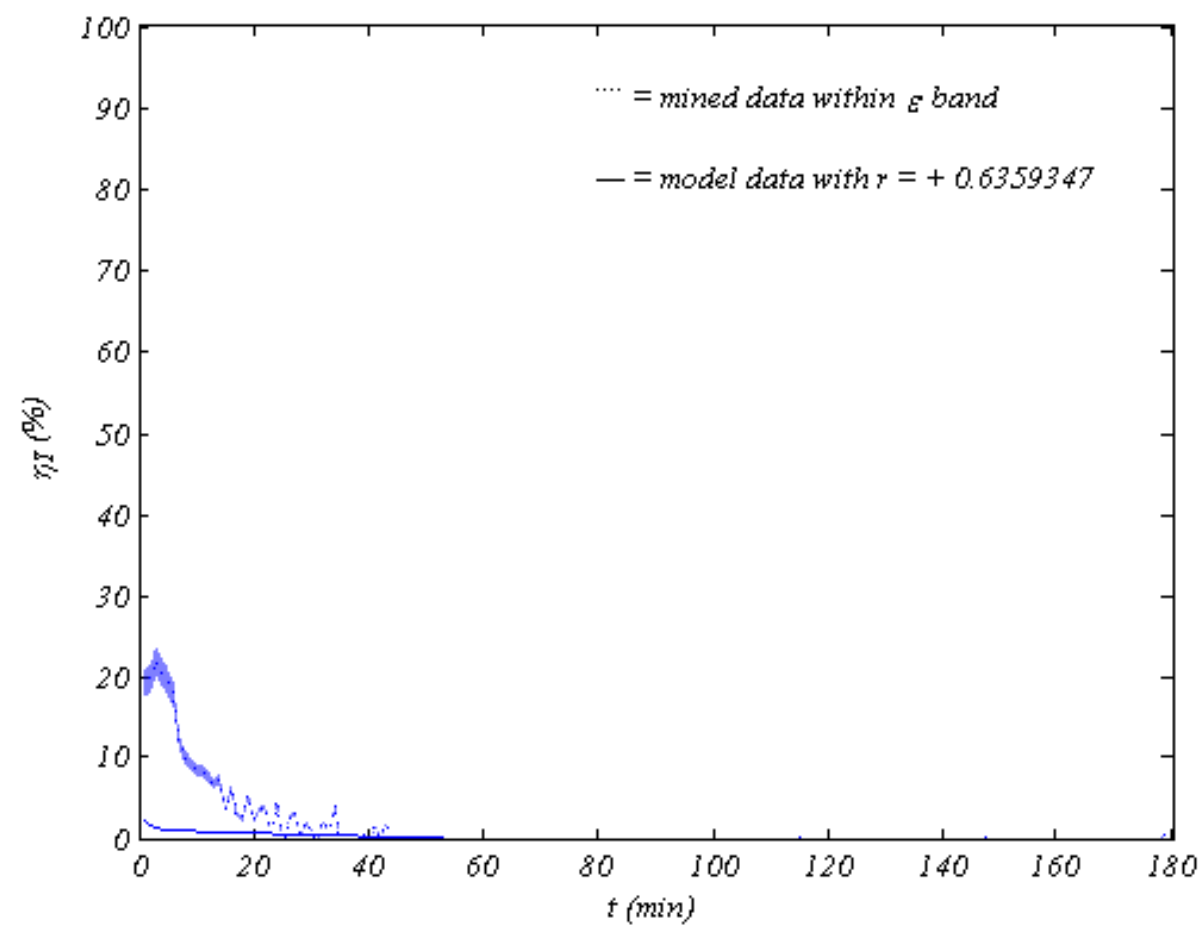

Figure 179. Thermal efficiency at $50^{\circ} \mathrm{C}$ flash and higher flow 


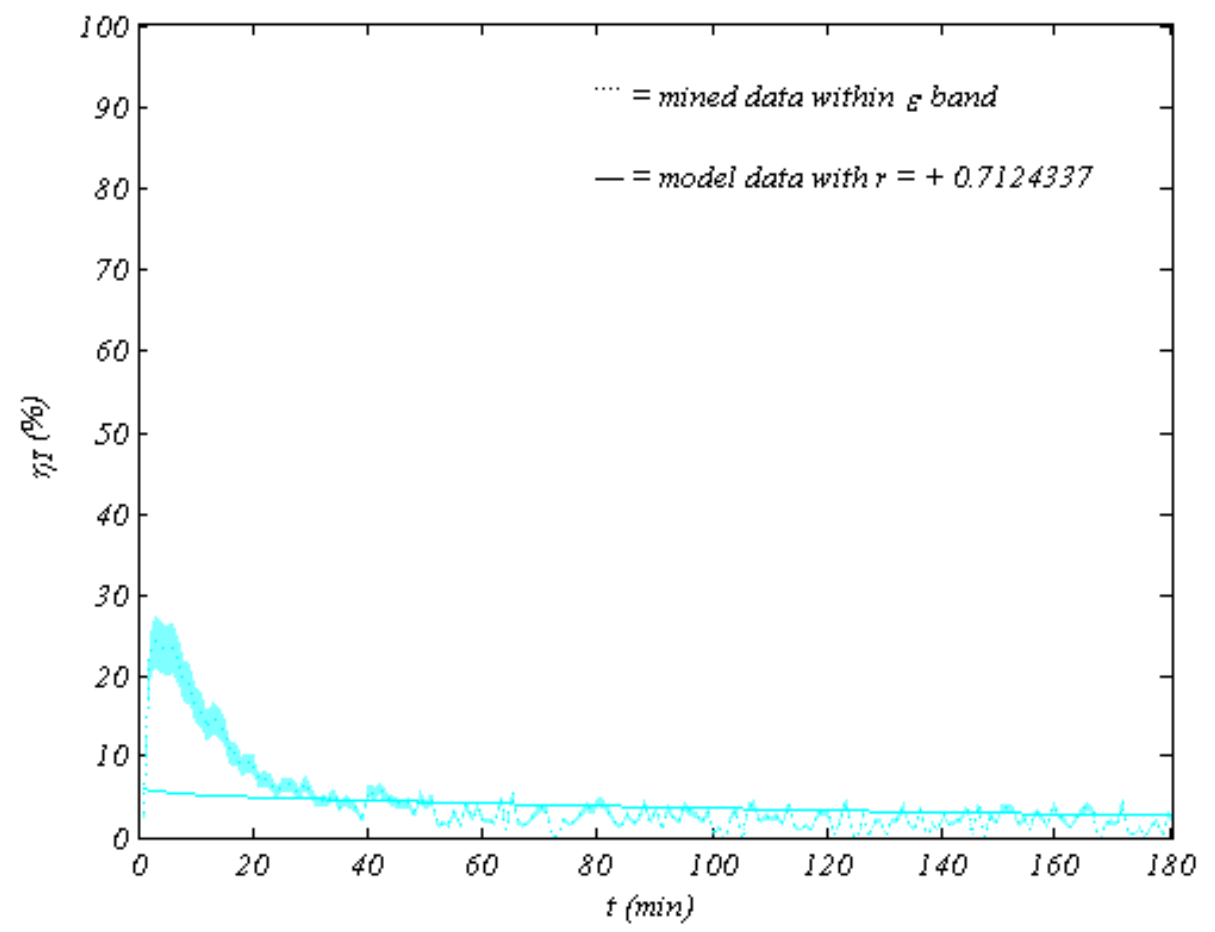

Figure 180 . Thermal efficiency at $60^{\circ} \mathrm{C}$ flash and lower flow

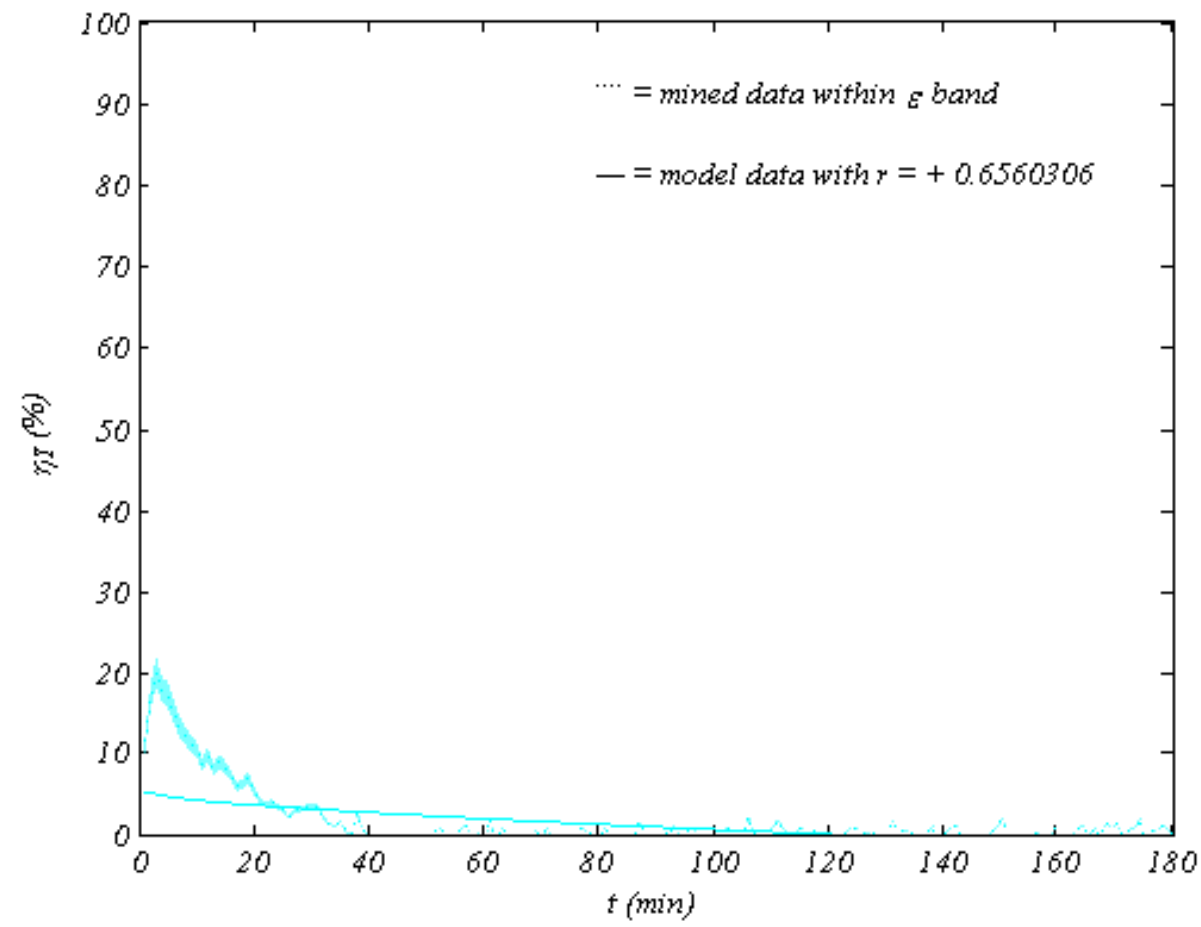

Figure 181 . Thermal efficiency at $60^{\circ} \mathrm{C}$ flash and higher flow 


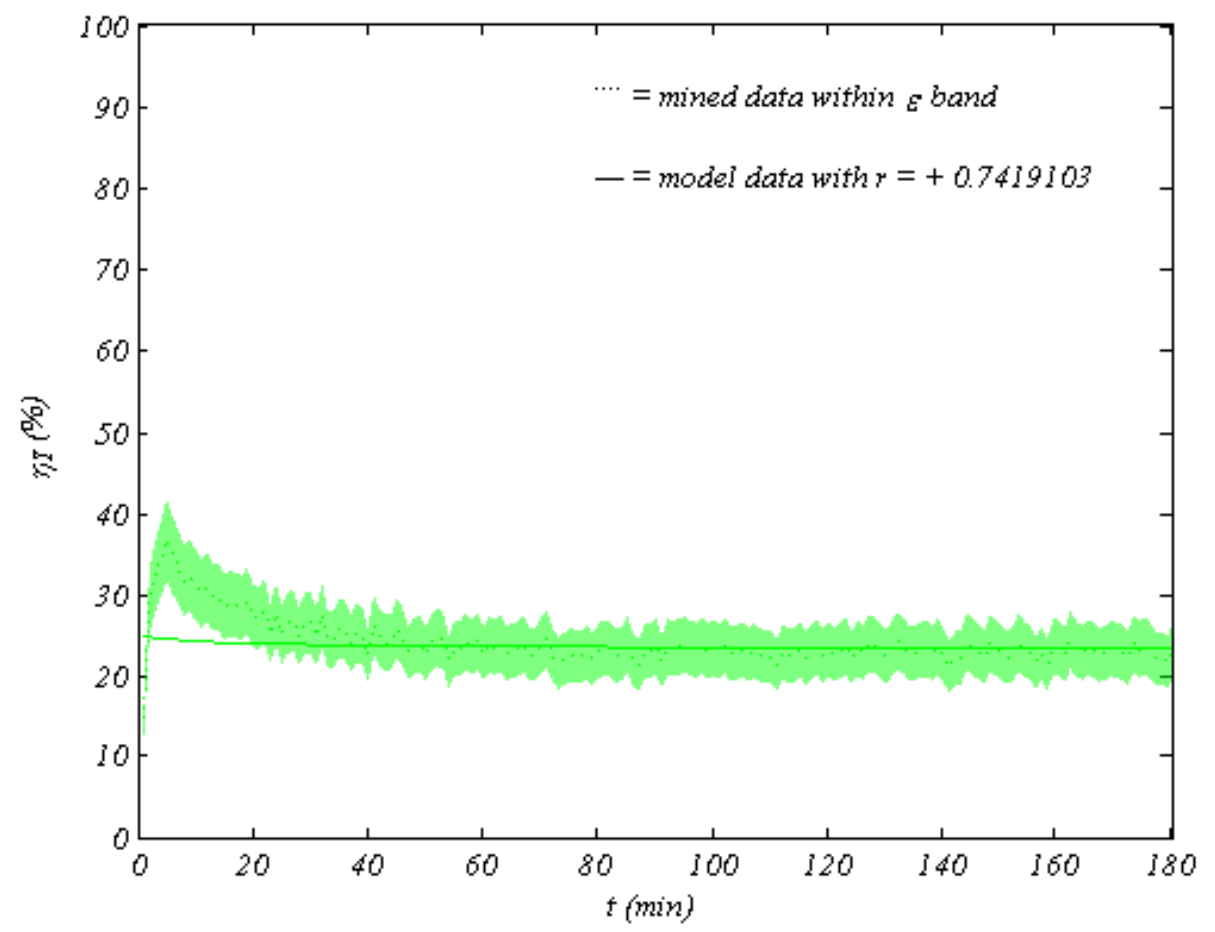

Figure 182 . Thermal efficiency at $70^{\circ} \mathrm{C}$ flash and lower flow

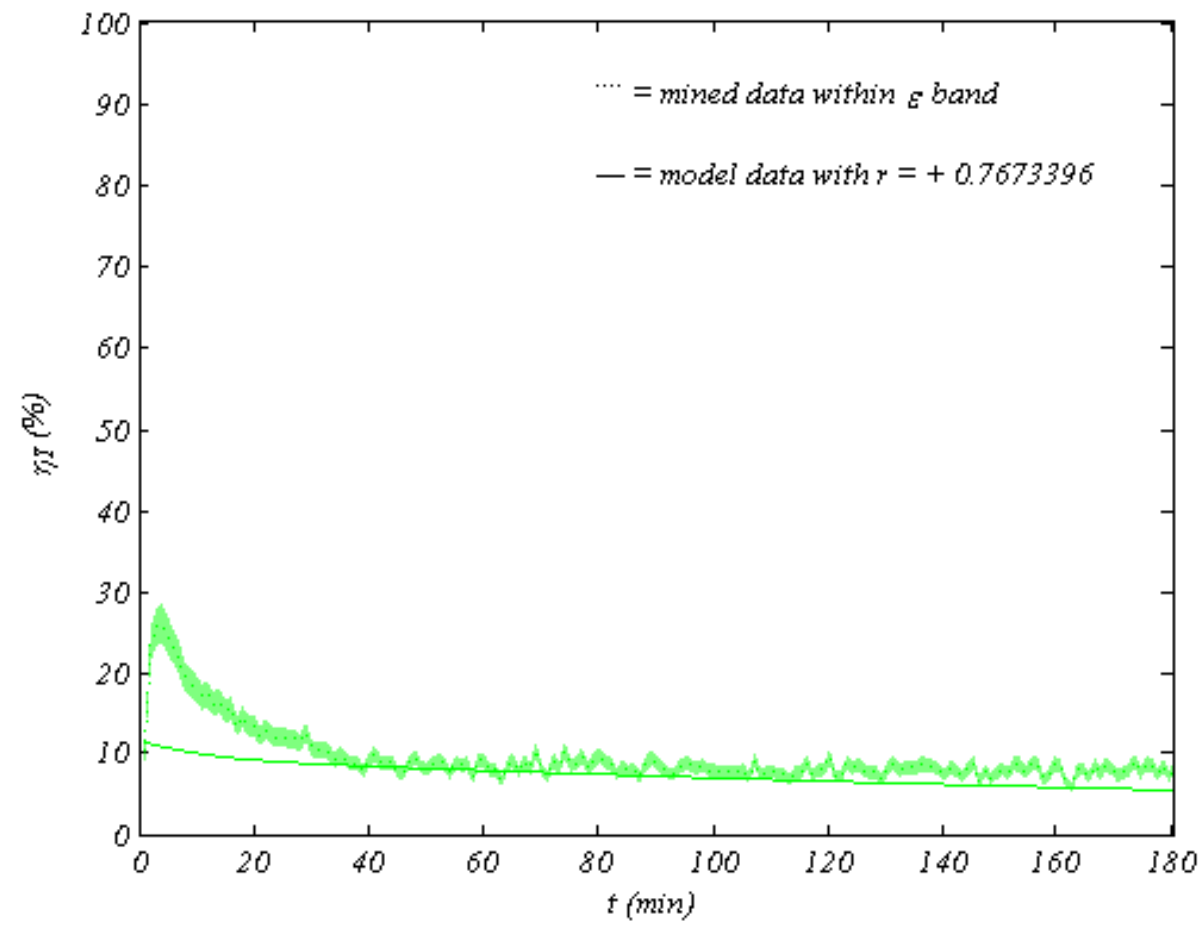

Figure 183 . Thermal efficiency at $70^{\circ} \mathrm{C}$ flash and higher flow 


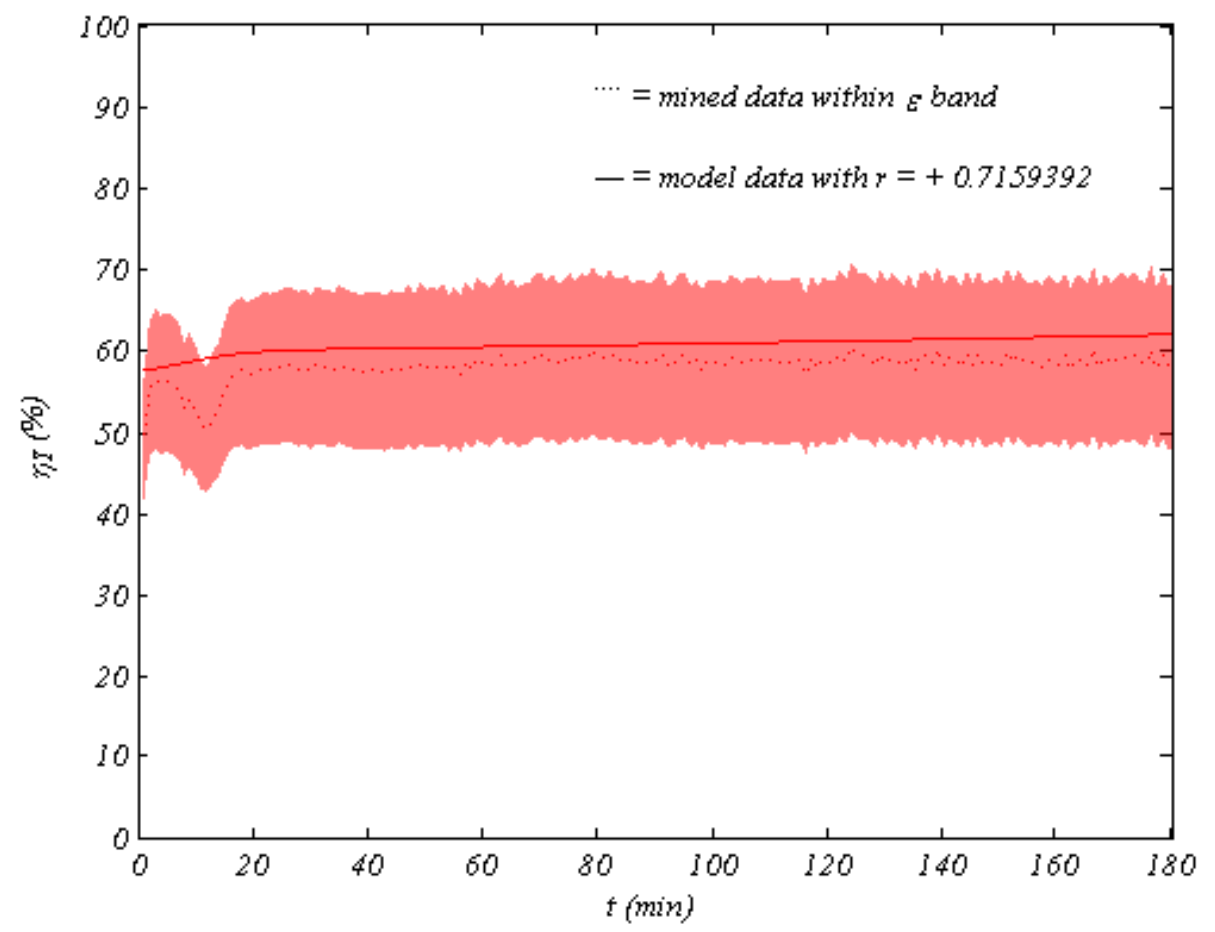

Figure 184. Thermal efficiency at $80^{\circ} \mathrm{C}$ flash and lower flow

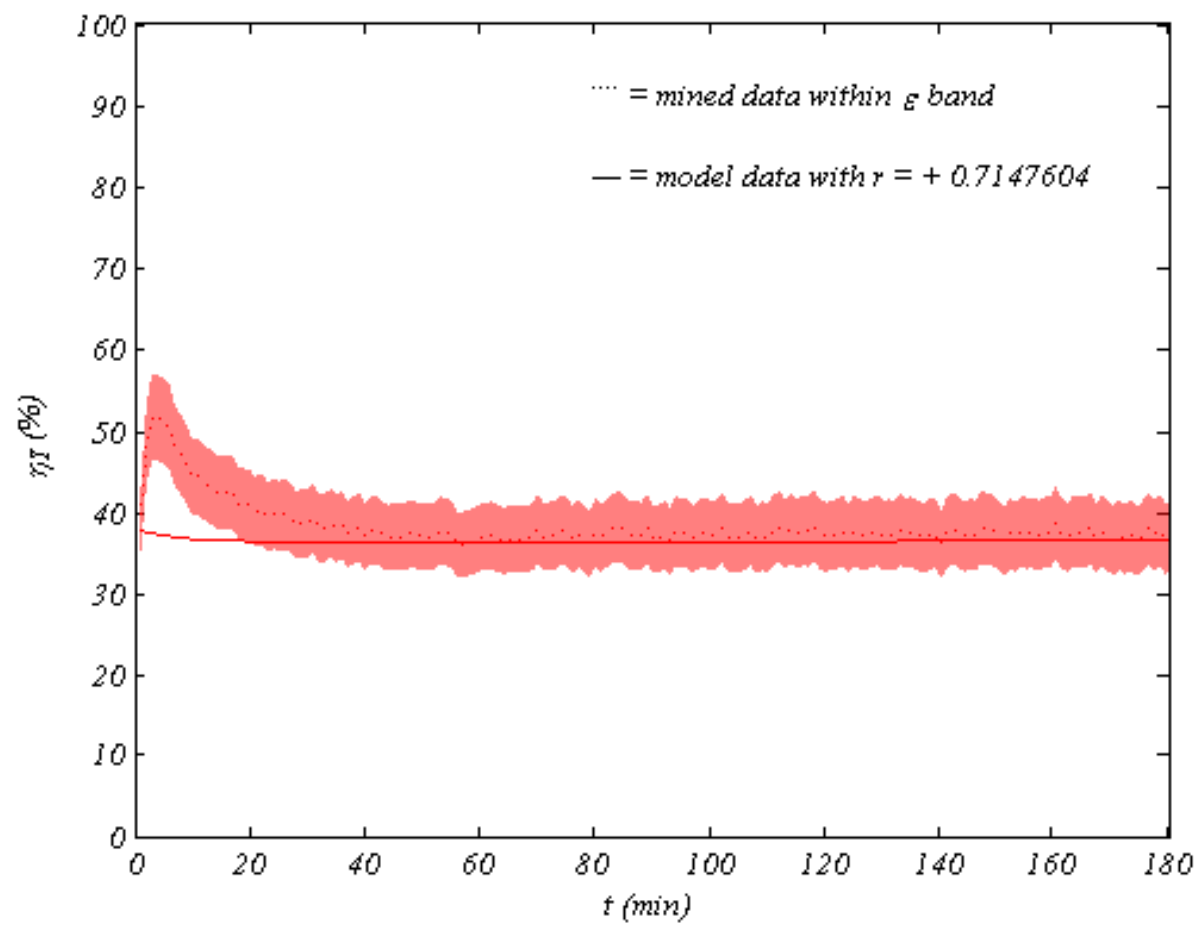

Figure 185. Thermal efficiency at $80^{\circ} \mathrm{C}$ flash and higher flow 


\subsection{Disambiguation}

The preceding discussion included references to three different types of data as it examined profiles of twelve time-varying system variables. Model data refer to data obtained by running the developed model with its alternate equations and adjusted parameter values and expressions. Experimental data refer to averaged temperature and pressure values recorded by the data acquisition system of three matching experiments; furthermore, experimental data also include observed values for seawater flow rate and fresh water amount. Pseudo-experimental data refer to results generated by a computer code composed of the developed model with its alternate equations but without adjusted parameter values and expressions; moreover, energy balance relations were deactivated, while experimental temperature and pressure, as well as recorded seawater flow rate and produced fresh water amount, were supplied to the computer code. The entire data mining procedure is illustrated in Figure 186.

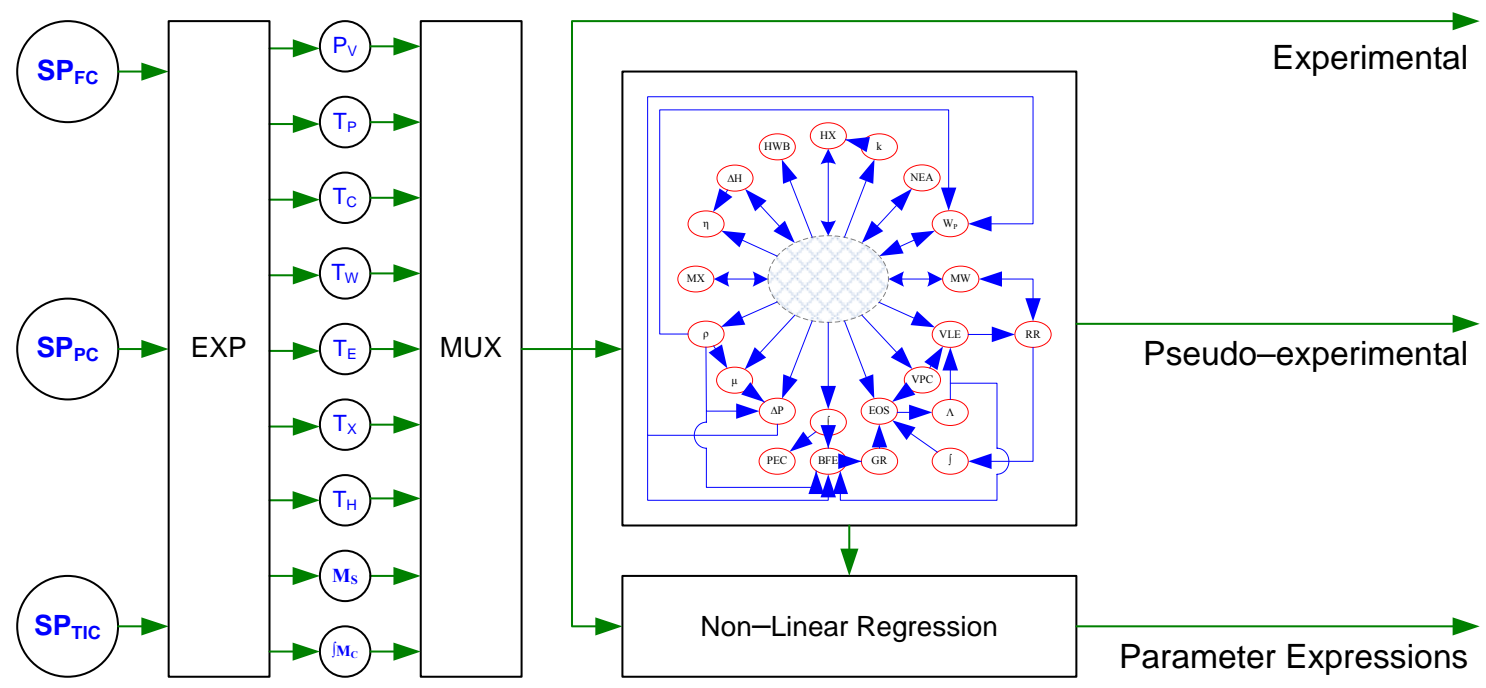

Figure 186. Experimental and pseudo-experimental data acquisition 


\section{CHAPTER 8. CONCLUSION}

\subsection{Summary}

A flash desalination process sustainable by natural forces of solar radiation and gravity has been proposed. In addition, experimental and theoretical simulations of the proposed desalination process have been carried out. The process includes a start-up procedure and continuous operation consisting of pumping seawater through a solar heater before flashing it under vacuum in an elevated chamber. The vacuum is passively created and subsequently maintained by hydrostatic balance between pressure inside the elevated flash chamber and outdoor atmospheric pressure.

Experimental simulations were carried out by a pilot unit depicting the proposed system but emulating solar heating and passive vacuum operations. Theoretical simulations were performed using a computer code comprising fundamental physical and thermodynamic laws plus numerous correlations and parameters. Experimental data were fed to an adapted computer code generating pseudo-experimental data; moreover, experimental and pseudo-experimental data were regressed, generating parametric values and correlations that were included in the developed computer model.

Experimental and theoretical simulations were run at varying operating conditions but at analogous circumstances, and their results were compared and analyzed to validate the developed model. Feasibility of the proposed system rapidly increased with flash temperature due to increased fresh water production and improved heat recovery. 


\subsection{Outcome}

Experimental and pseudo-experimental data were regressed, generating four correlations given in CHAPTER 6 that were included in the model. In addition, a dataset of $\mathrm{K}$-values was regressed to adjust parameter values of Henry's constant and vapor pressure correlations for non-condensable gases and water, respectively.

The developed correlation for the counter-current departure correction factor for the condenser tube yielded acceptable results as was seen in CHAPTER 6; however, preheat temperature computed by the model using that developed correlation yielded poor predictions as was seen in CHAPTER 7. The data mining code used the log mean temperature difference scheme to generate pseudo-experimental data of $F_{C T}$ that were regressed to generate the aforementioned correlation. This observation suggests that the $\log$ mean temperature difference scheme is probably not the best option to model the heat transfer operation across the condenser tube. Modeling a heat transfer operation with a phase change is extremely complex, especially in the presence of non-condensable gases. Precision of the model in predicting preheat temperature affects the quality of its evaluation of system performance as was seen in CHAPTER 7.

The developed correlation for the non-equilibrium allowance yielded average results as was seen in CHAPTER 6; consequently, equilibrium and concentrated brine temperatures computed by the model also yielded average predictions as was seen in CHAPTER 7. Heat loss of the evaporator was ignored, while non-equilibrium allowance correlation and energy balance around the evaporator were used to find equilibrium and the concentrated brine temperatures. This observation suggests that heat loss from the evaporator may need to be accounted for in the model. 
The developed correlation for the activity coefficient of water yielded excellent results as was seen in CHAPTER 6. The data mining code used the Rachford-Rice scheme along with experimental values of equilibrium temperature and system pressure, plus average reported composition of seawater to generate pseudo-experimental data of $\gamma_{\mathrm{H} 2 \mathrm{O}}$ that were regressed to generate the previously mentioned correlation. In addition, the data mining code included parameter values of Henry's constant and vapor pressure correlations for non-condensable gases and water obtained using the SUPERTRAPPTM code from NIST [31] as was mentioned in CHAPTER 4. The Rachford-Rice scheme and SUPERTRAPPTM code, plus the reported composition of seawater, are well recognized in literature for their accuracy; therefore, quality of the developed correlation for the activity coefficient of water is believed to be very high.

The developed correlation for the gas phase molecular content correction factor yielded good results, except for the case of higher seawater flow rates flashing at $50^{\circ} \mathrm{C}$ as was seen in CHAPTER 6. The data mining code used the ideal gas law with experimental values of equilibrium temperature and system pressure, plus a straightforward formula for calculating dynamic vacuum volume to generate pseudo-experimental data of $\psi$ that were regressed to generate the previous correlation. This observation suggests that flashing seawater at lower temperatures and higher flow rates rapidly increases the rate of accumulation of non-condensable gases. This phenomenon has been experimentally explored and theoretically modeled by Abtahi [32] via the molecular arrival rate concept. Results for $\psi$ seen in CHAPTER 6 indicate that deviation from ideal behavior increases with decreasing $P_{V} / P_{H 2 O}$ values due to rising temperature gradient between the hot and the cold sides of the flash chamber. This observation was also confirmed by Abtahi [32]. 
The results seen in CHAPTER 7 suggest that the proposed process becomes more feasible if operated at higher temperatures and more moderate flow rates. Higher flash temperatures will result in more evaporation and subsequent condensation, resulting in more fresh water production. In addition, the increased amount of heat reclaimed from condensing vapor reduced overall heater load and thus required less solar collection area. The collective outcome of increased fresh water output and decreased heater load is a significant decrease in prime energy consumption of the desalination unit, making it more economically viable. These observations are quantified in Figure 187 and Figure 188 for seawater conversion efficiency and prime energy consumption, respectively.

The results seen in CHAPTER 7 also suggest that most fresh water production occurs in the beginning of the operation, where vacuum pressure is lowest. The current experimental simulations were launched at a reasonably low vacuum; however, a much lower vacuum can be achieved using less energy if the proposed method of creating a passive vacuum is implemented. The collective outcome of applying passive vacuum and solar heating schemes is a significant decrease in prime energy consumption of the unit due to lower energy input and higher product output, furthering its feasibility.

The efficiency of the unit can also be boosted by exploiting the thermal energy of hot brine by either employing multi-stage schemes or including heat recovery provisions to increase the amount of reclaimed heat, effectively reducing prime energy consumption. The temperature difference manifested in the vapor pressure gradient between the two compartments of the flash chamber is the driving force of vapor transfer from the hot evaporator to the cold condenser; therefore, any attempt to exploit the thermal energy of the hot concentrated brine should be carefully applied as not to compromise that gradient. 


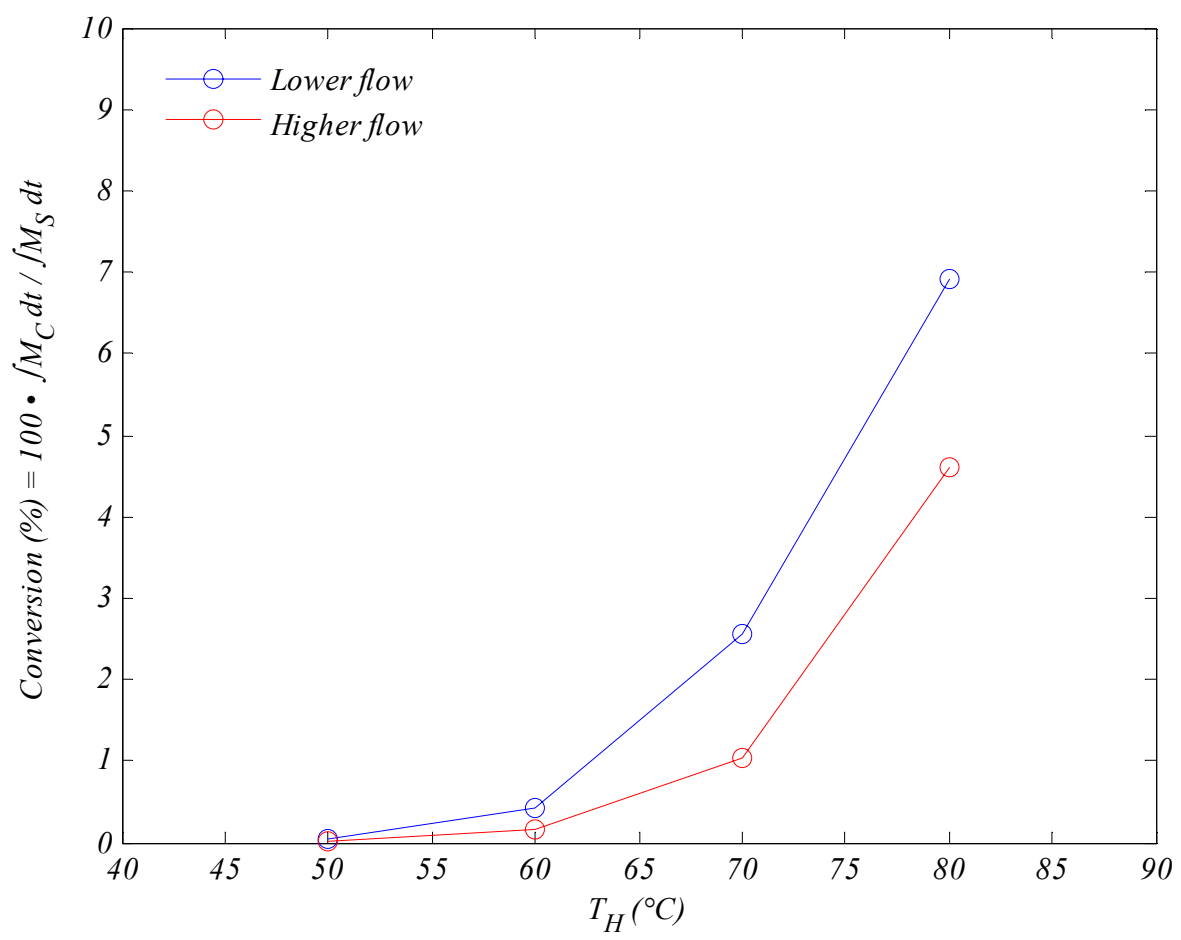

Figure 187. Seawater conversion dependence on flash temperature

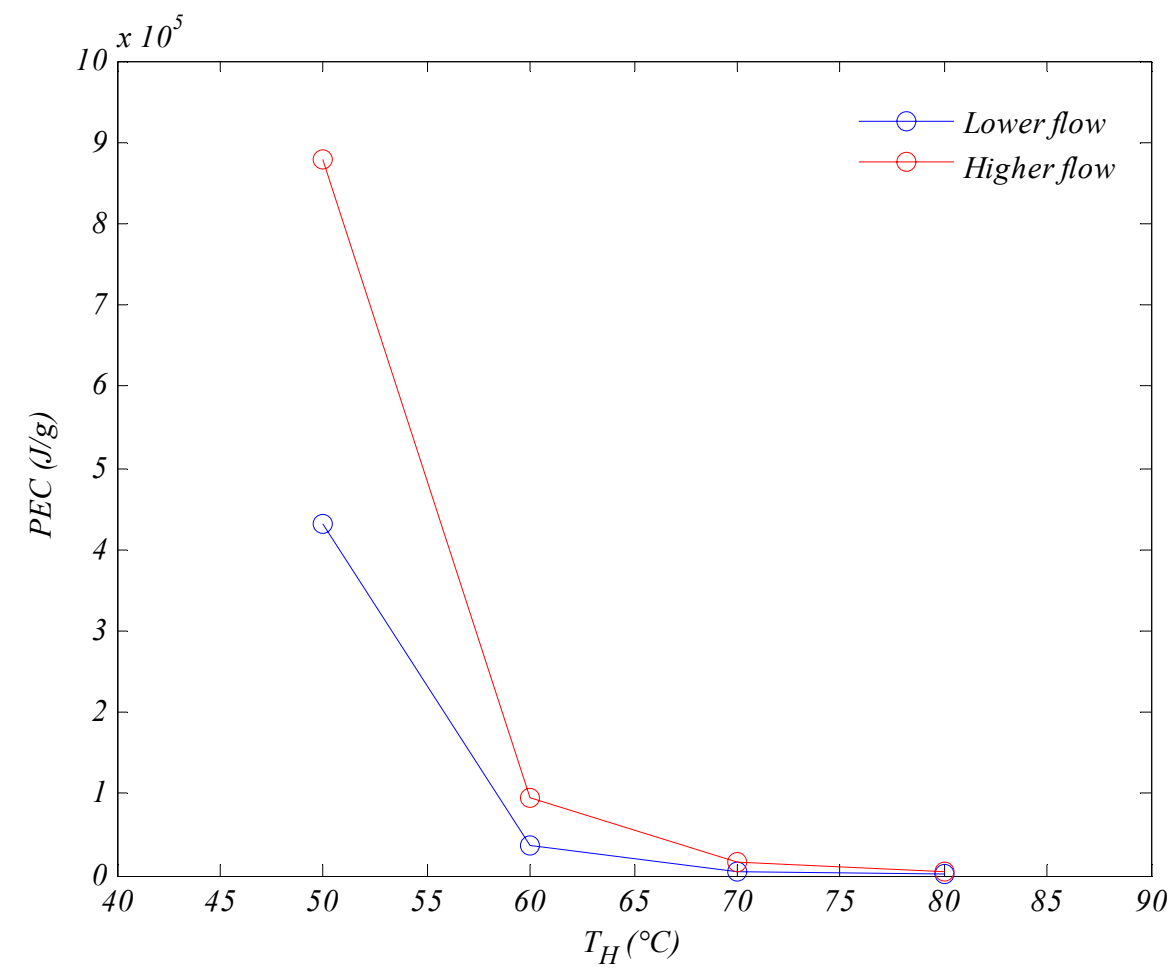

Figure 188. Prime energy consumption dependence on flash temperature 


\subsection{Prospects}

The results of this exploration demonstrate great potential for application of the proposed desalination system, especially in light of growing energy and water demands. This section is aimed at offering recommendations for prospective researchers seeking to optimize and further feasibility of the proposed desalination system.

CHAPTER 2 includes a brief overview of the most common conventional and solar desalination methods. An immense number of renewable energy driven desalination systems have been proposed and examined but never commercially materialized. The current study did not present those systems; nevertheless, including them in the literature review of future research would be supportive of the novelty of the current system. In addition, corrosion and scaling are major challenges to all desalination systems; however, they were overlooked in this study to keep the focus on simulation of the proposed desalination system, but they should be addressed in future investigations.

Moving the experimental unit to an outdoor setting would enhance replication of the proposed system. An outdoor unit can be furnished with a real solar heater and will enable the proposed passive vacuum generation by elevating the flash chamber to at least ten meters above ground. Implementing the proposed passive vacuum generation will also allow for much lower vacuums to be achieved using less energy, which translates to more fresh water production. In addition, vacuum erosion will be slower in an outdoor unit because vacuum volume will be increasing as system pressure increases due to the hydrostatic balance between the levels of the ground tanks and the flash chamber, which translates to more fresh water production due to lower pressures. Also, an outdoor unit will enable automatic flow control via a throttling valve or a variable-frequency drive. 
Accuracy of model output and pseudo-experimental data, as well as the precision of the regression, deeply rely on certainty of their input. There are slight discrepancies in literature when it comes to reporting seawater content; therefore, it is essential to investigate the seawater parameters given in CHAPTER 6 further, explicitly $\varphi_{i}$ and $\alpha_{i}$, especially those pertaining to carbon dioxide due to its complex kinetics.

Model convergence was difficult at times due to interdependence nature of its equations; therefore, other programming tools may be explored. In addition, the current model executes and integrates using one minute increments, yielding smooth results; however, easier convergence and smoother results can be obtained by decreasing increment size but that will also increase program run time.

Heat transfer relations of the current model did not produce very reliable results; consequently, they should be improved to predict heat loss of the flash chamber accurately as well as heat transfer across the condenser tube. Rigorous heat transfer computations will result in better predictions of equilibrium and brine temperatures resulting in superior flash calculations. More rigorous heat transfer computations will also result in better predictions of preheat temperature, resulting in enhanced performance evaluation due to regression of a more precise mined data.

The log mean temperature difference method should be substituted with a more appropriate heat transfer model capable of handling the complexity of phase change operations. Experimental data should always be used to fine-tune the parameters of the employed heat transfer model. In addition, if heat transfer computations remained imprecise, adjusting local and overall heat transfer coefficients should be explored as an alternative to adjusting the parameters of the heat transfer model. 
Abtahi [32] hypothesized that the non-condensable gas molecules accumulating in the condenser tend to amass around the cold surface of the condenser tube, in essence forming an insulation layer that hampers heat transfer from the condensing water vapor. In addition, some heat transfer texts include mass transfer parameters within heat transfer coefficient correlations in condensers as they try to model the diffusion of water vapor molecules through the non-condensable gas layer.

The current model assumes the total condensation of flashed water vapor and uses a regressed molecular content correction factor to account for rate of accumulation of non-condensable gases. Alternately, the distribution of non-condensable gases among flashed vapor, concentrated brine, and condensed water in the flash chamber can be estimated by assuming equilibrium among the three phases [33]. This approach may be more valid, but will exacerbate computations and hinder convergence.

A more accurate version of the correlation for the activity coefficient of water can be obtained by using a suitable activity coefficient model to write the activity coefficient formula, then adjusting its parameters using the data mining code mentioned earlier in CHAPTER 6 and found in the APPENDICES section.

The diffusion correlation developed in CHAPTER 4 includes two parameters that serve as conductance and resistance terms. In its current form, the model considers resistance to water vapor transfer to be pertinent only to diffusing and not flashing vapor. It would be more prudent to remove the resistance term, then readjust the conductance term in accordance with reported values [14]. Afterward, a resistance term pertaining to diffusing and flashing water vapor should be included in the model and adjusted using the data mining code mentioned in CHAPTER 6 and found in the APPENDICES section. 
The diffusion correlation development should be carried out alongside the above mentioned molecular arrival rate concept modeled by Abtahi [32]. This approach will make the adjusted conductance and resistance terms more consistent with the complex mass and heat transfer operations, which will ultimately result in improved vacuum pressure and preheat temperature predictions.

Feasibility of the proposed desalination process should be simply investigated via prime energy consumption, $P E C=\left(\int Q_{H}+\int W_{P}\right) / \int M_{C}$, and not via unnecessarily meticulous economic analyses. Process optimization is synonymous with prime energy consumption mitigation that is realized via minimizing the amount of energy exhausted or maximizing the amount of fresh water produced. Most of the exhausted energy of all thermal desalination processes is due to heat and not to power input as was seen earlier in CHAPTER 2 and as confirmed by the current experimental and theoretical simulations; therefore, optimizing the proposed desalination system should specifically revolve around reducing heater load and amplifying fresh water production rate. Detailed economic analyses can be performed by properly estimating capital and operating costs; however, these analyses can be quite cumbersome and should be carefully employed.

Seawater flash temperature is a set parameter; therefore, reducing heater load should be aimed for by increasing preheat temperature achieved via improved heat recovery in the condenser. Improving heat recovery in the condenser can be accomplished by improving the geometry of the condenser tube to harness the most of the latent heat of the condensing steam. Thermally insulating the condenser should be investigated to see its consequences on directing condensing steam away from the condenser wall and more towards the condenser tube. 
Amplifying the fresh water production rate is thermodynamically controlled via increasing equilibrium temperature and lowering system pressure. With the exception of increasing flash temperature set point, increasing equilibrium temperature is accomplished by minimizing heat loss of the evaporator via enhanced thermal insulation. System pressure is always increasing because of the eroding vacuum due to build up of non-condensable gases in the flash chamber; however, initial system pressure is a controlled variable, and lowering it would lower system pressure all through the desalination process. The proposed method of passively creating vacuums should accomplish very low vacuum pressures, in effect equaling ambient water vapor pressure. In addition, fresh water production rate can be significantly enhanced by employing the multi-stage scheme outlined earlier in CHAPTER 4. The current model should be extended from simulating single-stage to multi-stage desalination schemes. In addition, a qualitative sensitivity analysis of model parameters should be executed to evaluate the outcome of their variation on model results.

The proposed desalination system is meant to be driven by solar energy and average values for a generic solar collector were used to estimate solar collection area. Detailed solar computations should be used instead of the average values to broaden the applicability of the model to different geographies and different collectors. This would involve including several solar calculations that are widely available in literature [8].

Finally, experimental results discussed earlier have proven that some of the flashed vapor condenses prematurely in the evaporator before making it to the condenser; therefore, resistance to vapor transfer from the evaporator to condenser should be reduced to increase fresh water production and improve heat recovery. 


\section{REFERENCES}

1. National Atlas of the United States. Water Resources of the United States; U. S. Geological Survey: Reston, VA, 2005.

2. Minton, P. E. Handbook of Evaporation Technology; Noyes Publications: Westwood, NJ, 1986.

3. Maxwell, E., George R., Wilcox S. Climatological Solar Radiation Model; the National Climatic Data Center: Asheville, NC, 1998.

4. Culp, A. Principles of Energy Conversion; McGraw Hill: New York, NY, 1991.

5. Wangnick, K. Worldwide Desalting Plants Inventory; Report Number 15; International Desalination Association: Topsfield, MA, 1998.

6. Miller, J. E. Review of Water Resources and Desalination Technologies; Materials Chemistry Department; Sandia National Laboratories: Albuquerque, NM, 2003.

7. Kalogirou, S. A. Seawater Desalination Using Renewable Energy Sources, Progress in Energy and Combustion Science 2005, 31(3), pp 242-281.

8. Goswami, D. Y., Kreith, F., Kreider, J. F. Principles of Solar Engineering, second edition; Taylor \& Francis: Philadelphia, PA, 2000.

9. Delyannis, E. Historic Background of Desalination and Renewable Energies, Solar Energy 2003, 75(5), pp 357-366.

10. Tzen, E., Morris, R. Renewable Energy Sources for Desalination, Solar Energy 2003, 75(5), pp 375-379. 
11. Al-Kharabsheh, S. Theoretical and Experimental Analysis of Water Desalination System Using Low Grade Solar Heat, Doctoral Dissertation; University of Florida: Gainesville, Florida, 2003.

12. Maroo, S. C. Theoretical Analysis of Solar Driven Flash Desalination System Based on Passive Vacuum Generation, Master Thesis; University of Florida: Gainesville, Florida, 2006.

13. Rachford, H. H., Rice J. D. Procedure for Use of Electronic Digital Computers in Calculation Flash Vaporization Hydrocarbon Equilibrium, Petroleum Technology 1952, 4,9 .

14. Bemporad, G. A. Basic Thermodynamic Aspects of a Solar Energy Based Desalination Process, Solar Energy 1995, 54 (2), pp 125-134.

15. Geankoplis, C. J. Transport Processes and Separation Process Principles; Prentice Hall: Englewood Cliffs, NJ, 2003.

16. Turekian, K. K. Oceans; Prentice Hall: Englewood Cliffs, NJ, 1968.

17. Sander, R. Compilation of Henry's Law Constants for Inorganic and Organic Species of Potential Importance in Environmental Chemistry, www.henrys-law.org, 1999.

18. Perry, R. H., Green, D. Perry's Chemical Engineers' Handbook; McGraw-Hill: New York, NY, 1984.

19. Thibodeaux, L. J. Environmental Chemodynamics; John Wiley \& Sons: New York, NY, 1996.

20. Sinnott, R. K. Coulson and Richardson's Chemical Engineering; ButterworthHeinemann: Oxford, UK, 1996.

21. E1-Nashar, A. M., Qamhiyeh, A. A. Simulation of the Steady-State Operation of a Multi-Effect Stack Seawater Distillation Plant, Desalination 1995, 101(3), pp 231-243. 
22. Miyatake, O., Murakami, K., Kawata, Y., Fujii, T. Fundamental Experiments with Flash Evaporation, Heat Transfer - Japan Research 1973, 2(4), pp 89-100.

23. Coker, A. K. Chemical Process Design, Analysis, and Simulation; Gulf Publishing: Houston, TX, 1995.

24. Hermann M., Koschikowski J., Rommel M. Corrosion-Free Solar Collectors for Thermally Driven Seawater Desalination, Solar Energy 2002, 72(5), pp 415-426.

25. Granet, I. Fluid Mechanics; Prentice Hall: Englewood Cliffs, NJ, 1996.

26. Caldwell, D. R. The Thermal Conductivity of Seawater, Deep-Sea Research 1974, $21,131-138$.

27. Chopey, N. P. Handbook of Chemical Engineering Calculations; McGraw-Hill: New York, NY, 1994.

28. Millero, F. J., Poisson, A. International One Atmosphere Equation of State of Seawater, Deep-Sea Research 1981, 28, pp 625-629.

29. Sündermann, J. Numerical Data and Functional Relationships in Science and Technology - New Series Group V: Geophysics and Space Research, Oceanography 1986, 3(A), Springer-Verlag, Berlin, Germany.

30. Mamaev, O. I. Temperature-Salinity Analysis of World Ocean Waters; translation from Russian by Burton, R. J.; Elsevier Scientific Publishing: Amsterdam, Netherlands, 1975.

31. Chase, M. W. NIST-JANAF Themochemical Tables. Journal of Physical and Chemical Reference, Number 9; American Chemical Society: Washington, DC, 1998.

32. Abtahi, H. Investigation of local pressure characteristics in gas-loaded heat pipes, Proceedings of the ASME National Heat Transfer Conference, Houston, TX, 1988; American Society of Mechanical Engineers: New York, NY, 1988, A89 5325123 34, pp $347-352$. 
33. Abutayeh, M., Goswami, D. Y. Passive Vacuum Solar Flash Desalination, American Institute of Chemical Engineers [Online] 2009, pp 1547-5905. 
APPENDICES 
Appendix A. The operating procedure

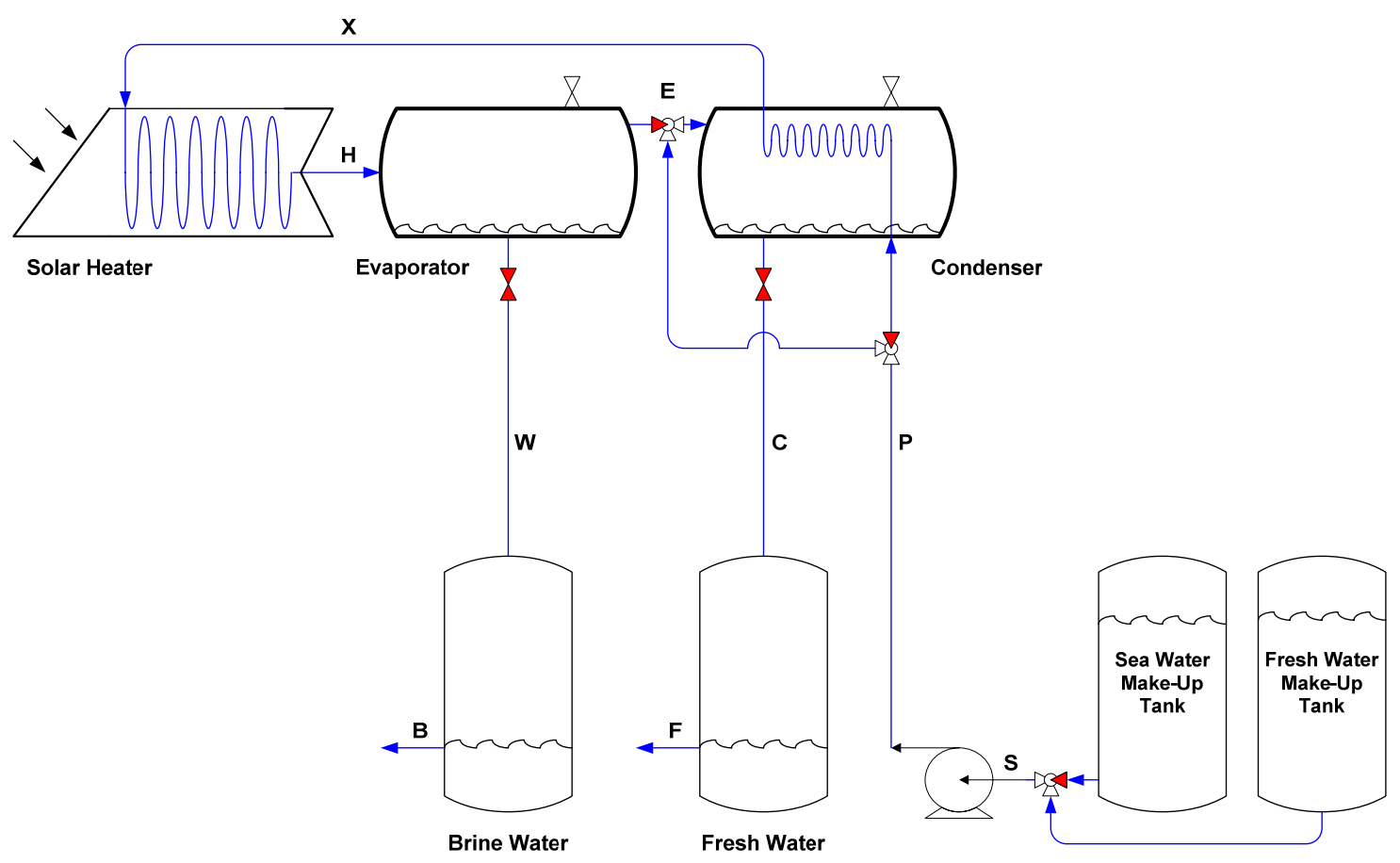

Figure 189. Preparing to fill up the condenser

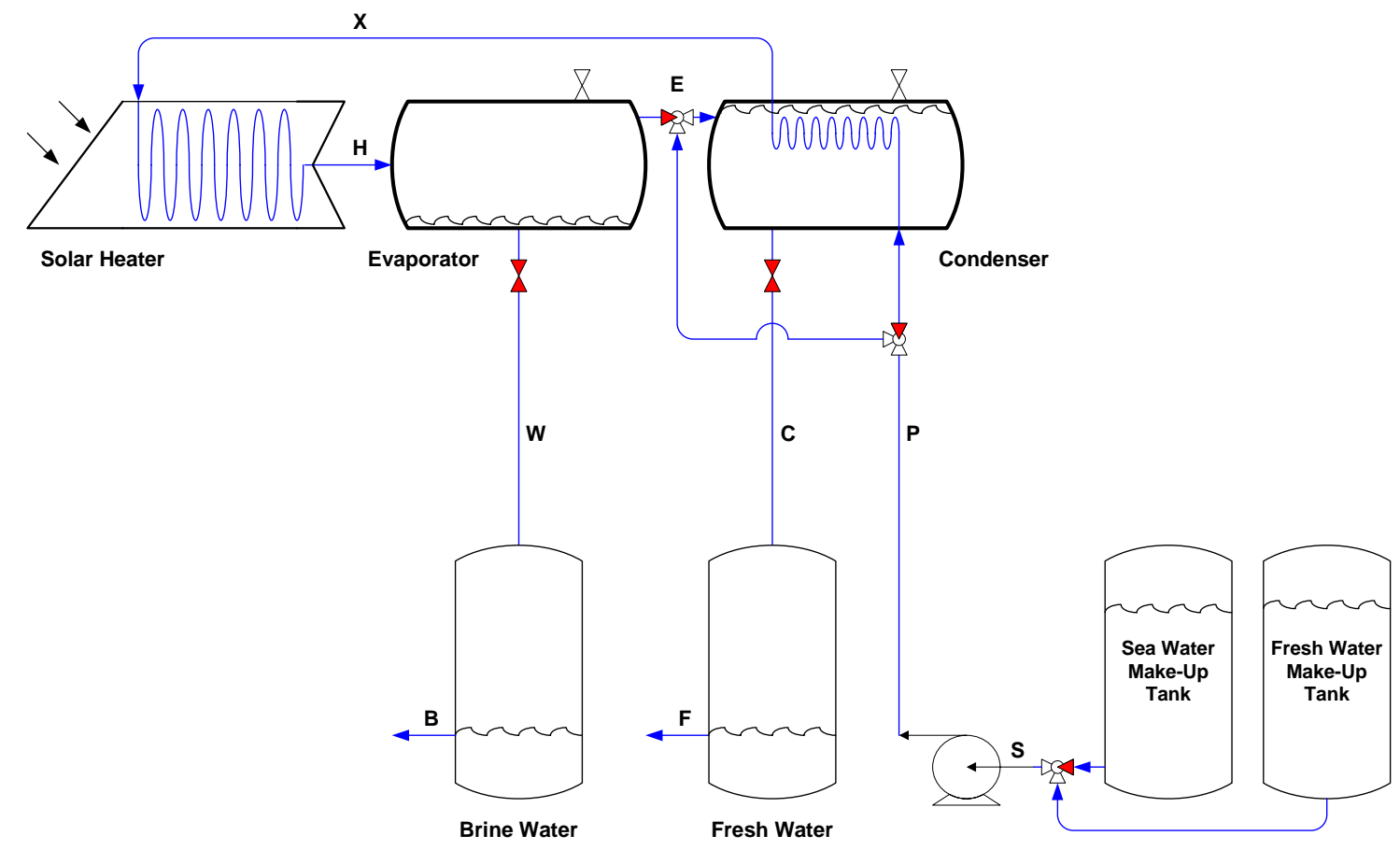

Figure 190. Condenser full of fresh water 
Appendix A (Continued)

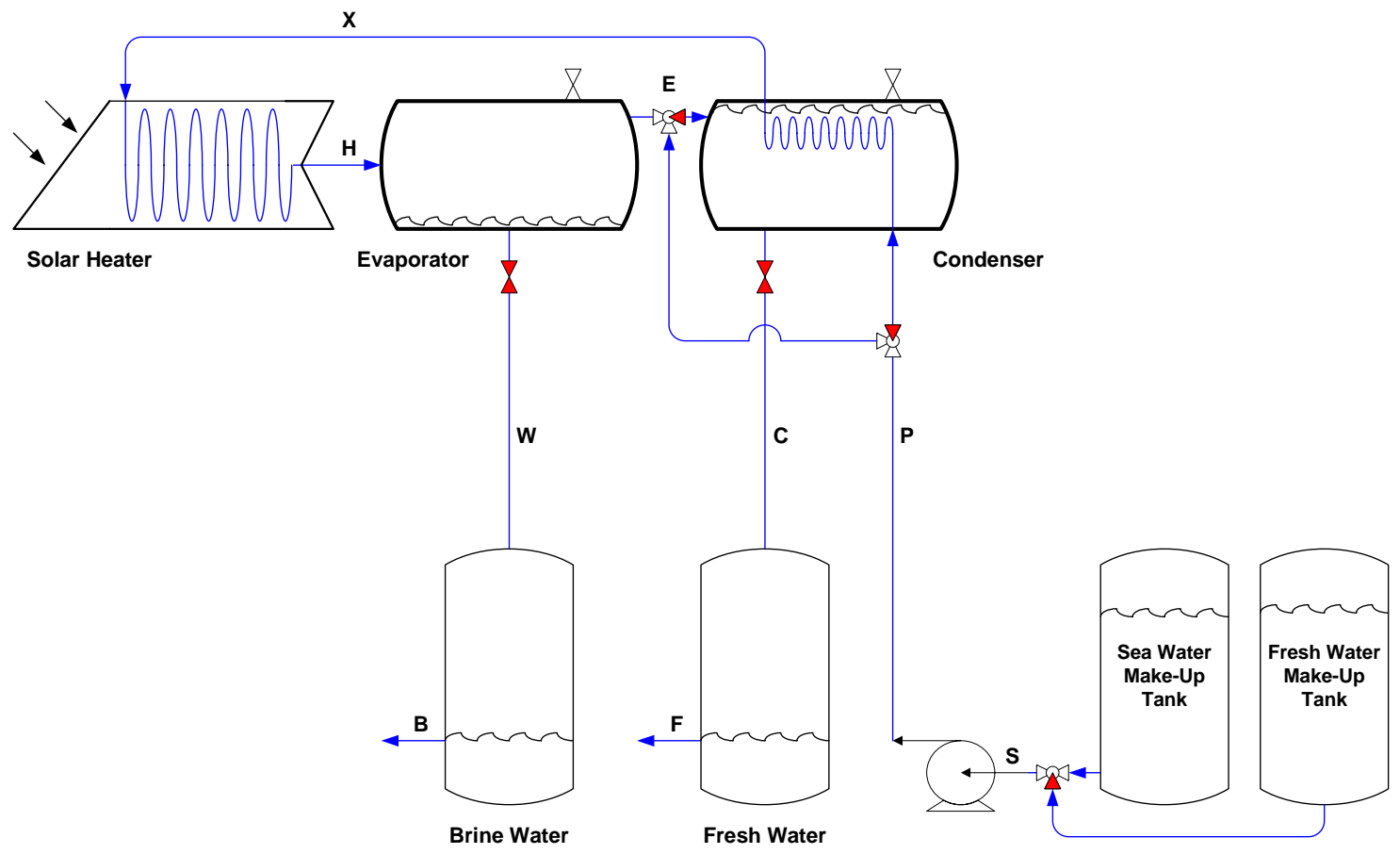

Figure 191. Preparing to fill up the evaporator

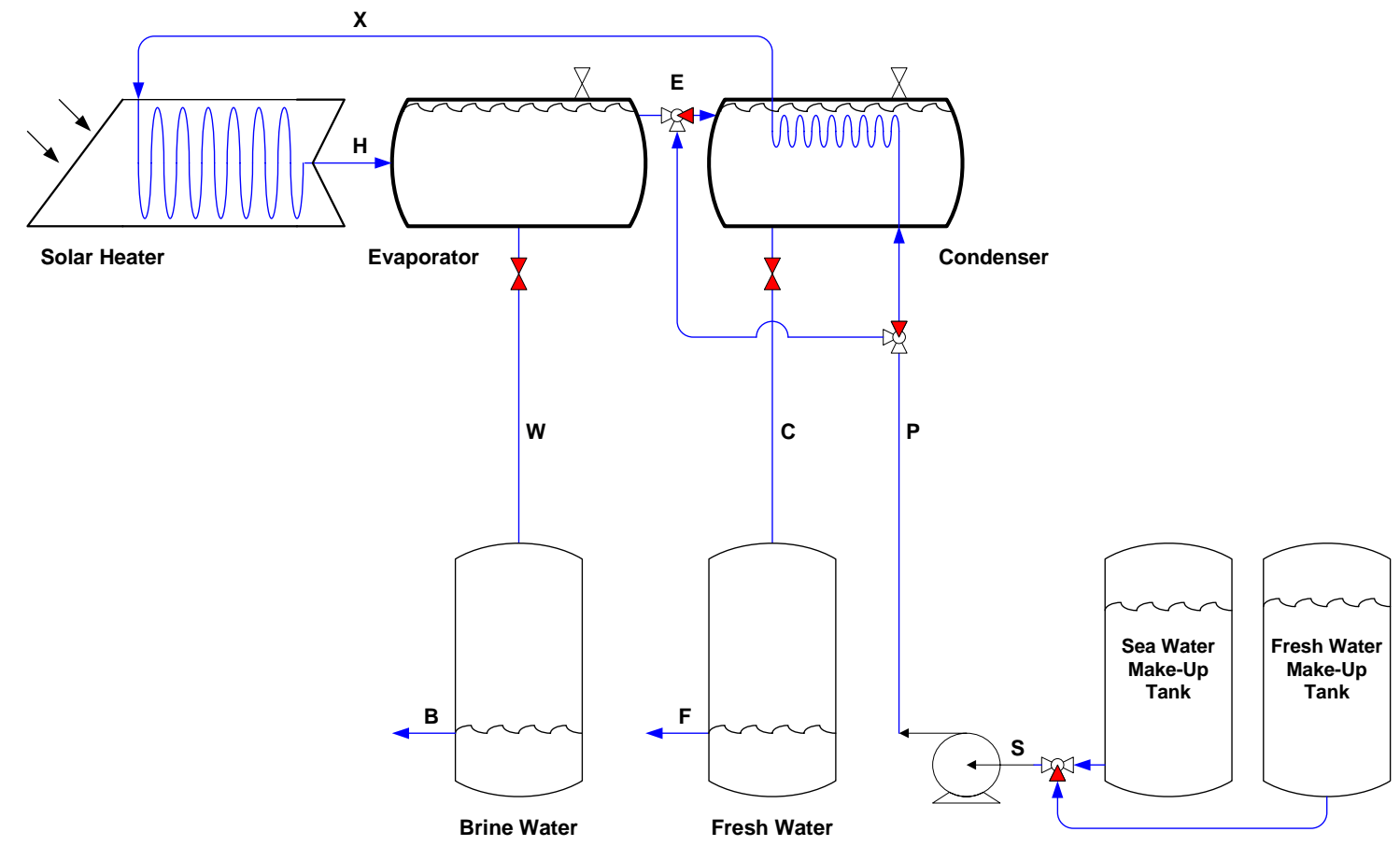

Figure 192. Evaporator full of seawater 
Appendix A (Continued)

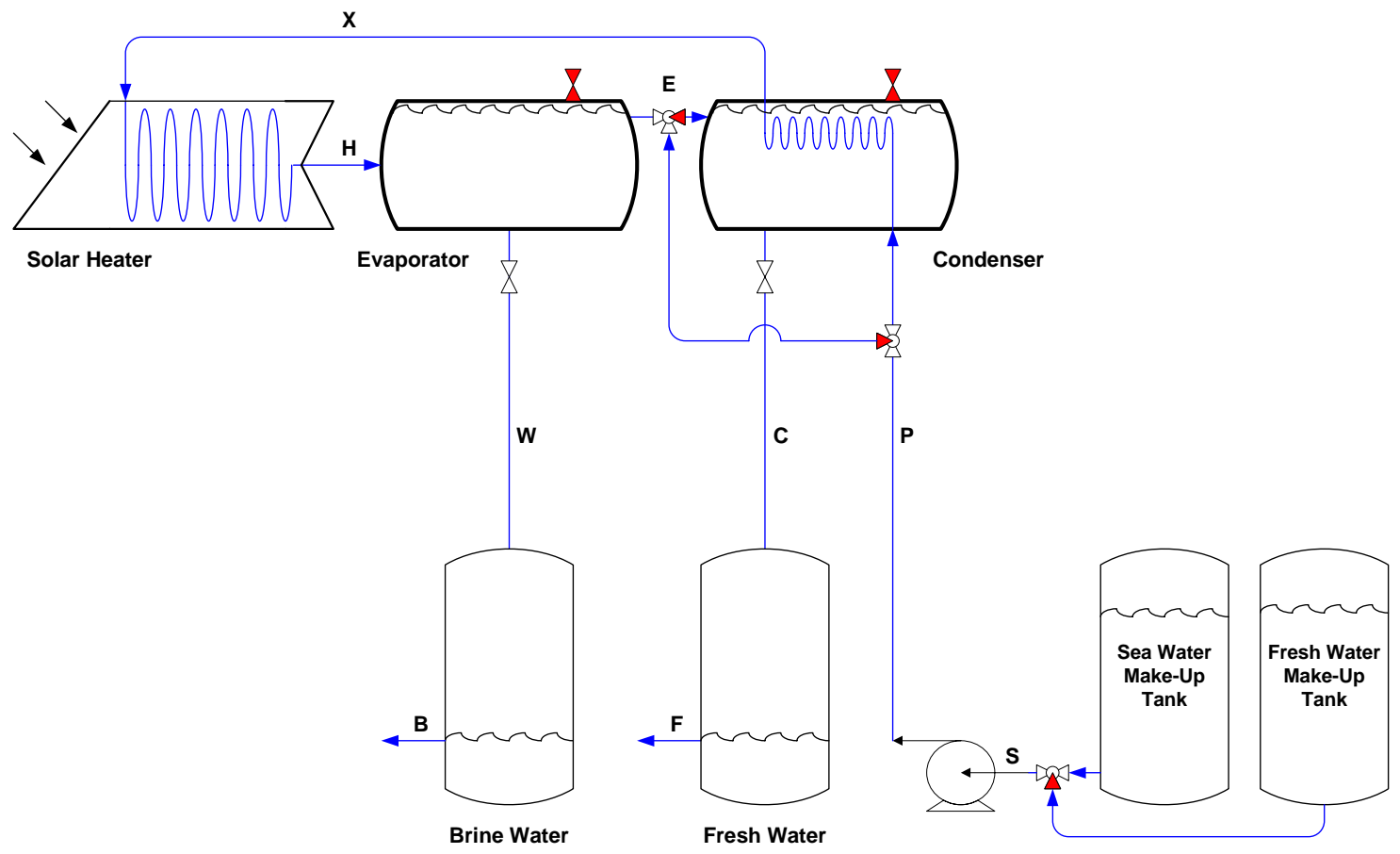

Figure 193. Switching the valve positions of the flash chamber

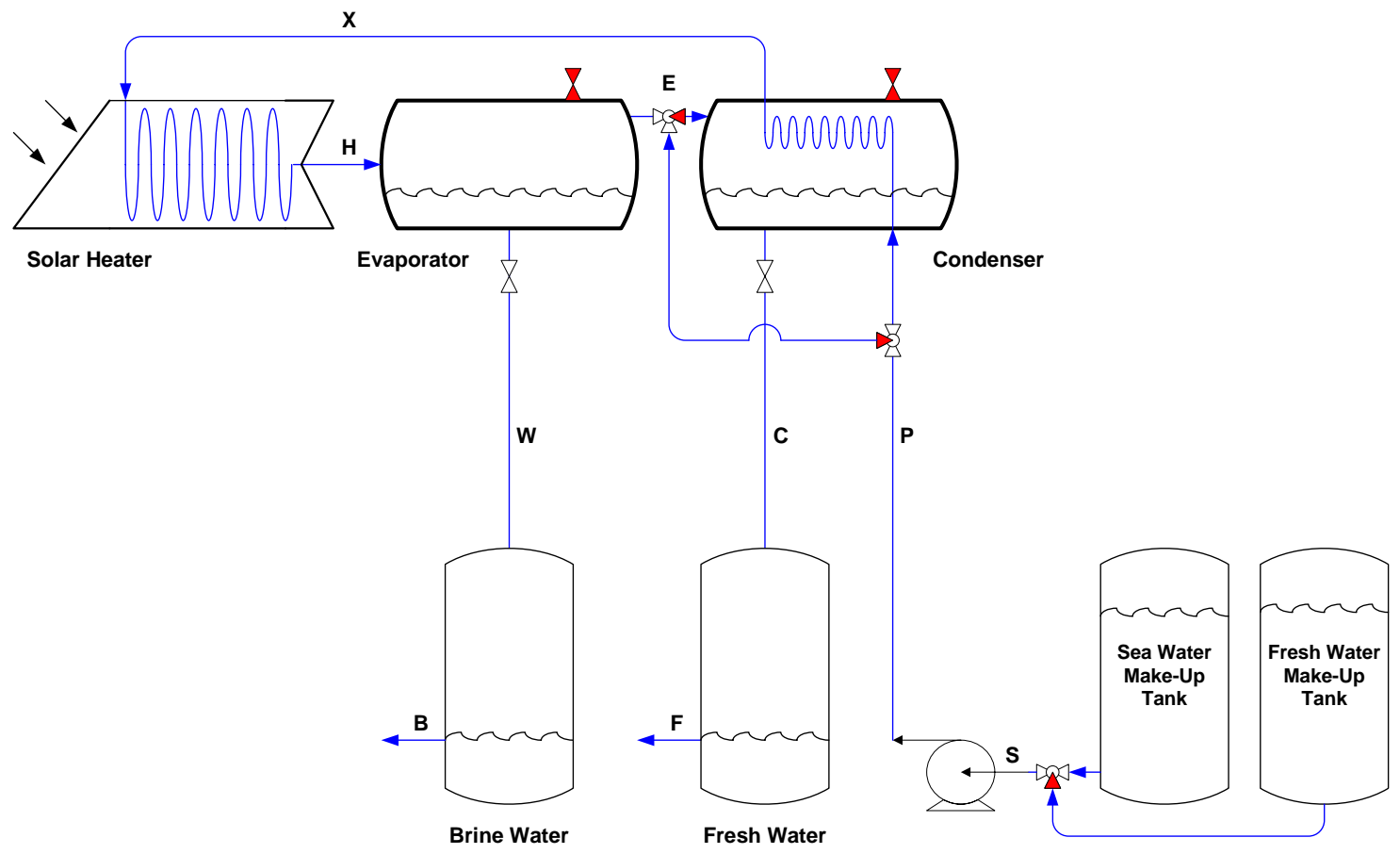

Figure 194. Flash chamber passively vacuumed 
Appendix A (Continued)

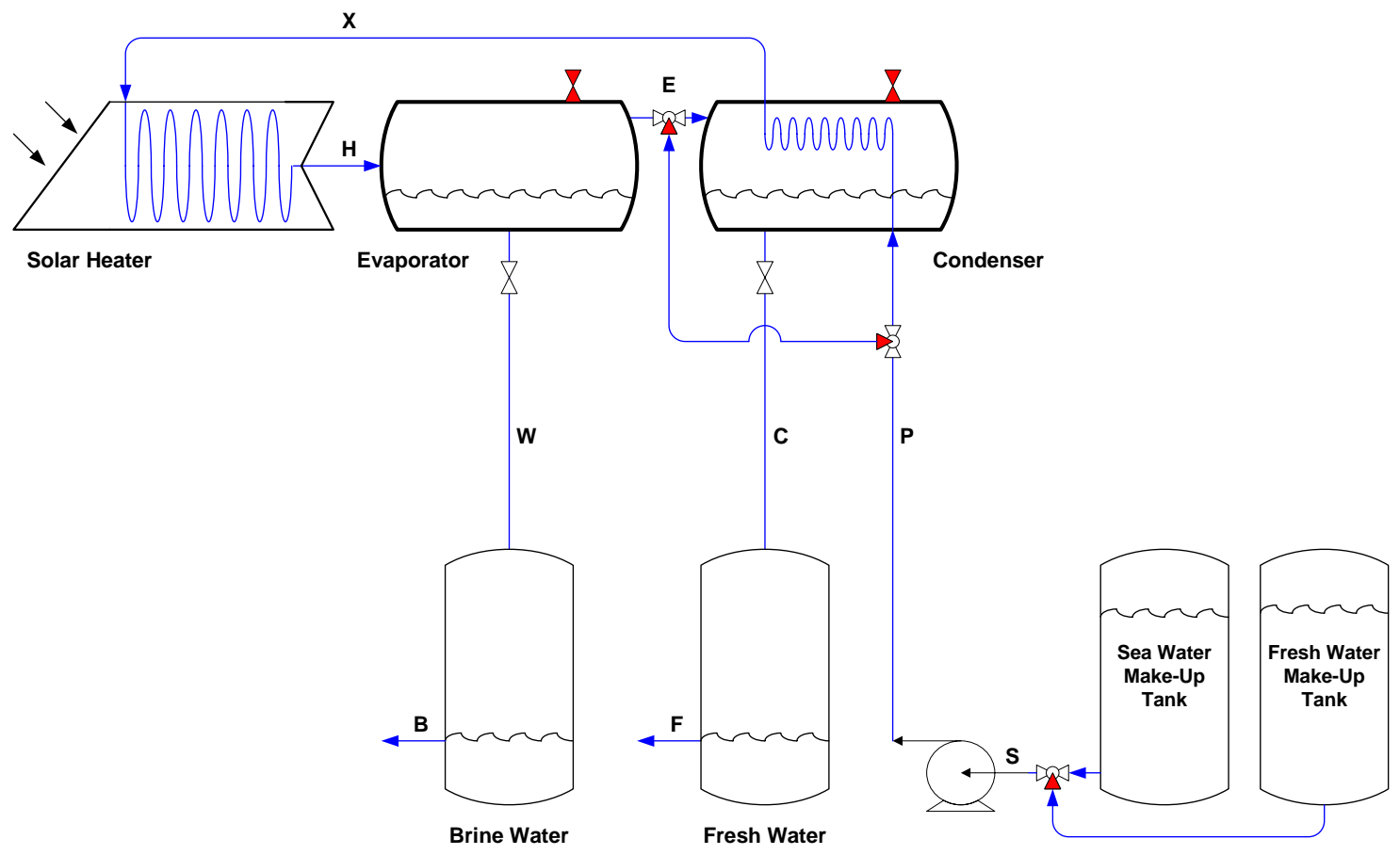

Figure 195. Preparing to start the desalination process

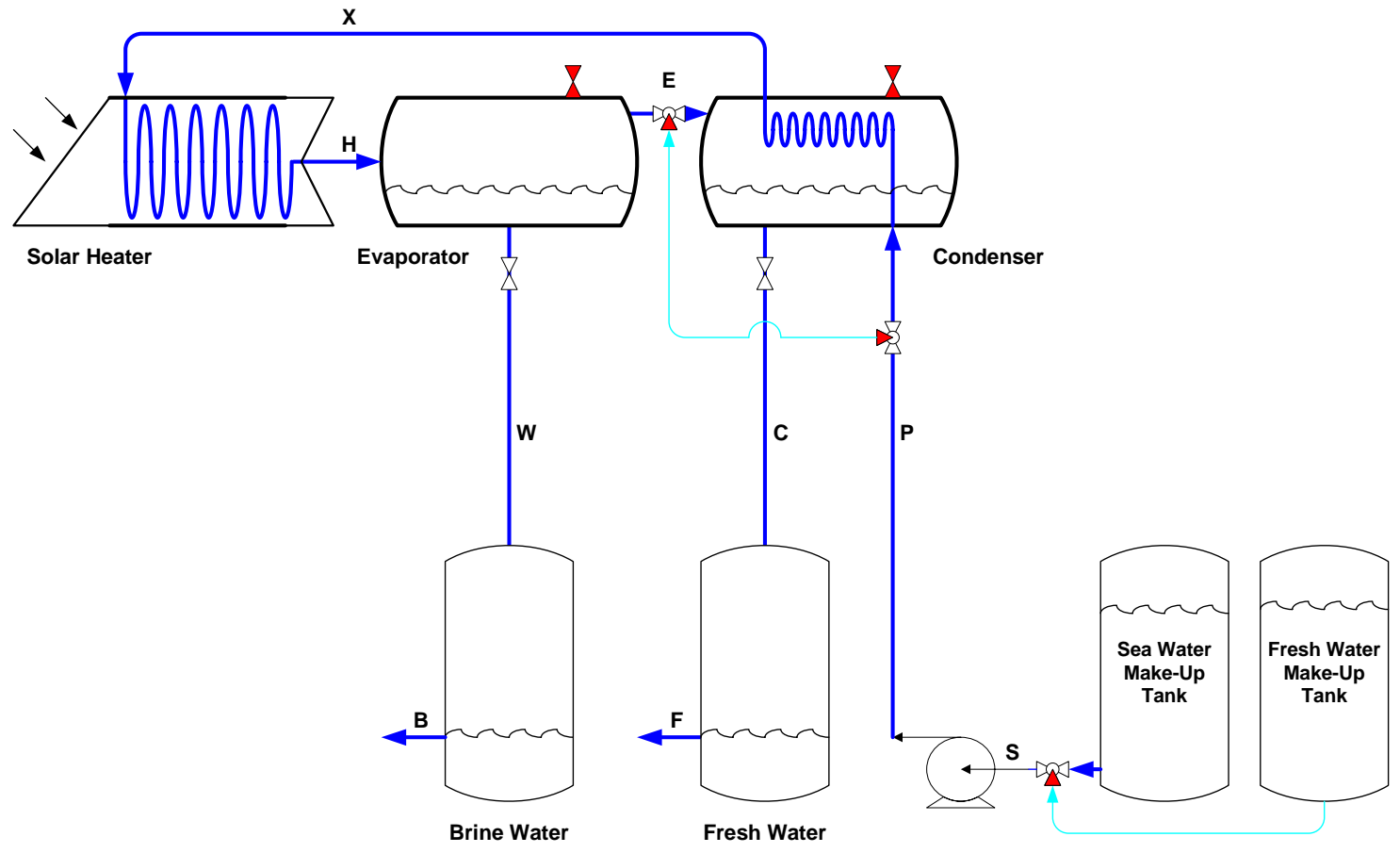

Figure 196. Desalination process taking place 
Appendix A (Continued)

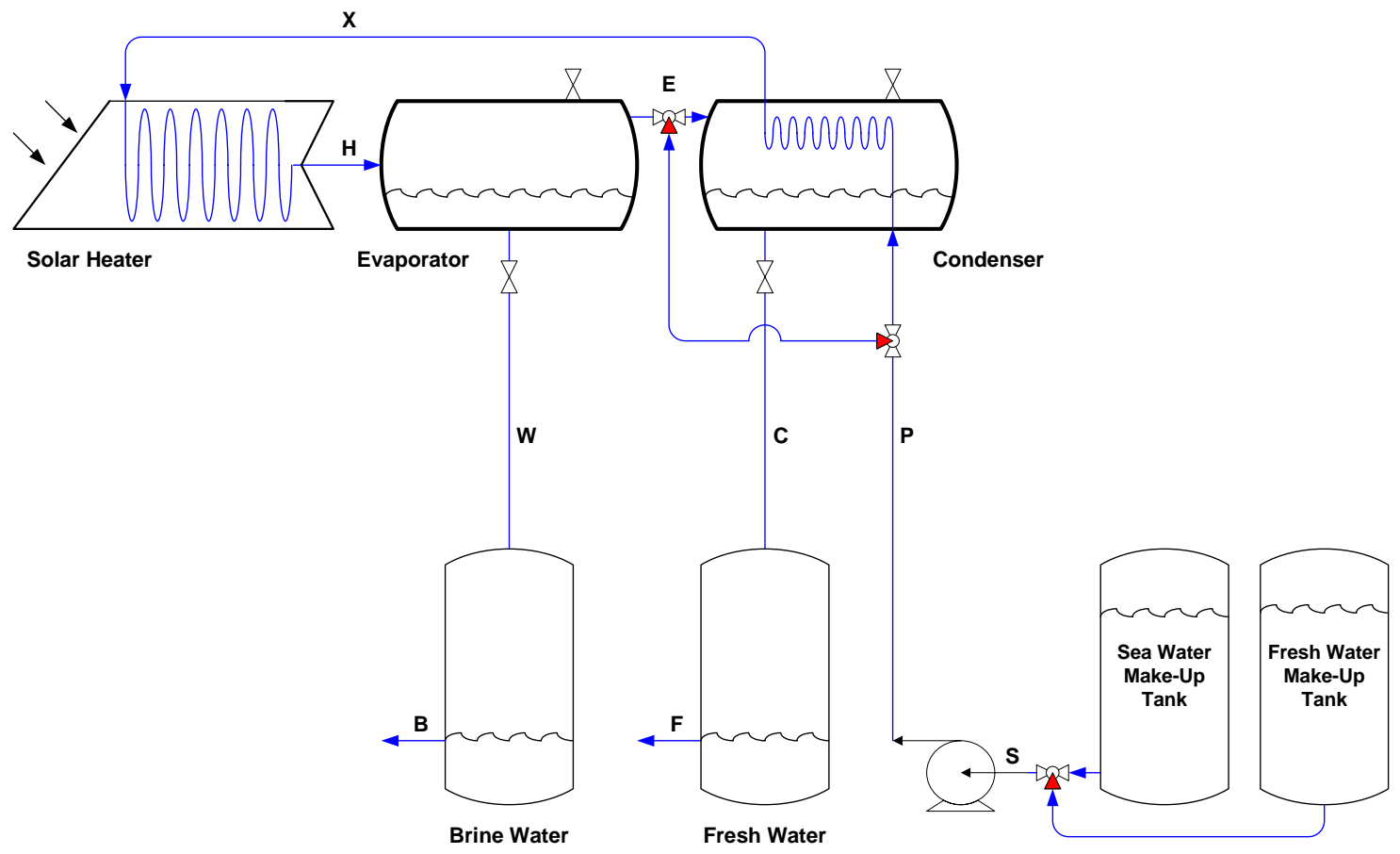

Figure 197. Flash chamber vented to terminate vacuum

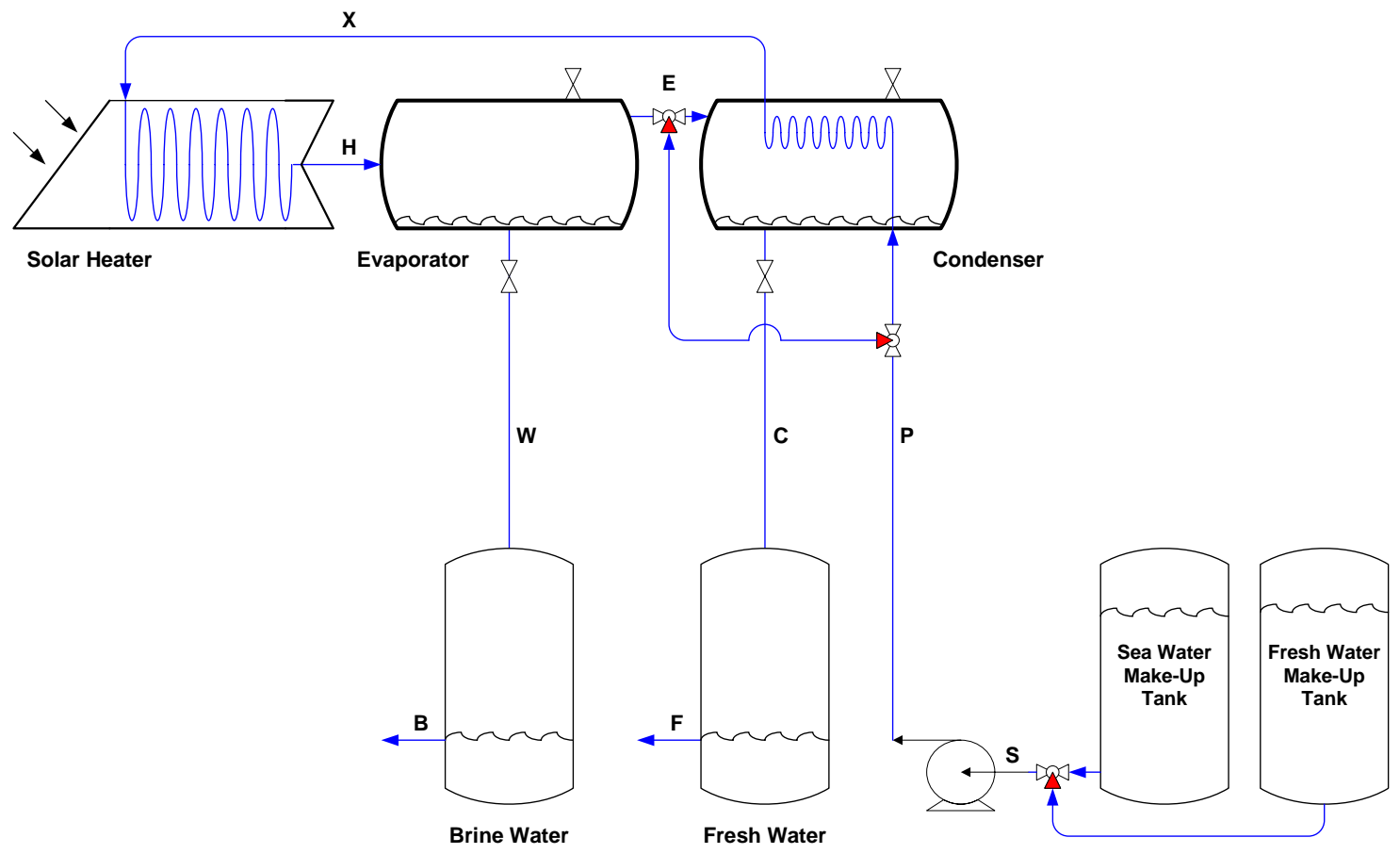

Figure 198. Flash chamber drained 


\section{Appendix B. SUPERTRAPPTM code to generate K-values}

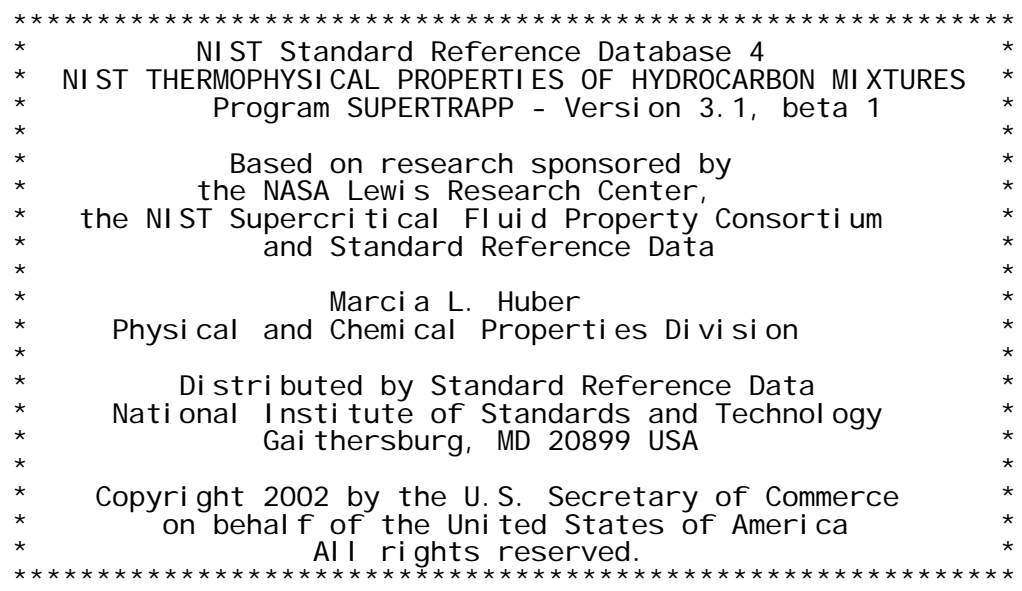

For help in response to any question, enter "?"

Press enter to continue.

Do you want to use default settings? ( $Y / N)$

(The default settings are whatever you last selected for units and file I/O.)

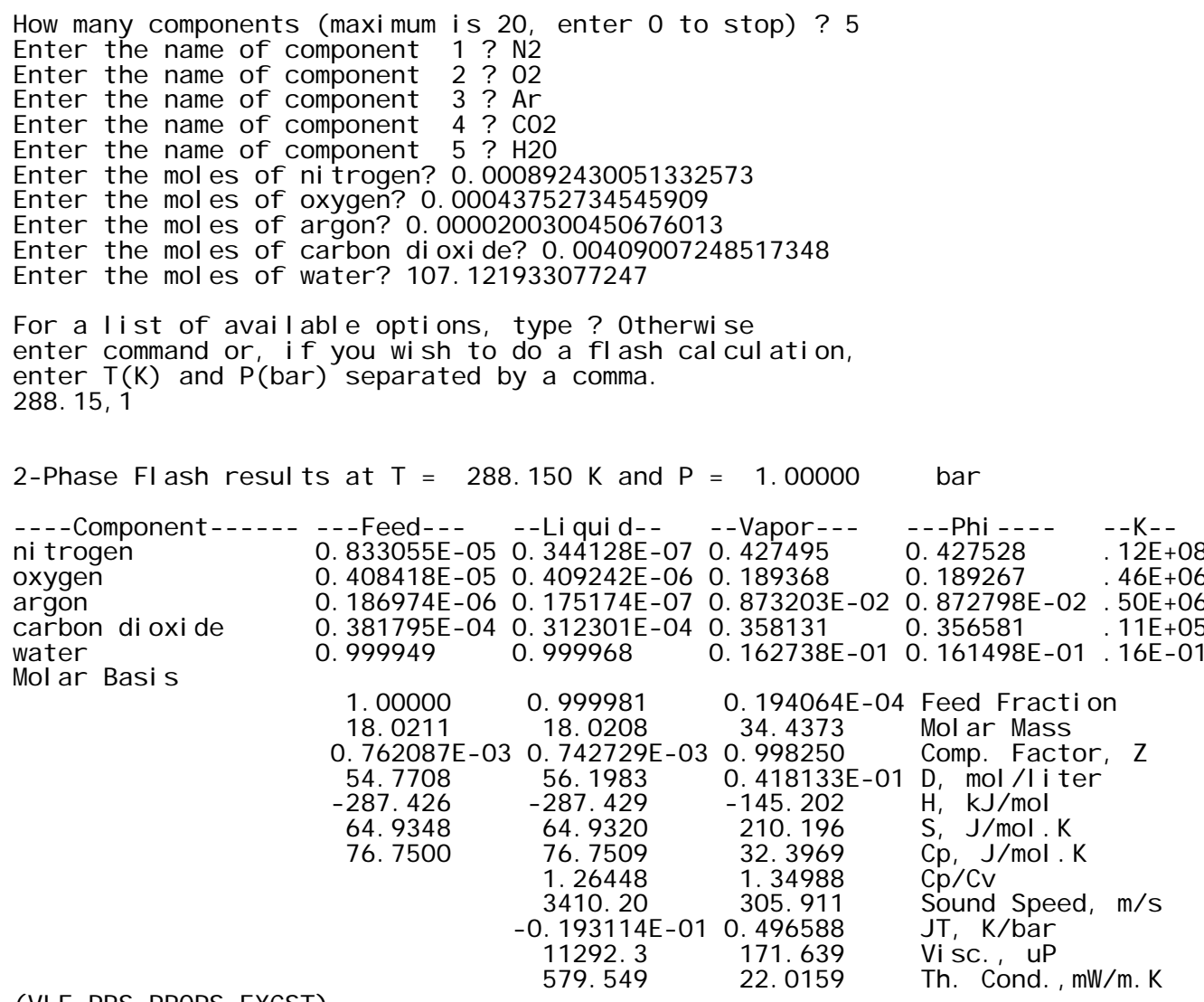

\begin{tabular}{|c|c|c|c|c|}
\hline $\begin{array}{l}833055 \mathrm{E}-05 \\
408418 \mathrm{E}-05 \\
186974 \mathrm{E}-06 \\
381795 \mathrm{E}-04 \\
999949\end{array}$ & $\begin{array}{l}0.344128 \mathrm{E}-07 \\
0.409242 \mathrm{E}-06 \\
0.175174 \mathrm{E}-07 \\
0.312301 \mathrm{E}-04 \\
0.999968\end{array}$ & $\begin{array}{l}0.427495 \\
0.189368 \\
0.873203 \mathrm{E} \cdot 02 \\
0.358131 \\
0.162738 \mathrm{E} \cdot 01\end{array}$ & $\begin{array}{l}0.427528 \\
0.189267 \\
0.872798 \mathrm{E}-02 \\
0.356581 \\
0.161498 \mathrm{E}-01\end{array}$ & $\begin{array}{l}12 \mathrm{E}+0 \\
46 \mathrm{E}+0\end{array}$ \\
\hline $\begin{array}{l}1.00000 \\
18.0211 \\
0.762087 \mathrm{E} \cdot 03 \\
54.7708 \\
-287.426 \\
64.9348 \\
76.7500\end{array}$ & $\begin{array}{l}0.999981 \\
18.0208 \\
0.742729 \mathrm{E}-03 \\
56.1983 \\
-287.429 \\
64.9320 \\
76.7509 \\
1.26448 \\
3410.20 \\
-0.193114 \mathrm{E}-01 \\
11292.3 \\
579.549\end{array}$ & $\begin{array}{l}0.194064 \mathrm{E}-04 \\
34.4373 \\
0.998250 \\
0.418133 \mathrm{E}-01 \\
-145.202 \\
210.196 \\
32.3969 \\
1.34988 \\
305.911 \\
0.496588 \\
171.639 \\
22.0159\end{array}$ & $\begin{array}{l}\text { Feed Fraction } \\
\text { Molar Mass } \\
\text { Comp. Factor, } \\
\text { D, mol / liter } \\
\text { H, kJ/mol } \\
\text { S, I / mol.K } \\
\text { Cp, J/mol. K } \\
\text { Cp/CV } \\
\text { Sound Speed, } \\
\text { JT, K/bar } \\
\text { Visc. uP } \\
\text { Th. Cond., mW/ }\end{array}$ & $\begin{array}{l}\text { Z } \\
\mathrm{m} / \mathrm{s} \\
\mathrm{V} / \mathrm{m} . \mathrm{K}\end{array}$ \\
\hline
\end{tabular}

( $V L E=P R S, P R O P S=E X C S T)$

For a list of available options, type? Otherwise

enter command or, if you wish to do a flash calculation.

enter $T(K)$ and $P($ bar) separated by a comma.

$293,15,1$ 


\section{Appendix B (Continued)}

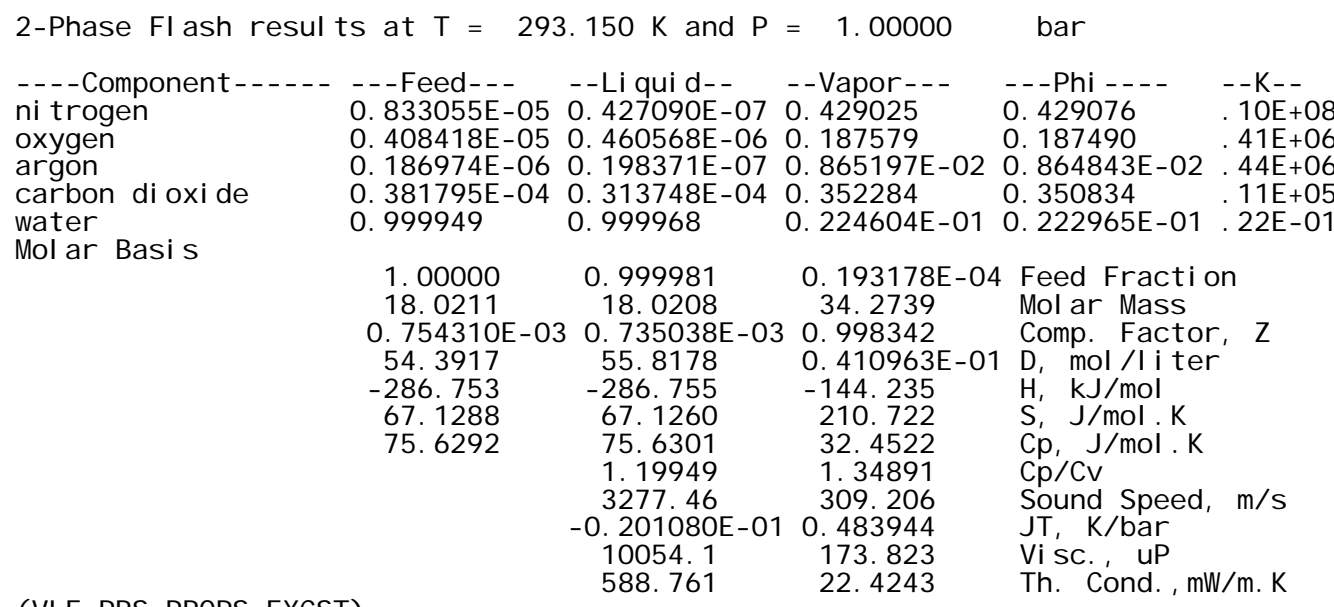

( $V L E=P R S, P R O P S=E X C S T)$

For a list of available options, type? Otherwise

enter command or, if you wish to do a flash calculation, enter $T(K)$ and $P$ (bar) separated by a comma.

$298.15,1$

\begin{tabular}{|c|c|c|c|c|c|}
\hline $\begin{array}{l}\cdots \text {. Component..... } \\
\text { nitrogen } \\
\text { oxygen } \\
\text { argon } \\
\text { carbon dioxide } \\
\text { water }\end{array}$ & $\begin{array}{l}\cdots \text { Feed-.. } \\
0.833055 \mathrm{E}-05 \\
0.408418 \mathrm{E}-05 \\
0.186974 \mathrm{E}-06 \\
0.381795 \mathrm{E}-04 \\
0.999949\end{array}$ & $\begin{array}{l}\cdots \text { Li quid-. } \\
0.525102 \mathrm{E}-07 \\
0.514592 \mathrm{E}-06 \\
0.222940 \mathrm{E}-07 \\
0.315055 \mathrm{E}-04 \\
0.999968\end{array}$ & $\begin{array}{l}\cdots \text { Vapor... } \\
0.429422 \\
0.185172 \\
0.854277 \mathrm{E}-02 \\
0.346245 \\
0.306178 \mathrm{E}-01\end{array}$ & $\begin{array}{l}\cdots P h i \ldots \\
0.429493 \\
0.185096 \\
0.853976 \mathrm{E}-02 \\
0.34488 \\
0.34488 \\
0.304033 \mathrm{E}-01\end{array}$ & $\begin{array}{l}-K- \\
82 E+07 \\
36 E+06 \\
38 E+06 \\
11 E+05 \\
31 E-01\end{array}$ \\
\hline & $\begin{array}{l}1.00000 \\
18.0211 \\
0.746899 \mathrm{E} \cdot 03 \\
54.0102 \\
-286.086 \\
69.2517 \\
74.5180\end{array}$ & $\begin{array}{l}0.999981 \\
18.0208 \\
0.727667 \mathrm{E}-03 \\
55.4377 \\
-286.089 \\
69.2490 \\
74.5188 \\
1.14872 \\
3164.64 \\
-0.209314 \mathrm{E}-01 \\
9000.45 \\
597.672\end{array}$ & $\begin{array}{l}0.192772 \mathrm{E}-04 \\
34.0849 \\
0.998416 \\
0.404041 \mathrm{E}-01 \\
-143.669 \\
211.245 \\
32.5133 \\
1.34788 \\
312.599 \\
0.474132 \\
175.802 \\
22.8360\end{array}$ & 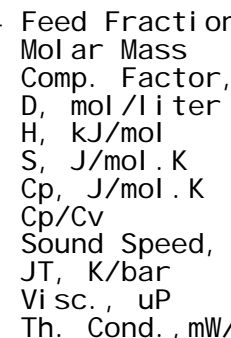 & $\mathrm{m} / \mathrm{s}$ \\
\hline
\end{tabular}

( $V L E=P R S, P R O P S=E X C S T$ )

$597.672-22.8360$

Th. Cond., mW/ m. K

For a list of available options, type? Otherwise

enter command or, if you wish to do a flash calculation.

enter $T(K)$ and $P$ (bar) separated by a comma.

303.15, 1

\begin{tabular}{|c|c|c|c|c|c|}
\hline $\begin{array}{l}\cdots \text { Component..... } \\
\text { nitrogen } \\
\text { oxygen } \\
\text { argon } \\
\text { carbon dioxide } \\
\text { water }\end{array}$ & $\begin{array}{l}\cdots \text { Feed-.. } \\
0.833055 \mathrm{E}-05 \\
0.408418 \mathrm{E}-05 \\
0.186974 \mathrm{E}-06 \\
0.381795 \mathrm{E}-04 \\
0.999949\end{array}$ & $\begin{array}{l}-. \mathrm{Li} q u i \mathrm{~d}-. \\
0.639249 \mathrm{E}-07 \\
0.570542 \mathrm{E}-06 \\
0.248543 \mathrm{E}-07 \\
0.316181 \mathrm{E}-04 \\
0.999968\end{array}$ & $\begin{array}{l}\cdots \text { Vapor... } \\
0.428310 \\
0.182049 \\
0.839976 \mathrm{E}-02 \\
0.339991 \\
0.412514 \mathrm{E}-01\end{array}$ & $\begin{array}{l}\ldots P h i \ldots \\
0.428401 \\
0.181984 \\
0.839729 \mathrm{E}-02 \\
0.338717 \\
0.409730 \mathrm{E}-01 .\end{array}$ & $\begin{array}{l}-K-. \\
67 E+07 \\
32 E+06 \\
34 E+06 \\
11 E+05 \\
41 E-01\end{array}$ \\
\hline al 50 & $\begin{array}{l}1.00000 \\
18.0211 \\
0.739394 \mathrm{E} \cdot 03 \\
53.6585 \\
-285.463 \\
71.2038 \\
73.5538\end{array}$ & $\begin{array}{l}0.999981 \\
18.0208 \\
0.720137 \mathrm{E}-03 \\
55.0934 \\
-285.465 \\
71.2010 \\
73.5546 \\
1.11477\end{array}$ & $\begin{array}{l}0.193005 \mathrm{E}-04 \\
33.8644 \\
0.998470 \\
0.397356 \mathrm{E}-01 \\
-143.617 \\
211.762 \\
32.5823 \\
1.34678\end{array}$ & 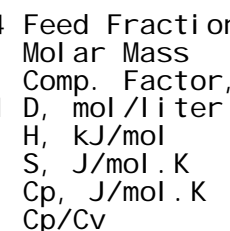 & , Z \\
\hline
\end{tabular}




\section{Appendix B (Continued)}

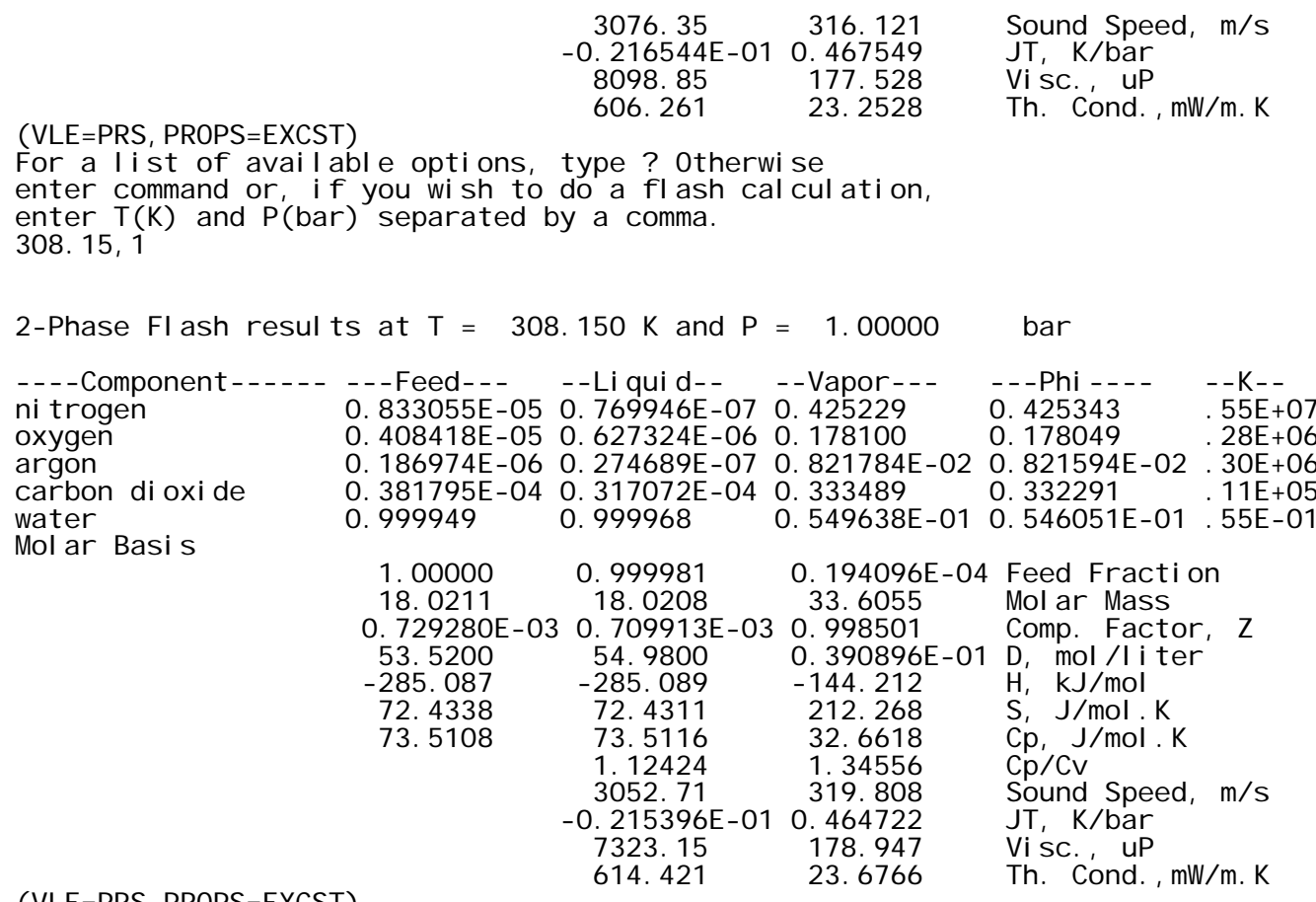

( VLE $=P R S, P R O P S=E X C S T$ )

For a list of available options, type? Otherwise

enter command or, if you wish to do a flash calculation,

enter $T(K)$ and $P(b a r)$ separated by a comma.

313.15, 1

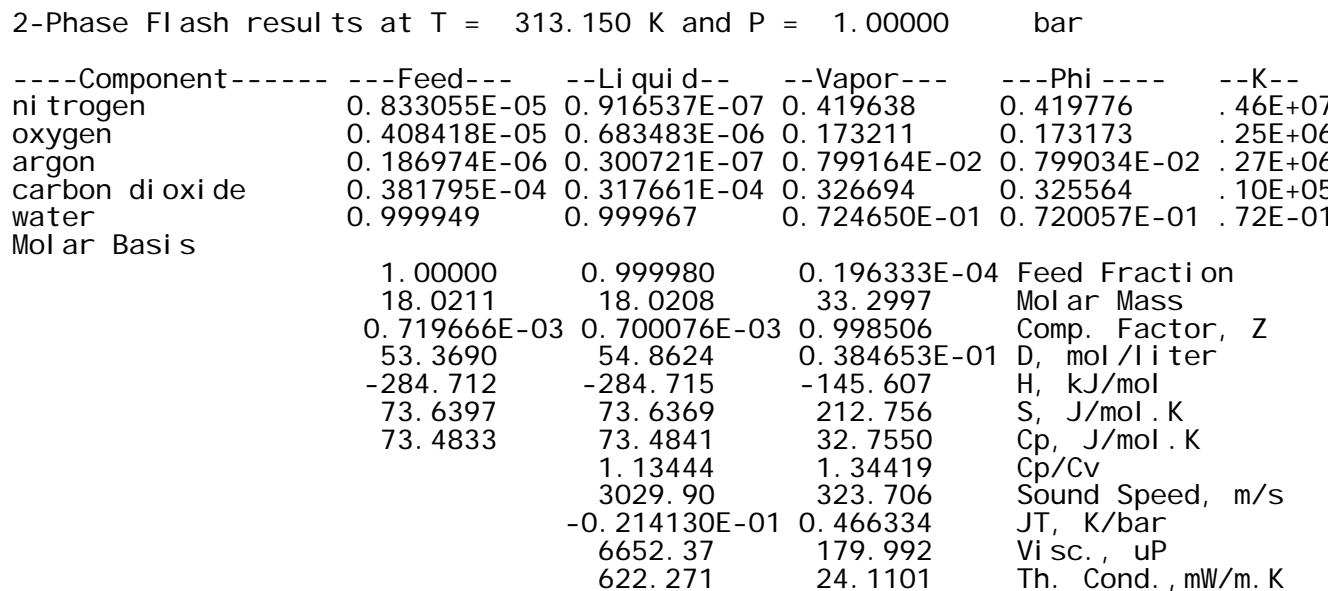

( $V L E=P R S, P R O P S=E X C S T)$

For a list of available options, type? otherwise

enter command or, if you wish to do a flash calculation.

enter $T(K)$ and $P(b a r)$ separated by a comma.

$318,15,1$

\begin{tabular}{|c|c|c|c|c|c|}
\hline $\begin{array}{l}\text {.. Component..... } \\
\text { nitrogen } \\
\text { oxygen } \\
\text { argon } \\
\text { carbon dioxide }\end{array}$ & $\begin{array}{l}\text {.. Feed-.. } \\
0.833055 \mathrm{E}-05 \\
0.408418 \mathrm{E}-05 \\
0.186974 \mathrm{E}-06 \\
0.381795 \mathrm{E}-04\end{array}$ & $\begin{array}{l}- \text { Li quid.. } \\
0.107669 \mathrm{E}-06 \\
0.737106 \mathrm{E}-06 \\
0.325762 \mathrm{E}-07 \\
0.317855 \mathrm{E}-04\end{array}$ & $\begin{array}{l}-. \text { Vapor... } \\
0.410902 \\
0.167256 \\
0.771537 \mathrm{E}-02 \\
0.319543\end{array}$ & $\begin{array}{l}\ldots \text { Phi.... } \\
0.411067 \\
0.167232 \\
0.771471 \mathrm{E}-02 \\
0.318473\end{array}$ & $\begin{array}{l}-K- \\
38 E+07 \\
23 E+06 \\
24 E+06 \\
10 E+05\end{array}$ \\
\hline
\end{tabular}




\section{Appendix B (Continued)}

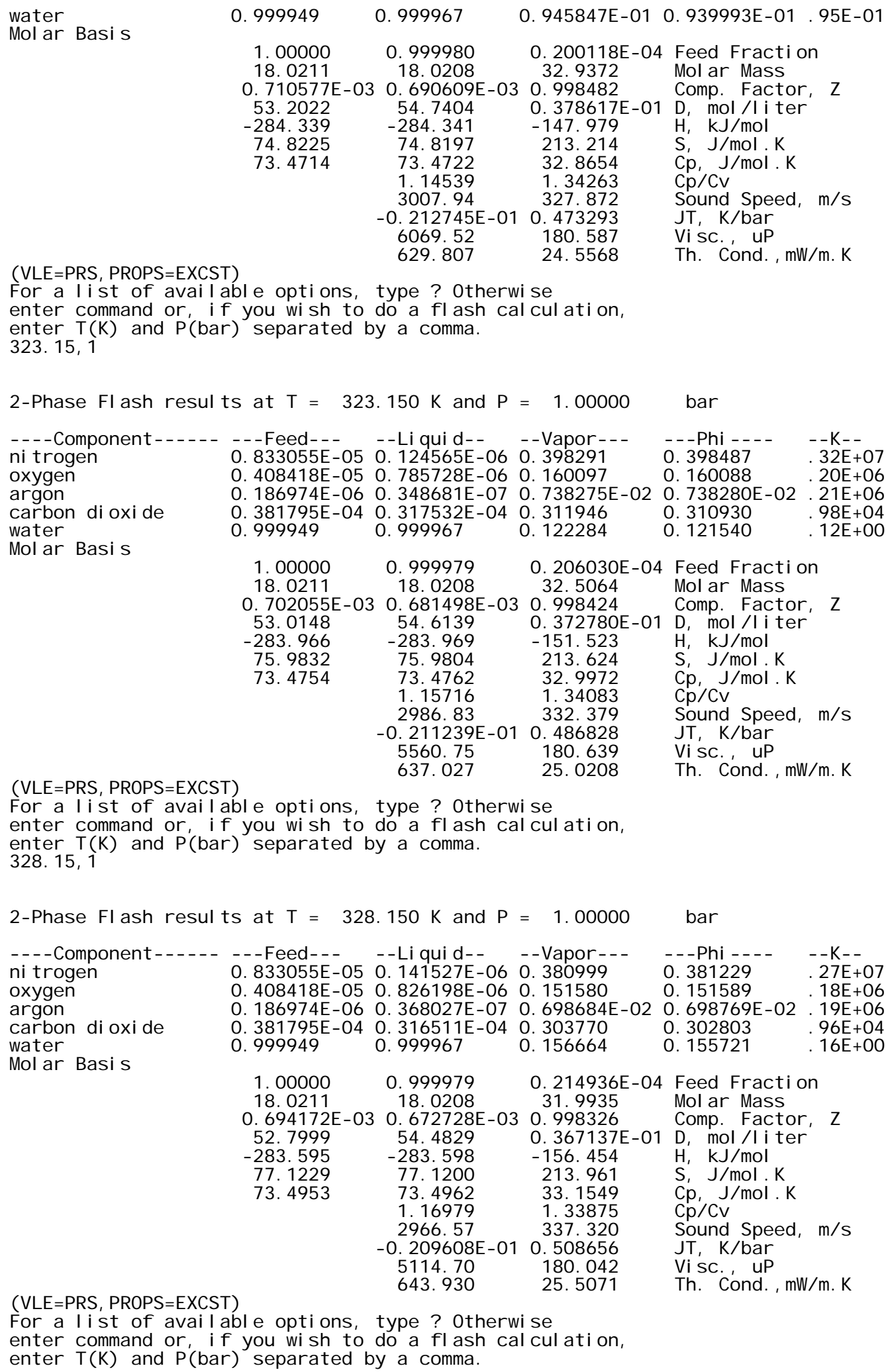




\section{Appendix B (Continued)}

$333,15,1$

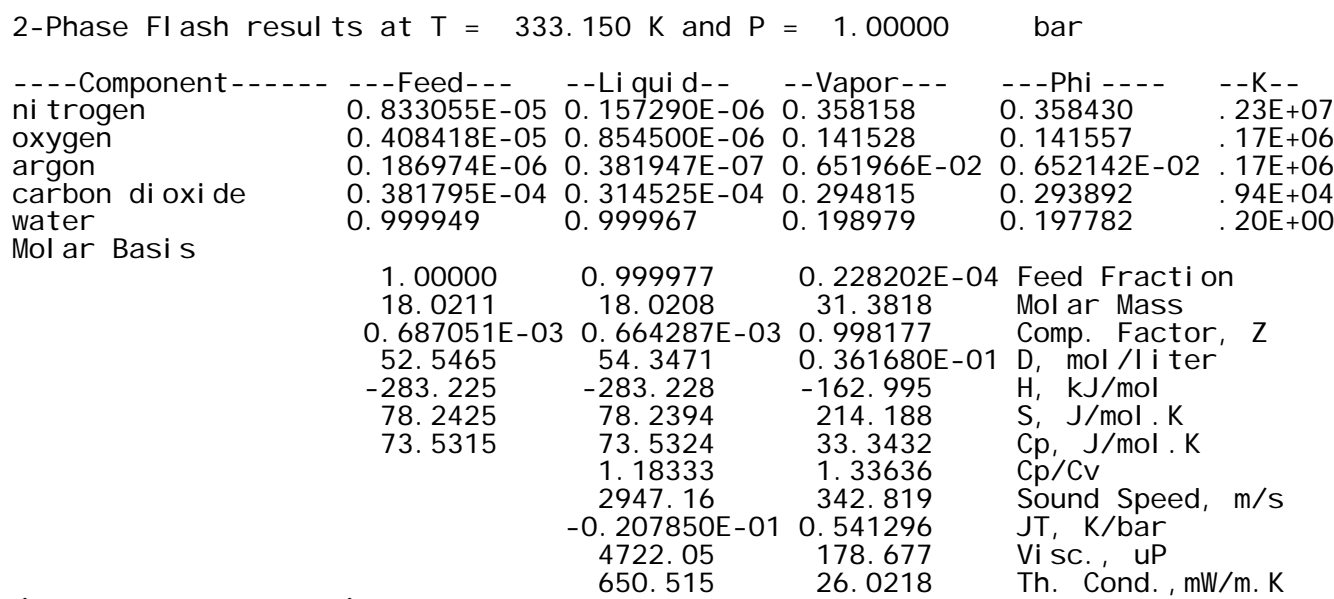

( $V L E=P R S, P R O P S=E X C S T$ )

For a list of available options, type? Otherwise

enter command or, if you wish to do a flash calculation, enter $T(K)$ and $P(b a r)$ separated by a comma.

$338,15,1$

\begin{tabular}{|c|c|c|c|c|c|}
\hline $\begin{array}{l}\text {.. Component..... } \\
\text { nitrogen } \\
\text { oxygen } \\
\text { argon } \\
\text { carbon dioxide } \\
\text { water }\end{array}$ & $\begin{array}{l}\cdots \text { Feed-.. } \\
0.833055 \mathrm{E}-05 \\
0.408418 \mathrm{E}-05 \\
0.186974 \mathrm{E}-06 \\
0.381795 \mathrm{E}-04 \\
0.999949\end{array}$ & $\begin{array}{l}\cdots \text { Li quid-. } \\
0.170017 \mathrm{E}-06 \\
0.865497 \mathrm{E}-06 \\
0.388077 \mathrm{E}-07 \\
0.311150 \mathrm{E}-04 \\
0.999968\end{array}$ & $\begin{array}{l}\text {. Vapor... } \\
0.328901 \\
0.129726 \\
0.597171 \mathrm{E} .02 \\
0.284758 \\
0.250644\end{array}$ & $\begin{array}{l}\cdots P h i \ldots \\
0.329223 \\
0.129778 \\
0.597455 \mathrm{E}-02 \\
0.283878 \\
0.249120\end{array}$ & $\begin{array}{l}-K- \\
19 E+07 \\
15 E+06 \\
15 E+06 \\
92 E+04 \\
25 E+00\end{array}$ \\
\hline B & $\begin{array}{l}1.00000 \\
18.0211 \\
0.680907 \mathrm{E} \cdot 03 \\
52.2366 \\
-282.856 \\
79.3429 \\
73.5839\end{array}$ & $\begin{array}{l}0.999975 \\
18.0208 \\
0.656162 \mathrm{E}-03 \\
54.2065 \\
-282.858 \\
79.3396 \\
73.5849 \\
1.19785 \\
2928.60 \\
-0.205963 \mathrm{E}-01 \\
4375.04 \\
656.778\end{array}$ & $\begin{array}{l}0.248116 \mathrm{E}-04 \\
30.6511 \\
0.997966 \\
0.356408 \mathrm{E}-01 \\
-171.361 \\
214.254 \\
33.5673 \\
1.33363 \\
349.043 \\
0.588638 \\
176.560 \\
26.5724\end{array}$ & $\begin{array}{l}\text { Feed Fraction } \\
\text { Molar Mass } \\
\text { Comp. Factor, } \\
\text { D, mol/IIiter } \\
H_{1}, \mathrm{~kJ} / \mathrm{mol} \\
S_{,} \text {J / mol. K } \\
\text { Cp, J / mol. K } \\
\text { Cp/CV } \\
\text { Sound Speed, } \\
\text { JT, K/bar } \\
\text { Visc. uP } \\
\text { Th. Cond., mW/ }\end{array}$ & $\mathrm{m} / \mathrm{s}$ \\
\hline
\end{tabular}

( $V L E=P R S, P R O P S=E X C S T$ )

For a list of available options, type? Otherwise enter command or, if you wish to do a flash calculation, enter $T(K)$ and $P$ (bar) separated by a comma.

$343,15,1$

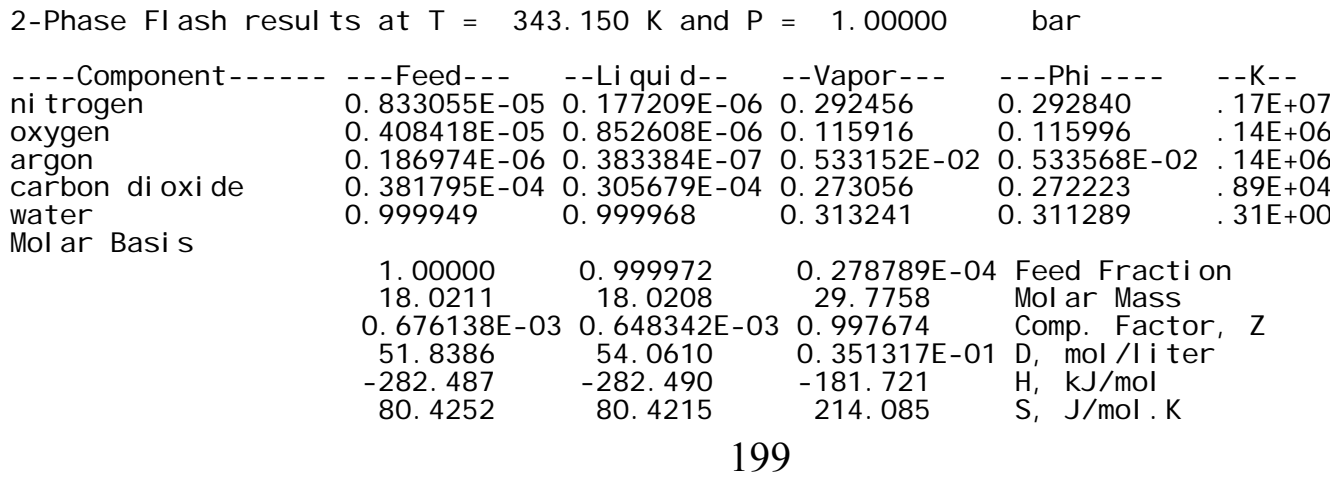




\section{Appendix B (Continued)}

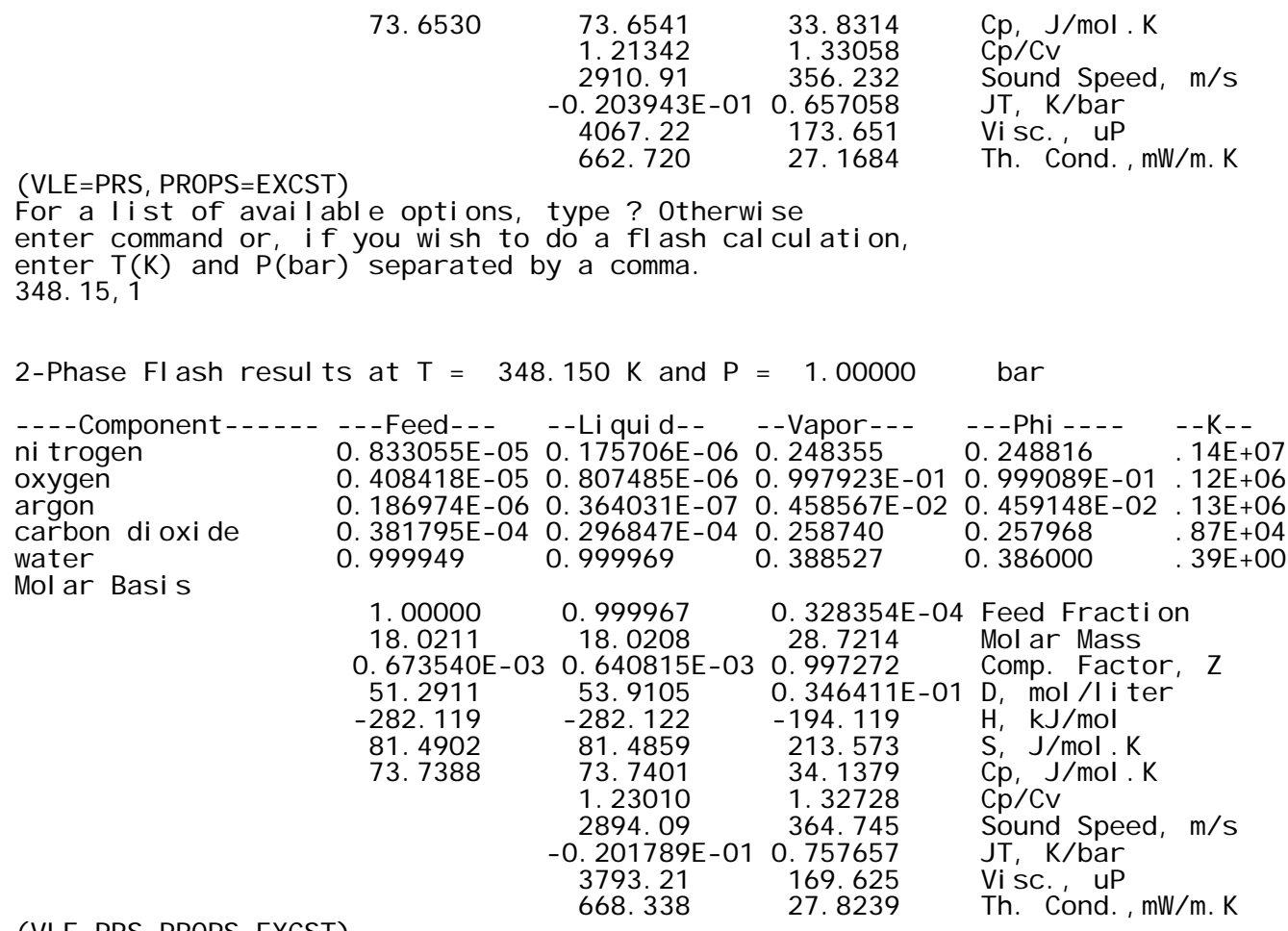

( $V L E=P R S, P R O P S=E X C S T$ )

For a list of available options, type? otherwise

enter command or, if you wish to do a flash calculation,

enter $T(K)$ and $P$ (bar) separated by a comma.

$353,15,1$

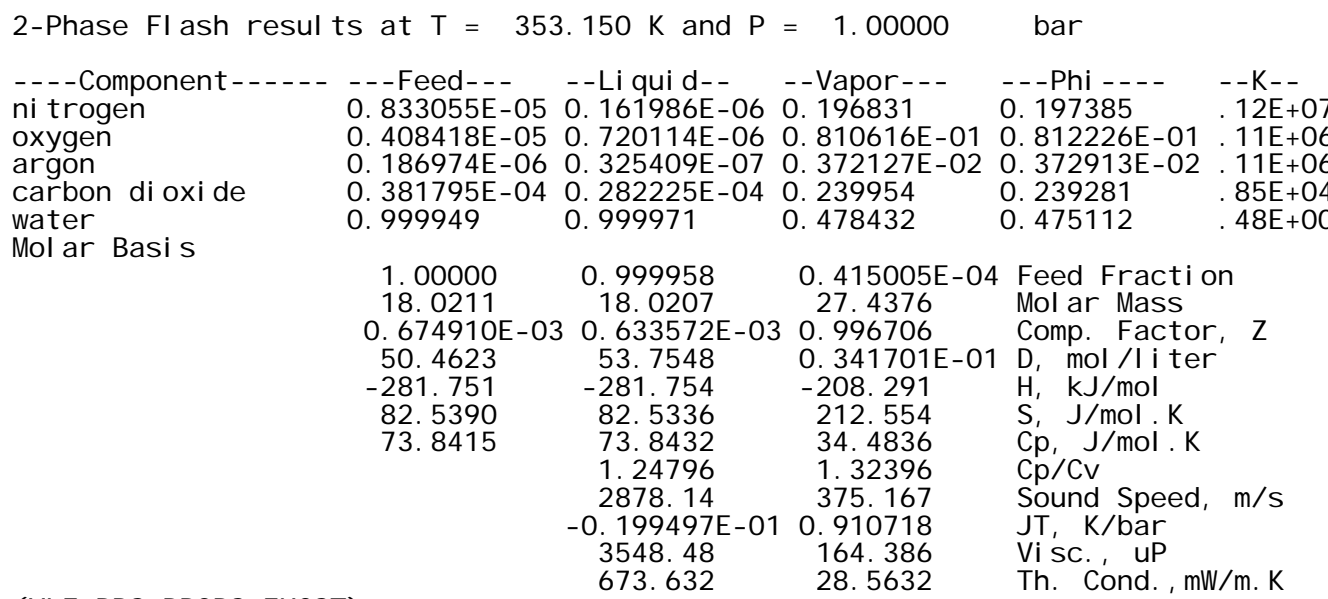

( $V L E=P R S, P R O P S=E X C S T$ )

$673.632 \quad 28.5632 \quad$ Th. Cond., mW/m. K

or a list of available options, type? otherwise

enter command or, if you wish to do a flash calculation,

enter $T(K)$ and $P$ (bar) separated by a comma.

$358,15,1$

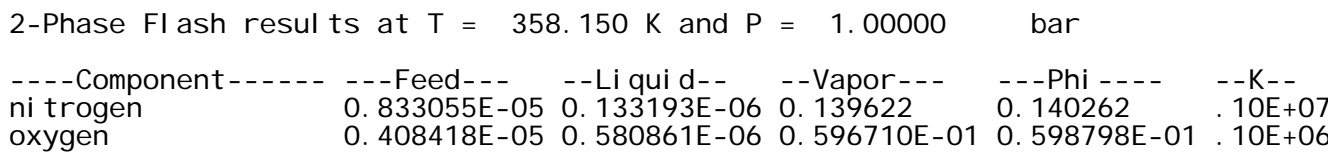




\section{Appendix B (Continued)}

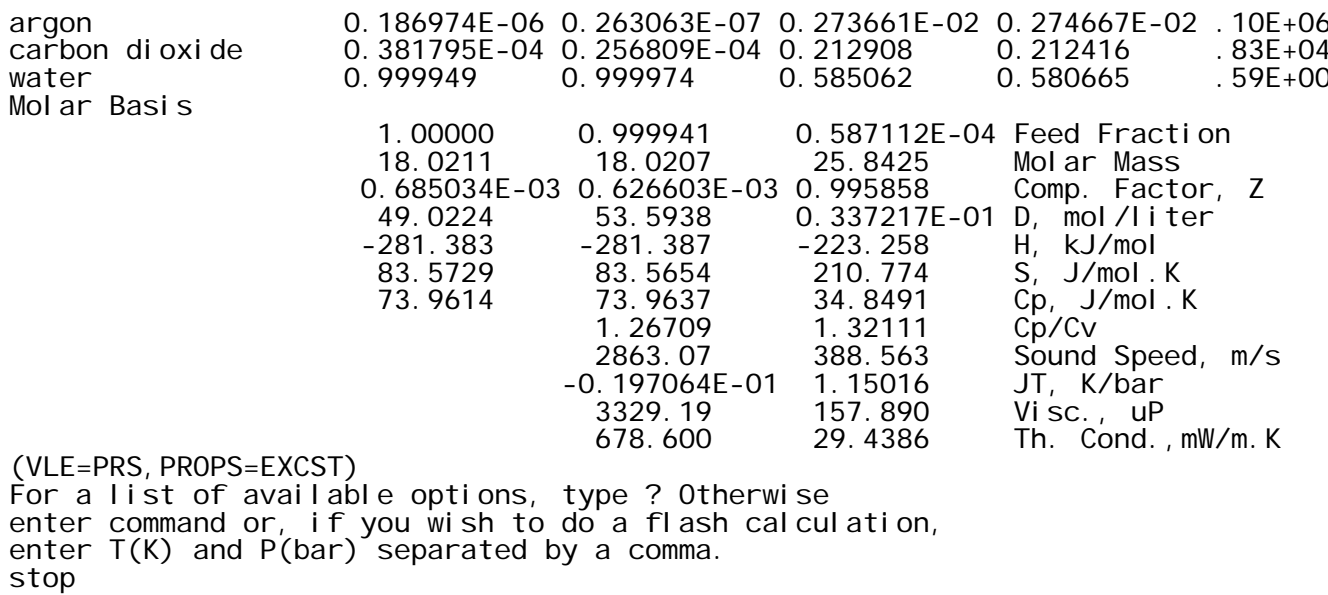




\section{Appendix C. Matlab code for $F_{C T}$ data regression}

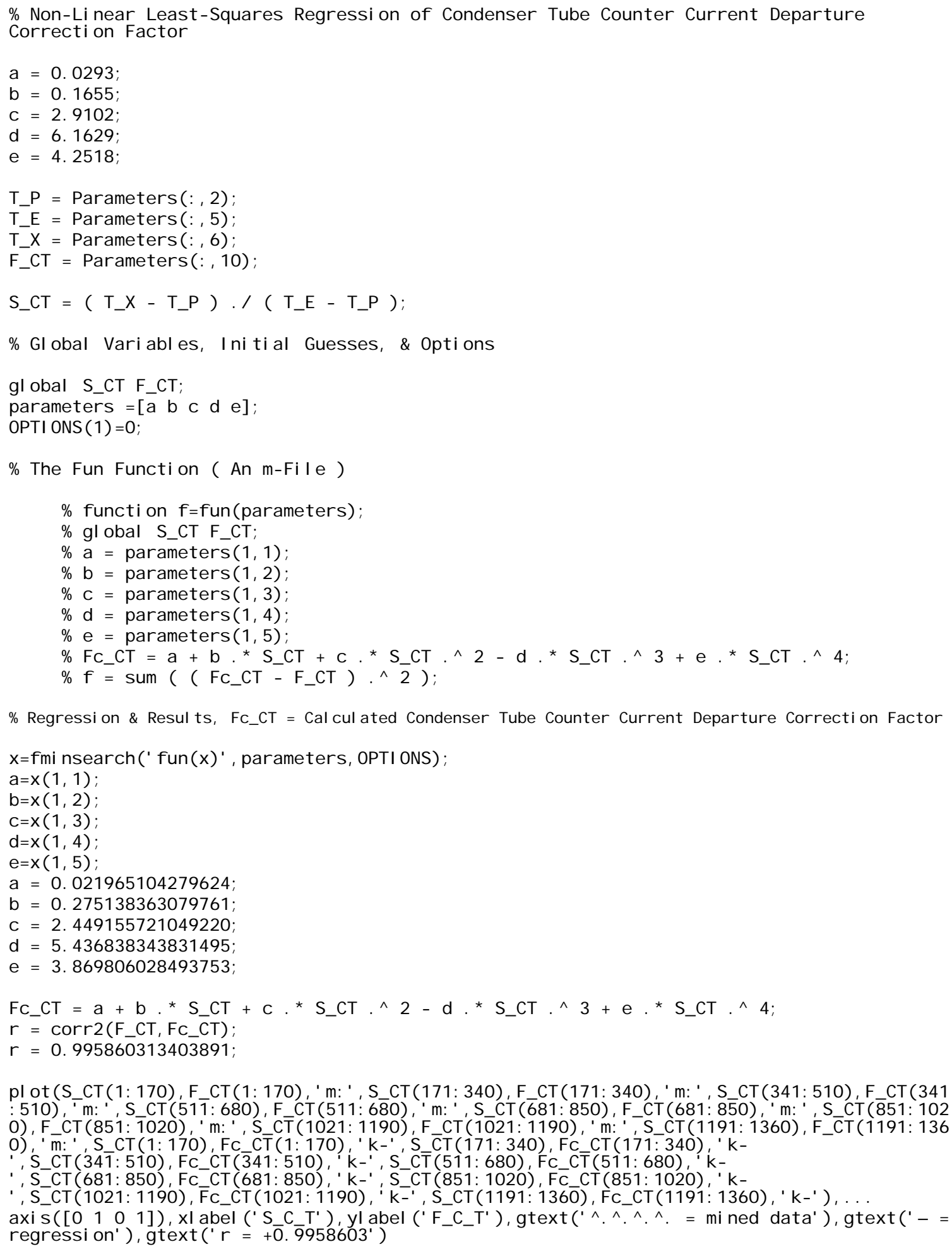

plot ( S CT(1:170), F CT(1:170), ' m: ', S CT(171:340), F CT(171:340), ' m: ', S CT(341:510), F CT(341

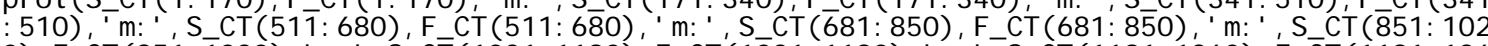

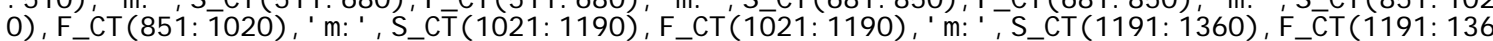

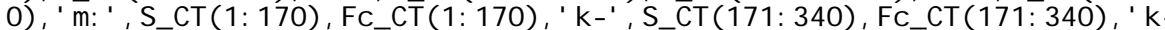

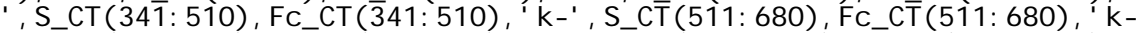
', $S^{-} C T(681: 850), F^{-} C T(681: 850)$,' $k-1, S^{-} C T(851: 1020), F \bar{c} C T(851: 1020), ' k$. ', $S^{-} C T(1021: 1190), \bar{F} C$ CT (1021:1190), 'k=1,S CT(1191:1360), FC CT(1191:1360), 'k-'), .

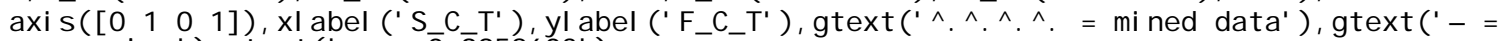
regression'), gtext(' $r=+\overline{0} . \overline{9} 958603$ ') 


\section{Appendix D. Matlab code for $N E A$ data regression}

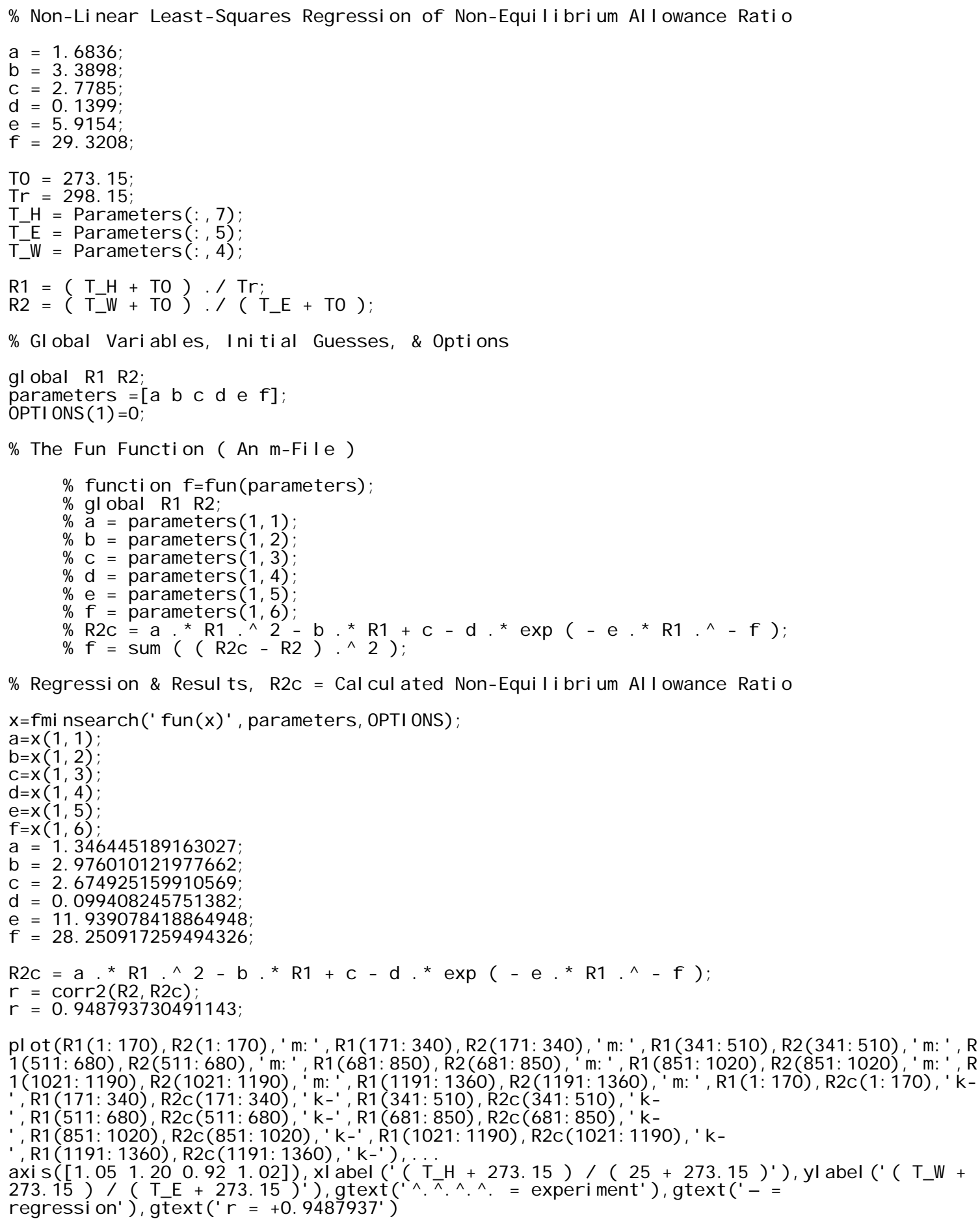




\section{Appendix E. Matlab code for $\gamma_{H 2 O}$ data regression}

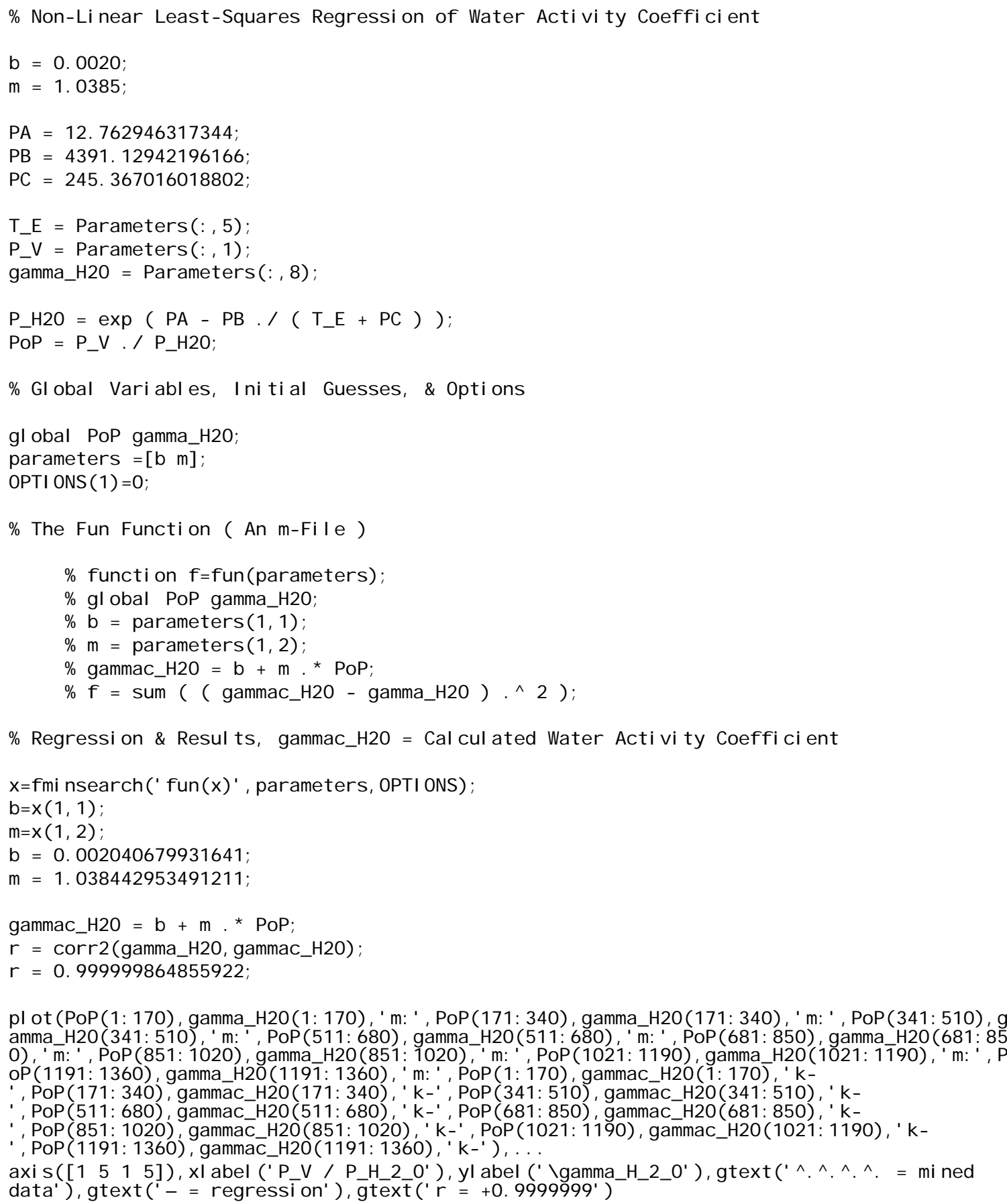




\section{Appendix F. Matlab code for $\psi$ data regression}

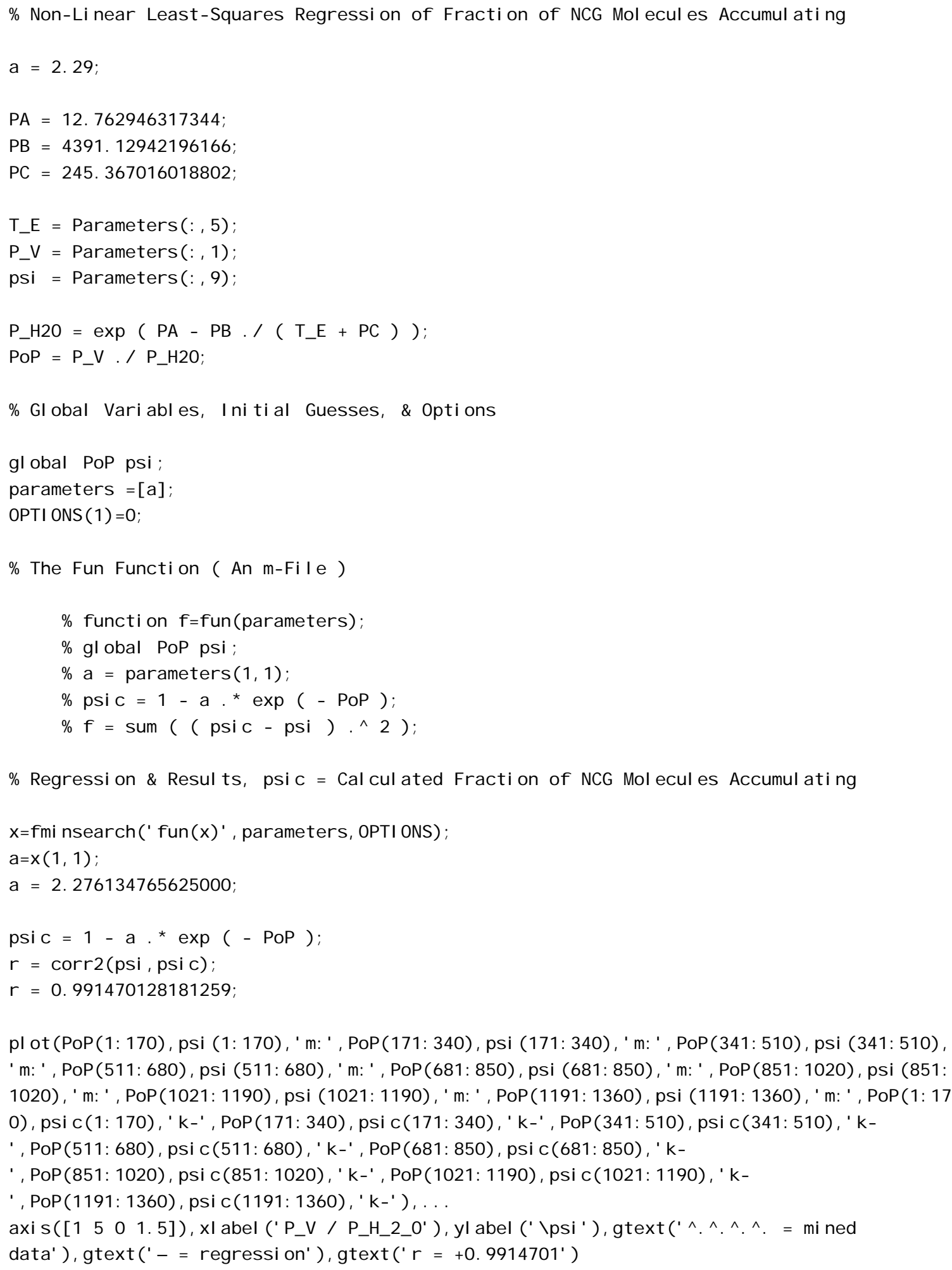




\section{Appendix G. Matlab code for $H C_{N 2}$ data regression}

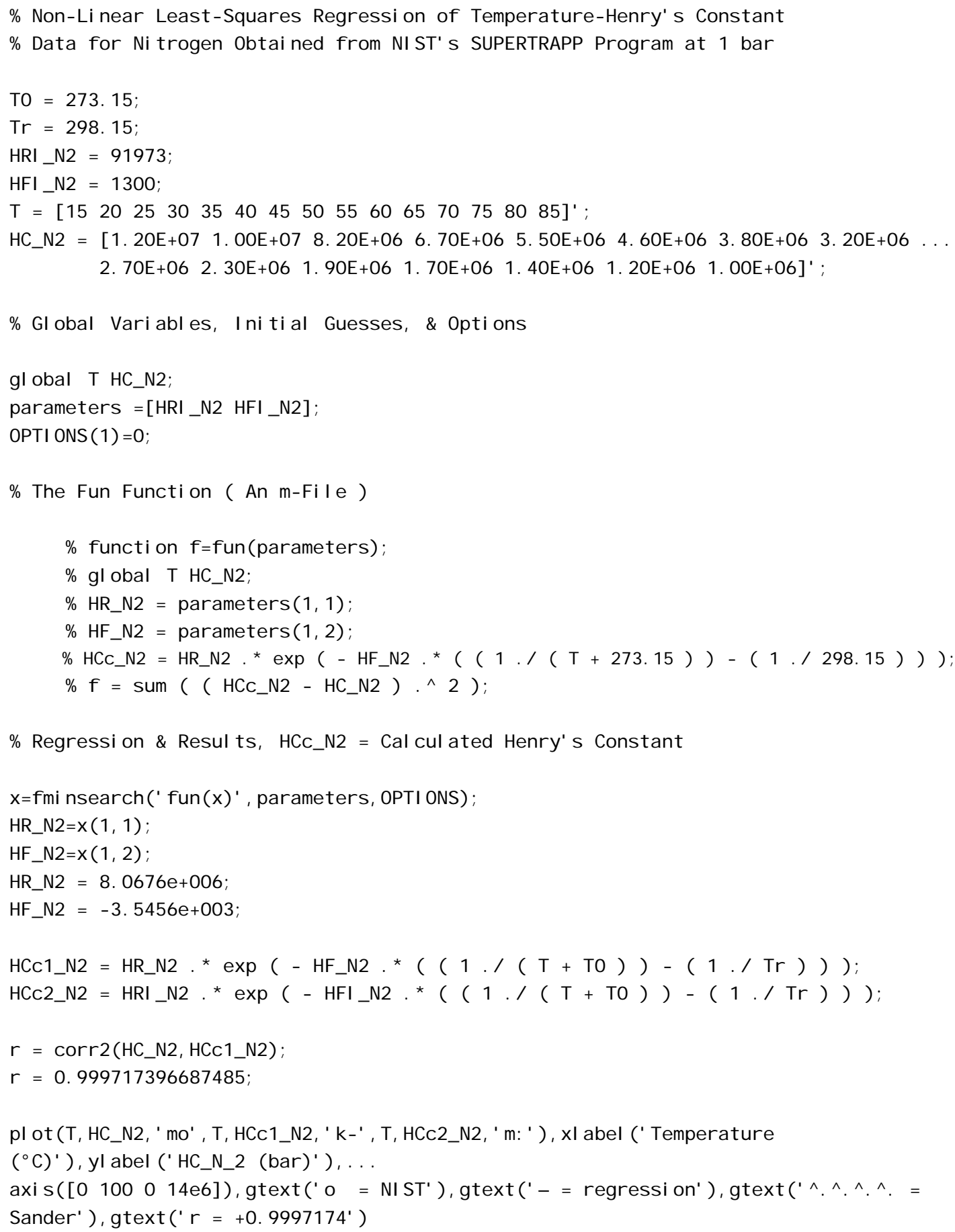




\section{Appendix H. Matlab code for $\mathrm{HC}_{\mathrm{O} 2}$ data regression}

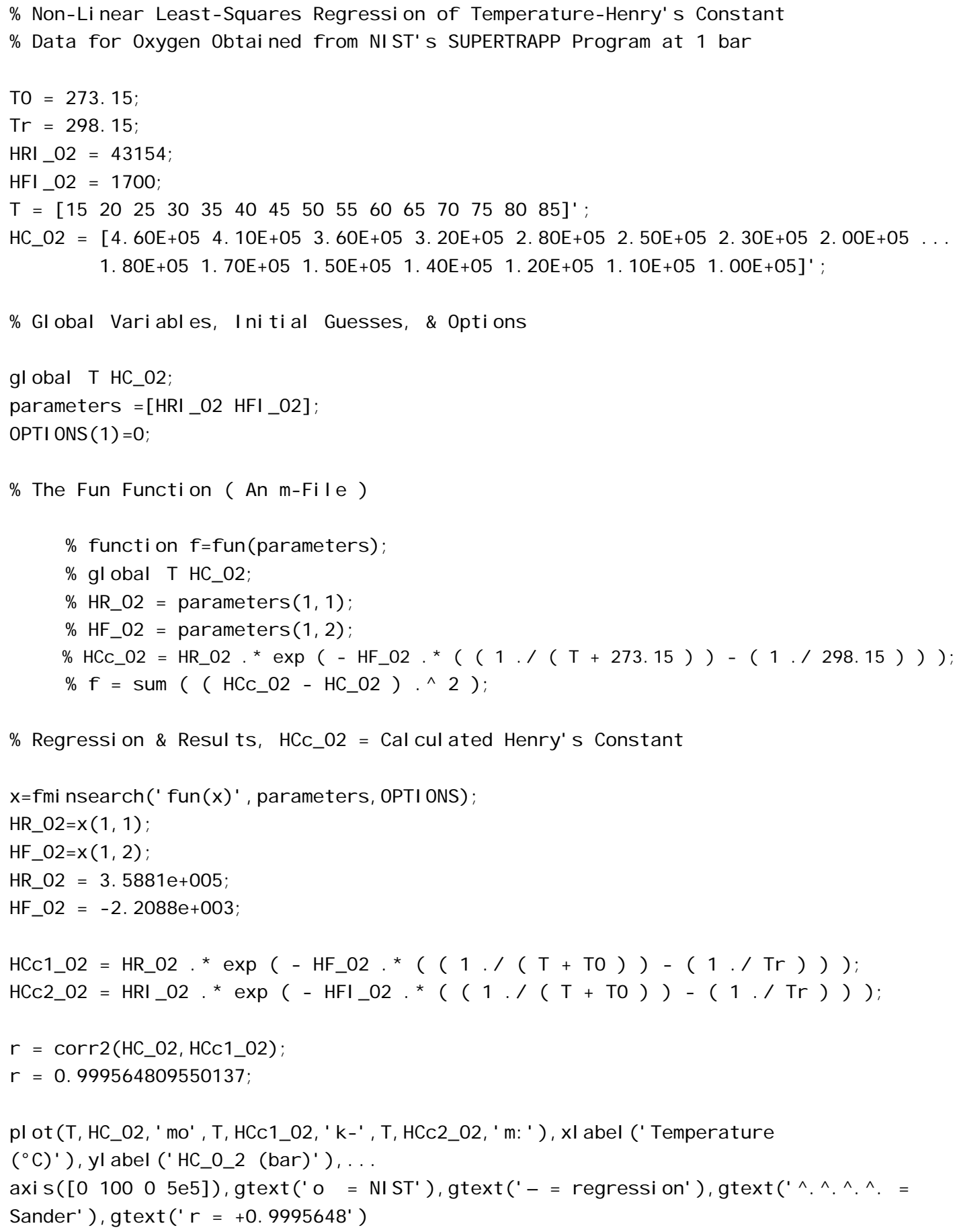




\section{Appendix I. Matlab code for $H C_{A r}$ data regression}

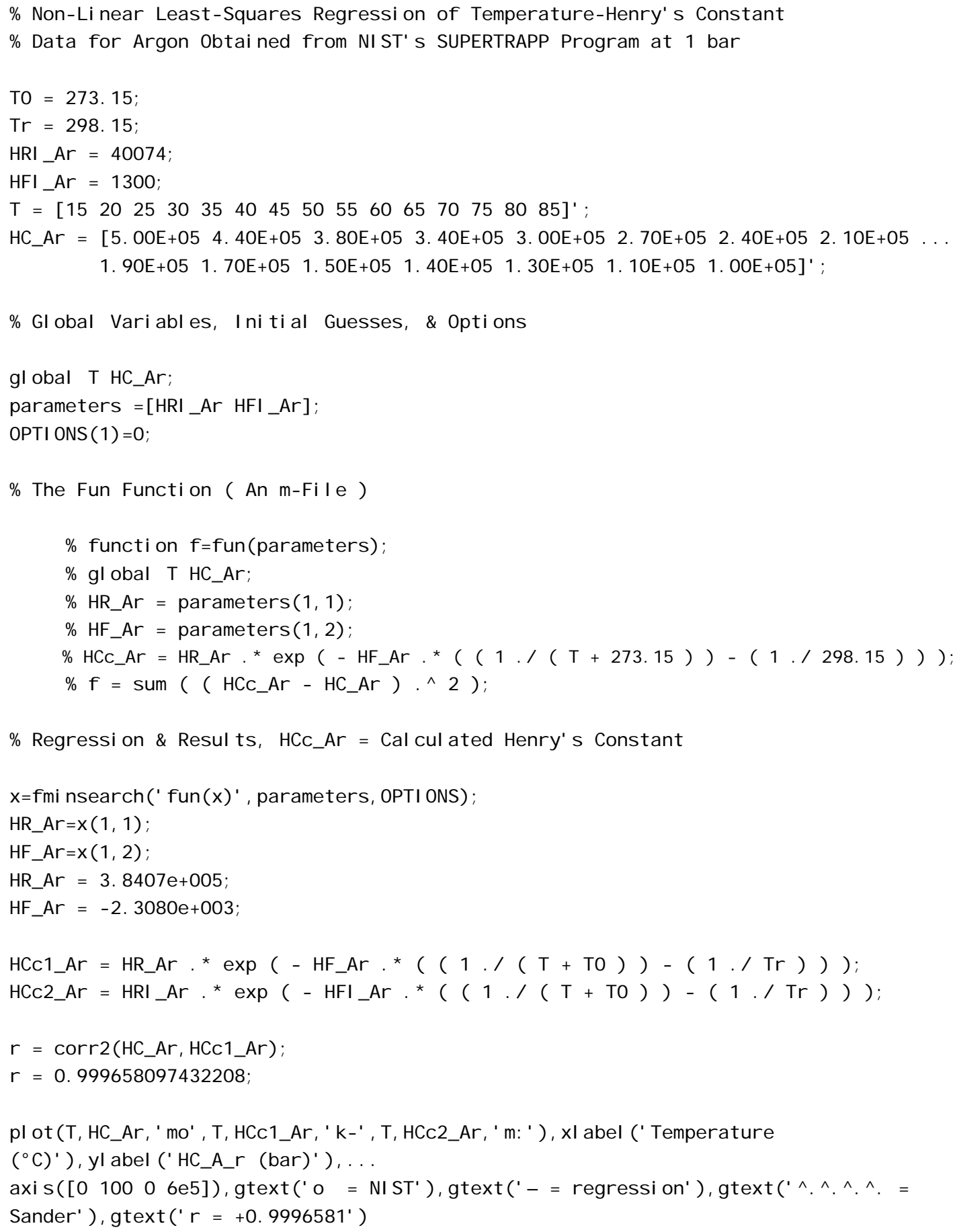




\section{Appendix J. Matlab code for $\mathrm{HC}_{\mathrm{CO} 2}$ data regression}

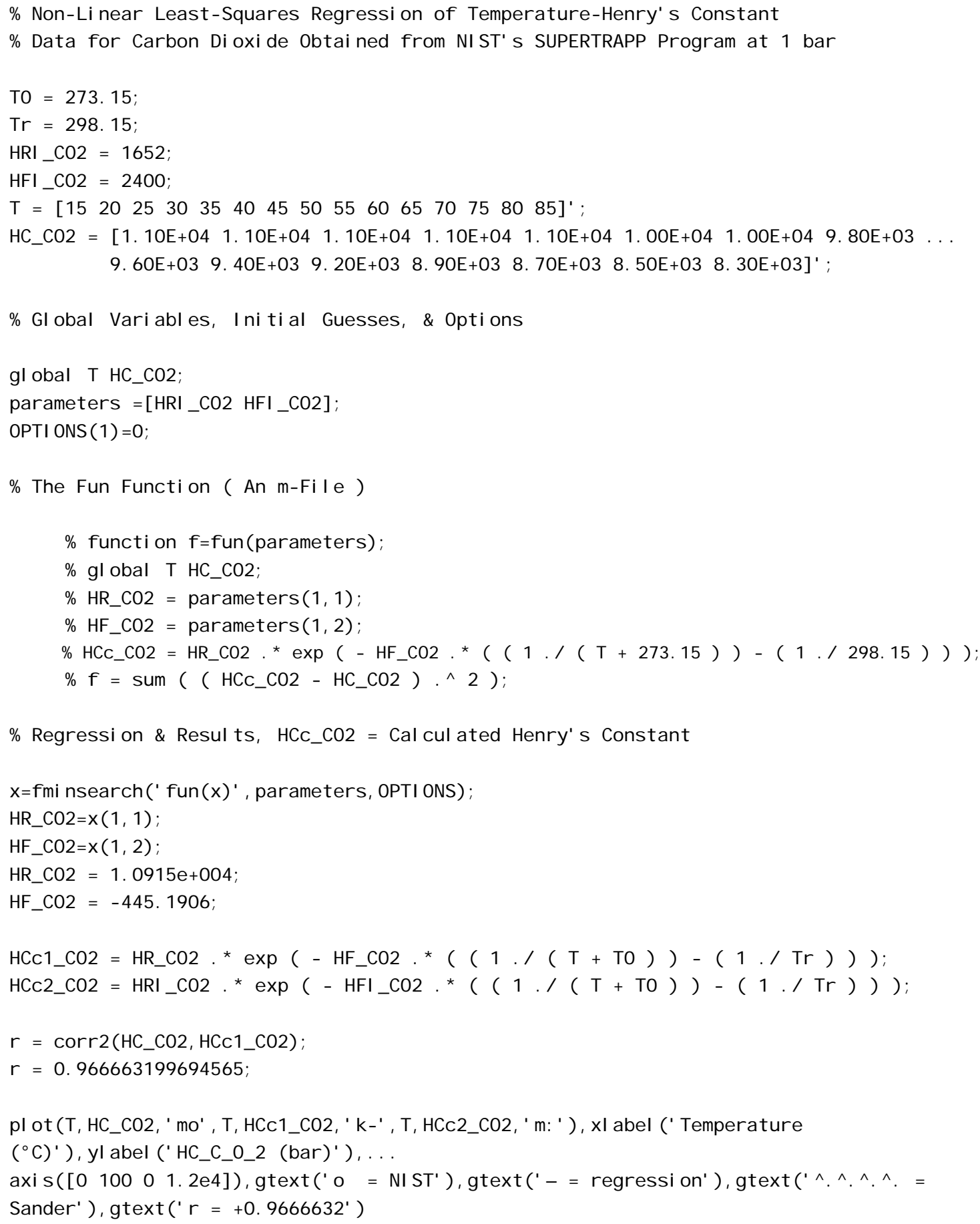




\section{Appendix K. Matlab code for $P_{H 2 O}{ }^{\text {sat }}$ data regression}

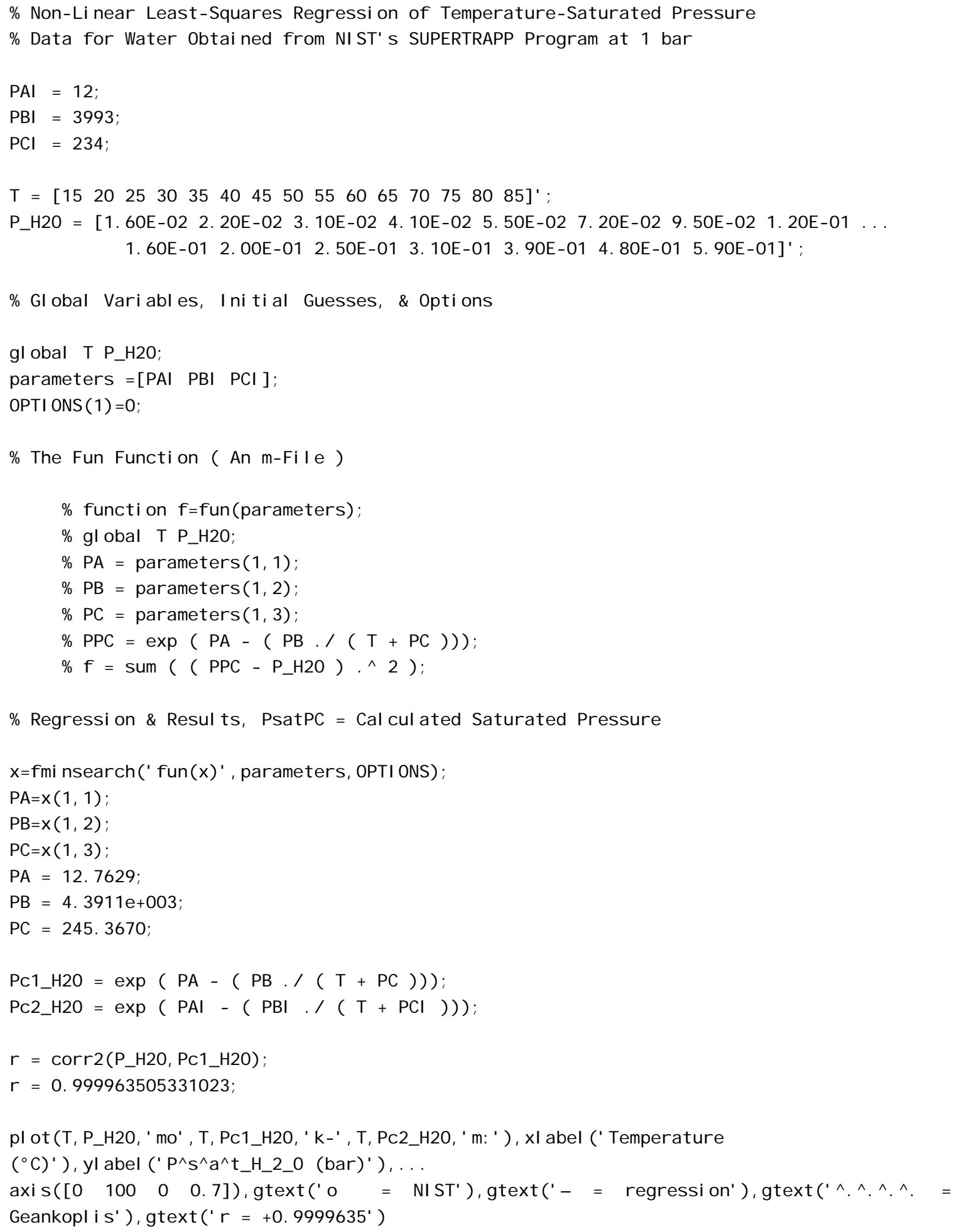


Appendix L. Sample TK Solver code for data mining

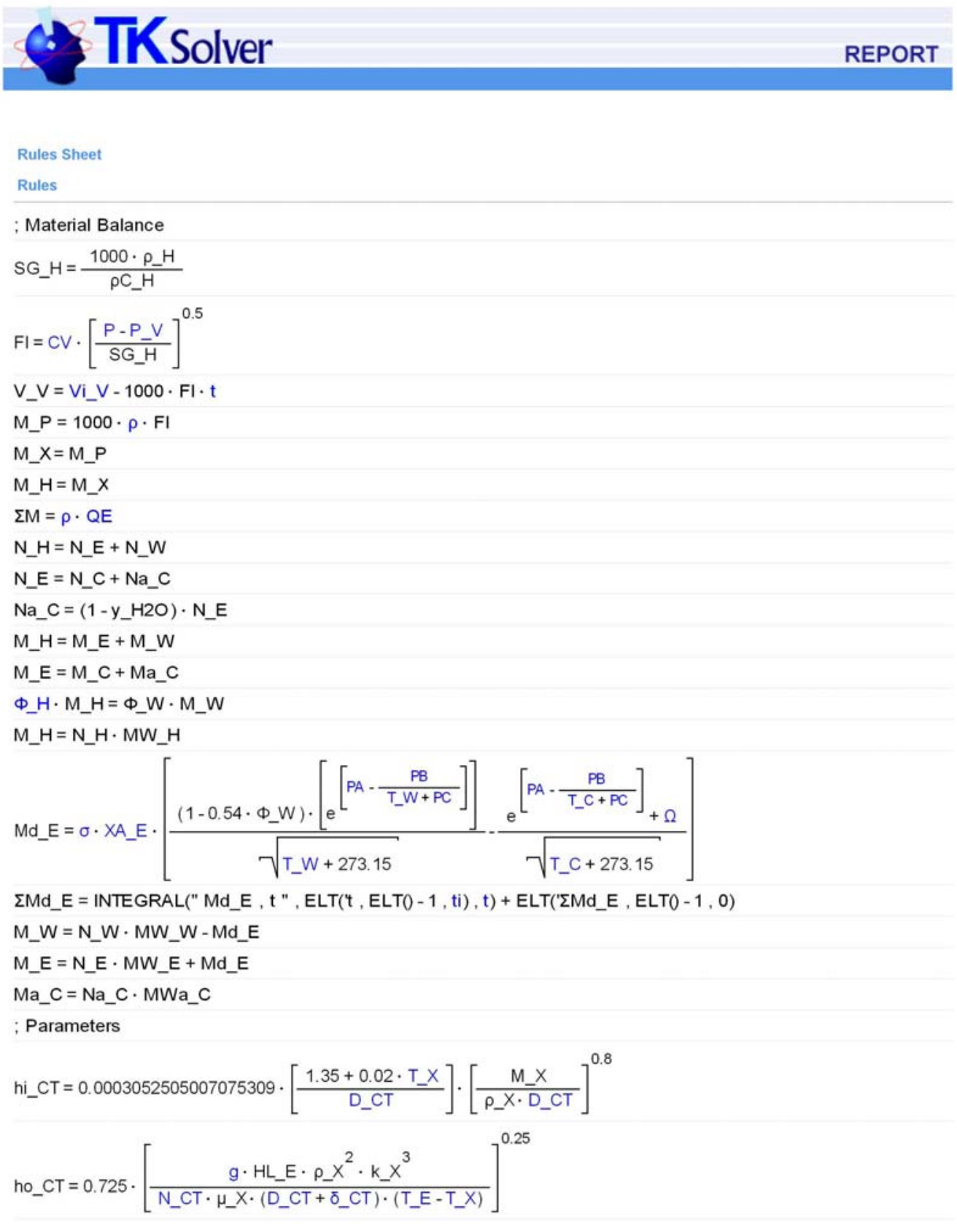




\section{Appendix L (Continued)}

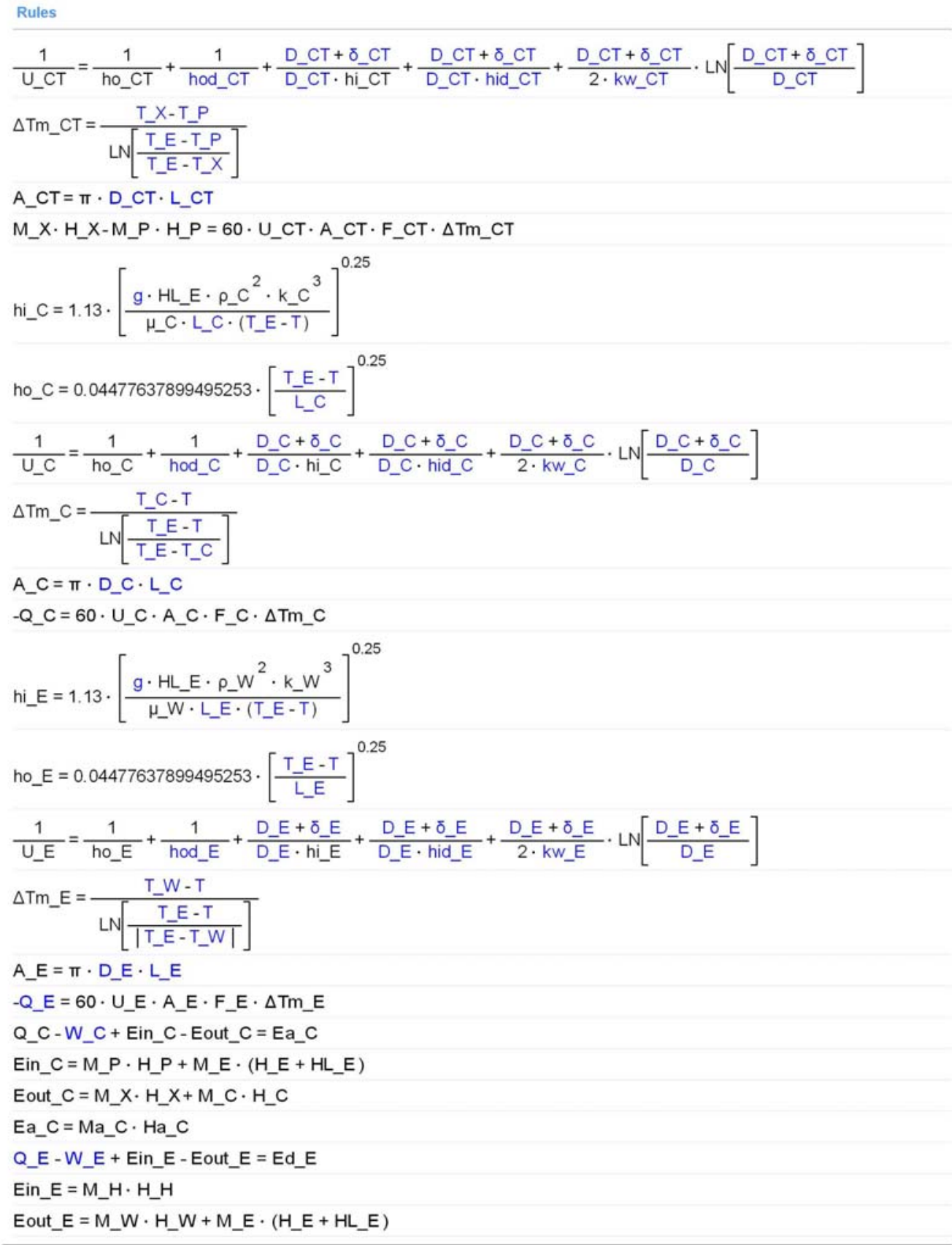




\section{Appendix L (Continued)}

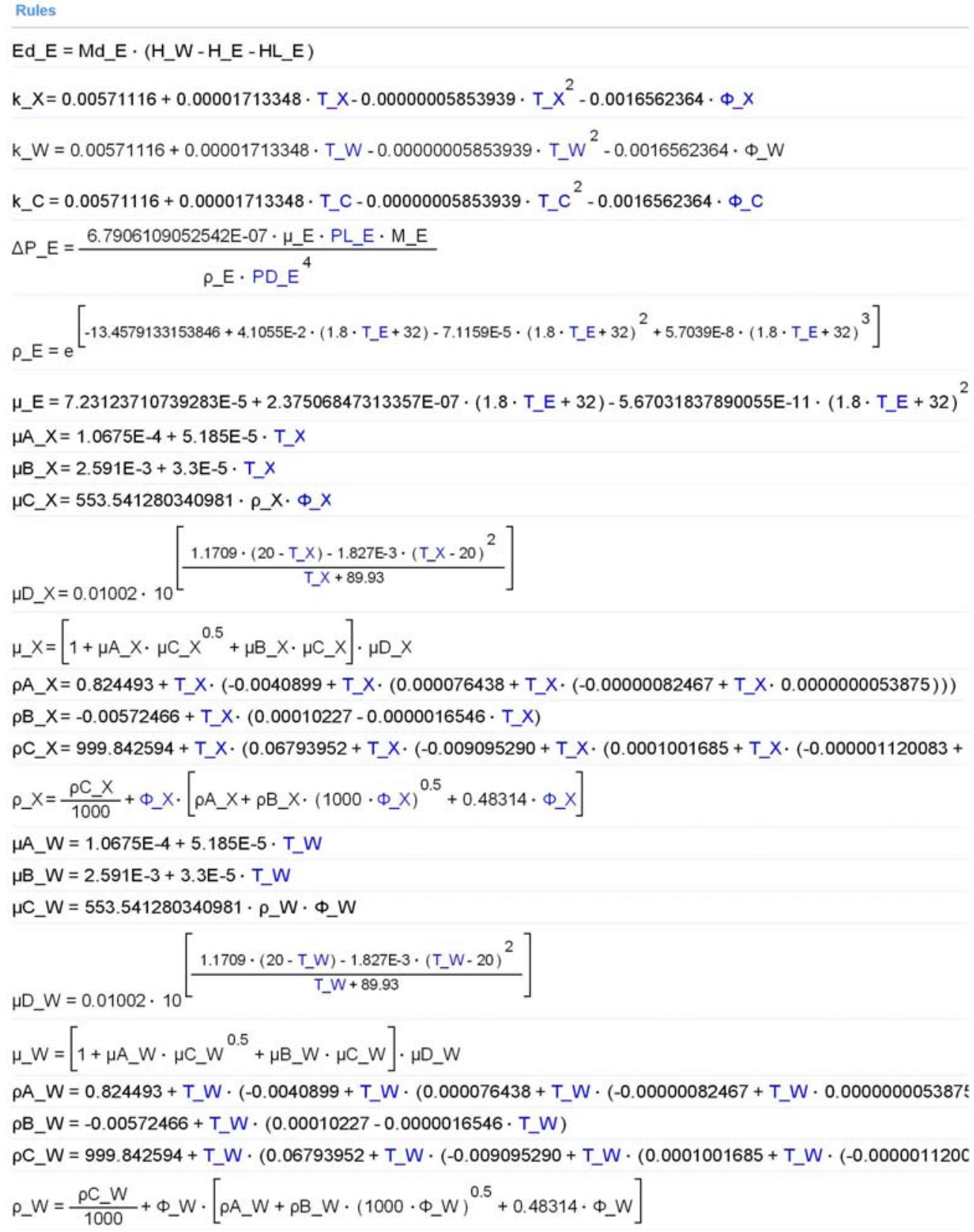




\section{Appendix L (Continued)}

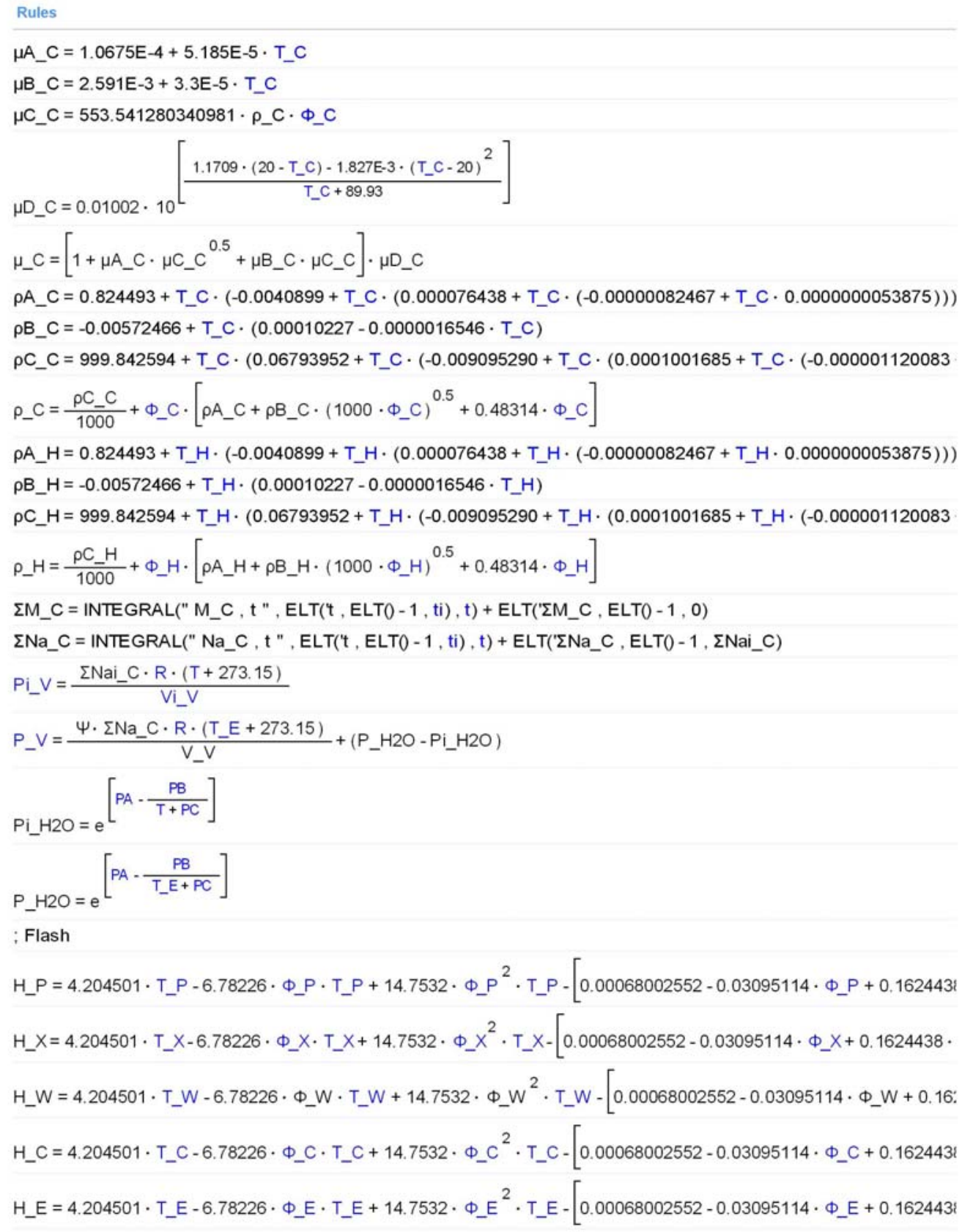




\section{Appendix L (Continued)}

Rules

$H L E=2496.21010517215-1.9534839865599 \cdot T \_E-0.00417809176330114 \cdot T_{-} E^{2}$

$H_{-} H=4.204501 \cdot T_{-} H-6.78226 \cdot \Phi_{-} H \cdot T_{-} H+14.7532 \cdot \Phi_{-} H^{2} \cdot T_{-} H-\left[0.00068002552-0.03095114 \cdot \Phi_{-} H+0.162443:\right.$ $H_{-} N 2=A \_N 2 \cdot\left(T \_E+273.15\right)+\left[\frac{B \_N 2}{2000}\right] \cdot\left(T \_E+273.15\right)^{2}+\left[\frac{C_{-} N 2}{3000000}\right] \cdot\left(T_{-} E+273.15\right)^{3}+\left[\frac{D \_N 2}{4000000000}\right] \cdot(T$. H_O2 $=A_{-} O 2 \cdot\left(T_{-} E+273.15\right)+\left[\frac{B_{-} O 2}{2000}\right] \cdot\left(T_{-} E+273.15\right)^{2}+\left[\frac{C_{-} O 2}{300000}\right] \cdot\left(T_{-} E+273.15\right)^{3}+\left[\frac{D_{-} O 2}{4000000000}\right] \cdot(T$ $H_{-} A r=A_{-} A r \cdot\left(T_{-} E+273.15\right)+\left[\frac{B_{-} A_{r}}{2000}\right] \cdot\left(T_{-} E+273.15\right)^{2}+\left[\frac{C_{-} A r}{3000000}\right] \cdot\left(T_{-} E+273.15\right)^{3}+\left[\frac{D_{-} A r}{400000000}\right] \cdot\left(T_{-} E\right.$ $H_{-} C_{C O}=A_{-} C O 2 \cdot\left(T_{-} E+273.15\right)+\left[\frac{B_{-} C O 2}{2000}\right] \cdot\left(T_{-} E+273.15\right)^{2}+\left[\frac{C_{-} C O 2}{3000000}\right] \cdot\left(T_{-} E+273.15\right)^{3}+\left[\frac{D_{-} C O 2}{4000000000} \cdot\right.$

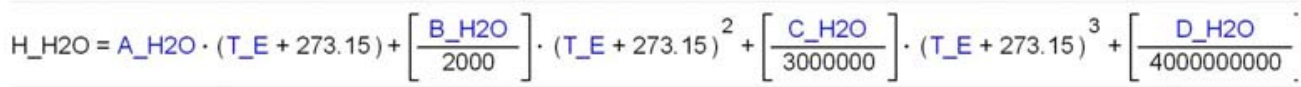
$\mathrm{Ha} \_\mathrm{C}=\frac{\mathrm{y} \_\mathrm{N} 2 \cdot \mathrm{H} \_\mathrm{N} 2+\mathrm{y} \_\mathrm{O} 2 \cdot \mathrm{H} \_\mathrm{O} 2+\mathrm{y} \_\mathrm{Ar} \cdot \mathrm{H} \_\mathrm{Ar}+\mathrm{y} \_\mathrm{CO} 2 \cdot \mathrm{H} \_\mathrm{CO} 2}{\left(1-\mathrm{y} \_\mathrm{H} 2 \mathrm{O}\right) \cdot \mathrm{MWa} \mathrm{C}^{\mathrm{C}}}$

$\mathrm{MW} \_\mathrm{H}=\mathrm{z} \_\mathrm{N} 2 \cdot \mathrm{MW} \_\mathrm{N} 2+\mathrm{z} \_\mathrm{O} 2 \cdot \mathrm{MW} \_\mathrm{O} 2+\mathrm{z} \_\mathrm{Ar} \cdot \mathrm{MW} \_\mathrm{Ar}+\mathrm{z} \_\mathrm{CO} 2 \cdot \mathrm{MW} \_\mathrm{CO} 2+\mathrm{z} \_\mathrm{Salt} \cdot \mathrm{MW} \_\mathrm{Salt}+\mathrm{z} \_\mathrm{H} 2 \mathrm{O} \cdot \mathrm{M}$ $\mathrm{MW} \_W=\mathrm{x}_{-} \mathrm{N} 2 \cdot \mathrm{MW} \_\mathrm{N} 2+\mathrm{x} \_\mathrm{O} 2 \cdot \mathrm{MW} \_\mathrm{O} 2+\mathrm{x} \_\mathrm{Ar} \cdot \mathrm{MW} \_\mathrm{Ar}+\mathrm{x} \_\mathrm{CO} 2 \cdot \mathrm{MW} \_\mathrm{CO} 2+\mathrm{x} \_\mathrm{S}$ alt $\cdot \mathrm{MW} \_\mathrm{Salt}+\mathrm{x} \_\mathrm{H} 2 \mathrm{O} \cdot \mathrm{N}$ $M W \_E=y \_N 2 \cdot M W \_N 2+y \_O 2 \cdot M W \_O 2+y \_A r \cdot M W \_A r+y \_C O 2 \cdot M W \_C O 2+y \_H 2 O \cdot M W \_H 2 O$ $\mathrm{MW} \_\mathrm{C}=\mathrm{MW} \_\mathrm{H} 2 \mathrm{O}$ $\mathrm{MWa} \mathrm{C}=\frac{\mathrm{y} \_\mathrm{N} 2 \cdot \mathrm{MW} \_\mathrm{N} 2+\mathrm{y} \_\mathrm{O} 2 \cdot \mathrm{MW} \mathrm{O}_{-}+\mathrm{y} \_\mathrm{Ar} \cdot \mathrm{MW} \_\mathrm{Ar}+\mathrm{y} \_\mathrm{CO} 2 \cdot \mathrm{MW} \_\mathrm{CO} 2}{1-\mathrm{y} \_\mathrm{H} 2 \mathrm{O}}$

$K_{-} N 2=\frac{H_{-} N 2 \cdot e^{\left[-H F_{-} N 2 \cdot\left[\frac{1}{T_{-} E+273.15} \cdot \frac{1}{298.15}\right]\right]}}{P_{-} V}$

$K_{-} \mathrm{O} 2=\frac{\mathrm{HC}_{-} \mathrm{O} 2 \cdot \mathrm{e}^{\left[-\mathrm{HF}_{-} \mathrm{O} 2 \cdot\left[\frac{1}{\mathrm{~T}_{-} \mathrm{E}+273.15} \cdot \frac{1}{298.15}\right]\right]}}{\mathrm{P}_{-} \mathrm{V}}$

$K_{-} A r=\frac{H C_{-} A_{r} \cdot e^{\left[-H F_{-} A r \cdot\left[\frac{1}{T_{-} E+273.15} \cdot \frac{1}{298.15}\right]\right]}}{P_{-} V}$

$K_{-} \mathrm{CO}_{2}=\frac{\mathrm{HC}_{-} \mathrm{CO} 2 \cdot e^{\left[-\mathrm{HF}_{-} \mathrm{CO} \cdot\left[\frac{1}{T_{-} E+273.15} \cdot \frac{1}{298.15}\right]\right]}}{P_{-} \mathrm{V}}$

$\mathrm{K}_{-} \mathrm{H}_{2} \mathrm{O}=\frac{\mathrm{Y}_{-} \mathrm{H} 2 \mathrm{O} \cdot \mathrm{P}_{-} \mathrm{H}_{2} \mathrm{O}}{\mathrm{P}_{-} \mathrm{V}}$ 


\section{Appendix L (Continued)}

Rules

$z_{-} N 2=\frac{\frac{\Phi_{-} \mathrm{N} 2}{\mathrm{MW} \_\mathrm{N} 2}}{\frac{\Phi_{-} \mathrm{N} 2}{\mathrm{MW} \_\mathrm{N} 2}+\frac{\Phi_{-} \mathrm{O} 2}{\mathrm{MW} \mathrm{O}_{-}}+\frac{\Phi_{-} \mathrm{Ar}}{\mathrm{MW} \_\mathrm{Ar}}+\frac{\Phi_{-} \mathrm{CO} 2}{\mathrm{MW} \_\mathrm{CO} 2}+\frac{\Phi_{-} \mathrm{H}}{\mathrm{MW} \text { Salt }}+\frac{\Phi_{-} \mathrm{H} 2 \mathrm{O}}{\mathrm{MW} \_\mathrm{H} 2 \mathrm{O}}}$

Ф_O2

$\mathrm{z}_{-} \mathrm{O} 2=\frac{\frac{\Phi_{-} \mathrm{N} 2}{\mathrm{MW} \_\mathrm{O} 2}}{\mathrm{MW}+\mathrm{N} 2}+\frac{\Phi_{-} \mathrm{O} 2}{\mathrm{MW} \_\mathrm{O} 2}+\frac{\Phi_{-} \mathrm{Ar}}{\mathrm{MW} \mathrm{Ar}_{-}}+\frac{\Phi_{-} \mathrm{CO} 2}{\mathrm{MW} \_\mathrm{CO} 2}+\frac{\Phi_{-} \mathrm{H}}{\mathrm{MW} \text { Salt }}+\frac{\Phi_{-} \mathrm{H} 2 \mathrm{O}}{\mathrm{MW} \mathrm{H} 2 \mathrm{O}}$

D_Ar

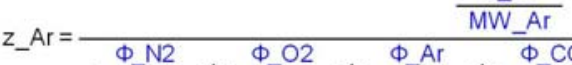

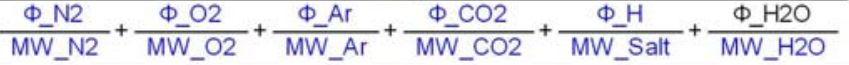

$\Phi \_\mathrm{CO} 2$

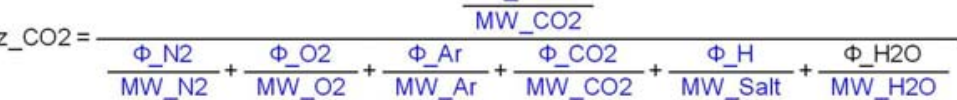

$\Phi$ H

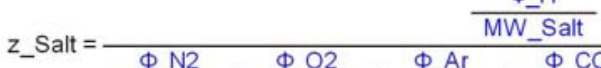

$\frac{\Phi \_\mathrm{N} 2}{\mathrm{MW} \mathrm{N}_{2}}+\frac{\Phi_{-} \mathrm{O} 2}{\mathrm{MW} \mathrm{O}_{-}}+\frac{\Phi_{-} \mathrm{Ar}}{\mathrm{MW} \mathrm{Ar}_{-}}+\frac{\Phi_{-} \mathrm{CO} 2}{\mathrm{MW} \_\mathrm{CO} 2}+\frac{\Phi_{-} \mathrm{H}}{\mathrm{MW} \text { Salt }}+\frac{\Phi_{-} \mathrm{H} 2 \mathrm{O}}{\mathrm{MW} \_\mathrm{H} 2 \mathrm{O}}$

$x_{-} \mathrm{N} 2=\frac{z_{-} N 2 \cdot N_{-} H}{N_{-} W+\text { N_E } \cdot a_{-} N 2 \cdot K_{-} N 2}$

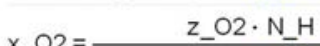

$\mathrm{x}_{-} \mathrm{O} 2=\frac{\mathrm{N}_{-} \mathrm{W}+\mathrm{N} \mathrm{E}_{-} \cdot \mathrm{a}_{-} \mathrm{O} 2 \cdot \mathrm{K} \_\mathrm{O} 2}{\mathrm{~N}}$

$x_{-} A r=\frac{z_{-} A r \cdot N_{-} H}{N_{-} W+N_{-} E \cdot a_{-} A r \cdot K_{-} A r}$

$\mathrm{Z} \_\mathrm{CO} 2 \cdot \mathrm{N} \_\mathrm{H}$

$x_{-} \mathrm{CO} 2=\frac{\mathrm{N}_{-} \mathrm{CO} \cdot \mathrm{N} \cdot \mathrm{N} \mathrm{N}_{-} \mathrm{CO} 2 \cdot \mathrm{K} \_\mathrm{CO} 2}{\mathrm{~N}}$

$x_{-}$Salt $=\frac{z_{-} \text {Salt } \cdot \text { N_H }}{N \text { - }}$

$x+\mathrm{H} 2 \mathrm{O}=\frac{\text { Z_H2O }}{\mathrm{N} \text { N } \_H}$

N_W + N_E $\cdot$ a_H $2 \mathrm{O} \cdot \mathrm{K} \_\mathrm{H} 2 \mathrm{O}$

$\mathrm{y} \_\mathrm{N} 2=\mathrm{x} \_\mathrm{N} 2 \cdot \mathrm{\alpha} \_\mathrm{N} 2 \cdot \mathrm{K} \_\mathrm{N} 2$

$\mathrm{y} \_\mathrm{O} 2=\mathrm{x} \_\mathrm{O} 2 \cdot \mathrm{a}_{-} \mathrm{O} 2 \cdot \mathrm{K} \_\mathrm{O} 2$

$\mathrm{y}_{-} \mathrm{Ar}=\mathrm{x} \_\mathrm{Ar} \cdot \mathrm{a}_{-} \mathrm{Ar} \cdot \mathrm{K} \_\mathrm{Ar}$

$\mathrm{y}_{-} \mathrm{CO} 2=\mathrm{x} \_\mathrm{CO} 2 \cdot \mathrm{a}_{-} \mathrm{CO} 2 \cdot \mathrm{K} \_\mathrm{CO} 2$

$\mathrm{y} \_\mathrm{H} 2 \mathrm{O}=\mathrm{x}_{-} \mathrm{H} 2 \mathrm{O} \cdot \mathrm{a}_{-} \mathrm{H} 2 \mathrm{O} \cdot \mathrm{K} \_\mathrm{H} 2 \mathrm{O}$

$\Phi \_\mathrm{N} 2+\Phi+\mathrm{O} 2+\Phi\left[\mathrm{Ar}+\Phi_{-} \mathrm{CO} 2+\Phi+\mathrm{H}+\Phi_{-} \mathrm{H} 2 \mathrm{O}=1\right.$

$\mathrm{z} \_\mathrm{N} 2+\mathrm{z} \_\mathrm{O} 2+\mathrm{z} \_\mathrm{Ar}+\mathrm{z} \_\mathrm{CO} 2+\mathrm{z}$ _Salt $+\mathrm{z} \_\mathrm{H} 2 \mathrm{O}=1$

x_N2 +x_O2+x_Ar +x_CO2 + x_Salt + x_H $2 \mathrm{O}=1$

$\mathrm{y} \_\mathrm{N} 2+\mathrm{y} \_\mathrm{O} 2+\mathrm{y} \_\mathrm{Ar}+\mathrm{y} \_\mathrm{CO} 2+\mathrm{y} \_\mathrm{H} 2 \mathrm{O}=1$

; Performance 
Appendix L (Continued)

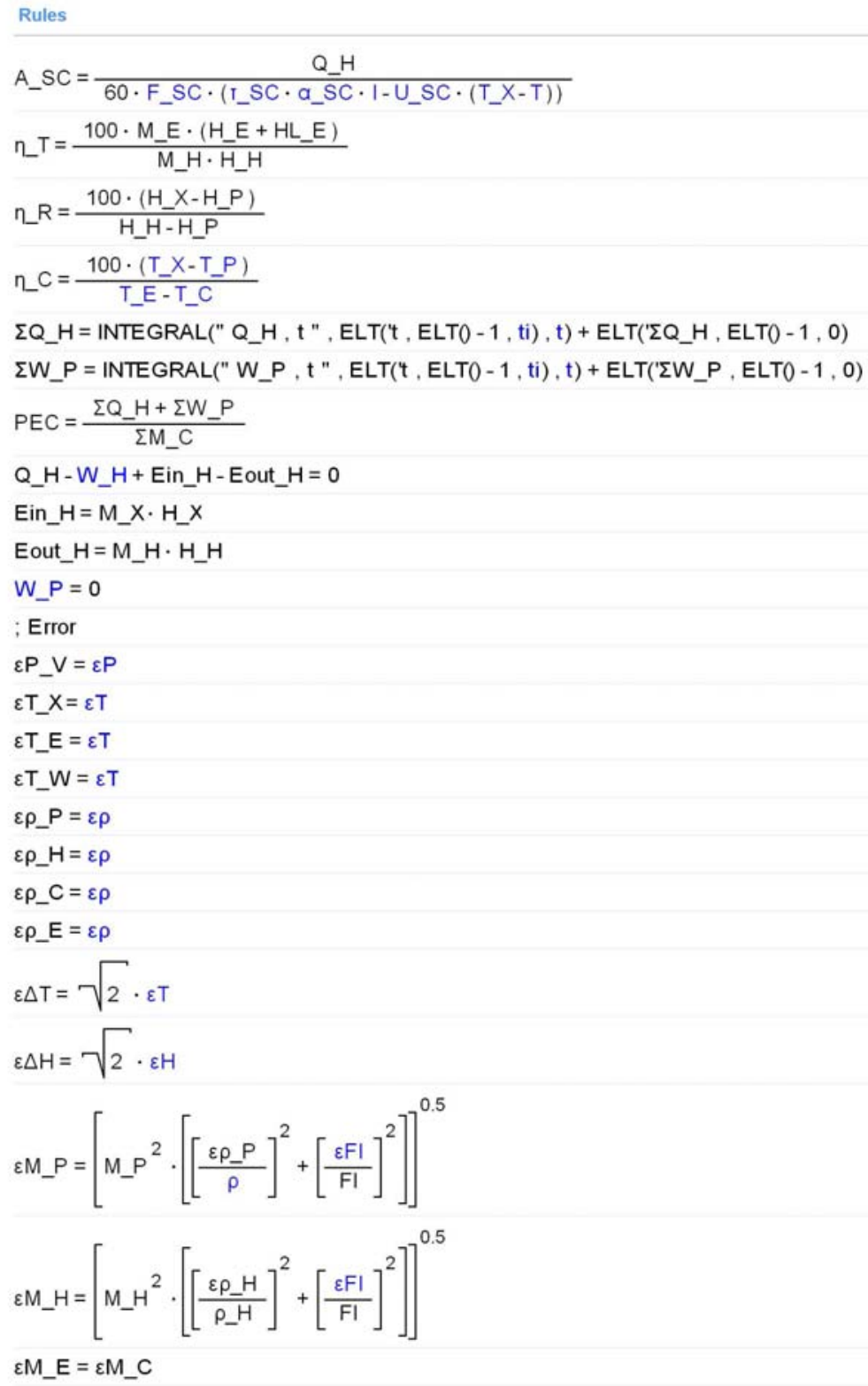


Appendix L (Continued)

Rules

$\varepsilon M_{-} C=\left[M_{-} C^{2} \cdot\left[\left[\frac{\varepsilon P_{-} C}{P_{-} C}\right]^{2}+\left[\frac{\varepsilon F I}{F I}\right]^{2}\right]\right]^{0.5}$

$\varepsilon \Sigma M_{-} C=\left[\sum M_{-} C^{2} \cdot\left[\left[\frac{\varepsilon \rho_{-} C}{\rho_{-} C}\right]^{2}+\left[\frac{\varepsilon Q E}{Q E}\right]^{2}\right]\right]^{0.5}$

$\varepsilon Q_{-} H=\left[Q_{-} H^{2} \cdot\left[\left[\frac{\varepsilon M_{-} P}{M_{-} P}\right]^{2}+\left[\frac{\varepsilon \Delta H}{H_{-} H-H_{-} X}\right]^{2}\right]\right]^{0.5}$

$\varepsilon \Sigma Q_{-} H=\varepsilon Q_{-} H$

$\varepsilon A_{-} S C=\left[A_{-} S C^{2} \cdot\left[\left[\frac{\varepsilon Q \_H}{Q_{-} H}\right]^{2}+\left[\frac{U_{-} S C \cdot \varepsilon T_{-} X}{I_{-} S C \cdot a_{-} S C \cdot 1-U_{-} S C \cdot\left(T_{-} X-T\right)}\right]^{2}\right]\right]^{0.5}$

$\varepsilon P E C=\left[P E C^{2} \cdot\left[\left[\frac{\varepsilon \Sigma Q_{2} H}{\Sigma Q_{-} H}\right]^{2}+\left[\frac{\varepsilon \Sigma M_{-} C}{\Sigma M_{-} C}\right]^{2}\right]\right]^{0.5}$

$\varepsilon \eta \_=100 \cdot\left[\left[\frac{\eta \subset C}{100}\right]^{2} \cdot\left[\left[\frac{\varepsilon \Delta T}{T_{-} X-T_{-} P}\right]^{2}+\left[\frac{\varepsilon \Delta T}{T_{-} E-T_{-} C}\right]^{2}\right]\right]^{0.5}$

$\varepsilon \eta_{-} R=100 \cdot\left[\left[\frac{\eta \_R}{100}\right]^{2} \cdot\left[\left[\frac{\varepsilon \Delta H}{H_{-} X-H_{-} P}\right]^{2}+\left[\frac{\varepsilon \Delta H}{H_{-} H-H \_P}\right]^{2}\right]\right]^{0.5}$

$\varepsilon \eta_{-} T=100 \cdot\left[\left[\frac{\eta \_T}{100}\right]^{2} \cdot\left[\left[\frac{\varepsilon M_{-} E}{M_{-} E}\right]^{2}+\left[\frac{\varepsilon H}{H_{-} E}\right]^{2}+\left[\frac{\varepsilon M_{-} H}{M_{-} H}\right]^{2}+\left[\frac{\varepsilon H}{H \_H}\right]^{2}\right]\right]^{0.5}$ 


\section{Appendix L (Continued)}

\begin{tabular}{|c|c|c|c|c|}
\hline \multicolumn{5}{|l|}{ Variables Sheet } \\
\hline Input & Name & Output & Unit & Comment \\
\hline 0 & $\mathrm{ti}$ & & $\min$ & Initial Time \\
\hline 1 & $\mathrm{t}$ & & $\min$ & Run Time \\
\hline 83.14472 & $\mathbf{R}$ & & $\begin{array}{l}\left(\mathrm{bar}-\mathrm{cm}^{\wedge} 3\right) /( \\
\left.\mathrm{mol}-{ }^{\circ} \mathrm{C}\right)\end{array}$ & Universal Gas Constant \\
\hline 980.0665 & $\mathrm{~g}$ & & $\mathrm{~cm} / \mathrm{s}^{\wedge} 2$ & Gravity Acceleration \\
\hline \multirow[t]{9}{*}{310922.658770} & Vi_V & & $\mathrm{cm}^{\wedge} 3$ & Initial Total Vacuum Volume \\
\hline & V_v & 310418.662453 & $\mathrm{~cm}^{\wedge} 3$ & Total Vacuum Volume \\
\hline & ENai_C & 1.785895 & moles & Initial NCG Molar Amount \\
\hline & $\Sigma \mathrm{Na} \_\mathrm{C}$ & 1.786259 & $\mathrm{~mol}$ & NCG Amount \\
\hline & $\mathrm{Na} \_\mathrm{C}$ & 0.000364 & $\mathrm{~mol} / \mathrm{min}$ & NCG Molar Accumulation Rate \\
\hline & Ma_C & 0.010799 & $\mathrm{~g} / \mathrm{min}$ & NCG Molar Accumulation Rate \\
\hline & $\Sigma M$ & 27.653921 & g & Actual Product Amount \\
\hline & $\Sigma M_{-} C$ & 5.071088 & g & Calculated Product Amount \\
\hline & $\mathrm{FI}$ & 0.503996 & $\mathrm{~L} / \mathrm{min}$ & Average Flow Indicator Value \\
\hline 27 & QE & & $\mathrm{cm}^{\wedge} 3$ & Average Quantitative Element Value \\
\hline 0.546461 & CV & & & Ball Valve Flow Coefficient \\
\hline 20 & $T$ & & ${ }^{\circ} \mathrm{C}$ & Ambient Temperature \\
\hline 1.01325 & $P$ & & bar & Ambient Pressure \\
\hline \multirow[t]{4}{*}{1.024219} & $\rho$ & & $\mathrm{g} / \mathrm{cm}^{\wedge} 3$ & Stream Density \\
\hline & k_X & 0.005988 & $\mathrm{~W} /\left(\mathrm{cm}-{ }^{\circ} \mathrm{C}\right)$ & Stream Thermal Conductivity \\
\hline & k_W & 0.005990 & $\mathrm{~W} /\left(\mathrm{cm}-{ }^{\circ} \mathrm{C}\right)$ & Stream Thermal Conductivity \\
\hline & k_C & 0.006043 & $\mathrm{~W} /\left(\mathrm{cm}-{ }^{\circ} \mathrm{C}\right)$ & Stream Thermal Conductivity \\
\hline .035 & $\Phi+P$ & & & Stream Salt Mass Fraction \\
\hline .035 & $\Phi \_x$ & & & Stream Salt Mass Fraction \\
\hline \multirow[t]{2}{*}{.035} & $\Phi \_H$ & & & Stream Salt Mass Fraction \\
\hline & Ф_W & 0.035348 & & Stream Salt Mass Fraction \\
\hline 0 & $\Phi \_C$ & & & Stream Salt Mass Fraction \\
\hline 0 & Ф_E & & & Stream Salt Mass Fraction \\
\hline 20 & T_P & & ${ }^{\circ} \mathrm{C}$ & Stream Temperature \\
\hline 21.022222 & $T_{-} \mathrm{x}$ & & ${ }^{\circ} \mathrm{C}$ & Stream Temperature \\
\hline 43.078889 & T_H & & ${ }^{\circ} \mathrm{C}$ & Stream Temperature \\
\hline 24.241111 & T_E & & ${ }^{\circ} \mathrm{C}$ & Stream Temperature \\
\hline
\end{tabular}




\section{Appendix L (Continued)}

\begin{tabular}{|c|c|c|c|c|}
\hline Input & Name & Output & Unit & Comment \\
\hline 21.240556 & T_W & & ${ }^{\circ} \mathrm{C}$ & Stream Temperature \\
\hline 20.850556 & T_C & & ${ }^{\circ} \mathrm{C}$ & Stream Temperature \\
\hline & $\mathrm{Pi} \_\mathrm{H} 2 \mathrm{O}$ & 0.022722 & bar & Initial Vapor Pressure \\
\hline & P_H2O & 0.029477 & bar & Vapor Pressure \\
\hline .14 & PI_V & & bar & Initial Vacuum Pressure \\
\hline 0.140585 & P_V & & bar & Vacuum Pressure \\
\hline 35 & D_E & & $\mathrm{cm}$ & Evaporator Nominal Diameter \\
\hline 160 & L_E & & $\mathrm{cm}$ & Evaporator Length \\
\hline 35 & $D_{-} C$ & & $\mathrm{~cm}$ & Condenser Nominal Diameter \\
\hline 160 & L_C & & $\mathrm{cm}$ & Condenser Length \\
\hline 26.092 & A_N2 & & & Nitrogen Molar Enthalpy Parameter \\
\hline 8.218801 & B_N2 & & & Nitrogen Molar Enthalpy Parameter \\
\hline-1.976141 & C_N2 & & & Nitrogen Molar Enthalpy Parameter \\
\hline .159274 & D_N2 & & & Nitrogen Molar Enthalpy Parameter \\
\hline .044434 & E_N2 & & & Nitrogen Molar Enthalpy Parameter \\
\hline-7.98923 & F_N2 & & & Nitrogen Molar Enthalpy Parameter \\
\hline 29.659 & A_O2 & & & Oxygen Molar Enthalpy Parameter \\
\hline 6.137261 & B_O2 & & & Oxygen Molar Enthalpy Parameter \\
\hline-1.186521 & C_O2 & & & Oxygen Molar Enthalpy Parameter \\
\hline .09578 & D_O2 & & & Oxygen Molar Enthalpy Parameter \\
\hline-.219663 & E_O2 & & & Oxygen Molar Enthalpy Parameter \\
\hline-9.861391 & F_O2 & & & Oxygen Molar Enthalpy Parameter \\
\hline 20.786 & A_Ar & & & Argon Molar Enthalpy Parameter \\
\hline 0.000000 & B_Ar & & & Argon Molar Enthalpy Parameter \\
\hline 0.000000 & C_Ar & & & Argon Molar Enthalpy Parameter \\
\hline 0.000000 & D_Ar & & & Argon Molar Enthalpy Parameter \\
\hline 0.000000 & E_Ar & & & Argon Molar Enthalpy Parameter \\
\hline-6.19735 & F_Ar & & & Argon Molar Enthalpy Parameter \\
\hline 24.99735 & A_CO2 & & & Carbon Dioxide Molar Enthalpy Parameter \\
\hline 55.18696 & B_CO2 & & & Carbon Dioxide Molar Enthalpy Parameter \\
\hline-33.69137 & C_CO2 & & & Carbon Dioxide Molar Enthalpy Parameter \\
\hline 7.948387 & D_CO2 & & & Carbon Dioxide Molar Enthalpy Parameter \\
\hline-136638 & E_CO2 & & & Carbon Dioxide Molar Enthalpy Parameter \\
\hline-10.0851 & $\mathrm{~F}_{-} \mathrm{CO} 2$ & & & Carbon Dioxide Molar Enthalpy Parameter \\
\hline 30.092 & A_H2O & & & Water Molar Enthalpy Parameter \\
\hline
\end{tabular}




\section{Appendix L (Continued)}

\begin{tabular}{|c|c|c|c|c|}
\hline Input & Name & Output & Unit & Comment \\
\hline 6.832514 & B_H2O & & & Water Molar Enthalpy Parameter \\
\hline 6.793435 & $\mathrm{C} \_\mathrm{H} 2 \mathrm{O}$ & & & Water Molar Enthalpy Parameter \\
\hline-2.53448 & D_H2O & & & Water Molar Enthalpy Parameter \\
\hline .082139 & E_H2O & & & Water Molar Enthalpy Parameter \\
\hline \multirow[t]{14}{*}{-9.054600} & $\mathrm{~F} \_\mathrm{H} 2 \mathrm{O}$ & & & Water Molar Enthalpy Parameter \\
\hline & H_N2 & -32.686204 & $\mathrm{~J} / \mathrm{mol}$ & Nitrogen Molar Enthalpy \\
\hline & $\mathrm{H} \_\mathrm{O} 2$ & -41.255674 & $\mathrm{~J} / \mathrm{mol}$ & Oxygen Molar Enthalpy \\
\hline & H_Ar & -15.778230 & $\mathrm{~J} / \mathrm{mol}$ & Argon Molar Enthalpy \\
\hline & H_CO2 & -31.084062 & $\mathrm{~J} / \mathrm{mol}$ & Carbon Dioxide Molar Enthalpy \\
\hline & $\mathrm{H} \_\mathrm{H} 2 \mathrm{O}$ & -24.962916 & $\mathrm{~J} / \mathrm{mol}$ & Water Molar Enthalpy \\
\hline & H_P & 79.740379 & $\mathrm{~J} / \mathrm{g}$ & Stream Enthalpy \\
\hline & H_X & 83.823279 & $\mathrm{~J} / \mathrm{g}$ & Stream Enthalpy \\
\hline & H_H & 172.043899 & $\mathrm{~J} / \mathrm{g}$ & Stream Enthalpy \\
\hline & H_W & 84.655639 & $\mathrm{~J} / \mathrm{g}$ & Stream Enthalpy \\
\hline & H_E & 101.548881 & $\mathrm{~J} / \mathrm{g}$ & Stream Enthalpy \\
\hline & HL_E & 2446.400305 & $\mathrm{~J} / \mathrm{g}$ & Stream Enthalpy of Vaporization \\
\hline & H_C & 87.369790 & $\mathrm{~J} / \mathrm{g}$ & Stream Enthalpy \\
\hline & $\mathrm{Ha} \_\mathrm{C}$ & -1.192302 & $\mathrm{~J} / \mathrm{g}$ & NCG Enthalpy \\
\hline & MW_H & 18.287997 & $\mathrm{~g} / \mathrm{mol}$ & Stream Molecular Weight \\
\hline & MW_W & 18.298045 & $\mathrm{~g} / \mathrm{mol}$ & Stream Molecular Weight \\
\hline & MW_E & 18.018971 & $\mathrm{~g} / \mathrm{mol}$ & Stream Molecular Weight \\
\hline & $M W \_C$ & 18.0148 & $\mathrm{~g} / \mathrm{mol}$ & Stream Molecular Weight \\
\hline & MWa_C & 29.655264 & $\mathrm{~g} / \mathrm{mol}$ & Stream Molecular Weight \\
\hline & N_H & 28.226315 & $\mathrm{~mol} / \mathrm{min}$ & Stream Molar Flow Rate \\
\hline & N_W & 27.210002 & $\mathrm{~mol} / \mathrm{min}$ & Stream Molar Flow Rate \\
\hline & N_E & 1.016312 & $\mathrm{~mol} / \mathrm{min}$ & Stream Molar Flow Rate \\
\hline & N_C & 1.015948 & $\mathrm{~mol} / \mathrm{min}$ & Stream Molar Flow Rate \\
\hline & M_P & 516.202756 & $\mathrm{~g} / \mathrm{min}$ & Stream Mass Flow Rate \\
\hline & M_X & 516.202756 & $\mathrm{~g} / \mathrm{min}$ & Stream Mass Flow Rate \\
\hline & M_H & 516.202756 & $\mathrm{~g} / \mathrm{min}$ & Stream Mass Flow Rate \\
\hline & M_W & 511.120869 & $\mathrm{~g} / \mathrm{min}$ & Stream Mass Flow Rate \\
\hline & M_E & 5.081887 & $\mathrm{~g} / \mathrm{min}$ & Stream Mass Flow Rate \\
\hline & M_C & 5.071088 & $\mathrm{~g} / \mathrm{min}$ & Stream Mass Flow Rate \\
\hline & Ein_E & 88809.534752 & $\mathrm{~J} / \mathrm{min}$ & Evaporator Energy Input \\
\hline & Eout_E & 56217.653939 & $\mathrm{~J} / \mathrm{min}$ & Evaporator Energy Output \\
\hline 0 & Q_E & & $\mathrm{J} / \mathrm{min}$ & Evaporator Heat Input \\
\hline
\end{tabular}




\section{Appendix L (Continued)}

\begin{tabular}{|c|c|c|c|c|}
\hline Input & Name & Output & Unit & Comment \\
\hline \multirow[t]{5}{*}{0} & W_E & & $\mathrm{J} / \mathrm{min}$ & Evaporator Work Output \\
\hline & Ea_C & -0.012876 & $\mathrm{~J} / \mathrm{min}$ & NCG Energy Accumulation \\
\hline & Ein_C & 54110.593734 & $\mathrm{~J} / \mathrm{min}$ & Condenser Energy Input \\
\hline & Eout_C & 43712.867337 & $\mathrm{~J} / \mathrm{min}$ & Condenser Energy Output \\
\hline & $Q_{-} C$ & -10397.739273 & $\mathrm{~J} / \mathrm{min}$ & Condenser Heat Input \\
\hline 0 & W_C & & $\mathrm{J} / \mathrm{min}$ & Condenser Work Output \\
\hline 0.000013 & Ф_N2 & & & Nitrogen Mass Fraction in Ambient Seawater \\
\hline 0.000008 & $\Phi \_\mathrm{O} 2$ & & & Oxygen Mass Fraction in Ambient Seawater \\
\hline 0.000000 & $\Phi \_$Ar & & & Argon Mass Fraction in Ambient Seawater \\
\hline \multirow[t]{19}{*}{0.000000} & $\Phi_{-} \mathrm{CO} 2$ & & & Carbon Dioxide Mass Fraction in Ambient Seawater \\
\hline & $\Phi \_\mathrm{H} 2 \mathrm{O}$ & 0.964979 & & Water Mass Fraction in Ambient Seawater \\
\hline & Z_N2 & 0.000008 & & Nitrogen Mole Fraction in Ambient Seawater \\
\hline & z_O2 & 0.000004 & & Oxygen Mole Fraction in Ambient Seawater \\
\hline & z_Ar & 0.000000 & & Argon Mole Fraction in Ambient Seawater \\
\hline & $z_{-} \mathrm{CO} 2$ & 0.000000 & & Carbon Dioxide Mole Fraction in Ambient Seawater \\
\hline & z_Salt & 0.020374 & & Salt Mole Fraction in Ambient Seawater \\
\hline & $\mathrm{z}-\mathrm{H} 2 \mathrm{O}$ & 0.979613 & & Water Mole Fraction in Ambient Seawater \\
\hline & $x \_$N2 & 0.000000 & & Nitrogen Mole Fraction in Brine Water \\
\hline & x_O2 & 0.000000 & & Oxygen Mole Fraction in Brine Water \\
\hline & $x \_$Ar & 0.000000 & & Argon Mole Fraction in Brine Water \\
\hline & $x_{-} \mathrm{CO} 2$ & 0.000000 & & Carbon Dioxide Mole Fraction in Brine Water \\
\hline & x_Salt & 0.021135 & & Salt Mole Fraction in Brine Water \\
\hline & $x_{-} \mathrm{H} 2 \mathrm{O}$ & 0.978865 & & Water Mole Fraction in Brine Water \\
\hline & y_N2 & 0.000228 & & Nitrogen Mole Fraction in Vaporized Water \\
\hline & y_O2 & 0.000122 & & Oxygen Mole Fraction in Vaporized Water \\
\hline & y_Ar & 0.000005 & & Argon Mole Fraction in Vaporized Water \\
\hline & y_CO2 & 0.000003 & & Carbon Dioxide Mole Fraction in Vaporized Water \\
\hline & $\mathrm{Y} \_\mathrm{H} 2 \mathrm{O}$ & 0.999642 & & Water Mole Fraction in Vaporized Water \\
\hline 28.0134 & MW_N2 & & $\mathrm{g} / \mathrm{mol}$ & Nitrogen Molecular Weight \\
\hline 31.998 & MW_O2 & & $\mathrm{g} / \mathrm{mol}$ & Oxygen Molecular Weight \\
\hline 39.94 & MW_Ar & & $\mathrm{g} / \mathrm{mol}$ & Argon Molecular Weight \\
\hline 44.009 & MW_CO2 & & $\mathrm{g} / \mathrm{mol}$ & Carbon Dioxide Molecular Weight \\
\hline 31.416538 & MW_Salt & & $\mathrm{g} / \mathrm{mol}$ & Approximated Seawater Salt Molecular Weight \\
\hline 18.0148 & $\mathrm{MW} \_\mathrm{H} 2 \mathrm{O}$ & & $\mathrm{g} / \mathrm{mol}$ & Water Molecular Weight \\
\hline 8067573.136086 & HC_N2 & & bar & Nitrogen Reference State Henry's Constant \\
\hline
\end{tabular}




\section{Appendix L (Continued)}

\begin{tabular}{|c|c|c|c|c|}
\hline Input & Name & Output & Unit & Comment \\
\hline 358814.929481 & HC_O2 & & bar & Oxygen Reference State Henry's Constant \\
\hline 384073.329688 & HC_Ar & & bar & Argon Reference State Henry's Constant \\
\hline 10914.952753 & HC_CO2 & & bar & Carbon Dioxide Reference State Henry's Constant \\
\hline-3545.567207 & HF_N2 & & ${ }^{\circ} \mathrm{C}$ & Nitrogen Henry's Coefficient \\
\hline-2208.812178 & HF_O2 & & ${ }^{\circ} \mathrm{C}$ & Oxygen Henry's Coefficient \\
\hline-2308.046425 & HF_Ar & & ${ }^{\circ} \mathrm{C}$ & Argon Henry's Coefficient \\
\hline-445.190581 & HF_CO2 & & ${ }^{\circ} \mathrm{C}$ & Carbon Dioxide Henry's Coefficient \\
\hline 12.762946 & PA & & & Water Vapor Pressure Coefficient \\
\hline 4391.129422 & PB & & ${ }^{\circ} \mathrm{C}$ & Water Vapor Pressure Coefficient \\
\hline 245.367016 & PC & & ${ }^{\circ} \mathrm{C}$ & Water Vapor Pressure Coefficient \\
\hline 1.21 & a_N2 & & & Nitrogen Relative Solubility \\
\hline 1.22 & a_O2 & & & Oxygen Relative Solubility \\
\hline 1.23 & a_Ar & & & Argon Relative Solubility \\
\hline 1.17 & a_CO2 & & & Carbon Dioxide Relative Solubility \\
\hline \multirow[t]{19}{*}{.9816} & a_H2O & & & Water Relative Vapor Pressure \\
\hline & K_N2 & 59153990.173289 & & $\begin{array}{l}\text { Evaporator-Based Nitrogen VLE Distribution } \\
\text { Coefficient }\end{array}$ \\
\hline & K_O2 & 2601014.888961 & & $\begin{array}{l}\text { Evaporator-Based Oxygen VLE Distribution } \\
\text { Coefficient }\end{array}$ \\
\hline & K_Ar & 2786476.236923 & & $\begin{array}{l}\text { Evaporator-Based Argon VLE Distribution } \\
\text { Coefficient }\end{array}$ \\
\hline & K_CO2 & 77936.106242 & & $\begin{array}{l}\text { Evaporator-Based Carbon Dioxide VLE Distribution } \\
\text { Coefficient }\end{array}$ \\
\hline & K_H2O & 1.040368 & & $\begin{array}{l}\text { Evaporator-Based Water VLE Distribution } \\
\text { Coefficient }\end{array}$ \\
\hline & PA_X & 0.765685 & & Stream Density Coefficient \\
\hline & PA_H & 0.742783 & & Stream Density Coefficient \\
\hline & PA_W & 0.765301 & & Stream Density Coefficient \\
\hline & PA_C & 0.765990 & & Stream Density Coefficient \\
\hline & $p B \_x$ & -0.004306 & & Stream Density Coefficient \\
\hline & $\rho B_{-} H$ & -0.004390 & & Stream Density Coefficient \\
\hline & $\rho B \_W$ & -0.004299 & & Stream Density Coefficient \\
\hline & $\rho B_{-} C$ & -0.004312 & & Stream Density Coefficient \\
\hline & $\rho C_{-} x$ & 997.990004 & & Stream Density Coefficient \\
\hline & $\rho C \_H$ & 991.010632 & & Stream Density Coefficient \\
\hline & $\rho C \_W$ & 997.942402 & & Stream Density Coefficient \\
\hline & $\rho C \_c$ & 998.027088 & & Stream Density Coefficient \\
\hline & $\rho_{-} X$ & 1.024489 & $\mathrm{~g} / \mathrm{cm}^{\wedge} 3$ & Stream Density \\
\hline
\end{tabular}




\section{Appendix L (Continued)}

\begin{tabular}{|c|c|c|c|c|}
\hline Input & Name & Output & Unit & Comment \\
\hline & P_H & 1.016691 & $\mathrm{~g} / \mathrm{cm}^{\wedge} 3$ & Stream Density \\
\hline & P_W & 1.024694 & $\mathrm{~g} / \mathrm{cm}^{\wedge} 3$ & Stream Density \\
\hline & P_C & 0.998027 & $\mathrm{~g} / \mathrm{cm}^{\wedge} 3$ & Stream Density \\
\hline & $\mu A_{-} X$ & 0.001197 & & Stream Viscosity Coefficient \\
\hline & $\mu A \_W$ & 0.001208 & & Stream Viscosity Coefficient \\
\hline & $\mu A \_C$ & 0.001188 & & Stream Viscosity Coefficient \\
\hline & $\mu B_{-} X$ & 0.003285 & & Stream Viscosity Coefficient \\
\hline & $\mu B \_W$ & 0.003292 & & Stream Viscosity Coefficient \\
\hline & $\mu \mathrm{B} \_\mathrm{C}$ & 0.003279 & & Stream Viscosity Coefficient \\
\hline & $\mu C_{-} x$ & 19.848398 & & Stream Viscosity Coefficient \\
\hline & $\mu C_{-} W$ & 20.049759 & & Stream Viscosity Coefficient \\
\hline & $\mu C_{-} \mathrm{C}$ & 0 & & Stream Viscosity Coefficient \\
\hline & $\mu D_{-} X$ & 0.009774 & & Stream Viscosity Coefficient \\
\hline & $\mu D_{-} W$ & 0.009722 & & Stream Viscosity Coefficient \\
\hline & $\mu D_{-} C$ & 0.009814 & & Stream Viscosity Coefficient \\
\hline & $\mu \_x$ & 0.010463 & $P$ & Stream Viscosity \\
\hline & H_W & 0.010417 & $P$ & Stream Viscosity \\
\hline & $\mu \_C$ & 0.009814 & $P$ & Stream Viscosity \\
\hline 1.27 & D_CT & & $\mathrm{cm}$ & Condenser Tube Diameter \\
\hline 475 & L_CT & & $\mathrm{cm}$ & Condenser Tube Length \\
\hline .2 & hid_CT & & $W /\left(\mathrm{cm}^{\wedge} 2-{ }^{\circ} \mathrm{C}\right)$ & Condenser Tube Inside Dirt Coefficient \\
\hline \multirow[t]{3}{*}{.5} & hod_CT & & $\mathrm{W} /\left(\mathrm{cm}^{\wedge} 2-{ }^{\circ} \mathrm{C}\right)$ & Condenser Tube Outside Dirt Coefficient \\
\hline & hi_CT & 0.051020 & $\mathrm{~W} /\left(\mathrm{cm}^{\wedge} 2-{ }^{\circ} \mathrm{C}\right)$ & Condenser Tube Inside Fluid Film Coefficient \\
\hline & ho_CT & 0.943999 & $\mathrm{~W} /\left(\mathrm{cm}^{\wedge} 2-{ }^{\circ} \mathrm{C}\right)$ & Condenser Tube Outside Fluid Film Coefficient \\
\hline .5 & hid_E & & $\mathrm{W} /\left(\mathrm{cm}^{\wedge} 2-{ }^{\circ} \mathrm{C}\right)$ & Evaporator Inside Dirt Coefficient \\
\hline \multirow[t]{3}{*}{.75} & hod_E & & $\mathrm{W} /\left(\mathrm{cm}^{\wedge} 2-{ }^{\circ} \mathrm{C}\right)$ & Evaporator Outside Dirt Coefficient \\
\hline & hi_E & 0.594382 & $\mathrm{~W} /\left(\mathrm{cm}^{\wedge} 2-{ }^{\circ} \mathrm{C}\right)$ & Evaporator Inside Fluid Film Coefficient \\
\hline & ho_E & 0.018067 & $\mathrm{~W} /\left(\mathrm{cm}^{\wedge} 2-{ }^{\circ} \mathrm{C}\right)$ & Evaporator Outside Fluid Film Coefficient \\
\hline .5 & hid_C & & $\mathrm{W} /\left(\mathrm{cm}^{\wedge} 2-{ }^{\circ} \mathrm{C}\right)$ & Condenser Inside Dirt Coefficient \\
\hline \multirow[t]{3}{*}{.75} & hod_C & & $\mathrm{W} /\left(\mathrm{cm}^{\wedge} 2-{ }^{\circ} \mathrm{C}\right)$ & Condenser Outside Dirt Coefficient \\
\hline & hi_c & 0.599330 & $\mathrm{~W} /\left(\mathrm{cm}^{\wedge} 2-{ }^{\circ} \mathrm{C}\right)$ & Condenser Inside Fluid Film Coefficient \\
\hline & ho_C & 0.018067 & $\mathrm{~W} /\left(\mathrm{cm}^{\wedge} 2-{ }^{\circ} \mathrm{C}\right)$ & Condenser Outside Fluid Film Coefficient \\
\hline 4 & N_CT & & & Number of Condenser Tube Vertical Rows \\
\hline
\end{tabular}




\section{Appendix L (Continued)}

\begin{tabular}{|c|c|c|c|c|}
\hline Input & Name & Output & Unit & Comment \\
\hline .125 & ס_CT & & $\mathrm{cm}$ & Condenser Tube Thickness \\
\hline 3.81 & kw_CT & & $\mathrm{W} /\left(\mathrm{cm}-{ }^{\circ} \mathrm{C}\right)$ & Condenser Tube Thermal Conductivity \\
\hline .25 & $\delta_{-} c$ & & $\mathrm{~cm}$ & Condenser Thickness \\
\hline .45 & kw_C & & $\mathrm{W} /\left(\mathrm{cm}-{ }^{\circ} \mathrm{C}\right)$ & Condenser Thermal Conductivity \\
\hline .25 & ס_E & & $\mathrm{cm}$ & Evaporator Thickness \\
\hline \multirow[t]{17}{*}{.001} & kw_E & & $\mathrm{W} /\left(\mathrm{cm}-{ }^{\circ} \mathrm{C}\right)$ & Evaporator Thermal Conductivity \\
\hline & U_CT & 0.033225 & $\mathrm{~W} /\left(\mathrm{cm}^{\wedge} 2-{ }^{\circ} \mathrm{C}\right)$ & Condenser Tube Overall Heat Transfer Coefficient \\
\hline & A_CT & 1895.165768 & $\mathrm{~cm}^{\wedge} 2$ & Condenser Tube Surface Area \\
\hline & $\Delta \mathrm{Tm} \_\mathrm{CT}$ & 3.706537 & ${ }^{\circ} \mathrm{C}$ & $\begin{array}{l}\text { Condenser Tube Logarithmic Mean Temperature } \\
\text { Difference }\end{array}$ \\
\hline & U_E & 0.005381 & $\mathrm{~W} /\left(\mathrm{cm}^{\wedge} 2-{ }^{\circ} \mathrm{C}\right)$ & Evaporator Overall Heat Transfer Coefficient \\
\hline & A_E & 17592.918860 & $\mathrm{~cm}^{\wedge} 2$ & Evaporator Surface Area \\
\hline & $\Delta T m_{-} E$ & 3.585132 & ${ }^{\circ} \mathrm{C}$ & $\begin{array}{l}\text { Evaporator Logarithmic Mean Temperature } \\
\text { Difference }\end{array}$ \\
\hline & U_C & 0.016486 & $\mathrm{~W} /\left(\mathrm{cm}^{\wedge} 2-{ }^{\circ} \mathrm{C}\right)$ & Condenser Overall Heat Transfer Coefficient \\
\hline & A_C & 17592.918860 & $\mathrm{~cm}^{\wedge} 2$ & Condenser Surface Area \\
\hline & $\Delta T m_{-} C$ & 3.799981 & ${ }^{\circ} \mathrm{C}$ & $\begin{array}{l}\text { Condenser Logarithmic Mean Temperature } \\
\text { Difference }\end{array}$ \\
\hline & $\mathrm{Y} \_\mathrm{H} 2 \mathrm{O}$ & 4.961777 & & Evaporator-Based Water Activity Coefficient \\
\hline & $\psi$ & 0.940570 & & Fraction of NCG Molecules Accumulating \\
\hline & F_CT & 0.150507 & & $\begin{array}{l}\text { Condenser Tube Counter Current Departure } \\
\text { Correction Factor }\end{array}$ \\
\hline & F_E & 0 & & $\begin{array}{l}\text { Evaporator Counter Current Departure Correction } \\
\text { Factor }\end{array}$ \\
\hline & $F_{-} C$ & 0.157232 & & $\begin{array}{l}\text { Condenser Counter Current Departure Correction } \\
\text { Factor }\end{array}$ \\
\hline & $\mu_{-} E$ & 0.000090 & $P$ & Stream Viscosity \\
\hline & P_E & 0.000022 & $\mathrm{~g} / \mathrm{cm}^{\wedge} 3$ & Stream Density \\
\hline 5.08 & PD_E & & $\mathrm{cm}$ & Stream Nominal Pipe Diameter \\
\hline 180 & PL_E & & $\mathrm{cm}$ & Stream Equivalent Pipe Length \\
\hline \multirow[t]{3}{*}{962.112750} & XA_E & & $\mathrm{cm}^{\wedge} 2$ & Evaporator Cross Sectional Area \\
\hline & $\Delta P_{-} E$ & 0.000004 & bar & Stream Pressure Drop \\
\hline & SG_H & 1.025913 & & Stream Specific Gravity \\
\hline .118 & $\Omega$ & & bar & Steam Diffusion Resistance \\
\hline \multirow[t]{4}{*}{2} & $\sigma$ & & $\begin{array}{l}\left(\mathrm{g}-{ }^{\circ} \mathrm{C}{ }^{\wedge} 0.5\right) /(\text { bar } \\
\left.-\min -\mathrm{cm}^{\wedge} 2\right)\end{array}$ & Steam Diffusion Coefficient \\
\hline & Md_E & -13.231018 & $g / \min$ & Steam Diffusion Rate \\
\hline & EMd_E & -13.231018 & $\mathrm{~g}$ & Total Steam Diffused \\
\hline & Ed_E & 32591.880813 & $\mathrm{~J} / \mathrm{min}$ & Steam Diffusion Energy \\
\hline \multirow[t]{3}{*}{0} & W_H & & $\mathrm{J} / \mathrm{min}$ & Heater Work Output \\
\hline & Q_H & 45539.727333 & $\mathrm{~J} / \mathrm{min}$ & Heater Heat Input \\
\hline & Ein_H & 43269.807420 & $\mathrm{~J} / \mathrm{min}$ & Heater Energy Input \\
\hline
\end{tabular}




\section{Appendix L (Continued)}

\begin{tabular}{|c|c|c|c|c|}
\hline Input & Name & Output & Unit & Comment \\
\hline & Eout_H & 88809.534752 & $\mathrm{~J} / \mathrm{min}$ & Heater Energy Output \\
\hline \multirow[t]{7}{*}{0} & W_P & & $\mathrm{J} / \mathrm{min}$ & Pump Work Output \\
\hline & $\Sigma W \_P$ & 0 & J & Power Input \\
\hline & $\Sigma Q_{-} H$ & 45539.727333 & $\mathrm{~J}$ & Heat Input \\
\hline & PEC & 8980.267181 & $\mathrm{~J} / \mathrm{g}$ & Prime Energy Consumption \\
\hline & $\eta R$ & 4.423341 & $\%$ & Recovery Efficiency \\
\hline & $\eta T$ & 14.579955 & $\%$ & Thermal Efficiency \\
\hline & $\eta_{L} \mathrm{C}$ & 30.149107 & $\%$ & Condenser Efficiency \\
\hline .92 & a_sc & & & Solar Collector Absorptance \\
\hline 9 & I_SC & & & Solar Collector Transmittance \\
\hline .000092 & U_SC & & $\mathrm{W} /\left(\mathrm{cm}^{\wedge} 2-{ }^{\circ} \mathrm{C}\right)$ & Solar Collector Heat Loss Conductance \\
\hline .82 & F_sC & & & Solar Collector Heat Removal Factor \\
\hline \multirow[t]{2}{*}{.06} & 1 & & $\mathrm{~W} / \mathrm{cm}^{\wedge} 2$ & Incident Insolation On Solar Collector \\
\hline & A_SC & 18666.660826 & $\mathrm{~cm}^{\wedge} 2$ & Solar Collector Area \\
\hline .005 & $\varepsilon P$ & & bar & Pressure Transducer Error \\
\hline 1 & $\varepsilon T$ & & ${ }^{\circ} \mathrm{C}$ & Thermocouple Error \\
\hline .045 & $\varepsilon \mathrm{FI}$ & & $\mathrm{L} / \mathrm{min}$ & Flow Indicator Error \\
\hline .2 & $\varepsilon Q E$ & & $\mathrm{~cm}^{\wedge} 3$ & Quantitative Element Error \\
\hline .000035 & $\varepsilon \rho$ & & $\mathrm{g} / \mathrm{cm}^{\wedge} 3$ & Density Correlation Error \\
\hline \multirow[t]{22}{*}{.045} & $\varepsilon \mathrm{H}$ & & $\mathrm{J} / \mathrm{g}$ & Enthalpy Correlation Error \\
\hline & $\varepsilon P_{-} V$ & .005 & bar & Vacuum Pressure Error \\
\hline & $\varepsilon T_{-} E$ & 1 & ${ }^{\circ} \mathrm{C}$ & Stream Temperature Error \\
\hline & $\varepsilon T$ EW & 1 & ${ }^{\circ} \mathrm{C}$ & Stream Temperature Error \\
\hline & $\varepsilon T_{-} X$ & 1 & ${ }^{\circ} \mathrm{C}$ & Stream Temperature Error \\
\hline & $\varepsilon \rho \_P$ & .000035 & $\mathrm{~g} / \mathrm{cm}^{\wedge} 3$ & Stream Density Error \\
\hline & $\varepsilon \rho \_H$ & .000035 & $\mathrm{~g} / \mathrm{cm}^{\wedge} 3$ & Stream Density Error \\
\hline & $\varepsilon \rho \_E$ & .000035 & $\mathrm{~g} / \mathrm{cm}^{\wedge} 3$ & Stream Density Error \\
\hline & $\varepsilon \rho_{C} C$ & .000035 & $\mathrm{~g} / \mathrm{cm}^{\wedge} 3$ & Stream Density Error \\
\hline & $\varepsilon \Delta \mathrm{T}$ & 1.414214 & ${ }^{\circ} \mathrm{C}$ & Temperature Gradient Error \\
\hline & $\varepsilon \Delta \mathrm{H}$ & 0.063640 & $\mathrm{~J} / \mathrm{g}$ & Enthalpy Gradient Error \\
\hline & $\varepsilon M \_P$ & 46.089872 & $\mathrm{~g} / \mathrm{min}$ & Stream Mass Flow Rate Error \\
\hline & EM_H & 46.089872 & $\mathrm{~g} / \mathrm{min}$ & Stream Mass Flow Rate Error \\
\hline & $\varepsilon M \_E$ & 0.452779 & $\mathrm{~g} / \mathrm{min}$ & Stream Mass Flow Rate Error \\
\hline & $\varepsilon M_{-} C$ & 0.452779 & $\mathrm{~g} / \mathrm{min}$ & Stream Mass Flow Rate Error \\
\hline & $\varepsilon \Sigma M_{-} C$ & 0.037564 & g & Product Amount Error \\
\hline & $\varepsilon Q_{-} H$ & 4066.209798 & $\mathrm{~J} / \mathrm{min}$ & Heater Heat Input Error \\
\hline & $\varepsilon \Sigma Q_{-} H$ & 4066.209798 & $J$ & Heat Input Error \\
\hline & $\varepsilon A \_S C$ & 1667.092631 & $\mathrm{~cm}^{\wedge} 2$ & Solar Collector Area Error \\
\hline & $\varepsilon P E C$ & 804.596245 & $\mathrm{~J} / \mathrm{g}$ & Prime Energy Consumption Error \\
\hline & $\varepsilon \eta C$ & 43.564826 & $\%$ & Condenser Efficiency Error \\
\hline & $\varepsilon \eta R$ & 0.069013 & $\%$ & Recovery Efficiency Error \\
\hline
\end{tabular}


Appendix L (Continued)

\begin{tabular}{lllll} 
Input & Name & Output & Unit & Comment \\
\hline & $\varepsilon \sqcap T$ & 1.839071 & $\%$ & Thermal Efficiency Error
\end{tabular}


Appendix M. Sample TK Solver code for model simulation

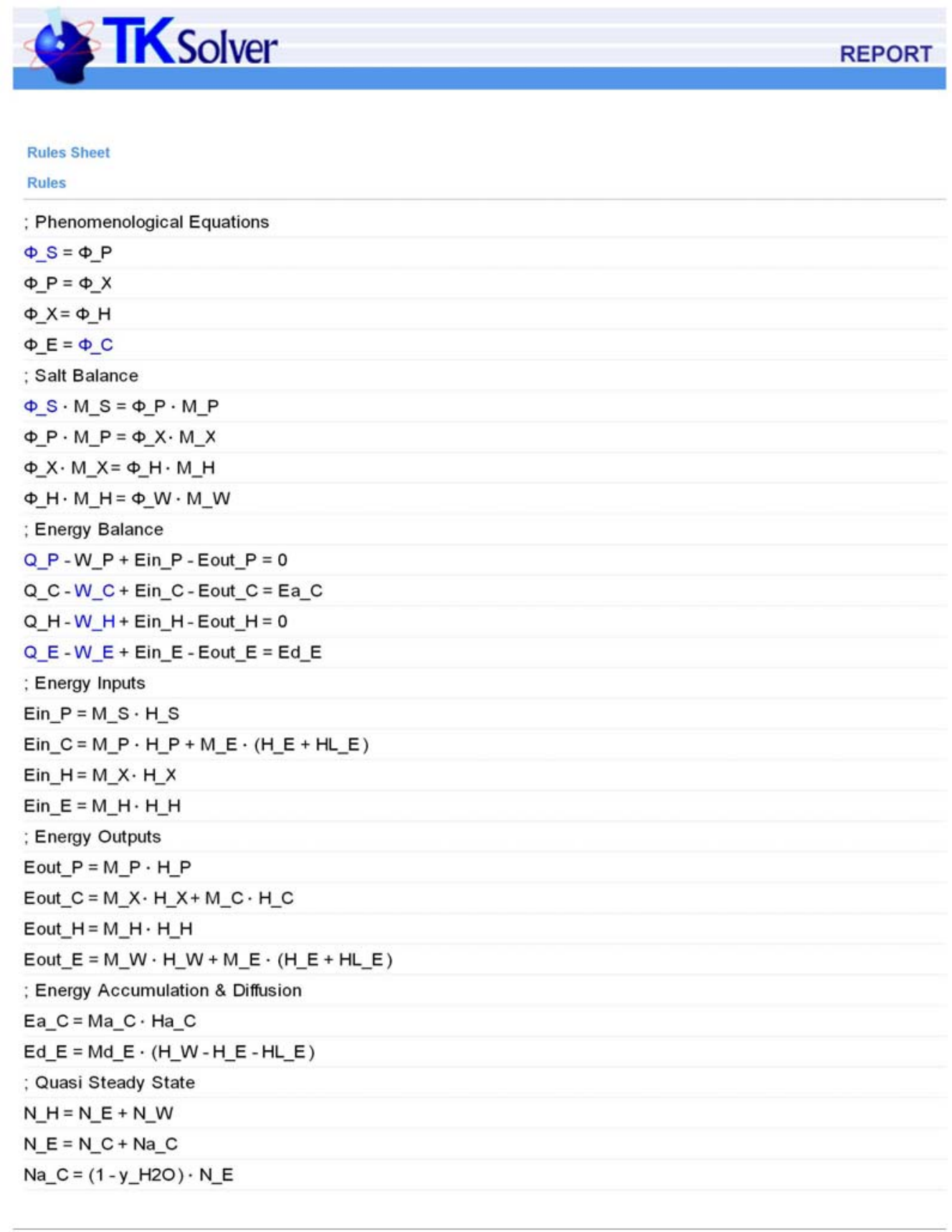




\section{Appendix M (Continued)}

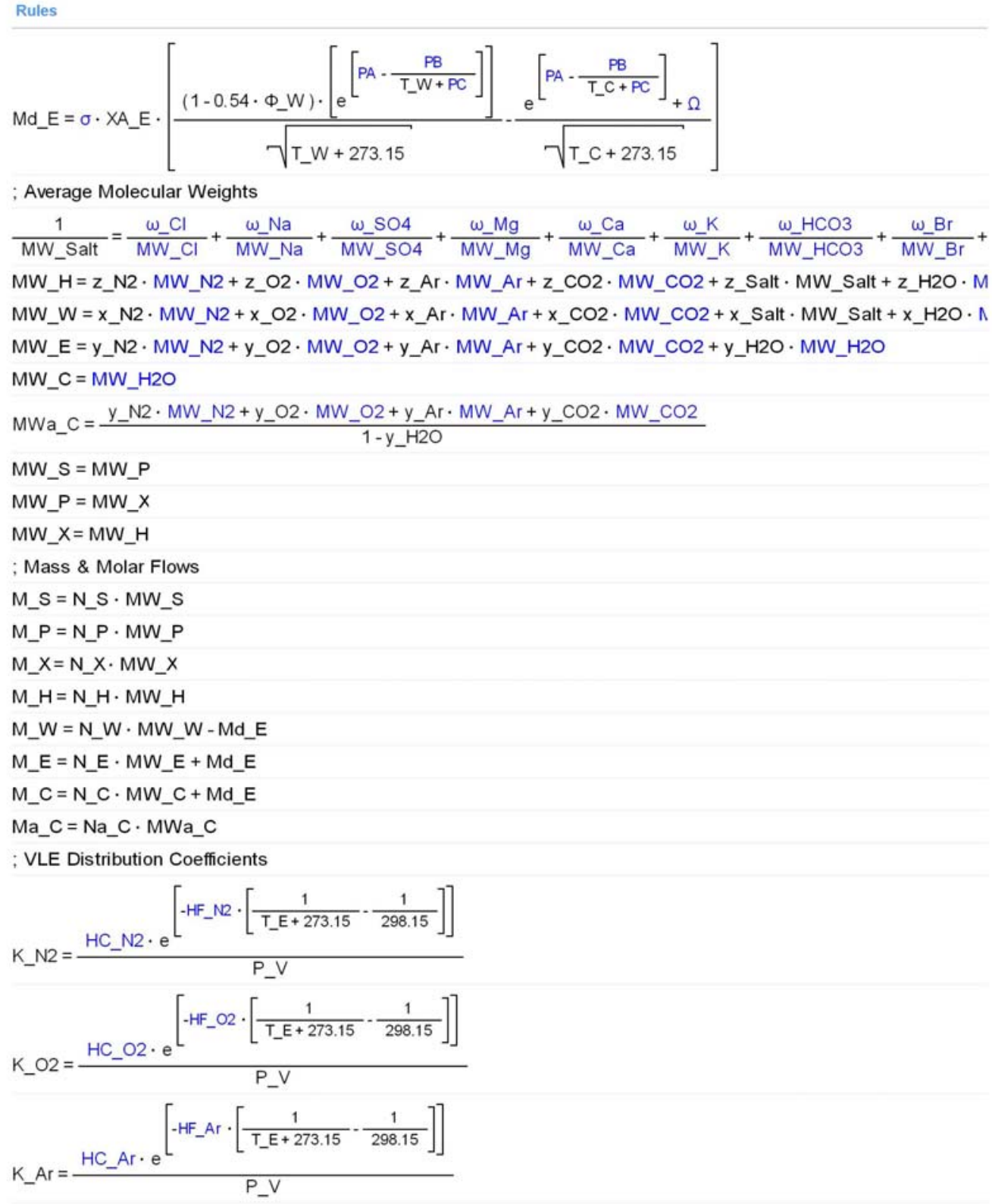




\section{Appendix M (Continued)}

Rules

$\mathrm{K}_{-} \mathrm{CO} 2=\frac{\mathrm{HC}_{-} \mathrm{CO} 2 \cdot \mathrm{e}^{\left[-\mathrm{HF}_{-} \mathrm{CO} 2 \cdot\left[\frac{1}{\mathrm{~T}_{-} E+273.15} \cdot \frac{1}{298.15}\right]\right]}}{\mathrm{P}_{-} \mathrm{V}}$

$\mathrm{K}_{-} \mathrm{H} 2 \mathrm{O}=\frac{\mathrm{Y}_{-} \mathrm{H} 2 \mathrm{O} \cdot \mathrm{P}_{-} \mathrm{H}_{2} \mathrm{O}}{\mathrm{P}_{-} \mathrm{V}}$

$Y_{-} H_{2} \mathrm{O}=0.0019583973+\frac{1.038491 \cdot P_{-} V}{P_{-} H_{2} \mathrm{O}}$

; Flash Calculations

Ф_N2

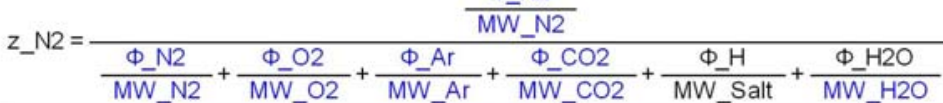

Ф_O2

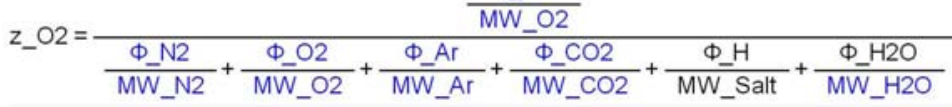

$\Phi \_$Ar

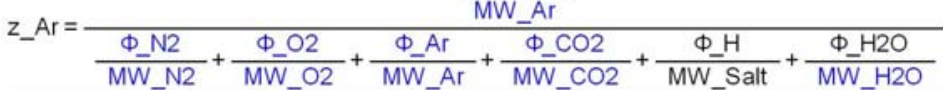

$\Phi \_\mathrm{CO} 2$

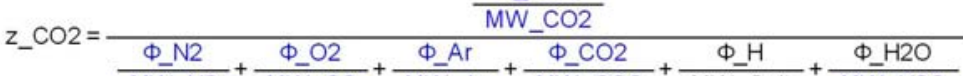

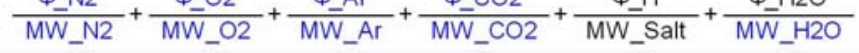

$\Phi \_$H

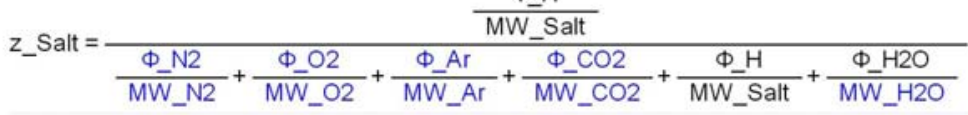

$\times \mathrm{Z}_{-} \mathrm{N} 2 \cdot \mathrm{N}$ - $\mathrm{H}$

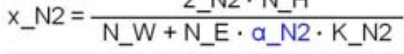

$x_{-} \mathrm{O} 2=\frac{z_{-}}{\mathrm{N}_{-} \mathrm{O} 2 \cdot \mathrm{N} \_\mathrm{H}}$

X_O2 $=\frac{\text { N_W }+ \text { N_E } \cdot \text { a_O2 } \cdot \text { K_O2 }}{\text { N }}$

$x_{-} A r=\frac{z_{-} A r \cdot N_{-} H}{\text { N_W }+ \text { N_E } \cdot a_{-} A r \cdot K_{-} A r}$

$x_{-} \mathrm{CO} 2=\frac{z_{-} \mathrm{CO} 2 \cdot \mathrm{N} \_H}{\mathrm{~N} H+\mathrm{N}}$

x_Salt $=\frac{z \_ \text {Salt } \cdot \text { N_H }}{\text { N_W }}$

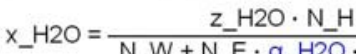

$y \_N 2=x \_N 2 \cdot a_{-} N 2 \cdot$ K_N2

$\mathrm{y}_{-} \mathrm{O} 2=\mathrm{x} \_\mathrm{O} 2 \cdot \mathrm{a}_{-} \mathrm{O} 2 \cdot \mathrm{K} \_\mathrm{O} 2$

$\mathrm{y} \_\mathrm{Ar}=\mathrm{x} \_\mathrm{Ar} \cdot \mathrm{a} \_\mathrm{Ar} \cdot \mathrm{K} \_\mathrm{Ar}$ 


\section{Appendix M (Continued)}

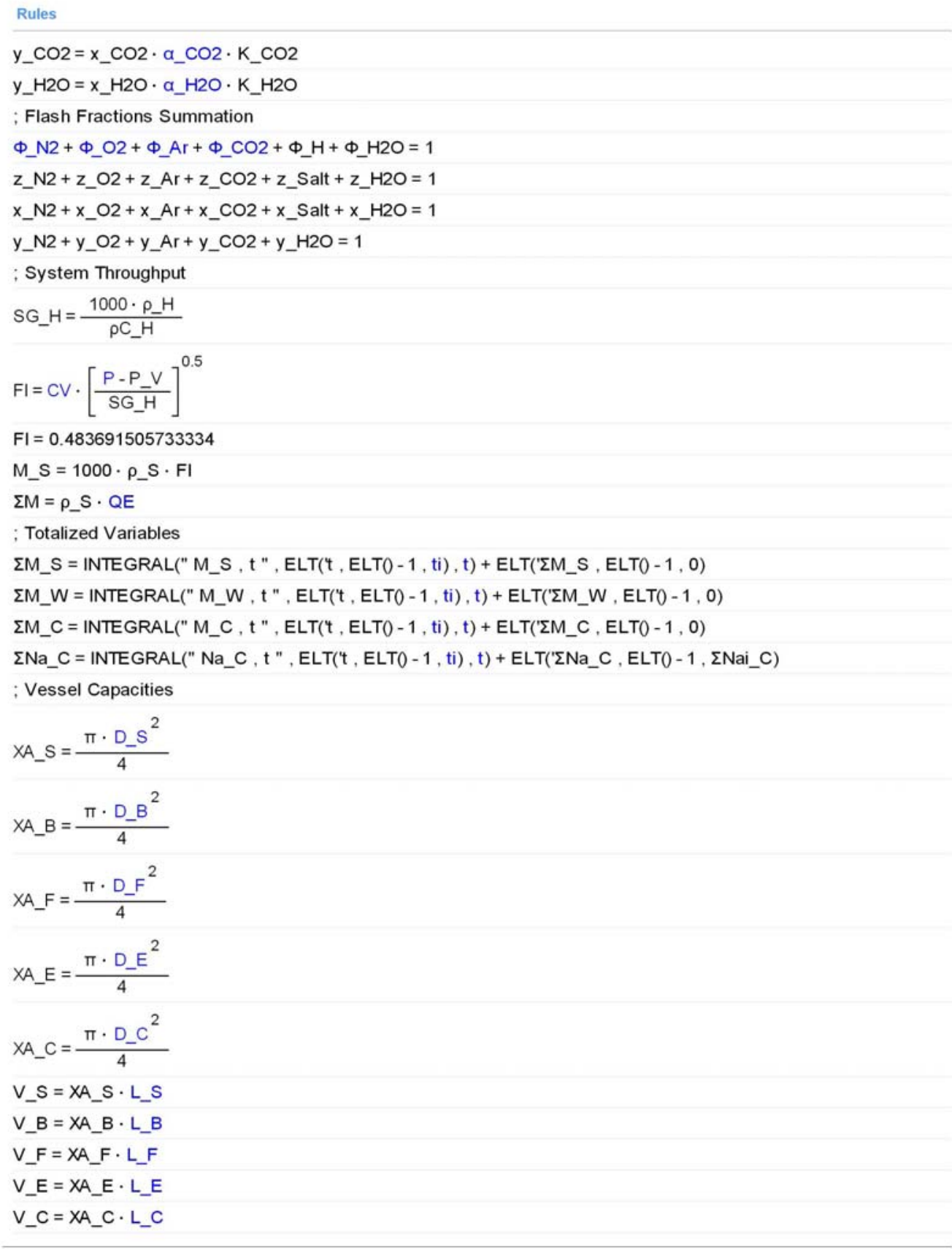




\section{Appendix M (Continued)}

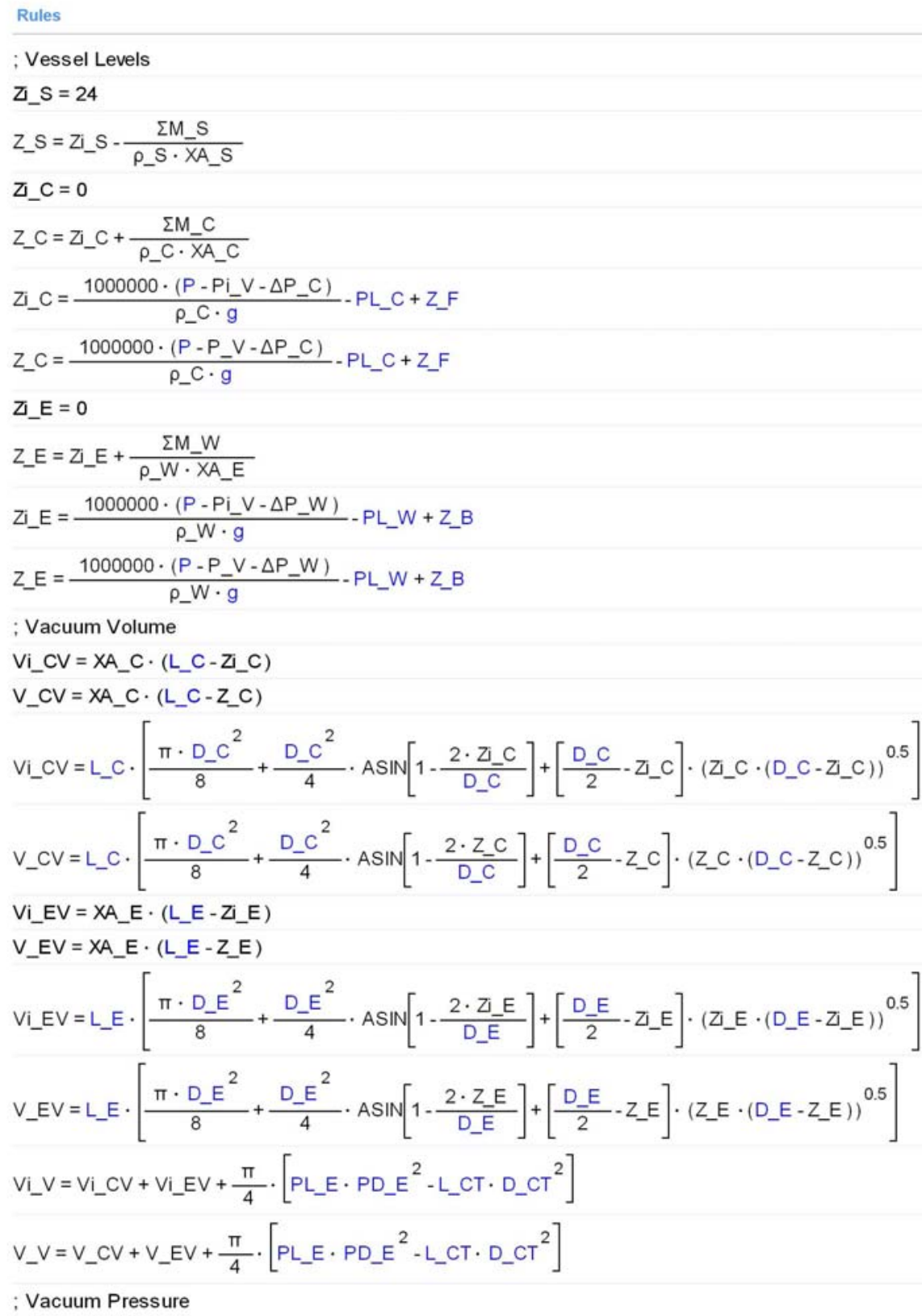




\section{Appendix M (Continued)}

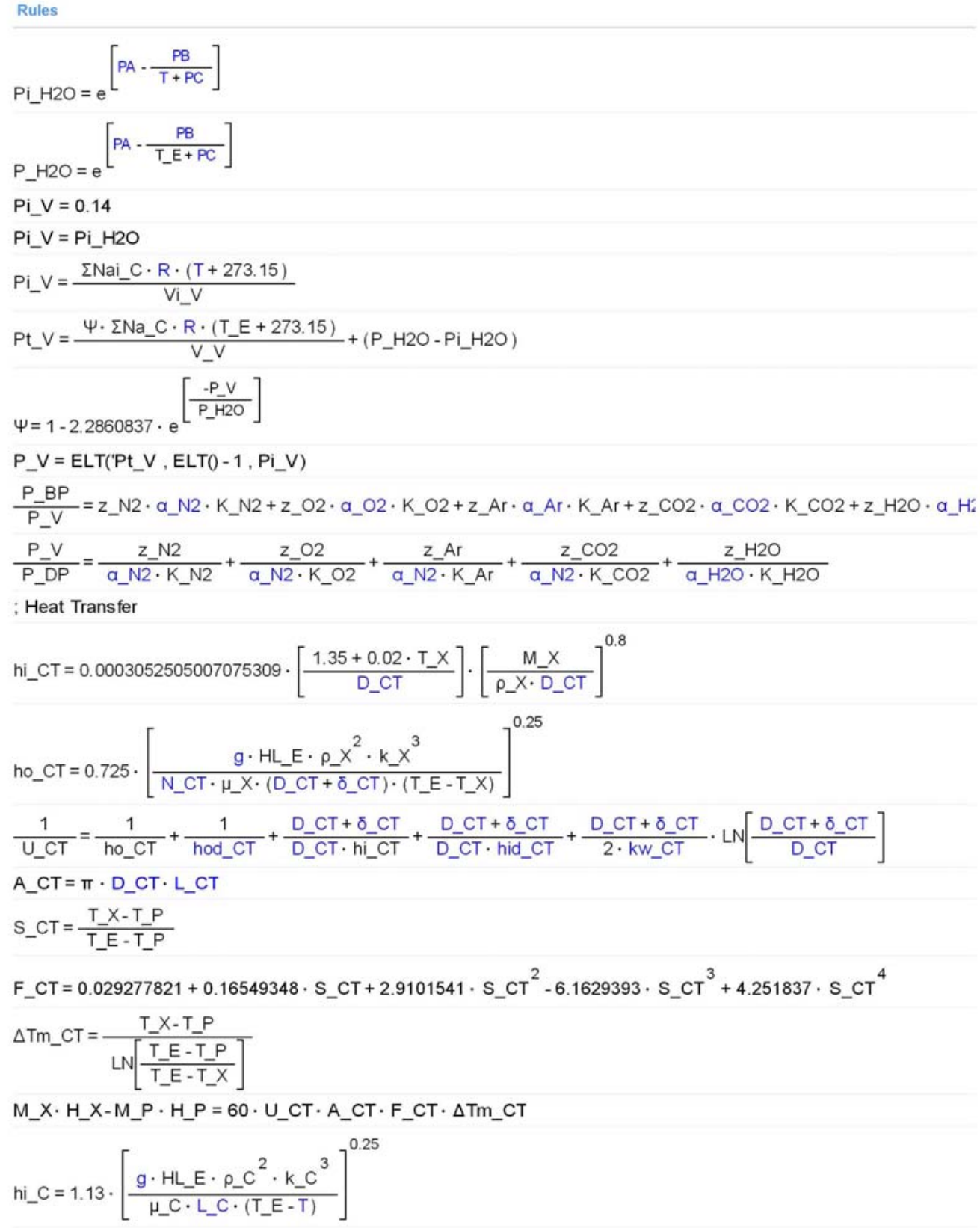




\section{Appendix M (Continued)}

Rules

ho_C $=0.04477637899495253 \cdot\left[\frac{\text { T_E }-\mathrm{T} T}{L_{-} C}\right]^{0.25}$

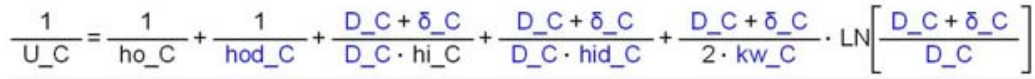

A_C $=\pi \cdot D_{-} C \cdot L_{-} C$

$S_{-} C=\frac{T_{-} C-T}{T_{-} E-T}$

; $F_{-} \mathrm{C}=\mathrm{f}\left(\mathrm{S}_{-} \mathrm{C}\right)$

$\Delta T m_{-} C=\frac{T_{-} C-T^{-}}{L N\left[\frac{T_{-} E-T}{T_{-} E-T_{-} C}\right]}$

$-Q_{-} C=60 \cdot U_{-} C \cdot A_{-} C \cdot F_{-} C \cdot \Delta T m_{-} C$

hi_E $=1.13 \cdot\left[\frac{g \cdot H L_{-} E \cdot P_{-} W^{2} \cdot K_{-} W^{3}}{H_{-} W \cdot L_{-} E \cdot\left(T \_E-T\right)}\right]^{0.25}$

ho_E $E=0.04477637899495253 \cdot\left[\frac{T_{-} E-T}{L_{-} E}\right]^{0.25}$

$\frac{1}{U_{-} E}=\frac{1}{h 0_{-} E}+\frac{1}{h o d_{-} E}+\frac{D_{-} E+\delta_{-} E}{D_{-} E \cdot h i_{-} E}+\frac{D_{-} E+\delta_{-} E}{D_{-} E \cdot h i d_{-} E}+\frac{D_{-} E+\delta_{-} E}{2 \cdot k W_{-} E} \cdot L_{N}\left[\frac{D_{-} E+\delta_{-} E}{D_{-} E}\right]$

$A_{-} E=\pi \cdot D_{-} E \cdot L_{-} E$

$S_{-} E=\frac{T-W-T}{T_{-} E-T}$

$; F_{-} E=f\left(S_{-} E\right)$

$\Delta T m_{-} E=\frac{T \_W-T}{L N\left[\frac{T_{-} E-T}{\left|T \_E-T \_W\right|}\right]}$

$-Q_{-} E=60 \cdot U_{-} E \cdot A_{-} E \cdot F \_E \cdot \Delta T m_{-} E$

; Equipment Sizing

HP_P $=\frac{-W_{-} P}{44741.994}$

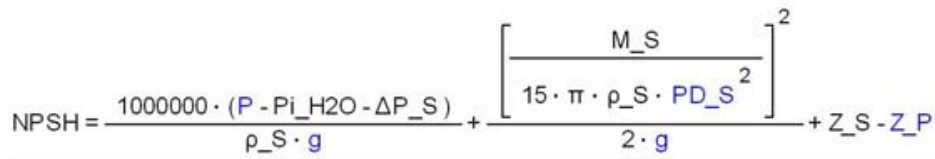

$\Delta \mathrm{P}=\Delta \mathrm{P}_{-} \mathrm{S}+\Delta \mathrm{P}_{-} \mathrm{P}+\Delta \mathrm{P}_{-} \mathrm{X}+\Delta \mathrm{P}_{-} \mathrm{H}+\Delta \mathrm{P} \_\mathrm{HT}+\Delta \mathrm{P} \_\mathrm{CT}$

$P \_O-P=\frac{-P_{-} P \cdot \eta_{-} P \cdot W_{-} P}{10 \cdot M_{-} P}-\frac{P_{-} P \cdot g \cdot\left(Z_{-} O-Z_{-} P\right)}{1000000}-\Delta P$

C_O $=\left[\frac{41318.7502427578}{M_{-} H}\right] \cdot\left(\rho_{-} H \cdot\left(P_{-} O-P_{-}{ }^{\prime}\right)\right)^{0.5}$ 


\section{Appendix M (Continued)}

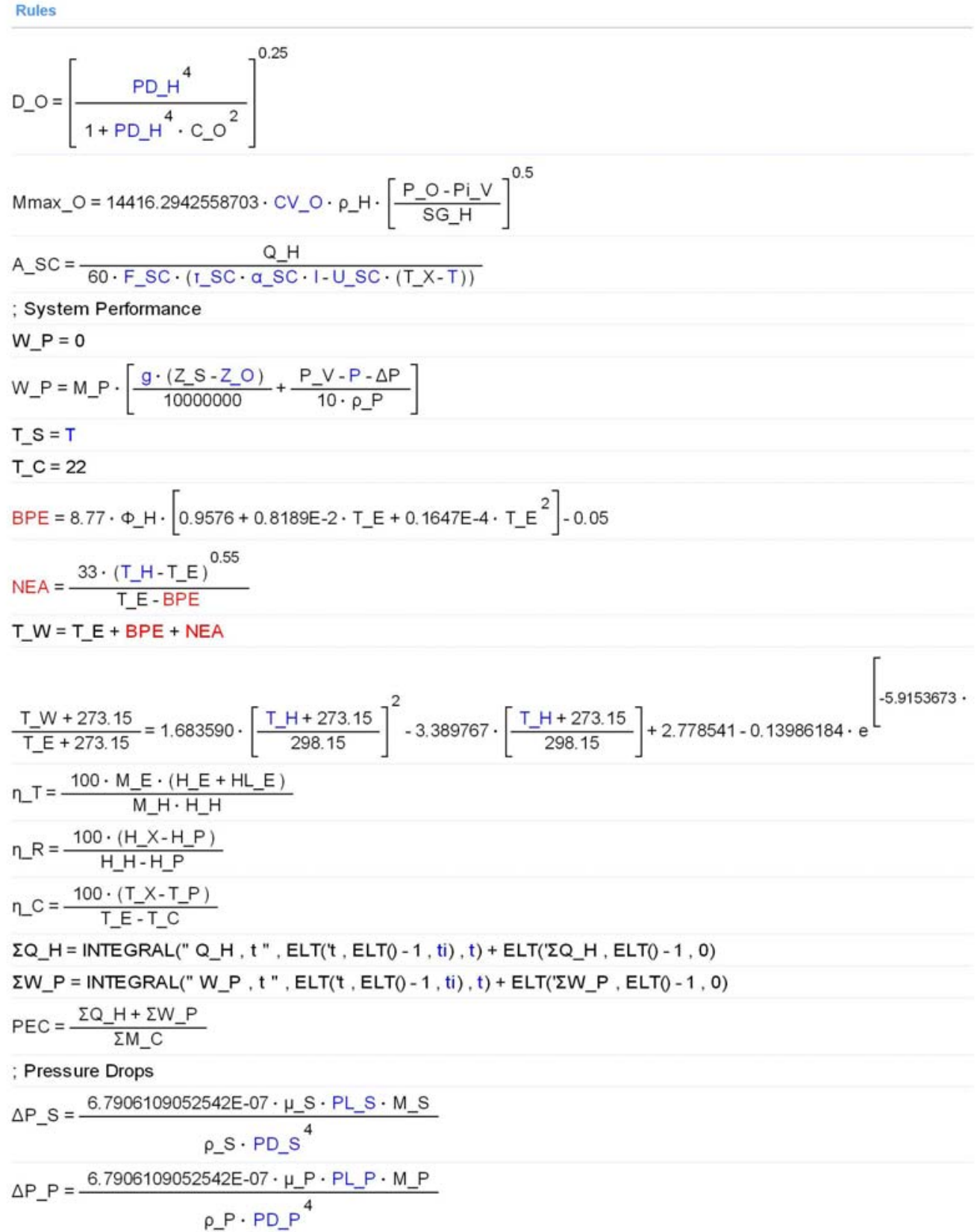




\section{Appendix M (Continued)}

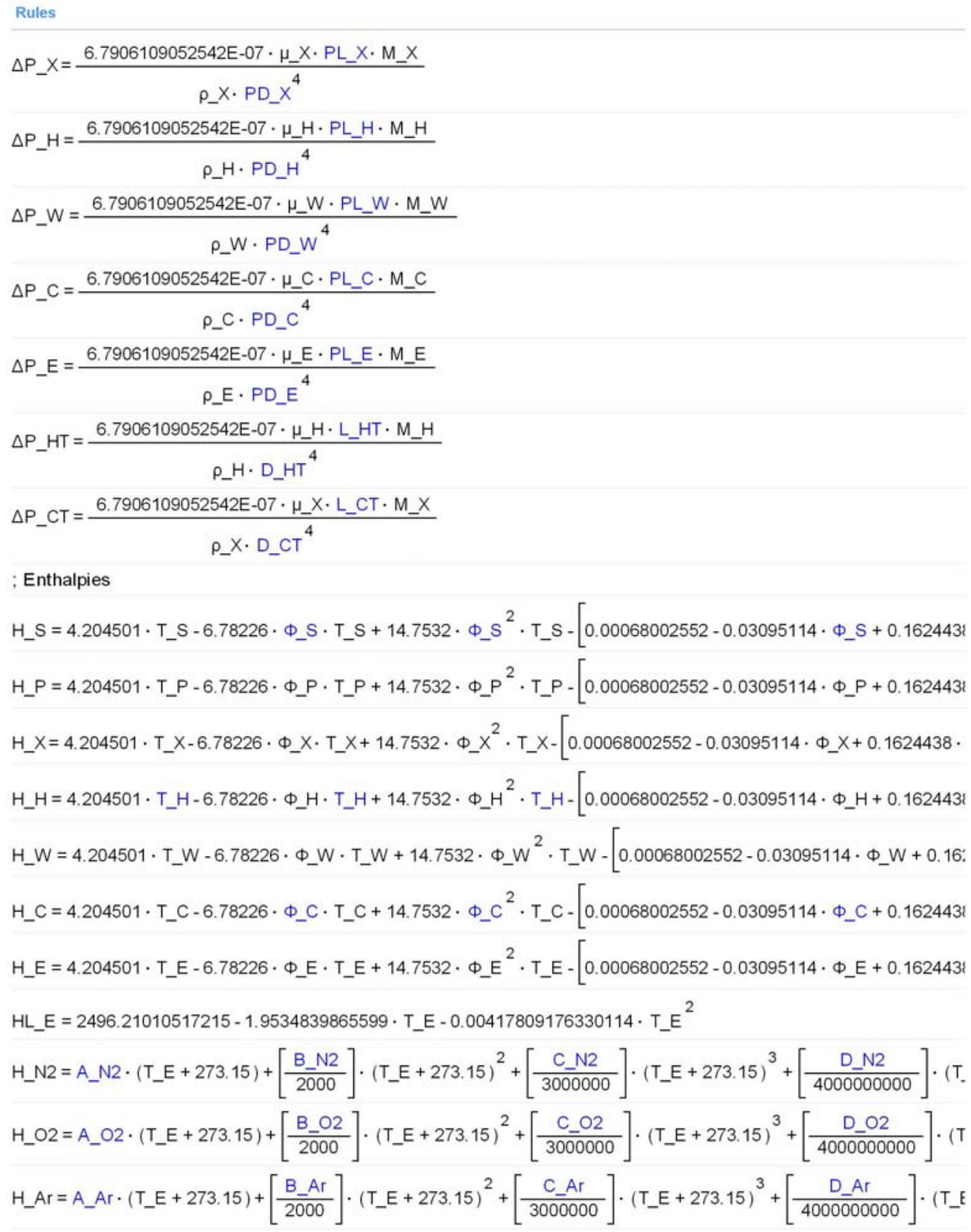




\section{Appendix M (Continued)}

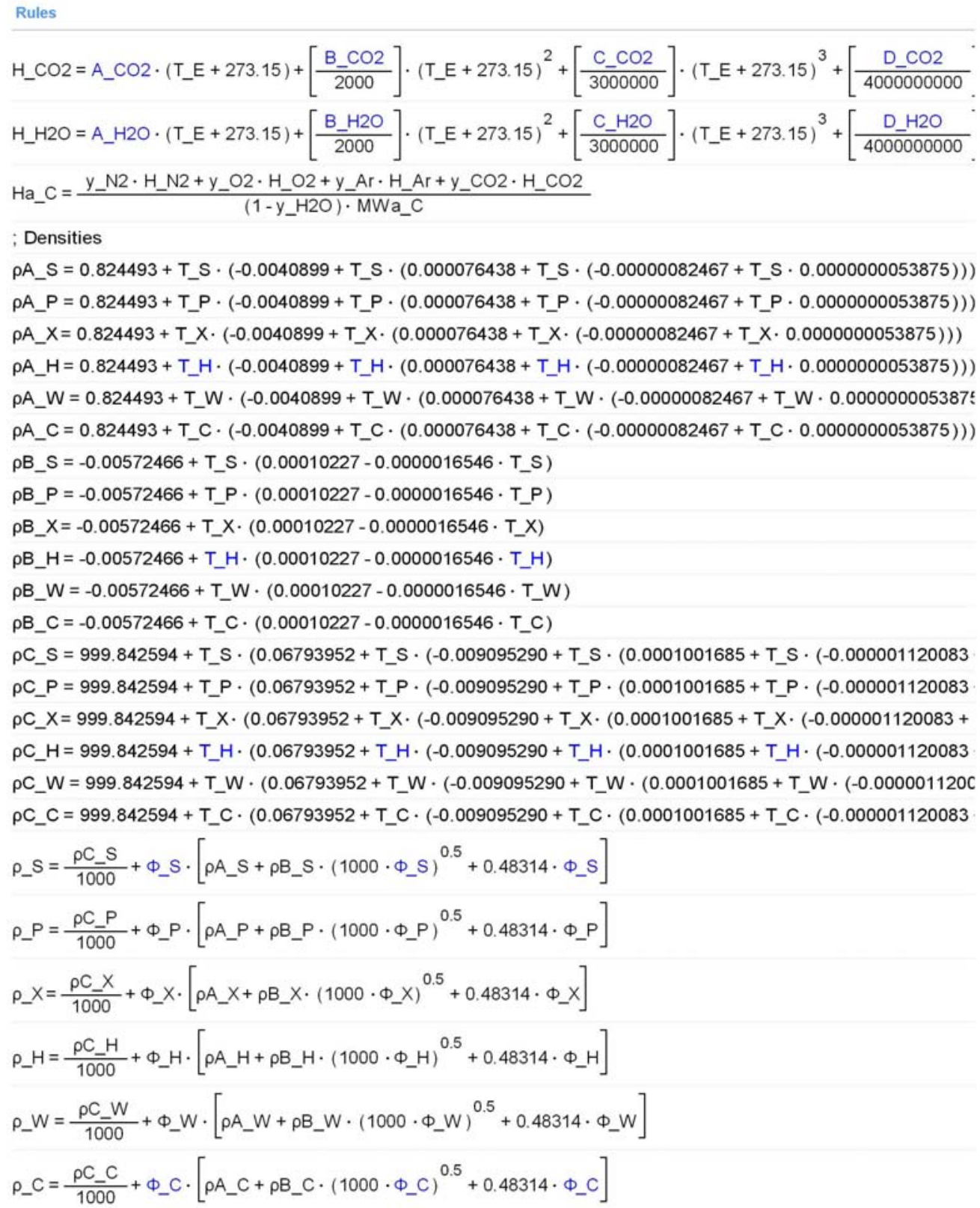




\section{Appendix M (Continued)}

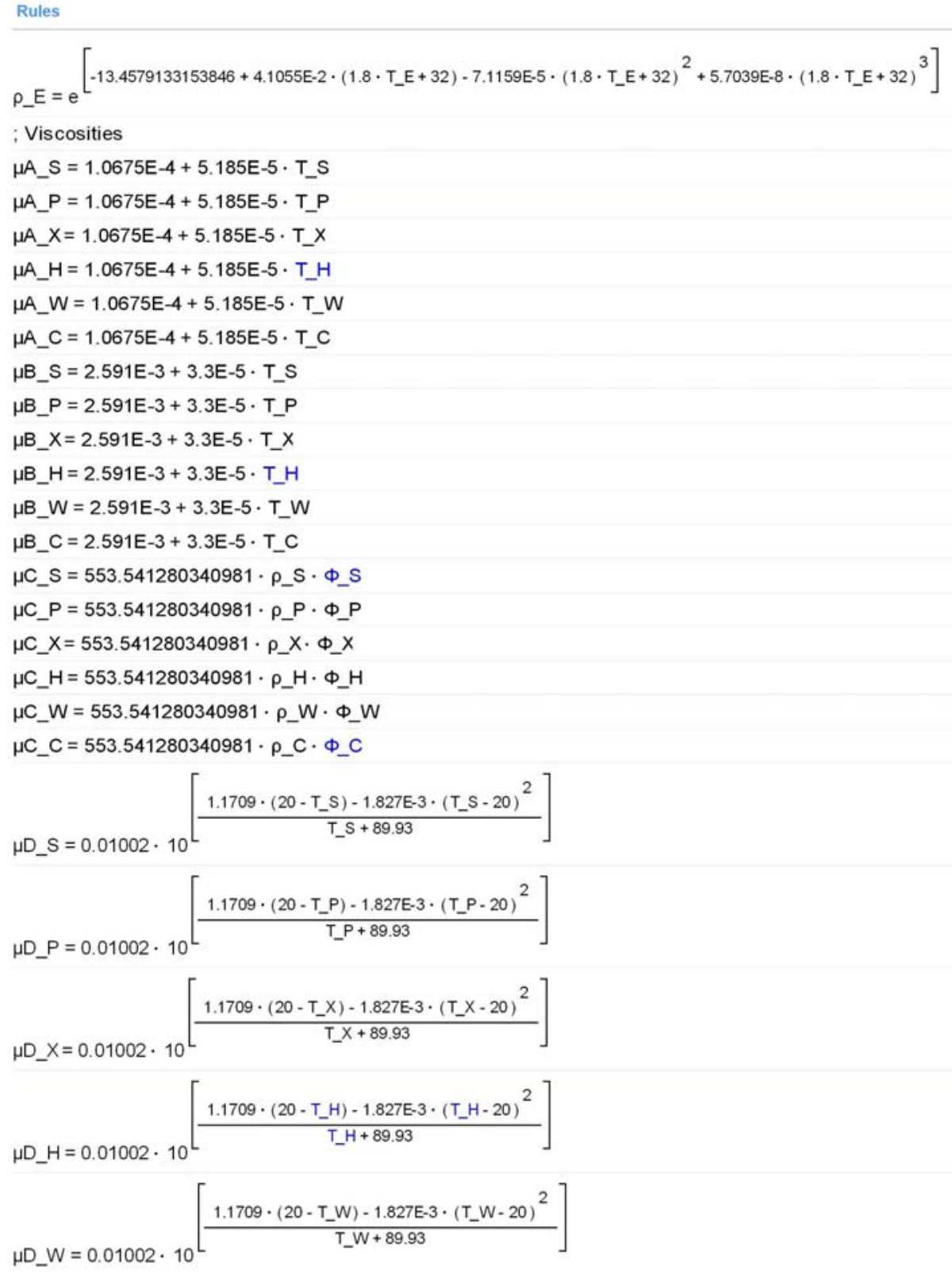




\section{Appendix M (Continued)}

Rules

$\mu D_{-} C=0.01002 \cdot 10\left[\frac{1.1709 \cdot\left(20-T_{-} C\right) \cdot 1.827 E-3 \cdot\left(T_{-} C-20\right)^{2}}{T_{-} C+89.93}\right]$

$\mu \_S=\left[1+\mu A_{-} S \cdot \mu C_{-} S^{0.5}+\mu B_{-} S \cdot \mu C_{-} S\right] \cdot \mu D_{-} S$

$\mu \_P=\left[1+\mu A_{-} P \cdot \mu C_{-} P^{0.5}+\mu B_{-} P \cdot \mu C_{-} P\right] \cdot \mu D_{-} P$

$\mu_{-} X=\left[1+\mu A_{-} X \cdot \mu C_{-} X^{0.5}+\mu B_{-} X \cdot \mu C_{-} X\right] \cdot \mu D_{-} X$

$\mu_{-} H=\left[1+\mu A_{-} H \cdot \mu C_{-} H^{0.5}+\mu B_{-} H \cdot \mu C_{-} H\right] \cdot \mu D_{-} H$

$\mu_{-} W=\left[1+\mu A_{-} W \cdot \mu C_{-} W^{0.5}+\mu B_{-} W \cdot \mu C_{-} W\right] \cdot \mu D_{-} W$

$\mu_{-} C=\left[1+\mu A_{-} C \cdot \mu C_{-} C^{0.5}+\mu B_{-} C \cdot \mu C_{-} C\right] \cdot \mu D_{-} C$

$\mu_{-} E=7.23123710739283 E-5+2.37506847313357 E-07 \cdot\left(1.8 \cdot T_{-} E+32\right)-5.67031837890055 E-11 \cdot\left(1.8 \cdot T_{-} E+32\right)^{2}$

; Thermal Conductivities

K_S $=0.00571116+0.00001713348 \cdot T \_S-0.00000005853939 \cdot T_{-} S^{2}-0.0016562364 \cdot \Phi \_S$

K_P $=0.00571116+0.00001713348 \cdot T \_P-0.00000005853939 \cdot T_{-} P^{2}-0.0016562364 \cdot \Phi \_P$

k_X $=0.00571116+0.00001713348 \cdot T \_X-0.00000005853939 \cdot T_{-} X^{2}-0.0016562364 \cdot \Phi \_X$

$k_{-} H=0.00571116+0.00001713348 \cdot T_{-} H-0.00000005853939 \cdot T_{-} H^{2}-0.0016562364 \cdot \Phi+H$

k_W $=0.00571116+0.00001713348 \cdot T \_W-0.00000005853939 \cdot T_{-} W^{2}-0.0016562364 \cdot \Phi \_W$

k_C $=0.00571116+0.00001713348 \cdot T \_C-0.00000005853939 \cdot T_{-} C^{2}-0.0016562364 \cdot \Phi+C$

$k a_{-} C=1.5207 E-13 \cdot\left(T_{-} E+273.15\right)^{3}-4.8574 E-10 \cdot\left(T_{-} E+273.15\right)^{2}+1.0184 E-06 \cdot\left(T_{-} E+273.15\right)-3.9333 E-06$

$K_{-} E=1.69430023512008 E-4+3.89120840469752 E-7 \cdot\left(1.8 \cdot T_{-} E+32\right)-5.8517701472412 E-10 \cdot\left(1.8 \cdot T_{-} E+32\right)^{2}+$ ; Reynolds Numbers

$\operatorname{Re} \_S=\frac{\text { M_S }}{15 \cdot \pi \cdot P D_{-} S \cdot \mu \_S}$

$\operatorname{Re} \_P=\frac{M \_P}{15 \cdot \pi \cdot P D_{-} P \cdot \mu_{-} P}$

$\mathrm{Re}_{-} \mathrm{X}=\frac{\mathrm{M}_{-} \mathrm{X}}{15 \cdot \pi \cdot \mathrm{PD} \mathrm{X}_{-} \cdot \mu_{-} \mathrm{X}}$

$\mathrm{Re} \_\mathrm{H}=\frac{\mathrm{M} \_\mathrm{H}}{15 \cdot \pi \cdot \mathrm{PD} \_\mathrm{H} \cdot \mu_{-} \mathrm{H}}$ 


\section{Appendix M (Continued)}

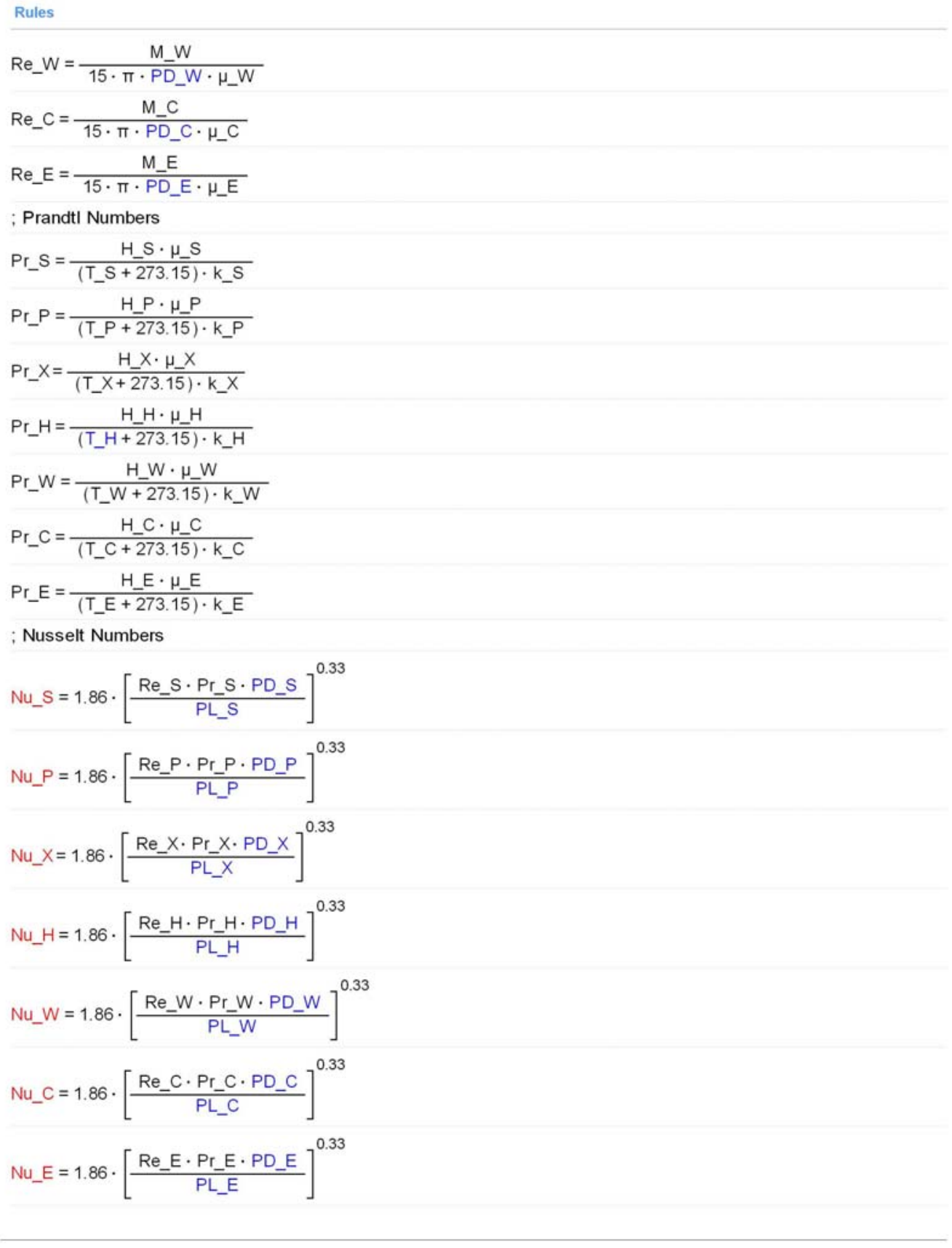




\section{Appendix M (Continued)}

\begin{tabular}{|c|c|c|c|c|}
\hline \multicolumn{5}{|c|}{ Variables Sheet } \\
\hline Input & Name & Output & Unit & Comment \\
\hline 0 & $\mathrm{ti}$ & & $\min$ & Initial Time \\
\hline 1 & $\mathrm{t}$ & & $\min$ & Run Time \\
\hline 20 & $T$ & & ${ }^{\circ} \mathrm{C}$ & Ambient Temperature \\
\hline \multirow[t]{2}{*}{1.01325} & $P$ & & bar & Ambient Pressure \\
\hline & $\mathrm{FI}$ & 0.483692 & $\mathrm{~L} / \mathrm{min}$ & Average Flow Indicator Value \\
\hline \multirow[t]{2}{*}{27} & QE & & $\mathrm{cm}^{\wedge} 3$ & Average Quantitative Element Value \\
\hline & $\Sigma M$ & 27.668601 & g & Actual Product Amount \\
\hline 83.14472 & $\mathbf{R}$ & & $\begin{array}{l}\left(\text { bar }-\mathrm{cm}^{\wedge} 3\right) /( \\
\left.\mathrm{mol}-{ }^{\circ} \mathrm{C}\right)\end{array}$ & Universal Gas Constant \\
\hline \multirow[t]{10}{*}{980.0665} & $\mathrm{~g}$ & & $\mathrm{~cm} / \mathrm{s}^{\wedge} 2$ & Gravity Acceleration \\
\hline & P_BP & 47.803156 & bar & Bubble Point Pressure \\
\hline & P_DP & 0.145822 & bar & Dew Point Pressure \\
\hline & $\sum M \_S$ & 495.669161 & g & Total Seawater Amount \\
\hline & EM_W & 495.911874 & $\mathrm{~g}$ & Total Brine Amount \\
\hline & $\Sigma M_{-} C$ & -0.253082 & g & Total Condensed Steam Amount \\
\hline & $\sum \mathrm{Na} \_\mathrm{C}$ & 1.786244 & $\mathrm{~mol}$ & Total NCG Amount \\
\hline & $\Sigma Q_{-} H$ & 41983.941375 & $\mathrm{~J}$ & Heat Input \\
\hline & $\Sigma W \_P$ & 0 & $\mathrm{~J}$ & Power Input \\
\hline & PEC & -165890.373805 & $\mathrm{~J} / \mathrm{g}$ & Prime Energy Consumption \\
\hline .5503 & $\omega_{-} \mathrm{Cl}$ & & & Chloride Mass Fraction in Seawater Salt \\
\hline .3059 & $\omega_{-} \mathrm{Na}$ & & & Sodium Mass Fraction in Seawater Salt \\
\hline .0768 & w_SO4 & & & Sulfate Mass Fraction in Seawater Salt \\
\hline .0368 & $\omega_{-} M g$ & & & Magnesium Mass Fraction in Seawater Salt \\
\hline .0118 & $\omega_{-} \mathrm{Ca}$ & & & Calcium Mass Fraction in Seawater Salt \\
\hline .0111 & $\omega_{-} k$ & & & Potassium Mass Fraction in Seawater Salt \\
\hline .0041 & $\omega_{-} \mathrm{HCO} 3$ & & & Bicarbonate Mass Fraction in Seawater Salt \\
\hline .0019 & $\omega_{-} B r$ & & & Bromide Mass Fraction in Seawater Salt \\
\hline .0008 & $\omega_{-} \mathrm{BO} 3$ & & & Borate Mass Fraction in Seawater Salt \\
\hline .0004 & $\omega \_S r$ & & & Strontium Mass Fraction in Seawater Salt \\
\hline .00003 & $\omega_{-} F$ & & & Fluoride Mass Fraction in Seawater Salt \\
\hline 35.453 & $\mathrm{MW} \_\mathrm{Cl}$ & & $\mathrm{g} / \mathrm{mol}$ & Chloride Molecular Weight \\
\hline 22.99 & MW_Na & & $\mathrm{g} / \mathrm{mol}$ & Sodium Molecular Weight \\
\hline 96.062 & MW_SO4 & & $\mathrm{g} / \mathrm{mol}$ & Sulfate Molecular Weight \\
\hline 24.305 & MW_Mg & & $\mathrm{g} / \mathrm{mol}$ & Magnesium Molecular Weight \\
\hline 40.078 & MW_Ca & & $\mathrm{g} / \mathrm{mol}$ & Calcium Molecular Weight \\
\hline 39.098 & MW_K & & $\mathrm{g} / \mathrm{mol}$ & Potassium Molecular Weight \\
\hline 61.016 & MW_HCO3 & & $\mathrm{g} / \mathrm{mol}$ & Bicarbonate Molecular Weight \\
\hline 79.904 & $\mathrm{MW} \_\mathrm{Br}$ & & $\mathrm{g} / \mathrm{mol}$ & Bromide Molecular Weight \\
\hline 58.808 & MW_BO3 & & $\mathrm{g} / \mathrm{mol}$ & Borate Molecular Weight \\
\hline 87.62 & MW_Sr & & $\mathrm{g} / \mathrm{mol}$ & Strontium Molecular Weight \\
\hline
\end{tabular}




\section{Appendix M (Continued)}

\begin{tabular}{|c|c|c|c|c|}
\hline Input & Name & Output & Unit & Comment \\
\hline 18.998 & MW_F & & $\mathrm{g} / \mathrm{mol}$ & Fluoride Molecular Weight \\
\hline 28.0134 & MW_N2 & & $\mathrm{g} / \mathrm{mol}$ & Nitrogen Molecular Weight \\
\hline 31.998 & MW_O2 & & $\mathrm{g} / \mathrm{mol}$ & Oxygen Molecular Weight \\
\hline 39.94 & MW_Ar & & $\mathrm{g} / \mathrm{mol}$ & Argon Molecular Weight \\
\hline \multirow[t]{2}{*}{44.009} & MW_CO2 & & $\mathrm{g} / \mathrm{mol}$ & Carbon Dioxide Molecular Weight \\
\hline & MW_Salt & 31.416538 & $\mathrm{~g} / \mathrm{mol}$ & Approximated Seawater Salt Molecular Weight \\
\hline 18.0148 & MW_H2O & & $\mathrm{g} / \mathrm{mol}$ & Water Molecular Weight \\
\hline 0.000013 & $\Phi \_N 2$ & & & Nitrogen Mass Fraction in Ambient Seawater \\
\hline 0.000008 & Ф_O2 & & & Oxygen Mass Fraction in Ambient Seawater \\
\hline 0.000000 & Ф_Ar & & & Argon Mass Fraction in Ambient Seawater \\
\hline \multirow[t]{19}{*}{0.000000} & $\Phi \_\mathrm{CO} 2$ & & & Carbon Dioxide Mass Fraction in Ambient Seawater \\
\hline & $\Phi \_\mathrm{H} 2 \mathrm{O}$ & 0.964979 & & Water Mass Fraction in Ambient Seawater \\
\hline & z_N2 & 0.000008 & & Nitrogen Mole Fraction in Ambient Seawater \\
\hline & z_O2 & 0.000004 & & Oxygen Mole Fraction in Ambient Seawater \\
\hline & Z_Ar & 0.000000 & & Argon Mole Fraction in Ambient Seawater \\
\hline & z_CO2 & 0.000000 & & Carbon Dioxide Mole Fraction in Ambient Seawater \\
\hline & z_Salt & 0.020374 & & Salt Mole Fraction in Ambient Seawater \\
\hline & $\mathrm{Z} \_\mathrm{H} 2 \mathrm{O}$ & 0.979613 & & Water Mole Fraction in Ambient Seawater \\
\hline & $\mathrm{X} \_\mathrm{N} 2$ & 0.000000 & & Nitrogen Mole Fraction in Brine Water \\
\hline & x_O2 & 0.000000 & & Oxygen Mole Fraction in Brine Water \\
\hline & X_Ar & 0.000000 & & Argon Mole Fraction in Brine Water \\
\hline & x_CO2 & 0.000000 & & Carbon Dioxide Mole Fraction in Brine Water \\
\hline & x_Salt & 0.020715 & & Salt Mole Fraction in Brine Water \\
\hline & $x \_\mathrm{H} 2 \mathrm{O}$ & 0.979285 & & Water Mole Fraction in Brine Water \\
\hline & y_N2 & 0.000500 & & Nitrogen Mole Fraction in Vaporized Water \\
\hline & y_O2 & 0.000268 & & Oxygen Mole Fraction in Vaporized Water \\
\hline & $\mathrm{y}_{-} \mathrm{Ar}$ & 0.000011 & & Argon Mole Fraction in Vaporized Water \\
\hline & y_CO2 & 0.000006 & & Carbon Dioxide Mole Fraction in Vaporized Water \\
\hline & $\mathrm{y}_{-} \mathrm{H} 2 \mathrm{O}$ & 0.999216 & & Water Mole Fraction in Vaporized Water \\
\hline 26.092 & A_N2 & & & Nitrogen Molar Enthalpy Parameter \\
\hline 8.218801 & B_N2 & & & Nitrogen Molar Enthalpy Parameter \\
\hline-1.976141 & C_N2 & & & Nitrogen Molar Enthalpy Parameter \\
\hline .159274 & D_N2 & & & Nitrogen Molar Enthalpy Parameter \\
\hline .044434 & E_N2 & & & Nitrogen Molar Enthalpy Parameter \\
\hline-7.98923 & F_N2 & & & Nitrogen Molar Enthalpy Parameter \\
\hline 29.659 & A_O2 & & & Oxygen Molar Enthalpy Parameter \\
\hline 6.137261 & B_O2 & & & Oxygen Molar Enthalpy Parameter \\
\hline
\end{tabular}




\section{Appendix M (Continued)}

\begin{tabular}{|c|c|c|c|c|}
\hline Input & Name & Output & Unit & Comment \\
\hline-1.186521 & C_O2 & & & Oxygen Molar Enthalpy Parameter \\
\hline .09578 & D_O2 & & & Oxygen Molar Enthalpy Parameter \\
\hline-.219663 & E_O2 & & & Oxygen Molar Enthalpy Parameter \\
\hline-9.861391 & F_O2 & & & Oxygen Molar Enthalpy Parameter \\
\hline 20.786 & A_Ar & & & Argon Molar Enthalpy Parameter \\
\hline 0.000000 & B_Ar & & & Argon Molar Enthalpy Parameter \\
\hline 0.000000 & C_Ar & & & Argon Molar Enthalpy Parameter \\
\hline 0.000000 & D_Ar & & & Argon Molar Enthalpy Parameter \\
\hline 0.000000 & E_Ar & & & Argon Molar Enthalpy Parameter \\
\hline-6.19735 & F_Ar & & & Argon Molar Enthalpy Parameter \\
\hline 24.99735 & A_CO2 & & & Carbon Dioxide Molar Enthalpy Parameter \\
\hline 55.18696 & B_CO2 & & & Carbon Dioxide Molar Enthalpy Parameter \\
\hline-33.69137 & C_CO2 & & & Carbon Dioxide Molar Enthalpy Parameter \\
\hline 7.948387 & D_CO2 & & & Carbon Dioxide Molar Enthalpy Parameter \\
\hline-.136638 & E_CO2 & & & Carbon Dioxide Molar Enthalpy Parameter \\
\hline-10.0851 & $\mathrm{~F} \_\mathrm{CO} 2$ & & & Carbon Dioxide Molar Enthalpy Parameter \\
\hline 30.092 & A_H2O & & & Water Molar Enthalpy Parameter \\
\hline 6.832514 & $\mathrm{~B} \_\mathrm{H} 2 \mathrm{O}$ & & & Water Molar Enthalpy Parameter \\
\hline 6.793435 & C_H2O & & & Water Molar Enthalpy Parameter \\
\hline-2.53448 & D_H2O & & & Water Molar Enthalpy Parameter \\
\hline .082139 & E_H2O & & & Water Molar Enthalpy Parameter \\
\hline \multirow[t]{14}{*}{-9.054600} & $\mathrm{~F}_{-} \mathrm{H} 2 \mathrm{O}$ & & & Water Molar Enthalpy Parameter \\
\hline & H_N2 & 408.621886 & $\mathrm{~J} / \mathrm{mol}$ & Nitrogen Molar Enthalpy \\
\hline & H_O2 & 402.549078 & $\mathrm{~J} / \mathrm{mol}$ & Oxygen Molar Enthalpy \\
\hline & H_Ar & 301.655669 & $\mathrm{~J} / \mathrm{mol}$ & Argon Molar Enthalpy \\
\hline & $\mathrm{H} \_\mathrm{CO} 2$ & 540.860997 & $\mathrm{~J} / \mathrm{mol}$ & Carbon Dioxide Molar Enthalpy \\
\hline & H_H2O & 488.461223 & $\mathrm{~J} / \mathrm{mol}$ & Water Molar Enthalpy \\
\hline & H_S & 79.740379 & $\mathrm{~J} / \mathrm{g}$ & Stream Enthalpy \\
\hline & H_P & 79.740379 & $\mathrm{~J} / \mathrm{g}$ & Stream Enthalpy \\
\hline & H_X & 115.076142 & $\mathrm{~J} / \mathrm{g}$ & Stream Enthalpy \\
\hline & H_H & 199.777682 & $\mathrm{~J} / \mathrm{g}$ & Stream Enthalpy \\
\hline & H_W & 160.594502 & $\mathrm{~J} / \mathrm{g}$ & Stream Enthalpy \\
\hline & H_C & 92.177531 & $\mathrm{~J} / \mathrm{g}$ & Stream Enthalpy \\
\hline & H_E & 165.347464 & $\mathrm{~J} / \mathrm{g}$ & Stream Enthalpy \\
\hline & HL_E & 2412.499766 & $\mathrm{~J} / \mathrm{g}$ & Stream Enthalpy of Vaporization \\
\hline 8067573.136086 & HC_N2 & & bar & Nitrogen Reference State Henry's Constant \\
\hline
\end{tabular}




\section{Appendix M (Continued)}

\begin{tabular}{|c|c|c|c|c|}
\hline Input & Name & Output & Unit & Comment \\
\hline 358814.929481 & HC_O2 & & bar & Oxygen Reference State Henry's Constant \\
\hline 384073.329688 & HC_Ar & & bar & Argon Reference State Henry's Constant \\
\hline 10914.952753 & HC_CO2 & & bar & Carbon Dioxide Reference State Henry's Constant \\
\hline-3545.567207 & HF_N2 & & ${ }^{\circ} \mathrm{C}$ & Nitrogen Henry's Coefficient \\
\hline-2208.812178 & HF_O2 & & ${ }^{\circ} \mathrm{C}$ & Oxygen Henry's Coefficient \\
\hline-2308.046425 & HF_Ar & & ${ }^{\circ} \mathrm{C}$ & Argon Henry's Coefficient \\
\hline-445.190581 & HF_CO2 & & ${ }^{\circ} \mathrm{C}$ & Carbon Dioxide Henry's Coefficient \\
\hline 12.762946 & PA & & & Water Vapor Pressure Coefficient \\
\hline 4391.129422 & PB & & ${ }^{\circ} \mathrm{C}$ & Water Vapor Pressure Coefficient \\
\hline 245.367016 & PC & & ${ }^{\circ} \mathrm{C}$ & Water Vapor Pressure Coefficient \\
\hline 1.21 & a_N2 & & & Nitrogen Relative Solubility \\
\hline 1.22 & a_O2 & & & Oxygen Relative Solubility \\
\hline 1.23 & a_Ar & & & Argon Relative Solubility \\
\hline 1.17 & $\mathrm{a}_{-} \mathrm{CO} 2$ & & & Carbon Dioxide Relative Solubility \\
\hline \multirow[t]{9}{*}{.9816} & a_H2O & & & Water Relative Vapor Pressure \\
\hline & K_N2 & 33181360.195354 & & $\begin{array}{l}\text { Evaporator-Based Nitrogen VLE Distribution } \\
\text { Coefficient }\end{array}$ \\
\hline & K_O2 & 1817195.406546 & & $\begin{array}{l}\text { Evaporator-Based Oxygen VLE Distribution } \\
\text { Coefficient }\end{array}$ \\
\hline & K_Ar & 1915296.044397 & & $\begin{array}{l}\text { Evaporator-Based Argon VLE Distribution } \\
\text { Coefficient }\end{array}$ \\
\hline & K_CO2 & 72743.446749 & & $\begin{array}{l}\text { Evaporator-Based Carbon Dioxide VLE Distribution } \\
\text { Coefficient }\end{array}$ \\
\hline & K_H2O & 1.039478 & & $\begin{array}{l}\text { Evaporator-Based Water VLE Distribution } \\
\text { Coefficient }\end{array}$ \\
\hline & T_S & 20 & ${ }^{\circ} \mathrm{C}$ & Stream Temperature \\
\hline & T_P & 20 & ${ }^{\circ} \mathrm{C}$ & Stream Temperature \\
\hline & $T_{-} \mathrm{x}$ & 28.842820 & ${ }^{\circ} \mathrm{C}$ & Stream Temperature \\
\hline \multirow[t]{4}{*}{50} & T_H & & ${ }^{\circ} \mathrm{C}$ & Stream Temperature \\
\hline & T_W & 40.218905 & ${ }^{\circ} \mathrm{C}$ & Stream Temperature \\
\hline & T_C & 22 & ${ }^{\circ} \mathrm{C}$ & Stream Temperature \\
\hline & T_E & 39.512635 & ${ }^{\circ} \mathrm{C}$ & Stream Temperature \\
\hline \multirow[t]{5}{*}{.035} & Ф_S & & & Stream Salt Mass Fraction \\
\hline & Q_P & .035 & & Stream Salt Mass Fraction \\
\hline & $\Phi_{-} \mathrm{X}$ & .035 & & Stream Salt Mass Fraction \\
\hline & Ф_H & .035 & & Stream Salt Mass Fraction \\
\hline & Ф_W & 0.034983 & & Stream Salt Mass Fraction \\
\hline \multirow[t]{4}{*}{0} & $\Phi \_C$ & & & Stream Salt Mass Fraction \\
\hline & Ф_E & 0 & & Stream Salt Mass Fraction \\
\hline & MW_S & 18.287997 & $\mathrm{~g} / \mathrm{mol}$ & Stream Molecular Weight \\
\hline & MW_P & 18.287997 & $\mathrm{~g} / \mathrm{mol}$ & Stream Molecular Weight \\
\hline
\end{tabular}




\section{Appendix M (Continued)}

\begin{tabular}{|c|c|c|c|c|}
\hline Input & Name & Output & Unit & Comment \\
\hline & $M W \_X$ & 18.287997 & $\mathrm{~g} / \mathrm{mol}$ & Stream Molecular Weight \\
\hline & MW_H & 18.287997 & $\mathrm{~g} / \mathrm{mol}$ & Stream Molecular Weight \\
\hline & MW_W & 18.292413 & $\mathrm{~g} / \mathrm{mol}$ & Stream Molecular Weight \\
\hline & $M W \_C$ & 18.0148 & $\mathrm{~g} / \mathrm{mol}$ & Stream Molecular Weight \\
\hline & MW_E & 18.023930 & $\mathrm{~g} / \mathrm{mol}$ & Stream Molecular Weight \\
\hline & M_S & 495.669161 & $\mathrm{~g} / \mathrm{min}$ & Stream Mass Flow Rate \\
\hline & M_P & 495.669161 & $\mathrm{~g} / \mathrm{min}$ & Stream Mass Flow Rate \\
\hline & M_X & 495.669161 & $\mathrm{~g} / \mathrm{min}$ & Stream Mass Flow Rate \\
\hline & M_H & 495.669161 & $\mathrm{~g} / \mathrm{min}$ & Stream Mass Flow Rate \\
\hline & M_W & 495.911874 & $\mathrm{~g} / \mathrm{min}$ & Stream Mass Flow Rate \\
\hline & M_C & -0.253082 & $\mathrm{~g} / \mathrm{min}$ & Stream Mass Flow Rate \\
\hline & M_E & -0.242713 & $\mathrm{~g} / \mathrm{min}$ & Stream Mass Flow Rate \\
\hline & N_S & 27.103524 & $\mathrm{~mol} / \mathrm{min}$ & Stream Molar Flow Rate \\
\hline & N_P & 27.103524 & $\mathrm{~mol} / \mathrm{min}$ & Stream Molar Flow Rate \\
\hline & $\mathrm{N} \_\mathrm{X}$ & 27.103524 & $\mathrm{~mol} / \mathrm{min}$ & Stream Molar Flow Rate \\
\hline & N_H & 27.103524 & $\mathrm{~mol} / \mathrm{min}$ & Stream Molar Flow Rate \\
\hline & N_W & 26.657744 & $\mathrm{~mol} / \mathrm{min}$ & Stream Molar Flow Rate \\
\hline & N_C & 0.445430 & $\mathrm{~mol} / \mathrm{min}$ & Stream Molar Flow Rate \\
\hline & N_E & 0.445779 & $\mathrm{~mol} / \mathrm{min}$ & Stream Molar Flow Rate \\
\hline & pA_S & 0.767535 & & Stream Density Coefficient \\
\hline & $\rho A \_P$ & 0.767535 & & Stream Density Coefficient \\
\hline & $\rho A_{-} X$ & 0.754059 & & Stream Density Coefficient \\
\hline & $\rho A_{-} H$ & 0.741681 & & Stream Density Coefficient \\
\hline & PA_W & 0.744091 & & Stream Density Coefficient \\
\hline & PA_C & 0.763992 & & Stream Density Coefficient \\
\hline & $\rho B \_S$ & -0.004341 & & Stream Density Coefficient \\
\hline & $\rho B \_P$ & -0.004341 & & Stream Density Coefficient \\
\hline & $\rho B_{-} X$ & -0.004151 & & Stream Density Coefficient \\
\hline & $\rho B \_H$ & -0.004748 & & Stream Density Coefficient \\
\hline & $\rho B \_W$ & -0.004288 & & Stream Density Coefficient \\
\hline & $\rho B_{-} C$ & -0.004276 & & Stream Density Coefficient \\
\hline & $\rho C \_s$ & 998.206319 & & Stream Density Coefficient \\
\hline & $\rho C_{-} P$ & 998.206319 & & Stream Density Coefficient \\
\hline & $\rho c_{-} x$ & 995.994512 & & Stream Density Coefficient \\
\hline & $\rho C \_H$ & 988.064493 & & Stream Density Coefficient \\
\hline & $\rho C \_w$ & 992.136617 & & Stream Density Coefficient \\
\hline & $\rho C_{-} c$ & 997.773037 & & Stream Density Coefficient \\
\hline
\end{tabular}




\section{Appendix M (Continued)}

\begin{tabular}{|c|c|c|c|c|}
\hline Input & Name & Output & Unit & Comment \\
\hline & p_s & 1.024763 & $\mathrm{~g} / \mathrm{cm}^{\wedge} 3$ & Stream Density \\
\hline & P_P & 1.024763 & $\mathrm{~g} / \mathrm{cm}^{\wedge} 3$ & Stream Density \\
\hline & 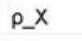 & 1.022119 & $\mathrm{~g} / \mathrm{cm}^{\wedge} 3$ & Stream Density \\
\hline & P_H & 1.013632 & $\mathrm{~g} / \mathrm{cm}^{\wedge} 3$ & Stream Density \\
\hline & P_W & 1.017871 & $\mathrm{~g} / \mathrm{cm}^{\wedge} 3$ & Stream Density \\
\hline & P_C & 0.997773 & $\mathrm{~g} / \mathrm{cm}^{\wedge} 3$ & Stream Density \\
\hline & P_E & 0.000049 & $\mathrm{~g} / \mathrm{cm}^{\wedge} 3$ & Stream Density \\
\hline & $\mu A \_S$ & 0.001144 & & Stream Viscosity Coefficient \\
\hline & HA_P & 0.001144 & & Stream Viscosity Coefficient \\
\hline & $\mu A \_X$ & 0.001602 & & Stream Viscosity Coefficient \\
\hline & HA_H & 0.002699 & & Stream Viscosity Coefficient \\
\hline & HA_W & 0.002192 & & Stream Viscosity Coefficient \\
\hline & $\mu A \_C$ & 0.001247 & & Stream Viscosity Coefficient \\
\hline & $\mu \mathrm{B} \_\mathrm{S}$ & .003251 & & Stream Viscosity Coefficient \\
\hline & $\mu B_{-} P$ & .003251 & & Stream Viscosity Coefficient \\
\hline & $\mu \mathrm{B}_{-} \mathrm{X}$ & 0.003543 & & Stream Viscosity Coefficient \\
\hline & $\mu B_{-} H$ & .004241 & & Stream Viscosity Coefficient \\
\hline & $\mu B_{-} W$ & 0.003918 & & Stream Viscosity Coefficient \\
\hline & $\mu \mathrm{B} \_\mathrm{C}$ & .003317 & & Stream Viscosity Coefficient \\
\hline & $\mu \mathrm{C} \_\mathrm{S}$ & 19.853702 & & Stream Viscosity Coefficient \\
\hline & $\mu C \_P$ & 19.853702 & & Stream Viscosity Coefficient \\
\hline & $\mu C_{-} X$ & 19.802474 & & Stream Viscosity Coefficient \\
\hline & $\mu \mathrm{C} \_\mathrm{H}$ & 19.638053 & & Stream Viscosity Coefficient \\
\hline & $\mu C \_W$ & 19.710527 & & Stream Viscosity Coefficient \\
\hline & $\mu C_{-} C$ & 0 & & Stream Viscosity Coefficient \\
\hline & $\mu D \_S$ & .01002 & & Stream Viscosity Coefficient \\
\hline & $\mu D_{-} P$ & .01002 & & Stream Viscosity Coefficient \\
\hline & $\mu D_{-} X$ & 0.008175 & & Stream Viscosity Coefficient \\
\hline & $\mu D_{-} H$ & 0.005471 & & Stream Viscosity Coefficient \\
\hline & $\mu D_{-} W$ & 0.006505 & & Stream Viscosity Coefficient \\
\hline & $\mu D_{-} C$ & 0.009547 & & Stream Viscosity Coefficient \\
\hline & $\mu \_s$ & 0.010718 & $P$ & Stream Viscosity \\
\hline & $\mu \_P$ & 0.010718 & $P$ & Stream Viscosity \\
\hline & $\mu \_x$ & 0.008807 & $P$ & Stream Viscosity \\
\hline & H_H & 0.005992 & $P$ & Stream Viscosity \\
\hline & H_W & 0.007070 & $P$ & Stream Viscosity \\
\hline & H_C & 0.009547 & $\mathrm{P}$ & Stream Viscosity \\
\hline
\end{tabular}




\section{Appendix M (Continued)}

\begin{tabular}{|c|c|c|c|c|}
\hline Input & Name & Output & Unit & Comment \\
\hline & H_E & 0.000096 & $P$ & Stream Viscosity \\
\hline & k_s & 0.005972 & $\mathrm{~W} /\left(\mathrm{cm}-{ }^{\circ} \mathrm{C}\right)$ & Stream Thermal Conductivity \\
\hline & k_P & 0.005972 & $\mathrm{~W} /\left(\mathrm{cm}-{ }^{\circ} \mathrm{C}\right)$ & Stream Thermal Conductivity \\
\hline & k_X & 0.006099 & $\mathrm{~W} /\left(\mathrm{cm}-{ }^{\circ} \mathrm{C}\right)$ & Stream Thermal Conductivity \\
\hline & k_H & 0.006364 & $\mathrm{~W} /\left(\mathrm{cm}-{ }^{\circ} \mathrm{C}\right)$ & Stream Thermal Conductivity \\
\hline & k_W & 0.006248 & $\mathrm{~W} /\left(\mathrm{cm}-{ }^{\circ} \mathrm{C}\right)$ & Stream Thermal Conductivity \\
\hline & k_C & 0.006060 & $\mathrm{~W} /\left(\mathrm{cm}-{ }^{\circ} \mathrm{C}\right)$ & Stream Thermal Conductivity \\
\hline & k_E & 0.000206 & $\mathrm{~W} /\left(\mathrm{cm}-{ }^{\circ} \mathrm{C}\right)$ & Stream Thermal Conductivity \\
\hline & Re_S & 772.754293 & & Stream Reynolds Number \\
\hline & Re_P & 772.754293 & & Stream Reynolds Number \\
\hline & $\operatorname{Re} X X$ & 940.429542 & & Stream Reynolds Number \\
\hline & $\mathrm{Re} \_\mathrm{H}$ & 1382.136449 & & Stream Reynolds Number \\
\hline & Re_W & 1171.973697 & & Stream Reynolds Number \\
\hline & Re_C & -1.771727 & & Stream Reynolds Number \\
\hline & Re_E & -10.539154 & & Stream Reynolds Number \\
\hline & Pr_S & 0.488137 & & Stream Prandtl Number \\
\hline & Pr_P & 0.488137 & & Stream Prandtl Number \\
\hline & Pr_X & 0.550268 & & Stream Prandtl Number \\
\hline & Pr_H & 0.582159 & & Stream Prandtl Number \\
\hline & Pr_W & 0.579965 & & Stream Prandtl Number \\
\hline & Pr_C & 0.492048 & & Stream Prandtl Number \\
\hline & Pr_E & 0.247172 & & Stream Prandtl Number \\
\hline & Nu_s & & & Stream Nusselt Number \\
\hline & Nu_P & & & Stream Nusselt Number \\
\hline & Nu_X & & & Stream Nusselt Number \\
\hline & $\mathrm{Nu} \_\mathrm{H}$ & & & Stream Nusselt Number \\
\hline & Nu_W & & & Stream Nusselt Number \\
\hline & Nu_C & & & Stream Nusselt Number \\
\hline & Nu_E & & & Stream Nusselt Number \\
\hline 1.27 & PD_S & & $\mathrm{cm}$ & Stream Nominal Pipe Diameter \\
\hline 1.27 & PD_P & & $\mathrm{cm}$ & Stream Nominal Pipe Diameter \\
\hline 1.27 & $P D_{-} X$ & & $\mathrm{~cm}$ & Stream Nominal Pipe Diameter \\
\hline 1.27 & PD_H & & $\mathrm{cm}$ & Stream Nominal Pipe Diameter \\
\hline 1.27 & PD_W & & $\mathrm{cm}$ & Stream Nominal Pipe Diameter \\
\hline 5.08 & PD_E & & $\mathrm{cm}$ & Stream Nominal Pipe Diameter \\
\hline .3175 & PD_C & & $\mathrm{cm}$ & Stream Nominal Pipe Diameter \\
\hline 95 & PL_S & & $\mathrm{cm}$ & Stream Equivalent Pipe Length \\
\hline 25 & $P L \_P$ & & $\mathrm{~cm}$ & Stream Equivalent Pipe Length \\
\hline 60 & PL_X & & $\mathrm{cm}$ & Stream Equivalent Pipe Length \\
\hline 75 & PL_H & & $\mathrm{cm}$ & Stream Equivalent Pipe Length \\
\hline
\end{tabular}




\section{Appendix M (Continued)}

\begin{tabular}{|c|c|c|c|c|}
\hline Input & Name & Output & Unit & Comment \\
\hline 30 & PL_W & & $\mathrm{cm}$ & Stream Equivalent Pipe Length \\
\hline 180 & PL_E & & $\mathrm{cm}$ & Stream Equivalent Pipe Length \\
\hline \multirow[t]{11}{*}{35} & PL_C & & $\mathrm{cm}$ & Stream Equivalent Pipe Length \\
\hline & $\Delta P_{-} S$ & 0.000129 & bar & Stream Pressure Drop \\
\hline & $\Delta P_{-} P$ & 0.000034 & bar & Stream Pressure Drop \\
\hline & $\Delta P_{-} X$ & 0.000067 & bar & Stream Pressure Drop \\
\hline & $\Delta P_{-} H$ & 0.000057 & bar & Stream Pressure Drop \\
\hline & $\Delta P_{-} W$ & 0.000027 & bar & Stream Pressure Drop \\
\hline & $\Delta P_{-} C$ & -0.000006 & bar & Stream Pressure Drop \\
\hline & $\Delta P_{-} E$ & 0.000000 & bar & Stream Pressure Drop \\
\hline & $\triangle P_{-} C T$ & 0.000530 & bar & Condenser Tube Side Pressure Drop \\
\hline & $\Delta P_{-} H T$ & 0.000010 & bar & Heater Tube Side Pressure Drop \\
\hline & $\Delta \mathrm{P}$ & 0.000826 & bar & Total Pressure Drop From Pump to Valve \\
\hline 90 & D_S & & $\mathrm{cm}$ & Seawater Tank Nominal Diameter \\
\hline 35 & D_B & & $\mathrm{cm}$ & Brine Water Tank Nominal Diameter \\
\hline 35 & D_F & & $\mathrm{cm}$ & Fresh Water Tank Nominal Diameter \\
\hline 35 & D_E & & $\mathrm{cm}$ & Evaporator Nominal Diameter \\
\hline 35 & $D_{-} \mathrm{C}$ & & $\mathrm{cm}$ & Condenser Nominal Diameter \\
\hline 30 & L_S & & $\mathrm{cm}$ & Seawater Tank Length \\
\hline 160 & L_B & & $\mathrm{cm}$ & Brine Water Tank Length \\
\hline 160 & L_F & & $\mathrm{cm}$ & Fresh Water Tank Length \\
\hline 160 & L_E & & $\mathrm{cm}$ & Evaporator Length \\
\hline \multirow[t]{15}{*}{160} & L_C & & $\mathrm{cm}$ & Condenser Length \\
\hline & XA_S & 6361.725124 & $\mathrm{~cm}^{\wedge} 2$ & Seawater Tank Cross Sectional Area \\
\hline & XA_B & 962.112750 & $\mathrm{~cm}^{\wedge} 2$ & Brine Water Tank Cross Sectional Area \\
\hline & XA_F & 962.112750 & $\mathrm{~cm}^{\wedge} 2$ & Fresh Water Tank Cross Sectional Area \\
\hline & XA_E & 962.112750 & $\mathrm{~cm}^{\wedge} 2$ & Evaporator Cross Sectional Area \\
\hline & XA_C & 962.112750 & $\mathrm{~cm}^{\wedge} 2$ & Condenser Cross Sectional Area \\
\hline & v_s & 190851.753706 & $\mathrm{~cm}^{\wedge} 3$ & Seawater Tank Volume \\
\hline & V_B & 153938.040026 & $\mathrm{~cm}^{\wedge} 3$ & Brine Water Tank Volume \\
\hline & V_F & 153938,040026 & $\mathrm{~cm}^{\wedge} 3$ & Fresh Water Tank Volume \\
\hline & V_E & 153938.040026 & $\mathrm{~cm}^{\wedge} 3$ & Evaporator Volume \\
\hline & $V_{-} C$ & 153938.040026 & $\mathrm{~cm}^{\wedge} 3$ & Condenser Volume \\
\hline & Zi_s & 24 & $\mathrm{~cm}$ & Initial Seawater Tank Level \\
\hline & Zi_E & 0 & $\mathrm{~cm}$ & Initial Evaporator Level \\
\hline & Zi_c & 0 & $\mathrm{~cm}$ & Initial Condenser Level \\
\hline & z_s & 23.923968 & $\mathrm{~cm}$ & Seawater Tank Level \\
\hline 5 & $Z_{-} B$ & & $\mathrm{~cm}$ & Brine Water Tank Level \\
\hline 5 & Z_F & & $\mathrm{cm}$ & Fresh Water Tank Level \\
\hline
\end{tabular}




\section{Appendix M (Continued)}

\begin{tabular}{|c|c|c|c|c|}
\hline Input & Name & Output & Unit & Comment \\
\hline & Z_E & 0.506391 & $\mathrm{~cm}$ & Evaporator Level \\
\hline & $z_{-} c$ & -0.000264 & $\mathrm{~cm}$ & Condenser Level \\
\hline & Vi_CV & 153938.040026 & $\mathrm{~cm}^{\wedge} 3$ & Initial Condenser Vacuum Volume \\
\hline & Vi_EV & 153938.040026 & $\mathrm{~cm}^{\wedge} 3$ & Initial Evaporator Vacuum Volume \\
\hline & Vi_V & 310922.658770 & $\mathrm{~cm}^{\wedge} 3$ & Initial Total Vacuum Volume \\
\hline & V_CV & 153938.293673 & $\mathrm{~cm}^{\wedge} 3$ & Condenser Vacuum Volume \\
\hline & V_EV & 153450.835048 & $\mathrm{~cm}^{\wedge} 3$ & Evaporator Vacuum Volume \\
\hline & $v_{-} v$ & 310435.707439 & $\mathrm{~cm} \wedge 3$ & Total Vacuum Volume \\
\hline .118 & $\Omega$ & & bar & Steam Diffusion Resistance \\
\hline \multirow[t]{19}{*}{2} & $\sigma$ & & $\begin{array}{l}\left(\mathrm{g}-{ }^{\circ} \mathrm{C}^{\wedge} 0.5\right) /(\text { bar } \\
\left.-\min -\mathrm{cm}^{\wedge} 2\right)\end{array}$ & Steam Diffusion Coefficient \\
\hline & Md_E & -8.277411 & $g / \min$ & Steam Diffusion Rate \\
\hline & Ed_E & 20008.593395 & $\mathrm{~J} / \mathrm{min}$ & Steam Diffusion Energy \\
\hline & $\psi$ & 0.685501 & & Fraction of NCG Molecules Accumulating \\
\hline & ENai_C & 1.785895 & moles & Initial NCG Molar Amount \\
\hline & Y_H2O & 2.061926 & & Water Activity Coefficient \\
\hline & $\mathrm{Pi} \_\mathrm{H} 2 \mathrm{O}$ & 0.022722 & bar & Initial Vapor Pressure \\
\hline & P_H2O & 0.070578 & bar & Vapor Pressure \\
\hline & Pi_V & .14 & bar & Initial Vacuum Pressure \\
\hline & Pt_V & 0.150395 & bar & Transitional Vacuum Pressure \\
\hline & P_V & .14 & bar & Vacuum Pressure \\
\hline & Ein_P & 39524.846869 & $\mathrm{~J} / \min$ & Pump Energy Input \\
\hline & Ein_C & 38899.169216 & $\mathrm{~J} / \mathrm{min}$ & Condenser Energy Input \\
\hline & Ein_H & 57039.694651 & $\mathrm{~J} / \mathrm{min}$ & Heater Energy Input \\
\hline & Ein_E & 99023.636026 & $\mathrm{~J} / \mathrm{min}$ & Evaporator Energy Input \\
\hline & Eout_P & 39524.846869 & $\mathrm{~J} / \mathrm{min}$ & Pump Energy Output \\
\hline & Eout_C & 57016.366136 & $\mathrm{~J} / \mathrm{min}$ & Condenser Energy Output \\
\hline & Eout_H & 99023.636026 & $\mathrm{~J} / \mathrm{min}$ & Heater Energy Output \\
\hline & Eout_E & 79015.042631 & $\mathrm{~J} / \mathrm{min}$ & Evaporator Energy Output \\
\hline \multirow[t]{3}{*}{0} & Q_P & & $\mathrm{J} / \mathrm{min}$ & Pump Heat Input \\
\hline & Q_C & 18117.338871 & $\mathrm{~J} / \mathrm{min}$ & Condenser Heat Input \\
\hline & Q_H & 41983.941375 & $\mathrm{~J} / \mathrm{min}$ & Heater Heat Input \\
\hline \multirow[t]{2}{*}{0} & Q_E & & $\mathrm{J} / \mathrm{min}$ & Evaporator Heat Input \\
\hline & W_P & 0 & $\mathrm{~J} / \mathrm{min}$ & Pump Work Output \\
\hline 0 & W_C & & $\mathrm{J} / \mathrm{min}$ & Condenser Work Output \\
\hline 0 & W_H & & $\mathrm{J} / \mathrm{min}$ & Heater Work Output \\
\hline \multirow[t]{3}{*}{0} & W_E & & $\mathrm{J} / \mathrm{min}$ & Evaporator Work Output \\
\hline & ka_c & 0.000272 & $\mathrm{~W} /\left(\mathrm{cm}-{ }^{\circ} \mathrm{C}\right)$ & NCG Thermal Conductivity \\
\hline & $\mathrm{Ha} \_\mathrm{C}$ & 13.689610 & $\mathrm{~J} / \mathrm{g}$ & NCG Enthalpy \\
\hline
\end{tabular}




\section{Appendix M (Continued)}

\begin{tabular}{|c|c|c|c|c|}
\hline Input & Name & Output & Unit & Comment \\
\hline & MWa_C & 29.655207 & $\mathrm{~g} / \mathrm{mol}$ & NCG Molecular Weight \\
\hline & Ma_C & 0.010369 & $\mathrm{~g} / \mathrm{min}$ & NCG Mass Accumulation Rate \\
\hline & $\mathrm{Na} \_\mathrm{C}$ & 0.000350 & $\mathrm{~mol} / \mathrm{min}$ & NCG Molar Accumulation Rate \\
\hline & $\mathrm{Ea} \_\mathrm{C}$ & 0.141950 & $\mathrm{~J} / \mathrm{min}$ & NCG Energy Accumulation \\
\hline 2 & Z_p & & $\mathrm{cm}$ & Pump Center Elevation \\
\hline \multirow[t]{8}{*}{250} & $z_{-} O$ & & $\mathrm{~cm}$ & Expansion Orifice Center Elevation \\
\hline & HP_P & 0 & HP & Pump Power \\
\hline & NPSH & 1008.068669 & $\mathrm{~cm}$ & Net Positive Suction Head \\
\hline & P_O & 0.763349 & bar & Expansion Orifice Inlet Pressure \\
\hline & c_o & 66.261451 & $1 / \mathrm{cm}^{\wedge} 2$ & Expansion Orifice Coefficient \\
\hline & D_O & 0.122846 & $\mathrm{~cm}$ & Expansion Orifice Nominal Diameter \\
\hline & SG_H & 1.025876 & & Stream Specific Gravity \\
\hline & Mmax_o & 7290.073786 & $g / \min$ & Maximum Flow Delivered By Needle Valve \\
\hline .64 & $\mathrm{CV}_{-} \mathrm{O}$ & & & Needle Valve Flow Coefficient \\
\hline 0.546461 & $\mathrm{CV}$ & & & Ball Valve Flow Coefficient \\
\hline 1.27 & $D_{-} H T$ & & $\mathrm{~cm}$ & Heater Tube Diameter \\
\hline 13 & L_HT & & $\mathrm{cm}$ & Heater Tube Length \\
\hline 1.27 & D_CT & & $\mathrm{cm}$ & Condenser Tube Diameter \\
\hline 475 & L_CT & & $\mathrm{cm}$ & Condenser Tube Length \\
\hline 2 & hid_CT & & $W /\left(\mathrm{cm}^{\wedge} 2-{ }^{\circ} \mathrm{C}\right)$ & Condenser Tube Inside Dirt Coefficient \\
\hline \multirow[t]{3}{*}{.5} & hod_CT & & $\mathrm{W} /\left(\mathrm{cm} \wedge 2-{ }^{\circ} \mathrm{C}\right)$ & Condenser Tube Outside Dirt Coefficient \\
\hline & hi_CT & 0.053853 & $W /\left(\mathrm{cm}^{\wedge} 2-{ }^{\circ} \mathrm{C}\right)$ & Condenser Tube Inside Fluid Film Coefficient \\
\hline & ho_CT & 0.737128 & $W /\left(\mathrm{cm}^{\wedge} 2-{ }^{\circ} \mathrm{C}\right)$ & Condenser Tube Outside Fluid Film Coefficient \\
\hline .5 & hid_C & & $W /\left(\mathrm{cm}^{\wedge} 2-{ }^{\circ} \mathrm{C}\right)$ & Condenser Inside Dirt Coefficient \\
\hline \multirow[t]{3}{*}{.75} & hod_c & & $\mathrm{W} /\left(\mathrm{cm}^{\wedge} 2-{ }^{\circ} \mathrm{C}\right)$ & Condenser Outside Dirt Coefficient \\
\hline & hi_C & 0.411422 & $W /\left(\mathrm{cm}^{\wedge} 2-{ }^{\circ} \mathrm{C}\right)$ & Condenser Inside Fluid Film Coefficient \\
\hline & ho_c & 0.026460 & $W /\left(\mathrm{cm}^{\wedge} 2-{ }^{\circ} \mathrm{C}\right)$ & Condenser Outside Fluid Film Coefficient \\
\hline .5 & hid_E & & $W /\left(c n^{\wedge} 2-{ }^{\circ} \mathrm{C}\right)$ & Evaporator Inside Dirt Coefficient \\
\hline \multirow[t]{3}{*}{.75} & hod_E & & $W /\left(\mathrm{cm}^{\wedge} 2-{ }^{\circ} \mathrm{C}\right)$ & Evaporator Outside Dirt Coefficient \\
\hline & hi_E & 0.458323 & $\mathrm{~W} /\left(\mathrm{cm} \wedge 2-{ }^{\circ} \mathrm{C}\right)$ & Evaporator Inside Fluid Film Coefficient \\
\hline & ho_E & 0.026460 & $W /\left(\mathrm{cm}^{\wedge} 2-{ }^{\circ} \mathrm{C}\right)$ & Evaporator Outside Fluid Film Coefficient \\
\hline 4 & N_CT & & & Number of Condenser Tube Vertical Rows \\
\hline .125 & 8_CT & & $\mathrm{cm}$ & Condenser Tube Thickness \\
\hline 3.81 & $\mathrm{~kW} \_\mathrm{CT}$ & & $\mathrm{W} /\left(\mathrm{cm}-{ }^{\circ} \mathrm{C}\right)$ & Condenser Tube Thermal Conductivity \\
\hline .25 & o_c & & $\mathrm{cm}$ & Condenser Thickness \\
\hline .45 & kw_C & & $\mathrm{W} /\left(\mathrm{cm}-{ }^{\circ} \mathrm{C}\right)$ & Condenser Thermal Conductivity \\
\hline .25 & ס_E & & $\mathrm{cm}$ & Evaporator Thickness \\
\hline \multirow[t]{4}{*}{.001} & kW_E & & $\mathrm{W} /\left(\mathrm{cm}-{ }^{\circ} \mathrm{C}\right)$ & Evaporator Thermal Conductivity \\
\hline & U_CT & 0.034173 & $W /\left(\mathrm{cm}^{\wedge} 2-{ }^{\circ} \mathrm{C}\right)$ & Condenser Tube Overall Heat Transfer Coefficient \\
\hline & A_CT & 1895.165768 & $\mathrm{~cm} \wedge 2$ & Condenser Tube Surface Area \\
\hline & S_CT & 0.453184 & & $\begin{array}{l}\text { Condenser Tube Counter Current Departure } \\
\text { Parameter }\end{array}$ \\
\hline
\end{tabular}




\section{Appendix M (Continued)}

\begin{tabular}{|c|c|c|c|c|}
\hline Input & Name & Output & Unit & Comment \\
\hline & F_CT & 0.307688 & & $\begin{array}{l}\text { Condenser Tube Counter Current Departure } \\
\text { Correction Factor }\end{array}$ \\
\hline & $\Delta \mathrm{Tm} \_\mathrm{CT}$ & 14.649078 & ${ }^{\circ} \mathrm{C}$ & $\begin{array}{l}\text { Condenser Tube Logarithmic Mean Temperature } \\
\text { Difference }\end{array}$ \\
\hline & U_C & 0.022796 & $\mathrm{~W} /\left(\mathrm{cm}^{\wedge} 2-{ }^{\circ} \mathrm{C}\right)$ & Condenser Overall Heat Transfer Coefficient \\
\hline & A_C & 17592.918860 & $\mathrm{~cm}^{\wedge} 2$ & Condenser Surface Area \\
\hline & S_c & 0.102498 & & Condenser Counter Current Departure Parameter \\
\hline & F_C & -0.040709 & & $\begin{array}{l}\text { Condenser Counter Current Departure Correction } \\
\text { Factor }\end{array}$ \\
\hline & $\Delta T m_{-} C$ & 18.494616 & ${ }^{\circ} \mathrm{C}$ & $\begin{array}{l}\text { Condenser Logarithmic Mean Temperature } \\
\text { Difference }\end{array}$ \\
\hline & U_E & 0.005925 & $\mathrm{~W} /\left(\mathrm{cm}^{\wedge} 2-{ }^{\circ} \mathrm{C}\right)$ & Evaporator Overall Heat Transfer Coefficient \\
\hline & A_E & 17592.918860 & $\mathrm{~cm} \wedge 2$ & Evaporator Surface Area \\
\hline & S_E & 1.036195 & & Evaporator Counter Current Departure Parameter \\
\hline & $F_{-} E$ & 0 & & $\begin{array}{l}\text { Evaporator Counter Current Departure Correction } \\
\text { Factor }\end{array}$ \\
\hline & $\Delta T m_{-} E$ & 6.092195 & ${ }^{\circ} \mathrm{C}$ & $\begin{array}{l}\text { Evaporator Logarithmic Mean Temperature } \\
\text { Difference }\end{array}$ \\
\hline & BPE & & ${ }^{\circ} \mathrm{C}$ & Boiling Point Elevation \\
\hline & NEA & & ${ }^{\circ} \mathrm{C}$ & Non Equilibrium Allowance \\
\hline \multirow[t]{4}{*}{75} & $\eta P$ & & $\%$ & Pumping Efficiency \\
\hline & nR & 29.437318 & $\%$ & Recovery Efficiency \\
\hline & $\eta T$ & -0.631847 & $\%$ & Thermal Efficiency \\
\hline & nC & 50.493944 & $\%$ & Condenser Efficiency \\
\hline .92 & a_sC & & & Solar Collector Absorptance \\
\hline .9 & T_SC & & & Solar Collector Transmittance \\
\hline .000092 & U_SC & & $\mathrm{W} /\left(\mathrm{cm}^{\wedge} 2-{ }^{\circ} \mathrm{C}\right)$ & Solar Collector Heat Loss Conductance \\
\hline .82 & F_SC & & & Solar Collector Heat Removal Factor \\
\hline \multirow[t]{2}{*}{.06} & 1 & & $W / \mathrm{cm}^{\wedge} 2$ & Incident Insolation On Solar Collector \\
\hline & A_SC & 17462.532227 & $\mathrm{~cm} \wedge 2$ & Solar Collector Area \\
\hline 21.022222 & T_Xe & & ${ }^{\circ} \mathrm{C}$ & Experimental Value of Stream Temperature \\
\hline 21.240556 & T_We & & ${ }^{\circ} \mathrm{C}$ & Experimental Value of Stream Temperature \\
\hline 24.241111 & T_Ee & & ${ }^{\circ} \mathrm{C}$ & Experimental Value of Stream Temperature \\
\hline 0.140585 & Pt_Ve & & bar & Experimental Value of Vacuum Pressure \\
\hline
\end{tabular}


Appendix N. Experimental record

\begin{tabular}{|c|c|c|c|c|c|c|c|c|c|c|}
\hline Number & Date & Start & Stop & $\begin{array}{c}t \\
\text { (minutes) }\end{array}$ & $\begin{array}{c}P E^{i} \\
\text { (bar) }\end{array}$ & $\begin{array}{c}V_{S}^{i} \\
\text { (Gallon) }\end{array}$ & $\begin{array}{c}V_{S}^{f} \\
\text { (Gallon) }\end{array}$ & $\begin{array}{c}M_{S} \\
(L P M)\end{array}$ & $\begin{array}{l}\text { TIC } \\
\left({ }^{\circ} \mathrm{C}\right)\end{array}$ & $\begin{array}{l}Q E \\
(m l)\end{array}$ \\
\hline 1 & $4 / 24 / 09$ & 3:20:00 PM & 6:20:00 PM & 180 & 0.140 & 40 & 17 & 0.48 & 50 & 30 \\
\hline 2 & $4 / 25 / 09$ & 1:15:00 PM & 4:15:00 PM & 180 & 0.140 & 40 & 17 & 0.48 & 50 & 15 \\
\hline 3 & $4 / 26 / 09$ & 2:09:00 РМ & 5:09:00 PM & 180 & 0.140 & 40 & 17 & 0.48 & 50 & 36 \\
\hline 4 & $4 / 27 / 09$ & 12:55:00 PM & 3:55:00 PM & 180 & 0.140 & 40 & 18 & 0.46 & 60 & 345 \\
\hline 5 & $4 / 28 / 09$ & 12:42:00 PM & 3:42:00 PM & 180 & 0.140 & 40 & 18 & 0.46 & 60 & 350 \\
\hline 6 & 4/29/09 & 12:37:00 PM & 3:37:00 PM & 180 & 0.140 & 40 & 18 & 0.46 & 60 & 360 \\
\hline 7 & $4 / 30 / 09$ & 1:17:00 PM & 4:17:00 PM & 180 & 0.140 & 40 & 19 & 0.44 & 70 & 2030 \\
\hline 8 & $5 / 1 / 09$ & 2:49:00 PM & 5:49:00 PM & 180 & 0.140 & 40 & 19 & 0.44 & 70 & 2050 \\
\hline 9 & $5 / 2 / 09$ & 1:07:00 PM & 4:07:00 PM & 180 & 0.140 & 40 & 19 & 0.44 & 70 & 2030 \\
\hline 10 & $5 / 3 / 09$ & 1:37:00 PM & 4:37:00 PM & 180 & 0.140 & 40 & 22 & 0.38 & 80 & 4880 \\
\hline 11 & $5 / 4 / 09$ & 1:17:00 PM & 4:17:00 PM & 180 & 0.140 & 40 & 22 & 0.38 & 80 & 4720 \\
\hline 12 & $5 / 5 / 09$ & 1:47:00 PM & 4:47:00 PM & 180 & 0.140 & 40 & 22 & 0.38 & 80 & 4560 \\
\hline 13 & $5 / 15 / 09$ & 1:13:00 PM & 4:13:00 PM & 180 & 0.140 & 40 & 7 & 0.69 & 50 & 13 \\
\hline 14 & $5 / 16 / 09$ & 1:04:00 PM & 4:04:00 PM & 180 & 0.140 & 40 & 7 & 0.69 & 50 & 25 \\
\hline 15 & $5 / 17 / 09$ & 2:24:00 PM & 5:24:00 PM & 180 & 0.140 & 40 & 7 & 0.69 & 50 & 27 \\
\hline 16 & $5 / 18 / 09$ & 12:24:00 PM & 3:24:00 PM & 180 & 0.140 & 40 & 8 & 0.67 & 60 & 190 \\
\hline 17 & $5 / 19 / 09$ & 12:30:00 PM & 3:30:00 PM & 180 & 0.140 & 40 & 8 & 0.67 & 60 & 205 \\
\hline 18 & $5 / 20 / 09$ & 12:59:00 PM & 3:59:00 PM & 180 & 0.140 & 40 & 8 & 0.67 & 60 & 200 \\
\hline 19 & $5 / 21 / 09$ & 12:24:00 PM & 3:24:00 PM & 180 & 0.140 & 40 & 9 & 0.65 & 70 & 1310 \\
\hline 20 & $5 / 22 / 09$ & 1:24:00 PM & 4:24:00 PM & 180 & 0.140 & 40 & 9 & 0.65 & 70 & 1180 \\
\hline 21 & $5 / 23 / 09$ & 1:39:00 PM & 4:39:00 PM & 180 & 0.140 & 40 & 9 & 0.65 & 70 & 1145 \\
\hline 22 & $5 / 24 / 09$ & 2:42:00 PM & 5:42:00 PM & 180 & 0.140 & 40 & 13 & 0.57 & 80 & 4995 \\
\hline 23 & $5 / 25 / 09$ & 1:04:00 PM & 4:04:00 PM & 180 & 0.140 & 40 & 13 & 0.57 & 80 & 4770 \\
\hline 24 & $5 / 26 / 09$ & 1:04:00 PM & 4:04:00 PM & 180 & 0.140 & 40 & 13 & 0.57 & 80 & 4365 \\
\hline
\end{tabular}




\section{Appendix O. Experimental equipment specifications}

\section{Seawater Tank-McMaster-Carr}

Polyethylene Troughs

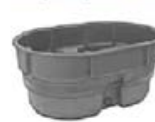

Pohethytene troughs are buil tough so they wont rust, dent, of leak-even when frozen. All are nestable.

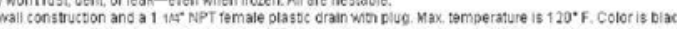

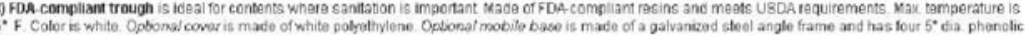
swivel casters. Base adds $67 \mathrm{TS}^{\circ}$ to trough height

\begin{tabular}{|c|c|c|c|c|c|c|}
\hline \multirow[b]{2}{*}{ Cap. } & O'all & \multicolumn{5}{|l|}{ Onil } \\
\hline & $\begin{array}{l}\text { Body Size, } \\
\text { Loxwo.t }\end{array}$ & Top Slize, & Oall & $\begin{array}{l}\text { Wall } \\
\text { Thick. }\end{array}$ & & Each \\
\hline \multicolumn{7}{|c|}{ Structural-Foam Trougyts } \\
\hline & $4910^{\circ} \times 28500^{\circ}$ & $52 \times 31^{-}$ & $12^{\prime}$ & 14 & 3673K49 & 1118.22 \\
\hline 100 & $4911^{\circ} \times 28112^{\circ}$ & $53 \times 31^{-1}$ & 25 & $20 a^{2}$ & $3673 k 52$ & 109.36 \\
\hline 150 & $55^{\circ} \times 36 \mathrm{us}^{\circ}$ & $58 \times 39$ & 25 & Q10 $10^{\circ}$ & $3673 K 53$ & 183.02 \\
\hline 300 & $66,14^{*} \times 60112^{*}$ & $68 \times 631 \mathrm{ma}$ & 25 & $010^{\circ}$ & $3673 K 54$ & 284.35 \\
\hline
\end{tabular}

1 Condenser-McMaster-Carr

ASHE Painted Steel Tanks Manulactured in accordance with ASME codes. Maximum pressure is 150 psi. Mazimum
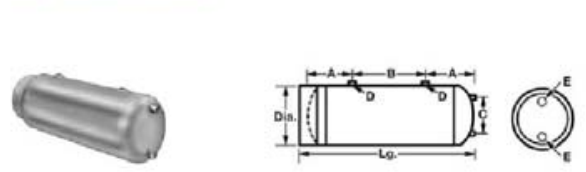
aperating temporature is $450^{\circ}$ F. Tanks have a gray enamel finith

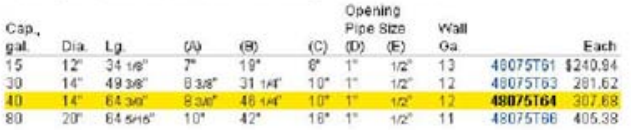

1 Evaporator-McMaster-Carr

Galvanized-Steel Tanks
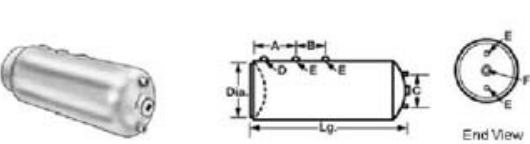

Maximum pressurs is 75 psi Msamum eperating temparants is $450^{\circ} F$. Tanks ar

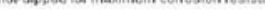

End Vew

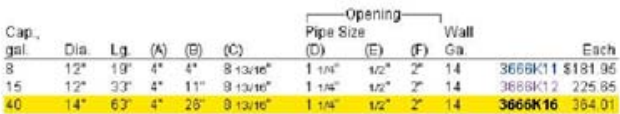

1 Electric Heater-McMaster-Carr

Water Heater Replacement Parts

Heater elements are made of zinc-plated capper and must be mounted horizontally, Screst in stive have 1 " NPSEX Dolts are not. Flange is stee

(hertiostats ara for 120 to $480 \mathrm{VAC}$. Temp. range is $120^{\circ}$ to $160^{\circ} \mathrm{F}$

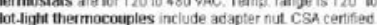

\begin{tabular}{|c|c|c|c|c|}
\hline \multicolumn{5}{|c|}{ Heater Elements } \\
\hline \multirow{2}{*}{\multicolumn{5}{|c|}{$\begin{array}{l}\text { Watis } \\
\text { Screw-In }\end{array}$}} \\
\hline & & & & \\
\hline 1500 & 120 (1) & 12.5 & 3 $12^{\circ}$ & $355555 \mathrm{~K} 31 \$ 9.12$ \\
\hline 1500 & $240(1)$ & 6.25 & $81 \varepsilon^{*}$ & $35555 K 3449.20$ \\
\hline 4600 & 2400 & 1876 & 17 & $36656 k 328.49$ \\
\hline \multirow{2}{*}{\multicolumn{5}{|c|}{ Flat Flange Bolt-ln }} \\
\hline & & & & \\
\hline 1500 & 120 (1) & 12.5 & $81 \alpha^{\circ}$ & $35555 \mathrm{~K} 2110.89$ \\
\hline 4500 & 240 (1) & 18.75 & 12 & $35555 K 2211.12$ \\
\hline \multirow{2}{*}{\multicolumn{5}{|c|}{ Rassed Fange Boll in }} \\
\hline & & & & \\
\hline 1500 & 120 (1) & 12.5 & $811 z^{\prime}$ & $\begin{array}{l}355555 \mathrm{~K} 41 \\
0.600\end{array}$ \\
\hline 4500 & $240(1)$ & 18.75 & & $35555 \mathrm{~K} 42 \quad 9.93$ \\
\hline \multicolumn{5}{|c|}{ PilotLight Thermocouples } \\
\hline \multicolumn{4}{|c|}{$\frac{\text { O'all La }}{18}$} & \\
\hline \multirow{2}{*}{\multicolumn{4}{|c|}{$\begin{array}{l}18 \\
24^{\circ}\end{array}$}} & $4148 K 15 \quad 5.93$ \\
\hline \multirow{2}{*}{\multicolumn{4}{|c|}{$\begin{array}{l}36^{\circ} \\
48^{\circ}\end{array}$}} & $4140 k 17 \quad 6.42$ \\
\hline & & & & $4148 \times 16 \quad 7.43$ \\
\hline
\end{tabular}

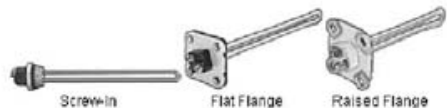

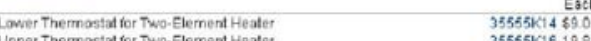

Ther thermostat for Two-Element Heater $\quad 3555551619.00$

$\begin{array}{lll}140 k 17 & 0.42\end{array}$ 
Appendix O (Continued)

CPVC Piping \& Fittings-McMaster-Carr

Plastic Pipe Fittings and Pipe

26 procucts match your salection:

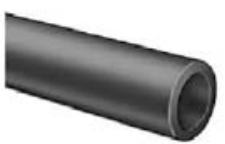

$$
\begin{aligned}
& \text { Shape Pipe } \\
& \text { Pipe Type Unithreade }
\end{aligned}
$$

Pipe to Pipa Connection Unthreadod (pipo

Svstern or Measurement inch

Schedule 80

Perroration Type Solic Pipe

Material CPVC

Spectications Het American Sociev for Testing and Materals (ASTM) and National
Sanitation foundation (NSF)

ASTM Specintason ASTM 01704 and NSTM F FA

NSF Specification NSF 61

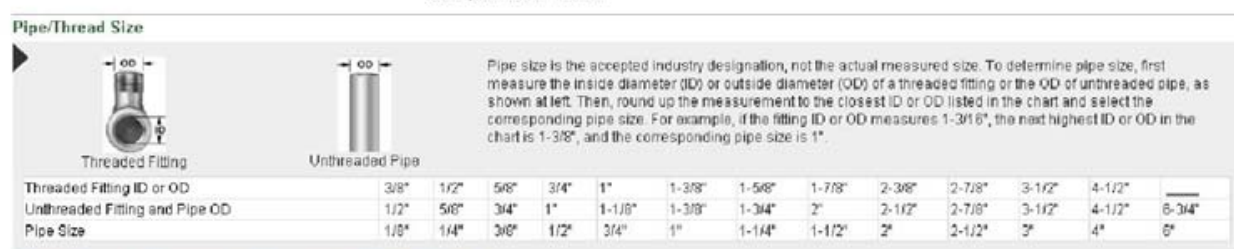

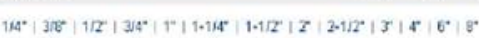

Maximum Pressure (psi)

the amcurt of pressure a pipe fitaing can endure

$L_{250}$ Low Pressure High Pressure

$\begin{array}{ll}250 & 520 \\ 200 & 630\end{array}$

\begin{tabular}{ll}
200 & 650 \\
330 & 690 \\
370 & 850 \\
400 & 920 \\
\hline
\end{tabular}

$420 \quad 1130$

Length

Plastic Pipe Fittings and Pipe
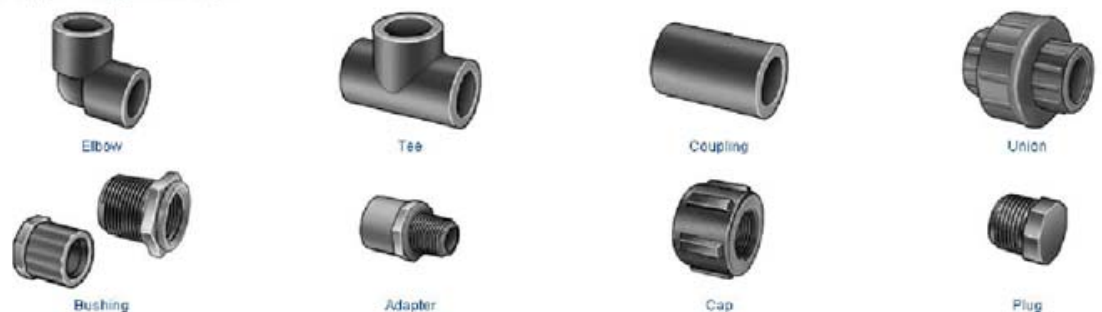

Adapter

cap

Plug

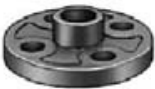

Flange

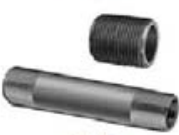

Nipple

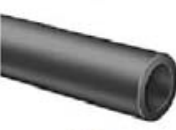

Pipe 


\section{Appendix O (Continued)}

Copper Piping \& Fittings-McMaster-Carr

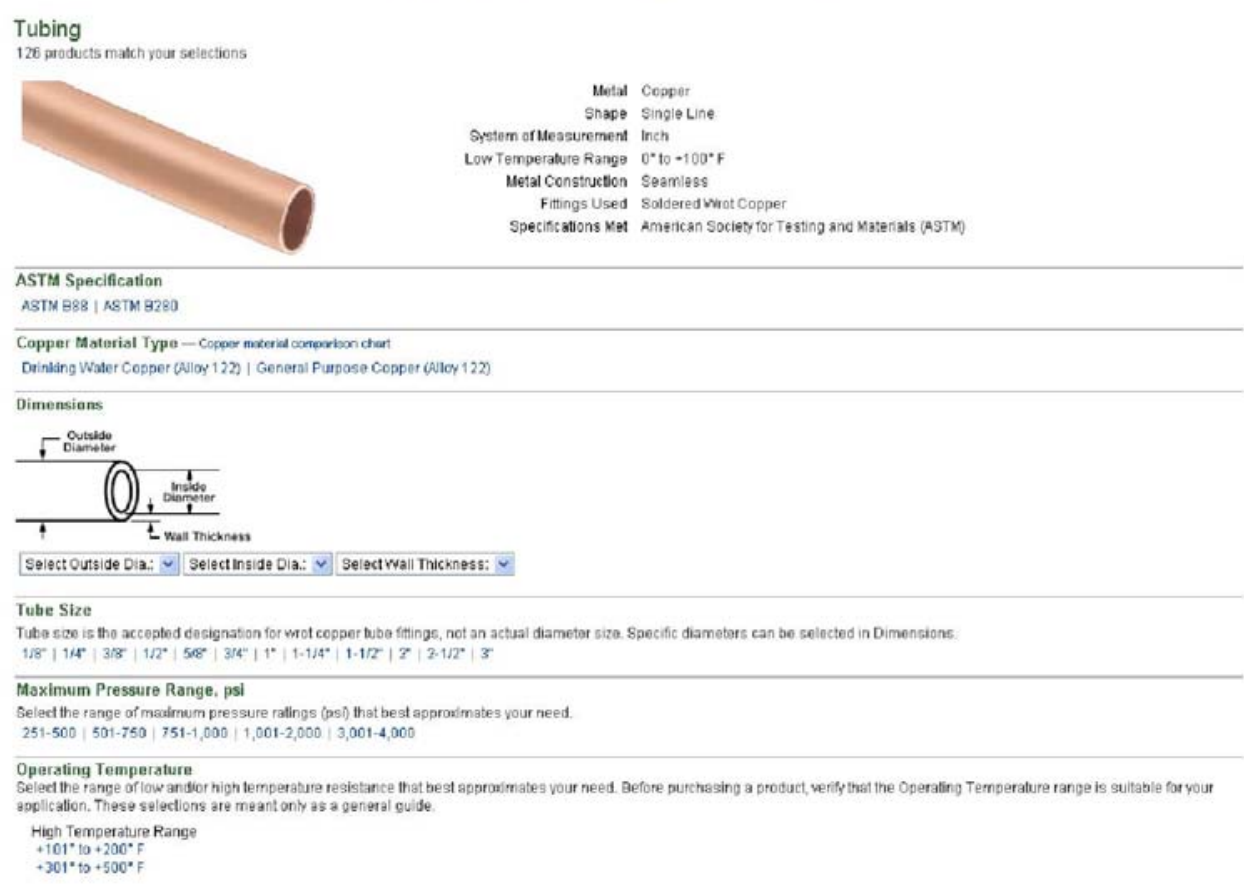

Copper Tube Fittings
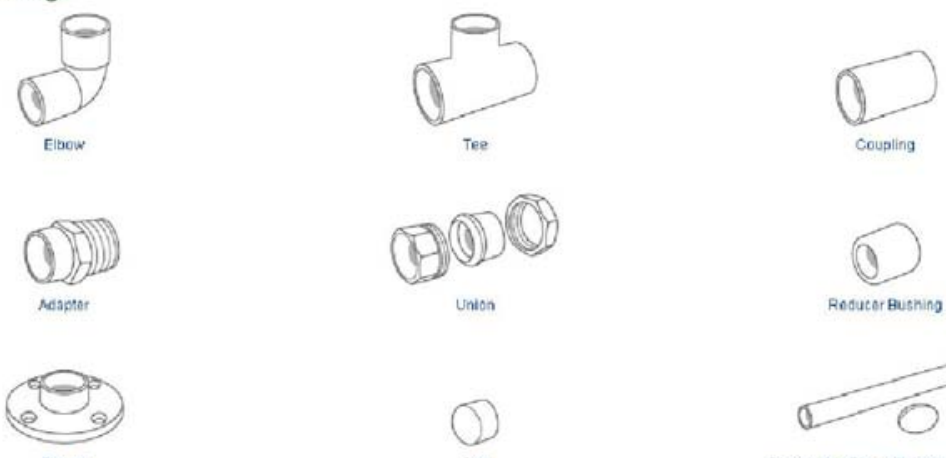

Flange
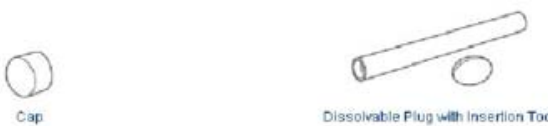

Dissolvatie Piug with Inserilon Tool 


\title{
Appendix O (Continued)
}

\author{
6 Ball Valves-McMaster-Carr \\ 4 1/2" Full Port with Lever Handle \\ $21 / 4 "$ Full Port with Lever Handle
}

Type 316 Stainless Steel Ball Valves

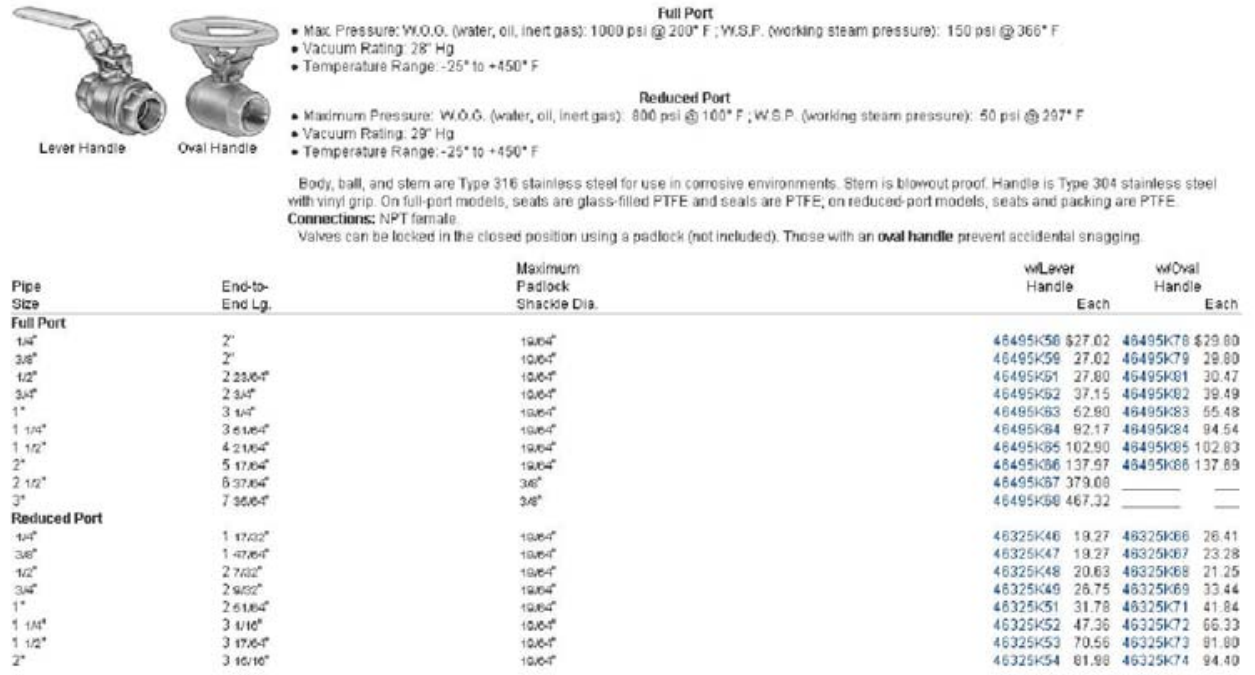

Insulation Material-McMaster-Carr

Plastics

This product matches all of your selections.

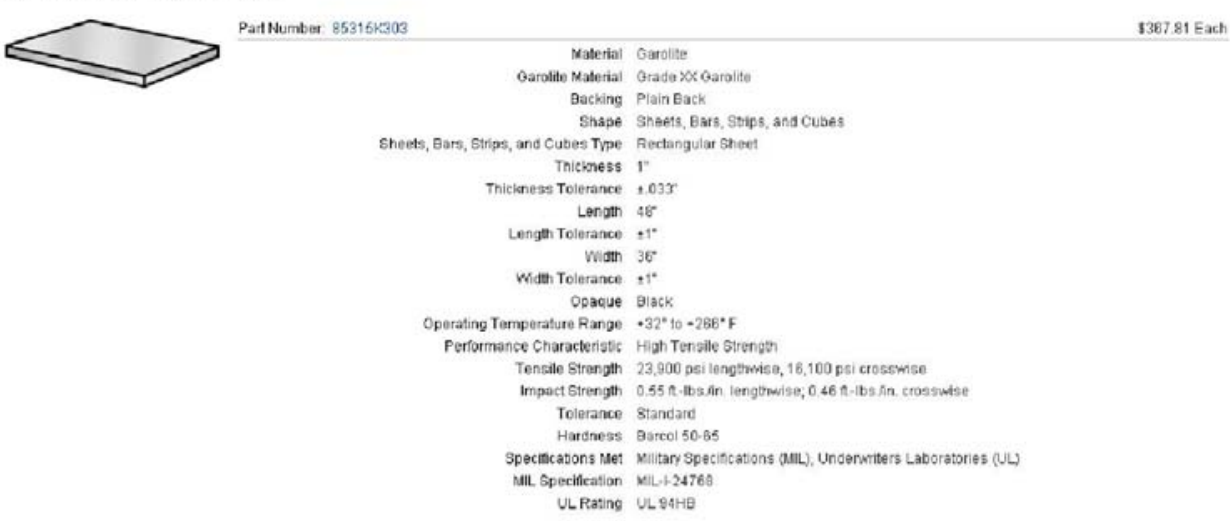




\section{Appendix O (Continued)}

Strut Channels-McMaster-Carr

\section{$21 "$ Solid 10' bars}

\section{$71 "$ Slotted Hole 10' bars}

\section{$20.5^{\prime \prime}$ Slotted-Hole 10' bars}

\section{Strut Channel}

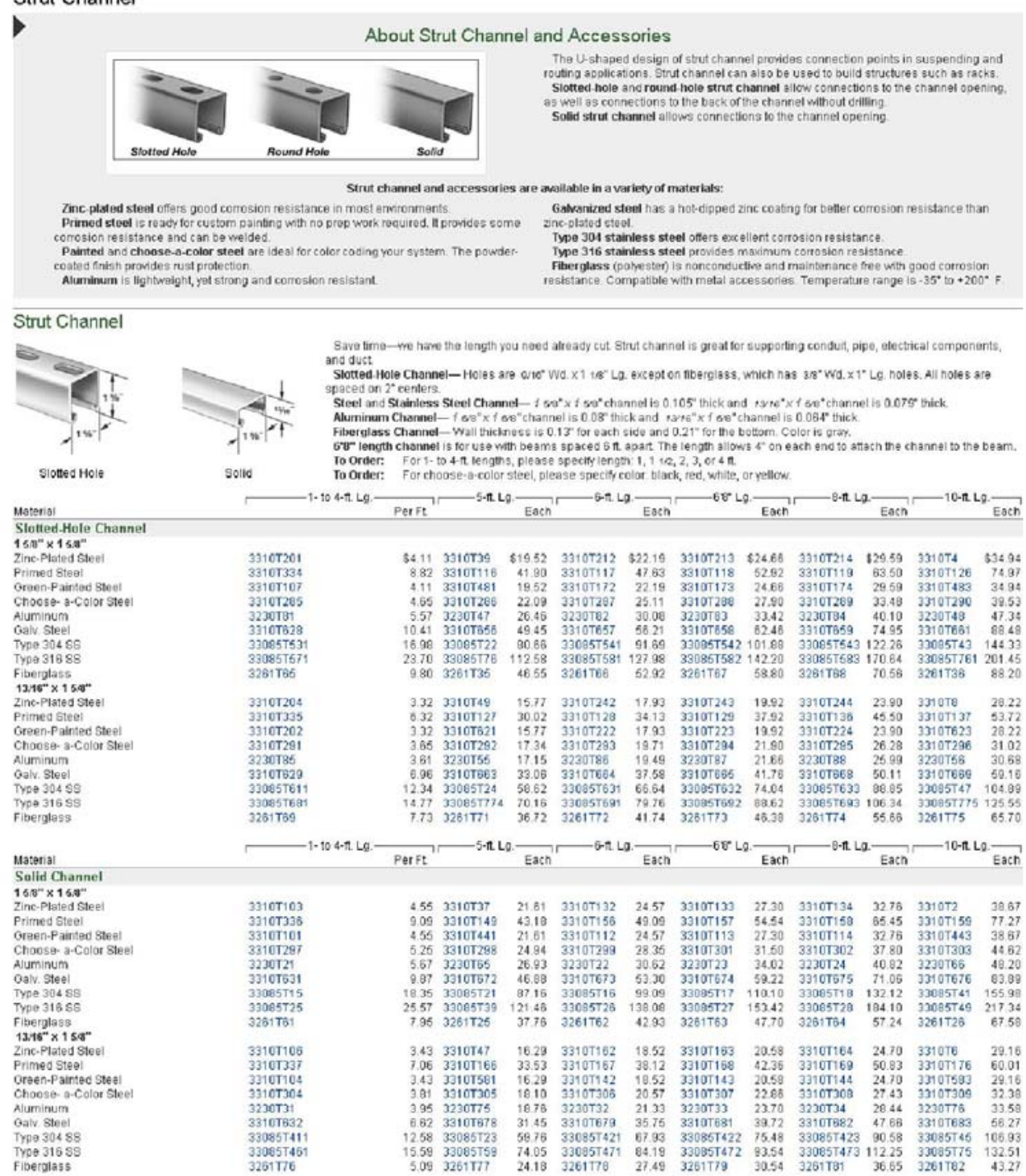




\section{Appendix O (Continued)}

15 lbs Sea Salt - Petco

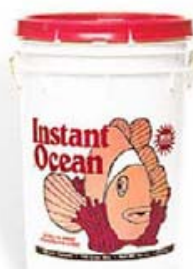

Aquarium Systems Instant Ocean

Aquarium Salt

Nitrate-free, phosphate-free, fast-dissolving

mix contains every important major, minor

and trace element necessary for the health

of your aquarium.

12 of $13(92 \%)$ of customers said they

would recommend this product to a friend.

Aquarium Systems Instant Dcean Aquarium Salt

15 lbs. - Makes 50 Gallons

SKU: 77780

Instant Ocean salt is the most carefully formulated and most carefully manufactured synthetic sea salt in the world. It's pharmaceutically blended and scientifically analyzed. This complete formula contains every major, minor, and trace element necessary to insure that even the most delicate marine fish, invertebrates, and plants will thrive.

Exceptional solubility provides a clear solution in minutes and can be used immediately ofter mixing. Reaches and maintains proper pH quickly. The convenient one-part form is easy to use for mixing complete package or small quantities*-no special additives to handle. Uniform particle size assures consistency throughhout package--no separation during packaging and shipping.

Nitrate-free, Phosphate-free.

Simply put, no other product outperforms Instant Ocean salt. It's the world's most popular brand.

1 Needle Valve-Cole-Parmer

\section{Plastic Needle Valves}

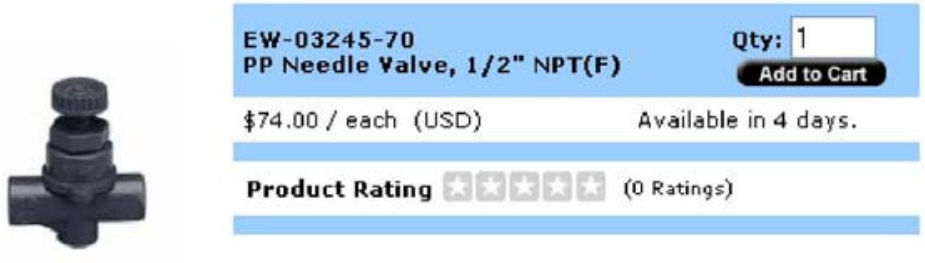

PVC and PP valves withstand up to 150 psi; PUDF up to 200 psi. Seats are made of PTFE and a-rings made of Viton ${ }^{6}$. Pipe thread connections.

Specifications

\begin{tabular}{|l|l|}
\hline NPT(F) & $1 / 2^{\prime \prime}$ \\
\hline Max gpm & 8 \\
\hline Max temperature & $250^{\circ} \mathrm{F}\left(121^{\circ} \mathrm{C}\right)$ \\
\hline Material & polypropylene \\
\hline
\end{tabular}


Appendix O (Continued)

1 Vacuum Gauge-Cole-Parmer

\section{WIKA Forged-Brass Liquid-Filled Gauge}
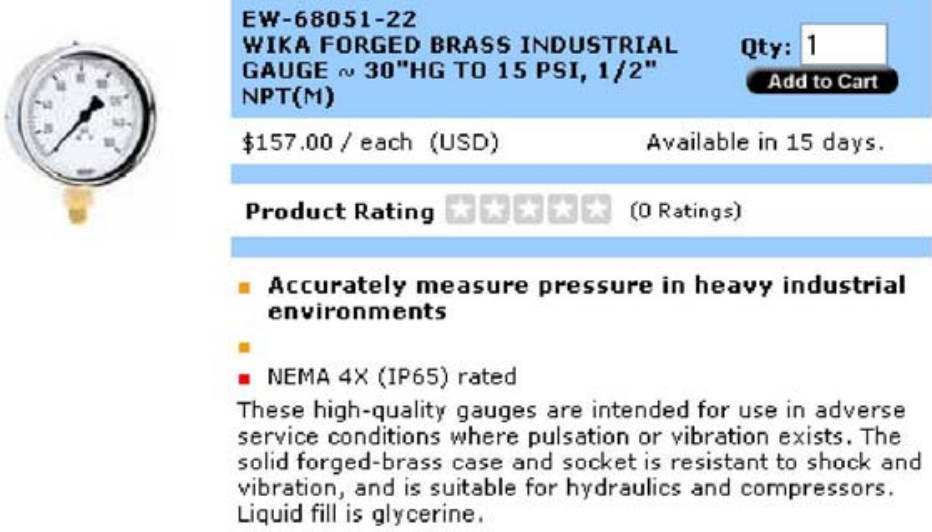

1 Pressure Transducer-Cole-Parmer

\section{Cole-Parmer High-Accuracy Pressure} Transducers
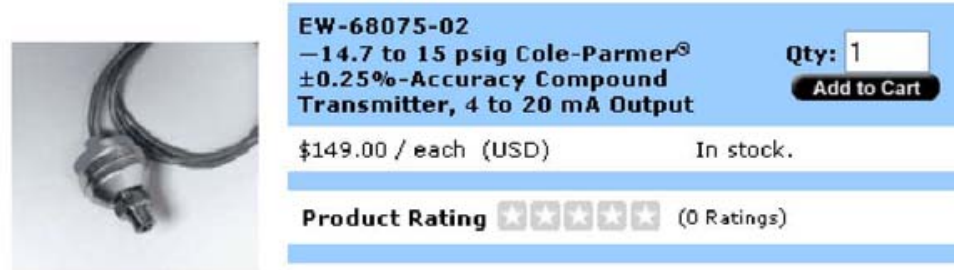

Transmitter, 4 to $20 \mathrm{~mA}$ Output

In stock.

$\$ 149.00 /$ each (USD)

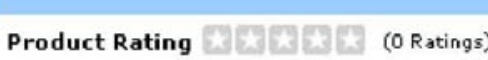

Specifications

\begin{tabular}{|l|l|}
\hline Output & 4 to $20 \mathrm{~mA}$ \\
\hline Accuracy & $\pm 0.25 \%$ full-scale \\
\hline Process connection & $1 / 4^{\prime \prime} \mathrm{NPT}(\mathrm{M})$ \\
\hline Power & 9 to $30 \mathrm{VDC}$ \\
\hline Electrical connections & 2 -ft cable \\
\hline Dimensions & $23 / 4$ "L $\times 11 / 2 "$ dia \\
\hline Wetted parts & $17-4 \mathrm{PH}$ stainless steel \\
\hline Range & -14.7 to 15 psig \\
\hline
\end{tabular}




\section{Appendix O (Continued)}

1 Flow Meter-Cole-Parmer

\section{Easy-View Acrylic In-Line Flowmeters}

EW-32477-02

Flowmeter for Liquids; 0.025 to 0.25 GPM, 3/8" NPT(F) Adapter

$\$ 98.00 /$ each (USD)

In stock.

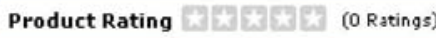

- Choose the high/low level alarm meters to monitor critical flow points.

- Units designed for in-line installation.

- General purpose liquid flowmeters feature easy-to-read dual scales (GPM/LPM).

- Polypropylene-reinforced fittings have aluminum stress rings for added strength.

- Machined high-quality acrylic rod stock, polished to a clearas-glass finish.

- Flow ranges up to 50 GPM with model 32477-82.

General-Purpose Acrylic Flowmeters are for liquids or air. Models for liquids have both English and metric directreading scales; models for air have English direct-reading reading scales;
scales only.

Large-Body Flowmeters are for use with liquids and are direct reading, with PVC floats and 11/2" NPT(M) PVC

adapters.

Flowmeters with High/Low Level Alarm are for use with liquids and feature an adjustable aluminum sensor beze clamp with an enclosed one-watt reed switch. Two lead wires are included for hookup to your own electronics.

Specifications

\begin{tabular}{|c|c|c|}
\hline $\begin{array}{l}\text { Flow } \\
\text { rate }\end{array}$ & water & $\begin{array}{l}0.025 \text { to } 0.25 \text { GPM }(0.1 \\
\text { to } 1 \text { LPM) }\end{array}$ \\
\hline \multicolumn{2}{|l|}{ Accuracy } & $\pm 5 \%$ of reading \\
\hline \multicolumn{2}{|c|}{ Repeatability } & $\pm 1 \%$ \\
\hline \multirow{3}{*}{ Material } & Housing & Acrylic \\
\hline & o-ring & Viton ${ }^{3}$ \\
\hline & float & 316 SS \\
\hline \multicolumn{2}{|c|}{ Max operating temperature } & $150^{\circ} \mathrm{F}$ \\
\hline \multicolumn{2}{|c|}{ Max pressure } & $150 \mathrm{psi}$ \\
\hline \multicolumn{2}{|c|}{ Dimensions } & $83 / 16^{\prime \prime} \mathrm{H} \times 11 / 4 " \mathrm{dia}$. \\
\hline \multicolumn{2}{|l|}{ Scale } & English/Metric \\
\hline fitting & $\begin{array}{l}\text { Polypropylene- } \\
\text { reinforced with } \\
\text { aluminum stress rings }\end{array}$ & \\
\hline \multicolumn{2}{|c|}{ Connections } & $\begin{array}{l}\text { 3/8" NPT(F), } \\
\text { polypropylene } \\
\text { reinforced, aluminum } \\
\text { stress rings }\end{array}$ \\
\hline \multicolumn{2}{|l|}{ Media } & Liquids \\
\hline
\end{tabular}




\section{Appendix O (Continued)}

1 Temperature Controller-Omega

1/16 DIN Autotune PID/On-Off Controllers with Modular Output Options

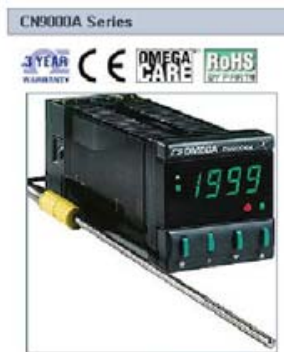

Click here for latgorimage.
- 1/16 Den Cutout

User Selectable Input from 9 Thermocouple Types or RTD Input

- Clear 312 Dight High Brightness Green LED Dkplay

- B.1 Resolution to 200

- Constant Setpoint Deviation Indication

- Auta Manual Output Control

- User Selest from Autotune PID, PI, PD, P or Qn/Orl Control

- Independent Second Setpoint and Output Hodels

- Comprehensive Alarm Features Deviation, Full Scale, Loop Break with Latching Option

Fault Indication for Sensor Bumout, Sensor Shert, Heater Break and
Process Diagnostics

IMinithax Data Storage and Autotune Diagnestics Eliminates Need for Chart Recorder

- Optional 24 Vac Power

- Field Replaceable Output Modules

The CN9000A digital temperature controllers feature high accuracy and reliablity, and the sophisticated Pto with aporasch control for optemal control during start ap and steady-state aperation. These ur

sophisticated autotune algorithm will calculate the optimum P1D values, and additionally recommends the best value for cycle time. For most applications, the user need only select the desired ingut type, simply by using the front pushibuttons. The autotune parameters can be changed by the operator at any time,

The microprocessor holds all data in non-volatile memory, with the ability to rotan data for 10 years with no power. The CN900OA has a large, $31 / 2$ digt green LED readout, with auxilary indicators for each cutput, and 3 LEO's to indicate deviation from setpont.

Selection of all operational controls is made through the keys on the front panel, with the display prompting the user through each stop. After the parameters have been sol, they can be locked in, parameters, display resolution $\left(1\right.$ or $\left.0.1^{\circ}\right)$, and units $\left({ }^{\circ} \mathrm{F} /{ }^{\circ} \mathrm{C}\right)$. The operator can also ut lize the ranging feature, which Imits the range in which the setpoint may be chosen, or lock out a user from changing output of the CNg000A modal can be set for proportional, on-off or latching limit control, and can be se set independently of the primary setpoint.

\section{$1 A D C-O m e g a$}

Portable Data Acquisition SystemsFor Notebook and Desktop PCs

\section{OMB.DAOBOOK Series}

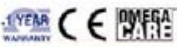

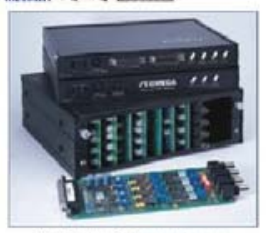

Links to Notebonk or Deshapop PCs vis a Standasd or Enhanced lel Port (EPP) or Optional PCMCIA Link

Operable From ac Adapter. Optinanal Mickel Cadmium Power

- Two 12 bit Analog Outputs

- 24 General purpose Digital 0 Lines, Expandable to 192

- Five Programmable 16 bit Countercimers

- DoS and Windows Drivers

Clock here for largor image

OME-DAQBOOK portable data acquisition systems for notebook and dasktop PCS offer 12- or 16 -bit, 100 $\mathrm{KHz}$ data acquisition. The OMB-DAQBOOK models provide $>700 \mathrm{kbyte} / \mathrm{s}$ bidirectional data communication

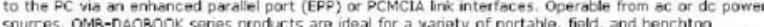
apglications. The OMB-DAQBOOKg' high perfomance A/D conversion and 100 bHz sampling make the particularly useful for applications with high accuracy and speed recuirements. Their extensive $\mathrm{N} / \mathrm{O}$ and signal conditioning capabaities, and low cost per channel also make them an offective altemative to more expensive stand alcne data loggers, less portable strip chart recorders, and less versatie logeing appication that allows you to set up rour acavist tion apolcations and sare acaured data logging application that alows you to set up your acquistion applications and save acquired data when used with an OMB-DBK19 themocouple card. The OMB-DAQBOOK products include dnvers for Visual Basic, Quick \&asic, $C$, and Pascal; they also include DoS cinvars that are compatible with DAS-16, pro-12, and Cim-as boards. Several graphical analysis and control sortware packages also suppart the 


\section{Appendix O (Continued)}

5 Single-Output Thermocouples-Omega

\section{Quick Disconnect Thermocouples with Miniature Connectors}

ПTMOSS Series JMOSS, KMOSS, EMOSS, TMOSS, NMOIN

MAOE IM PIDAS

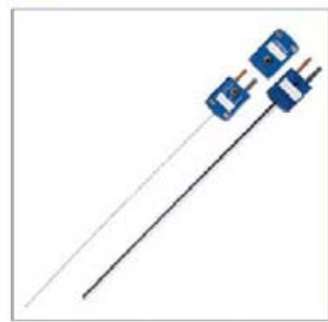

Click here for larqer imace.

$\$ 48.00$ JMQss-010G-6

- $6^{\prime \prime}$ and $12^{\prime \prime}$ Lengths in Stock

- Sheath Diameters from $0.010^{\prime \prime}$ to $0.125^{\prime \prime}$

- 304SS or 321SS Sheath

- Grounded, Ungrounded or Exposed Junction

- Color-Coded SAP Miniature Connector Termination

- Mating Connector and Cable Clamp Included FREE

- Custom Lengths Available

- Made from Special Limits of Error Material

- Glass Filled Nylon Connector Body Rated to $220^{\circ} \mathrm{C}$ $\left(425^{\circ} \mathrm{F}\right)$

\begin{tabular}{|c|c|c|c|}
\hline $\begin{array}{l}\text { Alloy/AnSI } \\
\text { Color Code }\end{array}$ & $\begin{array}{l}\text { Sheath Dia. } \\
\text { inches }\end{array}$ & $\begin{array}{l}\text { Model No. } \\
6^{*} \text { Length }\end{array}$ & $\begin{array}{l}\text { Model No. } \\
12 " \text { Length }\end{array}$ \\
\hline $\begin{array}{c}\text { J } \\
\text { Iron-Constantan } \\
\text { 304 SS Sheath }\end{array}$ & $\begin{array}{l}0.010 \\
0.020 \\
0.032 \\
0.040 \\
0.052 \\
0.125\end{array}$ & 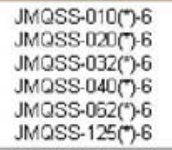 & $\begin{array}{l}\text { JMOSS-010(\%)-12 } \\
\text { JMOSS-020(7)-12 } \\
\text { JMOSS-032(")-12 } \\
\text { JMOSS-040(7)-12 } \\
\text { JMOSS-062(")-12 } \\
\text { JMOSS-125(7)-12 }\end{array}$ \\
\hline $\begin{array}{c}\text { K } \\
\text { CHROMEGAB-ALOMEGAB } \\
304 \text { SS Sheath }\end{array}$ & $\begin{array}{l}0.010 \\
0.020 \\
0.032 \\
0.040 \\
0.052 \\
0.125 \\
\end{array}$ & $\begin{array}{l}\text { KMOSS-010(\%)-6 } \\
\text { KMOSS-020(\%)-6 } \\
\text { KMOSS-032(\%-6 } \\
\text { KMOSS-040(\%)-6 } \\
\text { KMOSS-062(\%-6 } \\
\text { KMOSS-125(7)-6 }\end{array}$ & $\begin{array}{l}\text { KMOSS-010(\%)-12 } \\
\text { KMOSS-020(\%)-12 } \\
\text { KMOSS-032(\%)-12 } \\
\text { KMOSS.040(\%)-12 } \\
\text { KMOSS-062(\%)-12 } \\
\text { KMOSS.125(\%)-12 }\end{array}$ \\
\hline $\begin{array}{c}\text { K } \\
\text { CHROMEGARALOMEGANA } \\
\text { SUper OMEGACLADEX XL } \\
\text { Sheath } \\
\text { Sheath }\end{array}$ & $\begin{array}{l}0.010 \\
0.020 \\
0.032 \\
0.040 \\
0.062 \\
0.125\end{array}$ & $\begin{array}{l}\text { KMOXL-010(1)-6 } \\
\text { KMOXL-020\%-6 } \\
\text { KMOXL-032(")-6 } \\
\text { KMOXL-040\%)-6 } \\
\text { KMOXL-062(")-6 } \\
\text { KMOXL-125(")-6 }\end{array}$ & $\begin{array}{l}\text { KMOXL-010(")-12 } \\
\left.\text { KMOXL-020" }{ }^{*}\right)-12 \\
\text { 1MMOXL-032(")-12 } \\
\text { KMOXL-040(")-12 } \\
\text { KMOXL-062(")-12 } \\
\text { KMOXL-125(")-12 }\end{array}$ \\
\hline $\begin{array}{l}\mathbf{N} \\
\text { OMEGA-PGOMEGANE } \\
\text { Super OMEGACLADG XL } \\
\text { Sheath }\end{array}$ & $\begin{array}{l}0.020 \\
0.032 \\
0.040 \\
0.052 \\
0.125\end{array}$ & $\begin{array}{l}\text { NMOXLL-020(\%)-6 } \\
\text { NMOXL-032(\%)-6 } \\
\text { NMOXL-040\%)-6 } \\
\text { NMOXL-062(\%)-6 } \\
\text { NMOXL-125(7)-6 }\end{array}$ & $\begin{array}{l}\text { NMOXL-020(7)-12 } \\
\text { NMOXL-032(")-12 } \\
\text { NMOXL-040(")-12 } \\
\text { NMOXL-062(")-12 } \\
\text { NMOXL-125(7)-12 }\end{array}$ \\
\hline $\begin{array}{c}\text { E } \\
\text { CHROMEGAQ Constantan } \\
304 \text { SS Sheath }\end{array}$ & $\begin{array}{l}0.010 \\
0.020 \\
0.032 \\
0.040 \\
0.062 \\
0.125\end{array}$ & 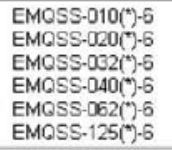 & $\begin{array}{l}\text { EMOSS-010(*)-12 } \\
\text { EMQSS-020( })-12 \\
\text { EMOSS-032( })-12 \\
\text { EMOSS-040(\%)-12 } \\
\text { EMQSS-062( })-12 \\
\text { EMOSS-125( }(\eta)-12\end{array}$ \\
\hline $\begin{array}{c}\text { T } \\
\text { Copper-Constantan } \\
\text { 304 SS Sheath }\end{array}$ & $\begin{array}{l}0.020 \\
0.032 \\
0.040 \\
0.062 \\
0.125\end{array}$ & $\begin{array}{l}\text { TMOSS-020(7)-6 } \\
\text { TMQSS-032(")-6 } \\
\text { TMQSS-040(7)-6 } \\
\text { TMQSS-052(7)-6 } \\
\text { TMOSS-125(7)-6 }\end{array}$ & $\begin{array}{l}\text { TMQSS-020(7)-12 } \\
\text { TMQSS-032(")-12 } \\
\text { TMQSS-040(")-12 } \\
\text { TMQSS-062(")-12 } \\
\text { TMQSS- } 125(7)-12\end{array}$ \\
\hline $\begin{array}{c}\text { N } \\
\text { OMEGALLOYQ } \\
\text { Inconel bOC Sheath }\end{array}$ & $\begin{array}{l}0.010 \\
0.020 \\
0.032 \\
0.040 \\
0.052 \\
0.125\end{array}$ & 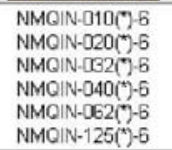 & 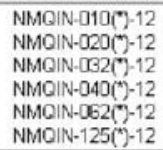 \\
\hline
\end{tabular}




\section{Appendix O (Continued)}

\section{Dual Element Thermocouple Assemblies with Standard Size Dual TC Connector}

\section{()XL or SS Series and ()IN Dual Element}

MADE IN

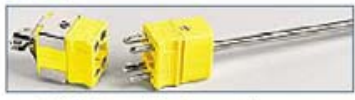

Click here for larger image.
$\$ 57.50$ ICIN-116E-12-DUAL

- Two Sensor Readings

- Allows Two Readings of a Single Point

- Continuous Monitor and Control

- Built-in Backup

- Made from Special Limits of Error Material

- Glass Filled Nylon Connector Rated to $220^{\circ} \mathrm{C}\left(425^{\circ} \mathrm{F}\right)$

- 12" Length Standard

- 1/16, 1/8, 3/16 and 1/4" Diameters Available

- Omegaclad XI. SS or Inconel Sheath

- DTC Type Dual Connector-Mating Connector Included

\begin{tabular}{|c|c|c|c|c|}
\hline $\begin{array}{r}\text { Thermoc } \\
\text { Allo }\end{array}$ & $\begin{array}{l}\text { Sheath } \\
\text { Dia. } \\
\text { mm (in.) }\end{array}$ & $\begin{array}{l}\text { Gro } \\
\text { Ju }\end{array}$ & $\begin{array}{c}\text { Ungro } \\
\text { Junc }\end{array}$ & $\begin{array}{l}\text { Ex } \\
\text { Ju }\end{array}$ \\
\hline $\begin{array}{l}\mathbf{J} \\
\text { IRON-CONSTANTAN } \\
\text { Inconel Sheath } \\
\end{array}$ & $\begin{array}{c}3.0\left(1 / 8^{\prime \prime}\right) \\
4.5\left(3 / 16^{\prime \prime}\right) \\
6.0\left(1 / 4^{* \prime}\right)\end{array}$ & $\begin{array}{l}\text { ICIN-116G-12-DUAL } \\
\text { ICIN-18G-12-DUAL } \\
\text { ICIN-316G-12-DUAL } \\
\text { ICIN-14G-12-DUAL }\end{array}$ & $\begin{array}{l}\text { ICIN-116U-12-DUAL } \\
\text { ICIN-18U-12-DUAL } \\
\text { ICIN-316U-12-DUAL } \\
\text { ICIN-14U-12-DUAL } \\
\end{array}$ & $\begin{array}{l}\text { ICIN-116 } \\
\text { ICIN-18 } \\
\text { ICIN-316 } \\
\text { ICIN-14 } \\
\end{array}$ \\
\hline $\begin{array}{l}\mathbf{J} \\
\text { IRON-CONSTANTAN } \\
304 \text { SS Sheath }\end{array}$ & $\begin{array}{l}1.5\left(1 / 16^{\prime \prime}\right) \\
3.0\left(1 / 8^{\prime \prime}\right) \\
4.5\left(3 / 16^{\prime \prime}\right) \\
6.0\left(1 / 4^{\prime \prime}\right)\end{array}$ & $\begin{array}{l}\text { 2-DUAL } \\
\text { 2-DUAL } \\
\text { 2-DUAL } \\
\text { 2-DUAL }\end{array}$ & $\begin{array}{l}\text { ICSS-116U-12-DUAL } \\
\text { ICSS-18U-12-DUAL } \\
\text { ICSS-316U-12-DUAL } \\
\text { ICSS-14U-12-DUAL }\end{array}$ & $\begin{array}{l}\text { ICSS-116 } \\
\text { ICSS-18E } \\
\text { ICSS-316 } \\
\text { ICSS-14E }\end{array}$ \\
\hline $\begin{array}{l}\text { K } \\
\text { EGAB-ALOMEGAB } \\
\text { conel Sheath }\end{array}$ & $\begin{array}{l}1.5\left(1 / 16^{\prime \prime}\right) \\
3.0\left(1 / 8^{\prime \prime}\right) \\
4.5\left(3 / 16^{\prime \prime}\right) \\
6.0\left(1 / 4^{\prime \prime}\right)\end{array}$ & \begin{tabular}{|l|} 
CAIN-116G-12-DUAL \\
CAIN-18G-12-DUAL \\
CAIN-316G-12-DUAL \\
CAIN-14G-12-DUAL
\end{tabular} & $\begin{array}{l}\text { CAIN-116U-12-DUAL } \\
\text { CAIN-18U-12-DUAL } \\
\text { CAIN-316U-12-DUAL } \\
\text { CAIN-14U-12-DUAL }\end{array}$ & $\begin{array}{l}\text { CAIN-11 } \\
\text { CAIN-18 } \\
\text { CAIN-31 } \\
\text { CAIN-1 }\end{array}$ \\
\hline $\begin{array}{c}\text { K } \\
\text { CHROMEGAE.ALOMEGAE } \\
\text { 304 SS Sheath }\end{array}$ & $\begin{array}{c}1.5\left(1 / 16^{\prime \prime}\right) \\
3.0\left(1 / 8^{\prime \prime}\right) \\
4.5\left(3 / 16^{\prime \prime}\right) \\
6.0\left(1 / 4^{\prime \prime}\right) \\
\end{array}$ & $\begin{array}{l}\text { CASS-116G-12-DUAL } \\
\text { CASS-18G-12-DUAL } \\
\text { CASS-316G-12-DUAL } \\
\text { CASS-14G-12-DUAL } \\
\end{array}$ & $\begin{array}{l}\text { CASS-116U-12-DUAL } \\
\text { CASS-18U-12-DUAL } \\
\text { CASS-316U-12-DUAL } \\
\text { CASS-14U-12-DUAL } \\
\end{array}$ & $\begin{array}{l}\text { CASS-1 } \\
\text { CASS-1 } \\
\text { CASS-3 } \\
\text { CASS-1 }\end{array}$ \\
\hline 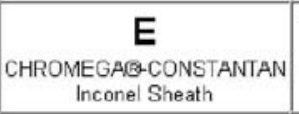 & $\begin{array}{l}1.5\left(1 / 16^{\prime \prime}\right) \\
3.0\left(1 / 8^{\prime \prime}\right) \\
4.5\left(3 / 16^{\prime \prime}\right) \\
6.0\left(1 / 4^{\prime \prime}\right)\end{array}$ & \begin{tabular}{|l|} 
CXIN-116G-12-DUAL \\
CXIN-18G-12-DUAL \\
CXIN-316G-12-DUAL \\
CXIN-14G-12-DUAL
\end{tabular} & $\begin{array}{l}\text { CXIN-116U-12-DUAL } \\
\text { CXIN-18U-12-DUAL } \\
\text { CXIN-316U-12-DUAL } \\
\text { CXIN-14U-12-DUAL }\end{array}$ & $\begin{array}{l}\text { CXIN-116E-12-DUAL } \\
\text { CXIN-18E-12-DUAL } \\
\text { CXIN-316E-12-DUAL } \\
\text { CXIN-14E-12-DUAL }\end{array}$ \\
\hline \begin{tabular}{|c|} 
E \\
$\begin{array}{c}\text { CHROMEGARCONSTANTAN } \\
304 \text { SS Sheath }\end{array}$ \\
\end{tabular} & $\begin{array}{c}1.5\left(1 / 16^{\prime \prime}\right) \\
3.0\left(1 / 8^{\prime \prime}\right) \\
4.5\left(3 / 16^{\prime \prime}\right) \\
6.0\left(1 / 4^{\prime \prime}\right)\end{array}$ & \begin{tabular}{|l|} 
CXSS-116G-12-DUAL \\
CXSS-18G-12-DUAL \\
CXSS-316G-12-DUAL \\
CXSS-14G-12-DUAL
\end{tabular} & $\begin{array}{l}\text { CXSS-116U-12-DUAL } \\
\text { CXSS-18U-12-DUAL } \\
\text { CXSS-316U-12-DUAL } \\
\text { CXSS-14U-12-DUAL } \\
\end{array}$ & $\begin{array}{l}\text { CXSS-116E-12-DUAL } \\
\text { CXSS-18E-12-DUAL } \\
\text { CXSS-316E-12-DUAL } \\
\text { CXSS-14E-12-DUAL }\end{array}$ \\
\hline 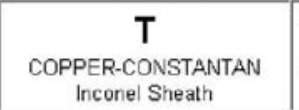 & $\begin{array}{c}1.5\left(1 / 16^{\prime \prime}\right) \\
3.0\left(1 / 8^{\prime \prime}\right) \\
4.5\left(3 / 16^{\prime \prime}\right) \\
6.0\left(1 / 4^{\prime \prime}\right)\end{array}$ & \begin{tabular}{|l|} 
CPIN-116G-12-DUAL \\
CPIN-18G-12-DUAL \\
CPIN-316G-12-DUAL \\
CPIN-14G-12-DUAL
\end{tabular} & $\begin{array}{l}\text { CPIN-116U-12-DUAL } \\
\text { CPIN-18U-12-DUAL } \\
\text { CPIN-316U-12-DUAL } \\
\text { CPIN-14U-12-DUAL }\end{array}$ & $\begin{array}{l}\text { CPIN-116E-12-DUAL } \\
\text { CPIN-18E-12-DUAL } \\
\text { CPIN-316E-12-DUAL } \\
\text { CPIN-14E-12-DUAL }\end{array}$ \\
\hline $\begin{array}{c}\text { T } \\
\text { COPPER-CONSTANTAN } \\
\text { 304 SS Sheath }\end{array}$ & $\begin{array}{c}1.5\left(1 / 16^{\prime \prime}\right) \\
3.0\left(1 / 8^{\prime \prime}\right) \\
4.5\left(3 / 16^{\prime \prime}\right) \\
6.0\left(1 / 4^{\prime \prime}\right)\end{array}$ & $\begin{array}{l}\text { CPSS-116G-12-DUAL } \\
\text { CPSS-18G-12-DUAL } \\
\text { CPSS-316G-12-DUAL } \\
\text { CPSS-14G-12-DUAL }\end{array}$ & 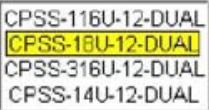 & $\begin{array}{l}\text { CPSS-116E-12-DUAL } \\
\text { CPSS-18E-12-DUAL } \\
\text { CPSS-316E-12-DUAL } \\
\text { CPSS-14E-12-DUAL }\end{array}$ \\
\hline
\end{tabular}


Appendix O (Continued)

1 Vacuum Pump - Robin Air

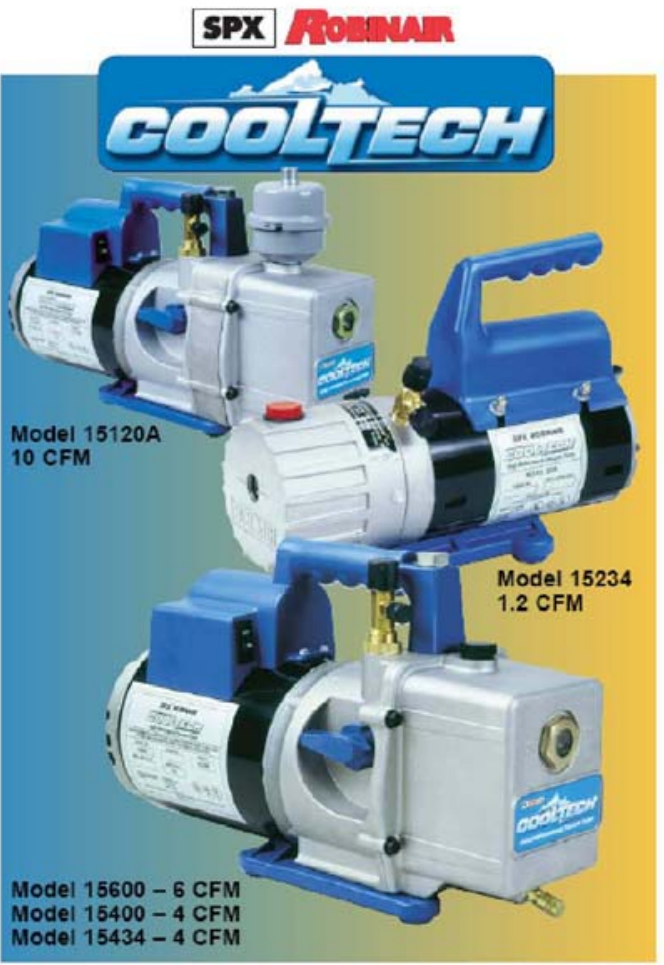

\begin{tabular}{|c|c|c|c|c|c|c|c|c|c|c|}
\hline Model & $\begin{array}{c}\text { Free Air } \\
\text { Displasement }\end{array}$ & $\begin{array}{l}\text { Factory } \\
\text { Mioton } \\
\text { Rating } \\
\end{array}$ & $\begin{array}{l}\text { No. of } \\
\text { Stages }\end{array}$ & Intake Fitting & Oil Capacity & $\begin{array}{r}\text { Motor } \\
\text { Size } \\
\end{array}$ & Voltage & Approvolis & Weight & Dimensions \\
\hline \begin{tabular}{|l|} 
Domes \\
isras
\end{tabular} & $10 \mathrm{Mcd} / \mathrm{s} \mathrm{Cm}$ & 50 microns & 2 & 114'MFL and T/2 ACME & 5 or $(148 \mathrm{~m} /)$ & $1 / 8 \mathrm{hp}$ & 115V 50, $180 \mathrm{~Hz}$ & $\mathrm{ut}$ & $10 \mathrm{bss}(45 \mathrm{ka})$ & $\begin{array}{c}85^{\circ} \mathrm{H} \times 43^{\circ} \mathrm{W} \times 95^{\circ} \mathrm{L} \\
215 \mathrm{~mm} \times 108 \mathrm{~mm} \times 240 \mathrm{~mm}\end{array}$ \\
\hline 15400 & 4 CFM & 20 misrons & 2 & 1.4" MFL and $1 / 2$ MFL. & 10 or $(445 \mathrm{ml})$ & $1 / 3 \mathrm{he}$ & $110 \mathrm{VeOHz}$ & $u$ & 27 lbs. (12.2 hot & $\begin{array}{l}0.5^{\circ} \mathrm{H} \times B .^{\circ} \mathrm{W} \times 10^{\circ} \mathrm{L} \\
247 \mathrm{~mm} \times 142 \mathrm{~mm} \times 381 \mathrm{~mm}\end{array}$ \\
\hline 15434 & 4 CFM & 20 misers & 2 & 1/4 MFL and $1 / 2^{\circ}$ ACME & $15 \mathrm{cz} .(445 \mathrm{ml})$ & $1 / 2 h$ & $115 \mathrm{~V} 00 \mathrm{~Hz}$ & $u$ & $27 \mathrm{kbs} .(12.2 \mathrm{~kg})$ & $\begin{array}{c}9.5^{\circ} \mathrm{H} \times 5.5^{\circ} \mathrm{W} \times 15^{5 \mathrm{~L}} \\
247 \mathrm{~mm} \times 142 \mathrm{mim} \times 381 \mathrm{~mm}\end{array}$ \\
\hline 15600 & OCFM & 20 microns & 2 & $1 / 4^{\circ} \mathrm{MFL}$ and $1 / 2^{\prime} \mathrm{MF}$ & $1602(445 \mathrm{~m})$ & $1 / 2 \mathrm{hp}$ & T15V EOHZ & Uit & $27 \mathrm{lbs}(12.2 \mathrm{~kg})$ & $\begin{array}{r}05^{\circ} \mathrm{H} \times 5^{\circ} \mathrm{W} \times 15^{\circ} \mathrm{L} \\
247 \mathrm{~mm} \times 142 \mathrm{~mm} \times 391 \mathrm{~mm}\end{array}$ \\
\hline $15120 \mathrm{~A}$ & 10 CFM & 20 mistons & 2 & 1:4" MFL and 1/2' MF- & 17 az. (500 $\mathrm{mil})$ & $1 / 2 \mathrm{hp}$ & $115 \mathrm{VeO} \mathrm{Hz}$ & $u$ & $36 \mathrm{ltb},(17.2 \mathrm{~kg})$ & $\begin{array}{c}10 \mathrm{H} \times 5.5^{\circ} \mathrm{W} \times 10^{\circ} \mathrm{L} \\
272 \mathrm{~mm} \times 142 \mathrm{~mm} \times 419 \mathrm{~mm}\end{array}$ \\
\hline $\begin{array}{l}\text { linternat } \\
15226\end{array}$ & 281 thersiminute & 50 microns & 2 & 11/4'MFL and $1 / 2^{*}$ ACME & $5 \propto[(148 \mathrm{~m})\}$ & $1 / 8 \mathrm{hp}$ & $220 \mathrm{~V} 50,60 \mathrm{~Hz}$ & GE & $10 \mathrm{bs}(45 \mathrm{~kg})$ & $\begin{array}{c}855^{\circ} \mathrm{H} \times 45^{*} \mathrm{~W} \times 95^{\circ} \mathrm{L} \\
215 \mathrm{~mm} \times 108 \mathrm{~mm} \times 240 \mathrm{~mm}\end{array}$ \\
\hline 15404 & 03 Litersiminute & 20 mionors & 2 & 1:4 MFL and 1/2' MFL & 1602 (445ml) & $1 / 3 \%$ & $\begin{array}{l}115 \mathrm{~V} / 220-250 \mathrm{~V} \\
6000 \mathrm{He}\end{array}$ & CE & $27 \mathrm{lbs} .(12.2 \mathrm{~kg})$ & $\begin{array}{c}0.5^{\circ} \mathrm{H} \times 5 . E^{\circ} \mathrm{W} \times 15^{\circ} \mathrm{L} \\
247 \mathrm{~mm} \times 142 \mathrm{~mm} \times 391 \mathrm{~mm}\end{array}$ \\
\hline 15424 & 93 itersminuts & 20 microns & 2 & 1/4- MFL and $1 / 2^{*}$ ACME & 15 or (445mis) & $1 / 3 / p$ & $\begin{array}{c}115 \mathrm{~V} / 220-250 \mathrm{~V} \\
5000 \mathrm{~Hz}\end{array}$ & CE & 27 ibs $\{12.2 \mathrm{~kg}\}$ & $\begin{array}{c}95^{\circ} \mathrm{H} \times 55^{\circ} \mathrm{W} \times 15^{\circ} \mathrm{L} \\
247 \mathrm{~mm} \times 142 \mathrm{~mm} \times 38 \mathrm{mmm}\end{array}$ \\
\hline 15601 & 142 thersiminute & 20 misrons & 2 & $1 / 4^{*}$ MFL and $1 / 2$ MFL & 16 oz (445m) & $1 / 3 \mathrm{hp}$ & $\begin{array}{l}115 \mathrm{~V} 2220-250 \mathrm{~V} \\
30000 \mathrm{~Hz}\end{array}$ & $C E$ & $27 \mathrm{lbs} .(12.2 \mathrm{~kg})$ & $\begin{array}{c}0.5^{\circ} \mathrm{H} \times 5.6^{\circ} \mathrm{W} \times 11^{\circ} \mathrm{L} \\
247 \mathrm{~mm} \times 142 \mathrm{~mm} \times 381 \mathrm{~mm}\end{array}$ \\
\hline $15121 \mathrm{~A}$ & 230 Hessiminue & 20 microms & 2 & 1/F MFL and $1 / 2-M F L$ & $1702 .(500 \mathrm{~m})$ & $1 / 2 \mathrm{hp}$ & $\begin{array}{l}115 \mathrm{~V} / 220 \mathrm{~V} \\
5090 \mathrm{~Hz}\end{array}$ & CE & $38 \mathrm{lts} .(17.2 \mathrm{~kg})$ & $\begin{array}{r}10^{\circ} \mathrm{H} \times 5.5^{\circ} \mathrm{W} \times 10^{\circ} \mathrm{L} \\
272 \mathrm{~mm} \times 142 \mathrm{~mm} \times 410 \mathrm{~mm}\end{array}$ \\
\hline
\end{tabular}




\section{Appendix O (Continued)}

\section{FBMI-IO Tech}

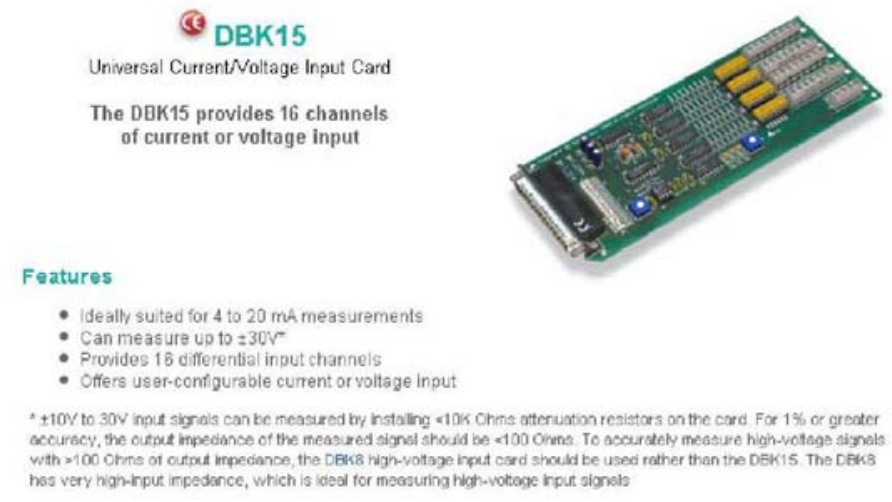

1 FBM2-IO Tech

(9) $\mathrm{DBK} 81, \mathrm{DBK} 82, \mathrm{DBK} 83$, and DBK84

7- \& 14-Channel, Low-Noise, High-Accuracy. Thermocouple/mV Expansion Options

\section{Features}

- Measures type J, K, S, T, E, B, R, and N

thermocouples as well as voltage up to $\pm 100 \mathrm{mV}$

- Low noise, high accuracy, high stability

- 200-k-2 maximum scan rate

- High noise immunity

- Open thermocouple detection per channel

- Overvoltage protectior

- Avallable in four form-factors:

-7-channel card wilh an-board screw terminals (DBK81)

14-channel card with on-board

screwi terminals (Delcaz)

-14-channel card with external

signal connection "pod" (DEK83)

-14-channel module, housed in

metal package with mini TC

connections (DEK84)
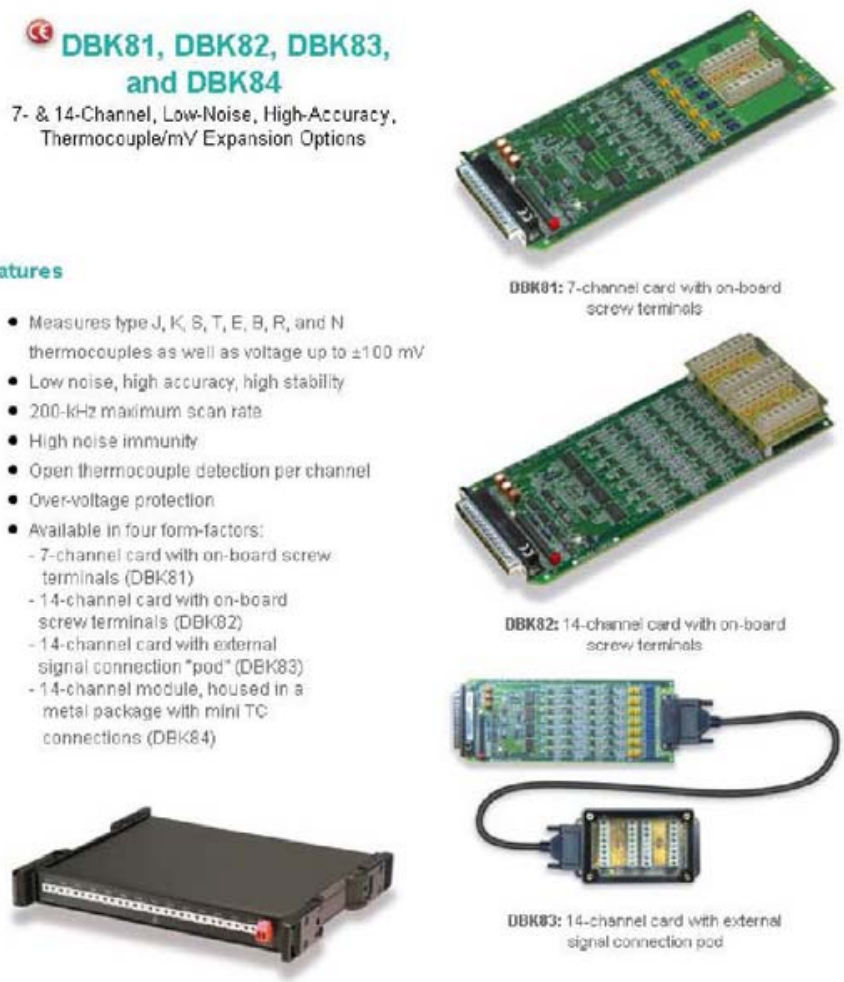

08k91: 7-channel card with on-boand screw terminals

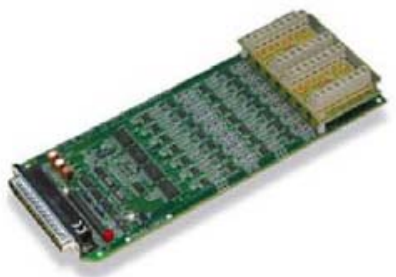

DBK82: 14-channe/ card with on-board screw terminals

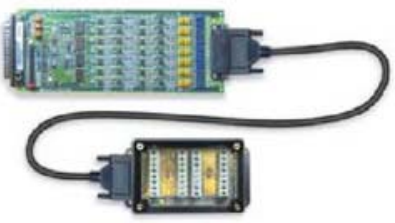

DBk83: 14-channel card wth externa signal connection pod

Dekes. 14-charne modte, houred in andal packape with mini TC carnections 
Appendix P. Error analysis

Experimental data are directly measured and entail specific errors provided by the manufacture of the measuring devices. In addition, physical properties determined by empirical relations also entail certain errors given by the developers of those correlations. Pseudo-experimental data are generated using the experimental data and the empirically determined physical properties; consequently, they entail indirect errors that are propagations of the direct errors of the experimental data and the physical properties.

The errors associated with the previously mentioned devices and correlations are given in Table 12 while formulas to calculate the propagation of error as functions of directly measured errors are given in Table 13. The rules of Table 13 can be multiplexed to represent the error of other variations as will be seen shortly.

Table 12. Device and correlation errors

\begin{tabular}{ccc|cccc}
\hline & \multicolumn{5}{c}{ Correlation } & \multicolumn{4}{c}{ Device } \\
\cline { 2 - 7 } & $\rho$ & $H$ & $T E$ & $P E$ & $F I$ & $Q E$ \\
& $\left(\mathrm{~g} / \mathrm{cm}^{3}\right)$ & $(\mathrm{J} / \mathrm{g})$ & $\left({ }^{\circ} \mathrm{C}\right)$ & $($ bar $)$ & $($ LPM $)$ & $\left(\mathrm{cm}^{3}\right)$ \\
\hline Error & $\pm 35 \cdot 10^{-6}$ & \pm 0.045 & \pm 1.000 & \pm 0.005 & \pm 0.045 & \pm 0.200 \\
\hline
\end{tabular}

Table 13. Propagation of error rules

\begin{tabular}{ll}
\hline Relationship & Compounded Error \\
\hline$Z=X+Y$ & $\varepsilon_{Z}^{2}=\varepsilon_{X}^{2}+\varepsilon_{Y}^{2}$ \\
\hline$Z=X-Y$ & $\varepsilon_{Z}^{2}=\varepsilon_{X}^{2}+\varepsilon_{Y}^{2}$ \\
\hline$Z=X \cdot Y$ & $\left(\varepsilon_{Z} / Z\right)^{2}=\left(\varepsilon_{X} / X\right)^{2}+\left(\varepsilon_{Y} / Y\right)^{2}$ \\
\hline$Z=X / Y$ & $\left(\varepsilon_{Z} / Z\right)^{2}=\left(\varepsilon_{X} / X\right)^{2}+\left(\varepsilon_{Y} / Y\right)^{2}$ \\
\hline$Z=X^{n}$ & $\left(\varepsilon_{Z} / Z\right)=n \cdot\left(\varepsilon_{X} / X\right)$ \\
\hline$Z=\ln (X)$ & $\varepsilon_{Z}=\left(\varepsilon_{X} / X\right)$ \\
\hline$Z=\exp (X)$ & $\left(\varepsilon_{Z} / Z\right)=\varepsilon_{X}$ \\
\hline
\end{tabular}


Many elements contribute to error in measurements; however, the average error values given above in Table 12 are considered inclusive of all errors since experiments were conducted at matching laboratory conditions and because a true measurement can never be claimed. The dynamic errors of the time-varying system variables that were graphically presented in CHAPTER 7 as error bands were computed using the values of Table 12 and the rules of Table 13 to generate the following perturbations

$$
\begin{aligned}
& \varepsilon_{F I}= \pm 0.045 \\
& \varepsilon_{Q E}= \pm 0.2 \\
& \varepsilon_{P_{V}}=\varepsilon_{P E}= \pm 0.005 \\
& \varepsilon_{T_{E}}=\varepsilon_{T_{W}}=\varepsilon_{T_{X}}=\varepsilon_{T E}= \pm 1 \\
& \varepsilon_{\rho_{P}}=\varepsilon_{\rho_{H}}=\varepsilon_{\rho_{C}}=\varepsilon_{\rho_{E}}=\varepsilon_{\rho}= \pm 35 \cdot 10^{-6} \\
& \varepsilon_{H_{P}}=\varepsilon_{H_{X}}=\varepsilon_{H_{H}}=\varepsilon_{H_{E}}=\varepsilon_{H}= \pm 0.045 \\
& \varepsilon_{\Delta T}= \pm \sqrt{2} \cdot \varepsilon_{T E} \\
& \varepsilon_{\Delta H}= \pm \sqrt{2} \cdot \varepsilon_{H} \\
& \varepsilon_{M_{P}}= \pm\left[M_{P}{ }^{2} \cdot\left(\left[\frac{\varepsilon_{\rho_{P}}}{\rho_{P}}\right]^{2}+\left[\frac{\varepsilon_{F I}}{F I}\right]^{2}\right)\right]^{\frac{1}{2}} \\
& \varepsilon_{M_{H}}= \pm\left[M_{H}{ }^{2} \cdot\left(\left[\frac{\varepsilon_{\rho_{H}}}{\rho_{H}}\right]^{2}+\left[\frac{\varepsilon_{F I}}{F I}\right]^{2}\right)\right]^{\frac{1}{2}} \\
& \varepsilon_{M_{C}}= \pm\left[M_{C}{ }^{2} \cdot\left(\left[\frac{\varepsilon_{\rho_{C}}}{\rho_{C}}\right]^{2}+\left[\frac{\varepsilon_{F I}}{F I}\right]^{2}\right)\right]^{\frac{1}{2}}
\end{aligned}
$$




$$
\begin{aligned}
& \varepsilon_{\int M_{C}}= \pm\left[\left(\int M_{C}\right)^{2} \cdot\left(\left[\frac{\varepsilon_{\rho_{C}}}{\rho_{C}}\right]^{2}+\left[\frac{\varepsilon_{Q E}}{Q E}\right]^{2}\right)\right]^{\frac{1}{2}} \\
& \varepsilon_{\int Q_{H}}=\varepsilon_{Q_{H}}= \pm\left[Q_{H}{ }^{2} \cdot\left(\left[\frac{\varepsilon_{M_{P}}}{M_{P}}\right]^{2}+\left[\frac{\varepsilon_{\Delta H}}{H_{H}-H_{X}}\right]^{2}\right)\right]^{\frac{1}{2}} \\
& \varepsilon_{A_{S C}}= \pm\left[A_{S C}{ }^{2} \cdot\left(\left[\frac{\varepsilon_{Q_{H}}}{Q_{H}}\right]^{2}+\left[\frac{U_{S C} \cdot \varepsilon_{T_{X}}}{\tau_{S C} \cdot \alpha_{S C} \cdot I-U_{S C} \cdot\left(T_{X}-T\right)}\right]^{2}\right)\right]^{\frac{1}{2}} \\
& \varepsilon_{P E C}= \pm\left[P E C^{2} \cdot\left(\left[\frac{\varepsilon_{\int Q_{H}}}{\int Q_{H}}\right]^{2}+\left[\frac{\varepsilon_{\int M_{C}}}{\int M_{C}}\right]^{2}\right)\right]^{\frac{1}{2}} \\
& \varepsilon_{\eta_{C}}= \pm 100 \cdot\left[\left(\frac{\eta_{C}}{100}\right)^{2} \cdot\left(\left[\frac{\varepsilon_{\Delta T}}{T_{X}-T_{P}}\right]^{2}+\left[\frac{\varepsilon_{\Delta T}}{T_{E}-T_{C}}\right]^{2}\right)\right]^{\frac{1}{2}} \\
& \varepsilon_{\eta_{R}}= \pm 100 \cdot\left[\left(\frac{\eta_{R}}{100}\right)^{2} \cdot\left(\left[\frac{\varepsilon_{\Delta H}}{H_{X}-H_{P}}\right]^{2}+\left[\frac{\varepsilon_{\Delta H}}{H_{H}-H_{P}}\right]^{2}\right)\right]^{\frac{1}{2}} \\
& \varepsilon_{\eta_{T}}= \pm 100 \cdot\left[\left(\frac{\eta_{R}}{100}\right)^{2} \cdot\left(\left[\frac{\varepsilon_{M_{E}}}{M_{E}}\right]^{2}+\left[\frac{\varepsilon_{H_{E}}}{H_{E}}\right]^{2}+\left[\frac{\varepsilon_{M_{H}}}{M_{H}}\right]^{2}+\left[\frac{\varepsilon_{H_{H}}}{H_{H}}\right]^{2}\right)\right]^{\frac{1}{2}}
\end{aligned}
$$

The above perturbations were included in the data mining code presented earlier to generate static error values for the primary variables and dynamic error values for the derived variables. The error values were then linked to their prospective variables to generate a translucent patch of error bars, or error bands, around their profiles as was presented earlier in CHAPTER 7. 


\begin{abstract}
ABOUT THE AUTHOR
Mohammad Abutayeh is originally from Kafr Sur, Palestine: a small West Bank village approximately thirty miles north of Jerusalem. He came to America in search of a better life almost twenty years ago and has been calling it home since.

He received a Bachelor of Science in Chemical Engineering from the University of South Florida in 1997 with Cum Laude distinction. He continued on obtaining his Master of Science in Chemical Engineering from the University of South Florida in 1999 where he wrote a thesis on predicting the citrate soluble-loss of the dihydrate process.

He then worked in several engineering areas designing process control systems, optimizing unit operations, customizing process equipment, administering US patent laws, and many other functions. In addition, he successfully completed the Fundamentals of Engineering examination of the Florida Board of Professional Engineers, attended numerous seminars, and acquired several other certifications.

He published several journal articles and presented his thesis and dissertation research findings at national and international venues. He graduated with a Doctor of Philosophy in Chemical Engineering from the University of South Florida in 2010 where he wrote a dissertation on simulating the passive vacuum solar flash desalination.
\end{abstract}

